UNIVERSITY OF SZEGED

FACULTY OF SCIENCE AND INFORMATICS

DOCTORAL SCHOOL IN BIOLOGY

\title{
The Osteological Evidence of Neolithic Populations from the Southern Great Plain of Hungary
}

An Insight into the Potential of Macroscopic Observations for the Demographic and Pathological Analyses of Past Populations

\section{Muriel Masson}

Ph.D. Thesis

Co-supervisors:

Dr. György PÁLFI, Associate Professor, Head of Department

Dr. Erika MOLNÁR, Assistant Professor

Department of Biological Anthropology, University of Szeged

Volume 1: Thesis

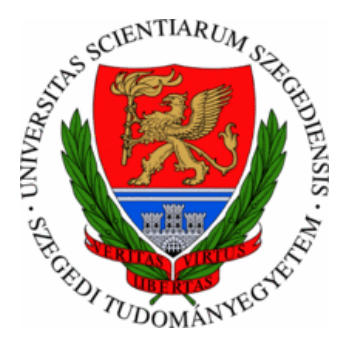

SZEGED 
Dedicated to the memory of Aonghas Staerck

A Hódmezővásárhely környékén élt újkőkori emberek emlékére

"The greatest tribute to the dead is not grief but gratitude." Thornton Wilder 


\section{Abstract}

This research aims to provide a unique contribution to our knowledge of the first farmers of Europe in general and the Neolithic populations from the Southern Great Plain of Hungary in particular, while demonstrating the potential of macroscopic observations for the demographic and pathological analyses of past populations.

A total of one hundred individuals from Hódmezővásárhely-Gorzsa and some neighbouring communities from Southern Hungary are analysed. The Early Neolithic sample comprises of twelve individuals from the Körös culture (5950 to 5400 BC), ten of whom are represented by their skull only. The Late Neolithic sample includes 86 individuals of the Tisza Culture ( 4970 to $4410 \mathrm{BC}$ ), with most of the remains excavated from the tell settlement of Hódmezővásárhely-Gorzsa (4970 to 4594 BC).

Current macroscopic methods for osteological analysis are critically reviewed, and several methods for ageing, sexing and stature estimation are compared. The results of the demographic analyses indicate that the Early Neolithic sample includes five female and seven male adults, without any juvenile. The Late Neolithic population is represented by a third of juveniles, 23 males and 31 females, nearly all the females coming from the site of Gorzsa. Stature estimations based on a third of the adults indicate an average height of $167 \mathrm{~cm}$ for males and $154 \mathrm{~cm}$ for females.

Ten juveniles show signs of pathology, including two cases of scurvy, four cases of infection, and six presenting non-specific stress indicators. Pathological analyses of the adult population reveal that mechanical changes (osteoarthritis, degenerative bony changes and musculoskeletal stress markers) are common in old adults and also in young females. Eleven individuals show non-specific stress indicators. Evidence of trauma is found in eleven individuals, presenting mainly as well-healed fractures and one case of spondylolysis. Ten individuals display signs of infection, including mastoiditis, chronic rhinitis, sacroiliitis, and four possible cases of meningitis of unknown aetiology. One case of tuberculosis with secondary hypertrophic osteoarthropathy and four further cases of tuberculosis are also confirmed. In addition, a potential case of leprosy and one of cancer are also discovered. Finally, dental disease including caries, calculus and tooth loss is widespread.

Despite the lack of contextual data, the damaged and fragmentary skeletal remains, and the restriction to morphological analyses, these early farmers are revealed as a mostly non-violent population, prone to infections including tuberculosis, with a physically stressful way of life and a diet leading to a high rate of dental disease and (at least occasionally) lack of vitamin C. 


\section{Table of Contents}

ABSTRACT

TABLE OF CONTENTS ii

LIST OF ILLUSTRATIONS $v i$

ACKNOWLEDGEMENTS $\quad$ ix

INTRODUCTION

1.1 Archaeological and Anthropological Background 1

1.1.1 Transition to farming - Early Neolithic Körös Culture 1

1.1.2 Increasingly complex societies - Late Neolithic Tisza Culture 2

1.1.3 Anthropological taxonomic evidence 3

1.2 Provenance of the osteological sample 4

1.2.1 Early Neolithic Sites of the Körös Culture 5

1.2.2 Late Neolithic Sites of the Tisza Culture 8

1.2.3 Unspecified Neolithic Sites 12

$\begin{array}{ll}1.3 \text { Research Aims, Objectives and Structure } & 13\end{array}$

PART I: PALAEODEMOGRAPHY 15

Chapter 2: Sexing Methods 16

2.1. Introduction 16

2.2. Juveniles $\quad 18$

$\begin{array}{ll}2.2 .1 \text { Skull } & 18\end{array}$

$\begin{array}{ll}2.2 .2 \text { Pelvis } & 25\end{array}$

2.2.3 Other methods 33

2.3. Adults $\quad 35$

2.3.1 Skull 36

2.3.2 Pelvis 46

2.3.3 Spine and Thorax $\quad 58$

2.3.4 Shoulders and Upper Limbs $\quad 61$

$\begin{array}{ll}\text { 2.3.5 Lower Limbs } & 70\end{array}$

$\begin{array}{ll}\text { 2.4. Conclusion } & 78\end{array}$

$\begin{array}{ll}\text { Chapter 3: Ageing Methods } & 81\end{array}$

3.1. Introduction $\quad 81$

3.2. Juveniles $\quad 83$

3.2.1 Dental Development $\quad 83$

$\begin{array}{lr}3.2 .2 \text { Bone dimensions } & 94\end{array}$

$\begin{array}{lr}3.2 .3 \text { Skeletal maturation } & 109\end{array}$ 
3.3. Adults 117

3.3.1 Skull 117

$\begin{array}{ll}3.3 .2 \text { Thorax } & 120\end{array}$

3.3.3 Spine 122

3.3.4 Pelvis 124

3.3.5 Dental Wear 132

3.3.6 Multiregional analyses 136

$\begin{array}{ll}\text { 3.4. Conclusion } & 139\end{array}$

Chapter 4: Stature Methods 143

4.1. Introduction 143

4.2. Estimating Juvenile Stature $\quad 143$

4.2.1. Fetal Stature 143

4.2.2. Postnatal Juvenile Stature 144

4.2.3. Growth studies $\quad 150$

4.3. Estimating Adult Stature $\quad 156$

4.3.1 First Studies 156

4.3.2 Post Second World War 156

4.3.3 Trotter and Gleser's method $\quad 157$

4.3.4 Sjøvold's method 162

4.3.5 Anatomical Stature 163

4.3.6 Comparison of long bone formulae and anatomic methods 166

$\begin{array}{ll}\text { 4.3.7 Other techniques } & 167\end{array}$

$\begin{array}{ll}\text { 4.4.Conclusion } & 170\end{array}$

Chapter 5: Osteological analysis - Palaeodemographic results 174

5.1. Introduction 174

5.2.Juveniles 174

5.2.1. Age estimates 174

5.2.2. Sex estimates $\quad 180$

5.2.3. Stature estimates 182

5.3. Adults 185

5.3.1 Sex-Morphological estimations 185

5.3.2 Sex-Osteometric estimations 188

5.3.3 Age estimates 201

5.3.4 Stature estimates 205

5.4.Conclusion $\quad 215$

\section{PART II: PALAEOPATHOLOGY 218}

Chapter 6: Palaeopathology - methodology and pathologies 219

6.1. Introduction $\quad 219$

6.2. Methodology 219 
6.3. Skeletal pathologies $\quad 220$

6.3.1 Trauma 221

6.3.2 Metabolic diseases $\quad 222$

6.3.3 Inflammatory/Immune / Infectious diseases 223

6.3.4 Mechanical changes 226

6.3.5 Anomalies / anatomic variations $\quad 228$

6.3.6 Non-Specific Stress Indicators $\quad 228$

6.4. Oral pathologies $\quad 229$

6.5.Conclusion 230

Chapter 7: Osteological analysis - Palaeopathological results 231

7.1. Introduction 231

7.2. Palaeopathology in the Early Neolithic $\quad 232$

7.2.1. Skeletal Pathologies $\quad 232$

$\begin{array}{ll}\text { 7.2.2. Oral Pathology } & 232\end{array}$

7.3. Palaeopathology among individuals from unspecified Neolithic sites 233

7.4. Palaeopathology in the Late Neolithic: Juveniles 234

7.4.1. Neonates 235

$\begin{array}{ll}\text { 7.4.2. Infants } & 237\end{array}$

$\begin{array}{ll}\text { 7.4.3. Children } & 238\end{array}$

7.4.4. Adolescents 240

7.5. Palaeopathology in the Late Neolithic: Adults 242

$\begin{array}{ll}\text { 7.5.1. Trauma } & 242\end{array}$

7.5.2. Non-specific Pathologies $\quad 245$

7.5.3. Specific Infections $\quad 247$

7.5.4. Multiple Pathology case study 259

7.5.5. Other Skeletal Pathologies 263

7.5.6. Oral Pathologies $\quad 266$

7.6. Significance for the palaeopathological record 268

7.6.1. Hypertrophic Pulmonary Osteopathy (HPO) / Hypertrophic Osteoarthropathy (HOA) 268

$\begin{array}{ll}\text { 7.6.2. Tuberculosis (TB) } & 269\end{array}$

$\begin{array}{ll}\text { 7.6.3. Scurvy } & 270\end{array}$

$\begin{array}{ll}\text { 7.6.4. Leprosy } & 272\end{array}$

$\begin{array}{ll}\text { 7.6.5. Cancer } & 273\end{array}$

$\begin{array}{ll}\text { 7.6.6. Mastoiditis } & 273\end{array}$

7.6.7. Spondylolysis 274

$\begin{array}{ll}\text { 7.7.Conclusion } & 275\end{array}$

DISCUSSION AND CONCLUSION 282

8.1. Palaeodemographic and palaeopathological analyses based on
macroscopic observations

8.1.1. Juvenile demographic characteristics 282 
8.1.2. Adult demographic characteristics

8.2. Early farmers of Europe

8.3. Areas for future research

8.4. Conclusion

APPENDICES in second volume 


\section{List of Illustrations}

Figures

1.1: Location of the sites 4

1.2: Multiple plots of recalibrated radiocarbon dates for Körös sites $\ldots$

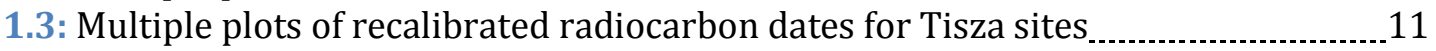

3.1: Timings of epiphyseal union

5.1: Juvenile growth in Late Neolithic Tisza populations

5.2: Stature estimates for Late Neolithic juveniles $\ldots$

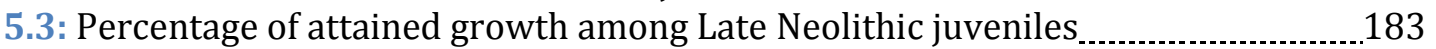

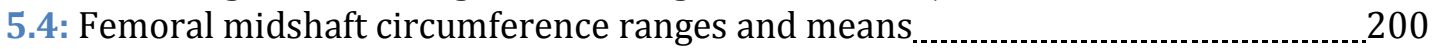

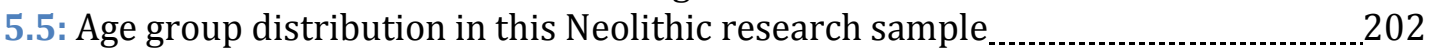

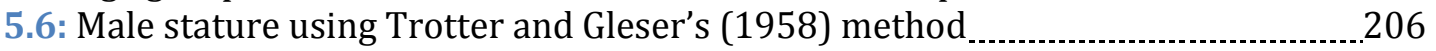

5.7: Male stature using Sjøvold's (1958) method $\ldots$

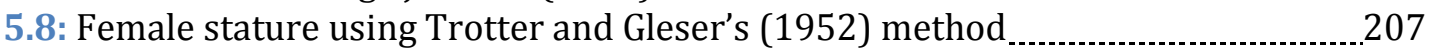

5.9: Female stature using Sjøvold's (1958) method _........................................ 207

5.10: Height estimates for males from the Tisza Culture using two methods ................ 210

5.11: Height estimates for females from the Tisza Culture using two methods ........... 210

5.12: Male stature estimates from average humerus length of the Tisza culture _....... 211

5.13: Male stature estimates from average femur length of the Tisza culture _............ 211

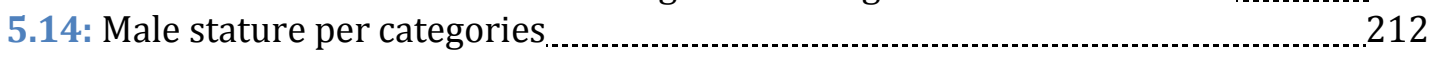

5.15: Female stature per categories $\ldots$

5.16: Comparison of stature estimates

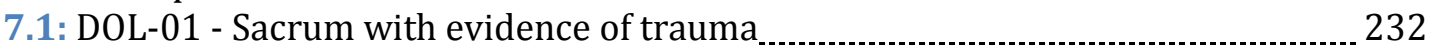

7.2: HKO-07 - Mandible with caries, alveolar resorption, abscesses, periodontal disease

and antemortem tooth loss $\ldots$

7.3: HKJ-01 - Unique tooth wear, probably activity-related

7.4: Skeletal remains of HGO-55

7.5: Signs of probable scurvy on HGO-55 236

7.6: Signs of probable scurvy on HGO-43

7.7: HGO-57 - Skeletal remains

7.8: Periostitis with woven bone formation on humeral shaft of HGO-57 ..................... 238

7.9: Signs of severe infection on HGO-57

7.10: Signs of infection on the skull of HGO-06

7.11: Developmental anomaly of the enamel of HGO-06 permanent teeth _................ 239

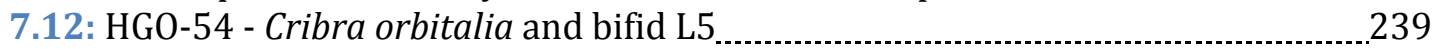

7.13: Pronounced cribra orbitalia on HGO-50 _ 240

7.14: Signs of strenuous physical activities on HKK-04 _............................ 241

7.15: HGO-26 - Porotic palate and anomalous fovea capitis __......................... 241

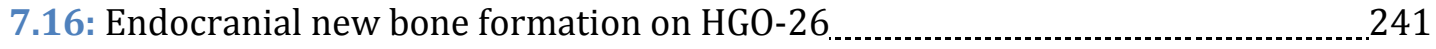

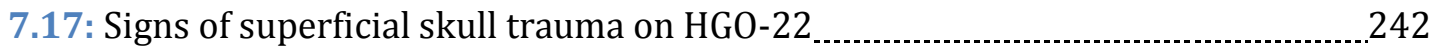

7.18: Skull of HGO-41

7.19: HGO-10 - Right MC1 with avulsion fracture $\ldots \ldots \ldots$

7.20: Pathological left ankle of HGO-12 
7.21: Avulsion fracture on the right talus of HGO-56

7.23: Traces of probable mastoiditis observed on HGO-44

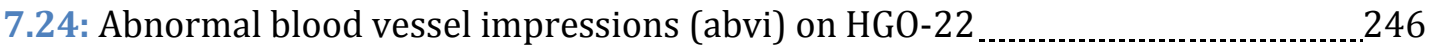

7.25: HGO-23 - abvi $\ldots$

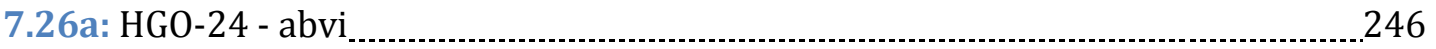

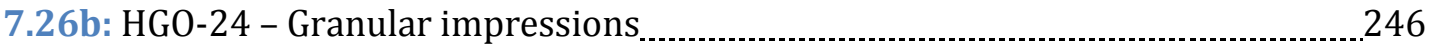

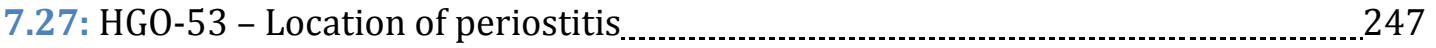

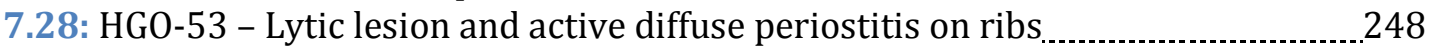

7.29: HGO-53 - Cavitations on two fragments of vertebral bodies _........................ 248

7.30a: HGO-53 - Periostitis on distal end of humerus _...................................... 249

7.30b: HGO-53 - Traces of widespread woven bone formation on ulna _................... 249

7.31a: HGO-53 - Periostitis on femur 250

7.31b: HGO-53 - Periostitis on tibia

7.32: HGO-53 - Active diffuse woven bone formation on fibula $\ldots \ldots \ldots \ldots . . . . . . . . . . . . . . . . . . .250$

7.33: HGO-53 - Cavitation on calcaneus $\ldots$

7.34: Results from DNA analyses on HGO-53

7.35: HGO-53 - Mycocerosate profile

7.36: HGO-08 - Resorptive lesions on thoracic and lumbar vertebrae $\ldots$

7.37: HGO-08 - Mycocerosate profile

7.38: HGO-10 - Hypervascularisation of thoracic vertebrae

7.39: HGO-10 - Mycocerosate profile

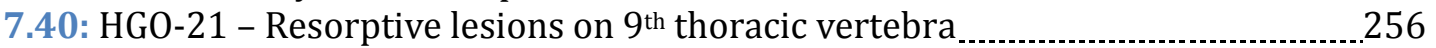

7.41: HGO-21 - Periostitis on two rib fragments $\ldots$

7.42: HGO-21 - Mycocerosate profile

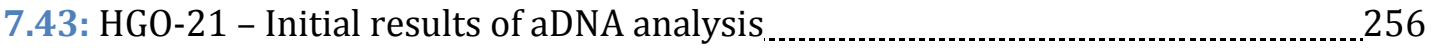

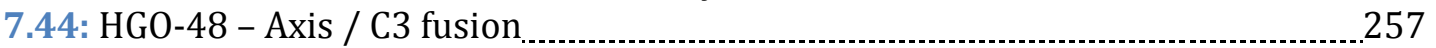

7.45: HGO-48 - Abnormal blood vessel impression in SES-like pattern _................... 257

7.46: HGO-48 - Mycocerosate profile

7.47: HGO-48 - Initial results of aDNA analysis $\ldots \ldots \ldots$

7.48: Mycolic acid profiles of all samples $\ldots$

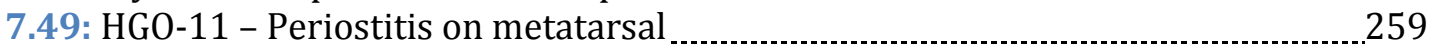

7.50: HGO-11 - Severe widespread active woven bone formation _._. _ 259

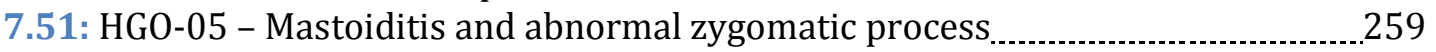

7.52: HGO-05 - Abnormal mastoid process with perforated tip $\ldots \ldots \ldots \ldots \ldots \ldots$

7.53: HGO-05 - Missing zygomatic process

7.54: HGO-05 - Left side of skull with missing zygomatic and facial asymmetry..........261

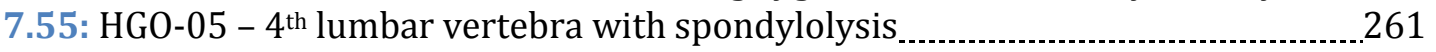

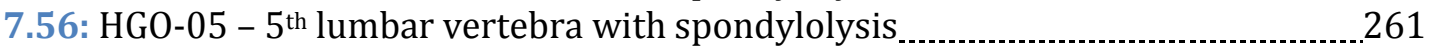

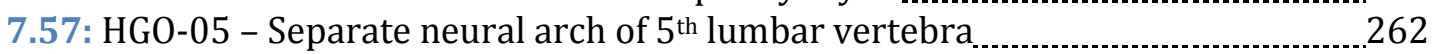

7.58: Advance OA with lipping on a vertebra

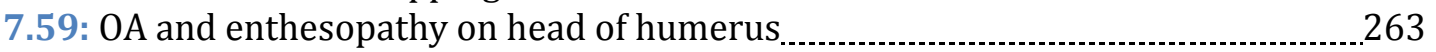

7.60: HGO-28 - Button osteoma

7.61: BKP-01 - Spina Bifida Occulta

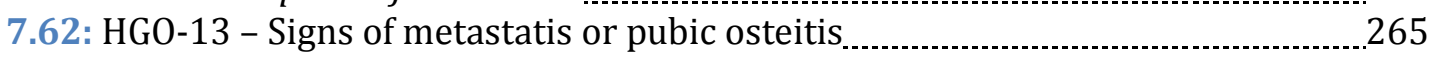

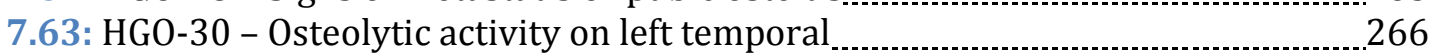

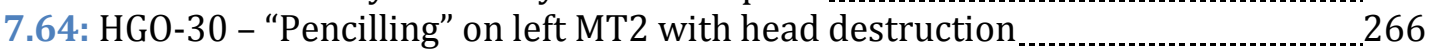

7.65: Severe carious lesions and alveolar resorption $\ldots$

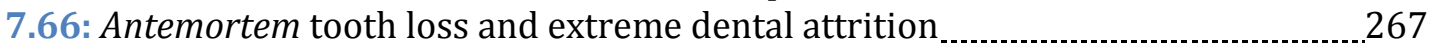


7.67: HGO-34 - Activity-related pattern of tooth wear

\section{Tables}

1.1: Radiocarbon dates for Körös sites

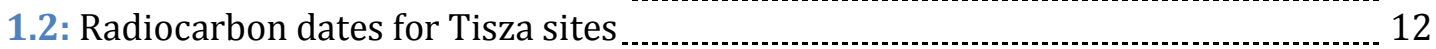

2.1: Skull sexual traits

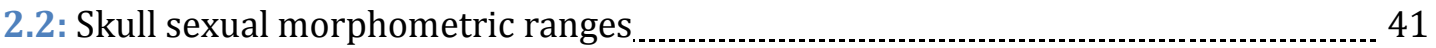

2.3: Pelvis sexual traits

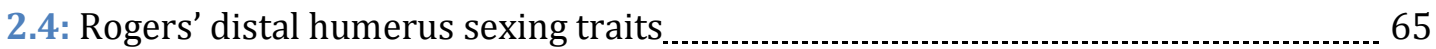

3.1: Stages of tooth mineralisation $\ldots$

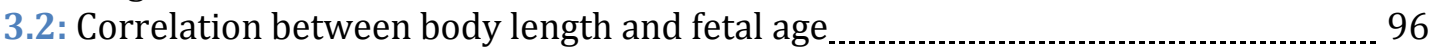

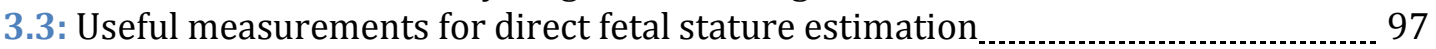

3.4: Comparison of the samples used by different methods for age estimation from diaphyseal bone length

3.5: Comparison of age estimation from diaphyseal length of the clavicle _................108

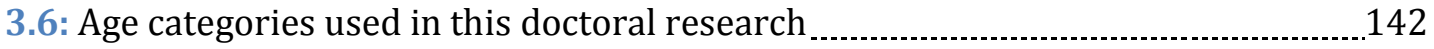

4.1: Regression equations for stature estimation for 3-10 year-old children _.............147

4.2: Summary table of samples utilised in Feldesman's 1972 study _....................... 149

4.3: Regression equations from single bones $\ldots$

4.4: Comparison of stature estimations from different authors $\ldots \ldots \ldots \ldots$

4.5: Revised regression equations from single bones $\ldots \ldots \ldots$

4.6: Weighted line of organic correlation formulae $\ldots \ldots \ldots$

5.1: Dental and skeletal age estimates of Late Neolithic infants _.......................... 175

5.2: Infants and children age estimates from dentition, skeletal maturation and

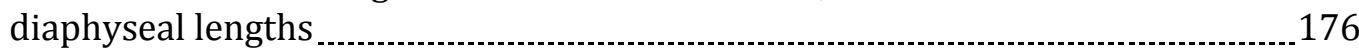

5.3: Ageing from diaphyseal measurements in Late Neolithic Tisza populations _.................... 178

5.4: Age estimates from partes basilaris of infantile occipital bone _......................... 179

5.5: Sex estimates from juveniles

5.6: Comparison of percentages of attained growth in European populations …............. 184

5.7: Baseline dataset for osteometric sex estimates of Tisza populations …................... 198

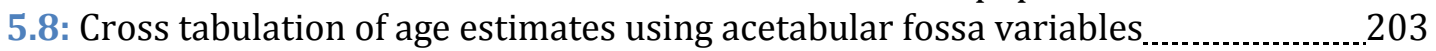

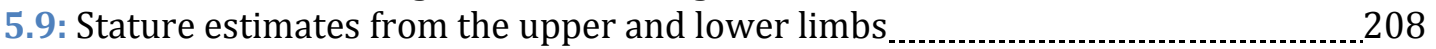

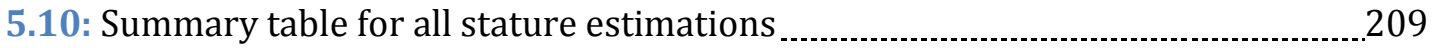

5.11: Mean stature estimates for three Tisza Culture sites ........................................213

5.12: Comparison of mean male stature between prehistoric and historic samples .... 215

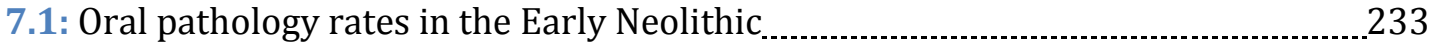

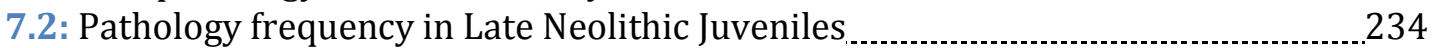

7.3: Oral pathology comparison between Early and Late Neolithic adults ..................2276 


\section{Acknowledgements}

I would like to thank Dr. Erika Molnár and Dr. György Pálfi for their supervision; Dr. Ferenc Horváth and Dr. Antónia Marcsik for access to the bones; Dr. Helen D. Donoghue, Centre for Clinical Microbiology and Centre for the History of Medicine, UCL, UK, for the DNA analyses; Prof. David E. Minnikin and Dr. Oona Lee, School of Biosciences, University of Birmingham, UK, for the lipid biomarkers analyses; Dr. Kocsis Gábor for verifying my dental pathology cases; the librarian of the department of Forensic Medicine at the University of Szeged for access to the library; everyone at the department of Biological Anthropology of the University of Szeged; members of the British Association for Biological Anthropology and Osteoarchaeology and members of the Paleopathology Association for their support and good advice throughout the years; Dr. Zsolt Bereczki, Dr. Magdalena Midgley and Dr. Tina Jakob for their continuous encouragements; and my family for their support.

I thank all my colleagues in the division of Governance and Quality Enhancement, Queen Margaret University, Edinburgh, for their encouragements over the last couple of years, especially in the last few months. I am particularly grateful for the amazing support of Hilary Glendinning, a great friend, fantastic colleague, and the best line manager one could ever wish for. I am also indebted to Prof. Alan Gilloran for his kind help and advice.

This PhD would not have been possible without the love and understanding of all my friends during these long years, including (but not limited to and in no particular order) Clionadh McGarry, Charles Burgoyne, Annie Scanlon, Alison Lynas, Lucy Clapson, Virag Hamori, France Dubanchet, Sandra Peddie, Dr. Sarah Mothulatshipi and her children Lere and Arabang, Alex Staerck and his children Oonagh and Aonghas, Brent Philion, Rose Drew, Ian Miller, John Grant, Robert Warman, Ili and Misi Kiss, Erika Molnár, Zsolt Bereczki, Gabriella Kajdocsi Lovász, Brigitta Ösz, László Paja, Tamás Herman, Norman Lockhart, Virginie Kastler, Nathalie and Joran De Zorzi, Ronald Scholten and my "nephews" Jayden and Tycho, Mike Kimber, and of course my wonderful odd daughter Amber Kimber. I owe them all a great deal and hope they know how thankful I am.

Finally, I would like to express my deepest gratitude to my friends Madeleine Stone and Ingeborg Scholten, and my neighbours Ann and James McCrae, who have always been there for me and without whom life would have been incredibly more difficult. I feel very privileged to have such wonderful and generous friends. 


\section{INTRODUCTION}

This research started in 2003 following on from the author's undergraduate dissertation on the human osteological evidence for the transition from huntinggathering to farming in Europe (Masson 2002). While searching for suitable samples in a smaller geographical area for a more in-depth analysis, access was offered to the osteological collections held at the Department of Biological Anthropology, University of Szeged, Hungary. Hungary is the ideal location to study the transition to farming with hunter-gatherers and agriculturalists cohabiting for a prolonged period at very close proximity (Tálas 1996). Unfortunately, the scarcity of human remains from the Mesolithic meant that the focus of this research had to be redirected to Early and Late Neolithic communities. Skeletal material from both the Körös (Early Neolithic) and the Tisza (Late Neolithic) cultures were then provided, constituting an exceptional sample of 100 individuals from the Southern Great Plain of Hungary.

\subsection{Archaeological and Anthropological Background}

\subsubsection{Transition to farming - Early Neolithic Körös Culture}

The debate on the origins of agriculture among archaeologists has been ongoing "for most of the discipline's history" (Barker 2006:382). In Europe, two main possibilities have been considered: the movement of people originally from the Levant bringing a new culture with them, or the adoption of this culture and way of life by indigenous Mesolithic populations following contacts with these early agrarian societies (also referred to as the diffusionist vs indigenist model by Makkay 1996; or as the colonisation vs acculturation hypothesis by Whittle 1996). In Hungary, the halt in the first wave of neolithisation with the Körös Culture in the Carpathian basin for several centuries before its spread north- 
and westwards, together with the recent Mesolithic archaeological evidence from this area has led to the conclusion that the local indigenous populations had resisted the adoption of this new culture (Kertész 1996; Makkay 1996; Kertész \& Sümegi 2001). It is unknown if the uncovered sites were occupied occasionally or on a permanent basis. The Körös Culture marked the arrival of the first domestic animals in the Carpathian basin, mostly with sheep and goat although cattle and pig also played a smaller role. These were parts of a complex economy involving cereal cultivation, animal husbandry, fishing, hunting and gathering (Paluch \& Tóth 2005, Bonsall et al. 2007). Insistence on concentrating their livestock on sheep and goats of southeastern origins not well suited to the local climate has been highlighted as probable evidence that this population had newly immigrated to the area (Bartosiewicz 2005). Körös individuals were buried in simple individual graves on the edges of settlements (Whittle 2003).

\subsubsection{Increasingly complex societies - Late Neolithic Tisza Culture}

It is thought that after the colonisation of the Southern Great Plain by the Körös population in the Early Neolithic, indigenous Mesolithic hunter-gatherer groups adopted this new way of life in the Middle Neolithic as demonstrated by the Alföld Linear Pottery (AVK) Culture (Paluch \& Tóth 2005). A complex system of tell settlements and smaller farmsteads followed with the Tisza Culture of the Late Neolithic (Kalicz \& Raczky 1987). In complete opposition to the Körös Culture, the Tisza population utilised their local resources with an intensive exploitation of wild animals, such as the large aurochs, reflecting the "culturally different attitude to animals and the wild in general" (Bartosiewicz 2005:61). Tisza individuals were buried within the settlement, and could be found between or under houses (Whittle 2003).

The tell-settlement of Hódmezővásárhely-Gorzsa spread over 5 hectares and accumulated a $180-200 \mathrm{~cm}$ thick layer during the Tisza Culture topped by subsequent layers from the following periods of occupation up to the Middle 
Ages. The site included a large, burnt, six-room house, the largest building from this period in Hungary. Tisza burials at this site were reported as small family grave groups in temporarily unoccupied parts of the settlements (Horváth 2005c), with an earlier publication mentioning serological analyses indicating that these represented "four successive generations of one genetic unit" (Horváth 1987b). An extensive range of contacts with surrounding cultures was observed in the lithic raw materials and the use of flax in textiles, while a rudimentary form of writing was also noted on finds (Horváth 2005c). The nearby site of Hódmezővásárhely-Kökénydomb was a less densely inhabited "tell-like settlement within a single-layer settlement" (Kalicz \& Raczky 1987). Horváth (2005c) described the shift to a pastoral economy as the cause for the disintegration of these large villages during the subsequent Copper Age with a "breakdown of the earlier socio-economic system" which may have otherwise led to urbanisation as seen in the Near East.

\subsubsection{Anthropological taxonomic evidence}

Since the 1980s, distance analyses have been utilised to find anthropological evidence of the transition from hunting-gathering to farming, particularly trying to establish if colonisation or acculturation had taken place. In a pan-European analysis of 286 Mesolithic individuals (including 62 from the Iron Gates on the Danube River between Romania and Serbia) and 280 Early and Middle Neolithic individuals (twenty from the Iron Gates), a decrease in robusticity with the first farmers was established, together with the cohabitation of two distinctive morphological types in the Mesolithic-Neolithic transition (Menk \& Nemeskéri 1989). This was understood to prove the coexistence of local and newly arrived populations for an extended period, with the Neolithic populations eventually (and only progressively) replacing the original Mesolithic locals.

Such gracilisation was also demonstrated in Early and Middle Neolithic Hungary in a similar study (Zoffmann 1994), albeit on populations from the Neolithic 
onwards only as Mesolithic skeletal remains have yet to be uncovered in this area. Results of this and several other Penrose distance analyses showed a continuity in the indigenous populations of the Carpathian Basin throughout Prehistory (Zoffmann 1980, 1984, 1992, 2000). The most recent analyses indicated that the Early Neolithic Körös populations originated from the South and the South-East, which would support the theory that early farmers had colonised the area, while the AVK Middle Neolithic populations of the Northern Great Plain had stronger links with North-Eastern Europe, which would confirm the later adoption of a Neolithic way of life by indigenous late hunter-gatherers (Zoffmann 2005). The Late Neolithic Tisza population appeared to be strongly linked genetically to the AVK Middle Neolithic population, although also introducing a new anthropological variant with a broader face not seen in the region previously.

\subsection{Provenance of the osteological sample}

A sample of 100 individuals from the Southern Great Plain of Hungary close to the Romanian Border was gathered for macroscopic analysis, mostly from the small geographical area between Szeged and Hódmezővásárhely, with a few individuals close to Békés slightly further North-East (see fig 1.1 below).

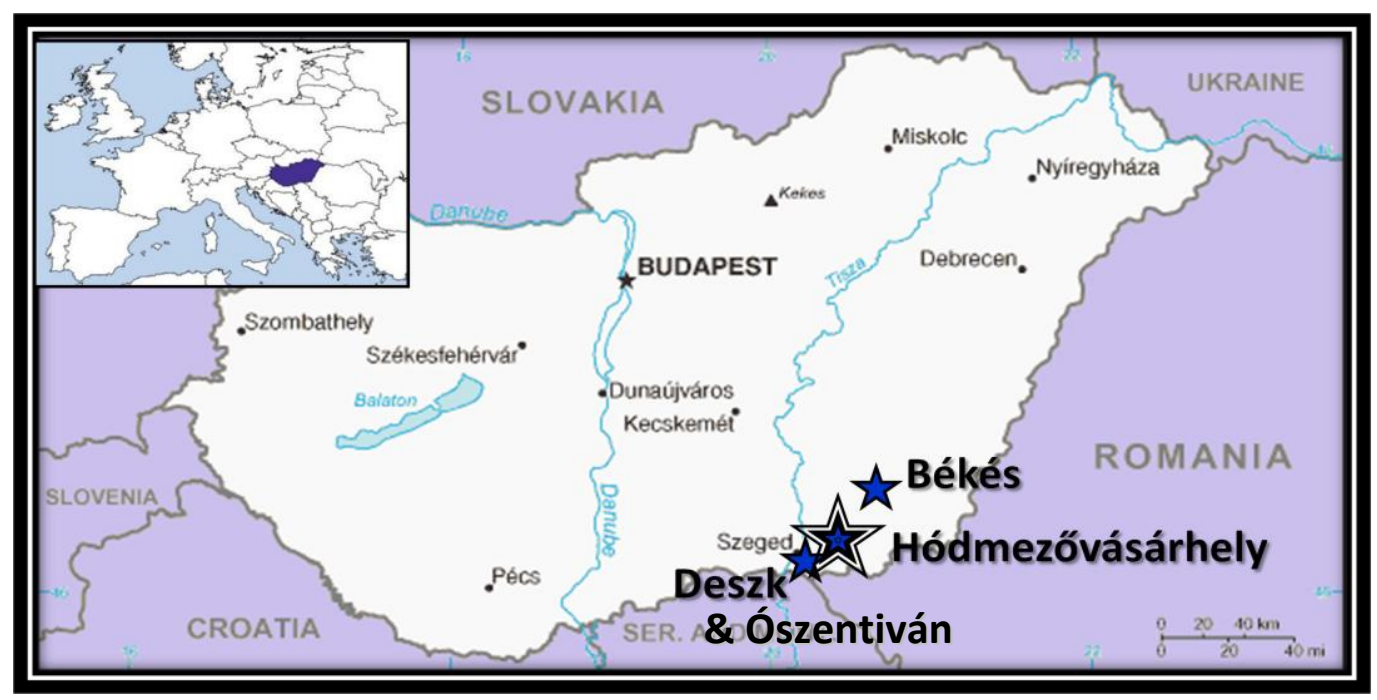

Figure 1.1: Location of the sites 


\subsubsection{Early Neolithic Sites of the Körös Culture}

\section{Deszk - Olajkút}

During rescue excavations in 1966, two skeletons belonging to the Körös culture were recovered by Trogmayer (1969). Lipták (1975) and Farkas $(1975,1977)$ analysed the remains concentrating mainly on metrics, morphology and taxonomy. Their data was later used by Szathmáry (1987) looking at human genetics and Zoffman (1992) for taxonomy using Penrose distance analyses. Both skeletons were examined for this doctoral research. The skull of one of the individuals (DOL-01) has since been used in a facial reconstruction (Kustár \& Árpás 2005).

\section{Hódmezővásárhely - Bodzáspart}

This site was excavated between 1937 and 1939 by Banner (1939, 1940a, 1940c), revealing four graves, one of them from the Körös culture. Nine new graves were found in 1948 and a further two in 1952 (Banner 1954). The Körös individual was analysed by Farkas $(1975,1977)$ and Zoffman $(1992)$ mostly for metric, morphological and taxonomic purposes. These remains were available for this study.

\section{Hódmezővásárhely - Kotac-part}

In 1932, Banner recovered six Neolithic graves from this site, followed by another sixteen in 1933 including remains from the Copper Age and Bronze Age, and eight more graves attributed to "Tisza III" in 1934 (Banner 1935, 1940a). Banner (1957) later re-identified these last eight graves as part of the Körös culture. Farkas $(1975,1977)$ analysed the remains of ten graves which were then used by Zoffman (1992) for Penrose distance analyses and Tóth (1987) to study nutrition based on weight estimation. The remains of eight of these graves were available for this doctoral research. 


\section{Hódmezővásárhely -Kovács-tanya}

The remains of one individual was found at this site by Banner (1940a) and analysed by Farkas (1977). Another Körös individual was found by Gazdapusztai (1957) and described briefly by Farkas (2005). Due to confusion in the recording system and lack of clarity in the archaeological data available, it is unclear which of these two skeletons was examined in this study.

\section{- Radiocarbon dates}

Hertelendi and colleagues (1998a; 1998b) dated the span of the Körös culture from 5950 to 5400 cal. BC (with a 68.3\% confidence interval), and Horváth (2003b) proposed dates of 5860 to 5310 cal. BC for the Early Neolithic. More specifically for the sites studied in this doctoral research, Horváth and Hertelendi (1994) placed Hódmezővásárhely-Kotac-part in the third of their eight periods dividing the Körös culture, dated 5500-5400 BC, with DeszkOlajkút in the third and fourth phases (5500-5300 BC) and HódmezővásárhelyKovács-tanya in the fourth phase (5400-5300 BC).

Uncalibrated radiocarbon dates have been published for the sites of DeszkOlajkút (one date in Ammerman \& Cavalli-Sforza 1971, labelled DOL14C-AC in the calibrations by the author; five dates in Horváth \& Hertelendi 1994, labelled DOL14C-HH1 to HH5) and Hódmezővásárhely-Kotac-part (one date in Ammerman \& Cavalli-Sforza 1971; one date in Horváth \& Hertelendi 1994). The author has recalibrated all these dates using the calibration curve IntCal04 for Northern Hemisphere (Reimer et al. 2004) in the dating programme OxCal 4.1 (Bronk Ramsey 2009). Results can be seen in Table 1.1 and Figure 1.2 below.

Pinhasi and Pluciennik (2004) also provided a single date of $6400 \mathrm{bp}$ based on 19 carbon dates from Deszk-Olajkút, Hódmezővásárhely-Kotac-part, VésztőMágor and Endrőd. In the author's calibrations using the methods described above, a 95\% confidence interval offers a range of 5466 to $5325 \mathrm{BC}$ for this date. 


\begin{tabular}{|c|c|c|c|c|}
\hline Labels & References & $\begin{array}{c}\text { Original } \\
\text { uncalibrated } \\
\text { date bp }\end{array}$ & $\begin{array}{l}\text { Calibrated range } \\
\text { using IntCal04 } \\
(68.2 \% \mathrm{Cl})\end{array}$ & $\begin{array}{c}\text { Calibrated range } \\
\text { using IntCal04 } \\
(95.4 \% \mathrm{Cl})\end{array}$ \\
\hline \multicolumn{5}{|c|}{ Deszk - Olajkút } \\
\hline DOL14C-AC & $\begin{array}{c}\text { Ammerman \& } \\
\text { Cavalli-Sforza } 1971\end{array}$ & 6572 & $5525-5490$ BC & $5540-5483$ BC \\
\hline DOL14C-HH1 & $\begin{array}{c}\text { Horváth \& Hertelendi } \\
1994\end{array}$ & $6605 \pm 100$ & $5623-5481$ BC & $5710-5374$ BC \\
\hline DOL14C-HH2 & $\begin{array}{l}\text { Horváth \& Hertelendi } \\
1994\end{array}$ & $6540 \pm 100$ & $5615-5381$ BC & $5643-5316$ BC \\
\hline DOL14C-HH3 & $\begin{array}{l}\text { Horváth \& Hertelendi } \\
1994\end{array}$ & $6410 \pm 120$ & $5486-5229$ BC & $5616-5074$ BC \\
\hline DOL14C-HH4 & $\begin{array}{l}\text { Horváth \& Hertelendi } \\
1994\end{array}$ & $6390 \pm 100$ & $5477-5299$ BC & $5551-5078$ BC \\
\hline DOL14C-HH5 & $\begin{array}{c}\text { Horváth \& Hertelendi } \\
1994\end{array}$ & $6260 \pm 100$ & $5326-5061$ BC & $5470-4993$ BC \\
\hline \multicolumn{5}{|c|}{ Hódmezővásárhely - Kotac-part } \\
\hline HK014C-AC & $\begin{array}{c}\text { Ammerman \& } \\
\text { Cavalli-Sforza } 1971 \\
\end{array}$ & 6450 & $5473-5383$ BC & $5475-5377$ BC \\
\hline НК014С-НH & $\begin{array}{c}\text { Horváth \& Hertelendi } \\
1994\end{array}$ & $6450 \pm 100$ & $5489-5319$ BC & $5613-5222$ BC \\
\hline
\end{tabular}

Table 1.1: Radiocarbon dates for Körös sites

(recalibration by the author from original data as referenced)

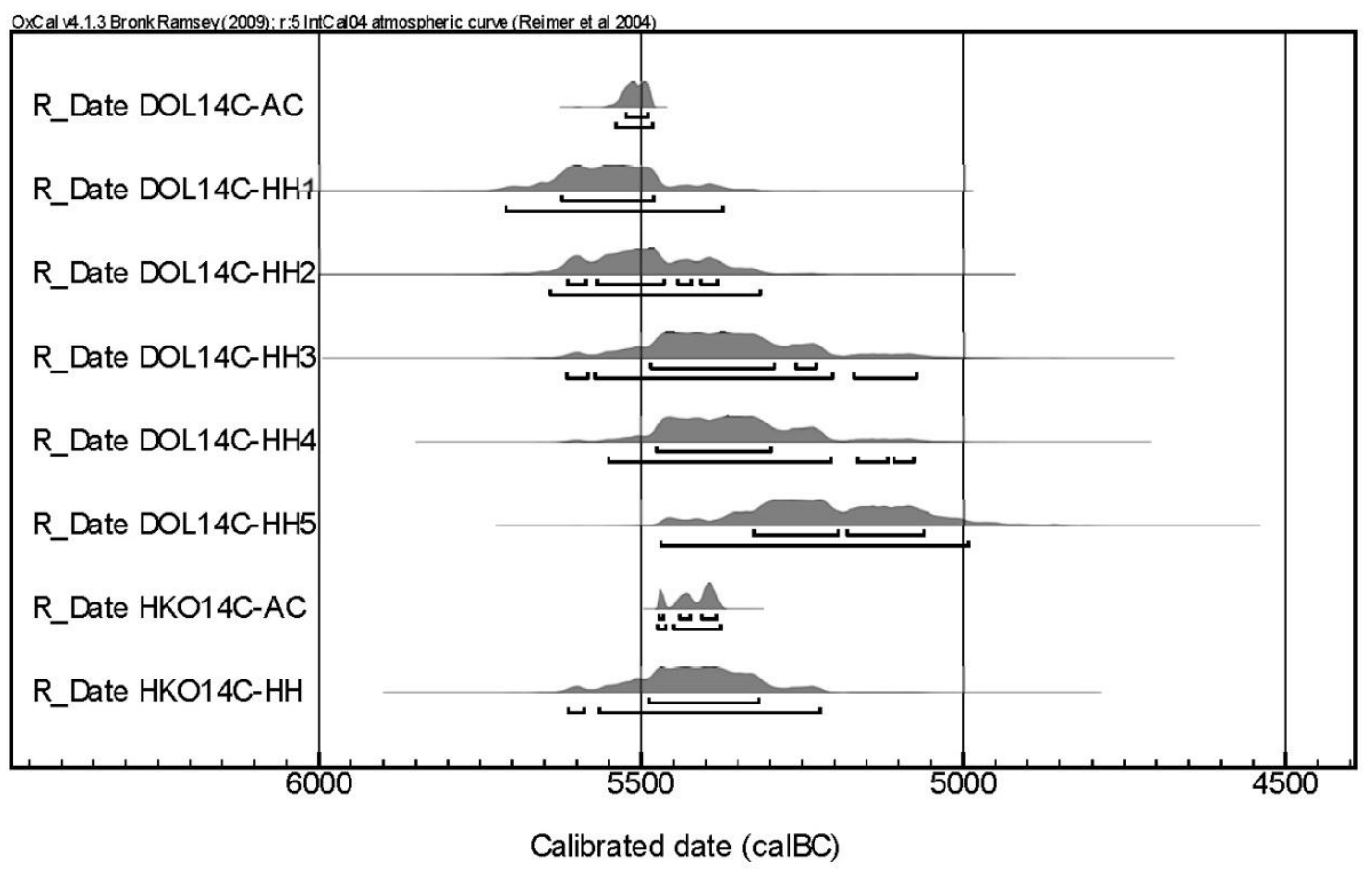

Figure 1.2: Multiple plots of recalibrated radiocarbon dates for Körös sites (by the author, see text above for references and methodology) 


\subsubsection{Late Neolithic Sites of the Tisza Culture}

\section{Békés - Povádzug}

The site was originally investigated by Banner (1930b, 1940c) in the first half of the twentieth century, with indications of Körös, Tisza and more recent cultures. Excavations in 1958 by Trogmayer $(1959,1962)$ revealed seven "prehistoric" graves, first thought to belong to the Körös culture but associated fifteen years later with the Tisza culture. Of these seven graves, only six could be analysed due to the damaged condition of the remains (Lipták \& Farkas 1967; Farkas 1975, 1977), mostly looking at the metric data for morphology and taxonomy. Five of these individuals were available for the author's own examinations.

\section{( Deszk - Ordos}

One skeleton, available to study for this research, was found at this site during the seventies (Horváth 1976), later analysed by Farkas \& Marcsik (1988) and utilised by Zoffman (1992) for her Penrose distance analyses.

\section{Hódmezővásárhely - Kökénydomb - Szabó-tanya}

Large scale excavations were undertaken by Banner with five campaigns from 1928 to 1944 (Banner 1930a, 1931, 1940b, 1942; Banner \& Foltiny 1945; Banner \& Korek 1949; Banner 1951) at this stratified site with multi-layered occupation. Nineteen graves from the Tisza culture, as well as graves from other time periods, were recovered over the two decades. The remains of two individuals were described in Farkas (1975) and later utilised by Zoffman (1992), four in total were made available for this doctoral research.

\section{Hódmezővásárhely - Kökénydomb}

During the eighties, further excavations by Horváth of the site described above revealed three more graves with four individuals. They were presented by 
Farkas and Szalai (1992) with emphasis on metrics and morphology, and all four were analysed in this study.

\section{Hódmezővásárhely - Kökénydomb - Vörös -tanya}

The remains of one individual was available for this research, excavated by Banner (1940a) and analysed first by Farkas (1975), then utilised by Zoffman (1992) for distance analyses.

\section{Hódmezővásárhely - Gorzsa}

The tell settlement of Hódmezővásárhely-Gorzsa, located in the Tisza-Maros angle $25 \mathrm{~km}$ North East of Szeged and 15km South West of Hódmezővásárhely, provided the majority of the sample under study. This site on a natural elevation was surrounded by streams and marshes during the Neolithic. Gorzsa was occupied through six settlement phases starting from the Early Tisza culture. Only two percent of the site has been investigated so far.

The site was first surveyed by Zalotay in 1953 and investigated by Gazdapusztai between 1955 and 1963 (Gazdapusztai 1963), with fourteen graves excavated in 1956-57. Some of these individuals were examined by Farkas (1975) but were unavailable for this doctoral research.

Systematic excavations were then undertaken by Horváth between 1978 and 1996 (Horváth 1982, 1987a, 1987b, 2003a, 2005b, 2005a). Seventy-one individuals were recovered in total, including 56 who had been buried in graves within the settlement and the partial remains of a further possible fifteen recovered from pits, ditches, houses and as stray finds. All were analysed in this study. Only the remains available from 25 graves uncovered during the 19781982 period have been published so far (Farkas \& Marcsik 1988; Kocsis 1988), corresponding to the new inventory numbers HGO-01 to HGO-20, minus HGO03 and HGO-14. Unfortunately, there are no published maps of the site, and 
there is no information currently available on the location of the graves and other remains in relation to the settlement and to each other.

\section{Radiocarbon dates}

Both Hódmezővásárhely-Kökénydomb and Hódmezővásárhely-Gorzsa provided numerous dates from their several layers of occupations. Gorzsa is now recognised as having been occupied for a time span of around three hundred years during the first half of the fifth millennium BC dated at 4970 to $4594 \mathrm{BC}$ (Hertelendi \& Horváth 1992; Horváth 2005a, 2005c) or between 4850 and 4550 cal BC (Hertelendi et al. 1998a; Hertelendi et al. 1998b) based on twenty samples from the site with a $68.3 \%$ confidence interval. Kökénydomb occupation during the Tisza culture is thought to have started around two hundred years earlier than Gorzsa, with a published span of 6200 to $5800 \mathrm{bp}$ (Hertelendi \& Horváth 1992). A radiocarbon date from Deszk-Ordos is also available around 5600 bp (Kalicz \& Raczky 1987; Hertelendi \& Horváth 1992).

The author has recalibrated dates available for HódmezővásárhelyKökénydomb (coded HKK14C), Hódmezővásárhely-Gorzsa (coded HG014C) and Deszk Ordos (coded DOR14C) from Kalicz and Raczky (1987, coded HR), and Hertelendi and Horváth (1992, coded HH) using the calibration curve IntCal04 for Northern Hemisphere (Reimer et al. 2004) in the dating programme OxCal 4.1 (Bronk Ramsey 2009). Results can be seen in Figure 1.3 and Table 1.2 below.

Overall ranges for the Tisza culture (Hertelendi et al. 1998a; 1998b) and the Late Neolithic of Hungary (Horváth 2003b) have been offered at 4970-4490 BC and 4970-4380 BC respectively. In their latest publication using new recalibrations, Yerkes and colleagues (2009) utilised 107 Late Neolithic samples to produce a range of dates from 5021 to $4402 \mathrm{BC}$ for the whole period. 


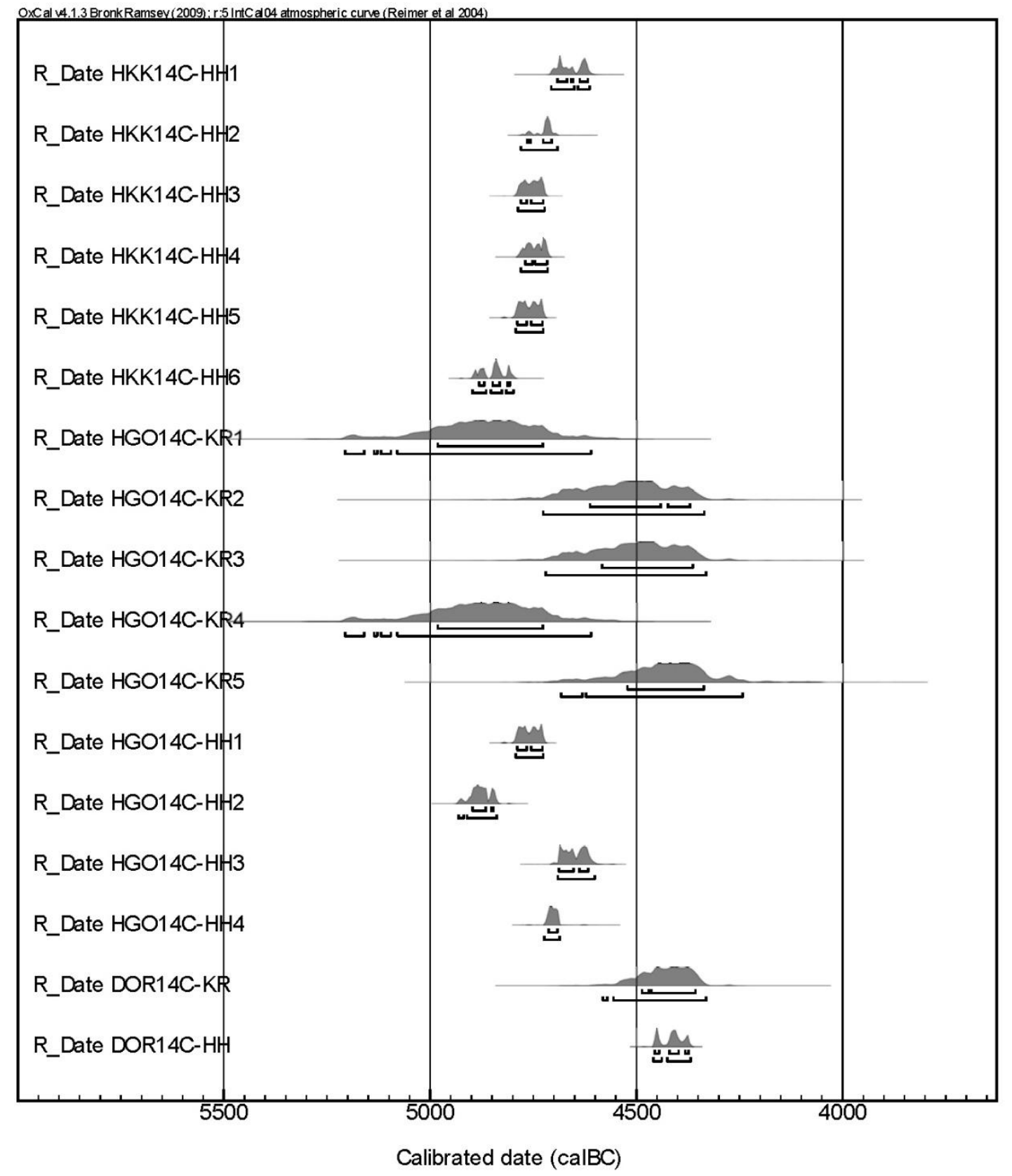

Figure 1.3: Multiple plots of recalibrated radiocarbon dates for Tisza sites (by the author, see text above for references and methodology) 


\begin{tabular}{|c|c|c|c|c|}
\hline Labels & References & $\begin{array}{c}\text { Original } \\
\text { uncalibrated } \\
\text { date bp }\end{array}$ & $\begin{array}{l}\text { Calibrated range } \\
\text { using IntCal04 } \\
(68.2 \% \mathrm{Cl})\end{array}$ & $\begin{array}{c}\text { Calibrated range } \\
\text { using IntCal04 } \\
(95.4 \% \mathrm{Cl})\end{array}$ \\
\hline \multicolumn{5}{|c|}{ Hódmezővásárhely-Kökénydomb } \\
\hline НКК14C-HH1 & $\begin{array}{l}\text { Hertelendi \& Horváth } \\
1992\end{array}$ & 5800 & $4692-4618$ BC & $4707-4614$ BC \\
\hline НКК14C-HH2 & $\begin{array}{l}\text { Hertelendi \& Horváth } \\
1992\end{array}$ & 5850 & $4765-4706$ BC & $4780-4692$ BC \\
\hline НКК14С-HН3 & $\begin{array}{l}\text { Hertelendi \& Horváth } \\
1992 \\
\end{array}$ & 5890 & $4780-4727$ BC & $4788-4723$ BC \\
\hline НКК14C-HH4 & $\begin{array}{l}\text { Hertelendi \& Horváth } \\
1992\end{array}$ & 5870 & $4770-4717$ BC & $4781-4716$ BC \\
\hline НКК14C-HH5 & $\begin{array}{c}\text { Hertelendi \& Horváth } \\
1992 \\
\end{array}$ & 5900 & $4789-4728$ BC & $4793-4726$ BC \\
\hline НКK14C-HH6 & $\begin{array}{l}\text { Hertelendi \& Horváth } \\
1992\end{array}$ & 5970 & $4882-4808$ BC & $4898-4799$ BC \\
\hline \multicolumn{5}{|c|}{ Hódmezővásárhely-Gorzsa } \\
\hline HG014C-KR1 & Kalicz \& Raczky 1987 & $5970 \pm 100$ & $4982-4727$ BC & $5207-4611 B C$ \\
\hline HG014C-KR2 & Kalicz \& Raczky 1987 & $5670 \pm 100$ & $4613-4371 B C$ & $4726-4336$ BC \\
\hline HG014C-KR3 & Kalicz \& Raczky 1987 & $5650 \pm 100$ & $4584-4364$ BC & 4721 - 4331 BC \\
\hline HG014C-KR4 & Kalicz \& Raczky 1987 & $5970 \pm 100$ & $4982-4727$ BC & $5207-4611$ BC \\
\hline HG014C-KR5 & Kalicz \& Raczky 1987 & $5580 \pm 100$ & $4523-4337$ BC & $4684-4243$ BC \\
\hline HG014C-HH1 & $\begin{array}{l}\text { Hertelendi \& Horváth } \\
1992\end{array}$ & 5900 & $4789-4728$ BC & $4793-4726$ BC \\
\hline HG014C-HH2 & $\begin{array}{l}\text { Hertelendi \& Horváth } \\
1992 \\
\end{array}$ & 5990 & $4898-4848$ BC & $4932-4839$ BC \\
\hline $\begin{array}{c}\text { HGO14C-HH3 } \\
* \\
\end{array}$ & $\begin{array}{c}\text { Hertelendi \& Horváth } \\
1992 \\
\end{array}$ & 5790 & $4688-4618$ BC & $4691-4602$ BC \\
\hline $\begin{array}{l}\text { HGO14C-HH4 } \\
* \\
\end{array}$ & $\begin{array}{l}\text { Hertelendi \& Horváth } \\
1992 \\
\end{array}$ & 5830 & $4713-4692$ BC & $4724-4687 B C$ \\
\hline \multicolumn{5}{|c|}{ Deszk-Ordos } \\
\hline DOR14C-KR & Kalicz \& Raczky 1987 & $5595 \pm 65$ & $4487-4358$ BC & $4582-4331$ BC \\
\hline DOR14C-HH & $\begin{array}{l}\text { Hertelendi \& Horváth } \\
1992\end{array}$ & 5600 & $4456-4374$ BC & $4460-4369$ BC \\
\hline
\end{tabular}

Table 1.2: Radiocarbon dates for Tisza sites (recalibration by the author from original data as referenced, dates with * obtained from sample in grave 16 corresponding to HGO-12 in our study)

\subsubsection{Unspecified Neolithic Sites}

\section{Hódmezővásárhely - Z bérház}

No information could be found on the individual analysed from this site, merely recorded as "Neolithic". 


\section{๑ Ószentiván}

This site was excavated by Korek (1943), revealing one grave. Radiocarbon dates were available in Horváth and Hertelendi (1994) placing it in the fourth Körös culture period between 5400 and 5300 BC, with uncalibrated dates around 6460 and $6270 \pm 80 \mathrm{bp}$. In the author's calibrations using the methods described above, a 95\% confidence interval would offer a range of 5611 to 5027 $\mathrm{BC}$ for this date. However, as the only information available on this individual indicates "Neolithic", no further precision can be achieved in this case.

\subsection{Research Aims, Objectives and Structure}

The osteological sample gathered for this doctoral research was unique in size for such a small geographical area in such a remote period, and at a time of particular archaeological and anthropological importance. The main site of Hódmezővásárhely-Gorzsa especially has the potential to provide a vast amount of information for this early period with its large number of recovered individuals.

Unfortunately, the lack of archaeological context and in particular funerary archaeological data, such as drawings of the graves and most importantly plans of the sites and grave locations as well as stratigraphy of the human remains, severely limited the scope of interpretation of the osteological data. The aims and means of this study had to be adapted accordingly from the many lines of research available in osteoarchaeology as well as funerary and social archaeology, to a purely osteological approach using macroscopic observations only. After decades of new and continually improving technology, macroscopic analyses of human bones often take a back seat, losing priority over more specialised scientific and mostly microscopic techniques. Nevertheless, a great deal of information may still be obtained in this manner. 
The main objective of this thesis was therefore to validate the potential of macromorphological analyses, even in worst-case scenarios reminiscent of this sample with ancient and severely fragmented or damaged remains lacking any contextual data, by providing crucial new palaeodemographic and palaeopathological information to help further our understanding of the First Farmers of Europe and more specifically of the Early and Late Neolithic Populations of the Southern Great Plain of Hungary.

Part One concentrates on establishing the palaeodemographic characteristics of these populations, including age, sex and growth/stature. In particular, methods available for macroscopic demographic analyses will be reviewed extensively to establish their suitability for the study of such archaeological remains, and to ensure that as much accurate information as possible can be retrieved from such material. The author will then utilise the most appropriate methods on this Neolithic sample, providing a comparison of results based on different methodologies when applicable.

Part Two presents the palaeopathological analysis of the individuals identified in the first part together with the significance of the results for the palaeopathological record in order to demonstrate that even the most fragmentary remains can offer a considerable amount of information when analysed thoroughly and systematically. These boxes of damaged fragments and incomplete bones can provide us with a better understanding of Neolithic populations, proving the potential of macromorphological studies at least as a first major step before undertaking complementary or more refined analyses, while providing new evidence of the transition to farming and the impact of the adoption of agriculture on Central European populations. 
PART ONE

PALAEODEMOGRAPHY 


\section{Chapter 2: Sexing Methods}

\subsection{Introduction}

Estimating the sex of an individual is the first step of any human osteological analysis. It is not only important in order to understand the demography of the population studied but also crucial as the basis for further information, such as estimating age.

Sex determination is based on elementary human sexual dimorphism. In general, males tend to be larger and more robust, while females tend to be smaller and more gracile, with a pelvis shaped for the requirements of reproduction, i.e. childbirth (Ubelaker 1984; Bass 1995; Mays 1998; Mays \& Cox 2000; White \& Folkens 2005).

In adults, the best indicators of an individual's sex are found first in the pelvis, followed closely by the skull, and then the long bones and the rest of the skeleton (Ferembach et al. 1980; Ubelaker 1984; Mays \& Cox 2000). The accuracy of sex determination varies depending on the completeness of the remains, starting with a minimum accuracy of 50\% from "random guessing" (White \& Folkens 2005). The most optimistic studies quote as high as 90 to $100 \%$ accuracy with a full skeleton, 90 to $95 \%$ based on complete pelvis alone, 80 to $90 \%$ with just a complete skull, 80 to $90 \%$ based on long bones only, $98 \%$ when both the skull and the pelvis are available, 90 to $95 \%$ for the long bones and the skull, and over 95\% accuracy from long bones and pelvis together (T.D. Stewart 1948; Meindl et al. 1985b; Krogman \& İşcan 1986; Byers 2008).

Sex is usually determined from the morphology with a "simple visual inspection of the bones" (Mays 1998:38). However, metric methods have also been applied since the second half of the $20^{\text {th }}$ century to improve reliability and to limit interobserver error by moving from a descriptive to a quantitative and statistical 
approach (Krogman \& İşcan 1986). Metric methods are often based on morphologic, sometimes also referred to as "anthroposcopic" (Byers 2008), methods, using measurements of sexually dimorphic features and applying discriminant function analyses to establish formulae. Measurements from the whole skeleton have been used, including the pelvis and the skull, as well as the long bones, but also hand and foot bones, ribs, and bones from the thorax and shoulder areas. Although it has been suggested that results from these metric studies on multiple postcranial bones provide "higher probability of accuracy than sex determination from the skull" (Byers 2008:194), the reliability of sex determination is rarely improved using morphometry (Mays 1998; Mays \& Cox 2000).

One major caveat of sexing archaeological remains is the variation between populations which are geographically, chronologically, or ancestrally distinct, as sexual dimorphism does vary between human groups (Buikstra \& Ubelaker 1994). This is especially true for sexing criteria linked to robustness or general morphometry. Variations also occur within the same population, as skeletal morphology may be influenced by genes, pathology, nutrition, or activity patterns, and there is often an overlap between male and female ranges (Ferembach et al. 1980; Ubelaker 1982; Mays 1998; Mays \& Cox 2000; Byers 2008).

Determining the sex of juveniles is much more difficult as sexual dimorphism, which is linked to testosterone levels and appears on a minute scale during fetal life, only becomes clearly visible with puberty (Mays 1998). Juvenile sexing is therefore rarely attempted and has been reported to have very low rate of accuracy, from $75-80 \%$ with pelvis present to a fifty-fifty chance if absent (Krogman \& İşcan 1986). However, researchers have recently tried to better these odds by creating new and/or more accurate methods, as will be seen below. 


\subsection{Juveniles}

Sex determination of juveniles has been described as the "largest single problem" (Scheuer \& Black 2004:19) and the "holy grail" (Lewis 2007:48) of osteological analyses of immature remains, whose "long history, frequently filled with hope, [...] eventually [ends] in discouragement" (Byers 2008:197). This rather bleak picture has not stopped numerous researchers studying sexual dimorphism in juveniles, mainly in the skull, the pelvis, and the dentition.

\subsubsection{Skull}

\section{Dentition}

Odontometric methods of sexing should provide a helpful advantage as once teeth are fully formed, they do not evolve further apart from changes attributed to dental attrition. However, teeth recovered from archaeological remains might not represent their contemporary living population due to the mortality bias, as ill health and malnutrition may cause developmental problems resulting in smaller teeth than in healthy individuals (Mays 1998; Mays \& Cox 2000).

One of the earliest and most complete studies on sexual dimorphism in the juvenile dentition was by Bailit and Hunt (1964). They utilised the radiographs of 25 female and 25 male clinic patients aged 7-12 years from Boston, USA. However, they could only reach 58\% accuracy, barely above guessing rate, when comparing dental developmental age to standards of the correct sex. Accuracy was increased to $70 \%$ by using the canine only, tooth chosen as it was considered to show the greatest sexual dimorphism and be the least likely to be missing or malformed. However, this higher accuracy was only achieved when chronological age was known. The requirement for radiographic facilities, the importance of known chronological age (information unlikely to be available when studying prehistoric archaeological remains), and the very low accuracy led to this method being discarded for the present research. 
A method that did not rely on x-rays was provided by Black (1978a; also quoted in Saunders 2008), who hoped that the human dentition could "provide reliable non-radiographic means for sexing immature skeletons". The analysis was based on the mesio-distal and bucco-lingual dimensions from the casts of right deciduous teeth of 133 white North American children taking part in the Growth Study of the University of Michigan, including 69 boys and 64 girls. One of the discriminant functions used twenty measurements and the other four each utilised a different set of ten measurements. Only fifteen out of twenty comparisons demonstrated larger teeth in boys, and sexual dimorphism was very small as well as less pronounced than in the permanent dentition. The best function, based on nine measurements from seven teeth, only managed to obtain an accuracy of 75\%, with other functions achieving between 64 and $68 \%$ accuracy. Due to the requirement for a mostly complete dentition, which would often be an issue when observing archaeological remains, and the low accuracy of the results, this method was also not deemed suitable for such Neolithic material.

A more recent study also based on dental metrics was provided by De Vito and Saunders (1990). They utilised all the forty measurements of the deciduous dentition of 80 girls and 82 boys aged three to four years, and further measurements for the first permanent molars from 45 girls and 39 boys from the same group of children now aged sixteen, taken from casts of the healthy and wealthy white subjects of the Burlington Growth Study, Ontario, Canada, between 1952 and 1972. The means of each measurement were always greater in males, although the actual male and female ranges of measurements overlapped considerably. Sexing accuracy from the functions obtained ranged from 75 to $90 \%$, while the pattern and the degree of sexual dimorphism of deciduous as well as permanent teeth were found to vary greatly between and within populations, highlighting the need for population specific data. In addition to the requirement for as complete a dentition as possible, this last 
point renders the method of limited use for archaeological material. Together with the considerable overlap in the range of measurements from both sexes, and the complexity of these measurements, with the diameters taken not maximum diameters but the "distance between the contact points, which are determined by the "ideal" anatomical relationship between the tooth position and the curve of the dental arch" (De Vito \& Saunders 1990:846), all led to this method being discarded for the current study.

Finally, discriminant function analyses were also carried out on a small archaeological sample of identified children from two historic AD 19 ${ }^{\text {th }}$ century pioneer cemeteries in Canada. Although six out of seven females were correctly identified using these functions, only three out of eight males were correctly sexed, demonstrating once again a very low accuracy (Saunders et al. 1992; Saunders 2008).

All research until now thus tend to agree that sexual dimorphism is present on deciduous and permanent teeth, but that this dimorphism, as well as varying between populations, is very low with the maximum ten percent difference in permanent teeth representing under $1 \mathrm{~mm}$ between female and male means and even less in deciduous teeth. This has proved too low in many cases to correctly determined the sex of some populations, such as with the remains from Spitalfields (Mays \& Cox 2000). Although Ferembach and colleagues thought that deciduous teeth were "the only factor useful for sex diagnosis" (1980:525), current research would best be summarised by Byers' conclusion that "the use of deciduous dental measurements to determine the sex of subadults probably is not warranted [yet]" (2008:200). Dentition will therefore not be used for estimating the sex of juveniles in these Neolithic populations.

\section{- Skull}

Since the 1990s, there have been several attempts at using the skull to establish the sex of juvenile individuals, mostly based on the mandible. 
Juvenile sexual dimorphism in the historic sample of Christ Church, Spitalfields, London, was studied by Schutkowski (1993), who examined the mandible and the ilium of 37 boys and 24 girls of known age and sex from the "Coffin Plate Sample". The majority of these children were aged from birth to five years, with only five individuals between six and eleven years old. Sexual differences were considered in three areas of the mandible: the protrusion of the chin region (more prominent, wide and angular in boys), the shape of the anterior dental arcade (rounded contour in girls, wider and U-shaped in boys) and the eversion of the gonial region (present in boys, no protrusion in girls). Although there were overlaps between both sexes with great variation within each sex, sexual dimorphism was clearly visible for the newborn to five years category. Unfortunately, the "girls" features were not restricted to female skeletons and would thus not be indicative of female sex. The "boys" features, however, were significantly attributed to males, with $94.1 \%$ of boys showing a prominent and angular chin, $82.6 \%$ with a wide anterior dental arcade, and $73.9 \%$ with gonial eversion. When these features are present on juvenile remains, it is therefore significantly likely that the individual was a boy, whereas when the "girls" features are observed, sex remains indeterminate.

These mandibular criteria were tested by Loth and Henneberg (2001) using their juvenile sample from the Dart collection comprising 62 mandible of white and black South Africans of known age (birth to nineteen years) and sex. They could only reach accuracies of 33\% for chin protrusion, $37 \%$ for gonial eversion and did not find any sexual dimorphism in the dental arcade shape. They then used the same sample to determine two different sexually dimorphic characters based on nineteen mandibles from children aged seven months to four years: the symphyseal base, rounded in females and with abrupt extension squaring off at the symphysis in males, and the shape of body, gradually curved in females and sharply angled in males. Their study highlighted more variations of shape in females than males, which agree with Schutkowski's findings. They recorded an accuracy of $82 \%$ in sexing juvenile mandibles using their method. 
However, accuracy for sexing girls was as low as $67 \%$ for both independent observers who took part in this study. Children from the age of six years could be sexed using the same sexually dimorphic characteristics as adults, such as the mandibular ramus flexure trait (Loth \& Henneberg 1996, described in more detail in section 2.3.1), which provided an equally low accuracy of $69 \%$ in sexing juveniles between six and nineteen years of age. Scheuer (2002) tested Loth and Henneberg's method on 27 male and 9 female mandibles of known age and sex from the Spitalfields collection, London, dating from the late eighteenth -early nineteenth centuries $\mathrm{AD}$, all under five years old. Overall accuracy only reached $64 \%$, with male sexing accuracy between 77 and $85 \%$ but with female results as low as 44\%, agreeing with Schutkowski's conclusions that such sexual traits on juvenile under 5 years of age could only establish either male or indeterminate sex.

The mandibular arcade shape still provided satisfactory results in other studies. Sutter (2003) tested eight mandibular and pelvic juvenile sexing traits on 30 female and 55 male autopsied pre-Columbian mummies from Northern Chile, aged using dental development and long bone lengths from birth to fifteen years. Using a scoring system of 1 (very male) to 5 (very female), 77.6\% accuracy was achieved when sexing juvenile mummies using the mandibular arcade shape. Sex estimation from different mandibular traits seemed to vary with age: for the newborn to one year age category, mandibular protrusion presented the expected sexual dimorphism. Between two and ten years of age, both mandibular protrusion and arcade shape were accurate sexing traits. Arcade shape was a better sexual character in the eleven to fifteen years old than protrusion. Gonial eversion, the last of Schutkowski's juvenile mandible traits, was the only one not to be statistically significant, and reached only $62 \%$ accuracy. However, the detailed results demonstrated a blurry distinction between sexes in the presence of what would be perceived as typical male or typical female traits. Nearly $96 \%$ of males presented with mandibular protrusion, but more than $64 \%$ of females also exhibited this trait. Therefore in 
this population, only the female smoother trait, rarely seen in boys, can be used as a high probability that the individual was a girl. The prominent chin, although present in the vast majority of boys, would not be a useful indicator due to its high frequency in girls as well. The mandibular arcade shape fared only slightly better, with around $92 \%$ of boys showing a rectangular shape, but with the same shape present in nearly half the girls. A rounded arcade shape would therefore very likely belong to a girl, whereas a rectangular shape, although more likely to indicate a male, could belong to either.

Based on these studies, mandibular morphological traits of the mandible including chin protrusion, shape of the dental arcade, gonial eversion and ramus angle do show potential for sexing juveniles. With clear descriptions and ease of use on archaeological remains, it was felt that these traits therefore warranted recording on these Neolithic populations, although extreme care would be needed when trying to establish sex as shown in the conflicting results above.

An alternative method of sexing juvenile based on skull morphological traits was provided by Molleson and colleagues (1998), also including chin protrusion (renamed mentum), and adding both mandibular angle and the orbit. These traits were scored from -2 (hyperfeminine) to +2 (hypermasculine), and results of the three traits summed to provide a "combined facial score (CFS)" which would indicate a female if negative and a male if positive. This scoring system was first applied to 34 females and 19 males of known age and sex, also from the Spitalfields Collection, and sex determination reached 89\% accuracy. Twenty juvenile crania of children aged one to fourteen years from Spitalfields, seventeen of whom were under five years of age, were then scored in the same way. Only thirteen boys and five girls could provide scores for the three indicators. Overall accuracy in sex determination is given at 78\%. However, when taking both sexes separately, only three out of the five females were sexed correctly, whereas eleven out of thirteen boys had been accurately determined. 
The same method was then applied to a sample of fourteen male and fourteen female adults from the cemetery of Wharram Percy, whose sex was not known but had been determined only from their pelvic bones. Like the Spitalfields sample, accuracy reached 89\%. Juveniles were aged using dental calcification. From an initial sample of 57 children between five and seventeen years old, only 26 could provide scores for all three indicators. These CFS results were then compared to the children's mandibular canine crown widths, and CFS male scores were found to be consistent with greater tooth measurements, and were considered by their authors to prove the validity of their sexing method. This method seems to offer more reliability with better accuracy in sexing juveniles, but may pose difficulties with ancient human remains where all three indicators might not be present on recovered juveniles. This method should therefore be of use for the Neolithic sample under study, although this will depend on the conditions of the remains.

Finally, an alternative osteometric method was provided recently by Franklin and colleagues (2007). They employed a geometric morphometric technique using a portable digitizer and the shape analysis software morphologika to establish if sexual dimorphism was present in juvenile mandibles using 38 bilateral three-dimensional landmarks. Their study sample included 18 male and 19 female black Americans from the Hamann-Todd collection, 25 male and 17 female black South Africans from the Dart collection, as well as ten male and seven female white Europeans from the Spitalfields Coffin Plate Sample, all aged between one and seventeen years. Their multivariate regressions analyses indicated differences in mandibular shape linked to age and ancestry. Although starting to be visible from fifteen years of age, sex determination using this technique was however statistically unsuccessful. Considering the need for a digitizer and specialised software in addition to the lack of statistical significance, it was decided not to use this method. 


\subsubsection{Pelvis}

\section{(-) Overall shape and measurements}

Sexual dimorphism has been studied on fetal pelves as early as the nineteenth century, with Verneau (1875) observing a wider sciatic notch in males and shallower in females, Thomson (1899) looking at the whole pelvis finding sexual differences in the shape of the sciatic notch and of the pubic angle, and Villemin (1937) demonstrating a greater sciatic notch length in female. However, such studies were based on extremely small samples, casting doubt on their reliability. Thomson's sample for example only consisted of four females and four males. Moreover, these studies had been undertaken on wet specimens with the cartilage still intact, prohibiting their use in osteoarchaeology. Thomson himself thought any examinations of the shape of dry bones would be worthless due to distortion during drying, and their measurements were too small to estimate accurate differences.

Data on sexual dimorphism in the juvenile pelvis from a larger sample was first provided by the Ohio Fels growth study, which was used as the basis for Reynolds' longitudinal study of the juvenile pelvis during the first year of life (1945) and from two to nine years of age (1947). For the first study, radiographs taken at birth, one, three, six, nine and twelve months of 46 male and 49 female healthy white Americans born in Ohio were used to provide twelve measurements and six indices. The second study included 78 of the individuals from the first study and comprised of fourteen measurements and four angles from the radiographs of 92 boys and 91 girls taken ten times between 15 months and 9.5 years. Both studies provided very similar results showing significant sexual dimorphism with larger measurements and indices in males for the outer pelvis and in females for the inner structure. Females also showed greater variation than males. Unfortunately, this study is of very limited use for archaeology as of all the measurements taken, only the pubic length might be available on osteological remains. All the other measurements and 
indices either involved the complete pelvis including cartilage or would be distorted due to the angled view on radiographs.

Much more recently, Rissech and colleagues' (2003) observed 327 pelves from individuals aged from birth to 97 years from the St Bride's collection in London, the Coimbra and the Lisbon collections from Portugal, and the Barcelona collection from Spain, all documented, including 48 male and 51 female juveniles. Ischium length proved to be larger in males than in females from five years of age. The ischium acetabular index $\frac{\text { vertical acetabular surface diameter }}{\text { horizontal acetabular surface diameter }}$ was very variable during growth, yielding higher means in girls until four years of age, then significantly higher means in boys from five to nine, and both sexes showing similar means from ten years of age. The osteological material utilised and its known sex and age would make this study the most appropriate to follow in this Neolithic sample. However, due to the great variability of its results and interpretations with age, it was decided not to use this method.

In their well-known standards, Buikstra and Ubelaker indicated that on an adolescent pelvis, the presence of female traits can "confidently be diagnosed as female" (1994:16) whereas a male pattern could indicate either a teenage male or a female who had not yet reached adult proportions, and should therefore remain inconclusive. This should be kept in mind throughout any attempt at sexing the juvenile pelvis.

\section{(-) Sciatic Notch}

In her initial study (1955) on the ilia of 13 male and 21 female British fetuses, Boucher measured the sciatic notch between "the points of contraflexure", using special gauges as the dimensions were so small. Both the sciatic notch index $\frac{\text { width of sciatic notch }}{\text { depth of sciatic notch }}$ and the relative percentage $\frac{\text { depth of sciatic notch } X 100}{\text { width of sciatic notch }}$ 
demonstrated sexual dimorphism among fetuses, the male sciatic notch being shorter and deeper, whilst longer and shallower in females. Although the follow-up study (Boucher 1957) was not directly applicable to osteoarchaeology as it was based wet dissected bones, the wider sample of 30 American white, 107 British white (including 33 from the first study), and 96 American black fetuses of known age, sex and ancestry, confirmed the results of the initial study. The complete pelves with cartilage still intact allowed Boucher to observe a significant sexual dimorphism in the subpubic angle. Indeed this dimorphism was even more marked than in adults. Sciatic notch index values ranged between 3.4 and 8.8, with separation between the sexes around 5, females over this threshold and males under it. Sciatic notch index accuracy in sexing the fetuses ranged from as high as $95 \%$ to as low as $57 \%$. Both the subpubic angle and the sciatic notch index revealed significant sexual dimorphism in American black and British white individuals, although not for American white fetuses, confirming population differences. Boucher also looked at the ischium pubis index $\frac{\text { pubis length } X 100}{\text { ischium length }}$, although its measurements are equally hard to locate, but no sexual differences were found.

Two decades later, Fazekas and Kósa (1978) utilised 61 male and 43 female Hungarian fetuses of known age and sex, from healthy parents, presenting no anomalies and either still born or having died within hours of birth. With the help of standard callipers filed down for more precision, they measured the length and width of the ilium, as well as the depth and length of the sciatic notch taken on the dorsal surface between the greatest protrusion of the posterior inferior iliac spine and the passage in the articular surface. The analyses demonstrated a deeper notch in males and a longer one in females with a highly significant sexual dimorphism, agreeing with Boucher's results. No sexual differences were detected in the ilium measurements. The accuracy of sex determination based on the sciatic notch indices reached 70 to $80 \%$, although only 44 to $50 \%$ when trying to establish sex with absolute certainty due to 
extensive overlaps, rendering this method no more accurate than guessing. The dimensions are so small that the actual difference between sexes in four out of six indices was less than one millimetre.

Although Boucher's measuring points were described as visible by eye, they are in reality very hard to find and will prove challenging without the availability of special gauges. However, with a high potential for sexing juvenile pelvis revealed in both methods, measurements will be recorded on these Neolithic populations using standard digital callipers in an attempt to establish Boucher sciatic notch index and Fazekas and Kósa's sciatic apertural index whenever possible.

Holcomb and Konigsberg (1995) tried a different approach by using image analysis on 55 female, 73 male and 5 unknown ilia from fetuses of known age from the Trotter Collection of Washington University. Photographs of the ventral side of the ilia were digitized and their trace coordinates analysed. Sexual dimorphism in the sciatic notch was found to be significant although not as marked as in adults. The sciatic notch index did not produce significant results, whereas the relative anterior-posterior position of the maximal depth of the notch provided the source of sexual dimorphism in fetuses. Unfortunately, the accuracy of this approach was very poor at below $60 \%$ with extensive overlaps between males and females. Therefore, although this method would have helped getting more accurate and objective measurements, the very poor accuracy barely above guessing rate and the need for digitalisation led to the decision not to try this new technique on this Neolithic material.

To utilise fully the potential of the sciatic notch, or other similarly dimorphic areas, as sex indicator whilst avoiding the issue of population variability, Mays (1998) proposed a new approach for archaeological samples with a large number of well-preserved juvenile bones. By taking the measurements and plotting them onto a graph, two clusters should appear if the area is suited to determine sex, representing the separate groups of boys and girls. Mays used 
his method on 36 perinates from Wharram Percy, plotting

$\frac{\text { depth of sciatic notch } X 100}{\text { width of sciatic notch }}$, which produced two distinct peaks for young infants. This method offers great potential for osteoarchaeology, and as already stated above, the sciatic notch will be recorded. Unfortunately, it is unlikely that enough juvenile remains would be available to use this methodology for this doctoral research.

Other studies utilised the morphological aspect of the sciatic notch rather than concentrating on measurements. In the study already described in the previous section, Schutkowski (1993) observed juvenile sexual dimorphism in the pelvis, specifically looking at the angle of the greater sciatic notch (around $90^{\circ}$ in boys, above $90^{\circ}$ in girls), sciatic notch depth (deep in boys, shallow in girls), the "arch criterion" (bordering the auricular surface in boys, crossing it in girls), and lastly the curvature of the iliac crest (marked S-shape in boys, much fainter in girls) on 29 boys and 22 girls of known age and sex aged from birth to five years. The best results were provided by the sciatic notch angle, with $95 \%$ of boys and $71 \%$ of girls correctly identified, followed by its depth, with $81 \%$ accuracy for boys and above $76 \%$ for girls. Even the arch criterion reached accuracy of $73 \%$ for boys and nearly $71 \%$ for girls. Although the iliac crest shape would not be a reliable indicator for girls only correctly identifying $62 \%$ of them, it is a useful sexual characteristic for boys with $81 \%$ accuracy.

This method was tested by Sutter (2003) on prehistoric known-sex mummies aged birth to fifteen years from Northern Chile (see section 2.1.1), including the sciatic notch angle and depth as well as the arch criteria. They reached an overall sexing accuracy of around 80\%. However, the same overlap between sexes in specific traits as seen in the skull also occurred in the pelvis: nearly $90 \%$ of the 37 males had a sciatic notch around $90^{\circ}$ but only $68 \%$ of the 25 females presented with an angle above $90^{\circ}$, a third of girls showing the male trait instead. This was even more accentuated for the sciatic notch depth with $36 \%$ of females showing a deep notch. The same pattern was also visible with 
the arch criteria. The fourteen girls and thirteen boys aged birth to five years seemed to demonstrate an opposite pattern with a larger proportion of girls showing female traits than boys with male traits. Therefore, to determine the sex of children five years and under with any certainty, the only traits to use would be a deep sciatic notch and a marked iliac crest, which would indicate a boy (only $7 \%$ of girls presented these features). To establish the sex of juveniles fifteen years and under, a bordering arch (only $8 \%$ of boys) as well as a sciatic notch angle shallow and above $90^{\circ}$ (both in only $10 \%$ of boys) would most probably indicate a girl.

Two of Schutkowski's sciatic notch sex indicators (depth and angle) were tested most recently by Vlak and colleagues (2008) on 23 female and 33 male juveniles under sixteen years of age from the modern Lisbon collection, with documented age and sex. Although sexual dimorphism was present along the same lines as Schutkowski's and Sutter's studies, their results were not as statistically significant, and seemed to correlate more strongly with age. For some age categories, accuracy of the sex determination obtained from their sample differed vastly from the British and Chilean samples, especially for the two to five years category, where only $25 \%$ of girls and $50 \%$ of boys were sexed accurately using the greater sciatic notch depth, as opposed to $88 \%$ for girls and 85\% for boys in Schutkowski's study, with $100 \%$ for girls and $85 \%$ for boys from Sutter's studies. Using the sciatic notch angle for the same age category, the Portuguese sample was correctly sexed in $38 \%$ of girls and $40 \%$ of boys, compared to $90 \%$ for girls and $82 \%$ for boys in the British sample, and $86 \%$ for both sexes in the Chilean sample. However, the same year, Wilson and colleagues' (2008) study yielded results in agreement with Schutkowski's and Sutter's findings, albeit on a smaller sample. Geometric morphometric methods were employed to produce discriminant functions on 25 ilia from 17 boys aged newborn to eight years and 8 girls aged newborn to three years from Christ Church, Spitalfields, The greater sciatic notch shape correctly identified $100 \%$ of males and $87.5 \%$ of females, while the greater sciatic notch angle was measured 
rather than placed in one of Schutkowski's two categories, with statistically significant results despite the small sample size. The marked curvature of the iliac crest also successfully indicated male sex in 82 to $88 \%$ of the boys, although as previous studies also demonstrated, would not be a useful sex indicator for females with only 25 to $37.5 \%$ accuracy. All results were greatly improved by excluding the infants under 0.1 year old, bringing accuracy up to an impressive $100 \%$ for both males and females for the greater sciatic notch shape as well as the iliac crest curve.

Therefore, Schutkowski's promising method will be employed in the analysis of the juvenile pelvic remains available from the Neolithic sites under study, although the potential population variations mean all results will only be considered as possible estimates.

\section{( Auricular Surface}

Weaver (1980) was the first to describe auricular surface elevation as a potential sex indicator in juveniles. The study utilised six metric and one nonmetric characters to identify sexual dimorphism on 71 female and 80 male fetuses and infants of known age and sex from the Smithsonian Institution collection, aged from six fetal months to six months post-partum. Measurements were taken directly from the bones using a millimetre grid, and the auricular surface was scored as elevated or non-elevated. Three indices were then calculated: $\quad$ sciatic apertural $\quad \frac{\text { depth of sciatic notch } X 100}{\text { width of sciaticnotch }}, \quad$ chilotic $\frac{\text { ilium posterior length } X 100}{\text { ilium anterior length }}$ and iliac breadth $\frac{\text { iliac width } X 100}{\text { iliac height }}$. It should be noted that Weaver's iliac height and width do not correspond to the standard iliac length and width as recommended by Fazekas and Kósa (1978), and in the Standards for Data Collection from Human Skeletal Remains (Buikstra \& Ubelaker 1994). No significant sexual dimorphism could be demonstrated from the indices. However, a non-elevated auricular surface correctly identified 91\% of fetal and six-month old males, although accuracy for sex determination of newborn males 
was lower at only $73 \%$. The elevation of the auricular surface did not provide accurate results for sexing female newborns (54\%) or six-month olds (43.5\%), albeit with better odds for female fetuses with a 75\% accuracy. Combining the three age categories, lack of auricular surface elevation therefore correctly identified $85 \%$ of males, whereas only $58 \%$ of females presented the elevated form.

Ten years later, Weaver's auricular elevation was tested by Hunt on 275 juveniles of unknown sex under seven years of age from an Arikara archaeological North American population. As sex was unknown, the sexing capability of the auricular surface elevation was only established by its closeness to an expected ratio of $1: 1$ in sex distribution. Results indicated a correlation with age and growth rather than sex, as ratios obtained shifted from 6:1 elevated to non-elevated ratio for newborns to a 1:4 ratio in teenagers, and thus "almost certainly" not reflecting sex variation (D.R. Hunt 1990:884). However, the use of male / female ratio on an archaeological population without any other indicators for sexing greatly limits the significance of this test study.

Results from both the above studies were compared by Mittler and Sheridan (1992) to 34 male and 24 female naturally mummified juveniles aged newborn to eighteen years from Kulubnarti, Nubia, dating from medieval Christian times. Once again, the proportion of boys correctly identified using auricular surface elevation (85\%) exceeded the results from girls, with only 58\% accuracy. A correlation with age was also visible in this sample, as observed with only a third of all girls under ten years showing the female elevation and just above $70 \%$ of males with non-elevated auricular area, whereas two thirds of the girls aged ten years and older had an elevated auricular surface and $100 \%$ of the boys in the same age category lacked auricular elevation. In the juveniles older than nine, $100 \%$ of the elevated auricular surfaces belonged to girls, whereas $75 \%$ of the non-elevated ilia belonged to boys. Although non-elevated auricular surfaces would therefore be more likely to identify males, a quarter of them 
would belong to females and might thus not be reliable enough as a sex indicator. However, according to the results of this study, the presence of auricular surface elevation on individuals from ten years of age would nearly certainly indicate that the juvenile was female. In a similary study described earlier, Sutter (2003) did not differentiate between these two age categories, results showing $60 \%$ of females aged from birth to fifteen years with elevated auricular area as opposed to nearly $78 \%$ of males lacking the elevation, with similar results in the birth to five years subcategory.

Finally, a recent study by Wilson and colleagues (2008) on children under nine years of age, also described above, revealed 94\% sexing accuracy for boys and over $62 \%$ for girls, increasing to $100 \%$ for boys and $50 \%$ for females excluding infants under 0.1 years, and when excluding infants under 0.5 year old reaching accuracies of $100 \%$ for males and $80 \%$ for females. These results would corroborate Mittler and Sheridan's findings for the under nine years as well, an elevated surface providing an accurate indicator of sex for females whereas a non-elevated surface would not give as high a probability of sexing males. Although any results for the Neolithic populations under study would need to be interpreted very cautiously, the potential for sexing based on auricular surface elevation, particularly on juveniles over 0.5 year old, advocates the recording of this trait to attempt sex estimation.

\subsubsection{Other methods}

\section{- Dental and skeletal age comparison}

An additional method of sexing was explored by Hunt and Gleiser (1955), based on the fact that female bones tend to mature earlier than males whereas the calcification and eruption of teeth follow a more similar rate in both sexes. Therefore, comparing age estimates from the skeleton and the dentition should give an insight into the sex of the juvenile. 


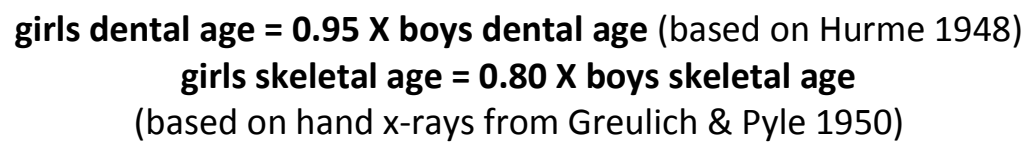

When tested on dental and skeletal radiographs of 49 boys and 45 girls from the Harvard longitudinal growth study, this method achieved 73\% sexing accuracy for two-year olds, $76 \%$ for five-year olds, and $81 \%$ for eight-year olds. In the study, hand bones were used to assess postcranial development, which would become an issue when analysing archaeological remains. However, this method would work equally well based on any other bones presenting marked sexual dimorphism in maturation rate, and the researchers singled out the knee joint as a well-suited alternative to obtain an age estimation post-cranially. This was confirmed by Sundick (1977; also quoted in Saunders 2008), who successfully applied Hunt and Gleiser's method to American Indian archaeological remains using the knee joint on individuals over twelve years old sexed from pelvic morphology and aged from diaphyseal lengths, although no sample size or accuracy rates were published. However, as this was obtained on an archaeological population with no record of age or sex, this confirmation should be considered with caution.

Following this method, estimates obtained using male standards should agree if the individual was a boy, or diverge if the child was a girl. Likewise, if female standards were used to get the age estimates, the child would be sexed as a girl if dental and skeletal estimates provided similar results, while a notable difference in these estimates would indicate that the individual was probably a boy. One major caveat of this method is that it gives an estimation of sex which will most likely vary between populations, and is itself based on two other estimations, each subject to population variability and inaccuracies. However, as age estimations from both skeleton and dentition are always a priority in juvenile remains, this comparison would not entail any additional measurements or observations and could therefore also be considered in osteological cases, albeit with a very limited chance of sexing the individual. 
Bailit and Hunt (1964) provided another method employing a similar idea based on mandibular canine development using male and female standards compared to known chronological age. The sex whose standards provided the results closest to known age correctly identified the individual in $70 \%$ of the case. Unfortunately, as it is highly unlikely that chronological age will be known in prehistoric populations, this method is of limited use in archaeology.

\section{Ossification order}

Another potential ageing technique was devised by Garn and colleagues (1966). They studied the order of ossification on 84 boys and 94 girls, all healthy white North American children taking part in a longitudinal growth study in Ohio. The radiographs revealed sexual dimorphism in the ossification order of the upper limbs, with capitulum radii fusion on the radius followed by medial epicondyle fusion on the humerus in $75 \%$ of males, while in $70 \%$ of females the medial epicondyle fusion occurred before capitulum radii fusion. Although this technique is better suited to radiological studies than observation on fragmentary and/or often incomplete juvenile skeletal remains, it might still be possible to apply it to skeletons complete enough to allow such analysis. Any estimates should also only be considered as a potential sign of the sex of the juvenile, as population variation would also be expected.

\subsection{Adults}

Adult remains can be sexed with much more confidence than juveniles, providing enough is left of the skeleton. In the best cases, sex estimates can reach near certainty. The pelvis, due to its function, is the most useful skeletal area to determine sex, followed closely by the skull, then the long bones and finally overall robusticity (Acsádi \& Nemeskéri 1970; Ferembach et al. 1980; Mays \& Cox 2000). 
Skeletal measurements have also been recognised as sexually dimorphic for a very long time. Pfitzner (1901; also quoted in Krogman \& İşcan 1986) established the proportions at $92 \%$ of male for females over a century ago, varying with the dimensions, stature proportion being set at $93.5 \%$, but arm length at $91.5 \%$ and head circumference at $96 \%$.

Krogman reported success rate of $100 \%$ in sexing 750 white and black adults from the Hamann-Todd collection when the full skeleton was available followed by $98 \%$ when combining long bones and pelvis (Krogman \& İşcan 1986). His accuracy of $98 \%$ based on pelvis-skull combination was also confirmed on archaeological remains using the $18^{\text {th }}$ to $19^{\text {th }}$ centuries documented collection of Spitalfields, London (Molleson \& Cox 1993) and on a modern North American sample with a slightly lower 97\% success rate (Meindl et al. 1985a). Estimates from the pelvis alone reached 95\% accuracy, mirroring Stewart's rate for the same area of 90-95\% (T.D. Stewart 1948, 1951). Estimates based on the skull alone achieved 92\% accuracy in the studies of Krogman and Meindl, although only $80 \%$ in Stewart's. Finally, long bones on their own can achieve 80 to $90 \%$ success rate (Krogman \& İşcan 1986; White \& Folkens 2005). Nevertheless, Acsádi and Nemeskéri (1970) warned of the strong variations found between individuals as well as populations, and of the influence of age with young males often more feminine and older individuals including females increasingly masculine in morphology, in addition to living conditions, physical activities and functional wear. Krogman also warned of the male bias in medical school collections used as a basis for numerous sexing methods with a male:female ratio of $15: 1$.

\subsubsection{Skull}

\section{- Cranium and facial bones}

The skull has been described as "the single most studied bone in physical anthropology" (Krogman \& İșcan 1986:191) and was often the only part of the 
skeleton to be kept for collections. Although measurements are frequently taken, morphological traits remain the most commonly utilised when estimating sex from a skull. Sexual dimorphism in the skull mostly stems from the difference in robusticity between males and females (Mays 1998). Male skulls evolve to their more robust form during puberty as females tend to retain their more gracile juvenile form, although factors including diet, health, activity pattern, and especially age and population variability all play a part (Ubelaker 1982; Krogman \& İşcan 1986; Mays \& Cox 2000; White \& Folkens 2005). The sexually dimorphic traits of the adult skull are detailed in table 2.1 below.

\begin{tabular}{|c|c|c|}
\hline Trait/character & Male & Female \\
\hline General shape & $\begin{array}{l}\text { Large, rugged, marked muscle } \\
\text { markings, heavy }\end{array}$ & Small, smooth and lighter \\
\hline $\begin{array}{l}\text { Nuchal crest* [or occipital } \\
\text { area] }\end{array}$ & $\begin{array}{l}\text { More rugged and pronounced } \\
\text { protuberances (with hook), } \\
\text { marked }\end{array}$ & $\begin{array}{l}\text { Smoother area, hook } \\
\text { uncommon, not marked }\end{array}$ \\
\hline Mastoid process* & $\begin{array}{l}\text { Medium to large, more } \\
\text { projecting }\end{array}$ & $\begin{array}{l}\text { Non-projecting, small to } \\
\text { medium, more pointed }\end{array}$ \\
\hline Supra orbital margin* & $\begin{array}{l}\text { Rounded, heavy, blunt, } \\
\text { protruding }\end{array}$ & Sharp, less rounded, thin \\
\hline Orbit & $\begin{array}{l}\text { Squared, lower, relatively } \\
\text { smaller with rounded margins }\end{array}$ & $\begin{array}{l}\text { Higher, more rounded, } \\
\text { relatively larger compared to } \\
\text { upper facial }\end{array}$ \\
\hline Frontal bone & Slanting & Globular \\
\hline $\begin{array}{l}\text { Glabella* [or supraorbital crest } \\
\text { /ridge] }\end{array}$ & $\begin{array}{l}\text { Prominent, large browridges } \\
\text { with slanted frontal, much } \\
\text { more strongly developed, } \\
\text { medium to large, raised }\end{array}$ & $\begin{array}{l}\text { Small to medium with high } \\
\text { rounded and more vertical } \\
\text { frontal }\end{array}$ \\
\hline Zygomatic arch [cheekbones] & Heavy or thick & Thin and moderate \\
\hline Nasal aperture & $\begin{array}{l}\text { Higher and narrower, sharp } \\
\text { margins }\end{array}$ & More rounded \\
\hline Palate & $\begin{array}{l}\text { Broad, long and vaulted, more } \\
\text { U-shaped, deep }\end{array}$ & $\begin{array}{l}\text { Shorter, rounder and flatter, } \\
\text { parabolic, shallow }\end{array}$ \\
\hline Mandible & $\begin{array}{l}\text { Larger, thicker, with great body } \\
\text { height, broader ascending } \\
\text { ramus, gonial angle less obtuse } \\
\left(<125^{\circ}\right) \text {, condyles larger }\end{array}$ & $\begin{array}{l}\text { Smaller, narrower ramus, wider } \\
\text { gonial angle, small condyles, } \\
\text { gracile, low symphysis, rounded } \\
\text { mandibular angle }\end{array}$ \\
\hline Mental eminence* [or chin] & Broad and almost square & $\begin{array}{l}\text { More pointed, one midline } \\
\text { point, more rounded }\end{array}$ \\
\hline
\end{tabular}

Table 2.1: Skull sexual traits, * indicates the five standard sexual indicators (table by the author, data from T.D. Stewart 1954; Brothwell 1963; Acsádi \& Nemeskéri 1970; Ferembach et al. 1980; Ubelaker 1984; Krogman \& İşcan 1986; Bass 1995; France 1998; Mays 1998; White \& Folkens 2005; Byers 2008) 
As a guide, sexually dimorphic features can be ranked starting with general size and architecture of the adult non-elderly skull as the most important indicator, followed by the glabella, mastoid process, nuchal crest, zygomatic arches, orbits, mandible and palate (Acsádi \& Nemeskéri 1970; Krogman \& İşcan 1986).

Sexual traits may be recorded by their presence or absence when not measured, although they are usually scored with five grades going from typical female / hyperfeminine to typical male / hypermasculine, either following a scale of -2 to +2 (Acsádi \& Nemeskéri 1970; Ferembach et al. 1980) or from 1 to 5 (Buikstra \& Ubelaker 1994; White \& Folkens 2005), with 0 and 3 as the respective grades for indeterminate or ambiguous individuals. Some researchers also match various scoring system, such as Acsádi and Nemeskéri (1970) as well as Ferembach and colleagues in their Recommendations (1980), who employed mostly the -2 to +2 system, but recommended Broca's (1875) six degrees of variation for the glabella and nuchal crest, 0-2 indicating females and 3-5 males.

The supra-orbital margin sexing trait was revisited by Graw and colleagues (1999), utilising a different scoring system with 1 to 3 indicating a male and 4 to 7 a female. However, the drawings of this new system, based on impressions of margins from 67 males and 41 females from twentieth century Germany, were not as clear as the basic male / female description (see table 2.1 above) or the more standard ranking using five grades instead of seven. In their assessment of reliability of standard cranial sex indicators on 42 Inuit crania from the University of Pennsylvania Museum, Walrath and colleagues (2004) found that the lack of clarity in the sexual traits' description caused the most difficulties. If the description was easily understandable, sex estimation would achieve much better rate of accuracy. Therefore, although supra-orbital margin will be evaluated in order to estimate the sex of the Neolithic individuals, the five more common grades (Acsádi \& Nemeskéri 1970; Ferembach et al. 1980; Buikstra \& Ubelaker 1994; White \& Folkens 2005) will be used rather than this newer but less effective method. 
The external occipital protuberance was examined in a very large sample including radiographs of five hundred female and five hundred male patients together with 371 male and 323 female historic Anatolian dry skulls by Gülekon and Turgut (2003) to evaluate the reliability of this sexual trait. This indicator provided accurate sex estimation only just above guessing rate, although the modern radiographic sample indicated that over $85 \%$ of females showed the less prominent "female" type as opposed to $18 \%$ of males, and "male" marked crest was observable in $63 \%$ of males but only $4.2 \%$ of females, which would tend to demonstrate its usefulness to estimate male sex. This trait will therefore still be recorded in the populations studied here.

Rogers (2005) tested seventeen cranial sex indicators on 46 skulls of known age and sex from a $19^{\text {th }}$ century cemetery in Belleville, Canada, and obtained $89 \%$ accuracy. Tooth size yielded the worst results, followed by orbit shape and position, frontal and parietal eminences, occipital condyle size, and with only slightly better accuracy palate size and shape. Other traits as described in table 2.1 achieved reasonably accurate sex estimates, and will be used in this analysis. A follow-up study was undertaken based on 25 male and 25 female skulls from the Bass collection (Williams \& Rogers 2006). Up to $96 \%$ accuracy was established when using all criteria, without age bias. Results agreed with the first studies, and females were more likely to be misclassified than males.

More recently, the accuracy of the five "standard" sex indicators for the skull (see table 2.1 above) was examined by using discriminant function analyses (Walker 2008). The sample included 164 males and 140 females from the Hamann-Todd, Terry and St Bride's Church collections of known age and sex, together with 156 Native Americans whose sex had been estimated from their pelvic morphology. Accuracy varied from 78\% for ancient Native American population to $89 \%$ using five traits (84\% when using only two) on modern skulls, proving once again the existence of marked population differences in sexual dimorphism. If using only one trait, the mastoid process and the glabellar 
area were the best sex indicators. The nuchal crest gave very poor results for Native Americans with a meagre 57\% accuracy. All five traits (nuchal crest, mastoid process, supra-orbiatl margin, glabella and mental eminence) will be recorded in the current study to establish the sex of the Neolithic individuals recovered from the area, although the nuchal crest indicator will need to be carefully interpreted.

\section{Osteometry}

Numerous measurements can be taken on the skull. The most commonly used include maximum length, maximum breadth, basion to bregma, basion to nasion, maximum zygomatic width, basion to prosthion, facial height (nasion to alveolar border at incisors), palatal breadth, and mastoid process length. Measurements and ranges from the studies described below can be found in a comparative table in Appendix 1, highlighting the difference in ranges between populations, and therefore the importance of using group-specific osteometric criteria in order to sex individuals.

Keen (1950) took 23 measurements on the skulls of fifty male and fifty female mixed race "coloured" adult South Africans of known sex. Only four of these measurements proved to be good sex indicator: maximum cranial length, maximum bizygomatic diameter for the facial part of the skull, depth of infratemporal fossa and length of mastoid process. Three traits were also useful to estimate sex: the supraorbital ridge, the external auditory meatus and the muscle markings on the occipital bone, with 84 out of 100 skulls correctly identified. Keen warned that measurements would not be as accurate a sex indicator on skulls of unknown origins, as a comparison of means between the two sexes needs to be known as a basis for sex estimation. With known populations, Keen claimed an $85 \%$ success rate. However, this is based on the calculated ranges, not the actual ranges which overlap greatly as can be seen in Table 2.2 on the next page. 


\begin{tabular}{|lcccccc|}
\hline $\begin{array}{c}\text { Measures } \\
(\mathrm{mm})\end{array}$ & $\begin{array}{c}\text { Actual } \\
\text { Range }\end{array}$ & $\begin{array}{c}\text { Calculated } \\
\text { range (mean } \\
\pm 1 \mathrm{SD})\end{array}$ & $\begin{array}{c}\text { Prob. } \\
\text { ( }\end{array}$ & $\begin{array}{c}\text { Actual } \\
\text { Range }\end{array}$ & $\begin{array}{c}\text { Calculated } \\
\text { range (mean } \\
\pm 1 \text { SD) }\end{array}$ & $\begin{array}{c}\text { Prob. } \\
+\end{array}$ \\
\hline Max.length & $168-198$ & $179.4-191.8$ & $>\mathbf{1 8 5}$ & $165-192$ & $171.7-185.5$ & $<\mathbf{1 7 9}$ \\
\hline $\begin{array}{l}\text { Bizygomatic } \\
\text { diam. }\end{array}$ & $116-140$ & $123.5-132.9$ & $>\mathbf{1 2 5}$ & $104-128$ & $114-125$ & $<\mathbf{1 2 3}$ \\
\hline $\begin{array}{l}\text { mastoid } \\
\text { process }\end{array}$ & $21-27$ & $25.7-32.9$ & $>\mathbf{2 9}$ & $19-33$ & $23.4-29.6$ & $<\mathbf{2 5}$ \\
\hline
\end{tabular}

Table 2.2: Skull sexual morphometric ranges (table by the author, data from Keen 1950).

Other researchers concentrated their studies on the measurements associated with the mastoid process, a proven morphological sexual trait as described in the previous section. Using French skulls of known sex from the Laboratoire d'anthropologie biologique de l'université Paris VII's collection, Demoulin (1972) observed on the 75 males and 56 females that the "length" (porionasterion) of the mastoid was much more discriminating than the "height", and that the most dimorphic dimensions of the skull were in order bizygomatic breadth, the mastoid length, breadth of zygomatic process and finally mastoid height. These measurements are all recorded in this doctoral analysis.

While Kemkes \& Göbel (2006) looked at the area of the same morphological trait, they utilised different measurements to assess sexual dimorphism of the mastoid triangle including the mastoidale-porion length and asterion, although the position of asterion was very variable. Their observations were based on two distinct populations: a German forensic sample of 25 females and 72 males at the Institute of Forensic Medicine in Mainz, and fifty males and fifty females from the Coimbra Collection, Portugal. Side asymmetry was noticed in the Portuguese sample. A nearly total overlap between sexes was observed when considering the minimum and maximum of thirteen lengths and the triangular area, although sexual dimorphism was statistically significant. Accuracy only reached $65 \%$ overall and showed great population variability, with an 
extremely poor $52 \%$ accuracy for females in the German sample but as much as $72 \%$ for their Portuguese counterparts. Therefore, although the sexual dimorphism of the mastoid process area was confirmed, the mastoid triangle itself would not be a useful sex indicator when sexing single individuals whose populations of origin are unknown, and will not be used in this analysis.

Several studies from around the world utilised discrimant function analyses on cranial measurements to provide mathematical functions for sex estimation. One of the earliest ones was from the East, where Hanihara (1959) utilised 64 male and 41 female modern Japanese skulls (and scapulae) of individuals aged 20-60 years from the Department of Anatomy and the Department of Legal medicine, at the University of Tokyo School of Medicine. Sexing accuracy ranged from $83 \%$ using three measurements to nearly $90 \%$ using four. However, his results also showed that long bones measurements were more accurate for sex estimation than cranial ones (see relevant sections below).

In the West, Giles and Elliot (1962) were the first to use similar function analyses. They used nine measurements from 103 white males, 79 white females, 113 black males and 108 black females aged 21 to 75 years from the Terry and the Hamann-Todd collections of medical school cadavers. Twenty-one different combinations managed to determinate sex with an accuracy of 82 to 89\%. A formula based on five measurements reached 82.9\% accuracy on 1022 individuals regardless of ancestry.

In a follow-up study, four hundred skulls originating from the initial sample were accurately sexed in $85 \%$ of the cases using nine measurements (Giles \& Elliot 1963). At least four measurements were necessary in order to reach accuracy of 83 to $88 \%$. This was tested on 670 American Indians of unknown sex, and accurately sexed 61 to $92 \%$ of individuals, demonstrating population variability. Giles (1970) concluded that cranial morphology and morphometry both provided the same accuracy. This was confirmed by Meindl and colleagues (1985a). The accuracy of two of Giles and Elliot functions ("14" and "7") were 
successfully confirmed to 83 and 85\% in a study by Boulinier (1968) based on fifty female and fifty male skulls from French individuals of diverse ancestry and known sex, aged 34 to 79 years, all from anatomical collections. Boulinier (1969) then checked the effect of age on sex estimates from the skull, using his 1968 study sample as well as Giles' sample of 187 white adults of known age. Although young males and old females were the most often misclassified, he concluded that as long as the age of the individuals in the study group was relatively homogeneous, its influence on sex determination would be weak.

Variation in the osteometric sexual dimorphism of the human skull was addressed by Johnson and colleagues (1989), although their sample was relatively small. They utilised 43 linear and eleven angular measurements on 34 australoid, thirty causadoid (from St Bride's Church, London), forty mongoloid and thirty negroid skulls, to establish that the best variables vary with ancestry. Caucasoid skulls were more accurately sexed from bizygomatic breadth, maximum length, nasal breadth and finally palatal length. All these measurements are utilised in this doctoral analysis.

Bizygomatic breadth was also the most dimorphic measurement with $80.2 \%$ sexing accuracy obtained from this measurement alone (demarking point of 125.5) in a study from South Africa (Steyn \& İșcan 1998). The twelve cranial and five mandibular measurements on 44 male and 47 female cadavers from the South African collections of Pretoria and Dart produced nearly 86\% accuracy in sex estimates from discriminant functions using six cranial measurements. Bigonial breadth was also strongly dimorphic. Both these measurements were recorded in this study.

In order to address the problem of incomplete skulls, different measurements were utilised by Holland (1986) to accurately sex 25 white male, 25 white female, 25 black male, and 25 black female fragmentary crania based on nine measurements of the cranial base. Accuracy of the estimates generated ranged from 71 to $90 \%$, with a similar 70 to $85 \%$ accuracy on a test sample of twenty 
additional crania from the Terry Collection. Gapert and colleagues (2009) recently tested a similar method on 75 male and 71 female skulls from $19^{\text {th }}$ century St Bride's Church, London, with a lower accuracy of 69 to $76 \%$. Although the interesting point of this method was to enable the use of fragmentary skulls where only the base could be measured, it still requires the occipital condyles and foramen magnum to be intact, which is an issue when studying ancient archaeological remains. Very few crania would provide the necessary measurements in this population, and this method will therefore not be used in this analysis.

Although these functions all provide ready-to-use formulae and relatively good accuracy, they require several measurements to be available. This is problematic with ancient and damaged osteological material, such as the Neolithic remains under study in this doctoral research, as such consistency in the variety of measurements required would be very difficult to achieve. Therefore, cranial measurements will be collected and analysed (see Appendices 17 and 18 for a full list of the cranial measurements utilised) but without recourse to established formulae.

\section{( Mandible}

Overall sexual dimorphism in the mandible is described in table 2.1 above. Its size, weight, chin development, shape, ramus height and width, mandibular angle, muscle markings at gonion area, size and development of condyles are all understood to be sex indicators, although care should be taken with mandibular angle over $125^{\circ}$ usually thought to indicate a female, if no other female signs can be observed as a wider angle might be assumed by males later in life following extensive tooth loss (Acsádi \& Nemeskéri 1970).

An additional morphological sexual trait named mandibular ramus flexure was established by Loth and Henneberg (1996). It corresponds to the "distinct angulation of the posterior border of the mandibular ramus at the level of the 
occlusal surface of the molars in adult males", while female adults tend to retain their juvenile straight shape. This trait was based on the examination of 116 male and 84 female non-pathologic mandibles from the South African Dart collection of mostly black individuals (with a near perfect 99\% accuracy), a further 59 males and 41 females from this collection with signs of pathology such as tooth loss (91\% accuracy), as well as 85 white and 96 black Americans from the Terry collection (91\% accuracy) and 66 Native Americans from the Smithsonian Institution, Washington DC (92\%). All samples combined reached a high $94.2 \%$ rate of accurate sex estimates. However, other researchers using Loth and Henneberg's mandibular ramus flexure trait were much less successful. Donnelly and colleagues (1998) could only reach $62.5 \%$ overall accuracy on two female and fourteen male white, black and hispanic Americans of known sex and age from the Tennessee forensic collection, in addition to 38 female and 42 male Native Americans sexed on the basis of their pelvic morphology. Males were accurately sexed in $80 \%$ of the cases, but female accuracy was unacceptably low at 37.5 to $50 \%$. Similar results were also obtained by Haun (2000) on 150 archaeological mandibles from the Tepe Hissar collection by comparing results of the mandibular flexure traits to results from other more recognized skull and pelvic sex indicators, and by Hill (2000) on 158 mandibles from the Hamann-Todd collection belonging to white and black Americans with documented age and sex, the last study also revealing high intraobserver error. This trait has much potential as a sexing indicator based on the accuracy of the original study, although care should be taken when interpreting results as shown in studies by other researchers. Therefore, the mandibular ramus flexure will be used in this analysis as a test compared to the other standards used currently, rather than a definite sex indicator.

Sexually dimorphic mandibular measurements from the studies already described above (Hanihara 1959; Giles 1964; Steyn \& İşcan 1998) are all included in the comparative table in Appendix 1. Discriminant functions analyses have also successfully been applied to the mandible. Hanihara (1959) 
obtained 85\% accuracy from four mandibular dimensions in the study described earlier. Giles (1964) correctly estimated the sex of 102 black males, 102 black females, 31 white males and 30 white females from the Terry collection with a similar $85 \%$ accuracy using nine discriminant functions. However, all these functions require several measurements for each individual and thus will not be used in this study.

\section{Dentition}

Sexual dimorphism can be found in the size of human tooth crowns, with up to $7.3 \%$ difference in the lower canines (Hillson 1996). However, differences between populations (White \& Folkens 2005) as well as within each sex, particularly in females (Krogman \& İşcan 1986), are high and overlaps are extensive between male and female dimensions (Ferembach et al. 1980). Moreoever, even though accuracy rate of 60 to $85 \%$ have been published (Owsley \& Webb 1983; Acharya \& Mainali 2008), the actual size differences for individual teeth only reach around half a millimetre (White \& Folkens 2005), adding potential for errors and difficulty in recording accurate enough measurements to provide clear sex distinctions. Although the potential of this method is not denied, its wide overlaps in range between sex, marked population variations and very minimal size difference, in addition to the requirements for the same teeth to be present in a significant number of individuals, means that tooth crown size will not be employed as a sexing criterion in this study.

\subsubsection{Pelvis}

The pelvis differs morphologically between males and females for the purpose of reproduction, and has been described as providing "the most abundant and accurate data for sex estimation" (Ubelaker 1984:42). Its sexual traits are summarised in table 2.3 below. 


\begin{tabular}{|c|c|c|}
\hline Trait/character & Male & Female \\
\hline Size & $\begin{array}{l}\text { Heavier and more robust, large } \\
\text { and rugged }\end{array}$ & $\begin{array}{l}\text { Broader, small and gracile, } \\
\text { smoother }\end{array}$ \\
\hline Ilium & High and vertical & Low and flat, laterally divergent \\
\hline Pelvic inlet & Heart shaped, narrow & Circular or elliptical \\
\hline Pubic shape & Narrow & Broad and square \\
\hline Sub-pubic angle & Narrow, V-shape, $<90^{\circ}$. & Wide, U-shaped, $>90^{\circ}$; rounded \\
\hline Ventral arc* & Absent, not well defined & Present, maybe well-defined \\
\hline Sub-pubic concavity* & $\begin{array}{l}\text { Absent (or angle even slightly } \\
\text { convex) }\end{array}$ & Present (large and obvious) \\
\hline $\begin{array}{l}\text { Medial border inferior pubic } \\
\text { ramus (or ischiopubic ramus)* }\end{array}$ & $\begin{array}{l}\text { Blunt / flat wide/dull, broad, no } \\
\text { ridge, slightly everted }\end{array}$ & $\begin{array}{l}\text { Ridge narrow / sharp, crestlike, } \\
\text { strongly everted }\end{array}$ \\
\hline Greater sciatic notch & $\begin{array}{l}\text { Deep and narrow ( } 30 \\
\text { degrees), small }\end{array}$ & $\begin{array}{l}\text { Wide ( } 60 \text { degrees) and } \\
\text { shallow, large }\end{array}$ \\
\hline Pre-auricular sulcus & $\begin{array}{l}\text { Rare, narrow or absent, thin } \\
\text { grooves }\end{array}$ & $\begin{array}{l}\text { Well developed, wide, more } \\
\text { frequent, large circular } \\
\text { depressions }\end{array}$ \\
\hline Auricular area & Flatter, not raised & $\begin{array}{l}\text { More elevated, sharper } \\
\text { auricular surface edge, raised }\end{array}$ \\
\hline Arc composé & Single curve & Double curve \\
\hline Postauricular space & Narrow & Wide, large \\
\hline Iliac tuberosity & $\begin{array}{l}\text { Large, mound-shape, not } \\
\text { pointed }\end{array}$ & $\begin{array}{l}\text { Small or absent, pointed or } \\
\text { varied }\end{array}$ \\
\hline Sacroiliac joint & Large & Small, oblique \\
\hline Iliac crest & S-curvature & More arched \\
\hline Acetabulum & Large, directed laterally, broad & Small, directed antero-laterally \\
\hline Obturator foramen & Large and ovoid, oval & $\begin{array}{l}\text { Small and triangular, low, wide } \\
\text { with sharp edges }\end{array}$ \\
\hline Sacrum & Long and narrow, more arched & $\begin{array}{l}\text { Short and broad, ventral curve } \\
\text { less distinct }\end{array}$ \\
\hline
\end{tabular}

Table 2.3: Pelvis sexual traits, * indicates Phenice's criteria (table by the author, data from Phenice 1969; Acsádi \& Nemeskéri 1970; Ferembach et al. 1980; İşcan \& Derrick 1984; Ubelaker 1984; Krogman \& İşcan 1986; Rogers \& Saunders 1994; Bass 1995; France 1998; Mays 1998; Bruzek 2002; White \& Folkens 2005; Byers 2008)

Apart from the characters which can only be marked as present or absent, the others are usually scored following the standard -2 to +2 or 1 to 5 scales, although the Data Collection Codebook (Steckel et al. 2005) required to score these traits between 1 (definitely male) and 9 (definitely female), 5 being indeterminate. Acsádi and Nemeskéri (1970) advised to use in order of importance the pubic angle, pubis and ischium length / index, and the greater sciatic notch. 
Seventeen morphological traits were tested by Rogers and Saunders (1994) on 49 right and left hip bones and sacra of adult skeletons from the $19^{\text {th }}$ century cemetery of Belleville, Ontario. Twelve of them gave accuracy results of $80 \%$ or over. Combination of two traits gave accuracy of 95 to $98 \%$. The best single traits were found to be sacrum shape, followed by obturator foramen shape, acetabulum size and orientation, and preauricular sulcus presence, all with over 90\% accuracy. Ventral arc presence, pubis shape, true pelvis size and shape, sciatic notch shape and size, subpubic concavity angle, ilium shape, ischiopubic ramus ridge and pelvic inlet shape, all yielded accuracy of $80 \%$ or over. The auricular surface rise was the only indicator not to fare as well with a limited $73 \%$ accuracy.

\section{( Phenice traits}

Phenice traits are three pelvic characteristics utilised by Phenice (1969) on twenty black males, 160 white males, 52 black females and 43 white females from the Terry collection. They include the ventral arc seen only in females, the subpubic concavity present in females and absent in males, and the sharp medial aspect of the ischiopubic ramus seen in females. Although some population variations were observed, sexing accuracy based on these three sexual characters reached above 95\%. White and Folkens described Phenice's method as the "most accurate method yet known for determining sex of an individual from the skeleton" (White \& Folkens 2005:395).

The accuracy of this method has been tested several times since its publication. Using twelve examiners to sex fifty pubic bones from thirteen male and 23 female white modern individuals aged 52-92 years, Lovell (1989) reported a lower accuracy of $83 \%$ in average, although the only professional physical anthropologist in the testing group had achieved an accuracy of $92 \%$. MacLaughlin and Bruce (1990) used the Phenice characters on European skeletal series with 52 males and 33 females from St Bride's Church in London, 
72 males and 64 females from the Department of Anatomy of Leiden, in addition to 28 males and 24 females from the Department of Anatomy of Aberdeen. The resulting accuracy rates were also much lower than in Phenice's original study, varying from $83 \%$ in the English sample to $68 \%$ in the Dutch collection and as low as $59 \%$ for the Scottish individuals, thus demonstrating significant population variations. Subpubic concavity was found to be the single most reliable indicator. Sutherland and Suchey (1991) obtained the expectedly high $96 \%$ accuracy using the ventral arc when they estimated the sex of 1284 pubic bones from the Los Angeles County Coroner's collection of individuals aged 11 to 99 years. They also noted that the ventral arc first appeared at 14, and only became marked from about 20 years. However they could only get an accuracy of $70 \%$ on the same sample based on the presence or absence of the ischiopubic ridge. Most recently, all three of Phenice's traits were tested on 99 males and 99 females from the Terry collection by Ubelaker and Volk (2002). The accuracy reached $88.4 \%$ overall although much higher for females with $97 \%$ correctly sexed compared to under $80 \%$ of males. Overall accuracy was increased to $96.5 \%$ when taking into account other pelvic indicators.

Despite the population variations, the high accuracies reported make these indicators a requirement for all analyses. Unfortunately, the pubic area involved is very often damaged in ancient skeletal remains, with all three indicators rarely observable on each individual. In this analysis, Phenice traits will therefore be recorded together under "shape of pubis", which will be used as one of the primary morphological indicators of sex.

\section{( Greater sciatic notch}

The greater sciatic notch might be more useful in archaeological remains as it is much stronger than the pubic symphysis and therefore survives longer (Mays 1998). The sciatic notch trait had previously been shown to reach $75 \%$ accuracy on one hundred white males, one hundred white females, fifty black males, and fifty black females from the Hamann-Todd collection (Washburn 1948), 
although the same study did not reveal any correlations between the pelvic traits. More recently, the validity of this sex indicator was tested by Walker (2005), using the standard recording scale of 1 to 5 on 56 black males, 58 white males, 47 black females and 50 white females from the Hamann-Todd and Terry collections, as well as 51 males and 34 females from St Bride's Church. London. Results indicated a clear sexual dimorphism. However, the scores did not follow the expectation of 1-2 indicating a female, 3 indeterminate, and 4-5 male. Instead, in this instance, grade 1 correctly identified $88 \%$ of the females, and $92 \%$ of the males scored 3-5, with 2 the intermediate grade with overlap of males and females. This recognised sexually dimorphic trait with proven accuracy will be used as one of the primary morphological indicators of sex in this analysis.

\section{( Pre-auricular sulcus}

This groove between the auricular surface and the sciatic notch was first described in 1866 and is still considered to appear nearly exclusively in females, although it may sometimes be observed on males (Ubelaker 1982; Cox 2000). On visual assessment of a very small sample of three black, ten white, and four Asiatic-white males together with three black, five white and two Asiatic white females, İșcan \& Derrick (1984) observed that the sulcus might disappear with age. Its accuracy as a sex indicator was established at $80 \%$ by Ferembach and colleagues (1980). Its presence was observed in most females from the documented collection of Coimbra but almost never in males (Arsuaga \& Carretero 1994). However, it has also now been correlated more successfully to pelvic size and shape rather than sex, associated with broad female pelvis and missing from the narrow male pelvis (Mays \& Cox 2000).

Some researchers have also made a distinction between two different types of groove found in both males and females. Houghton $(1974,1975)$ observed a "ligamentous groove" in both sexes and a "groove of pregnancy" in females, based on a sample of 54 male and 65 female pelves. Bruzek (2002) made a 
distinction between the preauricular groove associated with parturition and female sex, and the paraglenoid groove as well as the piriform tubercle, both associated with robusticity and high level of muscular activities which have been considered to indicate a male. This was based on the observations of 98 males and 64 females from a French collection in Paris, in addition to 106 males and 134 males from the collection of Coimbra, Portugal. However, although Bruzek quoted as the source for this distinction the work of Kurihara and colleagues (1996), this latter study described the preauricular sulcus and the paraglenoid sulcus as synonyms for the same groove often found in females but rarely in males. Therefore this groove seems to remain a female trait, and on the basis of all the evidence available at present, the preauricular sulcus will be recorded as one of the primary indicators of sex in these Neolithic populations.

\section{Arc composé (also known as composite arch)}

The accuracy of the composite arch as a sex indicator was reported as merely $60 \%$ by Ferembach and colleagues (1980). However, this feature was used as one of the five pelvic characters in Bruzek's (2002) new methods, based on the sample described in the paragraph above. His five traits included the preauricular surface, the greater sciatic notch's symmetry, the form of the composite arch, the morphology of the inferior pelvis, and ischiopubic proportions. Using these five traits together, sexing accuracy for this sample reached as high as 98\%. Bruzek's method was tested by Listi and Bassett (2006) on 876 left coxals from the Bass, Terry and Louisiana State University collections. Accuracy rates of $95 \%$ were obtained with traditional methods while Bruzek's system was able to correctly estimate the sex of the same individuals $90 \%$ of the time. Based on the lack of compelling evidence for this feature on its own, but the success of the method as a whole, the composite arch (or arc composé) as well as Bruzek's other four pelvic traits will be recorded on the populations under study but will be employed as alternative morphological sex indicators rather than using them for primary sex estimates. 


\section{- Osteometry}

Sexual dimorphism in pelvic measurements mainly results from the size difference between male and female pelves, but also from the difference in shape of the pelvic inlet (larger in females) and sciatic notch (broader in females). This was confirmed by Arsuaga and Carretero (1994) in a multivariate analysis of 34 measurements and ten morphological traits on 227 males and 191 females from the $19^{\text {th }}-20^{\text {th }}$ centuries Coimbra collection, Portugal.

One of the first metric studies in the pelvis was by Straus (1927), based on 244 pelves of white and black Americans of both sexes aged 21-50 years, although the choice of measurements did not correspond to modern standards, apart from the iliac width. Measurements and ranges from this and the studies described below can be found in a comparative table in Appendix 1.

Over $90 \%$ accuracy in sex estimates using pubic length and ischial length "from the point at which they meet in the acetabulum" and the ischium-pubis index $\frac{\text { pubis length } X 100}{\text { ischium length }}$ was obtained by Washburn (1948) on a sample of one hundred white males, one hundred white females, fifty black males and fifty black females from the Hamann-Todd collection. The same method was used in his follow-up study (Washburn 1949) in addition to the sciatic notch width, utilising 152 Bantu known-sex skeletons and 55 (!Kung) Bushman archaeological skeletons. The width of the sciatic notch produced $98 \%$ accuracy when sexing both Bantu and Bushman. However, there was still a vast overlap in the actual ranges (see Appendix 1). A few years later, his study on Eskimo pelvis (Hanna \& Washburn 1953) used the ischium pubis index and greater sciatic notch angle, with similar results to the original 1948 study. Xinzhi and colleagues (1982) used Washburn's method and additional dimensions, including acetabular diameter, on 115 male and 54 female from a contemporary Han population of China, from the collection at Xinjiang Medical college. However, as this population was undocumented, sex had first been determined 
based on pelvic morphology. Although this is the only metric sexing method for pelvis mentioned in several modern textbooks (France 1998), and is still described as "the most famous and effective" metric technique to estimate sex from the pelvis (White \& Folkens 2005:394), several major criticisms have been voiced over the years on the usefulness of Washburn's index. The main problem stems from the landmark used for both measurements, often subjective and hard to locate (Byers 2008; Drew 2013). There are also vast overlaps in the sexes between measurements, especially for the pubic length where the male range (60-88) actually includes the whole of the female range (63-86). Recently, a quarter of 36 known male individuals from the Mary Rose yielded female results based on Washburn's index (Drew 2013). The index will be recorded in the Neolithic populations under study but any sex estimate would need to be considered with caution.

The principle of the ischio-pubic index was adapted by Kelley (1979b) to fragmented ilia, utilising instead a sciatic notch / acetabular index $\frac{\text { greater sciatic notch width } \times 100}{\text { vertical diameter of acetabulum }}$. The method was applied to hundred white males, hundred black males, hundred white females and hundred black females from the Hamann-Todd collection, in addition to one hundred male and one hundred female Native Americans from California. Population variability was evident, and sciatic notch widths overlapped greatly. Nevertheless, accuracy was established at 90\%. The same index was used by MacLaughlin and Bruce (1986) on 71 males and sixty females from the documented St Bride's Church collection in London, together with 74 males and 66 females from the documented Leiden collection in the Netherlands. Population variations were visible between the Dutch and the English samples, and between these European samples and Kelley's (1979b) original American sample. Accuracy using the American based index sectioning point was very good for males with $96 \%$ of the English males correctly sexed and nearly $91 \%$ of the Dutch males. However, accuracy for the females based on the same criteria was less than $58 \%$ for Dutch females and as 
low as under $47 \%$ for English females. Using sample's specific sectioning points gave much more accurate results with nearly 77 and $76 \%$ of the English and Dutch females respectively correctly sexed, while English males were accurately sexed in nearly $82 \%$ of cases, and $77 \%$ of the Dutch males were correctly determined. This method will be utilised as it should prove particularly useful for damaged skeletal remains like this ancient osteological collection, while bearing in mind the importance of utilising population-specific data.

Other researchers utilised iliac measurements rather than concentrating on the pubic area. Camacho and colleagues' (1993) study was based on nine measurements from 15 female and 27 male pelvic bones in a modern Spanish skeletal collection of Madrid. Statistically significant sexual dimorphism was revealed in iliac crest rise, iliac crest arch, iliac width and iliac crest index $\frac{\text { iliac crest rise } X 100}{\text { iliac width }}$. Of these four, the least accurate was the most commonly measured iliac width whereas the two most accurate were the least often utilised iliac crest rise and iliac crest index. Unfortunately, such measurements require the ilia to be complete, which would be quite rare in ancient archaeological collections.

Attempts to provide discriminant functions for sexing the pelvis have been made on collections worldwide. Discriminant functions produced by Howells (1965) were based on Gaillard's skeletal collection of 75 males and 69 females. However, it appears that measurements were not taken in the same way as other studies, as his results differ greatly for pubic length and ischial length compared to other reported ranges (see Appendix 1). Discriminant functions were also produced by Kimura (1982b) using indices based on the pubic length, ischial length, and iliac width of 52 male and 51 female Japanese from Yokohama City University School of Medicine collection, in addition to fifty male white, 52 female white, 49 male black and 48 female black Americans from the Terry collection. Although the functions provided a high accuracy ranging from 74 to nearly $97 \%$, the shape of the pelvis was recommended as a better way to 
assess sex from pelvic bones, rather than size. Schulter-Ellis and colleagues' $(1983,1985)$ functions were based on fifty white male, fifty white female, fifty black male and fifty black female Americans from the Terry collection. These provided a very high 95\% accuracy using the acetabulum / pubis index and 97\% when combined with femoral head diameter. Unfortunately, all measurements utilised with the exception of the acetabular diameter were uncommon and would therefore not be readily available. Population variations were also noticeable.

All length and breadth/width measurements (particularly the strongly dimorphic acetabulum vertical diameter) available in this Neolithic collection will be recorded to utilise the material to its full potential. Ischium-pubis, sciatic notch, and notch-acetabular indices will be produced when the required measurements are available. However, any sex estimates provided from this osteometric data should be population specific in order to have a significant chance of accuracy.

\section{( Parturition}

Following Angel's (1969) study of eight pubic bones of known parity and age from nulliparous to multiparous women showing changes with lipping and erosion, maybe caused by haemorrhages and cyst formation (although also influenced by age), there were numerous studies throughout the seventies on signs of parturition on the human pelvis. If an indicator of parturition could clearly be established, it could consequently also be used as a female sex indicator, as although its absence could indicate either a male or female individual, its presence would definitely prove that the individual was female. Using a much more extensive sample than Angel, Stewart (1970) recorded evidence of scarring on the dorsal side of the pubic symphysis of 110 black and 60 white Americans from the Terry collection, aged 20-109 years. However, an unexpectedly high fifty percent of modern female pubic bones did not show any 
of the scarring generally associated with pregnancy and delivery. Moreover, individuals without scarring included cases with a record of childbirths.

Five metamorphic stages of parturition have been identified by Nemeskéri (1972), the first associated with one to two childbirths, the second with three to four childbirths, the third with four to five childbirths, the fourth for six to eight childbirths, and finally the fifth state for more than eight to ten childbirths. These stages were utilised by Ullrich (1975), together with an added "0" stage with five subgroups, on a sample of 39 males and 38 females from a Slavic cemetery in Sanzkow with 63 pubes, 70 ilia, and 49 sacra. These were promising as they would not only provide a useful sex indicator, but could contribute greatly to palaeodemography.

Evidence of parturition on the human pelvis was also described by Houghton (1975) as "smooth-walled pits or craters" on the dorsal surface of the pubic symphysis and at the preauricular groove, which Putschar (1976) attributed to the resorption and remodelling of the pubic symphysis accompanying pregnancy and the trauma of birth. The dorsal surface of the pubic symphysis of 68 females with medical records were then examined by Holt (1978), searching for evidence of scarring such as pits, cavities and depressions. Unfortunately, over a third of women who had never given birth were found to exhibit the typical "birthing" scars, strongly questioning the aetiology of these pathological signs and their use as indicator of parturition. This was confirmed by Suchey and colleagues' (1979) analysis of the pubic bones of 480 autopsied or donated females of known parturition, aged 13-99 years. Only a weak correlation was found between scarring and number of births in this sample, and age was also correlated. Indeed, seventeen females with no recorded parity showed evidence of "birth" scarring, whereas 22 females with a record of one to five pregnancies did not exhibit any signs on their pubis. In opposition to the results of these studies, the pelves of 75 white and 123 black females from the Hamman-Todd collection inspected by Kelley (1979a) for parturition scars were reported as 
significantly relating to parturition, although potentially becoming obliterated in old age. This was based on dorsal pubic pitting, interosseous grooves and preauricular grooves (since attributed to the difference in size and shape of the pelvis rather than parturition, see relevant section above). However, the percentage distribution of these traits between nulliparous and parous females lacked clarity, and another study on 87 males and 151 females with known parity from the same Hamann-Todd collection did not produce any significant association between pelvic scarring and parity (Andersen 1987). Instead, pelvic scars were thought to have been the produce of excessive movement of the pelvis, may it be from giving birth, trauma or activity.

Rather than limiting indicators of parturition to pelvic scars, Cox and Scott (1992) established a significant relationship between number of births and pubic tubercle extension on 94 adult females with documentation, including information on parity, buried during the $18^{\text {th }}$ and $19^{\text {th }}$ centuries at Christ Church, Spitalfields. This was thought to be linked to the function of the pubic tubercle as the attachment site of the rectus abdominis, particularly important during the last months of pregnancy and at birth. However, this extension was also present in as much as a third of nulliparous females and $87 \%$ of parous females. Moreover, neither preauricular sulcus nor pubic pitting correlated with parity status. As this was an historical sample, there was no certainty that all births had been recorded (Cox 2000), which could have created a bias in the number of women officially recorded nulliparous, or the number of births recorded for parous individuals. This was addressed in a more recent study by Snodgrass and Galloway (2003), based on 148 pelves from females aged 17 to 99 years autopsied at the Los Angeles County Department of the Coroner in the late 1970s, with more reliable documentation including parity status. However, tubercle height was not statistically correlated with the reported number of pregnancies in this sample either. Dorsal pitting was found to have a significant association with number of births for women under 50 , but in older individuals, the effect of age was more significant. 
Based on the unreliability of this trait and the controversial aetiology of its osteological evidence, it was decided not to use this potential sex indicator in this study.

\subsubsection{Spine and Thorax}

\section{- Spine}

Although the atlas has been described as the best vertebra to observe sexual dimorphism due to its larger dimensions in males (Acsádi \& Nemeskéri 1970), most studies have been undertaken on the sacrum, with its sexually dimorphic shape (see table 2.3 above) and dimensions linked to the different pelvic shape in males and females. In males, the body or promontory is wider than the alae, whereas in females, body width has been described as either equal to (Anderson 1962) or smaller than (France 1998) each ala or wing.

Numerous osteometric studies have been published. Strádalová's $(1974,1975)$ analysis of 72 male and 56 female sacra belonging to white Europeans from Charles University, Prague collection, provided a high sexing accuracy of 86.5 to 88.5\%. Unfortunately, the many measurements required in this method severely limit its use on damaged archaeological remains. Flander's (1978) study of fifty white male, fifty white female, fifty black male and fifty black female Americans from the Terry Collection was restricted to six measurements, which might still be difficult to collect from ancient populations. The discriminant functions he calculated correctly estimated the sex of $84 \%$ of the white individuals and $91 \%$ of the black individuals, although accuracy was much lower when ancestry was unknown, varying from 54 to $78 \%$, indicating the need for population-specific functions.

A simpler method was provided by Kimura (1982a), who studied the base-wing index $\frac{\text { wing widthX } 100}{\text { base width }}$ as an indicator of sex, thus only using two measurements which are more likely to be available in ancient osteological collections. Fifty- 
two male and 51 female Japanese from Yokohama City Medical School in addition to fifty white males, fifty white females, 49 black males and 48 black females from the Terry Collection were observed and their measurements analysed. $80.4 \%$ of the white Americans were correctly sexed with the formula:

$y=$ base width $-(0.604 X$ wing width $) \quad$ [ sectioning point $=9.494$ ]

Population differences were visible in the measurements and the indices, although female base widths were always lower than in males, and female wing widths were consistently larger than in males, demonstrating a crosspopulation sexual dimorphism. Kimura's base-wing index was tested against the sacral index $\frac{\text { max.breadth } X 100}{\text { max.height }}$ by Patel and colleagues (2005) on 32 male and 32 female sacra from the Department of Anatomy, Jamnagar, Saurashtra. This study provided poor accuracy rates when using a demarking point, with the sacral index identifying correctly only $62.5 \%$ of males and $69 \%$ of females, and Kimura's index performing extremely poorly with fewer than $19 \%$ of males and females accurately sexed. This highlights the need for population specific data.

The shape of the sacrum will therefore be utilised in this analysis, although only as an alternative morphological trait. Sacral and base-width indices will also be established when the relevant measurements are available and explore their potential as sex indicators, but without using demarking points provided by other populations.

\section{( Sternum}

Sexual dimorphism studies on the sternum are all based on its proportions. The sternum body has been indicated to often reach twice the length of the manubrium in males, while accounting for less than twice the manubrium length in females (Bass 1995). This follows Dwight's (1881) original findings of sexual dimorphism in the manubrium length to body length ratio at 49:100 in males and 52:100 in females (also quoted in Acsádi \& Nemeskéri 1970). 
Other studies mostly concentrated on the maximum combined sternal length. Macerated sterna from 312 male and 88 female Northern Indians were utilised by Jit and colleagues (1980) to establish that the width of the first and third sternebrae were "useless in sexing a sternum" (Jit et al. 1980:224), as so were the lengths of manubrium or mesosternum on their own, with a hundred percent overlap in female and male ranges. However, one hundred percent accuracy was reached on their sample for males with a combined manubrium and mesosternum length over $140 \mathrm{~mm}$ and for females with combined manubrium and mesosternum length under $131 \mathrm{~mm}$, while $131-140 \mathrm{~mm}$ was classified as an indeterminate area. They also tested Hyrtl's Law index $\frac{\text { manubrium length } X 100}{\text { mesosternal length }}$ used for 150 years, which only identified correctly $31 \%$ of males, although 89\% accuracy was achieved in females.

Another similar study on a North-American population also provided one hundred percent accuracy with a female combined length smaller than $121 \mathrm{~mm}$ and a male combined length larger than $173 \mathrm{~mm}$, although these measurements would need to be corrected for skeletal remains to take account of radiographic distortion. This study by McCormick and colleagues (1985) based on the plate radiographs of 617 autopsied adults also offered alternative measurements for lesser accuracies of 90 and 80\%, and resulted in an 85\% accuracy rate overall for sexing individuals by combining manubrio-mesosternal length and pattern of cartilage mineralisation.

The differences in measurements between these two studies however are vast (see Appendix 1). A high proportion of North Indian males would be determined as probably females based on North American radiographic measurements. This highlights the problem of population variability where sexual dimorphism remains the same in proportions but differs greatly in measurements, and advocates for population-specific comparison of indices and measurements, which will be undertaken in this analysis based on manubrium length, sternum body length and sternum index. 


\section{- Ribs}

Sexual dimorphism in the pattern of rib ossification has been confirmed by McCormick and Stewart (1983) based on the examination of the radiographs of 407 males and 244 females autopsied adults. However, as this process is only visible on radiographs with the cartilaginous parts still present, this method is unlikely to be of use for the majority of archaeological remains, and will not be used in this analysis.

Another alternative which does not require the use of radiographic facilities was provided by İşcan and Loth (1998). In their study, 83\% of 144 male and 86 female autopsied individuals were accurately sexed based on the dimensions of the sternal end of their fourth rib. Although male measurements were significantly larger, the individuals analysed had to be correctly aged first as accuracy changed drastically if the incorrect age category was selected, with more than $97 \%$ of young males classified as females when using old age group formulae, and over half the old females determined as males if young age category formulae were used. Moreover, this method also requires the fourth rib to be correctly identified, which can be very problematic for archaeological remains (Mays \& Cox 2000). The prerequisites of an accurate adult age estimate (see chapter 3, section 3.3) and correct identification of the fourth rib therefore also led to this method being discarded as a possible sexing tool in this study.

\subsubsection{Shoulders and Upper Limbs}

\section{- Clavicle}

In females, the clavicle is gracile, shorter, and has a small circumference, whereas in males it is longer, heavier, thicker, with a larger circumference (Acsádi \& Nemeskéri 1970, based on studies by Olivier in the 1950s). Eight measurements have been given by Thieme (1957, reproduced in Bass 1995) to estimate the sex of Black Americans based on their clavicle, although the clavicle 
length was reported as not very accurate. Jit and Singh (1966) also found that clavicle measurements were inadequate sex indicators on 116 male and 61 female Indian cadavers, with 80 to $92 \%$ of males not recognised by clavicle length. However, midshaft circumference was more successful with $72 \%$ of males correctly identified, whereas females were identified more accurately (though still poorly) from clavicle length than any other measurements. The maximum length, diameters and circumferences will be recorded, as well as the relevant indices when enough measurements are available, in order to attempt establishing the relevance of such osteometric data as a sex indicator for such ancient populations (see Appendix 20 for a full list of the data utilised).

\section{( ) Scapula}

The glenoid cavity length is the most commonly used sexually dimorphic measurement on the scapula, and tends to survive well in archaeological remains. The earliest studies of this metric sex indicator date back to the late $19^{\text {th }}$ - early $20^{\text {th }}$ centuries, when a study based on 63 males and 27 females established that the average male glenoid length was $3.92 \mathrm{~cm}$ and the average female glenoid length $3.36 \mathrm{~cm}$, with a cutting point of $3.6 \mathrm{~cm}$ (Dwight 1894, 1904-1905). As these measurements were taken on fresh bones with cartilage still intact, they would exceed any dimensions on dry bones.

However, the glenoid cavity length is not the only scapular measurement of use in the sex estimation of skeletal remains. The most dimorphic measurement has been given as the length of the scapular spine (Acsádi \& Nemeskéri 1970, based on studies by Olivier in 1960). Unfortunately, the spine is often found damaged in ancient osteological collections. Eleven measurements in total were also analysed by Bainbridge and Genovés (1956), based on European samples including 31 males and 41 females from St Bride's Church, London, 26 males and twenty females from Schooten cemetery, Belgium, and 46 males and thirteen females from the Department of Anatomy, Cambridge. They provided results with $99.9,92.4$ and $84.2 \%$ limits and utilised these documented samples 
to sex 52 left and 65 right scapulae of unknown sex. Unfortunately, they did not release the results obtained from the length of the glenoid cavity. Iordanidis (1961), like Bainbridge and Genovés, preferred the use of the glenoid cavity width to its length, although this dimension yielded more misclassification than scapular height, breadth or total spine length. Some of the studies were undertaken on extensive samples, such as Krogman's comparison of the scapular dimensions of thousands of individuals from different populations, including the scapular index (Krogman 1962, also quoted in Bass 1995). Byers (2008) chose Stewart's (1979) data on maximum length of scapula and length of glenoid cavity to use as sex indicators on the scapula. Finally, the Tennessee Data Bank (run by Jantz and Ousley, data in France 1998) provided a discriminant function suitable for white and black Americans with a high $94 \%$ accuracy.

All metric data from these studies is available in the comparative table in Appendix 1. Glenoid cavity length as well as scapular maximum length, maximum breadth, spine length and scapular index when available will be recorded to establish sexual dimorphism in the scapulae of the Neolithic individuals under study.

\section{Humerus}

Humeral measurements have been used as sex indicators by numerous researchers as early as the start of the $20^{\text {th }}$ century (Dwight 1905), with a marked sexual dimorphism observed on all populations. Available studies with full details of the samples they are based on can be found in Appendix 1, mostly consisting of head diameter, maximum length, midshaft circumference and epicondylar width. Head diameter, followed by the epicondylar width (also sometimes referred to as epicondylar breadth) are the most often utilised dimensions due to their high reported accuracy worldwide. Both transverse and vertical diameters of head have been described as "excellent" sex indicator with $89 \%$ accuracy, whereas epicondylar width, maximum and physiological 
lengths, midshaft minimum and maximum diameter yielded accuracies ranging from 75 to $85 \%$ on 173 male and 168 female Native Americans from California (kept at the Lowie Museum), sexed on the basis of Phenice's three pubic traits (Dittrick \& Suchey 1986). Measurements and formulae for other populations included studies on Chinese, Japanese and Thais (İşcan et al. 1998), as well as black and white South Africans (Steyn \& Iscan 1999). This last study, based on 55 white males, 49 white females, 43 black males and 45 black females from the Dart and the Pretoria collections, demonstrated that head diameter and epicondylar width were the most accurate to determine the sex of white individuals, with up to $96 \%$ accuracy. France $(1983,1985,1998)$ related that the best sex indicators were in the measurements of the proximal humerus, although she also confirmed all previous studies' findings that population variability was very high and activities could modify the original dimensions of the bone. She provided formulae for each measurement for black, white and Native Americans. The best results for white Americans were obtained from the head transverse diameter with $92.3 \%$ accuracy, followed by the vertical diameter with $89.4 \%$ accuracy, while the epicondylar width formula correctly identified only $85.5 \%$ of her sample. Care must be taken when using these equations as the measurements should be entered in centimetres as opposed to the standard millimetres and males are identified by results smaller than the cutoff point rather than above it. Other analyses also produced discriminant functions, such as Steel's (1972) study based on 27 males and 33 females of known age, sex and ancestry from St Bride's Church, London, which provided a function requiring three measurements.

The discriminant function with the highest accuracy was obtained in the Tennessee Data Bank (in France 1998, data from Jantz and Ousley), which reached $96 \%$ accuracy using three measurements from the combined results of 111 female and 151 male white and black Americans. 
A morphological method to estimate sex from the distal end of the humerus was published in the last few years, first for adults and most recently for adolescents (Rogers 1999, 2009). This method was based on 175 male and 27 female white adults aged 18-86 years from the Grant Collection, Toronto, and tested on 28 males and 7 females aged 20-90 years from the documented collection at the Department of Anthropology, New Mexico, in addition to 74 white males and 19 white females from the Bass Collection, aged 25-89 years. The four features and their accuracy on test samples are shown in table 2.4 below.

\begin{tabular}{ccccccc}
\hline $\begin{array}{c}\text { Trait/ } \\
\text { character }\end{array}$ & Male & $\begin{array}{c}\hat{0} \\
\text { accuracy }\end{array}$ & $\begin{array}{c}\text { Test } \\
\text { accuracy }\end{array}$ & Female & $\begin{array}{c}+ \\
\text { accuracy }\end{array}$ & $\begin{array}{c}\text { Test } \\
\text { accuracy }\end{array}$ \\
\hline $\begin{array}{c}\text { Olecranon } \\
\text { fossa }\end{array}$ & Shallow triangle & $83 \%$ & $88.6 \%$ & Deep oval & $88 \%$ & $80.2 \%$ \\
$\begin{array}{c}\text { Medial } \\
\text { epicondyle }\end{array}$ & $\begin{array}{c}\text { Flat / slightly } \\
\text { raised }\end{array}$ & $87 \%$ & $74.5 \%$ & Marked angle & $81 \%$ & $82 \%$ \\
$\begin{array}{c}\text { Trochlear } \\
\text { constriction }\end{array}$ & Less constricted & $87 \%$ & $56.5 \%$ & $\begin{array}{c}\text { More } \\
\text { constricted }\end{array}$ & $73 \%$ & $88.6 \%$ \\
$\begin{array}{c}\text { Trochlear } \\
\text { symmetry }\end{array}$ & Asymmetrical & $69 \%$ & $79.9 \%$ & Symmetrical & $77 \%$ & $83.2 \%$ \\
\hline
\end{tabular}

Table 2.4: Rogers' distal humerus sexing traits (table by the author, data from Rogers 1999; Falys et al. 2005)

However, it should be kept in mind that a very high proportion of the samples was male, so this methodology might have suffered from a male bias. The adolescent sample included five females and two males from Christ Church, Spitalfields, together with 17 females and 18 males aged 11-20 years from the Luis Lopes collection, Bocage, Portugal, all documented. Sex was estimated using the four features detailed above, and yielded 71\% accuracy for the English sample and $86 \%$ for the Portuguese sample, with $80 \%$ accuracy for all males and $82 \%$ for all females. In a test of Rogers' method on 184 males and 167 females from St Bride's Church, London, positive results were obtained in all but the trochlear constriction trait for males, producing an overall accuracy of $74.9 \%$ for males and $83.5 \%$ for females (Falys et al. 2005). 
Based on the overwhelming evidence for sexual dimorphism in the humerus proportions, together with population variability in the actual figures, all the measurements possible will be collected from the populations under study (see Appendix 20) to establish sexual dimorphism for this Neolithic group based on the size of their humeri. Rogers' promising morphological method for sexing based on distal humeral sexing traits will also be applied, although these will be considered as alternative traits rather than primary evidence for sex estimation.

\section{( Radius}

Measurements from available studies with sample details can be found in Appendix 1, mainly consisting of maximum length, midshaft circumference, head circumference, and distal end breadth. In the Tennessee Data Bank (France 1998, data from Jantz and Ousley), 73 female and 116 male white and black Americans were identified with a near perfect 99\% accuracy for females and $86 \%$ for males with a function based on their combined results for two less used dimensions. These and all other measurements will be recorded in this study.

\section{( Ulna}

Measurements from available studies with sample details can be found in Appendix 1, including maximum length, midshaft circumference and distal end breadth. In the Tennessee Data Bank (France 1998, data from Jantz and Ousley), seventy female and 96 male white and black Americans were identified with 91\% accuracy based on their combined results for two less used dimensions.

Three new measurements (the olecranon-coronoid angle in addition to the length and the width of the inferior medial trochlear notch) were also utilised more recently on one hundred male and sixty female dry ulnae from Madhya Pradesh (Purkait 2001). The olecranon-coronoid angle proved to be the best indicator with $85 \%$ accuracy and an independent test sample reached $90.6 \%$ 
accuracy. However, these measurements would be altered by any pathology such as lipping and would need most of the proximal end of the ulna to be undamaged, which might be an issue with archaeological remains. The aim is to record as many of all these measurements as possible during this analysis of Neolithic skeletal remains in order to establish the sexual dimorphism of this bone in such ancient populations.

\section{( Hands}

During the nineties, several studies were undertaken utilising the rarely used potential of hand bones for sexing skeletal remains. Measurements from all the studies described in this section can be found in the comparative table in Appendix 1. The first regression equations were provided by Scheuer and Elkington (1993) based on six measurements of the five metacarpals and first proximal phalanx of 33 male and 27 female British cadavers aged 19-86 from dissection rooms of medical schools. Their formulae employed all measurements for each metacarpals and the phalanx. Their equation for the second metacarpal was tested by Lazenby (1994) on 351 bones from 179 white individuals (only 47 with documented age and sex) from the $19^{\text {th }}$ century cemetery of Belleville, Ontario. Very good results for males were observed with over $90 \%$ identified, including one hundred percent of the known males, as wells as more accurate results based on the right side. Conversely, results were very poor for females with more than $60 \%$ of females misidentified as males, and the most accurate results obtained from the left side. Scheuer and Elkington's six measurements were then utilised by Stojanowski (1999) on 55 white males, 22 black males, thirty white females and fifteen black females from the collection of the University of New Mexico. However in this instance the calculated formulae did not require a full set of measurements, yet still provided a reasonably high accuracy ranging from 75 to $90 \%$, with the most consistent results provided by the fourth metacarpal. 
Two years after Scheuer and Elkington's original publication, Falsetti (1995) provided his own equations based on 51 black males, 56 black females, 58 white males and 47 white females from the Terry collection, and tested them on 33 individuals from the Royal Free Medical School in London as well as forty individuals from donated/forensic cases at University of New Mexico Maxwell Museum. The functions for the second, fourth and fifth metacarpals were demonstrated to be suitable for people of unknown ancestry. However, the functions for the first and third metacarpals showed a wide range of results depending on the population. The highest accuracy was achieved using the formulae for the second metacarpal. A year later, the hand bones of forty white males, forty white females, forty black males and forty black females from the Terry and Huntington collections were used to establish sex from their measurements with 89\% accuracy for the best formula (Smith 1996). However, it was advised that a full set of (undamaged) bones from one hand should be used, which would rarely be possible with ancient archaeological remains and would still only produce an accuracy ranging from 67 to $82 \%$.

The validity of Scheuer and Elkington's (1993), Falsetti's (1995) and Stojanowski's (1999) equations was tested by Burrows and colleagues (2003) on a limited sample of twelve male and eleven female white cadavers, bones cleaned of soft tissue. Their results achieved a lower accuracy than reported for the first two methods, and higher in Stojanowski's case, although the conclusion was very guarded about the use of metacarpals for sex determination in general. The potential of hand bones as sex indicator having been established, further research ensued.

The problem of incomplete or partially damaged hand remains was addressed by Barrio and colleagues (2006), whose method only required a single measurement, making it more appropriate for use as sex indicator on archaeological material. Their study utilised eight metacarpal measurements from 37 males and 42 females in a Spanish collection at the Complutense 
University of Madrid, who had died between 1975 and 1985 aged 20-91 years. Rates of accuracy obtained from the resulting two equations for each metacarpal using a single variable ranged from 81 to 91\%. Population variability would however still need to be taken into account.

The issue of activity-related changes in robusticity was then addressed by Case and Ross (2007) by only using the maximum length of metacarpals and phalanges. Rates of accuracy of their method based on 123 white females and 136 white males aged 18-72 years from the Terry Collection varied from 76 to $86 \%$. They also established that the left side gave better results than the right side, and that phalanges, especially from the distal row, provided the most accurate estimations. Unfortunately, unless the phalanges were recorded in situ when the skeleton was excavated, it is very difficult to distinguish precisely the side and the digit of each phalanx in archaeological remains, limiting this source of increased accuracy.

Finally, the sexing potential of carpal bones was also investigated on fifty males and fifty females from Christ Church, Spitalfields (Sulzmann et al. 2008). Five to nine measurements were taken on each carpal, with resulting accuracy ranging from 65 to nearly 89\%. Unfortunately, carpal bones from ancient archaeological remains are not always recovered and are often damaged.

The recent addition of hand bones as potential sex indicators for skeletal remains is most welcome. Ancient collections such as the one used in this study often include metacarpals and phalanges, which can sometime be one of the only sources of full measurements. As detailed above, specific equations from other studies might not be appropriate, particularly if they require full sets of measurements as hand skeletal remains are often incomplete. The use of carpal bones and correctly side phalanges would also be very limited in such collections. However, all measurements available will be recorded and sexual dimorphism of the metacarpals and proximal first phalanges of these Neolithic populations analysed based on these dimensions (see Appendix 21 for full list). 


\subsubsection{Lower Limbs}

\section{( Femur}

The femur has been described as one of the most studied, if not the most studied, postcranial bones (Krogman \& İșcan 1986; Bass 1995; Byers 2008), and its head diameter the best single measurement to estimate sex (T.D. Stewart 1979). Studies of this bone as a sex indicator go back to the start of the $20^{\text {th }}$ century, first with Dwight's (1905) measurements of femoral head diameters based on fresh bones including cartilage from two hundred males and two hundred females, followed by Parsons' (1914) analysis of more than three hundred undocumented femurs from Rothwell Church in England and his follow-up study on 31 males and 14 females of known identity, from hospitals dissecting rooms (Parsons 1915). All documented individuals were poor elderly people, with an average height of 5'6 for males and 5'1 for females, which might be closer to archaeological remains than contemporary healthy and well-fed individuals. Although the bones were still fresh, Parsons took into account the added cartilage so the measurements published would be suitable for dry bones. Parsons' research was quickly followed by Pearson and Bell's (1917/1919) thorough study of historic long bones, whose results were widely used in forensic anthropology for most of the 20 th century, and by Holtby's (1918) publication of femoral indices and measurements, based on 56 male and 44 female femurs from the Anatomical Department of Trinity College, Dublin. These measurements and those of other studies described in this section can all be found in the comparative table in Appendix 1 with details of their samples.

Discriminant function analysis were also utilised in the second half of the $20^{\text {th }}$ century. Two formulae using the same four measurements were produced by Hanihara (1958) based on the dimensions of 48 male and forty female contemporary Japanese femurs, yielding a very high 96\% accuracy. Hanira's data on Japanese individuals was added to Pons' (1955) data on white European by Giles (1970) to provide functions showing similar sexual dimorphism 
between the two populations, with accuracy just above 94\% using four measurements: the oblique length, head diameter, minimum transverse shaft diameter and bicondylar breadth. Schulter-Ellis and colleagues $(1983,1985)$ in their study of fifty black male, fifty black female, fifty white male and fifty white female pelves (see 2.3.2) also found the same high accuracy results regardless of ancestry using the femoral head diameter. A function to estimate sex on the basis of three femoral measurements was also produced by Steel (1972, see 2.3.4).

The femoral head diameter and distal breadth were demonstrated to achieve 87 and $90 \%$ accuracy on their own respectively, and when combined in a function increasing to $91 \%$ accuracy for white and 94\% accuracy for black individuals (İșcan \& Miller-Shaivitz 1984a). This was based on the observation of 56 white males, 55 white females, 52 black males and 61 black females from the HamannTodd collection.

A new method established the femoral midshaft circumference as another sex indicator, based on 63 male and 51 female Native Americans aged 20 years or over from the Libben Site, Ohio (T.K. Black, III 1978b). However, as this population was undocumented, sex of the individuals had first been established by Phenice's method (see 2.3.2) and age estimated by Lovejoy from six indicators (see 3.3). Sexual dimorphism in the femur midshaft circumference was demonstrated by a student t-test with an $81 \mathrm{~mm}$ section point providing 85\% accuracy, although femoral length provided a higher chance of correct identification at over 89\%. DiBennardo and Taylor (1979) tested Black's (1978b) new method on 35 female and fifty male white American femora of known age and sex from a collection at the American Museum of Natural History, New York, and established that the midshaft femoral circumference was as accurate as other femoral measurements for sexing. In a further study, they concentrated on femurs of black Americans (65 male and 65 female) from the Terry Collection and found that ancestry did not influence sex estimation 
based on femoral measurements, although accuracy for black individuals based on femoral circumference was much lower at $73 \%$. This would advocate the use of femoral dimensions as evidence of sexual dimorphism regardless of ancestry, but would also call for population-specific data in establishing cutting points.

Six out of nine femoral measurements were confirmed as good sex indicators by Dittrick and Suchey (1986), including the maximum head diameter with $89 \%$ accuracy, bicondylar width (between 86 and 89\% accuracy), midshaft circumference and diameters, as well as maximum and physiological lengths. Their study, like Black's method above, was based on undocumented 145 to 177 male bones and 143 to 173 females sexed on the basis of Phenice's three pubic traits, relative width of the pubic bone and configuration of the ventral rampart.

Numerous other metric studies have provided results for specific populations including Chinese (Liu 1989), white and black South Africans (Steyn \& Iscan 1997; Asala 2001), Spanish (Trancho et al. 1997), Thais (King et al. 1998), Germans (Mall et al. 2000), Croatians (Šlaus et al. 2003), Kosovans and Bosnians (Jantz et al. 2008), while the Tennessee Data Bank (run by Jantz and Ousley, data in France 1998) provided an equation based on four measurements suitable for white and black Americans with 91\% accuracy, based on 47 females and seventy males.

Finally, Purkait's triangle method was tested recently by Brown and colleagues (2007). This triangle is made of three measurements from the most lateral projecting point on the femoral head to the most medially projecting point on the greater trochanter $[\mathrm{AB}]$ to the most posteromedial point on the lesser trochanter [BC] and back to the most lateral projecting point on the head [AC]. The sexing accuracy on fifty white males, fifty white females, fifty black males and fifty black females was very poor for males between 54 to $65 \%$, but better for females ranging from an acceptable 85\% to an impressive $99 \%$ for the distance between the greater and the lesser trochanter. Measurements of this last trait provided means of $48.83 \pm 4.21$ for females and $45.95 \pm 3.93$ for males. 
Due to its potential, Purkait's greater to lesser trochanter length will be recorded in this study in addition to all other measurements mentioned in this section (see Appendix 23 for full list), before analysing the sexual dimorphism of this Neolithic population based on their femoral dimensions.

\section{( Patella}

The patella has also been shown to be sexually dimorphic, and might therefore potentially be utilised as a sex indicator. Seven patellar measurements were recorded on forty male and forty female Southern Italians from the collection of the Institute of Legal Medicine of Bari, who died around 1970 aged 25-80 years (Introna Jr et al. 1998). The highest accuracy in the equations produced was for a function using patella width and thickness, which correctly identified $83 \%$ of the sample. Six measurements on the patellae of sixty male and sixty female black South Africans belonging to the Dart Collection confirmed this dimorphism, with 85\% success from the best results (Dayal \& Bidmos 2005). The dimensions' means obtained in the latter study, although on a very different population, were very similar to those obtained by Introna Jr. and colleagues on their South Italian sample (see Appendix 1). Both maximum breadth and maximum length of the patella will be recorded in the populations under study.

\section{( Tibia}

Numerous metric studies on the tibia exist, with a range of sexing accuracies depending on the measurements used and the population under study. The highest reported accuracy of sex estimations based on the dimensions of the tibia was obtained by Hanihara (1958) in a contemporary Japanese sample of 48 males and forty females. His two formulae using the same four measurements provided a very high accuracy around 96\%. However, a later study on a similar contemporary Japanese sample of 47 males and 34 females from the Tokyo Collection by İşcan and colleagues (1994) revealed variations in 
accuracies when based on seven separate measurements. Accuracy for sexing males was observed to be constantly higher than for females, with $95 \%$ of males correctly identified from the proximal breadth though only $79.4 \%$ of females, an accuracy of $87 \%$ for males using distal breadth but still only $79.4 \%$ for females, and the widest gap in accuracy of estimates from the circumference at nutrient foramen with $87 \%$ of males and only $70.6 \%$ of females correctly identified.

In Europe, the St Bride's documented collection was utilised by Steel (1972) to produce a formula using three measurements. In North America, the Terry Collection was used by Symes and Jantz (1983) in addition to a post-contact burial sample of northern Plains Indians to establish that the proximal tibia sexing accuracy was $88.75 \%$, distal breadth $86.25 \%$ and circumference at nutrient foramen 82.5\%. However, İşcan and Miller-Shaivitz's (1984b, 1984c) analysis of forty white males, 39 white females, forty black males and forty black females also from the Terry Collection provided different results. In their sample, circumference at nutrient foramen offered $77 \%$ accuracy for white individuals, whereas length only reached $66 \%$ for the same group, together with $80 \%$ and $81 \%$ respectively for black adults. Proximal breadth presented the most significant sexual dimorphism for black and white individuals $(82.5 \%$ accuracy for white males and $92.3 \%$ accuracy for white females), followed by distal breadth (82.5\% for white males and $87.2 \%$ for white females) and circumference at nutrient foramen $(75 \%$ for white males and $79.5 \%$ for white females). Tibia length was the least significant dimension. Finally, the Tennessee Data Bank (run by Jantz and Ousley, data in France 1998) provided an equation based on two tibial measurements suitable for both white and black Americans with $86 \%$ accuracy, based on 102 females and 133 males.

Bass (1995) warned that any tibial measurements would be population-specific. This is visible in Appendix 1, which includes results of the studies mentioned in this section. Mean Japanese male dimensions would give a female estimate 
using Symes and Jantz (1983) data, or indeterminate based on İșcan and MillerShaivitz (1984), both based on white Americans. In this doctoral analysis, all measurements mentioned in this section (see Appendix 23 for full list) will be utilised to establish sexual dimorphism specifically for this Neolithic population.

\section{- Fibula}

Based on the metric results of a study of 224 male and eighty female cadavers from the Varasani region of India (Singh \& Singh 1976), fibular length has been observed as the strongest indicator of sex in the fibula. In order to maximise the potential of these ancient remains, both mediolateral and anteroposterior midshaft diameters as well as midshaft circumference will be recorded in addition to the maximum length.

\section{- Long bones sexual dimorphism overall}

According to Krogman (1955), there is a male-to-female ratio between long bones, ranging from 85 to $95 \%$, the ulna and the radius being the most dimorphic. This was based on his comparison of two documented individuals belonging to the Hamann- Todd collection, whom he had chosen as representing an "average" male and an "average" female. However, as these ratios were established on two individuals only and from a single collection, they should only be considered as a general indicator of sexual dimorphism in long bones.

Equations using diameters and circumferences of divers long bones were also provided by the Tennessee Data Bank (run by Jantz and Ousley, data in France 1998), based on 119 females and 150 males. They were suitable for both white and black Americans and provided very accurate results. These are of particular interest for ancient archaeological remains as they can provide sex estimates even if both ends of the bones are missing or too damaged to measure. 


\section{(๑) Feet}

Foot bones are rarely used as a sex indicator in archaeological remains. Yet they often survive, particularly the larger calcaneus and talus, and have been shown to be sexually dimorphic, as will be demonstrated in this section.

\section{$\underline{\text { Calcaneus }}$}

The first study highlighting the potential of the calcaneus for estimating sex was published by Steele (1976), who analysed five measurements of the calcanei of thirty white males, 29 white females, thirty black males and thirty black females from the Terry Collection. Male dimensions exceeded those of females regardless of ancestry, although all the ranges overlapped greatly. Same results were obtained on a Native American sample, albeit with smaller means, demonstrating sexual dimorphism in the proportions of the calcaneus, although measurements themselves will be population-specific. Two decades later, a European study on an extensive sample confirmed this sexual dimorphism, based on radiographs taken at the Clinic for Radiology of the University of Mainz, Germany, representing eight hundred Central Europeans aged 20-79 years (Riepert et al. 1996). Eighty percent accuracy was obtained with a section point of $86 \mathrm{~mm}$ for calcaneal length, with $8 \mathrm{~mm}$ differences between male and female means. Unfortunately, as these were radiographic measurements, they would not be directly applicable to osteological remains. However, the study of Introna and colleagues (1997) shortly thereafter was based on the osteological remains of forty males and forty females aged 20-80 years from the contemporary Southern Italian collection of Bari. Eight measurements on eighty right calcanei were used in univariate and multivariate analyses, resulting in $85 \%$ accuracy using three functions. Sex estimates from univariate discriminant function based on calcaneal maximum length also reached nearly $84 \%$. 
Equations suitable for white and black Americans were then provided in the Tennessee Data Bank (run by Jantz and Ousley, data in France 1998), based on two calcaneal measurements from fifty females and 82 males. These provided sex estimates with $84 \%$ accuracy. Finally, the calcanei of 53 white male, sixty white female, 58 black male and 58 black female South Africans from the Dart collection also all provided significantly sexually dimorphic measurements (Bidmos \& Asala 2003, 2004). Functions produced for white individuals yielded accuracy of 73 to $92 \%$ depending on the dimensions utilised. Accuracy for black South Africans ranged from 79 to $86 \%$ using combinations of variables, or a lower 64 to $79 \%$ when based on individual measurements.

$\underline{\text { Talus }}$

A strong sexual dimorphism was observed with very little population differences in Steele's (1976) study already described above, based on five talar measurements originating from the same sample as for their calcaneal analysis. Discriminant function analyses with a minimum of two variables per function reached accuracy of up to $88 \%$. This was increased to $89 \%$ when combining calcaneal and talar dimensions.

All talar measurements of sixty white male, sixty white female, sixty black male and sixty black female South Africans aged 18-70 years from the Dart Collection were also established to be significantly sexually dimorphic, with sexing accuracy varying between 80 and 89\% (Bidmos \& Dayal 2003, 2004).

\section{$\underline{\text { Metatarsals }}$}

Interarticular lengths and numerous other measurements recorded on forty white male, forty white female, forty black male and forty black female Americans from the Terry collection were analysed by Smith (1997) in an effort to establish the degree of sexual dimorphism in metatarsals and foot phalanges. Equations from metatarsal measurements correctly identified 77 to $84 \%$ of individuals, and formulae for the proximal phalanges accurately sexed between 
70 and $72 \%$. This was confirmed by another study the same year also on the Terry collection by Robling and Ubelaker (1997), based on complete sets of metatarsals from 48 white male, 48 white female, 52 black male, 52 black female Americans aged 21 to 85 years. Sex-specific functions were produced based on six measurements, including maximum lengths. For an unknown metarsal, it was recommended to use both female and male functions, with the highest score indicating the sex of the individual. Pooled-race results managed to correctly identify between 88 and $100 \%$ of the individuals. The Terry Collection also provided the sample of the most recent study (Case \& Ross 2007, described further above), where maximum length measurements of the metatarsals and the phalanges of 123 white females and 136 white males aged 18-72 years accurately sexed between 74 and $83 \%$ of individuals.

The strong sexual dimorphism observed in all the studies mentioned in this section resulted in the decision to analyse all relevant calcaneal, talar and metatarsal measurements (full list in Appendix 24) as a further potential indicator of sex for this Neolithic sample.

\subsection{Conclusion}

Numerous methods exist for sexing skeletal remains, although estimating the sex of juveniles is still a major issue. However, as was shown in this chapter, several methods are available to try distinguishing boys and girls from the earliest age. Although it is clear that these methods require to be developed further, need refinement, and can often only identify one specific sex, they do provide us with an opportunity to test our available knowledge and hopefully glance at a possible better understanding of juvenile archaeological remains.

Dental metrics was discounted as a sexing method for the juveniles of this Neolithic sample, but sciatic notch dimensions will be considered, and sciatic notch index (Boucher 1955) as well as sciatic apertural index (Fazekas \& Kósa 
1978; Weaver 1980) will be calculated. Should there be a substantial number of juvenile remains, a plotting method (Mays 1998) will be used for these dimensions. Dental age and skeletal age will be compared when possible, and for juveniles of the appropriate age groups, the sequence of epiphyseal fusion (Garn et al. 1966) will also be used as a potential indicator of sex. Several morphological traits will also be considered. On juvenile mandibles, chin protrusion, shape of the anterior dental arcade, gonial eversion and ramus angle will be recorded (Schutkowski 1993; Loth \& Henneberg 2001). Combined facial scores (Molleson et al. 1998) will be recorded when chin protrusion, mandibular angle and orbit are available for observation. Finally, the following morphological traits of the pelvis will be utilised: angle and depth of the greater sciatic notch, arch criterion, iliac crest curvature, and auricular elevation (Weaver 1980; Schutkowski 1993).

Although adult sex estimations are generally considered precise and accurate, concerns arise if the methodologies employed are examined critically. This thesis has demonstrated that in many cases, numbers of the original samples were very limited, their origins unclear or unsuitable for the material being analysed, and averaged accuracy rates hid discrepancies between the relevance of certain traits or measurements for each sex which might make a method perfectly appropriate for one sex but not the other. It is worth considering that a successful accuracy rate of eighty percent still means that one out of every five individuals will be incorrectly sexed. Methods should be investigated thoroughly and the results they provide should always be considered a possibility rather than a certainty, no matter how high the reported accuracy is. There is also always a place for new methods to be developed. Although they would need to be tested on known sex populations to ensure their precision, they might help researchers focus on more than the most commonly used features and hopefully lead to a better knowledge of sexual dimorphism in human remains of all ages. 
Sex of the adult individuals in this Neolithic sample will be established primarily on the basis of the five main cranial traits (which include the nuchal crest, mastoid process, supra-orbital margin, glabella, and mental eminence), in addition to two pelvic traits (greater sciatic notch and pre-auricular sulcus), overall pelvis shape, and shape of the pubis (the latter including sub-pubic angle, ventral arch, sub-pubic concavity and ischipubic ramus, often referred to as Phenice traits). In order to utilise to its full potential this precious material, alternative morphological traits will also be recorded including the mandible ramus flexure (Loth \& Henneberg 1996); distal humerus traits (Falys et al. 2005) with the fossa shape, epicondyle angle, trochlear constriction and trochlear symmetry; Bruzek (2002) pelvic traits of the composite arch, sciatic notch proportion, symmetry and AP line; and lastly the shape of the sacrum. These alternative traits will then be compared to the results of the primary sexing analysis.

Finally, the osteometric methods reviewed in this chapter and compared in Appendix 1 demonstrated the presence of sexual dimorphism in numerous measurements but also very significant population variability. To maximise the impact of these ancient skeletal remains, all sexually dimorphic measurements will be recorded, including hand and foot bones dimensions. Measurements from individuals sexed on the basis of conventional morphological skull and pelvic criteria (listed fully in Appendix 16) will then be statistically analysed and a baseline for this Neolithic sample established. Rather than relying on comparisons with published ranges of measurements from geographically and chronologically very distinct populations, this new Central European Late Neolithic dataset will then provide a much more appropriate platform to attribute potential sex estimates based on osteometric results when no morphological traits are available or have produced indeterminate estimates. 


\section{Chapter 3: Ageing Methods}

\subsection{Introduction}

Assessing age is a very important stage in the analyses of human skeletal remains. It has been described as "a most vital part of skeletal anthropology" (İşcan 1989:325) and "one of the fundamental biological parameters assessed as part of any skeletal analysis" (O'Connell 2004:18). Age-at-death provides us with more information about the individual excavated, the demography of the site and the demography of the area and period in general.

For a considerable amount of time, a common misconception was that most, if not all, people died "young" in the past. This was mainly a result of the limitations of ageing methods available before the 1980s. However, it was also exacerbated by a tendency from both specialists and the general public to assume that our modern way of life was the reason for our "extended" longevity. Although it is of course undeniable that our life expectancy has increased thanks to the advances of medicine, and to a certain extent by the much wider and easier availability of resources in some parts of the world, this should not be confused with a sudden change in human life span. This has been discussed in detail by several authors, including Jackes (2000) who referred to Sjøvold's (1978) comment on the "remarkable number of deaths between 70 and 80 years of age that were recorded in an Austrian village in the 250 years prior to 1852". A walk through long-standing local cemeteries can indeed give a very interesting insight into the ages reached by a significant part of the population. Montagu's (1994) study on the length of life in the ancient world showed that individuals born before 100BC lived as long as modern individuals who died before 1950. Cox (2000) mentioned Roman tombstones in addition to the more recent London Bills of Mortality of the $17^{\text {th }}$ to the $19^{\text {th }}$ centuries as a good source of evidence of long life spans (including numerous centenarians in the latter 
source). She also quoted the Psalms which referred to human life ending at approximately 70 years, and up to 80 years for the strongest individuals, which is still applicable today.

Different regions of the skeleton provide information for different age periods. For the fetal period, bone measurements and observation of dental development are the main indicators of age. Appearance of ossification centres which are unlikely to be seen in the archaeological record, and the morphology and size of bones are then added to bone measurements to estimate skeletal age at death for juveniles. During the later childhood and adolescent period, epiphyseal closure becomes the principal source of information for age estimation. Dental age is obtained from dental development and dental eruption until the late teens. Once the dentition is complete and the skeleton fully mature, observation of the much less reliable and less precise degenerative changes to the skeleton and the dentition, together with the highly controversial cranial sutures closure can be used. These factors are the only evidence from a morphological examination of the bones that can be interpreted as age indicators.

Although the morphological observation of human skeletal remains seems to provide, if not an extremely accurate, at least a reasonable estimation of age at death, it is necessary to be careful in the age assessment of any archaeological remains. Firstly, as noted by Meindl and colleagues, "no two humans show the effects in exactly the same way in different parts of the skeleton" (İşcan 1989:326). Secondly, those ageing methods have all been derived from recent samples, which might not be suitable for individuals from such a remote past. Thirdly, special attention must be paid to how those ageing methods were devised: some osteological collections and some methodologies have been proved to be either flawed or even completely inappropriate. For example, some cases in the Todd collection had been aged on the basis of the external appearance of corpses by anatomists (Mays 1998; Cox 2000), the Libben 
Collection from Ohio had no known age, just as most archaeological collections, and some methodologies excluded any incompatible cases, as will be shown throughout this chapter. Considering the wide variations in methodologies, reference material and results obtained, it was therefore decided to undertake a full review of the methods available in order to establish which particular methods should be used to analyse the skeletal remains of this Neolithic population. As previously discussed, only macroscopical observations were available for this doctoral research. There will therefore be no references in this chapter to the numerous other methods of ageing concerned with microscopical data, such as histological analyses of the bones and the teeth. Methods for ageing both juveniles (including fetuses) and adults are considered.

\subsection{Juveniles}

\subsubsection{Dental development}

Dental development is an extremely useful tool to age juvenile archaeological remains as teeth tend to be well preserved in inhumations and are also often present in the remains from cremations. Dental ageing has been utilised in the UK since the early part of the $19^{\text {th }}$ century when Saunders (1837) advised the Parliament to look at the state of tooth eruption to check the age of young factory workers, as registering births was not yet mandatory and work regulations differed with age. Dental development covers the whole juvenile period, from the embryonic stage to the late adolescence, and has been described by many as the best way to establish the age of non-adults (Bang 1989; Steckel et al. 2005). It was also demonstrated to be more accurate than skeletal age in a population of known chronological age (Bowman et al. 1992).

However, caution must still be applied, especially when analysing archaeological remains. There is a marked difference in tooth development and emergence between sexes, which also varies with individual tooth (the canine 
being the most strongly sexually dimorphic), with females usually more advanced in their development than males (Lewis 2007). This has a strong impact on the analyses of juvenile archaeological remains, as their sex cannot be easily determined. The later adolescent period relies on the third molar, with its root development seemingly the most reliable criterion (Bang 1989). However, root formation might not always be observable in macroscopic analyses of archaeological remains, while its time of eruption appears very variable.

Noticeable variations between populations have been reported, together with the influence of the environment and the health of the individual (El-Nofely \& İşcan 1989) as well as socioeconomic status (Hillson 1996). These are all very difficult to establish for archaeological populations. A recent investigation on Nigerian children (Folayan et al. 2007) showed that in this population, neither sex nor breastfeeding status affected the timing or sequence of teeth eruption. In addition, only the maxillary canines were affected by socioeconomic status, indicating how much populations can differ from each other with regard to sequence, timing and sex difference in dental development.

On a more technical point, there is also a difference between tooth emergence and tooth formation, and between gingival (or clinical) and alveolar tooth emergence. The majority of studies are usually based on gingival emergence, whereas by its nature, the observation of tooth emergence in archaeological populations will nearly always be restricted to alveolar emergence, as soft tissues very rarely survive. Both the timing and the sequence of gingival and alveolar emergences have been shown to vary (Hillson 1996). In addition, tooth emergence seems to be more affected by external factors than the actual tooth formation and therefore the latter is preferred by many authors (Ubelaker 1984; El-Nofely \& İşcan 1989). However, tooth eruption is often the only way of ageing skeletons from direct observation of the dentition, especially in the field or when no x-ray facilities are available. Finally, when selecting which methods to use, it is important to take into consideration how they were established, the 
sample used, any tests of the method since its publication and if it is based on macroscopical observations or radiographs, as tooth calcification usually shows up in x-rays at a later age (Lewis 2007).

\section{( Charts}

One of the oldest methods to estimate dental age is the study by Schour and Massler (1941). They utilised Logan and Kronfield's (1933) study to create a chart showing the time and sequence of both formation and emergence of teeth in 22 phases, later modified to 21 phases with standard deviation figures. Although originally designed for dentists (and still reprinted regularly by the American Dental Association), Schour and Massler's chart has been used widely by osteologists, as it offers a much simplified approach compared to other studies. An alternative and equally easy to use gross labial view was also published (Massler et al. 1941; also reproduced in El-Nofely \& İşcan 1989). Strong criticisms have been voiced against this method, mainly relating to the use of Logan and Kronfeld's data, this earlier study being based on twenty extremely ill children (Lewis 2007). Although these children were terminally ill, children recovered in archaeological context were "by definition, not healthy children. All died young, and it is most unlikely that their nutrition or healthcare approximated to that of modern middle-income Americans" (Hillson 1996:146). This method has been tested successfully several times (Hillson 1996, 2005), and was shown as giving the most accurate age estimation compared to other methods on a historical sample from Spitalfields of juveniles of known age under 5.4 years (Liversidge 1994). A recommendation was given to reduce the age estimate by three to six months for girls and to modify the age range after four years old to double the range by twelve years old, and to triple the range by thirteen years of age (Byers 2008).

Schour and Massler's chart was modified by Ubelaker (1978) to present a sequence of formation and eruption of teeth for Native American populations 
based on the Arikara population. The main difference in the updated chart was the increase in the provided age ranges, following the combination of data published by several authors from the 1940s to the 1970s, mostly from American Whites apart from the data on permanent teeth eruption. The combination of data for males and females into a single chart was justified by the lack of reliable methods to sex juvenile skeletal remains (Ubelaker 1984). The wider standard deviations presented took into account other populations' means for dental eruption, making it less precise but also applicable much more widely. It should be noted that eruption in this atlas refers to gingival emergence, not emergence through the alveolar bone. As this chart is mainly based on Schour and Massler's study, the same sample problems would apply (Lewis 2007, see above). However, this method has also been, and still is, very widely used and is recognised as a standard throughout the world (Ferembach et al. 1980; El-Nofely \& İșcan 1989; Buikstra \& Ubelaker 1994; Bass 1995; Hillson 1996).

\section{( Diagrams}

While the charts of Schour and Massler and Ubelaker are considered as standards in anthropology, Gustafson and Koch's diagram is a standard in forensic studies (Hillson 1996, 2005). This diagram was first published in the 1960s (Gustafson 1966), and again a decade later (Gustafson \& Koch 1974). It showed the time of the start of mineralisation, crown completion, eruption and root completion, for all deciduous and permanent teeth apart from the third molar. The data came from a combination of numerous published studies for both females and males, including histological and radiographic analyses. Several comparative studies found this method to estimate age within two months of true age, and valid for both sexes of any races and contemporary as well as historic populations (Gustafson \& Koch 1974; Crossner \& Mansfield 1983; Hägg \& Matsson 1985; Liversidge 1994). Although the stages are not as 
well defined as with Schour and Massler, it is a very good tool for archaeological material as it does not require all teeth to be present, is fairly easy and rapid to use, and gives an accurate age estimation.

Gustafson and Koch's (1974) data, together with the studies of Anderson and colleagues (1976), Levesque and colleagues (1981) and Garn and colleagues (1962) for the third molar, were utilised to produce a single diagram showing the development of all permanent teeth, including approximate eruption age (Stermer Beyer-Olsen \& Risnes 1994: figure 2; also reproduced in Mays 1998).

\section{( $)$ Stages}

A study on the formation of teeth and the resorption of their roots was published in the 1960s by Moorrees, Fanning and Hunt (MFH). Their research was based on serial radiographs of White American males and females, including 246 deciduous teeth and 99 permanent teeth of 48 males and 51 females from Boston, as well as 136 males and 110 females from Ohio (Moorrees et al. 1963). The fourteen distinctive stages of formation described have been used very widely and are also recognised as standards (Buikstra \& Ubelaker 1994).

\begin{tabular}{|c|c|c|c|}
\hline 1 & Initial cusp formation & 8 & Initial cleft formation \\
\hline 2 & Coalescence of cusps & 9 & Root length $1 / 4$ \\
\hline 3 & Cusp outline complete & 10 & Root length $1 / 2$ \\
\hline 4 & Crown $1 / 2$ complete & 11 & Root length 3/4 \\
\hline 5 & Crown $3 / 4$ complete & 12 & Root length complete \\
\hline 6 & Crown complete & 13 & Apex $1 / 2$ closed \\
\hline 7 & Initial root formation & 14 & Apex closed \\
\hline
\end{tabular}

Table 3.1: MFH stages of tooth mineralisation 
Despite some reservations mainly due to the small sample used (Byers 2008), MFH stages have proved to be more accurate for age estimation than skeletal age on samples with known chronological age at death (Bang 1989), to within six months of the true age in a sample from Ontario and on the children from Christ Church, Spitalfields (Hillson 1996).

MFH fourteen stages of development were utilised in a study of 121 white males and 111 white females from Canada, starting at three years of age (D.L. Anderson et al. 1976). This research has been criticized by many for consistently overestimating the age of young children and underestimating the age of older children (Hillson 1996). However, the data provided in the study were used in recent summarising studies, together with Gat and colleagues' (1984) study on four to fourteen years old.

MFH fourteen stages of development were also employed in the analysis of the permanent dentition of almost one thousand American children (300 white males, 355 white females, 151 black males and 184 black females) between 3.5 and thirteen years old (Harris \& McKee 1990). The tables produced during the study was recommended and reproduced in Byers' (2008) textbook for forensic anthropology.

In the 1990s, MFH data was simplified into one table for females and one table for males (Smith 1991). Fifteen stages were given for permanent mandibular teeth, based on MFH original fourteen stages with the addition a stage of $2 / 3$ root completion for the first and second incisors (Hillson 1996; Scheuer \& Black 2000). Averaging the results of females and males for the analyses of juvenile of unknown sex resulted in very little error, and was therefore deemed acceptable. Tests of this method have given better results than with the original method of Moorrees and colleagues, and it has been recommended by several authors, including Lewis (2007). It must be noted however that the same tests also showed that results from this method were not as good as the results obtained from the Schour and Massler's chart (Hillson 2005). More recently, MFH 
graphical data was transformed into a table for permanent teeth, including means and standard deviations for both sexes following the original thirteen stages for single-rooted teeth and fourteen stages for multi-rooted teeth (Harris \& Buck 2002).

\section{๑) Formulae}

Regression formulae using the length of the teeth rather than their developmental stages are also available. These were developed from direct observation of the developing deciduous and permanent teeth on around seventy children of known age buried between 1729 and 1859 at Christ Church, Spitalfields (Liversidge et al. 1993; Liversidge 1994; Liversidge et al. 1998; Liversidge \& Molleson 1999b, 1999a). These equations were tested on a modern sample, revealing a general underestimation of age, as well as a decrease in the accuracy of estimates with age (Liversidge et al. 2003). However, these were differences of a few months only. A follow-up study was undertaken on 121 documented and undocumented remains for Christ Church, Spitalfields, in addition to the radiographs of 61 healthy living two to five years old, and skeletal material from two Scottish medieval sites (Liversidge \& Molleson 2004). Age of the undocumented cases had been estimated from their tooth lengths, and mean ages were calculated for each tooth separately according to Demirjian's eight stages of development (Demirjian et al. 1973). Results showed no significant differences between population groups for either tooth formation or tooth eruption.

This method was recently criticised for counting mandibular and maxillary teeth together, as well as for the inadequacy of the statistical test employed (Cardoso 2007). Liversidge's regression formulae were also tested by Cardoso on 52 pairs of deciduous teeth and 63 pairs of permanent teeth from 57 Portuguese individuals aged from birth to nineteen years who had died during the $20^{\text {th }}$ century AD. Although some differences were noted in the deciduous 
teeth, with the age from maxillary teeth being overestimated compared to mandibular teeth, the permanent teeth only showed a consistently underestimated age when the estimation was based on the second molars (maxillary and mandibular). Those estimations were still very close to known age, leading to good results and an overall positive assessment of this method which requires only a single tooth measurement taken on any deciduous or permanent tooth from birth to about twenty years of age, without the need for the sex of the individual to be known.

\section{Other methods}

Several other studies have been published, nearly all based on tooth formation rather than tooth emergence. A study based on the radiographs of children from Helsinki provided ageing tables (Haavikko 1970; tables also reproduced in Schaefer et al. 2009:93). This method was tested and found to be very close to true age (by three to six months) and is widely used in Scandinavia. It is also very helpful for archaeological remains as it distinguishes between the stage of alveolar emergence and that of clinical (gingival) emergence.

A method using the composite score of the development of all teeth was produced in the 1970s from the study of nearly 3000 children (Demirjian et al. 1973; Demirjian \& Goldstein 1976; Demirjian \& Levesque 1980). Unfortunately, this method cannot be used when some teeth are missing, and thus would not be suitable for most archaeological material.

A new simplified method of ageing the dentition was also published in the last decade (Muller-Bolla et al. 2003). The study was based on the radiographs of 5848 white patients from Nice, France, aged one to fifteen years. A simple count of all fully erupted teeth (having reached the occlusal surface), third molars excluded, was shown to provide the age of the individual. Regrettably, this easyto-use method also relies on complete dentitions, which might be unavailable in the study of ancient human remains. 


\section{(4) The third molar}

The third molar is often omitted from charts, diagrams and tables as its development is extremely variable between individuals. However, it has attracted a lot of interest especially in the field of forensic science, as there is a growing need worldwide for precise ageing in the late teens for legal reasons, for example with refugees, illegal immigrants, or adults trying to pass as juveniles for legal purposes.

Several publications established the start of alveolar emergence of the third molar at thirteen years of age for females and fourteen years for males. These include the studies of over 3000 males and nearly 500 females from India between 6 and 21 years old (Shourie 1946); 990 black Ugandan males aged 626 years (Chagula 1960); 523 male and 548 female black Nigerians aged 11-21 years (Otuyemi et al. 1997); and 410 male and 106 female black South-Africans between 12 and 26 years old (Olze et al. 2007).

However, a twelve-year longitudinal study based on the radiographs of 140 healthy white children from Ohio (USA) demonstrated no difference based on sex in the third molar's developmental course, with complete developmental autonomy (Garn et al. 1962). Complete emergence was established regardless of sex between 16 and 18 (Otuyemi et al. 1997) or 19 years of age (Olze et al. 2007). This latter study based on radiographs distinguished between alveolar and gingival emergence. A difference of two to three years was established between the two, whereas an analysis of 4640 radiographs of French Canadians aged 7-25 years provided a difference of only 1.3 years between alveolar and clinical emergence (Levesque et al. 1981).

Other studies employed Demirjian's eight stages of tooth development, affording direct comparisons. A study for the American Board of Forensic Odontology on thousands of radiographs of 823 Americans of both sexes $180 \%$ white including 271 males and 323 females, and 19\% black) showed that if the 
third molar was in stages A to D, the individual was most likely under 18 years of age, while a fully complete third molar in stage $\mathrm{H}$ could confidently age the individual to 18 years or over (Mincer et al. 1993). However, a similar study on 1282 Japanese patients of both sexes aged 14 to 24 years revealed that in this population, stages A to D indicated an individual under 20 years old, while stage $\mathrm{H}$ aged the individual to at least 16 years of age with a 99\% certainty (Arany et al. 2004). This latter study also established stage $\mathrm{F}$ (when the root length is at least equal to the crown length) as an indicator that the individual was at least 14 years with 97\% confidence. Population variations and sexual dimorphism were further demonstrated in 637 American blacks and 563 American whites, all aged 14-24 years, with a 90\% likelihood of being at least 18 years of age when M3 had reached stage $H$ for white males, but varying to $93 \%$ for white females as well as black males, compared to only $84 \%$ for black females (Blankenship et al. 2007).

These limitations were echoed in the Canadian study of Levesque and colleagues (1981) described above, where bilateral absence (agenesis) of the third molars was found in $9 \%$ of the cases and no constant sexual differences could be established, and in the American study of Mincer and colleagues (1993) where males were confirmed to mature faster, with left/right and mandibular/maxillar asymmetry. In the latter study, the third molar was also frequently absent, malformed, impacted or extracted and the authors described it as "the most variable tooth in the dentition as regards size, time of formation and time of eruption" (Mincer et al. 1993:386).

In the guidelines of the Institute of Field Archaeologists (IFA) and the British Association of Biological Anthropology and Osteoarchaeology (BABAO, see Connell 2004), the third molar root mineralisation was timed at between 18 and 25 years (based on Schour \& Massler 1940: 18-25 years; Haavikko 1970: 19-21 years; D.L. Anderson et al. 1976: 18-19 years; Smith 1991: 19-20 years), coinciding with its gingival emergence in the late teens-early twenties, or more 
specifically between 17 and 25 years (based on Brown 1985; Hillson 1996). Most recently, the specialists in juvenile development Scheuer and Black (2000, 2004) as well as Lewis (2007) placed the eruption of the third molar at around 17 years of age. It is clear from all the above studies that a wide variation can be seen in the mineralisation as well at the emergence of the third molar, so interpretation of the state of development of this tooth should be carefully considered, especially for unknown past populations.

\section{(- The use of multiple methodologies}

The use of as many teeth as possible for separate age estimations to obtain a mean age estimation using atlases and charts for infancy and early childhood (Schour \& Massler 1941; Gustafson \& Koch 1974; Ubelaker 1978) was recommended in Scheuer and Black's exhaustive books on juvenile osteology (2000, 2004). For other age groups, it was recommended to select the least variable mandibular teeth in order to get the greatest accuracy. These vary with age, with the first molar, second molar, first premolar and central incisor most accurate for individuals under 10 years of age, while the second molar, first premolar and canine were preferred for those over 10 years old. Such selections however would be difficult in incomplete remains, and particularly for individuals with no known age at death. The formulae and tables of Moorrees, Fanning and Hunt, Liversidge and colleagues, Demirjian and colleagues, Haavikko and Ubelaker were also included in the latest "Field and Laboratory Manual" (Schaefer et al. 2009).

It should always be kept in mind that, as Hillson noted: "different methods may produce different age estimates for the same material" (Hillson 1996:146). However, for the investigation of past populations, it is usually hard to use only one method, as some teeth might still be in situ, some available but not in the jaws anymore, some damaged, and others missing altogether. Therefore several methods will be required to make the best use of all the evidence available. This 
can also be seen in the guidelines of BABAO and the IFA with the recommendation of several methods (Brickley 2004). It is clear that for unknown past populations, dental age will only be a guide rather than a very precise indicator of age.

\subsubsection{Bone dimensions}

Stature is closely correlated with age in juveniles (Scheuer \& Black 2000) and barely influenced by sexual dimorphism for infants and young children (Byers 2008). Diaphyseal measurements, or the length of juvenile long bones, have therefore been the "common sources of skeletal age estimates from before birth to mid-teens" (S.R. Saunders 2000:146).

Studies available include data from modern radiographic samples, forensic observations of dry bones and measurements from archaeological remains. However, caution is required as several limiting factors exist. Study samples are often small and based on modern individuals, which might differ from archaeological populations (Brickley 2004). Growth and the length of diaphyses are also closely related to the individual general state of health or the mother's during the fetal period and thus might be negatively affected during periods of illness and malnutrition (Lewis 2007). In the BABAO/IFA guidelines, researchers were also reminded that juveniles recovered from archaeological context had died prematurely and therefore had a "high chance of having suffered from a debilitating illness" (Brickley 2004:22), an issue already raised over forty years earlier by Johnston $(1962,1968)$.

Despite these limitations, diaphyseal length can be considered the best age indicator when no other criteria, such as dental age, are available (Ubelaker 1984). This estimated skeletal age can also be compared to dental age as an indicator of stress, growth defect and population variation (S.R. Saunders 2000; Lewis 2007). 


\section{(- Fetal period}

While after birth, the accuracy of age estimation from long bone lengths is reduced, it is considered a reliable method for prenatal ageing due to the linear relationship between age and long bones throughout the fetal period (Byers 2008). Formulae to estimate fetal stature or body length, and thus age, were published as early as the 1920s (Balthazard \& Dervieux 1921; and see Chapter 4 , section 4.2.1, for more details on the early studies), and some as recently as the last decade (Sherwood 2000).

The work of Fazekas and Kósa, which includes the dimensions of numerous bones in addition to the more common long bone diaphyses, remains "the definitive reference" (Krogman \& İşcan 1986:89) and "by far the most comprehensive account of fetal bones" (Scheuer \& Black 2000:2). Their study was based on 138 fetuses aged three to ten lunar months (12 to 40 weeks of gestation), which included 71 males and 67 females, all stillborn or dead shortly after birth and all from healthy parents (Fazekas \& Kósa 1978; Kósa 1989). Body length, also known as crown-heel distance, was measured after straightening the fresh corpses, and ranged from 9 to $55 \mathrm{~cm}$.

Pregnancy is typically counted from the first day of the last menstruation and would usually conclude with the normal delivery of a 50 to $51 \mathrm{~cm}$ neonate 280 to 283 days after this date or 270 days after conception. A frequent variation can be seen in the period of pregnancy of \pm 10 days as well as in mature body length which can vary between 48 and $52 \mathrm{~cm}$ (Fazekas \& Kósa 1978). As the exact date of conception was unknown in several cases, the fetuses were grouped in half-lunar month intervals following Haase's rule of correlation between body length and age. The rule states that until the $5^{\text {th }}$ lunar month, the body length in $\mathrm{cm}$ corresponds to the number of lunar months of pregnancy squared. From the $5^{\text {th }}$ lunar month, body length corresponds to the number of months multiplied by 5 . Although this is not applicable to the first two months, this ageing system from the third month of pregnancy was confirmed by 
Scammon and Calkins (1929), subsequently verified in forensic sciences, and is still widely accepted. The table below summarises Fazekas and Kósa's groupings of fetal body length in their study sample following Haase's correlation between body length and age.

\begin{tabular}{rccrc}
\hline $\begin{array}{c}\text { Body length } \\
(\mathbf{c m})\end{array}$ & $\begin{array}{c}\text { Age } \\
\text { (lunar month) }\end{array}$ & $\begin{array}{c}\text { Body length } \\
(\mathrm{cm})\end{array}$ & $\begin{array}{c}\text { Age } \\
\text { (lunar month) }\end{array}$ \\
\hline $\mathbf{9}$ & $(8-10)$ & $\mathbf{3}$ & $\mathbf{3 5}(34-36)$ & $\mathbf{7}$ \\
$\mathbf{1 6}(11-14)$ & 3.5 & $(37-38)$ & 7.5 \\
$(15-18)$ & 4 & $40(39-41)$ & $\mathbf{8}$ \\
$\mathbf{2 5}(24-26)$ & 4.5 & $(42-43)$ & 8.5 \\
$(24-28)$ & $\mathbf{5}$ & $\mathbf{4 5}(44-46)$ & $\mathbf{9}$ \\
$\mathbf{3 0}(29-31)$ & 5.5 & $(47-48)$ & 9.5 \\
$(32-33)$ & 6 & $50(49-51)$ & 10 \\
\hline
\end{tabular}

Table 3.2: Correlation between body length and fetal age (table by the author, data from Fazekas \& Kósa 1978:30-31)

In order to increase precision, the length and width of all dry bones were then taken using the direct measurement between two points rather than using the perimetry, which is taken with a tape around the bone. All characteristic measurements were then summarised in tables and analysed. Equations were calculated which revealed that a very precise determination of fetal body length, and therefore age, could be obtained using bone dimensions, with no more than half a lunar month of error possible. All available equations have been collected and are presented in Appendix 2.

In addition to summary tables and calculations, photographs were also presented with drawings and average measurements for each half lunar month for males and for females to assess age rapidly without the need for any calculation. Several bone dimensions were also found to be directly proportional to fetal body length, which is of great use when age assessment is required swiftly or in the field, and have been reproduced overleaf. 


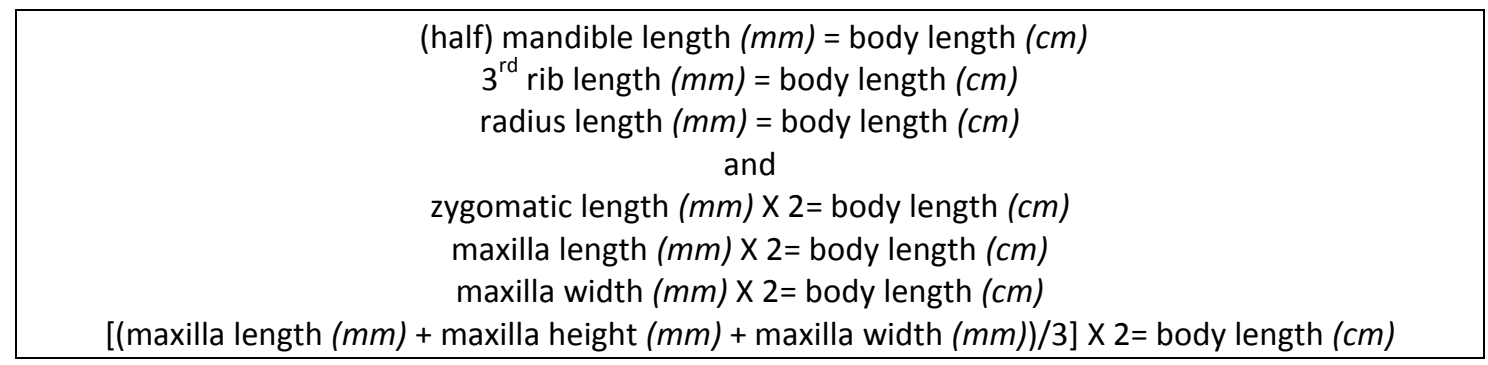

Table 3.3: Useful measurements for direct fetal stature estimation

Fetal physical development can be influenced by a number of factors which include the mother's state of health, age, and number of previous pregnancies. Nutrition was also believed to have a crucial role in fetal development and could therefore seriously impact the results of osteological analyses of archaeological populations, as these individuals did not have access to the same resources as modern populations. However, several studies undertaken during the Second World War demonstrated that only a very slight decrease in fetal weight was noted on average during that time, and that the role of nutrition was therefore "except for certain cases [...] not so essential as had been believed earlier" (Fazekas \& Kósa 1978:308).

Bone shrinkage from burning, which is regularly encountered in archaeological remains, was also considered. To take into account the average reduction in size of burnt bone by 10\%, Fazekas and Kósa (1978:371) recommended that the fetal age estimation from bone measurements "should be established 1 lunar month older than the age calculated from the dimensions of the burnt bones".

Most criticisms of Fazekas and Kósa's method of fetal age estimation stem from the lack of precisely known age-at-death in the study sample (Scheuer \& Black 2000; Piercecchi-Marti et al. 2002; Scheuer \& Black 2004; Lewis 2007). Fazekas and Kósa's equations have also been tested on six fetuses (Ubelaker 1989a), including two from the archaeological remains of a proto-historic site of South Dakota and four from the Smithsonian collection. Although the one-half lunar month accuracy was not attained in this very small sample, the average estimates measured up well with the known age of those fetuses, and average 
estimates from several bones were recommended to obtain the best results. More recently, a study on the radiographic determination of fetal age also demonstrated the compatibility of Fazekas and Kósa's data and equations with radiographic measurements of American fetuses (Warren 1999). Kósa's research on fetal age estimation still continues based on more bone measurements and bone morphology (see Castellana \& Kósa 2001; and Kósa \& Castellana 2005 for some examples of works in progress).

A new study on fetal age estimation was published in the last decade based on 72 fetuses aged between 15 and 42 weeks from known last menstruation (Sherwood 2000). Results showed that neither maternal age, number of previous pregnancies or fetal sex affected fetal growth significantly. Skeletal measures were the most accurate, especially those of the femur, tibia and ulna. The best age estimator was obtained from the length of the femur but was still severely restricted with a range of two lunar months ( \pm 4 weeks) with a $95 \%$ confidence interval. Inaccuracy in age estimation also increased with pathology.

femur length $(\mathrm{mm}) \times 0.38+10.91=$ age (gestational weeks)

Fazekas and Kósa's (1978) study is still rightly recognised as "the most detailed text on fetal osteology" (Scheuer \& Black 2000:2), and their tables have been included in the latest juvenile osteology manual (Schaefer et al. 2009). This method will be used to estimate body length and age of all fetal / neonatal remains in the Neolithic populations under study in this doctoral research.

\section{- Postnatal period - diaphyseal length}

Studies on growth and ageing estimation from bone measurements after birth are nearly all restricted to long bone lengths, mostly based on the diaphyses before epiphyseal fusion occurs. 


\section{Modern populations}

The most complete longitudinal growth study of the $20^{\text {th }}$ century was based on healthy white middle class American children taking part in the Child Research Council, Denver, Colorado (Maresh 1943, 1955, 1961, 1970). A total of 244 juveniles including 121 females and 123 males took part in the study, from age two months to eighteen years. Measurements were taken directly from radiographs without correction for magnification. Diaphyseal length was used from two months to twelve years, while the maximum length including both epiphyses was recorded from ten to eighteen years. Data for males and females were presented separately. In the 1955 publication, $10^{\text {th }}, 25^{\text {th }}, 50^{\text {th }}, 75^{\text {th }}$ and $90^{\text {th }}$ percentiles were given in addition to a range of values for successive age categories. In the 1970 publication, means and standard deviations were given for the successive age categories, together with the updated $10^{\text {th }}, 50^{\text {th }}$ and $90^{\text {th }}$ percentiles, but the ranges were omitted.

The same study provided the data for Hoffman's (1979) study, which included 334 healthy middle and upper middle class North American children from 1927 to 1967 aged few months to twelve years. Maresh's published data was corrected for x-ray magnification estimated at 2 to $3 \%$ greater than true anatomical length, based on the difference between the measurements of sample dry bones which were subsequently radiographed. However, instead of applying a fixed numerical correction, only the female data were utilised as they provided a larger and more varied sample with 65 to 86 individuals per age cohort with an overall smaller size which might "counteract the slight magnification error resulting from radiography" (Hoffman 1979:462). All the data was presented in the form of graphs of maximum diaphyseal length variation with age for each long bone, without any other tables or numerical data. Age estimates from the lengths of the radius and femur were compared to dental age based on other published sources of white American female tooth eruption age from other study groups. Results demonstrated less variability in 
diaphyseal length and in tooth eruption age, with increasing variability for both as chronological age increases. The femur was found to be the best indicator of age. Its growth curve in Hoffman's study differed from Stewart's (1976 and earlier editions) with greater mean lengths of 40 to $60 \mathrm{~mm}$, corresponding to a difference in ageing of two to three years between both methods. Hoffman's graphs based on female data only have been recommended as "the best data for determining age at death of both males and females [...] providing a reasonable middle ground" between direct radiographic measurements too long for females and too short for males (Byers 2008:205).

Another study was based on the radiographs of 67 males and 67 females from birth to eighteen years, all part of the Longitudinal Studies of Child Health and Development of the Harvard School of Public Health (M. Anderson et al. 1963; 1964). Results of the measurements corrected for radiographic magnification for each age group were presented in separate tables and graphs for each sex. Unfortunately, total maximum length including developing epiphyses was used and those results are therefore inappropriate for most archaeological contexts.

Growth standards covering the ages of one month to eighteen years for males and females have also been published based on data from the Fels longitudinal growth study of "several hundred" living white well-nourished and healthy North American children from Ohio (Gindhart 1973). Measurements, corrected to take into account the magnification from radiography, were only taken for the radius and the tibia. Measurements for all ages were restricted to the maximum diaphyseal length excluding any epiphyses, which would therefore be difficult to replicate exactly macroscopically on skeletal remains. Results indicated a growth spurt for females between nine and twelve years, and for males between twelve and fifteen years. No significant difference was noted before the age of thirteen years in growth for the tibia between males and females. However, significant differences were observed between both sexes in the growth of the radius through most of the age cohorts. 


\section{Archaeological populations}

The Archaic Indian Knoll population of Kentucky, dated around 3000 BC, was the basis of several American archaeological growth studies. An initial publication concentrated on 165 foetuses, infants and young children up to 5.5 years old, whose age had been estimated using skeletal age and tooth eruption following Schour and Massler dental chart and skeletal age (Johnston 1962). Total length of the diaphyses of the six long bones diaphyses of each individual were recorded, and means for each age group provided with standard deviations regardless of sex due to the uncertainties of sexing juvenile archaeological remains. Direct measurements of 128 American Indians from the same Indian Knoll population were later analysed (Sundick 1972) and compared with a Central European historic population of 82 Caucasians from Altenerding near Munich, Germany, dating from AD $6^{\text {th }}$ to $7^{\text {th }}$ centuries (Sundick 1978). The age estimation of both populations was based on Schour and Massler dental formation and dental eruption. Rather than giving a chronological age, eighteen dental age stages were given, with a table showing the corresponding age ranges from Schour and Massler for comparison. Measurements included the maximum length of the diaphyses on their own, diaphyses with one fused epiphysis, and total length with both epiphyses fused. In addition to the six long bones, the same measurements were provided for the clavicle, the ilium, the talus, the calcaneus, the cuboid and the first metatarsal. Both populations yielded very similar results until puberty, then slightly larger for the Altenerding historic population. This was shown to correspond to the difference in average adult height, estimated from Trotter and Gleser's equations for the femur and the fibula at $167 \mathrm{~cm}$ for Indian Knoll males and $173 \mathrm{~cm}$ for Altenerding males.

Another North American archaeological population utilised for a growth study was the historic population of the Protohistoric Arikara Indians from South Dakota, dated from AD $18^{\text {th }}$ century (Merchant \& Ubelaker 1977). The age of the 
193 skeletons complete enough for analysis was estimated using Moorrees and colleagues' tooth formation graphs, averaging male and female data. Maximum diaphyseal lengths of the six long bones and the ilium were measured and presented as mean, standard deviation and range for each 1-year cohort. Results were similar to the Indian Knoll populations until at least ten years of age, while adult average stature was around $2 \mathrm{~cm}$ higher for the Arikara at $169 \mathrm{~cm}$. Due to the extremely low number of individuals in the twelve years and over categories, it was advised to use principally the younger cohorts to estimate the age of Indian juveniles "with relative confidence" (Merchant \& Ubelaker 1977:71). The tables and data from this study have been reproduced several times in later publications by Ubelaker and are widely used to estimate juvenile age from diaphyseal length, mostly in North America. A large standard deviation of plus or minus two years was added in these later publications to enable the use of this data on other populations. Due to the very limited number of individuals per age categories sometimes as low as one or even zero, it has been advised that this method should be relied on mostly for the period from birth to 2.5 years only (Lewis 2007).

The Late Woodland Indian juveniles from the Yokem mounds, Illinois, dating from AD $13^{\text {th }}$ century, provided the basis for another North American study on linear growth (P.L. Walker 1969). The diaphyseal length of 43 children aged between birth and twelve years of age from their tooth formation and eruption were plotted into graphs and compared to another prehistoric population of the same area. Early childhood growth was found to be similar, but some differences were noted during adolescence.

A study on 24 Aleuts, 81 western Eskimos and four unspecified Eskimo or Aleut skeletons from Kodiak Island, dating before AD $19^{\text {th }}$ century, presented separate results for both sexes despite being based on non-documented archaeological populations (Y'Edynak 1976). Sex of the 47 male and 47 female juveniles had been estimated from the size of the sciatic notch, the pubo-ischial index, 
presence or absence of pre-auricular sulcus and size of femoral neck. Age was estimated in two-year intervals from dental eruption and skeletal age. The maximum diaphyseal length of long bones was recorded and the means presented for each two-year category.

The method recommended by the WEA - Workshop of European Anthropologists (Ferembach et al. 1980) and still widely used in Central Europe is based on juvenile long bone lengths of ancient Slavic populations (Stloukal \& Hanáková 1978). The skeletal remains of 304 juveniles aged from birth to fifteen years dating from AD 9th century Mikulčice in Southern Moravia, Czech Republic, with an average adult stature of $171 \mathrm{~cm}$ for males and $161.5 \mathrm{~cm}$ for females, were utilised together with 25 juveniles from Nové Zámky and seven juveniles from Virt, these two latter Slovakian sites dating from AD $7^{\text {th }}$ and $8^{\text {th }}$ centuries. The maximum diaphyseal length of the long bones was recorded and presented in a single group, as the sex of the children could not be established. The stage of individual tooth development was used to estimate the children's age rather than tooth eruption to ensure more accuracy, first from macroscopical examinations by Stloukal and Hanáková themselves and then confirmed by radiological examinations undertaken by stomatologists from Brno. The children were then divided into nineteen age categories of half-years for the first three years of life, and one year from three to fifteen years, with a standard error of two months for the first three months of life, increasing to between six and nine months after that. None of the children provided complete measurements of all six long bones. When both sides were present, an average of both measurements was used. There were 237 femora, 220 humeri, 186 tibiae, 136 radii, 117 ulnae and only 60 fibulae. For each age group, the average of each diaphyseal length was calculated and then presented in tables with the total range for each class showing minimum and maximum measurements. Once the total number of juveniles was divided into all the age groups, the number of individuals for each category, which varied between 1 and 23, was lower than what would be required for statistical purposes. To solve this problem, 
mathematical corrections were applied and the new corrected average and range given in the same tables next to the original data. This data is suitable for use on archaeological juvenile remains, particularly if dental age is unknown, either using the tables with corrected average maximum length and/or range, or using the graphs of the range provided. Although the age estimation might not be very precise, it will provide a general idea of the child's age from birth to fourteen years old. Long bone lengths of this historical population were also compared by Stloukal and Hanáková to modern data. Results indicated that until seven years of age, the Ancient Slavic children were taller than modernday children. However, between eight and ten years of age, they were both similar, and from eleven years, modern-day children were taller than their ancestors.

Another European study was based on the population of Wharram Percy, Yorkshire, dated from $\mathrm{AD} 10^{\text {th }}$ to $16^{\text {th }}$ centuries (Mays 1999). Femoral diaphyseal lengths from 117 juveniles aged from dental calcification between one and seventeen years were presented and compared to other populations already described above, including Mikulčice, Czech Republic, AD 9 $9^{\text {th }}$ century (Stloukal \& Hanáková 1978), Belleville, Canada, AD. 19 th century (S.R. Saunders et al. 1993) and Denver, U.S.A., AD 20th century (Maresh 1955). The pre-19th century populations were shown to have a very similar "growth", while all later measurements were larger.

\section{Multiple methodologies}

Comparative studies between different methods using diaphyseal bone length have been undertaken by several authors. Eleven different standards were utilised to compare age at death from six femurs, including Maresh (1943), Hoffman (1979), Walker (1969), Johnston (1962), Sundick (1972) and Merchant and Ubelaker (1977), the latter twice using different ageing methods, first Schour and Massler's standard and then Moorrees and colleagues' method. Results showed that "the variability of the estimate [was] considerable and 
[increased] dramatically with the size of the bone" (Ubelaker 1989a:66). Another comparison between ageing methods using long bones was undertaken more recently (Di-Loreto 2006; Zakrzewski \& Di-Loreto 2008), this time including Ubelaker, Maresh (1970), Fazekas \& Kósa (1978) and Scheuer \& Black (1980), the latter two restricted to fetuses and perinates, together with a comparison of dental age. This was applied to two historical skeletal collections with 28 juveniles from Écija in Spain between AD $8^{\text {th }}$ and $12^{\text {th }}$ centuries, and 25 juveniles from Great Chesterford in England dating from the Anglo-Saxon period. Fazekas \& Kósa's method and Scheuer \& Black's gave very similar results. Although Ubelaker's and Maresh's methods gave close age estimation for young children, estimations gave higher results after six years of age using Ubelaker's, with Maresh's results greatly underestimating age compared to dental age, especially during adolescence. Interestingly, no differences in the long bone length/dental development were found between juveniles with visible pathology on the skeleton such as non-specific stress markers, and those without. This was also the conclusion of a study on 234 Anglo-Saxon, late medieval and post medieval juveniles from England, where 21 of the juveniles from Broadgate and Christ Church presented with rickets yet had no statistical difference in long bone growth compared to the other juveniles (Pinhasi et al. 2006). In another study on early and late Medieval English skeletons, no statistical growth differences were found either between juveniles showing non-specific stress indicators and juveniles without (Ribot \& Roberts 1996).

For the purpose of this doctoral research, several methods will be compared in the analyses in Chapter 5 on available direct diaphyseal measurements. However, the method of choice for estimating age will be that of Stloukal and Hanáková (1978), as the origin of the population, the methodological approaches and the sample number seem the most appropriate for this type of archaeological analyses. Table 3.4 overleaf summarises the main methods available, including a revealing comparison of the number of individuals per cohort/ age group. 


\begin{tabular}{|c|c|c|c|c|c|c|c|c|c|c|c|c|c|c|c|c|c|c|c|c|}
\hline \multirow{3}{*}{ UPPER LIMBS } & \multirow{3}{*}{ Population } & \multirow{3}{*}{ Period } & \multirow{3}{*}{ Method } & \multirow{3}{*}{$\begin{array}{l}\text { Total } \\
\text { \# of } \\
\text { ind. }\end{array}$} & \multirow{3}{*}{$\begin{array}{l}\text { Max. } \\
\text { Age }\end{array}$} & \multicolumn{5}{|c|}{ Humerus } & \multicolumn{5}{|c|}{ Radius } & \multicolumn{5}{|c|}{ Ulna } \\
\hline & & & & & & \multirow{2}{*}{$\begin{array}{l}\# \text { coh } \\
\text { pres. }\end{array}$} & \multicolumn{4}{|c|}{ \# of individuals per cohort } & \multirow{2}{*}{$\begin{array}{l}\text { \# coh } \\
\text { pres. }\end{array}$} & \multicolumn{4}{|c|}{ \# of individuals per cohort } & \multirow{2}{*}{$\begin{array}{l}\text { \# coh } \\
\text { pres. }\end{array}$} & \multicolumn{4}{|c|}{ \# of individuals per cohort } \\
\hline & & & & & & & Mean & Med. & Min. & Max. & & Mean & Med. & Min. & Max. & & Mean & Med. & Min. & Max. \\
\hline $\begin{array}{c}\text { Stloukal \& } \\
\text { Hanáková (1978) }\end{array}$ & $\begin{array}{l}\text { Caucasian, Czech } \\
\text { Republic/Slovakia }\end{array}$ & $\begin{array}{l}A D 7^{\text {th }}- \\
9^{\text {th }} c .\end{array}$ & $\begin{array}{l}\text { Dry bone } \\
\text { measurements }\end{array}$ & 336 & $15 y r$ & $19 / 19$ & 11.7 & 11 & 6 & 21 & $19 / 19$ & 7.2 & 7 & 1 & 14 & $19 / 19$ & 6.2 & 6 & 1 & 13 \\
\hline $\begin{array}{c}\text { Sundick: } \\
\text { Altenerding (1978) }\end{array}$ & $\begin{array}{l}\text { Caucasian, } \\
\text { Germany }\end{array}$ & $\begin{array}{l}A D 6^{\text {th }}- \\
7^{\text {th }} c .\end{array}$ & $\begin{array}{l}\text { Dry bone } \\
\text { measurements }\end{array}$ & 82 & $18 \mathrm{yr}$ & $14 / 18$ & 2.9 & 3 & 1 & 6 & $14 / 18$ & 2.8 & 3 & 1 & 6 & $11 / 18$ & 2.4 & 2 & 1 & 4 \\
\hline $\begin{array}{l}\text { Sundick: Indian } \\
\text { Knoll }(1972,1978)\end{array}$ & $\begin{array}{l}\text { Amerindian, } \\
\text { North America }\end{array}$ & $3000 \mathrm{BC}$ & $\begin{array}{l}\text { Dry bone } \\
\text { measurements }\end{array}$ & 128 & $18 \mathrm{yr}$ & $15 / 18$ & 5.6 & 6 & 1 & 11 & $18 / 18$ & 4.9 & 5.5 & 1 & 9 & $15 / 18$ & 4.9 & 6 & 1 & 9 \\
\hline Johnston (1962) & $\begin{array}{l}\text { Amerindian, } \\
\text { North America }\end{array}$ & $3000 \mathrm{BC}$ & $\begin{array}{l}\text { Dry bone } \\
\text { measurements }\end{array}$ & 165 & $5 y r$ & $7 / 7$ & 22.1 & 9 & 6 & 71 & $7 / 7$ & 16.4 & 7 & 5 & 60 & $7 / 7$ & 16 & 7 & 4 & 54 \\
\hline $\begin{array}{c}\text { Merchant \& } \\
\text { Ubelaker (1977) }\end{array}$ & $\begin{array}{l}\text { Amerindian, } \\
\text { North America }\end{array}$ & $\begin{array}{l}\text { AD } 18^{\text {th }} \\
\text { c. }\end{array}$ & $\begin{array}{l}\text { Dry bone } \\
\text { measurements }\end{array}$ & 193 & $18 \mathrm{yr}$ & $13 / 19$ & 10.4 & 4 & 1 & 49 & $12 / 19$ & 10.3 & 4 & 1 & 47 & $11 / 19$ & 10.4 & 4 & 2 & 47 \\
\hline Gindhart (1973) & $\begin{array}{l}\text { Caucasian, } \\
\text { Ohio, U.S.A. }\end{array}$ & $\begin{array}{l}A D 20^{\text {th }} \\
\text { c. }\end{array}$ & $\begin{array}{l}\text { Radiographs } \\
\text { (corrected) }\end{array}$ & $100 s$ & $18 \mathrm{yr}$ & $\mathrm{n} / \mathrm{a}$ & $\mathrm{n} / \mathrm{a}$ & $\mathrm{n} / \mathrm{a}$ & $\mathrm{n} / \mathrm{a}$ & $\mathrm{n} / \mathrm{a}$ & $33 / 33$ & 236.4 & 220 & 28 & 376 & $\mathrm{n} / \mathrm{a}$ & $\mathrm{n} / \mathrm{a}$ & $\mathrm{n} / \mathrm{a}$ & $\mathrm{n} / \mathrm{a}$ & $\mathrm{n} / \mathrm{a}$ \\
\hline Maresh (1970) & $\begin{array}{l}\text { Caucasian, } \\
\text { Colorado, U.S.A. }\end{array}$ & $\begin{array}{l}\text { AD } 20^{\text {th }} \\
\text { c. }\end{array}$ & $\begin{array}{l}\text { Radiographs } \\
\text { (uncorrected) }\end{array}$ & 244 & $12 \mathrm{yr}$ & $26 / 26$ & 150.6 & 152 & 124 & 161 & $26 / 26$ & 150.6 & 151.5 & 124 & 161 & $26 / 26$ & 150.5 & 151.5 & 124 & 161 \\
\hline Hoffman (1979) & $\begin{array}{l}\text { Caucasian, } \\
\text { Colorado, U.S.A. }\end{array}$ & $\begin{array}{l}\text { AD } 20^{\text {th }} \\
\text { c. }\end{array}$ & $\begin{array}{l}\text { Adapted from } \\
\text { Maresh }\end{array}$ & $?$ & $12 \mathrm{yr}$ & Var & ing bet & en 6 & $d 86 p$ & cohort & number & unpubli & hed but & nould $c$ & rrespor & to fem & le data $f$ & om Mar & sh's stu & \\
\hline \multirow{3}{*}{ LOWER LIMBS } & \multirow{3}{*}{ Population } & \multirow{3}{*}{ Period } & \multirow{3}{*}{ Method } & \multirow{3}{*}{$\begin{array}{l}\text { Total } \\
\text { \# of } \\
\text { ind. }\end{array}$} & \multirow{3}{*}{$\begin{array}{l}\text { Max. } \\
\text { Age }\end{array}$} & \multicolumn{5}{|c|}{ Femur } & \multicolumn{5}{|c|}{ Tibia } & \multicolumn{5}{|c|}{ Fibula } \\
\hline & & & & & & \multirow{2}{*}{$\begin{array}{l}\# \text { coh } \\
\text { pres. }\end{array}$} & \multicolumn{4}{|c|}{$\#$ of individuals per cohort } & & \multicolumn{4}{|c|}{ \# of individuals per cohort } & & \# of $i$ & dividua & per co & nort \\
\hline & & & & & & & Mean & Med. & Min. & Max. & pres. & Mean & Med. & Min. & Max. & pres. & Mean & Med. & Min. & Max. \\
\hline $\begin{array}{c}\text { Stloukal \& } \\
\text { Hanáková (1978) }\end{array}$ & $\begin{array}{l}\text { Caucasian, Czech } \\
\text { Republic/Slovakia }\end{array}$ & $\begin{array}{l}A D 7^{\text {th }}- \\
9^{\text {th }} c .\end{array}$ & $\begin{array}{l}\text { Dry bone } \\
\text { measurements }\end{array}$ & 336 & $15 y r$ & $19 / 19$ & 13.1 & 14 & 6 & 23 & $19 / 19$ & 9.9 & 10 & 3 & 18 & $17 / 19$ & 3.5 & 3 & 1 & 6 \\
\hline $\begin{array}{c}\text { Sundick: } \\
\text { Altenerding (1978) }\end{array}$ & $\begin{array}{l}\text { Caucasian, } \\
\text { Germany }\end{array}$ & $\begin{array}{l}A D 6^{\text {th }}- \\
7^{\text {th }} c .\end{array}$ & $\begin{array}{l}\text { Dry bone } \\
\text { measurements }\end{array}$ & 82 & $18 \mathrm{yr}$ & $13 / 18$ & 4 & 4 & 1 & 9 & $14 / 18$ & 3.4 & 3 & 1 & 8 & $10 / 18$ & 2.8 & 3 & 1 & 6 \\
\hline Mays (1999) & $\begin{array}{l}\text { Caucasian, } \\
\text { England }\end{array}$ & $\begin{array}{l}\text { AD 10- } \\
16^{\text {th }} \mathrm{c} .\end{array}$ & $\begin{array}{l}\text { Dry bone } \\
\text { measurements }\end{array}$ & 117 & $17 \mathrm{yr}$ & $14 / 14$ & 8.4 & 8 & 2 & 21 & $\mathrm{n} / \mathrm{a}$ & $\mathrm{n} / \mathrm{a}$ & $n / a$ & $\mathrm{n} / \mathrm{a}$ & $\mathrm{n} / \mathrm{a}$ & $\mathrm{n} / \mathrm{a}$ & $\mathrm{n} / \mathrm{a}$ & $\mathrm{n} / \mathrm{a}$ & $\mathrm{n} / \mathrm{a}$ & $\mathrm{n} / \mathrm{a}$ \\
\hline $\begin{array}{l}\text { Sundick: Indian } \\
\text { Knoll }(1972,1978)\end{array}$ & $\begin{array}{l}\text { Amerindian, } \\
\text { North America }\end{array}$ & $3000 \mathrm{BC}$ & $\begin{array}{l}\text { Dry bone } \\
\text { measurements }\end{array}$ & 128 & $18 \mathrm{yr}$ & $18 / 18$ & 5.7 & 7 & 1 & 11 & $18 / 18$ & 5.4 & 6 & 1 & 10 & $17 / 18$ & 4.9 & 5 & 1 & 9 \\
\hline Johnston (1962) & $\begin{array}{l}\text { Amerindian, } \\
\text { North America }\end{array}$ & $3000 \mathrm{BC}$ & $\begin{array}{l}\text { Dry bone } \\
\text { measurements }\end{array}$ & 165 & $5 y r$ & $7 / 7$ & 20.7 & 11 & 6 & 64 & $7 / 7$ & 20.4 & 10 & 6 & 65 & $7 / 7$ & 12 & 6 & 1 & 37 \\
\hline $\begin{array}{c}\text { Merchant \& } \\
\text { Ubelaker (1977) }\end{array}$ & $\begin{array}{l}\text { Amerindian, } \\
\text { North America }\end{array}$ & $\begin{array}{l}\text { AD } 18^{\text {th }} \\
\text { c. }\end{array}$ & $\begin{array}{l}\text { Dry bone } \\
\text { measurements }\end{array}$ & 193 & $18 \mathrm{yr}$ & $14 / 19$ & 10 & 3.5 & 1 & 51 & $15 / 19$ & 8.5 & 3 & 1 & 47 & $16 / 19$ & 7.1 & 3 & 1 & 37 \\
\hline Gindhart (1973) & $\begin{array}{l}\text { Caucasian, } \\
\text { Ohio, U.S.A. }\end{array}$ & $\begin{array}{l}A D 20^{\text {th }} \\
\text { c. }\end{array}$ & $\begin{array}{l}\text { Radiographs } \\
\text { (corrected) }\end{array}$ & $100 s$ & $18 \mathrm{yr}$ & $\mathrm{n} / \mathrm{a}$ & $\mathrm{n} / \mathrm{a}$ & $\mathrm{n} / \mathrm{a}$ & $\mathrm{n} / \mathrm{a}$ & $\mathrm{n} / \mathrm{a}$ & $33 / 33$ & 175.3 & 199 & 23 & 308 & $\mathrm{n} / \mathrm{a}$ & $\mathrm{n} / \mathrm{a}$ & $\mathrm{n} / \mathrm{a}$ & $\mathrm{n} / \mathrm{a}$ & $\mathrm{n} / \mathrm{a}$ \\
\hline Maresh (1970) & $\begin{array}{l}\text { Caucasian, } \\
\text { Colorado, U.S.A. }\end{array}$ & $\begin{array}{l}A D 20^{\text {th }} \\
\text { c. }\end{array}$ & $\begin{array}{l}\text { Radiographs } \\
\text { (uncorrected) }\end{array}$ & 244 & $12 \mathrm{yr}$ & $26 / 26$ & 150.6 & 152 & 124 & 161 & $26 / 26$ & 150.7 & 152 & 124 & 161 & $26 / 26$ & 150.7 & 152 & 124 & 161 \\
\hline Hoffman (1979) & $\begin{array}{l}\text { Caucasian, } \\
\text { Colorado, U.S.A. }\end{array}$ & $\begin{array}{l}A D 20^{\text {th }} \\
\text { c. }\end{array}$ & $\begin{array}{l}\text { Adapted from } \\
\text { Maresh }\end{array}$ & $?$ & $12 \mathrm{yr}$ & & $n$ & 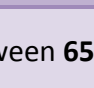 & 10 & 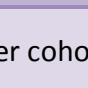 & pum & , & bad & Ild & Sח & S & th & $A_{2}$ & $=t$ & \\
\hline
\end{tabular}

Table 3.4: Comparisons of the samples used by different methods for age estimation from diaphyseal bone length 
All the data from the original articles of the methods described above were also gathered and interpreted, including tables, graphs and subsequent reproductions, in order to make them comparable and usable for archaeological remains. The results are presented in Appendix 3 with one table for each of the six long bones including mean maximum diaphyseal length and range of measurements at three and six months, one, 1.5, and two years, and yearly afterwards.

\section{(- Postnatal period - other measurements}

Pars basilaris bone growth can provide an indication of age for young juveniles. A list of mean width, sagittal and maximum length has been published based on the study of 48 juveniles aged from birth to five years old from the documented historical collections of St Bride's and Spitalfields, England (Scheuer \& MacLaughlin-Black 1994). Although there was only one to three individuals per cohort, the age categories themselves were very small. Once grouped in oneyear cohorts, they would correspond to a median number of thirteen individuals until three years of age, and a median number of four individuals between three and five years. Of particular interest is the shift of proportions at five months between maximum width and maximum length, with the width first inferior to the maximum length, but having superior dimensions by five months. This table was reproduced in the later juvenile osteology publications of the same authors.

Clavicle maximum diaphyseal length was also investigated in the comparative study between the Altenerding and Indian Knoll populations (Sundick 1978), and another study based on 143 individuals from the documented skeletal collections of Spitalfields, St Bride's and St Barnabas in England, including 87 under sixteen years of age, as well as the Portuguese collection of the Museu Bocage (Black \& Scheuer 1996). The results from both those studies have been summarised in table 3.5 overleaf, which shows very similar results between those geographically and chronologically different populations. 


\begin{tabular}{|c|c|c|c|c|c|c|}
\hline \multirow[b]{2}{*}{$3 \mathrm{mths}$} & \multicolumn{2}{|c|}{$\begin{array}{c}\text { Sundick: } \\
\text { Altenerding }\end{array}$} & \multicolumn{2}{|c|}{$\begin{array}{c}\text { Sundick: } \\
\text { Indian Knoll }\end{array}$} & \multicolumn{2}{|c|}{ Black \& Scheuer } \\
\hline & $n=1$ & 51.0 & $n=7$ & 44.9 & $n=11$ & 44.4 \\
\hline & & & & $41-49$ & & $39-55$ \\
\hline \multirow[t]{2}{*}{$6 \mathrm{mths}$} & & & & & $n=9$ & 54.1 \\
\hline & & & & & & $48-61$ \\
\hline \multirow[t]{2}{*}{$1 \mathrm{yr}$} & $n=1$ & 51.0 & $n=5$ & 57.7 & $n=11$ & 59.5 \\
\hline & & & & $54-62$ & & $54-66$ \\
\hline \multirow[t]{2}{*}{1.5 yrs } & $n=1$ & 66.0 & $n=5$ & 63.4 & $n=4$ & 63.0 \\
\hline & & & & $61-67$ & & $61-65$ \\
\hline \multirow[t]{2}{*}{2 yrs } & & & $n=5$ & 66.2 & $n=13$ & 66.5 \\
\hline & & & & $62-70$ & & $59-73$ \\
\hline \multirow[t]{2}{*}{3 yrs } & $n=1$ & 70.0 & $n=8$ & 68.7 & $n=7$ & 73.4 \\
\hline & & & & $62-76$ & & $69-77$ \\
\hline \multirow[t]{2}{*}{4 yrs } & $n=3$ & 76.7 & $n=3$ & 70.0 & $n=8$ & 74.4 \\
\hline & & $75-80$ & & $65-74$ & & $65-82$ \\
\hline \multirow[t]{2}{*}{5 yrs } & & & $n=2$ & 74.5 & $n=2$ & 75.9 \\
\hline & & & & $67-82$ & & $75-77$ \\
\hline \multirow[t]{2}{*}{6 yrs } & $n=3$ & 90.7 & $n=3$ & 84.8 & $n=4$ & 86.5 \\
\hline & & $87-96$ & & $77-96$ & & $85-89$ \\
\hline 7 yrs & $n=3$ & 92.3 & $n=5$ & 88.3 & $n=1$ & 89.5 \\
\hline \multirow[t]{2}{*}{8 yrs } & \multirow{2}{*}{\multicolumn{2}{|c|}{$87-102$}} & \multirow{2}{*}{\multicolumn{2}{|c|}{$81-97$}} & $n=3$ & 89.0 \\
\hline & & & & & & $79-99$ \\
\hline 9 yrs & $n=2$ & 104.5 & $n=9$ & 97.3 & & \\
\hline \multirow[t]{2}{*}{10 yrs } & \multirow{2}{*}{\multicolumn{2}{|c|}{$101-108$}} & \multirow{2}{*}{\multicolumn{2}{|c|}{$90-107$}} & $n=2$ & 103.7 \\
\hline & & & & & & 103-104 \\
\hline \multirow[t]{2}{*}{11 yrs } & $n=1$ & 110.0 & & & $n=2$ & 105.0 \\
\hline & & & & & & $105-105$ \\
\hline \multirow[t]{2}{*}{12 yrs } & $n=3$ & 106.0 & $n=2$ & 101.5 & $n=3$ & 106.4 \\
\hline & & $102-110$ & & $95-108$ & & $103-111$ \\
\hline \multirow[t]{2}{*}{13 yrs } & $n=5$ & 112.6 & $n=7$ & 104.1 & $n=2$ & 118.6 \\
\hline & & $111-123$ & & $96-114$ & & $117-120$ \\
\hline \multirow[t]{2}{*}{14 yrs } & $n=10$ & 123.2 & $n=11$ & 121.6 & $n=2$ & 118.5 \\
\hline & & $100-147$ & & $105-139$ & & $114-124$ \\
\hline \multirow[t]{2}{*}{15 yrs } & $n=1$ & 123.0 & $n=7$ & 127.9 & \multirow{2}{*}{$n=3$} & \\
\hline & & & & $114-144$ & & 137.7 \\
\hline 16 yrs & & & $n=6$ & 126.5 & & \\
\hline & & & & 117-135 & & $12 /-154$ \\
\hline 17 yrs & $n=2$ & 135.5 & $n=4$ & 133.5 & & \\
\hline & & $127-144$ & & $118-143$ & & \\
\hline 21 yrs & $n=1$ & 141.0 & $n=9$ & 132.3 & & \\
\hline & & & & $125-140$ & & \\
\hline
\end{tabular}

Table 3.5: Comparison of age estimation from diaphyseal length of the clavicle 
Scapular dimensions have also proved of interest for juvenile age estimation. The documented AD 19th century collection of eighty juveniles from St Thomas' Church, Ontario (S.R. Saunders et al. 1993) unfortunately yielded a very low number of individuals per cohort (median $n$ under three years $=12$, median $n$ above three years=2). A further study based on 31 individuals from the Scheuer collection in Dundee provided equations to predict age from several scapular measurements (Rissech \& Black 2007).

Mandibular ramus height was also found to be a good indicator of age for young juveniles from birth to two years in a collection of 53 skeletons from the Smithsonian's Southwest Collection (Norris 2002), although the ranges of dimension for each six month-cohorts overlapped greatly with 17-27mm, 21$34 \mathrm{~mm}, 29-34 \mathrm{~mm}$, and 32-39mm respectively.

Numerous studies have also been devoted to the correlation between age and metacarpals or hand phalanges dimensions. Regrettably, it is often impossible to identify exactly which phalanges and metacarpals are present in juvenile archaeological remains, unless they were recorded directly on site.

\subsubsection{Skeletal maturation}

The level of skeletal maturation, particularly the fusion of different parts of bones such as the skull bones during infancy and childhood and the union of epiphyses to their respective diaphyses during adolescence, is another very useful tool to estimate age at death on skeletal remains. Although the appearance of the epiphyses in childhood can also be timed quite accurately, these small bones are rarely recovered from the field and are best seen in radiographs of full bodies. Most of the information available comes from longitudinal radiographic studies of living children during the $20^{\text {th }}$ century, mostly healthy white Europeans and North Americans. Those studies are unlikely to be duplicated as they involved the repeated exposure to x-rays at regular intervals, often once a year if not more. This would now be deemed far 
too dangerous as the risks of radiography are better known. The repeated exposure to x-rays in those earlier studies might also have influenced the natural growth of the children involved (Scheuer \& Black 2004:10). Some difference exists as well in the time of observation of appearance and fusion of epiphyses on radiographs compared to macroscopical observation of dry bones.

There are several general issues associated with skeletal maturation as an age indicator. Different methods regarding epiphyseal union utilise various numbers of recorded stages of fusion. This wide range of stages number renders comparisons of data from different studies very difficult, varying from a simple "unfused/fused" to the more common three stages "open/(partial) fusion/complete union" (as recommended in Buikstra \& Ubelaker 1994:41), the four stages of "no fusion/commencement of fusion/advanced fusion/complete fusion", and even up to nine distinctive stages. The timings of epiphyseal fusion also show a marked difference between sexes, with girls often in advance of approximately two years in comparison to boys. This is very problematic for archaeological remains as attributing sex to juveniles is always very speculative (see Chapter 2). Towards the later years of adolescence, sexual dimorphism may become more obvious, and epiphyseal union timings are therefore usually given separately for males and females. However, in most cases, it will be necessary to apply an increased time range to cover the earlier female start of fusion to the usually later male end of fusion. Differences in populations and socio-economic status could also have an impact on skeletal maturation (Lewis 2007; Cardoso 2008), although this is still debated (Krogman \& İșcan 1986).

\section{Early studies}

The first study of epiphyseal union on a relatively large scale was published in the 1920s, based on the osteological observation of four stages of union of the bones of 110 skeletons from the Western Reserve collection, a dissecting room sample (Stevenson 1924). Known age ranged from 15 to 28 years and the 
sample included an equal proportion of black and white individuals, mostly males. Unfortunately, although those numbers were much higher than any previous studies, it still only amounted to a median of only 2.5 individuals per year. In addition, half of the year categories for females were not represented by any individual, and only four individuals were seventeen years or under. Four stages of union were recorded. A comparison of the sequence of epiphyseal union between two populations was provided ten years later, based on 33 Pueblo Indians and 41 Eskimos, all from collected natural burials kept at the National Museum (Stewart 1934). Sex was estimated from pelvic morphology. Age was unknown and was not estimated, as only the sequence of union was observed, rather than the precise timing of specific fusions. Differences between races were established, especially compared to Stevenson's results and mostly for the earliest epiphyses to fuse. However, those population differences were less marked than the variability present within each population (Krogman \& İşcan 1986).

\section{( $20^{\text {th }}$ century methodologies}

Most methodologies used in the $20^{\text {th }}$ century and still commonly employed today were based themselves on a mixture of data from different authors. The "History of Human Life Span and Mortality" (Acsádi \& Nemeskéri 1970) reproduced the data of Vallois (1960) for age of appearance of ossification centres and age of completion of union, with a single set of data for both sex and the advice that state of fusion should be recorded using five stages. A comparison of the data from Todd (1930b), Flecker (1942) and Johnston (1961) on the epiphyseal union times was also given, with separate results for females and males. However, it should be noted that only the ages of beginning of fusion were given in this publication, and that there are issues with all the original studies themselves. The basis for Todd's study of nine stages of fusion (as well as the later publications by Krogman 1939, 1955) was the Hamann-Todd Collection, whose age-at-death although recorded as "known" is also still 
debated as was described earlier. Flecker's study was based on radiographs of hospital patients in Australia from European decent, from birth to 29 years of age. Data about the radiographic observation of the appearance and fusion of ossification centres had been given in a table, including earliest age of appearance/fusion, age when appearance/fusion was noted in at least half of the individuals, and age of the oldest subjects without appearance/fusion. This information was provided separately for both sexes and compared to other studies of populations from around the world. Unfortunately, due to the sample used, this was a cross-sectional rather than a longitudinal study and the number of individuals per age varied widely, with most age categories represented by less than ten individuals. Finally, Johnston's study was based on a limited sample of 35 males and 27 females from the prehistoric Indian Knoll population, with state of fusion recorded following three stages. Results had showed a similar sequence of union to Stevenson's and Stewart's populations described above, and female maturation was determined at around two years earlier than males. However, the age of this population had been estimated from the calcification of the mandibular third molars, as observed in x-rays, and "adjusted to compensate from the earlier ages of tooth eruption observed among American Indians" (Johnston 1961:72). As the development of the third molar is highly variable (see above, section 3.2.1), this age estimation might not correspond to actual chronological age, which would influence any comparison of these results with samples of known age. Moreover, the issue of sexing prehistoric populations, and in particular juvenile remains, has already been discussed in the previous chapter and casts further doubt on the reliability of this material as a basis for ageing other populations.

The recommendations for ageing according to the timing of epiphyseal union published following the workshop of European Anthropologists (Ferembach et al. 1980) was based on data by Brothwell (1965), Wolff-Heidegger (1954), Rauber and Kopsch (1952), Haret and colleagues (1927) and Gray's Anatomy 
(1967). The same data was also presented in the German anthropology textbook of Knußmann and Martin (1988) widely used in Europe, although different results were given for the feet. Although Brothwell $(1963,1965,1972)$ has often been quoted as a source of data for times of epiphyseal union, his data was itself gathered from various sources which were not referenced, and no distinction was offered between male and female fusion times.

Ubelaker's (1984) textbook on the excavation, analysis and interpretation of human skeletal remains provided a table of the age of initial union of epiphyses, with separate data for males and females. It referred to McKern and Stewart's (1957) study as the most complete, although still only applicable to North American males. This study was based on a large sample of 450 casualties of the Korean War, all young males between seventeen and fifty years of age of clearly established age-at-death, with five stages of epiphyseal union recorded. Unfortunately, due to the specificities of the study sample, an artificially lower limit of seventeen years was imposed on all observation and no female data could be obtained for comparison. As noted by Ubelaker (1989a), this data clearly showed that epiphyseal union was a process that took several years.

The "Standards for Data Collection from Human Skeletal Remains" (Buikstra \& Ubelaker 1994) offered a graph reproduced in numerous other field manuals and text books (Bass 1995; White \& Folkens 2000, 2005; and Byers 2008 to name only a few). The data used had been taken from Krogman and İscan (1986), McKern and Stewart (1957, see above for a detailed description), Redfield (1970), Suchey and colleagues (1984) and Ubelaker (1989a, 1989b). All the data was for males only apart from the medial and distal epiphyses of the humerus. For female individuals, it was advised that maturation would occur one to two years earlier and that the range should be amended accordingly.

Mays' (1998) textbook for archaeological human remains utilised Flecker's (1942) data of earliest fusion and age of oldest subjects without fusion to produce times of epiphyseal unions, except from the iliac crest and medial 
clavicle for which the data came from Webb and Suchey (1985). The limitations of Flecker's small sample have already been described above. While Webb and Suchey's ageing study concentrated solely on the anterior iliac crest and the medial clavicle, it was based on large numbers of individuals of both sexes, ensuring the strength of their results, with observations from 605 male and 254 female Americans of all races, aged eleven to forty years, autopsied in Los Angeles County in the late 1970s. A different four-stage system had been employed, including a stage where epiphyses were absent and one where they were present but non-united. Results indicated little difference between males and females, varying by a couple of years at the most. Unfortunately, they also highlighted the wide age ranges for each union stage of these two bones.

\section{(- New methodology for the $21^{\text {st }}$ century}

New studies are published regularly, generally with a focus on a specific bone. In recent years, the study for forensic purposes of the remains of thousands of civilian as well as military casualties from recent conflicts and tragedies in Europe and the rest of the World has greatly increased our knowledge of bone formation and epiphyseal fusion, albeit on a modern sample. More osteological studies have also been undertaken on historical and modern skeletal remains with known age-at-death, such as the cemetery remains of Christ Church, Spitalfields, England or the documented Portuguese collections. Those recent publications are too numerous to be reviewed here. However, they have been taken into account in the newest data from Schaefer, Black and Scheuer (2009), and some of these newest results have been added to the table in Appendix 4. Sequence of fusion has also been shown as a very useful tool for commingled remains to highlight the potential presence of more than one individual (Schaefer \& Black 2007).

The extensive studies on juvenile osteology by Scheuer and Black's (2000, 2004) gathered information from over 300 years and were strictly restricted to 
skeletal material with known age-at-death to avoid any inaccuracies. Both these "excellent texts" (Lewis 2007:47) have been praised by other authors, and were described in the BABAO/IFA guidelines as "the most comprehensive review of information on development and fusion of bones across the skeleton currently available" (Brickley 2004:21). The research did not stop with these two volumes but was taken further with the addition of a third colleague, culminating with the publication of an updated laboratory and field manual which took into account all the latest data as mentioned above (Schaefer et al. 2009).

In order to ensure that the most appropriate ageing methods were employed in this doctoral research, it was decided to go back to the original studies on which available methodologies are based. Through this research, the inadequacies of these studies in either sample and / or methods were brought to light, as can be seen in this section. A comprehensive table of the age ranges available for skeletal maturation was also compiled and is presented in Appendix 4. This table can be used to check ageing results of different populations or studies to ensure that these are comparable. The assertion that "a wide variety of ages can be assigned depending on the bone or standard being used" (Lewis 2007) was demonstrated in the age ranges of the different standards and methodologies compared in this table. The very extensive data and strict methodology of Black, Scheuer and Schaefer rose above all other standards. It was therefore decided to adhere in this thesis to the latest reference of these researchers for juvenile skeletal maturation (Schaefer et al. 2009). Their data on epiphyseal union timings was also adapted by the author into a single easy-to-use figure, presented below (see figure 3.1). For age of fusion differing with sex, a range from the earliest to the latest ages presented will be utilised for juveniles unless these individuals can be confidently sexed. In the cases of unsexed or indeterminate juveniles, the earliest of the ages given for both sexes will also be used for unfused ("open") epiphyses, and the latest age for cases of complete union. 


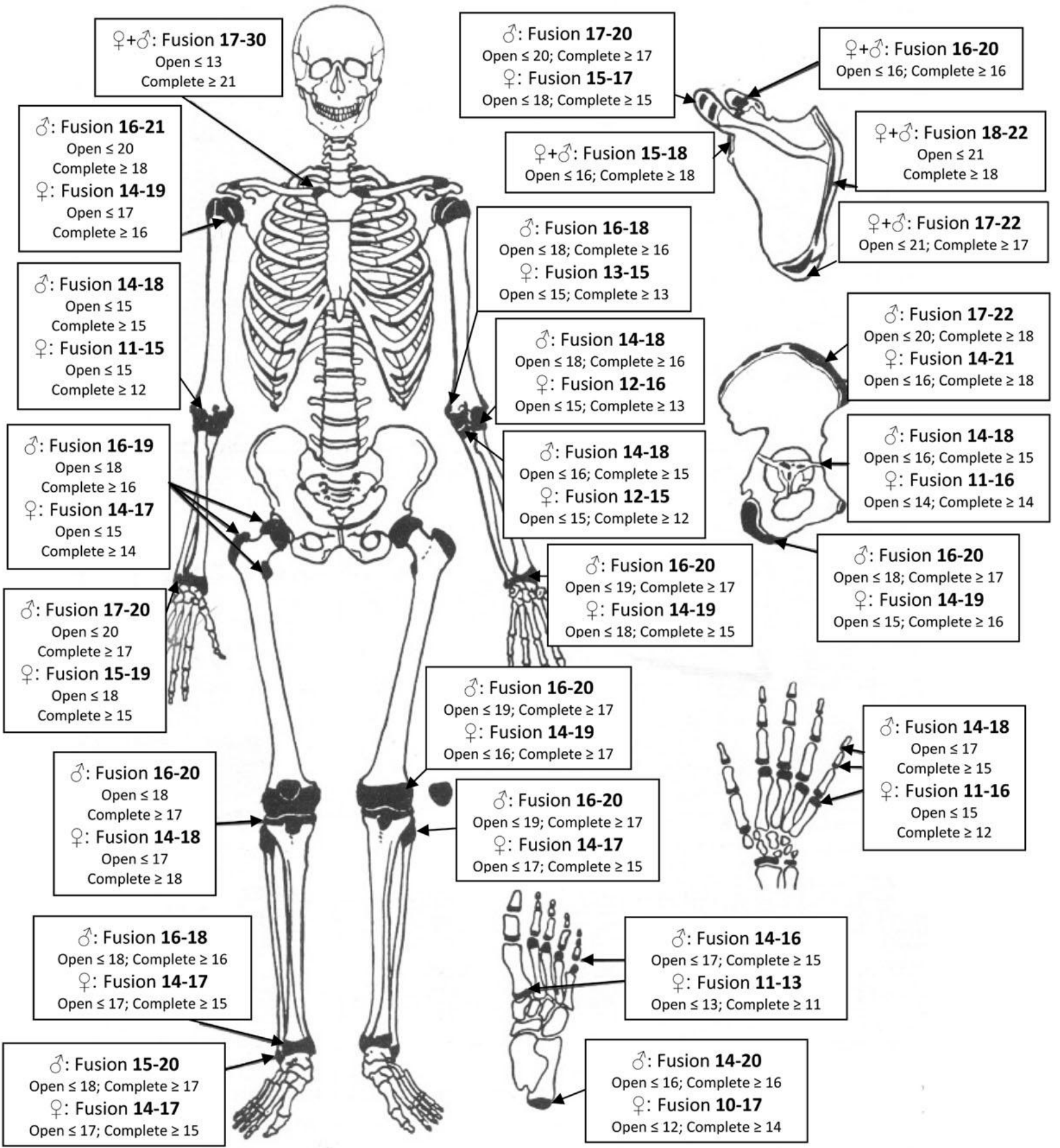

Figure 3.1: Timings of epiphyseal union (figure by the author, data from Schaefer, Black and Scheuer 2009) 


\subsection{Adults}

Although several epiphyseal fusions still occur during adulthood, they are limited and restricted to young adulthood. Once the skeleton is fully mature, only degenerative changes are left to help estimate adult age. These changes can themselves be influenced by sex, population, activity, nutrition, environment, social status and numerous other possible factors, and are therefore extremely variable. Methods used for ageing adults may depend on the population studied, on the country of origin and languages utilised by the researchers, or by their area of professional interest. Reviewed below are the skeletal age indicators in adult and the methods related to them, within the limits of strict macroscopical observations without access to radiographs, histology or microbiology.

\subsubsection{Skull}

\section{(-) Cranial sutures}

The skull has always played an important role in anthropology, especially when the discipline first began, and numerous early "skeletal" collections are represented solely by skulls. Unsurprisingly, this was also one of the first skeletal areas investigated in an attempt to provide information on age at death, and "of all age indicators, cranial sutures have been the most widely used" (Masset 1989:71). After a previous Portuguese study on 1000 skulls (Ferraz de Macedo 1892) and a Spanish publication based on over 2000 skulls (Aranzadi de 1913) went unnoticed, Martin's (1928) chapter on cranial sutures based on an earlier study of 287 skulls (Frédéric 1906) became the work of reference in the German-speaking world. English-speaking researchers were more likely to follow Todd and Lyon's (1924, 1925a, 1925b, 1925c) studies on cranial sutures closure, based on 514 skulls of white and black individuals of both sexes but strongly marred by the "segregation of anomalous skulls" (Todd \& Lyon 1924:333) leading to the rejection of up to $35 \%$ of the original sample. 
A study of Korean War casualties also investigated cranial suture closures with the conclusion that "so erratic [was] the onset and progress of closure that an adequate series [would] provide just about any pattern at any age level" (McKern \& Stewart 1957:37). A collection of 285 crania of males and females of known age was later utilised to develop an equation in order to estimate age from endocranial suture closure (Acsádi \& Nemeskéri 1970). This was part of a multifactorial approach for age estimation, which will be described further below. However, the distribution of material according to stages of closure and chronological age as detailed in the publication presented considerable overlaps in all age ranges. In the 1980s, another method was published involving a composite score from ten segments of different sutures (Meindl \& Lovejoy 1985). This was based on the observation of 236 skulls from the Hamann-Todd collection with uncertain age-at-death. Yet again, the age ranges covering up to fifty years each overlapped to such an extent as to render the use of this method totally inadequate for age estimation. More recently, a further study (Hershkovitz et al. 1997b) on a vast sample of 3636 skulls from the HammanTodd and the Terry collections revealed that the sagittal suture closure was not linked to age. The different stages of closure were indeed not progressive stages but "independent permanent phenoma" (Hershkovitz et al. 1997b:398), influenced by sex. Sexual differences were also demonstrated by Masset (1989). This review of the available research on this specific age indicator thus agrees with Cox's own conclusions that cranial suture closure is "unequivocally [...] not a reliable method of ageing adults" (Cox 2000:66), and this criterion will therefore not be used in this doctoral research.

\section{- Jugular growth plate (petroexoccipital articulation)}

Fusion of the jugular growth plate may be used to facilitate estimation of adult age. Maat and Mastwijk's (1995) study was referenced in the BABAO/IFA guidelines with a caution that the data originated from a small group. This study was based on the Utrecht Collection comprising of 124 skeletons buried in a 
Dutch cemetery during the late 1950s-early 1960s with documented sex and age-at-death. The sample included 98 skulls of individuals between 14 and 92 years. The crania showed no fusion before 22 years, unilateral fusion between 22 and 34 years, and complete bilateral fusion over 34 years for females and 36 years for males (data in Appendix 4). A further study on a much larger sample of 1869 individuals from the Hamann-Todd collection demonstrated that fusion could occur as late as fifty years of age, with $9 \%$ of individuals showing no fusion at all (Hershkovitz et al. 1997a). Fusion of this articulation was nevertheless recognised as a useful indicator of young adults, with less than $13 \%$ of individuals over forty presenting a completely open suture. However, any ageing linked to the Hamann-Todd collection is marred by the lack of known age-at-death (Cox 2000:66, also mentioned previously).

\section{- Spheno-occipital synchondrosis}

This fusion site has widely been used to estimate adult age, with main anatomical texts such as Gray's Anatomy placing fusion between 18 and 25 years. Redfield (1970) is commonly referenced as indicating that this fusion occurs between 20 and 29 years of age (Krogman \& İşcan 1986; Buikstra \& Ubelaker 1994). However, referring to Redfield's original publication, this was the age-range at which $100 \%$ of individuals showed complete fusion, whilst half of the 13 to 19 years also exhibited complete fusion, and thus demonstrated only that individuals with open synchondrosis were less than twenty years of age. Furthermore, Redfield's study was based on 109 juveniles and 41 young adults from AD 15th c. Mistihalj in Serbia, whose age was not known and had only been estimated based on their dentition and level of epiphyseal fusion. Thankfully, other studies based on large samples of individuals of known age are available. These include the analyses of laminagrams of 205 males aged eight to 21 years and 193 females aged six to eighteen years (Powell \& Brodie 1963), and more recently a study of an Indian sample of 50 males and 34 females aged ten to twenty years obtained from autopsies, and 46 male and 27 
female living subjects aged ten to nineteen years (Sahni et al. 1998). Both these studies and Scheuer, Black and Schaefer's publications indicate that this fusion occurs during adolescence rather than early adulthood (see Appendix 4 for precise age ranges), and is therefore a useful tool for juvenile ageing rather than an indicator of adult age.

\subsubsection{Thorax}

\section{( Clavicle}

The medial (or sternal) end of the clavicle only begins to fuse during early adulthood and is therefore a useful indicator of age for adults. The main studies of this epiphyseal fusion include those of McKern and Stewart (1957) and Webb and Suchey (1985), already described in section 3.2.3., in addition to Black and Scheuer's (1996) study. The latter work was based on 65 individuals with a documented age-at-death of eleven to thirty years, including 21 clavicles from Spitalfields and 18 from St Brides, England, as well as 26 clavicles from Portugal. Results were included in Scheuer, Black and Schaefer subsequent volumes on juvenile osteology, which were recommended in the BABAO/IFA guidelines. The data from these and several other publications including those mentioned above are presented in Appendix 4.

\section{( ) Ribs}

In the 1980s, a new method to age adults was devised following the morphological changes of the sternal end of the right fourth rib (İșcan et al. 1984, 1985; İşcan \& Loth 1986a, 1986b; Loth \& İşcan 1989). These studies were based on a sample of 277 ribs from autopsied individuals of known age, sex and race. Nine phases (0 to 8 ) were recorded, with separate results by sex and race. Ranges increased with age, encompassing from two to twelve years per phase. Marked racial differences were observed, which were corroborated in a study of 
339 ribs of black South Africans resulting in new phases being introduced with modified criteria and age ranges (Oettlé \& Styn 2000). Finally, ribs 2 to 7 were found likely to be within one phase of rib 4, which was confirmed in a later study on 231 ribs from the Terry collection (Yoder et al. 2001). An independent test observed the same results on 101 ribs from the Hamann-Todd collection of uncertain age, albeit with distinct racial differences from the original studies (Russell et al. 1993). More recently, İşcan method was applied successfully to 39 ribs using multislice computed tomography (Dedouit et al. 2008). Even though age ranges are still much extended, this method may be satisfactory for forensic purposes. However, where ancient remains are concerned, a major issue arises with the identification of the $4^{\text {th }}$ rib, which is extremely difficult in most archaeological contexts, particularly with damaged and fragmentary remains. This was demonstrated by experienced anthropologists working on the Spitalfields sample in England and the Belleville sample in Ontario (Cox 2000:70). Quantitative methods of rib seriations have been published with encouraging results (Hoppa \& Saunders 1998; Owers \& Pastor 2005). Unfortunately, these methods also rely on a complete set of mostly undamaged ribs and therefore do not solve identification problems for most archaeological remains.

To answer this identification issue, a new method of ageing from the first rib, which is easily recognisable, has recently been published (Kunos et al. 1999). This method does not provide phases and age ranges, but offers tabulated brief descriptions of morphological changes for the sternal end, the head and the tubercle facet. Unfortunately, it was also based on the Hamann-Todd collection with no known age at death. Tests of this method have been undertaken first on 29 individuals of J.C.B. Grant cadaver collection in Toronto (Kurki 2005), and later on 470 Bosnian males of known age, casualties of the war in former Yugoslavia (DiGangi et al. 2009). The former study revealed high inaccuracy (10.4 years) and bias (4.7 years), whilst still showing positive results compared to other adult ageing methods. The latter study introduced a scoring system 
between 1 and 5 for each of the eleven variables presented on the three observed sites and demonstrated that the geometric shape of the costal facet and the surface texture of the tubercle facet presented age-related morphological changes. Although the method was described as promising, the age ranges including lower and upper $95 \%$ of higher posterior density spanned several decades, spreading over the whole adult period from late adolescence to senility, with up to sixty years in the same score combination. This warranted the recommendations in the article for further tests and studies of this method on more collections of known age. Such research would be an absolute necessity before the use of this methodology on archaeological remains, and the first rib will therefore not be used as an age indicator in the Neolithic populations under study.

\subsubsection{Spine}

\section{- Vertebrae}

Fusion of epiphyseal annular rings on the vertebrae has been used to estimate adult age. A study of a large sample with limited age range based on 259 males aged 17 to 25 years (McKern \& Stewart 1957), and another with a much smaller sample but a wider age range based on 55 autopsied black and white individuals of both sexes aged 11 to 32 years (Albert \& Maples 1995), both concluded that complete epiphyseal union of all vertebrae was achieved by 24 to 25 years. Complete union in any vertebra could be seen around eighteen years, which is in agreement with later findings from Scheuer and Black $(2000,2004)$. The latest extensive studies on hundreds of casualties from the war in former Yugoslavia (Schaefer et al. 2009) also confirmed Scheuer and Black's start of fusion observed at puberty, with partial union visible in adolescence as early as fourteen years of age. Therefore, only complete union can be used as an indicator of (young) adult age. 


\section{(๑) Sacrum}

Fusion between the sacral vertebral bodies has also been investigated. In one of the latest studies, 486 sacra from the Sassari collection, Italy, and 418 sacra from Coimbra, Portugal were employed in the observation of four degrees of fusion, graded 0-3 (Belcastro et al. 2008). No specific age could be estimated from these results. Nonetheless, young adults 20 to 34 years of age could be identified from incomplete sacral vertebrae fusion, based on non-union or less than $50 \%$ fusion between any sacral bodies. Complete fusion from the second to fifth sacral vertebrae with less than $50 \%$ fusion between the first and second sacral vertebrae would indicate a young to mature adult age, thus below fifty years of age. However, complete fusion of all sacral vertebrae bodies could only be used as a sign of adulthood, without further distinction, concurring with Scheuer, Black and Schaefer's (2009) data. All data available for age-ranges of fusion of all vertebrae including the sacrum are presented in Appendix 4.

\section{( Lipping}

Lipping, or osteophytes, of the vertebrae, has also been examined as a potential age indicator. A study of 368 males from the Korean War aged seventeen to fifty years, together with 37 males and 17 females aged 38 to 84 years from the Terry Collection, observed these degenerative changes on a scale of 0 to 3 (Stewart 1958). Results indicated that these changes could not provide precise age estimations, although the absence of osteophytes at level 2 would indicate an individual under thirty while extensive lipping at level 2 or 3 would point towards an age of more than forty years. A Japanese sample of 138 male and 87 female autopsied individuals aged 20 to 88 years also demonstrated a significant correlation between the presence of osteophytes and the ageing process, with osteophytosis starting around thirty years of age (Watanabe \& Terazawa 2006). Two sex-specification equations were provided for age estimation from the index of osteophyte formations, although the distribution of 
those indexes was exceedingly widespread after thirty, particularly in male individuals. In addition, trauma (Ubelaker 1984) and of course mobility (Loth \& İşcan 1989) are strongly connected with these degenerative changes, which might therefore be influenced by population and activity-related stress, providing only very limited use for this method as an indicator of age in archaeological populations.

\subsubsection{Pelvis}

The pelvis is not only the primary source of information for sex estimation, but also a very useful indicator of age. The fusion of its different parts is very valuable for estimating juvenile age (see Appendix 4 for all the data). The changes observable on the pubic symphysis and auricular surface have also been considered some of the best indicators of adult age, with the addition more recently of acetabular changes. In this section, the author will look back to the original studies in order to understand how these methods were designed and confirm the accuracy of these key age indicators.

\section{Pubic symphysis morphology}

The oldest ageing method based on the morphology of the pubis dates back to the 1920s and is still in use today. It was based on the study on 306 American white males from the Hamann-Todd collection (Todd 1920) and has been reproduced in numerous textbooks (Buikstra \& Ubelaker 1994; Bass 1995; White \& Folkens 2000, 2005). This system identified ten phases starting at the age of eighteen and finishing with 50+. Limited age ranges varying between two and five years for each phase before $50+$ helped give a false sense of precision and reliability. However, several serious issues should be noted in connection with this study. Firstly, the reliability of "known age at death" of this collection has been questioned repeatedly (Mays 1998; Cox 2000). Secondly, all individuals who did not fit with ageing standards were discarded from the 
study. Finally, some phases were represented by a very small number of individuals, such as Phase $1(n=4)$, phase $2(n=2)$ and phase $4(n=6)$ while $43 \%$ of the study sample belonged to Phase 10 (50+ years). This method was tested on two distinct series of Native Californian Indians with 194 males and 177 females, and a sample from the Hamann-Todd collection of 103 males and 82 females which included most of the individuals utilised in the original study (Brooks 1955). The Californian sample came from archaeological remains of populations from different periods, whose sex had been estimated from the ischio-pubic index of the pelvis, and whose age had been estimated from Todd's cranial sutures and pubic symphysis ageing system. Age estimation from cranial sutures was found to be "an unreliable age indicator" (Brooks 1955:583), while age estimation from the pubis tended to overage individuals. Age ranges with minor corrections for all ten phases were presented in what seems to be, however, a circular argument as the only sample described of known age was from the Hamann-Todd collection of uncertain age at death, and included the original sample, while the comparative sample was itself aged according to Todd's system. A test on a sample of 96 individuals from the the Hamann-Todd collection showed that all methods underaged the pubis regardless of sex, and that Todd's method with Brooks' corrections was the most reliable of all the methods tested (Meindl et al. 1985). The original method was revised further in order to increase accuracy, regrouping Todd's ten phases into five biological stages that were considered equally valid for both sexes. This modified version of Todd's method was recently described as providing the "best estimates of age at death for adults of any method developed to date" (Byers 2008:222). However, as Meindl and colleagues' sample was also taken from the HamannTodd collection, their study and results suffer from the same pitfalls as the original method.

Observation of the morphological changes of the pubic symphysis as an ageing method was expanded in the 1950s with the distinction of five developmental stages for three distinct components of the symphysis, the two halves of the 
symphyseal surface and the rim (McKern \& Stewart 1957). Ranks of developmental stage for each component were added to obtain a total score which could be used to estimate age at death. The age ranges provided were slightly broader than Todd's and some overlapped. This study already described above was based on casualties of the Korean War, and was therefore restricted to white males between seventeen and fifty years of age, the majority of them in their twenties. The same components were later employed to establish standards for females as opposed to the earlier male-only sample, based on 180 females said to be of known age, including 140 with known parity status (Gilbert \& McKern 1973). However, no details were given as to the provenance of these individuals. No morphological changes were observed after 55 years, and the sample was accordingly reduced to 103 females aged 13 to 57 years. Differences with male results were evident and varied with each component, some changing faster than others. Parity status did not seem to affect morphological stages. The age ranges for each total score were given with substantial standard deviations and repeated overlapping, covering up to twenty years per score. This method was tested with the examination of eleven pubic bones from autopsied females of known age by 23 forensic anthropologists (Suchey 1979). Only 51\% of the estimated broad age ranges included true known age of the specimen, and interobserver differences in age estimation were great, resulting in a strongly negative feedback for this method.

A scheme of five morphological phases regardless of sex was produced partly based on Todd's system and described as useful to age an individual "considerably below 50 years, about 50 years, or over that age" (Acsádi \& Nemeskéri 1970:127). When tested against the American methods, this system was found to give age estimates mostly between 45 and 60 years (Cox 2000:69). This is not surprising as three out of their five phases offered a mean age between 46 and 58 years. Another serious concern arises from the difference between the calculated ranges. These included three standard deviations, spreading between ten and fifteen years, while the actual ranges covered nearly 
the whole of adulthood, spreading over six decades. This system is part of Acsádi and Nemeskéri's "Complex Method", detailed below and recommended during the Workshop of European Anthropologists (Ferembach et al. 1980). Following a comparative study of different methods to age the male pubis that proposed as the most appropriate system of ageing Todd's method grouped in six phases (Katz \& Suchey 1986), a new method was produced similar to Acsádi and Nemeskéri's system (Brooks \& Suchey 1990). This method was based on the study of 1225 pubic bones from individuals autopsied in Los Angeles between 1977 and 1979, without any exclusion. Age-at-death was clearly documented, ranging from 14 to 92 years for males and 13 to 99 years for females. A comprehensive range including $95 \%$ of the individuals yielded very broad results with considerable overlapping between most of the six phases, covering as much as sixty years. Results were presented separately for males and females. In a later work on pubic age determination, twenty females exactly 28 years old were compared (Suchey \& Katz 1998). Phases 2, 3, 4 and 5, as well as a blade-thin symphysis, were all observed in this very specific age group, proving once again the morphological variability of this skeletal location.

Despite these issues, the Suchey-Brooks method was compared favourably to those of McKern-Stewart and Gilbert-McKern based on a forensic autopsied sample from Florida comprising of 202 females and 116 males (Klepinger et al. 1992), albeit with a caution to always use two standard deviations and to expect more errors with advanced age. The same observations was made on a Serbian forensic sample based on 33 females and 52 males from autopsies, aged 17 to 91 years (Djurić et al. 2007). In this case, 83\% of males and 75\% of females were assigned to the correct age phase, a positive result although yet again with a severe underestimation of age in the last phase. The Suchey-Brooks method was also employed on the remains of 212 male and 84 female Balkan casualties of the War in Former Yugoslavia aged 15 to 98 years, and on 1560 males and 518 females aged 15 to 102 years from American collections, revealing no population difference in males, and only a slight difference in females 
(Kimmerle et al. 2008). Conversely, the Suchey-Brooks method was tested with disappointing results on the documented archaeological collections of Belleville, Ontario (S.R. Saunders et al. 1992) and of Spitalfields, England (Cox 2000), with some male individuals still falling out of the considerably wide age range provided by this system. A further test of this method on 404 individuals with well-distributed sex and age-at-death from the documented Sassari collection in Italy revealed no bias for males until forty years of age or for females under thirty (Hens et al. 2008). However, overestimation of up to six years was observed in females 30 to 39 years of age, changing to underestimation after forty years. This underestimation increased considerably after sixty, with inaccuracies of up to 32 years. The problem of ageing older individuals is slowly being addressed, most recently using 104 individuals from the William Bass Donated Collection in Tennessee and 85 individuals casualties of the War in Former Yugoslavia, all females. These samples were utilised to refine the Suchey-Brooks method, modifying slightly phases 5 and 6, and adding a new phase 7 for individuals in their mid-seventies (Berg 2008). Although this is a promising start which might help resolve the problem of older individuals, it is currently only applicable to females and requires to be tested further. Unfortunately, this review of the methods presently available for age estimation based on the pubis must therefore conclude in agreement with Jackes' statement that "there is no certainty of adult age based on pubes" (2000:433).

\section{(-) Auricular surface of the ilium}

This other area of the pelvis was investigated in the 1980s and presented as a valuable indicator of age for archaeological remains as this part of the bone is stronger than the pubic symphysis and thus more likely to survive (Meindl et al. 1983; Lovejoy et al. 1985a; Lovejoy et al. 1985b; Meindl \& Lovejoy 1989). Eight distinct phases were described following morphological changes starting at twenty years of age, with a five-year range for the first six phases, ten years for phase 7 and the final phase recorded as $60+$. This single set of eight phases and 
age-ranges was given as applicable regardless of sex and race, to be used in seriations of whole populations rather than just single cases. This method was based on a selection of 512 individuals from the Hamann-Todd collection, a further 250 individuals from the Libben population and fourteen forensic cases with known sex and age-at-death. Inherent to this method therefore are the issues of the lack of accurate known age-at-death of the Hamann-Todd collection mentioned several times above, as well as the selective process following Todd's and other osteologists' assessment of which individuals had been "correctly" aged. This resulted in the loss of any individuals who did not seem to fit the norm when first analysed by those osteologists, skewing any results towards a fictitious population with a much lesser range of variability than the actual assemblage available. Unfortunately, tests of the method by its authors were also based on individuals from the Hamann-Todd collection.

However, another test of Lovejoy's method on 189 individuals of known age, sex and race from the Terry collection with equal proportions of white and black individuals of both sexes was undertaken by different researchers (Murray \& Murray 1991). This study concurred with the lack of influence of sex or age on degenerative changes observed in this area, but also revealed an overestimation of individuals under 34 and an underestimation of those over forty, reaching on average 24 years for the oldest sample. Only $44 \%$ of individuals were aged correctly or within one age category. The method was deemed unsuitable as a single ageing factor due do the great variability of the degenerative changes together with the very wide estimation error, although it could potentially be used in conjunction with other ageing techniques to provide additional information. The same unsatisfactory results were observed using this method on a sample of 49 individuals from Belleville, Ontario (S.R. Saunders et al. 1992), and on 215 individuals from the Coimbra Collection, Portugal (Santos, in Jackes 2000). A further test of Lovejoy's method on 204 males aged 18 to 86 years and 200 females aged 17 to 98 from the Sassari Collection in Italy demonstrated overestimation of age under 39 by one to four years, and underestimation of at 
least twelve years for individuals over forty (Hens et al. 2008). Although both bias and inaccuracy were high, this method produced marginally better results than the Suchey-Brooks pubic symphysis method.

Lovejoy's method was recently transformed into a quantitative scoring system (Buckberry \& Chamberlain 2002). The study sample included 180 individuals from the Spitalfields collection which resulted in wider age ranges. New mean ages and standard deviations for composite scores and the corresponding auricular surface stages were also provided. This revised method was tested on 309 individuals from the Terry and Huntington Collections (Mulhern \& Jones 2005), on 167 individuals from St Bride's, London (Falys et al. 2006), and on 160 individuals from the William Bass Donated Collection (Shirley \& Bethard 2007). Mulhern and Jones' test established that the Buckberry and Chamberlain's system was less accurate than Lovejoy's original method for the 20-to-49-year age group, but more accurate in the 50 to 69 years category, with both methods applicable to black as well as white individuals of both sexes. Falys and colleagues' comparative study demonstrated such a wide variation in auricular changes that only three broad phases could be separated statistically. Although this implies that the method cannot be used for forensic purposes, the first and the last phases can still help to distinguish between adults below fifty years and those above sixty in archaeological populations. Shirley and Bethard's most recent analysis also concluded that seven phases were not needed, although Falys and colleagues' recommended reduction to as little as three phases was not considered suitable.

Lovejoy's eight-phase system for age estimation from the auricular surface was further reconsidered based on 72 individuals from the William Bass Donated Collection in Tennessee, and 194 individuals from the Terry Collection in Washington DC. (Osborne et al. 2004). Inaccuracy of up to 39 years in age estimation was observed using the original method, with only a third of individuals aged correctly using the five-year range, increasing to $42 \%$ by 
amalgamating three consecutive phases together to produce an age range of fifteen years. With a 95\% prediction interval, the age ranges were dramatically increased and all overlapped, with phases 4 to 7 covering virtually the whole of adulthood. A new six-phase system was presented by combining phases $1+2$, and phases 5+6, with new means, standard deviations, and much broader suggested age ranges.

A completely new method has also recently been designed based on the skeletal remains of 438 male and 262 female Japanese individuals (Igarashi et al. 2005). The presence or absence of nine surface features were recorded, including four relief and five texture categories, and four further features of hypertrophic bone. Multiple regression analyses produced six models of age-at-death estimation. This method was tested on 75 males and 59 females from the William Bass Donated Collection, unfortunately revealing a very poor correlation between true age and age-at-death estimated from the six regressions (Bethard \& Shirley 2007).

\section{- Acetabulum}

The acetabulum has recently become the focus of new research to help establish age-at-death. The initial study sample of thirty Spanish and French males aged 30 to 81 years (Rougé-Maillart et al. 2004) was extended to 242 males aged 16 to 96 from the Coimbra collection in Portugal (Rissech et al. 2006). The method was then tested on 57 males from the Museo Bocage Lisbon collection in Portugal, eighteen males from the UAB collection of Barcelona in Spain, and 77 males from St Bride's collection in England (Rissech et al. 2007). Morphological stages were given for seven variables: the acetabular groove, acetabular rim shape and porosity, apex activity, and acetabular fossa outer edge activity, general activity and porosity. Full descriptions, scores and photographs were provided for each stage of each variable. Although overlapping was present between the grades of all features, an estimated age within ten years of true age 
was achieved in over $89 \%$ of the cases in Coimbra, and within 5 years in $67 \%$ of individuals. Results were equally positive in the other Portuguese, Spanish and English samples when estimated age was based on their own population. Those results were lower when compared to age estimates based on other populations, proving that better accuracy can be obtained when the reference collection is close to the sample. This would be problematic for archaeological material, especially for more ancient populations. However, wider estimation age range of twenty or even thirty years would still give appreciable information for such archaeological populations, while encompassing far less than the six decades of several age ranges of other more commonly used methods focusing on other parts of the pelvis. This new method will therefore also be utilised in this doctoral analysis of Neolithic samples.

\subsubsection{Dental wear}

Dental wear has been studied regularly since the $19^{\text {th }}$ century on different living as well as past populations. Although degree of dental wear varies widely between and within populations, this feature has also often been used as an age indicator. The rate of tooth wear on juveniles has been used to age adult individuals since the 1950s by observing the degree of wear of the first molar at eruption of the second molar, which should mark a difference of around six years. Degree of wear of the first two molars at eruption of the third molar was also noted, corresponding to a further six to ten year gap. Rate of wear could then be deduced from those differences (Zuhrt 1955). Patterns of wear of all teeth were observed on more than 190 Australian aboriginal skulls from the collection of the University of Adelaide, with no age estimates given (Murphy 1959). Rate of wear was also analysed on the dentitions of 190 Anglo-Saxons skulls from England, dated AD 700-900, but this time providing age estimates (Miles 1962). Thirty-two skulls came from juvenile individuals, corresponding to roughly two skulls for every year between six and nineteen, and were therefore given a dental age to form a baseline using Schour and Massler's 
(1941) work and that of Gleiser and Hunt (1955). The rest of the skulls was then organised by comparison with the baseline following the 6:6.5:7 ration, meaning that the same degree of wear would be reached by M1 in 6 years, by M2 in 6.5, and by M3 in 7 years. Miles was careful to highlight that the further away from the baseline the dentitions stood, the less reliable their estimation of age would be. Past an estimated age of 45 to 50 years, the teeth would also be so worn that no more estimation of age could be made. 157 skulls in total were aged using this system. No comparison with skeletal age was undertaken as the postcranial remains were not available for study. The diagram of molar wear as an age indicator presented in Miles' study has been reproduced several times since its original publication (including Brothwell 1989; and Mays 1998), and this method has been used successfully on different populations from around the world (see Brothwell 1989 for a review of these studies). Miles' method was also mentioned, albeit with a cautionary advice, in the Recommendations of the Workshop of European Anthropologists (Ferembach et al. 1980).

Tooth wear of British archaeological populations was studied further in the 1960s extending from the Neolithic to the Middle Ages (Brothwell 1963, 1965). Very little change was observed in the rate of attrition during this long period. For the series of Maiden Castle, dating from the Iron Age, dental wear was also compared to age estimations from the pubic symphysis. Brothwell diagram of dental wear, restricted to molars and divided into four age periods (17-25, 25$35,35-45$ and $45+$ years), is still regularly reproduced in textbooks and other manuals (including Bass 1995; and Mays 1998), sometimes replaced by his numerical classification of twelve stages graded from 1 to 7 with subdivisions. Results of the analysis of 328 early British individuals ranging from the Bronze Age to the Anglo-Saxon period were later presented as a sequence of nine phases, scored 1 to 9 (Brothwell 1989). These were comparable to the phases derived from Australian aborigines described above (Murphy 1959). Total dental wear score was obtained by adding the score of each of the three molars. 
Brothwell's method was tested on the molars of 298 individuals from Poundbury Camp in England, dating from the Romano-British period (Mays et al. 1995). Tooth wear was recorded by taking two crown-height measurements, one in the mesial and one in the distal quadrant of the mandibular buccal side and the maxillary lingual side, these areas all suffering the maximum effect of dental wear. Results confirmed the connection between tooth wear and age, and crown heights showed that Brothwell's stages occurred in the right order, proving the potential of this method for age estimation. However, some stages were found to have different durations, which could impair calibration from juveniles and bring a potential for underageing. It was recommended that the first two categories be amalgamated, creating three mains periods: young adults between 17 and 35, mature adult between 35 and 55, and over 55 years for individuals having lost the majority of their teeth.

A test of both this newly developed system and Brothwell's original method was carried out on a documented AD $19^{\text {th }}$ century skeletal series from the Netherlands (Mays 2002). The sample included 47 males and 55 females aged eight to 89 years. Results showed a correlation between crown height and age, and no evidence of differences between molar wear rates. Carious lesions were not found to affect crown height but the importance of ante mortem tooth loss was noted. While in most studies, those lost teeth were merely discounted as they were unavailable for observation, the direct link between age and extensive tooth loss would skew results towards younger individuals. It was suggested that for dentition with more than half of all teeth lost ante mortem, age should be recorded as being over fifty years. Brothwell's system would provide more accuracy for past populations than this modern sample however as tooth wear was greater before the advent of more refined food processes, and all stages of wear would therefore be present including extreme wear rarely seen in modern populations. This was confirmed by a recent study on 298 permanent maxillary and mandibular molars of 38 individuals from two Brazilian prehistoric sites, where Brothwell's four period-classification was 
found to be suitable for analysing the dental wear of this ancient population (Oliveira et al. 2006). Lower and upper age range limits of both this method and skeletal ageing method differed by less than eight years.

Another classification of degrees of wear was introduced in the 1970s with eight categories for all teeth (Molnar 1971). This study did not try to estimate age but demonstrated a positive correlation between dental wear and populations or cultures, linked to different diets and different use of the teeth, often as tools. Molnar's classification was expanded into ten grades of wear to be recorded separately for each quadrant of the tooth, resulting in a new scoring technique with totals ranging from 4 to 40 (Scott 1979; reproduced in Buikstra \& Ubelaker 1994). Another method was also developed to study the link between dental wear and age based on the casts of 46 female and 39 male modern Igloolik Eskimo populations (Tomenchuk \& Mayhall 1979). Specific equipment was required to calculate a tooth wear index from cusp height and demonstrated a correlation between dental wear and age, together with a strong sexual dimorphism in rate of wear springing from biological as well as occupational differences. Dental wear as an age estimator was also researched on the Libben archaeological population (Lovejoy 1985). The dentitions of 332 adults were seriated and gathered into groups of degree of wear per functional dental area. Seriation was then used within those groups, resulting in a total of nine stages for the maxillary and ten stages for the mandibular dentition. Rate of wear was estimated from juvenile dentition as described above. The resulting age estimations from dental wear were then compared to skeletal age. However, the latter had itself been estimated from other skeletal areas after seriation, such as the pelvis and cranial sutures.

Murphy's original eight stages were utilised again in the 1980s to compare dental wear between five groups of hunter-gatherers represented by 298 individuals, and five groups of early agriculturalists comprising 365 individuals (Smith 1984). These groups were spread across the world and over time, from 
prehistoric to modern times. Angle of wear was also recorded with a custommade instrument, and a clear pattern of flat wear for hunter-gatherers as opposed to oblique wear for agriculturalists emerged from this study. This was linked to food consistency and preparation, and highlighted as a helpful indicator of transition between hunting-gathering and agriculture. A recent study also compared 306 hunter-gatherers to 87 early agriculturalists (Deter 2009). These populations were concentrated in North America, and their ages had been estimated from Brooks and Suchey's (1990) pubic symphysis method and Lovejoy and colleagues' (1985) auricular surface method. Dental wear was recorded using digital imaging as the percentage of dentine exposure to the total occlusal surface, which was found to be greater in hunter-gatherers than in agriculturalists. Smith's eight stages for incisors, canines and premolars were reproduced in the "Standards for Data Collection from Human Skeletal Remains" (Buikstra \& Ubelaker 1994) and most recently utilised on 374 males aged fifteen to ninety years and 46 females aged 19 to 88 years from Kosovo, all casualties of the war in Former Yugoslavia (Prince et al. 2008). These stages successfully provided estimates of age-at-death for this specific modern population with the use of Bayesian statistics. Based on the success of this method, dental wear will be recorded based on Smith's system for the incisors, canine and premolars, while the equally successful Brothwell's method will be used for molar attrition.

\subsubsection{Multiregional analyses}

Multiregional analyses covering different regions of the skeleton, such as thorax, pelvis and dentition, are seen as the best approach to estimate age in order to maximise accuracy and to limit otherwise broad age-ranges. They also produce a "more balanced reflection of ageing" (İşcan 1989:327). Specific multiregional methods have been devised based on distinct approaches for different parts of the body as described above. 


\section{The "Complex Method"}

This method was designed from 105 Hungarian autopsied specimens of both sexes (Nemeskéri et al. 1960; Acsádi \& Nemeskéri 1970). Different age estimation methods were first employed separately, using cranial suture closure, pubic symphyseal metamorphosis and the changes of spongious bone in the proximal epiphysis of the humerus and the femur, the latter relying on radiographs of the limb bones and thus not described. Both sexes were analysed as a single group, not taking into consideration any sexual dimorphism or differences in stages of ageing. The differences between age estimates from these methods and true age were found to differ "considerably" when used separately (Acsádi \& Nemeskéri 1970:123). A complex method was then extrapolated using six possible phases for the humerus and the femur, and five phases for the cranial sutures and the pubic symphysis (described further above). If most indicators pointed towards a young age, the lower limits of the estimations from those methods were added together. If most indicators indicated an advanced age, the upper limits would be used instead. If no particular age indication could be seen, the means of all age estimations would be utilised. The resulting sum was divided by the number of methods used. Several possible scenarios for a different mix of methods depending on availability were also given. Using this "complex method", a rate of 80 to $85 \%$ of age estimations within five years of true age was achieved on the study sample.

This method was recommended at the Workshop of European Anthropologists (Ferembach et al. 1980), and has been described as the "best attempt at [developing] multiregional and multimethodological method" (İşcan 1989:333). It is still employed extensively across continental Europe. However, the lack of distinction between males and females and the lack of accuracy of the separate methods raise serious concerns about this methodology. This was confirmed when the Complex Method was tested on the Spitalfields sample of known age at death, where systematic underestimation over seventy years of age and 
overestimation under seventy were observed (Molleson \& Cox 1993). Less than $30 \%$ of age estimations yielded results within five years of true age.

\section{(- The "Multifactorial Method"}

This method was devised to provide a "summary age" (Lovejoy et al. 1985a) by weighting five age indicators: pubic symphysis (from Meindl et al. 1985), auricular surface (from Lovejoy et al. 1985b), dental wear (from Lovejoy et al. 1985a) and cranial suture (from Meindl \& Lovejoy 1985), all described above, together with radiographs of the proximal femur (R.A. Walker \& Lovejoy 1985). The population studied, assembled in this instance to approximate a chosen survivorship curve from the Todd collection of debated age, was first seriated by arranging all individuals in an ageing sequence as shown by their age indicators. All indicators present on the individuals were used and a weighted average by principal component analysis of all the separate age estimations provided the final age estimation. In the study sample, dental wear was employed to indicate age without any juvenile dentition to calibrate the results of adults, with a very low rate of wear overall, different origins, heavy tooth loss and presence of clinical dental care. Yet it was reported that dental wear had a high accuracy and no bias. Dental wear also provided the most accurate age estimation on the Libben archaeological population, although this population was itself of unknown age. This multifactorial method was tested on 54 males and one female, all with verified age at death, from the Grant Collection in Toronto (Bedford et al. 1993). Accuracy was improved by at least one year when combining several age indicators, with a mean error average of 8.7 years. However, the age of younger adults was overestimated consistently with all age indicators, and underestimated in older individuals.

The merits of "comprehensive" approaches considering multiple age estimation techniques to estimate the age of one individual, have also been demonstrated on a sample of nineteen autopsied individuals from France aged 19 to 54 years 
(Baccino et al. 1999). More recently, another comparative study based on 218 white and black individuals of both sexes from the Terry collection revealed that the best accuracy for all ages was obtained by principal component analysis, with a strong loss of accuracy by all methods above sixty years of age (Martrille et al. 2007). Although this last study confirmed the importance of utilising as many indicators as possible, it also recommended taking into consideration the general age of the individuals as either a young or old adult before finalising age estimation, employing the most appropriate age indicators for the resulting age category. Whilst recognising the benefits of multiregional approaches to limit inaccuracy, the BABA0/IFA guidelines also warn of the need to consider "the fundamental issues of innate inaccuracies in each of the individual approaches involved" (O'Connell 2004:19). Both the "Complex" and the "Multifactorial" methods are seriously hindered by the severe limitations of the individual methods they are combining as described separately in the section above, and these specific methods will therefore not be utilised in this doctoral research.

\subsection{Conclusion}

Age estimation of the remains of undocumented past populations is hindered by numerous factors, including sampling and representativeness issues, inter- and intra-population variations, and in the majority of cases the lack of any similar documented sample to use as a reference. Additionally, the variability of bone preservation, with skeletons often incomplete and damaged, must also be considered. Indeed, "age determination is ultimately an art, not a precise science" (Maples 1989:323), a quote often repeated in publications regarding ageing methodology.

With complete and mostly undamaged skeletons, juvenile and somewhat less precisely young adult ageing can be undertaken reasonably accurately from dental development and skeletal maturation, within the constraints of an unknown population. However, this chapter demonstrates that, even during this 
most valuable period, results from different methods vary markedly. State of preservation, which can fluctuate between individuals of the same site, might also influence which methods can be applied. No suitable documented population will ever be available in periods as remote as the Neolithic to help ascertain the most appropriate reference sample. Therefore, even juvenile ageing for a sample similar to that used in this doctoral research will never be very accurate. Even if precise ages are produced from analyses, it is crucial to always bear in mind that these will only ever be estimates and may not actually represent chronological age.

In this doctoral study, the dental age of all available juvenile dentition will be estimated using all methods available for such ancient and incomplete remains, including Ubelaker and Schour and Massler charts, Gustafson and Koch diagrams, and MFH/Smith stages of development for comparison. Gustafson and Koch's (1974) method in particular should prove the most appropriate for such remains, for all deciduous and permanent teeth excluding the third molar, and affording estimates from partial and damaged dentitions. Bone dimensions will be utilised to estimate body length and associated age of all foetal and neonatal remains based on Fazekas and Kósa's (1978) method (see Appendix 2). For children, diaphyseal measurements will be used to estimate skeletal age according to Stloukal and Hanáková's (1978) method which was demonstrated the most appropriate for this type of analyses, based on the origin of their population, their methodological approach and sample number. In order not to restrict the amount of information available in this rare population, these results will also be compared with those obtained from all other methods available for archaeological remains (see table 3.4 and Appendix 3). For adolescents and young adults, skeletal maturation will be utilised and particularly the stage of epiphyseal union based on the most complete and upto-date work of Schaefer, Black and Scheuer (2009, which has been summarised in figure 3.1). A comprehensive table of all data available has also been provided in Appendix 4 for ease of comparison with other methods and populations. 
From the age of thirty, concerns in estimating age increase drastically. Methods need to be carefully chosen from those positively tested on large samples with documented age-at-death. Furthermore, broad age-ranges should also be presented instead of merely giving the mean estimate. The same issue of sampling and preservation as those concerning juvenile individuals also apply. Therefore the only solution for unknown archaeological population is to utilise as many age indicators as can possibly be observed. Unfortunately, despite numerous methodological advances in the last thirty years, Ubelaker's statement that "degenerative changes in the skeleton serve only as very general indicators of age at death" (1984:60) still stands. The factor of age in the variability of those skeletal changes has been approximated at "no more than 30\%" (Jackes 2000:451), and age ranges estimated from those changes have been rightly described as "within unacceptably large age brackets with a considerable and unacceptable degree of overlap" (Cox 2000:75). Even the definitions of age categories vary between authors, as can be seen in Appendix 5. Therefore, age classes should always be specified in publications and no assumption made on the age ranges covered when those are not detailed.

In this study, skeletal and dental development will be utilised as described above in the age estimation of adult remains, particularly of young adults. Neither cranial sutures (see section 3.3.1.) nor ribs (see section 3.3.2) will be used, as these have been demonstrated not to be suitable age indicators for such analyses. No precise age estimates will be given based on degenerative changes of the skeleton for the reasons highlighted throughout section 3.3 and reiterated above. However, the phases of the pubic symphysis (Todd 1920; Brooks \& Suchey 1990) and the auricular surface (Lovejoy et al. 1985b), acetabular stages (Rissech et al. 2006, 2007), and dental wear (Brothwell 1963, 1968; Smith 1984; Brothwell 1989) will all be recorded in order to compare these results and provide a general age estimation. The broad age classes indicated overleaf will therefore be used, while the author will also endeavour to provide more precise age estimates for individuals less than thirty years of age whenever possible. 


\begin{tabular}{|c|c|}
\hline Age Category & \multicolumn{1}{|c|}{ Age Span } \\
\hline Juvenile & Under $\mathbf{1 8}$ years \\
\hline Foetus & $\begin{array}{r}\text { 3 foetal months to birth } \\
\text { Birth to one month } \\
\hline \text { Neonate }\end{array}$ \\
\hline Infant & Birth to one year \\
\hline Child & 1 to 12 years \\
\hline Young child & 1 to 5 years \\
\hline Middle child & 6 to 12 years \\
\hline Adolescent & 13 to 17 years \\
\hline Adult & $18+$ years \\
\hline Young adult & 18 to 34 years \\
\hline Mature adult & 35 to 49 years \\
\hline Old adult & $50+$ years \\
\hline \hline
\end{tabular}

Table 3.6: Age categories used in this doctoral research

(see Appendix 5 for a full comparison of age categories in different works)

This chapter might portray a very bleak picture of the potential for success of age estimation on archaeological remains. This is unavoidable in the current state of research, and implies severe limitations for any analyses of the demography and health status of past populations. Nevertheless, even the broad definition of infant, child, adolescent, young adult and mature or old adult can be sufficient to at least create a window on the life and death of those former communities. In actual fact, the unknown culture of these mostly illiterate and innumerate prehistoric societies might not have valued precise chronological age to the extent that we do today. Whilst we, in our present society, have legal age restrictions for schooling, marriage, driving and so forth, different stepping stones based on physical as well as emotional capabilities would have been more critical to the role, status or possibilities of an individual in those past communities. 


\section{Chapter 4: Stature Methods}

\subsection{Introduction}

Since the first stature estimations in the late $19^{\text {th }}$ century from long bone lengths (Rollet 1888) and the first stature regression equations (Pearson 1899), several stature estimation formulae have been published. They are based on skeletal remains, cadavers or living subjects of different modern populations. The methods also differ in using a single side, both left and right, or an average of both. Results are obtained either by averaging the estimates from all available bones or restricting the result to the estimation with the least standard error.

The problem of stature estimation of juveniles will be addressed in the first instance, a research topic rarely investigated as stature studies tend to focus on adults. The first studies of adult stature estimations will then be considered, followed by a review of the two most widely-used techniques: Trotter and Gleser's $(1952,1958)$ method, which requires the sex and race of the individual to be known, and Sjøvold's (1990) method, which can be applied without this information and thus more appropriate for archaeological samples, especially for very ancient material and fragmentary remains. Finally, the potential of other methods using skeletal height, fragmentary bones, the lengths of metacarpals and metatarsals, and femur/stature ratio will also be examined.

\subsection{Estimating Juvenile Stature}

\subsubsection{Fetal stature}

Fetal stature is the most often estimated in the juvenile range due to its close correlation with age, and therefore its use in the estimation of fetal age. The first formulae to estimate fetal skeletal stature from the length of the humerus, 
femur, and tibia were published in 1921 (Balthazard \& Dervieux). The age of the fetus in days was then obtained from a further formula. Eighteen years later, a similar method to estimate fetal stature was published also utilising the diaphyseal length of the humerus, radius and tibia, and adding the length of the clavicle (S. Smith 1939). Later, Olivier and Pineau (1958, 1960; Olivier 1969) reworked Balthazard and Dervieux earlier formulae to give new regression equations based on the humerus, radius, ulna, femur, tibia and fibula. The diaphyses of the humerus and the femur were also utilised by Mehta and Singh (1972) in a study of thirty male and twenty female fetuses, but this time to establish formulae that estimated crown-rump length rather than full body length. Finally, the most utilised and probably most accurate formulae available at present come from the work of Fazekas and Kósa (Fazekas \& Kósa 1978; Kósa 1989), which is described in details in section 3.2.2 above.

Formulae for long bones from all the studies mentioned above were partially reprinted in Krogman and İşcan (1986). For a comprehensive list of Fazekas and Kósa's (1978) formulae for all bones, see Appendix 2. It is worth noting here again two bones extremely useful for osteoarchaeologists: the (half) mandible and the radius, which can both be used to estimate fetal stature without any calculation, simply by converting their length from $\mathrm{mm}$ to $\mathrm{cm}$.

(half) mandible length $(\mathrm{mm})=$ body length $(\mathrm{cm})$ radius length $(\mathrm{mm})=$ body length $(\mathrm{cm})$

\subsubsection{Postnatal juvenile stature}

\section{- Stature estimation from long bone lengths}

One of the first studies on the estimation of stature of juveniles was undertaken in the 1960s, based on radiographs of children from Helsinki (Palkama et al. 1962; Telkkä et al. 1962; Virtama et al. 1962). Children were divided into three groups, each requiring different regression equations: the under-one year old represented by 68 males and 51 females for all six limbs; the one to nine years 
old based on 201 male femora, 253 males for the other five limb bones, 162 female femora, and 224 females for the other five limb bones; and finally the ten to fifteen years old with twenty male femora, forty males for the other five limb bones, 26 female femora, and 39 females for the other five limb bones. The average of the maximum lengths of both sides was used in the calculations, and results were given separately for males and females. The results for the under1-year age group showed that the regression in these cases was not linear, and a logarithmic formula was used instead. This was also the case for the equations using the femur in the group of 1-9 years. The largest standard errors were found in the oldest group (10-15 years).

Several issues have been highlighted by other authors since this study was published. First, the "maximum length" of the tibia was taken at an oblique angle on the radiographs, and would therefore not correspond to most researchers' tibial diaphysis maximum length. Secondly, no correction or adjustment was made for radiographic distortion, and the measurements used to obtain the formulae were obtained directly from the radiographs. This could have led to a magnification of $3 \%$ to over $5 \%$, which would skew the results towards a lower stature when using direct skeletal measurements. Therefore this method should only be used very cautiously on ancient skeletal remains, particularly if only the tibia is available due to the different measurement taken.

New equations were published most recently for the estimation of stature of children aged three to ten years old (S.L. Smith 2007). Data from 31 boys and 36 girls from the Denver's growth study were utilised to produce new sex-specific as well as unknown-sex calculations, the latter particularly useful for archaeological cases, where the sex of juveniles is usually unknown. Diaphyseal lengths were measured from radiographs, without correction for magnification, and used in "inverse calibration" regressions (stature on long bone length) for each of the six long bones and a femur-tibia combination. Standard errors obtained were quite low, although it was indicated that for a full $95 \%$ 
confidence $( \pm 2 \mathrm{SE}$ ), the interval should be doubled and could then cover between 8 and $12 \mathrm{~cm}$. Further analyses showed that repeated long bone measurements obtained from longitudinal studies, such as the Denver growth study, did not affect the precision of the calculations. Overall, boys tended to have longer forearms compared to girls, which would underestimate boys' stature slightly when using equations for ulna and radius for individuals of unknown sex. In general, it would seem more appropriate to use sex-specific equations when possible as the standard errors were lower than those for unknown sex, although this will not result in a marked difference in the estimates.

These results were compared to Maresh's study, also derived from the Denver data (see Chapter 3, section 3.2.2), and the work of Telkkä, Palkama and Virtama, based on Finnish children as previously detailed. All the methods utilised measurements that were not adjusted for magnification. Smith's method obtained very similar results to Maresh's study, first selecting the age from one table using long bone lengths and then using the relative bone length by age to estimate the stature from a second table. However, there was a difference with the Finnish group, whose standard errors of stature estimation equations also covered a much broader range of estimates. This was attributed this to the difference in measurements of maximum lengths, and also possibly to an increased magnification effect due to the shorter focal length of the radiographs. Although it was recommended to adjust for magnification, it was also noted that the corresponding change in estimate was minimal, and thus an adjustment was not absolutely necessary. The estimated height obtained without correction would therefore tend to be slightly higher than would have been estimated with adjusted measurements. The importance of the role of juvenile stature estimation to highlight any growth stunting by comparison with dental or skeletal age was also emphasized. However, it should be noted that this comparison can also be undertaken based on the long bone lengths themselves without the added error potential of the stature estimation. 


\begin{tabular}{|llcc|} 
Coefficient & Measurement & $\alpha$ & SE \\
0.4658 & Humerus & +27.053 & $\pm 3.00 \mathrm{~cm}$ \\
0.6229 & Radius & +27.500 & $\pm 3.16 \mathrm{~cm}$ \\
0.5898 & Ulna & +23.742 & $\pm 2.91 \mathrm{~cm}$ \\
0.2928 & Femur & +36.923 & $\pm 2.46 \mathrm{~cm}$ \\
0.3519 & Tibia & +38.614 & $\pm 2.24 \mathrm{~cm}$ \\
0.3620 & Fibula & +37.273 & $\pm 2.24 \mathrm{~cm}$ \\
0.1612 & Femur + Tibia & +36.981 & $\pm 1.97 \mathrm{~cm}$ \\
\hline
\end{tabular}

Table 4.1: Regression equations for stature estimation for 3-10 year-old children of unknown sex, adapted from Smith (2007:539, Table 1).

All bone measurements are maximum diaphyseal lengths in $\mathrm{mm}$.

Although Smith's equations seem more appropriate for use on archaeological remains than Telkkä, Palkama and Virtama's equations, they are also unfortunately restricted to children aged three to ten years, so could not be utilised on children younger or older than this age group. Age must also have been correctly estimated, which could limit its potential on ancient skeletal remains as described in more details in the previous chapter.

\section{Stature estimation from tables and from metacarpal lengths}

A table was published in the late sixties affording direct approximation of stature from the femoral diaphyseal length without the need for any calculation (Olivier 1969). Unfortunately, no references were given as to the origins of the data used. This table was reprinted in Krogman \& İşcan (1986) with a warning that wide discrepancies could be seen between stature estimation from this table and actual living stature. A specific case was detailed where a difference of up to $15 \mathrm{~cm}$ was observed between the known stature of a missing girl and that estimated from this table (Krogman \& İșcan 1986:339). The lack of information as to the origins of Olivier's data, and the demonstrated wide discrepancies led to this method being completely discarded.

A more promising study was published in the late seventies based on 1597 radiographs of 372 boys and 338 girls from Guatemala, aged one to seven years, to calculate regression equations based on the maximum diaphyseal length of 
the second metacarpal (Himes et al. 1977). The resulting standard errors of around $4 \mathrm{~cm}$ were very similar to formulae using long bone diaphyseal measurements.

Boy stature $(\mathrm{cm})=8.80+2.90(\mathrm{MC2}$ maximum length in $\mathrm{mm}) \pm 3.99 \mathrm{~cm}$ Girl stature $(\mathrm{cm})=7.90+2.89(\mathrm{MC2}$ maximum length in $\mathrm{mm}) \pm 3.90 \mathrm{~cm}$

Unfortunately, these formulae for young juveniles are sex-specific and more importantly, identification of the second metacarpal can be very difficult until ossification is complete in later years. In addition to the limited age range and requirement for accurate age estimates, this method therefore also had to be discounted for the analysis of the Neolithic remains under study.

\section{Juvenile stature and body size}

In a study on the estimation of child body size, Visser (1998) utilised the data from the 113 children aged between six months and twelve years in Maresh's (1943, 1961) studies and the 280 males and 225 females aged between 3 and 18 years in the work by Tanner and colleagues (1981) to compare it with the data from the long bones of 37 children from a destitute children's asylum in Sydney during the second half of the $19^{\text {th }}$ century AD. Shrinkage of dry bones and magnification from radiography were taken into account to develop regression equations to estimate stature from the lengths of the humerus, tibia and femur. No details were given about the type of length used: although diaphyseal maximum length would generally be assumed, this might not necessarily be the case. Formulae to estimate body weight from the midshaft anteroposterior diameter of the humerus, tibia and femur were then given, followed by equations to estimate weight taking stature into account. However, the regression equations used to estimate stature presented vast standard errors, with a difference of over $\pm 12 \mathrm{~cm}$ for the humerus and femur, and nearly $\pm 10 \mathrm{~cm}$ for the tibia. Despite this, a comparison of stature estimates obtained with this method and estimates using other equations mentioned above would still be of 
interest and will be undertaken in this research, as Visser's formulae have the advantage of being appropriate for dry bones and for juveniles of all ages.

New age-specific formulae have also been offered in a recent study based on twenty individuals from the Denver Growth Study sample, measured between the ages of one and seventeen years (Ruff 2007). In addition to the small sample size, this method requires the age of the individual and is thus unlikely to be of use for archaeological remains, although it was tested by Ruff on the skeleton of the Homo ergaster Nariokotome boy, known as KNM-WT 15000 from his inventory record number. Equations given in this study were further tested on 186 juveniles from Ohio (Sciulli \& Blatt 2008). Although relatively accurate estimations were reported, a number of results by age class produced estimates with errors as large as $\pm 15 \mathrm{~cm}$, further limiting the use of this method.

\section{Femur/Stature ratio}

The juvenile femur/stature ratio was investigated by Fedelsman (1992), using the "true anatomical length" with both epiphyses, corrected for radiographic magnification. This study was based on four child growth studies, all employing different radiographic techniques, with samples detailed in the table below including children suffering from poliomyelitis and 51 individuals used twice.

\begin{tabular}{|c|c|c|c|c|c|}
\hline \multirow{2}{*}{$\begin{array}{l}\text { Growth } \\
\text { study }\end{array}$} & \multicolumn{2}{|c|}{ Males } & \multicolumn{2}{|c|}{ Females } & \multirow[t]{2}{*}{ Notes } \\
\hline & $\mathbf{n}$ & Age & $\mathbf{n}$ & Age & \\
\hline $\begin{array}{l}\text { Maresh } \\
(1970)\end{array}$ & 75 & $\begin{array}{l}2 \text { months - } \\
18 \text { years }\end{array}$ & 65 & $\begin{array}{l}2 \text { months - } \\
18 \text { years }\end{array}$ & $\begin{array}{c}\text { Total length only available from } 10 \\
\text { years of age }\end{array}$ \\
\hline \multirow[t]{2}{*}{$\begin{array}{l}\text { Anderson } \\
\text { et al. (1963) }\end{array}$} & 50 & $10-18$ years & 50 & $8-16$ years & $\begin{array}{l}\text { Included } 51 \text { "normal" children }+41 \\
\text { with paralytic poliomyelitis }\end{array}$ \\
\hline & 30 & 8-10 years & 25 & $16-18$ years & $\begin{array}{l}\text { Lengths from both sets modified to } \\
\text { fit length used in Feldesman's study }\end{array}$ \\
\hline $\begin{array}{l}\text { Anderson } \\
\text { et al. (1964) }\end{array}$ & 67 & $1-18$ years & 67 & $1-18$ years & $\begin{array}{l}\text { Included the } 51 \text { "normal" children of } \\
\text { the previous study by same authors }\end{array}$ \\
\hline $\begin{array}{l}\text { Tupman } \\
(1962)\end{array}$ & \multicolumn{2}{|c|}{$\begin{array}{c}202 \text { male and female } \\
\text { children }\end{array}$} & \multicolumn{2}{|c|}{$7-16$ years } & \\
\hline
\end{tabular}

Table 4.2: Summary table of samples utilised in Feldesman's (1972) study 
Gender-specific femur/stature ratios applicable to adolescents between twelve and eighteen were successfully established at 27.44 for males and 27.16 for females. However, as this method requires the sex of the individual to be known, as well as the complete maximum length including the proximal and the distal epiphyses, its use for archaeological material is severely restricted. For this reason as well as the issues regarding the study samples, this method will therefore not be employed in the analysis of the ancient and damaged remains of the Neolithic populations under study.

\subsubsection{Growth studies}

Growth studies have been a very important area of research in medicine and physical anthropology. Several long term longitudinal growth studies were carried out in the $20^{\text {th }}$ century by taking radiographs of individuals throughout their childhood and adolescence at regular intervals, as described in the previous chapter. These are unlikely to be repeated in the future as awareness of the possible damaging effects of radiography has since increased considerably. More recent cross-sectional rather than longitudinal studies have been based on hospital radiographs.

Growth is also a very interesting field of study for osteoarchaeology, as it has been linked repeatedly to health and environmental factors. This has been seen as an ideal way of studying the transition from certain environments or ways of life to others, demonstrating for example the transition to agriculture, the settlement to different areas during migrations, or the beginning of industrialisation. This can be done by comparing dental age to skeletal age, or dental age to diaphyseal bone lengths or stature, although bone length is usually preferred to avoid adding more error potential from stature estimates. More recently, percentage of attained growth has also been used for growth studies as a way to avoid population-specific differences. As only a very limited number of historic collections with known-age are available, most studies utilise estimated 
ages, especially those based on prehistoric populations.

\section{- Comparative studies}

Numerous studies have been carried out comparing archaeological sites with different subsistence and settlement systems, such as sedentary versus mobile hunter-gatherers (Mensforth 1985), agricultural versus pastoral post-neolithic populations (Piontek et al. 2001), the same sites at different periods (Jantz \& Owsley 1984), or sites from the same period located in different environments or geographical areas. The studies were based on populations from across the world, including Africa, Eastern and Western Asia, Europe and America. In Europe, they contained the aforementioned populations of Altenerding in Germany; Ancient Slavic countries; Wharram Percy, Spitalfields, St Brides and St Martin in England; as well as the Portuguese collections. In North America, study samples comprised of the European-decent population of Belleville, Ontario, in addition to the Native American populations of Indian Knoll in Kentucky, Libben in Ohio and Arikara in South Dakota. Although these studies are too numerous to detail here, a few publications are worth mentioning as they give a new insight into this specific topic of research in osteoarchaeology.

A study of the environmental effects on skeletal and dental development based on 58 females and 68 males under 21 years old from Lisbon, Portugal, all with detailed biographic information, confirmed that there was less environmental impact on dental rather than skeletal development (Cardoso 2007). It established that those differences were more pronounced when considering the location of residence rather than social status, although it also demonstrated that environmental factors could influence dental development as well. The growth status of this population had been obtained from the differences between skeletal and chronological age, and between dental and chronological age. Skeletal age was based on femoral length compared to Maresh's (1970) standards, as earlier research had shown that the timing of epiphyseal union 
was not as sensitive to the environment as long bone length and stature. Dental age was established using the methods of Moorrees and colleagues, Demirjian, and Smith (see Chapter 3, section 3.2.1 for explanations and references).

However, Mays, Brickley and Ives' (2008) study on growth in England during the Industrial Revolution raised great concerns about the use of growth studies as an indicator of health in past populations. This study was based on the comparison between diaphyseal long bone lengths and dental age in two English populations during the beginning of industrialisation. One of these populations was rural (church and churchyard of Wharram Percy) whilst the other one was urban (St Martin's churchyard in Birmingham), and both were buried roughly during the same period. Even though the detrimental effects on health of the transition from a rural agrarian to an urban industrialised environment are well established, results of these analyses did not show any statistically significant differences between both sites in long bone length relative to age for any of the limb bones. This would agree with an earlier study of the archaeological Indian Knoll, Libben, Eskimo, Aleut, and Altenerding populations compared to Iron Age and modern samples from South Africa (Steyn \& Henneberg 1996). No significant differences could be found in juvenile growth between those geographically and temporally widespread populations either. It was therefore decided to discard this method for the analysis of the Neolithic populations in this doctoral research.

\section{( Percentage of attained growth}

Growth is influenced by genetics, health, climate, environment and culture, the latter including effect from breastfeeding, supplementary feeding patterns, food allocation, infant mobility, population density and waste management, and can therefore vary widely between distinct populations (Humphrey 2003; and as demonstrated in the comparative studies directly above). 
In order to study linear growth without the restrictions of population variability, the rate of attained growth can be used instead to provide an insight into periods of growth faltering and catch-up growth, and this growth pattern can then be studied in relation to health status and to other populations. This was first demonstrated on the juvenile Libben population of twelve years and under (Lovejoy et al. 1990). The rate of attained growth was established by dividing the length of juvenile diaphyses by the mean adult maximum length of the same bones, and presenting the results as a percentage. Several archaeological studies have since utilised the same method to study the growth of past populations.

This method would appear more appropriate for archaeological material as it is based on the population studied rather than modern examples. However, caution must still be applied, as archaeological remains represent by nature a cross-sectional view of a population. Therefore, a growth curve cannot strictly speaking be created from such material, as the adult stature used in comparison is not from the same individual whose juvenile stature is observed, but from the mean of adult statures or measurements in the population studied. Additionally, the difficulty of establishing the sex of juvenile skeletal remains entails that the midpoint between female and male means must be used, rendering those studies much less precise. Some populations also present an adolescent growth spurt, which tends to occur two years earlier in females than in males (Humphrey 1998; Bogin 1999) whereas others show a prolonged growth until as late as 29 years (Mays 1999). This, together with increased sexual dimorphism with age, has led several authors to limit their observations to children of twelve years old or under only. Age of the individuals will also have been estimated in most collections, adding another source of potential error from the age estimates as well as differences from the methods used to obtain those results (see chapter 3 ). 
A major issue is also raised by the methodology itself. Although the precise measurements taken are not always described in growth studies, presentation of growth rate or percentage of attained growth are often based on different measurements and might therefore not be comparable. Whilst many authors follow Lovejoy and colleagues' example using the maximum length of the juvenile diaphyses divided by the overall mean adult maximum length (Okazaki 2004; Temple 2007, 2008), a wide range of different approaches has been employed. For example, a growth study on historical collections from England utilised measurements of both juvenile and adult diaphyses (Humphrey 1998). Adult diaphyseal lengths were estimated from the difference in the long bones of young adults between maximum length and the diaphyseal length still visible radiographically. This correction was then applied to the rest of the adults, with half of the correction used when only one epiphysis was fused. However, in a later comparative studies of human remains from collections across the world by the same author, a different methodology was utilised (Humphrey 2003). In order to compare percentages of attained growth, results from twelve former studies were recalculated by dividing femoral diaphyseal femoral length in each juvenile age group by the estimated adult maximum femoral length including both fused epiphyses. Estimated adult mean sizes were also taken at different ages depending on the collections, as young as sixteen years for females from Maresh's (1970) data and over twenty years of age for nearly all the other populations. Most recently, another study reverted to using diaphyseal measurements only, but adult diaphyseal lengths in this case were obtained by subtracting the length of the fused epiphyses instead (MacCord 2009).

Stature rather than measurements has also been used as the basis for percentages of attained growth. In a medieval case study from England, Feldesman's (1992) femur/stature ratio described above was utilised to estimate juvenile stature (Mays 1999). As this method requires full length of the 
long bone including epiphyses, juvenile diaphyses recovered with both unattached epiphyses were observed and results were consistent with Maresh's (1955) indications that diaphyseal length represented 91\% of total femoral length. Total lengths of juvenile diaphyses were then estimated using this result, and stature estimates were obtained from the newly calculated total femur length to stature ratio. Adult stature was estimated from Trotter and Gleser's equations (see section 4.3.3). The juvenile stature estimates were then compared to the average of male and female mean adult stature estimates to provide a growth profile. Mays' study was successful in showing prolonged growth in this medieval population by comparing percentage of attained growth at ten years of age in the Wharram Percy population, established at $71 \%$ of adult height, to modern children achieving $81 \%$ by the age of ten (Tanner et al. 1966). However, this conclusion was based on so many different estimates from a population whose age was itself estimated by dental methods, that it should only be interpreted very carefully.

The method of using stature-based percentage of attained growth has good merits for archaeological remains, as using stature rather than single bones, incomplete skeletons which might not all provide a specific bone, such as the femur, can therefore still be utilised for stature estimation. Population variability is also taken into account as the results are population-specific. Unfortunately in archaeological populations, age can only be estimated. Moreover, without accurate sex estimates (see Chapter 2 for the difficulties associated with sexing juvenile remains), percentages of attained growth can be given separately for both sexes but without certainty of which results apply, as well as potentially representing a mixture of both sexes. A safer alternative is to use the average of both male and female stature means as the basis of the percentage, particularly for younger juveniles before puberty and more marked sexual dimorphism. Although these results might not be directly comparable with other reported populations, they might still be able to provide some information on growth in past populations. 


\subsection{Estimating Adult Stature}

\subsubsection{First studies}

As early as the late $19^{\text {th }}$ century, fifty male and fifty female cadavers from France were utilised to estimate stature from long bone length, averaging results from both sides (Rollet 1888). This data was then re-used excluding cadavers over sixty years of age (Manouvrier 1892, 1893). Regression formulae were produced a few years later based on humid bones with cartilage and cadaver stature of a population sample averaging sixty years, using the right side only where available (Pearson 1899). These formulae were then tested on seven French criminals, resulting in estimates on average $2.73 \mathrm{~cm}$ below actual stature. Three decades later, Rollet's method was employed to produce formulae based on 48 cadavers of Chinese males of unknown age (Stevenson 1929). These equations were then compared to Pearson's, revealing that racespecific formulae did not produce satisfactory results on different populations. Finally, Pearson's statistical methods were again utilised, but this time based on 2,400 German male living subjects, including 1,400 from an athletic competition and 1,000 students (Breitinger 1937). The measurements were therefore taken using bony prominences of the limbs instead of the more accurate direct measurements of the bones themselves.

\subsubsection{Post Second World War}

All this previous literature was reviewed after the Second World War (Telkkä 1950). The reviewer added his own results from the study of 115 male and 39 female cadavers from Finland. During the same period, it was suggested based on stature estimates of Europeans from the $8^{\text {th }}$ to $10^{\text {th }}$ centuries $\mathrm{AD}$ that if at possible, measurements of the overall length of the skeletal remains in situ would be preferable to equations based on more recent populations (Kurth 1950). Comparison of cadaver stature and bone lengths of 400 individuals from 
the Todd Osteological Collection, including 100 white males, 100 white females, 100 black males and 100 black females, resulted in general formulae from the weighted averages of Pearson's data and this new sample (Dupertuis \& Hadden Jr. 1951). The same year, a study was published on the effects of ageing on stature using the Terry Anatomical Collection, followed a year later by a comparison between stature and bone lengths, as well as new equations (Trotter \& Gleser 1951, 1952). Shortly thereafter, Dupertuis and Hadden's (1951) ten general formulae were reproduced with the recommendation to use "as many formulae as possible and then adding and dividing the results to obtain a mean value", as this would provide the "most satisfactory estimate of stature" (Boyd \& Trevor 1953; quoted in Trotter \& Gleser 1958:115). However, this has been criticized by several authors since, including Keen $(1953,1955)$, as well as Trotter and Gleser (1958).

\subsubsection{Trotter and Gleser's method}

\section{(- Trotter and Gleser's equations (1952)}

Trotter and Gleser's research was based on military personnel casualties of the Second World War, including 1,115 white males and 85 black males mainly in their late teens and early twenties, and the Terry Skeletal Collection comprising of 255 white males, 360 black males, 63 white females and 177 black females. The stature of the military personnel was recorded at the time of their induction (living stature) and the bones of the dead measured once the bodies were returned to the USA after the war. Only individuals 18 or over were included, and a correction formula, subtracting [0.06 X (age - 30)] $\mathrm{cm}$ to the stature, was applied to the older individuals of the Terry Collection. All measurements were first taken in inches and then converted to the nearest centimetre. The maximum length of the six long bones was utilised. When both sides were available, the average between the two was calculated. If only one bone was available, that single measurement was used instead. To correct the difference 
between the recorded living stature of the military personnel compared with the cadaver stature of the collection, the average difference was taken from the equations for estimation of stature of white males from both sources. Final equations were then presented to estimate the living stature of white and black individuals of both sexes and all ages from the length of their long bones (see table 4.3 below).

\begin{tabular}{|cccc|ccccc|}
\multicolumn{4}{c|}{ White males $(1952)$} & \multicolumn{4}{c|}{ White females (1952) } \\
Coef. & Max.length & $\boldsymbol{\alpha}$ & SE & Coef. & Max.length & $\boldsymbol{\alpha}$ & SE \\
\hline 3.08 & Humerus & +70.45 & $\pm 4.05 \mathrm{~cm}$ & 3.36 & Humerus & +57.97 & $\pm 4.45 \mathrm{~cm}$ \\
3.78 & Radius & +79.01 & $\pm 4.32 \mathrm{~cm}$ & 4.74 & Radius & +54.93 & $\pm 4.24 \mathrm{~cm}$ \\
3.70 & Ulna & +74.05 & $\pm 4.32 \mathrm{~cm}$ & 4.27 & Ulna & +57.76 & $\pm 4.30 \mathrm{~cm}$ \\
2.38 & Femur & +61.41 & $\pm 3.27 \mathrm{~cm}$ & 2.47 & Femur & +54.10 & $\pm 3.72 \mathrm{~cm}$ \\
2.52 & Tibia & +78.62 & $\pm 3.37 \mathrm{~cm}$ & 2.90 & Tibia & +61.53 & $\pm 3.66 \mathrm{~cm}$ \\
2.68 & Fibula & +71.78 & $\pm 3.29 \mathrm{~cm}$ & 2.93 & Fibula & +59.61 & $\pm 3.57 \mathrm{~cm}$ \\
\hline
\end{tabular}

Table 4.3: Regression equations from single bones, adapted from Trotter \& Gleser (1952:495, Table 13)

This study showed a smaller standard error of estimates from the bones of the lower limbs compared to the upper limbs, with the best estimates of stature obtained from the femur, tibia and fibula. The use of an average between bones was discouraged due to its higher inaccuracy than estimations from a single lower limb bone. These equations were tested on a further one hundred cases with data of paired arm, thigh and leg bones from the 368 white males from military personnel with random long bones missing, concluding that "these equations may be applied without reservation to the entire population of American White males" (Trotter \& Gleser 1952:501). Results on those one hundred additional white males from the military were also compared to equations of previous research, with “Manouvrier's, Pearson's and Telkkä's equations [resulting] in stature estimates which [were] much too low for American military males while those of Dupertuis and Hadden [were] too high" (Trotter \& Gleser 1952:505, see table 4.4 below). 


\begin{tabular}{|lcccc|}
\hline \multicolumn{5}{|c|}{ Estimation of stature from length of the femur (white males) } \\
\hline \multicolumn{1}{|c|}{ Authors } & Coef. & Max.length & $\boldsymbol{\alpha}$ & SE \\
\hline Breitinger (1937) & 1.64 & Femur & +94.31 & $\pm 4.8 \mathrm{~cm}$ \\
Dupertuis and Hadden (1951) & 2.12 & Femur & +77.05 & $\pm 3.4 \mathrm{~cm}$ \\
Pearson (1899) & 1.88 & Femur & +81.31 & $\pm 3.2 \mathrm{~cm}$ \\
Telkkä (1950) & 2.10 & Femur & +71.85 & $\pm 4.9 \mathrm{~cm}$ \\
Trotter and Gleser (1952) & 2.38 & Femur & +61.41 & $\pm 3.3 \mathrm{~cm}$ \\
\hline \multicolumn{5}{|c|}{ Estimation of stature from length of the humerus (white males) } \\
\hline \multicolumn{1}{|c}{ Authors } & Coef. & Max.length & $\boldsymbol{\alpha}$ & SE \\
\hline Breitinger (1937) & 2.72 & Humerus & +83.21 & $\pm 4.9 \mathrm{~cm}$ \\
Dupertuis and Hadden (1951) & 2.27 & Humerus & +98.34 & $\pm 4.6 \mathrm{~cm}$ \\
Pearson (1899) & 2.89 & Humerus & +70.64 & $\pm 3.2 \mathrm{~cm}$ \\
Telkkä (1950) & 2.80 & Humerus & +75.28 & $\pm 5.0 \mathrm{~cm}$ \\
Trotter and Gleser (1952) & 3.08 & Humerus & +70.45 & $\pm 4.0 \mathrm{~cm}$ \\
\hline
\end{tabular}

Table 4.4: Comparison of stature estimations from different authors, adapted from Trotter \& Gleser (1952:505, Table 15)

However, a difference was noted between the white males from the military and those from the Terry Anatomical collection, with black males shorter than white military males, but taller than white males from the collection. This was attributed to "recent secular trends in stature [which] may partially account for these apparently contradictory findings" (Trotter \& Gleser 1952:508). Equations and all relevant calculations for black females were also corrected in 1977 after an inconsistency with radial measurements was brought to their attention (Trotter \& Gleser 1977).

\section{( ) Trotter and Gleser's equations (1958)}

A re-evaluation of Trotter and Gleser's stature estimation was published based on the study of the remains of American military personnel killed during the Korean War (Trotter \& Gleser 1958). All were males, with 4,672 white, 577 black, 92 mongoloid, 112 Mexican and 64 Puerto Rican individuals. This time, long bone measurements were obtained from moist as well as dry bones and instead of averaging between left and right sides as was done in the first study, they considered both sides separately. It was acknowledged that due to the 
large number of people involved in taking the measurements of stature and long bone lengths, the conversion between inches and centimetres and the way all the data were recorded, there would inevitably be compounded errors. Measurements of white and black individuals under 18 years were disregarded as in the first study.

Mean stature of the total white series was found to differ only very slightly from the first study at $173.95 \mathrm{~cm}$ compared to $174.23 \mathrm{~cm}$, whereas the mean stature of American black individuals exceeded the previous result with $173.43 \mathrm{~cm}$ compared to $172.14 \mathrm{~cm}$. The stature of the other three groups, which were not available in the first study, was shorter with a mean of $168.73 \mathrm{~cm}$ for the Mongoloids, $168.65 \mathrm{~cm}$ for the Mexicans and $166.64 \mathrm{~cm}$ for the Puerto Ricans. In order to compare these results to the first study, the new equations from right and left bone lengths were combined into a single equation. Results for both white male series had very similar results, apart from the length of the tibia, which was attributed either to a change in stature-tibia ratio or more likely to a variation in the way the tibia was measured. In both studies, multiple regressions hardly increased the precision of stature estimates compared to those from single long bones of the lower limb, leading to the conclusion that "multiple regression equations [were] not necessarily the most desirable for stature prediction" (Trotter \& Gleser 1958:99). However, there was also a significant increase in stature from 17 to 23 years of age in this series. This was not the case in the first study and it was attributed to a prolonged growth in the vertebral column rather than a delayed epiphyseal union, as the lengths of the long bones were not noticeably different. Equations were then recalculated using only individuals 21 years old or over, and a formula created to adapt stature estimates for younger people.

It was concluded that "the corresponding equations of the two studies [were] quite consistent and also that they [differed] between the two racial groups" (Trotter \& Gleser 1958:100). Furthermore, the use of general formulae was 
criticised as both studies showed that in order to get the greatest precision in estimates it was necessary to apply different equations to different racial groups, and also within the same racial group but over successive generations. The regression equations presented in the last table combined both sides and were provided in order of preference according to the standard errors of estimate. A warning was given several times of the dangers of estimating stature by averaging estimates from several equations, recommending that "for any individual remains [...] only one equation must be used and that this one should be the equation with the least standard of error of estimate according to the bones which are available" (Trotter \& Gleser 1958:121see table 4.5 below).

\begin{tabular}{|cccc|cccc|}
\multicolumn{4}{c|}{ White males (1958) } & \multicolumn{4}{|c|}{ Black males (1958) } \\
Coef. & Max.length & $\boldsymbol{\alpha}$ & SE & Coef. & Max.length & $\boldsymbol{\alpha}$ & SE \\
\hline 2.89 & Humerus & +78.10 & $\pm 4.57 \mathrm{~cm}$ & 2.88 & Humerus & +75.48 & $\pm 4.23 \mathrm{~cm}$ \\
3.79 & Radius & +79.42 & $\pm 4.66 \mathrm{~cm}$ & 3.32 & Radius & +85.43 & $\pm 4.57 \mathrm{~cm}$ \\
3.76 & Ulna & +75.55 & $\pm 4.72 \mathrm{~cm}$ & 3.20 & Ulna & +82.77 & $\pm 4.74 \mathrm{~cm}$ \\
2.32 & Femur & +65.53 & $\pm 3.94 \mathrm{~cm}$ & 2.10 & Femur & +72.22 & $\pm 3.91 \mathrm{~cm}$ \\
2.42 & Tibia & +81.93 & $\pm 4.00 \mathrm{~cm}$ & 2.19 & Tibia & +85.36 & $\pm 3.96 \mathrm{~cm}$ \\
2.60 & Fibula & +75.50 & $\pm 3.86 \mathrm{~cm}$ & 2.34 & Fibula & +80.07 & $\pm 4.02 \mathrm{~cm}$ \\
\hline
\end{tabular}

Table 4.5: Revised regression equations from single bones, adapted from Trotter \& Gleser (1958:120, Table 12)

In the fourth edition of his Field Manual, Bass (1995) only mentioned Trotter and Gleser's method, with the 1958 equations used to estimate male stature and the 1952 equations for female stature. Although these formulae were also reproduced in White and Folkens (2005)'s Human Bone Manual, the equation using tibial measurements was omitted as studies had since shown that the original measurements of the tibiae did not include the mallaeolus, creating an overestimation of nearly $3-4 \mathrm{~cm}$ when the tibia was correctly measured (see Jantz et al. 1994, 1995). Numerous other studies and reports since have also stopped using any Trotter and Gleser's equations based on tibial length. A recent study on the stature of ancient Egyptians (Raxter et al. 2008) also confirmed that limb length to stature proportions varied between populations. 
Therefore, whenever possible, regression equations should always be calculated for a specific population either based on known stature or the reconstructed anatomical stature detailed in section 4.3 .5 below, as would most likely be the case in well-preserved archaeological samples.

\subsubsection{Sjøvold's method}

In the late $20^{\text {th }}$ century, a new method of estimating stature was presented by Sjøvold using a weighted line of organic correlation instead of least squares regression equations. This research was based on the data of over 10,000 individuals from all over the world, including all previously analysed material, and ranging from anatomical collections to war casualties (army personnel as well as civilians whose stature had been recorded) and living subjects (Rösing 1988).

The first calculations on white males showed a "strong dependence between the mean statures and the mean bone length" (Sjøvold 1990:441). Different formulae for males and females had usually been used to take into account the difference in body proportions between both sexes, but further research on sexual dimorphism showed that "body proportions are adapted to the stature itself and that this adaptation is more important than sexual modification of these proportions" (ibid:442). Weighted lines of organic correlation were calculated using between nine and 23 samples, involving 2,308 to 8,577 individuals for each of the lines.

A second study to compare different ethnic groups included 14 to 44 samples with 3,232 to 10,573 individuals. The differences between the weighted lines of organic correlation of the first and the second studies were minimal. The same lines of organic correlation were successfully tested further on a much smaller Japanese population with a mean stature of $156.5 \mathrm{~cm}$ for males and $146.6 \mathrm{~cm}$ for females. This confirmed the universality of Sjøvold's equations with regards to sex as well as ethnic group. Overall, the natural (or bicondylar) length of the 
femur was found to be a reliable measurement for estimating stature, whereas the physiological length of the tibia should be avoided.

\begin{tabular}{|cccc|}
\hline Coef. & Measurement & $\alpha$ & SE \\
\hline 4.62 & Humerus & +19.00 & $\pm 4.89 \mathrm{~cm}$ \\
3.78 & Radius & +74.70 & $\pm 5.01 \mathrm{~cm}$ \\
4.61 & Ulna & +46.83 & $\pm 4.97 \mathrm{~cm}$ \\
2.71 & Femur & +45.86 & $\pm 4.49 \mathrm{~cm}$ \\
3.01 & Femur2 & +32.52 & $\pm 3.96 \mathrm{~cm}$ \\
3.29 & Tibia & +47.34 & $\pm 4.15 \mathrm{~cm}$ \\
3.59 & Fibula & +36.31 & $\pm 4.10 \mathrm{~cm}$ \\
\hline
\end{tabular}

Table 4.6: Weighted line of organic correlation formulae for all ethnic groups independent of sex, adapted from Sjøvold (1990:445, Table 2).

All measurements are maximum lengths apart from Femur2 which is the bicondylar length.

Regression equations need to be race- and sex-specific to ensure a good result, and they still tend to overestimate populations of small stature and underestimate tall ones. On the contrary, Sjøvold's equations are applicable to individuals of either sex regardless of race. This new method is therefore extremely useful for archaeology as information on the sex and race of human skeletal remains is often scarce, based on estimations or completely unavailable. Sjøvold's method was therefore chosen as the main method for estimating the stature of the adults from the Neolithic populations under study in this doctoral research. This method has also already been used in a comprehensive study on the stature of 4,305 males and 3,735 females from 330 burial sites of the Carpathian Basin, based on their humeri, radii, femora and tibiae (Éry 1998). This large sample ranged in date from the Neolithic period, represented by one hundred males and 84 females, to the $16^{\text {th }}-17^{\text {th }}$ centuries AD.

\subsubsection{Anatomical stature}

Anatomical methods of stature estimations take into account the whole of the skeleton rather than just concentrating on the long bones. They can therefore be much more accurate, but also require many more undamaged skeletal elements to be present. 


\section{(- Fully's Technique}

This anatomical method (Fully 1956) estimates the stature from the skeletal height with an added soft tissue correction. Skeletal height is obtained by adding together the basi-bregmatic height of the cranium, the maximum midline height of the vertebral bodies from the second cervical vertebra to the fifth lumbar vertebra, the anterior height of the first sacral vertebra measured in the midline, the bicondylar length of the femur, the length of the tibia (omitting the intercondylar eminence), and the height of the articulated talus/ calcaneus. To adjust for soft tissue loss, the following are then added to the skeletal height to obtain the living stature of the individual: $10 \mathrm{~cm}$ to skeletal heights of $153.5 \mathrm{~cm}$ or less, $10.5 \mathrm{~cm}$ to skeletal heights between 153.6 and $165.5 \mathrm{~cm}$, or $11.5 \mathrm{~cm}$ to skeletal heights of $165.6 \mathrm{~cm}$ or more.

This technique has been since been revised with the publication of new formulae to calculate living stature from skeletal height based on the study of 119 adult black and white males and females of known cadaveric statures from the Terry Collection. Skeletal height was obtained from revised and more precise measurements, given as follows (Raxter et al. 2006:382-3):

\footnotetext{
SKELETAL HEIGHT = Maximum length between bregma and basion + most superior point of the dens to the most inferior point of the anterioinferior rim of the vertebral body of the second cervical vertebra (axis) + maximum height of the vertebral body, measured in its anterior $1 / 3$, medial to superiorly curving edges of the centrum of the $3^{\text {rd }}$ to $7^{\text {th }}$ cervical vertebrae + maximum height of the vertebral body, anterior to rib articular facets and pedicles, of the thoracic vertebrae + maximum height of the vertebral body, anterior to the pedicles, not including any swelling of the centrum due to the pedicles, of the lumbar vertebrae + maximum height between the anterior-superior rim of the body of S1 and its point of fusion/articulation with S2 + physiological (bicondylar) length of femur + maximum length of the tibia with malleolus and without the proximal spines + articulated taluscalcaneus height
}

This study also demonstrated that Fully's soft tissue correction factors were underestimated by about $2.4 \mathrm{~cm}$. This was used by Byers (2008) to amend Fully's original correction factors in the section on anatomical stature estimation in his Forensic Anthropology textbook, together with separate correction factors for females, estimated at $92 \%$ of male values. This gave a total 
of six correction factors. Rather than attempting to correct all those factors and adding further potential for error, Raxter and colleagues calculated a formula including the soft tissue element, without the need for further calculations. This formula was shown to be equally valid for both sexes, without any need for correction or confirmation of the sex of the individual analysed.

As in previous studies, living stature was found to change with age, and two formulae for living stature estimation were thus given. One only required the skeletal height whereas their preferred formula factored in the age of the individual if known. In a further paper (Raxter et al. 2007), the risk of an underestimation of up to $1 \mathrm{~cm}$ for individuals much younger than fifty years of age and a possible overestimation for individuals much older than fifty when using the formula only based on height was emphasized. It was therefore recommended to use a "mean age-adjusted Fully Estimated Stature" when exact age is unknown but can be estimated in a broad age range (for example: age 22.5 for someone between 20 and 25 years, age 35 for an individual between thirty and forty, or age 45 for an individual between thirty and sixty years old). This would provide a better and more accurate estimation of living stature.

LIVING STATURE $=1.009 \times$ Skeletal height $-0.0426 \times$ age +12.1

or LIVING STATURE $=0.996 \times$ Skeletal height +11.7

\section{- Skeletal length in the grave}

Another method to estimate living stature from individuals buried in the supine position was published recently, with the in situ skeletal length measured from "a point above the cranial point farthest from the body to the most distal point of the talus" (Petersen 2005:106). Trotter and Gleser's linear regression equations from white males and females of the Terry collection were then used as a comparison. Skeletal length in the grave overestimated anatomically reconstructed stature on average by a mere $0.08 \mathrm{~cm}$. 


\subsubsection{Comparison of long bone formulae and anatomic methods}

The very small difference observed above between stature estimates using Fully's updated technique and skeletal length in the grave demonstrated the success of the latter method. The same study however revealed that Trotter and Gleser's (1952) femur-based stature yielded a very high overestimation of 4.15 cm on average (Petersen 2005). Trotter and Gleser's (1958) formulae did not provide better results, with an even larger overestimation of $4.90 \mathrm{~cm}$ for ten male skeletons in the Ribe sample, compared to $4.05 \mathrm{~cm}$ using the Terry Anatomical Collection formula. It was recommended that "in order to obtain unbiased estimates of mean stature of prehistoric and early historic populations for which there are no written records of stature, but where the dead are buried in an extended supine position, one should, whenever possible, measure skeletal length in the grave during excavation, with talus as foot end landmark" (Petersen 2005:113).

Anatomic methods, regression equations and lines of organic correlation were also tested on a small but well-preserved European Neolithic sample of 39 males and 27 females (Formicola 1993). Compared to anatomical methods of stature estimation, Pearson's equations (1899) and Trotter and Gleser's equations for black individuals (1952) gave a very good estimates for females, while Trotter and Gleser's formulae for white individuals (1952) gave "very unsatisfactory evaluations, except in specimens taller than $180 \mathrm{~cm}$, and usually overestimate stature both in male and in female skeletons" (Formicola 1993:351). The best results of new statistical approaches compared to anatomical estimations were obtained using Sjøvold's formulae.

This was further confirmed in a follow-up study on a European Neolithic sample of 33 males and 27 females (Formicola \& Franceschi 1996). Stature estimates from Fully's anatomical method, which used the sum of all skeletal components 
of height with a correction for soft tissue, and from least squares regression equations such as Trotter and Gleser's, as well as from major axis regressions techniques such as Sjøvold's method, were all compared. The results of regression equations confirmed that the femur and tibia (after correction) were the most suitable bones for stature estimations. Trotter and Gleser's equations for white individuals (1952) resulted in overestimates while their equations for black individuals after adjusting tibial length were more accurate. Model II equations like Sjøvold's were shown to yield very accurate estimates.

\subsubsection{Other techniques}

The use of standard regression equations (as well as lines of organic correlation) on archaeological material is often impaired by the need for complete long bones. In order to address this issue, other techniques have been developed, utilising fragmentary long bones, complete metacarpals and metatarsals, and simple ratio calculations.

\section{- Fragmentary long bones}

The first study on the potential of fragmentary long bones for stature estimation dates back to the 1930s (Müller 1935). Fifty radii, one hundred humeri and one hundred tibiae of white Europeans from a collection in Zellendorf, Austria were analysed. The numbers of possible segments varied per bone with five segments for the humerus, four for the radius and seven for the tibia. The proportions of each segment in comparison to the total bone were given, and stature could then be estimated from the recreated maximum bone length by using any regression equation.

A follow-up research, based on 81 male and 36 female prehistoric American Indians, concentrated on four femoral segments, five tibial fragments and four humeral fragments (Steele \& McKern 1969). Left and right sides were pooled together into a single sample. Formulae were given to utilise these segments to 
evaluate first the parallel length of the complete bone, which could then be converted to the maximum length of the same bone, which in turn could then be used in standard regression equations to estimate the living stature of the individual. All the regression tables were misprinted in the original article although later corrected in Krogman and İşcan's (1986) publication after consultation with the authors. A further study yielded regression equations based on the same segments (Steele 1970). These formulae could then be used to estimate living stature directly from the segment lengths without the need to estimate the maximum length of the complete bone first. Different standards were also given for white and black individuals, and for both sexes.

Recently, information from all the studies available were compiled to produce a description of the segments and the percentages of total bone length (Byers 2008). Steele's method with sex-specific percentages was utilised for the femur, tibia and humerus, and Müller's technique was employed for the radius with a single percentage for both sexes. Although this is indeed very helpful for archaeological populations, these methods can not cover every type of fragments and thus are still heavily dependent on the fragmentation of the skeletal remains recovered.

\section{- Metarcarpal length}

A method of estimating stature from the physiological (inter-articular) length of the metacarpals was based on the radiographs and stature measurements in $\mathrm{cm}$ of male and female adult patients who attended the A\&E of Bristol Royal Infirmary (Musgrave \& Harneja 1978). Twenty female left, 26 female right, 53 male left, and 67 male right hands were included. Their metacarpal interarticular lengths were measured from radiographs and the measurements then corrected for distortion during radiography. According to this study, the results of the regression equations from metacarpal length compared favourably to the measured stature of a study sample of ten male patients. However, there were 
major discrepancies in males of short stature, and the results indicated an underestimation for the stature of tall people and an overestimation for individuals of short stature. In the ten individuals studied, the difference between measured stature and the average stature estimates from metacarpals extended from $0.9 \mathrm{~cm}$ to $21.7 \mathrm{~cm}$ for each individual. Moreover, the difference between the stature estimates from the lengths of the five metacarpals of the same individual varied from $0.9 \mathrm{~cm}$ to $6.2 \mathrm{~cm}$. This was also the case when compared on modern and fossil skeletal subjects to stature estimates from Trotter's 1970 equations, with a difference of up to $3 \%$ in the results. The authors concluded that if this margin of error was deemed acceptable, this method could then be used for "obtaining a rough and ready estimate of living stature when all else fails" (Musgrave \& Harneja 1978:117).

A further study based on a much larger sample and using the same interarticular measurements as Musgrave and Harneja was undertaken in the 1990s (Meadows \& Jantz 1992). Formulae for white and black males and females were obtained utilising the metacarpals of the right hand only. These equations were reprinted in Byers' (2008) Forensic Anthropology textbook as the standard for stature estimation from metacarpal lengths. However, a warning was added indicating that due to the very large standard errors, this method should only be used when no long bones are available as a $95 \%$ confidence interval would give possible errors of up to a considerable $20 \mathrm{~cm}$ in stature.

\section{- Metatarsal length}

Studies on metatarsal length for stature estimation have also been undertaken. Unfortunately, the possible errors were as large as for estimates obtained from metacarpal length and even larger in some cases, especially for white males. This is not surprising as different sizes of feet and hands are common in humans and not necessarily proportional to the individual's stature. The standard for this technique quoted in Byers' (2008) Forensic Anthropology textbook is a 
study based on 57 white males, 51 white females, 13 black males and nine black females utilising the same measurements for the five metatarsals (Byers et al. 1989). An extra measurement for the fifth metatarsal derived from the morphological length, including the tip of the tuberosity, was also added. Living stature corrections were applied and an average of the measurements from both sides used. A clear warning was added concerning the need to restrict this method strictly to cases where no other limb bones were available.

\section{Femur / Stature ratio}

As mentioned above in section 4.2.2. on juvenile stature, femur/stature ratio has been investigated as a method of stature estimation (Feldesman et al. 1990). Based on the analysis of over 13,000 individuals from all over the world, a femur/stature ratio of $26.74 \%$ was established for adults, regardless of sex or ethnicity. Stature could thus be estimated by a simple calculation:

Stature $(\mathrm{cm})=$ maximum femur length $(\mathrm{cm}) \times 3.74$

Even though this very extensive study presented accurate results, the use of femur/stature ratio to estimate stature has been criticized by Meadows and Jantz (1995), whose study indicated that the femur was positively allometric, and by Konigsberg and colleagues, who went as far as describing this ratio as an “inadmissible stature estimator" (Konigsberg et al. 1998:89).

\subsection{Conclusion}

The best method to estimate fetal stature available at present remains the work of Fazekas and Kósa. Smith's method showed a great potential for postnatal juveniles as it can be applied regardless of sex, yet is restricted to the age of three to ten years of age. Himes' method using metacarpal measurements could also be extremely useful when other bones are unavailable for observation. 
Unfortunately, it is sex-specific and can also only be applied to children aged one to seven years. Feldesman's femur/stature ratio would help fill the gap in the juvenile range as it is aimed at individuals between twelve and eighteen years. However, the requirement for total long bone length renders it very limited for skeletal remains without having to resort to more estimations and possible added error. Visser's method also demonstrated potential, despite being applicable to three long bones only, and yet was let down by large standard errors. Percentage of attained growth might have resolved all these issues. Regrettably, it has been marred by the extremely variable methodologies utilised. Any such percentage would therefore need to be precisely explained, limited to the least amount of other estimations possible and might still not be directly comparable to results from other studies. The most recent growth studies have concluded that population differences are not as marked as was once believed, casting doubts over the purpose of such studies and the need to estimate juvenile stature or growth rate.

Estimation of adult stature is by no means more straightforward. Although Trotter and Gleser's regression equations have been the standards of adult stature estimation for many years mostly in North America and the UK, this chapter has shown that a wide variety of other methods are available. Unfortunately, they are often based on very different samples, from living subjects to cadavers as well as skeletal remains whose age had been estimated, and employ techniques ranging from direct bone measurements to radiographs or a mixture of both on different single or set of bones. This, together with different sample size and measurements, make a comparison between these methods difficult and the decision of which methods to apply on skeletal remains problematic.

For stature estimates based on long bone length, the numerous problems of Trotter and Gleser's regression equations highlighted in other studies are unavoidable, together with the general overestimation as well as occasional 
underestimation (such as in the case of modern Balkan populations, see Ross \& Konigsberg 2002), required knowledge of sex and race, and the very specific target population of their method. Conversely, the demonstrated reliability of Sjøvold's formulae and their ideal use for archaeological skeletal populations clearly points to Sjøvold's as the best method for the estimation of stature for adults, despite the slightly larger standard of errors. The need for age correction on such calculations, in addition to the adequacy of the correcting formulae, is also still debated (Giles 1991; Vercellotti et al. 2009). In prehistoric populations, maximum adult height rather than the actual stature at death would be sufficient thus age correction would not play a pivotal role in archaeological studies. The adult femur/stature ratio held great promise but has also been highly criticised. Despite being seemingly particularly suitable for the study of skeletal remains, methods based on fragmentary long bones suffer from the different systems of fragmentation utilised by different authors and the highly variable state of preservation within a single archaeological population.

The numerous issues with stature estimation methods not restricted to long bones have also been described in this chapter. Although metacarpals and metatarsals may still be analysed in comparison to stature in specific population, the methods and formulae available based on their measurements present standard errors too excessive for direct stature estimations. Even the reliability of anatomical stature following Fully's original or revised method, which has been and still is considered the most accurate of any stature estimator for skeletal population, has recently been tarnished (Bidmos 2005). This method would also only be applicable on complete skeletons, which are a rare find in prehistoric skeletal populations. Finally, stature estimation from length in the grave might thus seem the only redeeming accurate indicator of living stature. Sadly most archaeological populations will neither be complete enough, undisturbed, in the correct position or adequately measured in the field for this method to be applicable. 
Following this extensive review of stature estimation methods currently available and for the reasons highlighted above, the following will therefore be used in the analysis of the skeletal remains of the Neolithic populations under study in this doctoral research:

- Skeletal measurements of neonates will be utilised to estimate their stature based on Fazekas and Kósa's (1978) method, still currently unsurpassed.

- Diaphyseal lengths will be employed to estimate the stature of juveniles. Although Visser's (1998) equations will be preferred due to their suitability to dry bones of all ages, Smith's (2007) as well as Telkkä, Palkama and Virtama's (1962) respective formulae for the appropriate age categories will also be used as a comparison to ensure that this rare osteological collection yields as much information as possible.

- Percentage of attained growth will be provided to enable future comparisons with similar populations when these become available, albeit with the cautionary notes clearly set out in this chapter.

- For older adolescents with fully fused limb bones and for all adult individuals, long bone lengths will be utilised following Sjøvold's (1990) universal line of organic correlation method. A comparison will also be undertaken with Trotter and Gleser's regression equations, as these are still more commonly used in the UK and North America despite the serious concerns described in this chapter.

- Although additional methods were also shown to be promising in this chapter, such as the more accurate anatomical methods, the nature of such a fragmentary and damaged ancient osteological collection unfortunately excludes their use in this doctoral research. 


\section{Chapter 5: Osteological analysis - Palaeodemographic results}

\subsection{Introduction}

As this collection includes remains excavated by various archaeologists over several decades, its recording system was confusing with some individuals recorded with an inventory number and a grave number, some with a grave number but no inventory number, and others without any recording number, often all from the same sites. Before analysing all osteological observations, all remains available were therefore re-inventoried to give them a consistent numbering throughout. This new inventory can be found in Appendix 6. All the remains were fragmentary and damaged, with some skeletons mostly complete while other individuals were only represented by a few bones. All juveniles were recovered from Late Neolithic sites of the Tisza culture.

\subsection{Juveniles}

\subsubsection{Age estimates}

Estimates of age for juveniles based on skeletal development (including skeletal maturation and epiphyseal union) were obtained using the data of Schaefer, Scheuer and Black (2009, see section 3.2.3). This data is available in full in Appendix 4, and has been highlighted in bold throughout the table to easily distinguish it from the other comparative studies also presented.

\section{Neonates}

The three neonates were aged between 10 lunar months and overmature based on estimation of body length from their bone measurements following Fazekas 
and Kósa's (1978) method (see Appendix 7 for all estimations). Bone formation of the most complete skeleton, HGO-55, indicated an age from birth to six months, and its dental development to within two months of birth.

\section{( Infants}

Eight infants were identified on the basis of their dental and skeletal development, as summarised in table 5.1. Estimates from dental age from the three methods agreed with each other and corresponded well with skeletal age.

\begin{tabular}{|c|c|c|c|c|c|c|c|c|}
\hline & HKS-04 & HGO-02 & HGO-25 & HGO-33 & HGO-37 & HGO-42 & HGO-43 & HGO-57 \\
\hline $\begin{array}{l}\text { Skeletal } \\
\text { Age }\end{array}$ & c.1 year & $\begin{array}{l}6 \\
\text { months- } \\
1 \text { year }\end{array}$ & c. 1 year & $\begin{array}{l}6 \\
\text { months- } \\
1 \text { year }\end{array}$ & $\begin{array}{l}\text { Very } \\
\text { young } \\
\text { infant }\end{array}$ & $\begin{array}{l}\text { Just } \\
\text { under } 1 \\
\text { year }\end{array}$ & $\begin{array}{l}\text { c. } 6 \\
\text { months }\end{array}$ & $\begin{array}{l}6 \\
\text { months }\end{array}$ \\
\hline $\begin{array}{l}\text { Dental } \\
\text { Age } \\
\text { Ubelaker/ } \\
\text { Schour \& } \\
\text { Massler }\end{array}$ & $\mathrm{n} / \mathrm{a}$ & $\begin{array}{l}6 \\
\text { months } \\
\pm 3 \\
\text { months }\end{array}$ & $\begin{array}{l}1 \text { year } \pm \\
3 \\
\text { months }\end{array}$ & $\begin{array}{l}6 \\
\text { months } \\
\pm 3 \\
\text { months }\end{array}$ & $\mathrm{n} / \mathrm{a}$ & $\begin{array}{l}9 \\
\text { months } \\
\pm 3 \\
\text { months }\end{array}$ & $\begin{array}{l}6 \\
\text { months } \\
\pm 3 \\
\text { months }\end{array}$ & $\begin{array}{l}6 \\
\text { months } \\
\pm 3 \\
\text { months }\end{array}$ \\
\hline $\begin{array}{l}\text { Dental } \\
\text { Age } \\
\text { Smith }\end{array}$ & $\mathrm{n} / \mathrm{a}$ & $\begin{array}{l}\text { c. } 6 \\
\text { months }\end{array}$ & $\begin{array}{l}\text { c. } 10 \\
\text { months }\end{array}$ & $\begin{array}{l}\text { c. } 5.5 \\
\text { months }\end{array}$ & $\mathrm{n} / \mathrm{a}$ & $\begin{array}{l}\text { c. } 8.5 \\
\text { months }\end{array}$ & $\begin{array}{l}\text { c. } 6.5 \\
\text { months }\end{array}$ & $\begin{array}{l}\text { c. } 6 \\
\text { months }\end{array}$ \\
\hline $\begin{array}{l}\text { Dental } \\
\text { Age } \\
\text { Gustafson }\end{array}$ & $\mathrm{n} / \mathrm{a}$ & $\begin{array}{l}\text { Birth to } \\
\text { one year }\end{array}$ & $\begin{array}{l}6 \\
\text { months } \\
\text { to } 1 \\
\text { year }\end{array}$ & $\begin{array}{l}\text { c. } 6 \\
\text { months }\end{array}$ & $\mathrm{n} / \mathrm{a}$ & $\begin{array}{l}\text { c. } 8 \\
\text { months }\end{array}$ & $\begin{array}{l}\text { c. } 6 \\
\text { months }\end{array}$ & $\begin{array}{l}\text { c. } 6 \\
\text { months }\end{array}$ \\
\hline
\end{tabular}

Table 5.1: Dental and skeletal age estimates of Late Neolithic infants

(Data and table by the author, see section $\mathbf{3 . 2}$ for dental methods explanations and references)

Diaphyseal measurements were available for five of the infants and compared to different methods and samples (see table 5.2 overleaf). Gindhart's method consistently underestimated age compared to dental and skeletal development. HGO-57, an infant around six months old, was underaged by all methods at 3 months old. As this individual had been suffering from a severe infection (see section 7.4.2.), it is possible that growth had been delayed. The age of HGO-33 was also mostly underestimated by 3 months based on diaphyseal length. 


\begin{tabular}{|c|c|c|c|c|c|c|c|c|c|c|c|c|}
\hline Individual & Dental Age & $\begin{array}{l}\text { Skeletal } \\
\text { Age }\end{array}$ & $\begin{array}{c}\text { Diaphyseal } \\
\text { measurements }\end{array}$ & $\begin{array}{l}\text { Stloukal \& } \\
\text { Hanáková }\end{array}$ & $\begin{array}{l}\text { Sundick: } \\
\text { Altenerding }\end{array}$ & $\begin{array}{l}\text { Sundick: } \\
\text { Ind. Knoll }\end{array}$ & Johnston & $\begin{array}{c}\text { Merchant } \\
\& \\
\text { Ubelaker }\end{array}$ & Hoffman & Maresh & Gindhart & Mays \\
\hline HGO-33 & 6 months & $\begin{array}{l}6 \text { months } \\
-1 \text { year }\end{array}$ & Humerus $=76$ & 3 months & 3 months & 3 months & 6 months & 3 months & 6 months & 3 months & & \\
\hline \multirow{2}{*}{ HGO-57 } & \multirow{2}{*}{6 months } & \multirow{2}{*}{$\begin{array}{c}\text { c. } 6 \\
\text { months }\end{array}$} & $U / n a=63.4$ & 3 months & 3 months & 3 months & 3 months & 3 months & 3 months & 3 months & & \\
\hline & & & Tibia $=73.2$ & 3 months & 3 months & 3 months & 3 months & 3 months & 3 months & 3 months & 3 months & \\
\hline HGO-42 & 9 months & $\begin{array}{c}\text { Just }<1 \\
\text { year }\end{array}$ & $U / n a=73.6$ & 6 months & 1 year & 6 months & 6 months & 6 months & 6 months & 6 months & & \\
\hline \multirow{2}{*}{ HKS-04 } & \multirow{2}{*}{$\mathrm{n} / \mathrm{a}$} & \multirow{2}{*}{ c. 1 year } & Femur $=133.5$ & 1 year & & 1 year & 1 year & 1 year & 1 year & 1 year & & 1 year \\
\hline & & & Tibia $=107$ & 1.5 years & & 1 year & 1 year & 1 year & 1 year & 1 year & 6 months & \\
\hline \multirow{2}{*}{ HGO-25 } & \multirow{2}{*}{10 months } & \multirow{2}{*}{ c. 1 year } & Humerus $=93$ & 6 mths- $1 \mathrm{yr}$ & 1 year & 1 year & 1 year & 1 year & 1 year & 6 months & & \\
\hline & & & Tibia $=95.3$ & 1 year & & 1 year & 1 year & 1 year & 6 mths- $1 \mathrm{yr}$ & 6 months & 6 months & \\
\hline \multirow{3}{*}{ HGO-49 } & \multirow{3}{*}{$\mathrm{n} / \mathrm{a}$} & \multirow{3}{*}{$2-3$ years } & Humerus $=117.5$ & 2 years & & $1.5-3$ years & 2 years & 1 year & $1.5-2$ years & 1.5 years & & \\
\hline & & & Radius $=97.5$ & 3 years & 2-3 years & $2-3$ years & 3 years & $2-3$ years & 1.5- 2 years & 2 years & 2 years & \\
\hline & & & $U \ln a=97.5$ & 2 years & & 1.5 years & 2 years & 2 years & 1.5- 2 years & $1-1.5$ years & & \\
\hline HGO-06 & 5 years & $5-6$ years & $n / a$ & & & & & & & & & \\
\hline \multirow{3}{*}{ НКК-01 } & \multirow{3}{*}{7 years } & \multirow{3}{*}{ Child } & Humerus $=166$ & $5-7$ years & 5 years & $6-8$ years & & 5 years & $4-5$ years & 4.5 years & & \\
\hline & & & Femur $=228.5$ & $5-7$ years & $5-6$ years & 6 years & & 5 years & 4-5 years & 4 years & & 7 years \\
\hline & & & Tibia $=178$ & $5-7$ years & 5 years & 5 years & 5 years & 5 years & 3-4 years & 4 years & 3 years & \\
\hline HGO-09 & 8 years & $\geq 6$ years & $n / a$ & & & & & & & & & \\
\hline HGO-45 & $11-12$ years & $<14$ years & $n / a$ & & & & & & & & & \\
\hline \multirow{3}{*}{ HGO-54 } & \multirow{3}{*}{$11-12$ years } & \multirow{3}{*}{$\begin{array}{l}\text { Middle } \\
\text { child }\end{array}$} & Humerus $=219$ & $11-13$ years & 9-10 years & 9-13 years & & 9 years & $7-10$ years & $8-9$ years & & \\
\hline & & & Femur $=325$ & $12-15$ years & $11-14$ years & 9-13 years & & 11 years & $8-11$ years & $8-10$ years & & c.13 years \\
\hline & & & Tibia $=254$ & $11-14$ years & 9-11 years & 9-11 years & & 8 years & $7-10$ years & $8-9$ years & $7-8$ years & \\
\hline HGO-36 & $\mathrm{n} / \mathrm{a}$ & Child & $n / a$ & & & & & & & & & \\
\hline HKK-03 & $\mathrm{n} / \mathrm{a}$ & Child & Humerus $=160$ & $5-7$ years & 4 years & $5-6$ years & 5 years & 5 years & 3-5 years & 4-5 years & & \\
\hline
\end{tabular}

Table 5.2: Infants and children age estimates from dentition, skeletal maturation and diaphyseal lengths

(Data and table by the author, see section 3.2.2 and appendix 3 for explanations of the methods used and their references) 


\section{Children}

Ten children with a wide age range were identified from their dental and skeletal development. As with the infants, dental estimates from different methods were all in agreement, so only one dental age is given here.

Diaphyseal lengths available were compared to the other studies mentioned in section 3.2.2. (see table 5.2 on the previous page, which includes all children's age estimates). Estimates based on Gindhart's, Hoffman's and Maresh's data consistently underaged the Tisza sample, as did to a lesser extent both of Sundick's sets of ranges and Johnston's. Mays' as well as Stloukal and Hanáková's data consistently agreed with the skeletal and dental age estimates for children, although the former only provided estimates from femoral length. Until more juveniles are recovered and analysed from Tisza sites providing a larger range of ages and dimensions, it would therefore be recommended to use Stloukal and Hanáková's (1978) range to age juveniles lacking more accurate dental and skeletal data, such as HKK-03.

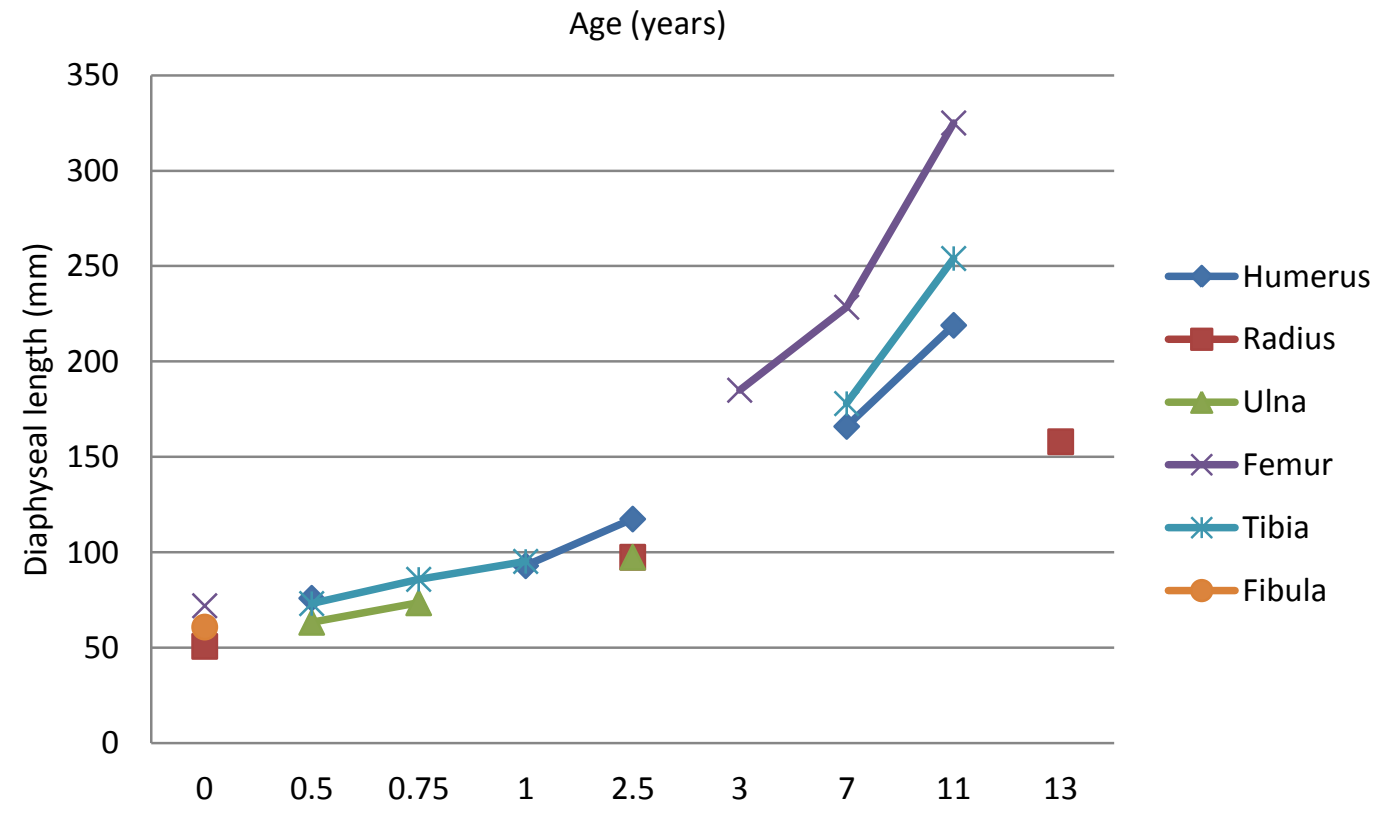

Figure 5.1: Juvenile growth in Late Neolithic Tisza populations 
The diaphyseal lengths of this Late Neolithic juvenile sample from the Tisza Culture could also be used to aid juvenile age estimation in similar populations, as provided in table 5.3 below. However, the very limited sample size and high proportion of pathology would restrict its use as a guideline only.

\begin{tabular}{|cccccc|}
\hline $\begin{array}{c}\text { Dental and } \\
\text { Skeletal Age }\end{array}$ & Humerus & Radius & Ulna & Femur & Tibia \\
\hline $\mathbf{6}$ months & 76 & & 63 & 73 & \\
\hline $\mathbf{9 - 1 2}$ mths & 93 & & 74 & & 91 \\
\hline $\mathbf{1}$ year & & & & 134 & 107 \\
\hline $\mathbf{2 - 3}$ yrs & 118 & 98 & 98 & 185 & \\
\hline $\mathbf{7}$ years & 166 & & & 229 & 178 \\
\hline $\mathbf{1 1 - 1 2}$ yrs & 219 & & 325 & 254 \\
\hline
\end{tabular}

Table 5.3: Ageing from diaphyseal measurements $(\mathrm{mm})$ in Late Neolithic Tisza populations

Comparing the results of table 5.3 above to the ranges of diaphyseal lengths per age provided in Appendix 3, the long bone dimensions of these Late Neolithic juveniles would fit well with the prehistoric (3000 BC) Indian Knoll population from Kentucky, the early historic (AD 7-9th c.) population from the Czech Republic and the medieval (AD 10-16 ${ }^{\text {th }}$ c.) population from England. However, they were unsurprisingly much smaller than contemporary American juveniles.

\section{- Adolescents}

Three adolescents aged around seventeen years were identified from the state of fusion of their epiphyses. The remains of a fourth adolescent [HGO-50] provided a dental age estimate of 12-15 years, and a skeletal age estimate of around 12-13 years for a female or 14-15 years if this individual was a boy. However, his or her radius maximum diaphyseal length of $158 \mathrm{~mm}$ produced much younger estimates of 8-11 years old using Stloukal and Hanáková's (1978) ranges. Traces of advanced cribra orbitalia were found in the orbits, and it is 
therefore possible that this young adolescent could have suffered from an unknown disease or inadequate diet which may have stunted his or her growth.

\section{- Estimates of age based on other measurements}

No mandibular ramus heights were available for juveniles from birth to two years, and the clavicle length could not be measured on any infant or child.

The partes basilaris from the occipital bones of four infants provided suitable dimensions to compare with those given by Scheuer and MacLaughlin-Black (1994). These can be seen in table 5.4. However, as the same measurements can be found at a wide range of ages, it did not clarify the original age estimates.

\begin{tabular}{|c|c|c|c|c|c|}
\hline & $\begin{array}{l}\text { Dental } \\
\text { Age }\end{array}$ & $\begin{array}{l}\text { Skeletal } \\
\text { Age }\end{array}$ & $\begin{array}{l}\text { Pars basilaris } \\
\text { max.width }\end{array}$ & $\begin{array}{l}\text { Pars basilaris } \\
\text { sagittal length }\end{array}$ & $\begin{array}{l}\text { Pars basilaris } \\
\text { max. length }\end{array}$ \\
\hline \multirow{3}{*}{ HGO-55 } & & & $15.2 \mathrm{~mm}$ & & \\
\hline & 0-1 month & Neonate & $\begin{array}{c}2 \text { weeks to } 3 \\
\text { months }\end{array}$ & & \\
\hline & & & $20.2 \mathrm{~mm}$ & $15.7 \mathrm{~mm}$ & $19.5 \mathrm{~mm}$ \\
\hline \multirow[t]{2}{*}{ HGO-02 } & 6 months & $\begin{array}{l}6 \text { months } \\
-1 \text { year }\end{array}$ & $\begin{array}{c}8 \text { months to } 1 \\
\text { year } 4 \\
\text { months }\end{array}$ & $\begin{array}{c}1 \text { year } 2 \text { months } \\
\text { to } 4 \text { years } 7 \\
\text { months }\end{array}$ & $\begin{array}{l}8 \text { months to } 1 \\
\text { year } 4 \text { months }\end{array}$ \\
\hline & & & $18.1 \mathrm{~mm}$ & $13.2 \mathrm{~mm}$ & $18 \mathrm{~mm}$ \\
\hline \multirow[t]{2}{*}{ HGO-33 } & 6 months & -1 year & $\begin{array}{c}5 \text { months to } 1 \\
\text { year }\end{array}$ & 3 to 5 months & $\begin{array}{c}5 \text { months to } 1 \\
\text { year }\end{array}$ \\
\hline & & & $16.5 \mathrm{~mm}$ & $13.5 \mathrm{~mm}$ & $17.2 \mathrm{~mm}$ \\
\hline HGO-57 & 6 months & 6 months & $\begin{array}{c}3 \text { weeks to } 5 \\
\text { months }\end{array}$ & 3 to 5 months & $\begin{array}{c}3 \text { weeks to } 1 \\
\text { year }\end{array}$ \\
\hline
\end{tabular}

Table 5.4: Age estimates from partes basilaris of infantile occipital bone (Data and table by the author, estimates from Scheuer \& MacLaughlin-Black's (1994) dimensions)

Only the scapula of one juvenile, neonate HGO-31, was complete enough to measure both length and width. These measurements were lower than the birth to six months example (based on only one individual, age not given) from Saunders and colleagues (1993), which could correspond to a newborn. Scapular measurements from eight juveniles were suitable for use with Rissech and Black's (2007) functions to provide age estimates. However, results were 
unsatisfactory, substantially underageing or overageing both infants and children. This method would therefore not be recommended on such populations. Full results are given in Appendix 8.

\subsubsection{Sex estimates}

The three adolescents in their late teens described above will be analysed with the adult population (section 5.3.1). All the other juveniles were considered for sexing using appropriate methods for their age (see section 2.2). Orbits could not be used as all juvenile cranial remains were very damaged and fragmentary, and the combined facial score (CFS) technique (see section 2.2.1) could therefore not be applied. Pelves were also very fragmentary so no acetabulum could be measured. Twelve juveniles in total were able to provide sex estimates from their mandible and / or pelvis.

The ilia of one neonate, one infant, three children and one adolescent were complete enough to enable measurements of the sciatic notch to be taken. This proved to be very difficult due to the very small dimensions in infants and the use of standard unmodified digital callipers. Results are presented in the first table of Appendix 9. These results should only be considered as a possibility and not a certainty as they are based on the proportions given in Fazekas and Kósa (1978) as seen in their fetal material. Morphological sex indicators were also observed on these ilia and two additional ones from a neonate and an infant. Results can be found in the second table of Appendix 9. All criteria on each single pelvis provided estimates for the same sex.

The mandibles of five infants, two children and one adolescent were also complete enough for examination of sexual traits, as well as the mandible of one neonate which could only provide an indeterminate result. The eight other mandibles produced concurring results for all criteria, and can also be seen in the second table of Appendix 9. 
Few individuals were able to provide sex estimates from both mandible and pelvis, and fewer also yielded information from their sciatic notch dimensions. Unfortunately, only four of the twelve analysed individuals could give estimates for both pelvis and mandible. Although all criteria were in agreement in each bone, only two of the four individuals with both mandible and pelvis had estimates of the same sex from both regions. One of these whose sciatic notch could also be measured showed a disagreement between morphological and metric pelvic traits, and only in one out of six pelves which could be measured did the sex estimate from the sciatic notch dimensions match morphological estimates. This lack of consistency can clearly be seen in table 5.5 below.

\begin{tabular}{|c|c|c|c|c|}
\hline & \multirow{2}{*}{$\begin{array}{l}\text { Age } \\
\text { group }\end{array}$} & \multicolumn{2}{|c|}{ Morphology } & \multirow{2}{*}{$\begin{array}{l}\text { Sciatic } \\
\text { notch } \\
\text { index }\end{array}$} \\
\hline & & Mandible & Pelvis & \\
\hline HGO-14 & NEO & & $\mathrm{F}$ & $M$ \\
\hline HGO-55 & NEO & & $\mathrm{F}$ & \\
\hline HGO-02 & INF & $M$ & & \\
\hline HGO-33 & INF & $M$ & M & $F$ \\
\hline HGO-42 & INF & $\mathrm{F}$ & & \\
\hline HGO-43 & INF & $M$ & & \\
\hline HGO-57 & INF & $M$ & $M$ & \\
\hline HGO-06 & YC & $\mathrm{F}$ & & \\
\hline HGO-49 & YC & & $F$ & M \\
\hline HGO-09 & $M C$ & & $M$ & $M ?$ \\
\hline HGO-54 & $\mathrm{MC}$ & $F$ & $M$ & $F ?$ \\
\hline HGO-50* & ADO & $\mathrm{F}$ & $M$ & $\mathrm{~F}$ \\
\hline
\end{tabular}

Table 5.5: Sex estimates from juveniles

$M=$ male, $F=$ female, $M$ ? = possibly male, $F$ ? = possibly female

$\mathrm{NEO}=$ neonate, $\mathrm{INF}=$ infant, $\mathrm{YC}=$ young child, $\mathrm{MC}=$ middle child, $\mathrm{ADO}=$ adolescent

*According to Garn et al. (1996) sequence of epiphyseal fusion, HGO-50 would likely be a male

Until further research is undertaken on large documented collections of juvenile remains, it cannot be ascertained which of the characters are more likely to correctly estimate sex and this juvenile sample will therefore remain unsexed at the moment. However, the visibility of these indicators on such ancient remains 
has at least demonstrated the potential of such methods and the need for further studies. Therefore it would be recommended to record such morphological traits in archaeological populations in the hope that one day more information on documented collections will help verify the results. Morphological pelvic traits would be particularly useful as taking sciatic notch measurements without specialised equipment proved to be particularly difficult during analysis. Mays' method would also have been particularly useful for such an ancient sample (see section 2.2.2), but unfortunately requires numerous individuals and therefore could not be utilised on this limited sample.

\subsubsection{Stature estimates}

Stature was estimated for the fourteen juveniles who could provide diaphyseal measurements, using the methods described in Chapter 4. All measurements and height estimates are reproduced in Appendix 10, and the results can be seen in figure 5.2 .

Figure 5.2: Stature estimates for Late Neolithic juveniles

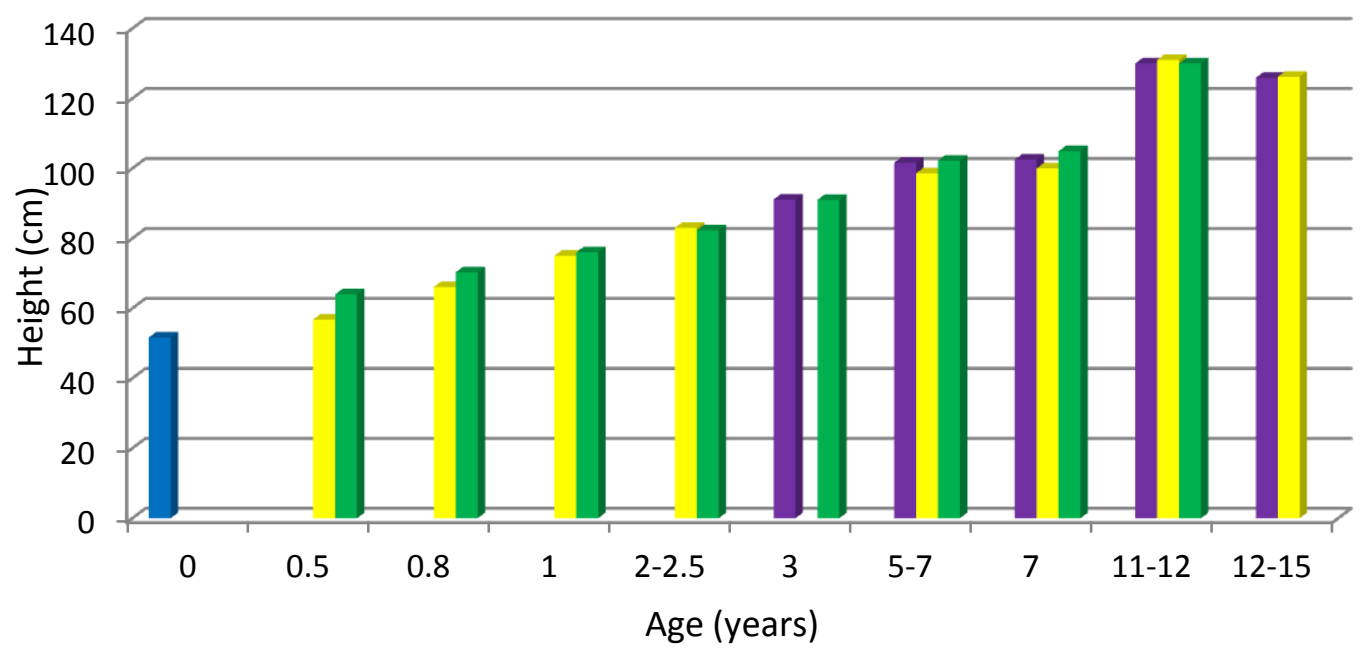

— Fazekas and Kósa (1978) — Smith (2007) — Telkkä, Palkama \& Virtama (1962) — Visser (1998)

Although suitable from birth to fifteen years, there were several issues with Telkkä, Palkama and Virtama's (1962) method as noted in section 4.2. This 
included the lack of correction or adjustment to take into account radiographic magnification as well as the tibia length taken at an oblique angle. Smith's (2007) formulae were only suitable for children aged 3-10 years, and no amendments were made to the measurements for radiographic distortion. Visser's (1998) equations on the other hand had been formulated after accounting for shrinkage of dry bones and magnification of radiographs, although the standard deviation given was extensive. In this juvenile sample, results from Smith's and Visser's calculations were very similar. Estimates obtained with Visser's formulae were chosen based on the thoroughness of the method and its wider age range from birth to eighteen years, and utilised to calculate percentage of attained growth in this juvenile Tisza population. These final stature results are presented in figure 5.3 below.

Figure 5.3: Percentage of attained growth among Late Neolithic juveniles

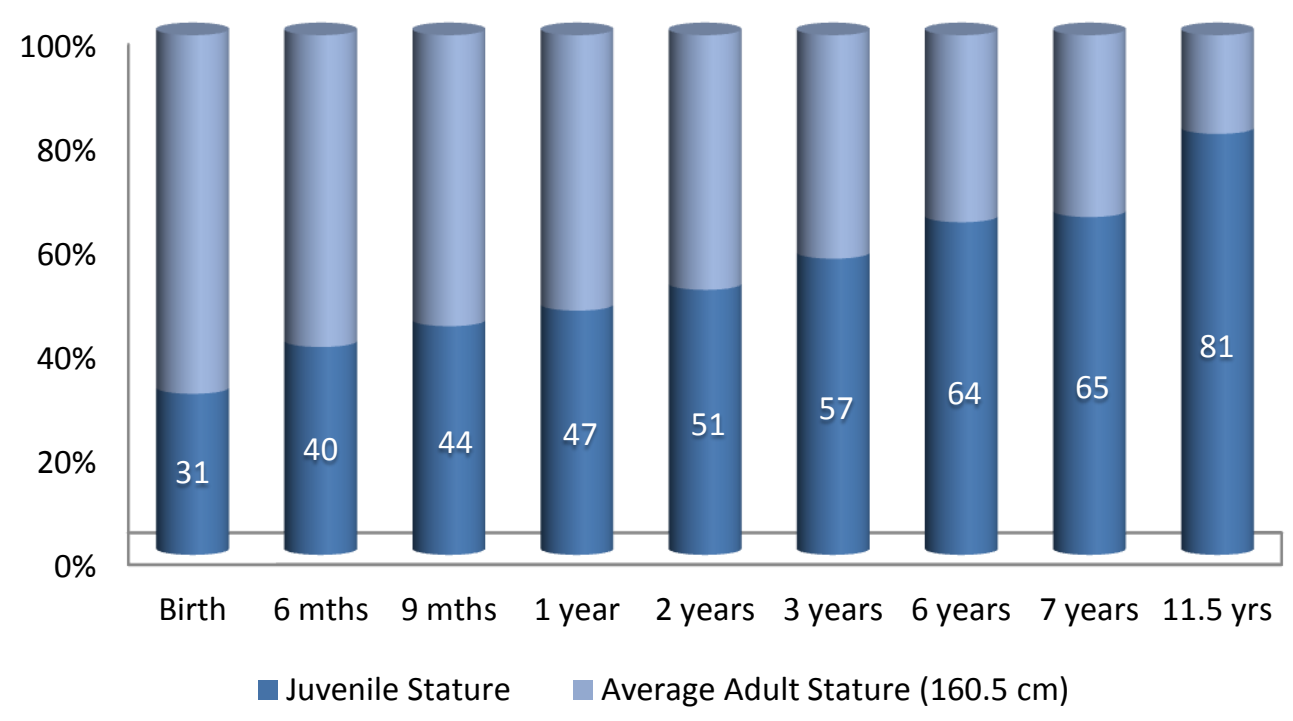

This percentage of attained growth can then be compared to other populations. Table 5.6 overleaf presents a comparison of this Late Neolithic Tisza population with the AD 7-9th c. population from the Czech Republic (Stloukal \& Hanáková 1978), the medieval population of Wharram Percy, England (Mays 1998, 1999) 
and a contemporary sample from England (Tanner et al. 1966). The percentage of attained growth in this archaeological sample was calculated using the diaphyseal length means reported to estimate juvenile stature utilising Visser's equations, against the average of both male and female adult stature for each population. Tanner presented percentages of attained growth for both sexes separately, so results from this doctoral study were also averaged accordingly.

\begin{tabular}{|c|c|c|c|c|}
\hline \multirow{2}{*}{$\begin{array}{c}\text { Age } \\
\text { (years) }\end{array}$} & \multicolumn{4}{|c|}{ Percentage of attained growth } \\
\hline & $\begin{array}{c}5^{\text {th }} \text { millennium } \\
\text { BC Hungary }\end{array}$ & $\begin{array}{c}\text { AD 7-9 }{ }^{\text {th }} \text { c. } \\
\text { Czech Republic }\end{array}$ & $\begin{array}{c}\text { AD } 10-16^{\text {th }} \mathrm{c} . \\
\text { England }\end{array}$ & $\begin{array}{c}\text { England } \\
1960 \text { s }\end{array}$ \\
\hline 1 & $47 \%$ & $44 \%$ & $45 \%$ & $45 \%$ \\
\hline 2 & $51 \%$ & $49 \%$ & $50 \%$ & $51 \%$ \\
\hline 3 & $57 \%$ & $54 \%$ & $54 \%$ & $56 \%$ \\
\hline 6 & $64 \%$ & $62 \%$ & $61 \%$ & $68 \%$ \\
\hline 7 & $65 \%$ & $65 \%$ & $66 \%$ & $71 \%$ \\
\hline 11.5 & $81 \%$ & $76 \%$ & $78 \%$ & $86 \%$ \\
\hline
\end{tabular}

Table 5.6: Comparison of percentages of attained growth in European Populations

The results agree with Mays' (1999) own conclusion that a prolonged growth could be seen in earlier populations. Compared to contemporary England, the Late Neolithic populations of Hungary grew slightly faster in the first three years, but by age six, growth was slightly slower, i.e. if the age estimates are correct, Late Neolithic juveniles would reach their full adult height slightly later than modern populations. It is interesting to note however that in these samples, growth rate was closer to contemporary populations during the Late Neolithic than during the Middle Ages, and particularly compared to early historic populations. As stated in section 4.2.3, these results should only be considered with extreme caution due to the use of several estimations and the limited sample available, particularly in this Late Neolithic populations. 


\subsection{Adults}

\subsubsection{Sex - Morphological estimations}

The twelve adults from Early Neolithic sites of the Körös culture and the two adults from unspecified Neolithic sites were sexed according to their cranial remains. DOL-01, a female from the Körös site, was complete enough to also enable the analysis of her pelvic remains. Five main cranial traits as well as two traits and two overall shape characteristics on the pelvis were graded between 1 (female) and 5 (male), allowing for half grades where necessary. The average of all results in each individual was then used to estimate sex, with results under 3 showing a female, and results above 3 indicating a male. The Early Neolithic sites revealed three females, two probable females, three males, one probable male and three possible males. The unspecific Neolithic sites were represented by two females, one probable and one possible. Detailed results are presented in Appendix 11.

The full results for the Late Neolithic Tisza Culture sample are also presented in the same appendix. These initial sex determinations uncovered eleven males, seven probable males, one possible male, fourteen females, seven probable females and one possible female, as well as two indeterminate individuals.

Additional methods of sexing described in Chapter 2 were then tested against these newly identified individuals, including the mandible ramus flexure trait from Loth and Henneberg (1996, see section 2.3.1), distal humerus traits from Falys and colleagues (2005, see section 2.3.4), pelvic traits from Bruzek (2002, see section 2.3.2) and sacral shape (see section 2.3.3). The initial impressions of accuracy in the colour-coded results table of Appendix 12 were confirmed by statistical analyses using the SPSS version 15 package. As the results were not normally distributed, only non-parametric statistics were utilised. 


\section{(-) Mandibular ramus flexure}

As described in section 2.3.1, mandibular ramus flexure has suffered from criticisms for its unreliability by several researchers having tested the method. This trait was also found to be difficult to recognise at first on this Neolithic sample. However, results of chi-square tests demonstrated that this characteristic was indeed strongly correlated to sex at a significance level of 0.001 and a phi coefficient of 0.610 . Fisher's Exact Probability Test also demonstrated a highly significant correlation at 0.001 level. This method was as reliable a sex indicator as other more commonly used traits, with $78.9 \%$ of females and $83.3 \%$ of males correctly identified, accurately sexing $81.1 \%$ of individuals overall. Results are presented in Appendix 13.

\section{Distal humerus morphological traits}

Sex indicators from the distal humerus were initially thought to be a promising sexing method, due to the ease of use of the methodology and the robusticity of this bone which regularly survives in archaeological context. Although the distal end may be too damaged for measurements, this morphological method would enable even fragmented ends to contribute to the analyses. Unfortunately, results using this method were very disappointing. None of the traits were found to be statistically significant and accuracy was extremely low. The fossa shape for example only correctly identified 35\% of females. Several individuals had to be labelled indeterminate when the sex estimates differed between both sides for individuals who had both humeri available for analysis. Full results can be found in Appendix 14. Kappa measure of agreement tests were also conducted and revealed very little agreement between the four various traits found in the distal humerus. 


\section{- Bruzek pelvic traits}

Bruzek's combination of four pelvic traits also produced several indeterminate individuals due to differing estimates from both sides of the same person. Full results can be seen in Appendix 15.

Results from the composite arch were poor with only $31.3 \%$ of females identified from the female trait although $77.8 \%$ of males were sexed correctly.

Sciatic notch proportion identified $100 \%$ of the males correctly, although only $64.3 \%$ of females. When indeterminate individuals were excluded, Fisher's Exact test revealed a high correlation with sex with a significance of 0.001 .

Sciatic notch symmetry identified $87.5 \%$ of males correctly but only $28.6 \%$ of females. None of the males showed the female trait.

Although the sciatic notch trait linked to the AP line was statistically significant when indeterminate individuals were excluded with 0.009 significance using Fisher's exact test as well as none of the males showing female traits, results including all individuals were poor with only $64.3 \%$ of females and $62.5 \%$ of males correctly identified.

Therefore in this pelvic method, the best estimations would only apply to female sciatic notch traits which could not be found in males, whereas a male character might indicate an individual of either sex.

\section{Sacral shape}

Finally, the sacrum shape trait was only observed in seven individuals, with the male shape only seen once and correctly identifying a male. Of the six female traits, five were correctly attributed to female individuals. However, this sample is too low to make any further observation as to the relevance of this sex indicator in this Neolithic sample. 


\subsubsection{Sex - Osteometric estimations}

As discussed in section 2.3 and as can be seen in Appendix 1, measurements of certain bones or specific parts of bones show strong sexual dimorphism but are also influenced greatly by population variability. Rather than applying standards derived from populations very distant both chronologically and geographically, a new baseline dataset for these Neolithic populations was created based on the individuals whose sex could be estimated from their cranial and pelvic morphological features as presented in Appendix 11. All measurements obtained from the osteological analysis undertaken by the author can be found in Appendix 16.

The samples were not normally distributed and therefore a Mann-Whitney $U$ test was utilised in all the statistical analyses below. As the postcranial remains of only two Early Neolithic individuals were available and a chronological difference of several hundred years existed between the two cultures, only postcranial measurements from the Tisza Late Neolithic population were analysed, while cranial measurements were analysed separately for both time periods.

\section{Skull}

\section{$\underline{\text { Körös (Early Neolithic) Culture }}$}

There were differences in means for all skull measurements and indices between males and females of the Köros culture group, but the actual ranges were overlapping. A Mann-Whitney Test confirmed that none of these differences reached statistical significances, although the sample number was very low. Means, ranges and standard deviations of these dimensions are presented in Appendix 17. 


\section{$\underline{\text { Tisza (Late Neolithic) Culture }}$}

The Tisza sample was larger and provided statistically significant results between both sexes with Mann-Whitney $U$ tests for six cranial and two mandibular traits:

- skull maximum height: males (median $=145, \mathrm{n}=3$ ), females (median = 139, $\mathrm{n}=5), U=000, z=-2.249, p=0.024, r=0.795$

- Cranial breadth-height index: males (median $=110.9, \mathrm{n}=3$ ), females (median $=98.6, \mathrm{n}=4$ ), $U=000, z=-2.121, p=0.034, r=0.802$

- Minimum frontal breadth: males (median $=99.5, \mathrm{n}=8$ ), females (median =93, $\mathrm{n}=11$ ) $, U=11.000, z=-2.734, p=0.006, r=0.627$

- Mastoid length: males $($ median $=32.8, \mathrm{n}=10)$, females $($ median $=29.8$, $\mathrm{n}=17), U=39.000, z=-2.311, p=0.021, r=0.445$

- Nasal height: males (median $=50, n=7$ ), females (median $=45, n=8), U$ $=6.000, z=-2.557, p=0.011, r=0.660$

- Palatal breadth: males (median $=36, \mathrm{n}=9$ ), females $($ median $=34, \mathrm{n}=$ 6), $U=5.500, z=-2.545, p=0.011, r=0.657$

- Mandible maximum length: males (median $=79, \mathrm{n}=10$ ), females (median $=72.5, \mathrm{n}=12$ ), $U=14.000, z=-3.042, p=0.002, r=0.649$

- Mandibular ramus height: males (median $=57, n=7$ ), females (median = 53, $\mathrm{n}=13), U=19.000, z=-2.118, p=0.034, r=0.474$

Although not the most statistically significant due to the low sample numbers, both the skull maximum height and its related cranial breadth-height index did not present any overlap in ranges of measurements between sexes. All the results are presented in Appendix 18.

The only statistically significant difference between the skull dimensions of Körös (median $=67.1, \mathrm{n}=4$ ) and Tisza (median $=70.1, \mathrm{n}=5$ ) cultures were found in the frontoparietal index of females performing a Mann-Whitney $U$ test: $U=2.000, z=-1.960, p=0.050, r=0.653$. 


\section{( Thorax and Spine}

In this limited sample, only the length of the manubrium was found to be significantly sexually dimorphic with $U=0.500, z=-2.178, p=0.029, r=0.77$, males median of $53.5 \mathrm{~mm}(\mathrm{n}=4)$ and females median of $45 \mathrm{~mm}(\mathrm{n}=4)$. Results are presented in Appendix 19.

\section{Shoulders and Upper Limbs}

The clavicle and scapula provided three significantly dimorphic dimensions:

- Clavicle length: males (median $=151, \mathrm{n}=4)$, females $($ median $=128.8, \mathrm{n}$ =10), $U=000, z=-2.828, p=0.005, r=0.756$. This dimension did not provide any overlap in measurements between both sexes.

- Clavicle midshaft circumference: males (median $=39.5, \mathrm{n}=7$ ), females (median $=35, \mathrm{n}=9$ ), $U=11.500, z=-2.152, p=0.031, r=0.538$. However, this dimension yielded large overlap in ranges between sexes.

- Glenoid cavity length: males (median $=39.6, \mathrm{n}=10$ ), females (median $=34.3, \mathrm{n}=15), U=4.500, z=-3.914, p=0.000, r=0.783$

The humerus had five dimensions that were recognised as significantly sexually dimorphic:

- Humerus head diameter: males (median $=46, \mathrm{n}=11$ ), females (median $=39.5, \mathrm{n}=16), U=10.000, z=-3.853, p=0.000, r=0.742$

- Humerus maximum length: males (median $=325.8, \mathrm{n}=9$ ), females ( median $=287.5, \mathrm{n}=15$ ), $U=8.000, z=-3.549, p=0.000, r=0.724$

- Humerus midshaft minimum diameter: males (median $=19.3, \mathrm{n}=11$ ), females (median =17.9, $\mathrm{n}=15), U=36.500, z=-2.392, p=0.017, r=0.469$

- Humerus midshaft maximum diameter: males (median $=21.9, \mathrm{n}=11$ ), females (median = 19, $\mathrm{n}=15), U=32.000, z=-2.624, p=0.009, r=0.515$

- Humerus epicondylar width: males (median $=64.5, \mathrm{n}=9$ ), females (median $=55.5, \mathrm{n}=14$ ) $, U=3.000, z=-3.781, p=0.000, r=0.788$ 
The radius produced three sexually dimorphic dimensions:

- Radius head diameter: males (median $=23.9, \mathrm{n}=4$ ), females (median = 20.1, $\mathrm{n}=12$ ), $U=000, z=-2.913, p=0.004, r=0.728$. Additionally, this dimension provided no overlap in measurements between both sexes.

- Radius maximum length: males (median = 250.5, $\mathrm{n}=7$ ), females (median $=212, \mathrm{n}=13$ ) $, U=8.000, z=-2.976, p=0.003, r=0.665$

- Radius midshaft antero-posterior diameter: males (median $=12, \mathrm{n}=6$ ), females (median $=10.3, \mathrm{n}=12$ ) $, U=13.500, z=-2.114, p=0.021, r=0.498$

Finally, the ulna yielded significant results for two dimensions, both also providing no overlap in measurements between sexes:

- Ulna maximum length: males (median $=270, \mathrm{n}=7$ ), females (median = 232, $\mathrm{n}=11), U=000, z=-3.496, p=0.000, r=0.824$

- Proximal ulna inferior medial trochlear notch width: males (median $=18.1, \mathrm{n}=2$ ), females (median $=14, \mathrm{n}=9$ ), $U=0.000, z=-2.126$, $p=0.033, r=0.641$

The five dimensions with the most significant sexual dimorphism in the shoulders and upper limbs were the glenoid cavity length, humerus head diameter, humerus maximum length, humerus epicondylar width and ulna maximum length, all with $\mathrm{p}<0.001$. All results can be found in Appendix 20 .

\section{(- Hands}

Twelve measurements from hand bones were found to be statistically significant for sexual dimorphism. Appendix 21 contains all results. 
On the first metacarpal:

- MC1 maximum length: males (median $=47, \mathrm{n}=5)$, females (median = 41.2, $\mathrm{n}=11), U=4.000, z=-2.662, p=0.008, r=0.666$

- MC1 proximal medio-lateral breadth: males (median $=16, \mathrm{n}=5$ ), females (median $=14.2, \mathrm{n}=7$ ), $U=0.500, z=-2.766, p=0.006, r=0.798$

- MC1 midshaft antero-posterior diameter: males (median $=9, n=3$ ), females (median $=7, \mathrm{n}=11$ ) $U=0.500, z=-2.511, p=0.012, r=0.671$

- MC1 distal medio-lateral breadth: males (median $=16, \mathrm{n}=5$ ), females (median $=13.9, \mathrm{n}=6$ ) $, U=2.000, z=-2.373, p=0.028, r=0.715$

- MC1 distal antero-posterior breadth: males (median $=13.6, \mathrm{n}=2$ ), females (median $=11.5, \mathrm{n}=6$ ) $, U=0.000, z=-2.012, p=0.044, r=0.711$. Additionally, this dimension provided no overlap in measurements between both sexes.

On the second metacarpal:

- MC2 maximum length: males (median $=66.7, \mathrm{n}=8$ ), females (median = 61.5, $\mathrm{n}=11$ ), $U=11.500, z=-2.685, p=0.007, r=0.616$

- MC2 proximal medio-lateral breadth: males (median $=18.6, \mathrm{n}=5$ ), females (median $=16, \mathrm{n}=11$ ), $U=1.000, z=-3.002, p=0.003, r=0.751$

- MC2 midshaft medio-lateral diameter: males (median $=8, \mathrm{n}=9$ ), females (median $=7.2, \mathrm{n}=10$ ), $U=20.000, z=-2.048, p=0.041, r=0.469$

On the third metacarpal:

- MC3 proximal antero-posterior breadth: males (median $=16.9, \mathrm{n}=2$ ), females (median = 15.7, $\mathrm{n}=7$ ) $, U=0.000, z=-2.058, p=0.040, r=0.683$

- MC3 distal antero-posterior breadth: males (median = 13.9, $\mathrm{n}=7$ ), females (median $=13, \mathrm{n}=7$ ) $, U=0.000, z=-2.084, p=0.037, r=0.557$

Neither of these dimensions yielded overlapping ranges between sexes. 
On the fifth metacarpal:

- MC5 maximum length: males (median $=55, \mathrm{n}=5$ ), females (median $=$ $48, \mathrm{n}=9), U=5.000, z=-2.333, p=0.020, r=0.624$

- MC5 midshaft medio-lateral diameter: males (median $=7.3, \mathrm{n}=5$ ), females (median $=6.7, \mathrm{n}=9$ ) $, U=4.000, z=-2.506, p=0.012, r=0.670$

On the first proximal phalanx:

- PP1 proximal medio-lateral breadth: males (median $=16.2, \mathrm{n}=4$ ), females (median $=14.6, \mathrm{n}=8$ ) $, U=0.000, z=-2.722, p=0.006, r=0.786$

- PP1 proximal antero-posterior breadth: males (median $=6.2, \mathrm{n}=4$ ), females (median $=5.3, \mathrm{n}=8$ ), $U=0.000, z=-2.000, p=0.046, r=0.577$

- PP1 midshaft medio-lateral diameter: males (median $=9.3, \mathrm{n}=4$ ), females (median = 8.2, $\mathrm{n}=8$ ), $U=0.000, z=-2.732, p=0.006, r=0.789$

- PP1 midshaft medio-lateral diameter: males $($ median $=11.7, \mathrm{n}=2)$, females (median $=10.7, \mathrm{n}=6), U=3.500, z=-2.134, p=0.033, r=0.754$

Additionally, the first two of these dimensions had no overlap in ranges.

\section{() Pelvis}

From the measurements that could be taken, three dimensions were significantly sexually dimorphic, with the strongest dimorphism observed in the acetabulum vertical diameter. All results can be found in Appendix 22.

- Os coxal (innominate) maximum length: males (median $=221, \mathrm{n}=2$ ), females (median $=188, \mathrm{n}=10$ ) $, U=.000, z=-2.152, p=0.031, r=0.621$. This dimension yielded no overlap in measurements between sexes.

- Acetabulum vertical diameter: males (median $=53.2, \mathrm{n}=9$ ), females (median $=45.3, \mathrm{n}=14$ ) $, U=2.000, z=-3.843, p=0.000, r=0.801$

- Sciatic notch-acetabular index: males (median $=38.1, \mathrm{n}=6)$, females (median $=53.3, \mathrm{n}=12$ ), $U=13.500, z=-2.108, p=0.035, r=0.497$ 


\section{- Lower limbs}

Thirteen dimensions demonstrated statistically significant sexual dimorphism, with the femoral head diameter and tibial midshaft anteroposterior diameter producing the strongest results. Appendix 23 includes the results from all lower limb bones.

The femur yielded six significantly dimorphic measurements:

- Femur maximum head diameter: males (median $=46.3, \mathrm{n}=7$ ), females ( median $=40, \mathrm{n}=14$ ), $U=4.000, z=-3.368, p=0.001, r=0.735$

- Femur maximum length: males (median $=451.5, \mathrm{n}=6$ ), females (median $=408.5, \mathrm{n}=12$ ), $U=10.000, z=-2.436, p=0.015, r=0.574$

- Femur midshaft circumference: males (median $=90, \mathrm{n}=5$ ), females $($ median $=81.3, \mathrm{n}=16), U=15.000, z=-2.074, p=0.038, r=0.453$

- Femur midshaft mediolateral diameter: males (median=26.6, $\mathrm{n}=10$ ), females (median=25, $\mathrm{n}=15$ ), $U=21.500, z=-2.975, p=0.003, r=0.595$

- Femur midshaft anteroposterior diameter: males (median=28.3, $\mathrm{n}=$ 10), females (median $=26.5, \mathrm{n}=15$ ) $, U=35.000, z=-2.223, p=0.026$, $r=0.445$

- Femur bicondylar width: males (median $=83, \mathrm{n}=5$ ), females (median = $72, \mathrm{n}=7), U=4.500, z=-2.115, p=0.034, r=0.611$

Interestingly, the greater to lesser trochanter length, part of Purkait's triangle method (see section 2.3.5) was found not to be statistically significant. Moreover, the ranges overlap nearly completely, with a female range of 46.6 to 77.7 , and a male range of 44.6 to 65.9 . Although the sample was low, the means also differed markedly from these in a recent study (Brown et al. 2007) which reported means of $48.83 \pm 4.21$ for females and $45.95 \pm 3.93$ for males, compared to $55.6 \pm 10.45$ for Tisza females and $56.9 \pm 9$ for Tisza males. This casts doubt on the usefulness of this measurement as a sex indicator. 
Statistically significant sexual dimorphism was achieved by six dimensions of the tibia:

- Tibia maximum length: males (median $=369, \mathrm{n}=8$ ), females (median = 333, $\mathrm{n}=12$ ), $U=11.000, z=-2.856, p=0.004, r=0.639$

- Tibia proximal breadth: males (median $=79, \mathrm{n}=3$ ), females (median = 67.7, $\mathrm{n}=9$ ), $U=0.000, z=-2.501, p=0.012, r=0.722$. This dimension provided no overlap in the measurements ranges between both sexes.

- Tibia circumference at nutrient foramen: males (median=96.5, $n=9$ ), females (median =84.5, $\mathrm{n}=14$ ) $, U=18.500, z=-2.806, p=0.005, r=0.585$

- Tibia midshaft mediolateral diameter: males (median $=22, \mathrm{n}=10$ ), females (median $=20, \mathrm{n}=11$ ) $, U=18.500, z=-2.579, p=0.010, r=0.563$

- Tibia midshaft anteroposterior diameter: males (median=33.3, $\mathrm{n}=10$ ), females (median $=27.9, \mathrm{n}=11), U=2.000, z=-3.739, p=0.000, r=0.816$

- Tibia distal breadth: males $($ median $=53, n=5)$, females $($ median $=43$, $\mathrm{n}=9), U=5.000, z=-2.336, p=0.019, r=0.624$

No statistical significance was found in patellar measurements and only one measurement obtained statistical significance in the fibula:

- Fibula maximum length: males (median $=360.5, \mathrm{n}=2$ ), females $($ median $=314.5, \mathrm{n}=6), U=0.000, z=-2.000, p=0.046, r=0.707$

\section{(๑) Feet}

Nine measurements provided statistically significant results for sexual dimorphism, including two measurements each for the talus and the calcaneus, four measurements from the metatarsals and one measurement for the phalanges. All results are provided in Appendix 24. 
The calcaneus provided two sexually dimorphic dimensions, both of them showing no overlap between both sexes in the ranges of their measurements:

- Calcaneus maximum length: males (median $=85, \mathrm{n}=5$ ), females (median $=70.9, \mathrm{n}=6$ ), $U=0.000, z=-2.751, p=0.006, r=0.829$

- Calcaneus maximum height: males (median $=47.2, \mathrm{n}=4$ ), females (median $=41.7, \mathrm{n}=4$ ), $U=0.000, z=-2.309, p=0.021, r=0.816$

The talus produced two sexually dimorphic dimensions:

- Talus maximum width: males (median $=47.5, \mathrm{n}=3$ ), females (median $=$ $39, \mathrm{n}=5), U=0.000, z=-2.236, p=0.025, r=0.791$

- Talus maximum length: males (median $=61.5, \mathrm{n}=5$ ), females (median $=50, \mathrm{n}=5$ ) $, U=0.000, z=-2.611, p=0.009, r=0.826$. In addition to being strongly significant, this dimension also provided no overlap between the measurement ranges of both sexes.

The metatarsals and phalanges provided five sexually dimorphic dimensions:

- First metatarsal maximum length: males (median $=60, n=7$ ), females ( median $=57.5, \mathrm{n}=7$ ), $U=0.000, z=-2.441, p=0.015, r=0.652$

- Second metatarsal maximum length: males (median $=72.5, \mathrm{n}=3$ ), females (median $=66, \mathrm{n}=5$ ) $, U=0.000, z=-2.249, p=0.024, r=0.795$

- Fourth metatarsal proximal mediolateral breadth: males (median = 14.7, $\mathrm{n}=3$ ),females(median=13, $\mathrm{n}=3$ ), $U=0.000, z=-1.964, p=0.050, r=0.802$

- Fifth metatarsal maximum length: males (median= 74.1, $\mathrm{n}=2$ ), females (median $=64.6, \mathrm{n}=6), U=0.000, z=-2.000, p=0.046, r=0.707$

- First proximal phalanx midshaft anteroposterior diameter: males ( median $=9.7, \mathrm{n}=3$ ), females (median $=8.5, \mathrm{n}=4$ ), $U=0.000, z=-2.121$, $p=0.034, r=0.802$.

The last three of these dimensions provided no overlap between sexes in the ranges of their measurements. 
Although all these results should be considered with great caution as this sample was limited in numbers and the sexed base group was itself estimated from cranial and pelvic morphology, this exhaustive analysis has confirmed the presence of well-known sexual dimorphic features such as glenoid cavity length acetabulum diameter, humerus and femur heads, as well as less recognized sexually dimorphic measurements, for example based on the hands and feet. Calcaneus and talus lengths are rarely used yet their ranges for both sexes did not overlap at all, and these robust bones are often recovered in archaeological settings.

\section{- Tisza baseline dataset and comparisons with other populations}

A new baseline dataset was created from all the results presented above and in the relevant appendices. This is based on the bone measurements from the 41 Late Neolithic individuals of the Tisza Culture who could be sexed based on standard cranial and pelvic morphological traits, including 19 males and 22 females (see Appendix 11). All dimensions significantly dimorphic at $p \leq 0.05$ level were included while unsuccessful dimensions were discarded. Sample numbers, minimum and maximum measurements as well as means and their standard deviations were given separately for both sexes for each dimension. Differences in levels of significance were also indicated, with dimensions of statistically significant sexual dimorphism at $p \leq 0.01$ level given in bold, while those with the strongest significance of $p \leq 0.001$ were also highlighted in yellow. This new baseline dataset is presented in table 5.7 overleaf.

This dataset can be utilised on single individuals or stray finds of similar Neolithic populations when no morphological sex indicators are present, or can be employed for comparison with other populations. It should be used with caution however due to the low sample numbers and the inherent lack of known sex in such ancient remains. It would also be advisable to look at the ranges of measurements rather than means only to ensure as accurate an estimate as possible. 


\begin{tabular}{|c|c|c|c|c|c|c|c|c|c|c|}
\hline \multirow{2}{*}{ Dimension / index } & \multicolumn{5}{|c|}{ Females } & \multicolumn{5}{|c|}{ Males } \\
\hline & $n$ & Min. & Max. & Mean & S.D & $n$ & Min. & Max. & Mean & S.D. \\
\hline Skull maximum height & 5 & 132 & 140 & 137.2 & \pm 3.6 & 3 & 143 & 152 & 146.7 & \pm 4.7 \\
\hline Cranial breadth-height index & 4 & 76.8 & 104.5 & 94.6 & \pm 12.4 & 3 & 109 & 116.9 & 112.3 & \pm 4.1 \\
\hline Minimum frontal breadth & 11 & 89 & 97 & 92.9 & \pm 3.2 & 8 & 93 & 106 & 98.8 & \pm 3.9 \\
\hline Mastoid length & 17 & 26 & 33 & 30 & \pm 2.3 & 10 & 26 & 39 & 33.6 & \pm 3.9 \\
\hline Nasal height & 8 & 39 & 50 & 44.7 & \pm 3.5 & 7 & 47 & 56 & 50 & \pm 3.2 \\
\hline Palatal breadth & 6 & 32 & 35 & 33.9 & \pm 1 & 9 & 33 & 39 & 36.2 & \pm 1.7 \\
\hline Mandible max. length & 12 & 61 & 77 & 72.1 & \pm 4.4 & 10 & 72 & 84 & 78.8 & \pm 4.2 \\
\hline Mandible ramus height & 13 & 47 & 61 & 52.8 & \pm 4 & 7 & 52 & 67 & 58 & \pm 5.4 \\
\hline Manubrium length & 4 & 39 & 52 & 45.2 & \pm 5.7 & 4 & 52 & 57 & 54 & \pm 2.2 \\
\hline Clavicle length & 10 & 121 & 141 & 130.5 & \pm 6.4 & 4 & 143 & 160.5 & 151.4 & \pm 7.6 \\
\hline Clavicle midshaft circumference & 9 & 34 & 40 & 36.4 & \pm 2.2 & 7 & 35 & 45 & 39.9 & \pm 3.4 \\
\hline Glenoid cavity length & 15 & 32 & 39 & 34.4 & \pm 1.7 & 10 & 36 & 42 & 39.8 & \pm 2.1 \\
\hline Humerus head diameter & 16 & 34 & 44 & 39.4 & \pm 2.1 & 11 & 39 & 52 & 46.6 & $\pm \mathbf{3 . 7}$ \\
\hline Humerus max. length & 15 & 246 & 309 & 283.8 & \pm 18.4 & 9 & 290 & 342 & 321.1 & \pm 17 \\
\hline Humerus midshaft min. diameter & 15 & 15 & 21 & 17.8 & \pm 1.7 & 11 & 18 & 22 & 19.4 & \pm 1.4 \\
\hline Humerus midshaft max. diameter & 15 & 17 & 22 & 19.2 & \pm 1.9 & 11 & 18 & 24 & 21.41 & \pm 1.8 \\
\hline Humerus epicondylar width & 14 & 52 & 60 & 55.5 & \pm 2.2 & 9 & 58 & 71 & 64.1 & $\pm \mathbf{3 . 8}$ \\
\hline Radius head diameter & 12 & 17.9 & 21.4 & 19.9 & \pm 1.1 & 4 & 23 & 24.5 & 23.8 & \pm 0.6 \\
\hline Radius max. length & 13 & 185 & 242 & 212.4 & \pm 15.5 & 7 & 214 & 264 & 242.9 & \pm 16.4 \\
\hline Radius midshaft AP diameter & 12 & 9 & 11.7 & 10.5 & \pm 0.8 & 6 & 10 & 13 & 11.7 & \pm 1.1 \\
\hline Ulna max. length & 11 & 204.5 & 253 & 233 & \pm 14.7 & 7 & 257 & 278 & 269.4 & \pm 8.4 \\
\hline $\begin{array}{l}\text { Prox. ulna inferior medial trochlear } \\
\text { notch width }\end{array}$ & 9 & 11.8 & 15.4 & 13.8 & \pm 1.2 & 2 & 16.9 & 19.3 & 18.1 & \pm 1.7 \\
\hline MC1 max. length & 11 & 35 & 45 & 40.8 & \pm 3 & 5 & 42 & 51 & 46.7 & \pm 3 \\
\hline MC1 prox ML breadth & 7 & 13 & 15 & 14.1 & \pm 0.9 & 5 & 15 & 18 & 16.3 & \pm 1 \\
\hline MC1 midshaft AP diameter & 11 & 6.8 & 8.2 & 7.3 & \pm 0.5 & 3 & 8.2 & 9.2 & 8.8 & \pm 0.5 \\
\hline MC1 distal ML breadth & 6 & 13 & 15 & 14 & \pm 0.7 & 5 & 14 & 18 & 16.1 & \pm 1.2 \\
\hline MC1 distal AP breadth & 6 & 10.7 & 12.7 & 11.7 & \pm 0.8 & 2 & 13.2 & 13.9 & 13.6 & \pm 0.5 \\
\hline MC2 max. length & 11 & 55 & 69 & 61.5 & \pm 4 & 8 & 61 & 72 & 67.1 & \pm 3.4 \\
\hline MC2 prox. ML breadth & 11 & 14.5 & 18 & 15.9 & \pm 1.1 & 5 & 17.2 & 19 & 18.4 & \pm 0.7 \\
\hline MC2 midshaft ML diameter & 10 & 7 & 9 & 7.5 & \pm 0.7 & 9 & 8 & 10 & 8.4 & \pm 0.7 \\
\hline MC3 prox. AP breadth & 7 & 13.8 & 16.3 & 15.5 & \pm 0.9 & 2 & 16.5 & 17.2 & 16.9 & \pm 0.5 \\
\hline MC3 distal AP breadth & 7 & 11.9 & 13.3 & 12.7 & \pm 0.5 & 2 & 13.7 & 14.1 & 13.9 & \pm 0.3 \\
\hline MC5 max. length & 9 & 43.2 & 54 & 48.5 & \pm 3.8 & 5 & 50.1 & 59.5 & 54.5 & \pm 3.7 \\
\hline MC5 midshaft ML breadth & 9 & 6.5 & 7.2 & 6.8 & \pm 0.2 & 5 & 7 & 9 & 7.7 & \pm 0.9 \\
\hline $\begin{array}{l}\text { Hand 1st prox.phalanx prox. ML } \\
\text { breadth }\end{array}$ & 8 & 13.2 & 15.5 & 14.4 & \pm 0.8 & 4 & 15.7 & 16.5 & 16.2 & \pm 0.4 \\
\hline $\begin{array}{l}\text { Hand 1st prox.phalanx prox. AP } \\
\text { breadth }\end{array}$ & 6 & 9.7 & 11.2 & 10.6 & \pm 0.6 & 2 & 11.5 & 11.9 & 11.7 & \pm 0.3 \\
\hline Hand 1st prox.phalanx midshaft & & & & & & & & & & \\
\hline $\begin{array}{l}\text { ML diameter } \\
\text { Hand 1st prox.phalanx midshaft }\end{array}$ & 8 & 7 & 9 & 8.15 & \pm 0.5 & 4 & 9 & 10 & 9.4 & \pm 0.5 \\
\hline AP diameter & 8 & 5 & 7 & 5.5 & \pm 0.6 & 4 & 6 & 7 & 6.4 & \pm 0.4 \\
\hline Coxal max. length & 10 & 179 & 202 & 187.9 & \pm 8 & 2 & 218 & 224 & 221 & \pm 4.2 \\
\hline Acetabulum vertical diameter & 14 & 41.2 & 52.2 & 45.5 & \pm 3.1 & 9 & 50.9 & 62.5 & 55.1 & \pm 4 \\
\hline Sciatic notch-acetabular index & 12 & 34.2 & 64.8 & 51.1 & \pm 8.3 & 6 & 31.3 & 52.1 & 40.8 & \pm 8.7 \\
\hline Femur head diameter & 14 & 37 & 45 & 40.3 & \pm 2.1 & 7 & 42 & 52 & 47.3 & \pm 3.6 \\
\hline Femur max. length & 12 & 348 & 443 & 405.63 & \pm 21.5 & 6 & 403 & 499 & 450.8 & \pm 31.3 \\
\hline Femur midshaft circumference & 16 & 74 & 90 & 81.53 & \pm 3.9 & 5 & 81 & 95 & 87.9 & \pm 5.7 \\
\hline Femur midshaft ML diameter & 15 & 21 & 29 & 24.9 & \pm 1.6 & 10 & 25 & 29 & 26.8 & \pm 1.4 \\
\hline Femur midshaft AP diameter & 15 & 22 & 28 & 25.9 & \pm 2 & 10 & 24 & 36 & 28.5 & \pm 3.3 \\
\hline Femur bicondylar width & 7 & 69 & 75 & 71.8 & \pm 2.3 & 5 & 70 & 90.5 & 82 & \pm 7.9 \\
\hline Tibia max. length & 12 & 303 & 359 & 332.8 & \pm 16.4 & 8 & 325 & 385 & 364.4 & \pm 18.9 \\
\hline Tibia prox. breadth & 9 & 64 & 70 & 67.4 & \pm 1.8 & 3 & 74 & 85 & 79.17 & \pm 5.3 \\
\hline $\begin{array}{l}\text { Tibia circumference at nutrient } \\
\text { foramen }\end{array}$ & 14 & 74 & 95 & 84.5 & \pm 5.7 & 9 & 82 & 104 & 95 & \pm 7.3 \\
\hline Tibia midshaft ML diameter & 11 & 18 & 22 & 19.9 & \pm 1.2 & 10 & 18 & 26 & 22 & \pm 2.1 \\
\hline Tibia midshaft AP diameter & 11 & 25 & 30 & 27.5 & \pm 1.3 & 10 & 28 & 37 & 32.6 & \pm 2.4 \\
\hline Tibial distal breadth & 9 & 40 & 48.5 & 43.1 & \pm 3.1 & 5 & 43.6 & 63 & 53.1 & \pm 7.8 \\
\hline Fibula max. length & 6 & 298 & 325 & 314 & \pm 10.2 & 2 & 360 & 361 & 360.5 & \pm 0.7 \\
\hline Calcaneus max. length & 6 & 68.5 & 76.5 & 71.6 & \pm 3.1 & 5 & 82 & 88 & 84.4 & \pm 2.5 \\
\hline Calcaneus max. height & 4 & 37.7 & 45 & 41.5 & \pm 3 & 4 & 45.3 & 54 & 48.4 & \pm 3.9 \\
\hline Talus max. width & 5 & 29 & 46 & 37.4 & \pm 6 & 3 & 46 & 51 & 48.1 & \pm 2.9 \\
\hline Talus max. length & 5 & 49 & 53 & 50.5 & \pm 1.5 & 5 & 60 & 64 & 62.2 & \pm 1.9 \\
\hline MT1 max. length & 7 & 53 & 60 & 57.2 & \pm 2.6 & 7 & 59 & 68 & 61.8 & \pm 3.4 \\
\hline MT2 max. length & 5 & 65 & 72 & 68 & \pm 3.2 & 3 & 72 & 79 & 74.6 & \pm 3.8 \\
\hline MT4 prox. ML breadth & 3 & 13 & 13 & 12.9 & \pm 0.3 & 3 & 14 & 15 & 14.7 & \pm 0.5 \\
\hline MT5 max. length & 6 & 61.6 & 68 & 64.7 & \pm 2.9 & 2 & 70.7 & 77.5 & 74.1 & \pm 4.8 \\
\hline $\begin{array}{l}\text { Foot 1st prox. phalanx midshaft AP } \\
\text { diameter }\end{array}$ & 4 & 8 & 9 & 4 & \pm 0.7 & 3 & 10 & 10 & 9.8 & \pm 0.2 \\
\hline
\end{tabular}

Table 5.7: Baseline dataset for osteometric sex estimates of populations from the Tisza Culture All significant at $p \leq 0.05$ level. In bold, measurements with $p \leq 0.01$. Highlighted yellow, significance $p \leq 0.001$ 
This new baseline dataset was then utilised to re-examine the measurements of twelve previously unsexed individuals and two individuals sexed as indeterminate from the Tisza sample, as presented in Appendix 25. This yielded sex estimates for all but one case (HGO-78), adding a total of nine females (HGO03, HGO-11, HGO-13, HGO-32, HGO-47, HGO-75, HGO-76, HGO-51 and HKS-02) and four males (HGO-16, HGO-20, HGO-74 and HGO-82) to the sexed group of this sample. HGO-27 had been sexed as a probable male from morphology. However, as all the measurements suggest strongly that this individual was indeed a female, it was decided to record this person as indeterminate. Therefore overall, it was possible to estimate that the remains of these Late Neolithic populations included 22 males and 31 females.

Comparing the new baseline dataset presented in table 5.7 to Appendix 1 confirmed the influence of population variability on bone dimensions. This was particularly the case with cranial measurements. Skull maximum height was higher in this sample, and all females would have been classified as males based on current published data. Mastoid length was also higher, whereas nasal height was lower. Mandibular length was much lower in this sample and all male individuals would have been classified as females following published standards.

Measurements of both Körös females with complete skeletons corresponded well to the new dataset of table 5.7 with very few exceptions (such as two metacarpals in one of the females). One notable exception was the skull maximum height at $130 \mathrm{~mm}$ for the largest of the two females, and $127 \mathrm{~mm}$ for one of the males. These measurements were below that of the Tisza population, and more alike to the mostly modern populations available in Appendix 1.

Unfortunately, the Alföld Linear Pottery culture (AVK) mature female discovered at Ecsegfalva 23, Hungary did not provide any cranial measurements (Guba et al. 2007). It would have very interesting to see if the cranial height and other cranial measurements of this Middle Neolithic female closer to the Early 
Neolithic or the Late Neolithic samples in this study. However, this Middle Neolithic individual had a maximum ulnar length of $251 \mathrm{~mm}$ and a maximum humeral length of $300 \mathrm{~mm}$, with a maximum midshaft diameter of $18.5 \mathrm{~mm}$. These upper limb measurements, like those of the Körös females in this study, all fitted within the ranges provided for females in the Tisza baseline dataset.

Comparable data for skeletal robusticity from femoral midshaft circumference was also available from Northeastern Hungary for the Neolithic (Ubelaker et al. 2006, 23 males and 15 females), Copper Age (Ubelaker \& Pap 2009, 35 males and 33 females) and Iron Age (Ubelaker \& Pap 1998, 29 males and 44 females). There was very little difference in means, mostly linked to wider ranges. The Tisza males appeared slightly more gracile although this might be due to the low sample number, while the females seemed slightly more robust than the other samples. Sexual dimorphism in robusticity in the Tisza sample was less marked than in the other samples, with less difference in the means of both sexes.

Figure 5.4: Femoral midshaft circumference ranges and means (in $\mathrm{mm}$ )

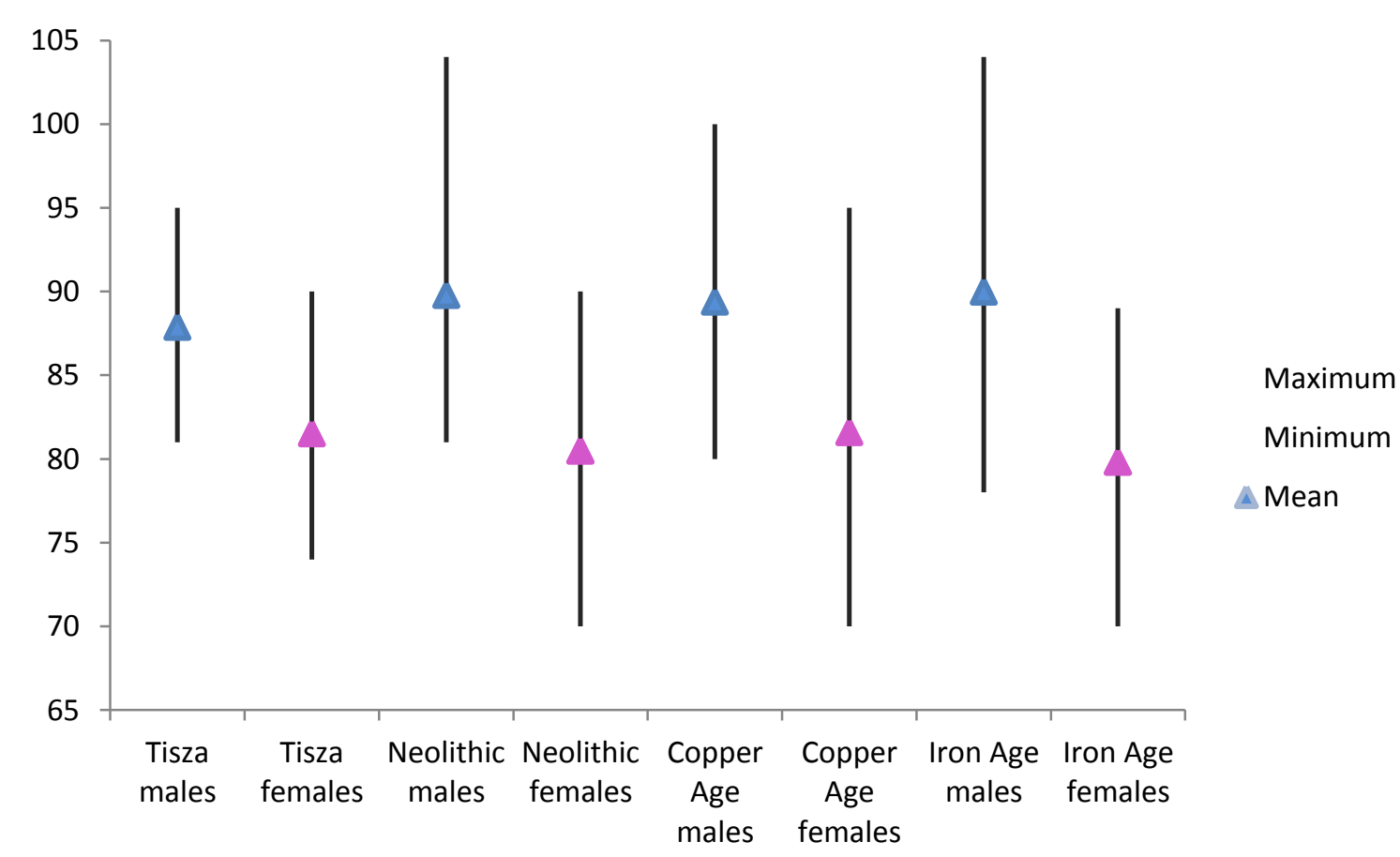




\subsubsection{Age estimates}

Epiphyseal fusion does not end with adolescence but continues throughout the twenties until the individual reaches skeletal maturation during the early thirties. Although there are much fewer elements to look for than in adolescents, young adults should therefore provide enough information for general ageing assuming most of the skeleton is recovered, at least to within a decade if not more precisely. Appendix 4 provides all information required on ageing from bone development and fusion, while methodologies have been discussed in Chapter 3. Seventeen young adults from the Tisza Culture and a further Neolithic young adult were identified based mostly on the state of fusion of their skeletal remains. Thirteen of them were aged more precisely to their late teens - early twenties.

However, once the skeleton is fully mature, it becomes much more difficult to find reliable and accurate age markers, as was described in section 3.3. Moreover, most individuals presented incomplete and fragmentary remains, making age estimations more difficult and less accurate. Some remains were so damaged that eleven individuals could not even be determined as being juvenile (adolescent) or adult. The Köros sample was the worst affected, mostly caused by the lack of postcranial remains, with only one individual out of twelve categorised as an old adult, with two females and six males only categorised as adults. In addition, two females and one male could not be aged at all. Thankfully, the Late Neolithic sample fared better with three mature females and eleven old adults identified, including seven females and four males. Seven females and eight males from the Tisza culture could only be distinguished as adults, as well as one Neolithic female.

Several methods were used to obtain the age estimates, each described in Chapter 3. Skeletal and dental development was considered first as the most reliable indication of age for adults. The pubic symphysis (Todd 1920; Brooks \& Suchey 1990) and the auricular surface of the ilium (Lovejoy et al. 1985) were 
also observed when available, although these results should only be used with caution as explained in section 3.3.4, and used to provide age categories rather than chronological age. Results of all age estimations for all adults or unknown individuals from this Neolithic study sample are presented in Appendix 26. The proportions of age categories of all the individuals analysed in this Neolithic sample are shown in figure 5.5 below.

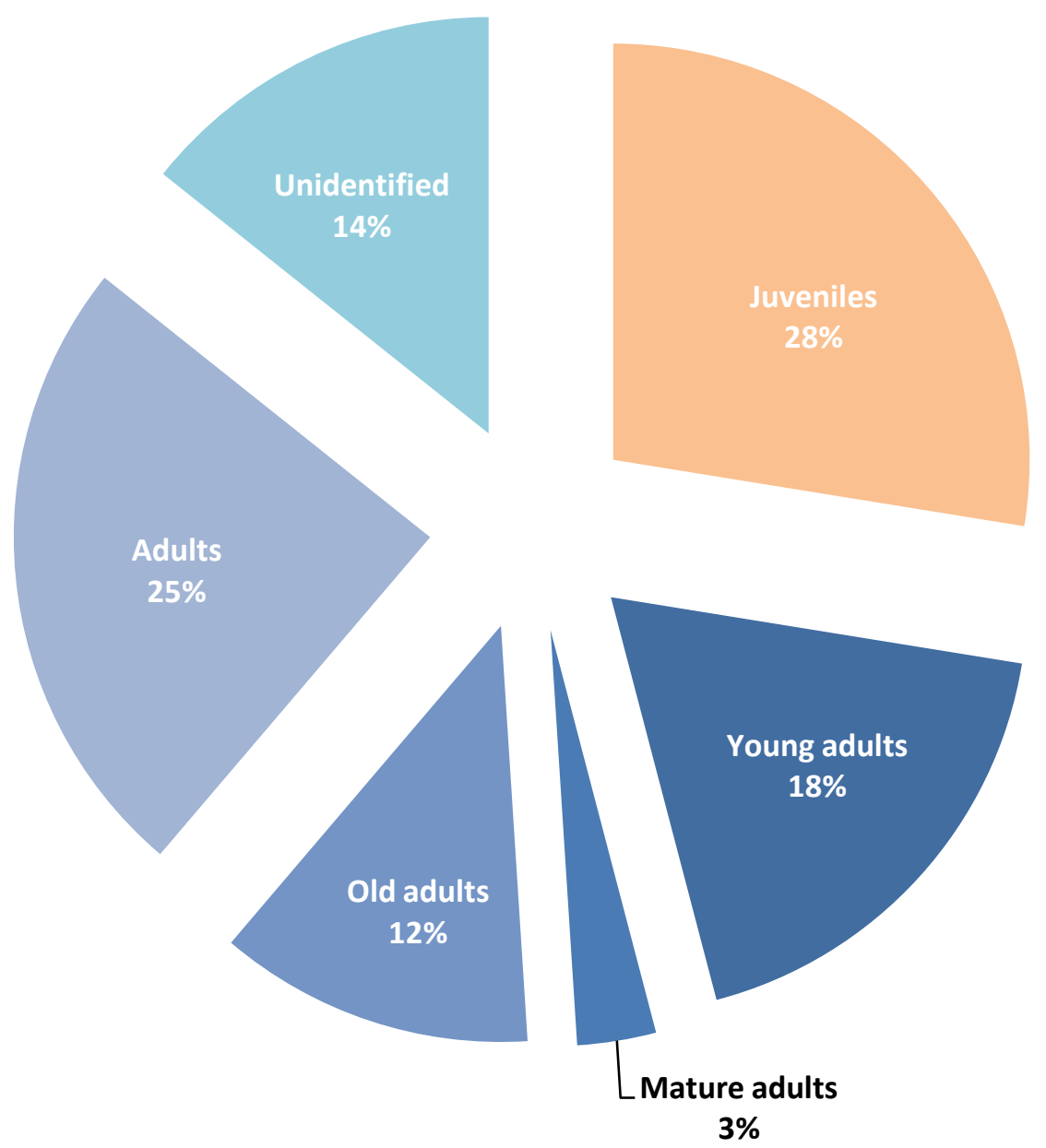

Figure 5.5: Age group distribution of this Neolithic sample

Dental wear was also recorded following the methods of Brothwell (1963, 1968) and Smith (1984), chosen as they do not require the complete dentition to be present, and seven variables of the acetabular fossa (Rissech et al. 2006) were also graded. 


\section{( Age estimation from the acetabular fossa}

All results obtained from the acetabular variables are presented in Appendix 27. They were further investigated using the SPSS (version 15) statistical package. The Kappa Measure of Agreement value between age estimates using the three adult sub-categories gave a value of only 0.294 demonstrating little agreement, and failing to achieve significance with $p=0.057$. The cross tabulation is presented in table 5.8 below. This does not necessarily signify that the method is not accurate, but rather that the age estimates from the original study population cannot be applied to this Neolithic population. As no documented collection from such ancient populations is likely to ever be available, new calculations based on the presence of these variables on individuals of known age in similar populations will not be possible and thus this method will not be utilised further in this doctoral research on Neolithic populations.

\begin{tabular}{|cl|c|c|c|c|}
\hline \multirow{2}{*}{$\begin{array}{c}\text { Original age estimate from dental } \\
\text { and skeletal development }\end{array}$} & \multicolumn{4}{|c|}{ Age estimate from acetabulum } \\
\cline { 2 - 6 } YA & Count & 7 & 2 & 0 & 9 \\
\cline { 2 - 6 } & \% within Original estimate & $77.8 \%$ & $22.2 \%$ & $0.0 \%$ & $100 \%$ \\
MA & Count & 2 & 1 & 0 & 3 \\
\cline { 2 - 6 } OA & \% within Original estimate & $66.7 \%$ & $33.3 \%$ & $0.0 \%$ & $100 \%$ \\
& \% within Original estimate & 1 & 3 & 2 & 6 \\
Total & Count & $10.7 \%$ & $50.0 \%$ & $33.3 \%$ & $100 \%$ \\
& \% within Original estimate & $55.6 \%$ & $33.3 \%$ & $11.1 \%$ & $100 \%$ \\
\hline
\end{tabular}

Table 5.8: Cross tabulation of age sub-group estimates from dental and skeletal development and from the means of estimates based on the seven acetabular fossa variables (Rissech et al. 2006) $\mathrm{YA}=$ young adult, $\mathrm{MA}=$ mature adult, $\mathrm{OA}=$ old adult 


\section{- Age estimates from dental wear}

Statistical analyses were also undertaken on both methods of recording tooth wear, with all results presented in Appendix 28.

The relationship between Smith tooth wear grades and age estimates obtained from this Neolithic sample was investigated using Spearman's Rank Order Correlation (rho), preliminary analyses having confirmed that results were not normally distributed. There was a very strong positive correlation between age and dental wear recorded in this way, with a rho value of $0.832, \mathrm{n}=20$, and $p$ $<0.0005$. This is of particular interest for this sample and any similar populations as this method can be applied to loose teeth easily, and can be used on any other teeth than the molars. Cross tabulation indicated that in this small sample, grades 2, 3 and 4 were exclusively found in the young adults, while grades 7 and 8 were observed exclusively on old adults. Grades 5 and 6 were found across the age groups, although it is also interesting to note that all mature adults only showed these latter two scores.

Brothwell's widely used grading of molar attrition was also found to strongly correlate with age estimates in this sample using Spearman's Rank Order Correlation (rho), with a rho value of $0.792, \mathrm{n}=29$, and $p<0.0005$. However, there were more overlaps between the groups. Two categories were of particular interest to use in this and similar sample when no other age indicators can be observed: the 17-25 years pattern of wear was found exclusively in the young adults of this Neolithic population, and the $45+$ years dental wear was only observed in old adults. The two middle categories were of less use as they could be seen in all three groups.

Utilising Smith dental wear grades 2, 3, 4 with Brothwell 17-25 dental wear pattern as an indicator of young adult age, and Smith dental wear grades 7, 8 with Brothwell 45+ years category indicating an old adult, three adults of the 
Körös sample could be reclassified, one as a young adult (HKO-02) and two as old adults (DOL-02 and HKO-05). One of the adults of the Tisza sample (HGO46) could also be re-categorised as an old adult.

A similar extensive dental attrition was found on ten individuals from VésztőMágor and six individuals from Kisköre-Gát, with a rate of tooth wear faster at both sites than more recent cultures (Molnár \& Molnár 1985). Although these sites have been attributed to the Tisza Culture, they were only dated to the first half of the third millennium $\mathrm{BC}$, so their dating remains uncertain.

\subsubsection{Stature estimates}

The remains of the population studied in this thesis were too damaged and too fragmentary for anatomical methods of living stature reconstruction. Although it was demonstrated earlier (see section 4.3) that Sjøvold's was currently the best method for estimating stature from long bone lengths, a comparison with Trotter and Gleser's method was undertaken to ascertain if the same overall differences appeared in this population, and to confirm that the results from Sjøvold's equations should be used to estimate the living stature of this Neolithic European population.

In order to avoid added error potential, only complete long bones with their epiphyses fully fused were utilised. Thirty-six sexed individuals provided at least one maximum length measurement. Only two individuals were dated from the Körös culture. Although their measurements did not differ from the later period, they were not added to the Late Neolithic remains for this study to ensure that all the individuals analysed were both chronologically and geographically related. One of the Late Neolithic individuals was sexed as indeterminate and was therefore removed from the sample. This late Neolithic study group thus included 34 Neolithic individuals: four males and one female from Békés-Povádzug, one male from Hódmezővásárhely-Kökénydomb, and 
seven males and twenty females from Hódmezővásárhely-Gorzsa, and. All measurements available are reproduced in Appendix 29.

All stature estimates were plotted on graphs (figs. 5.6 to 5.9). Disparity between the results was evident and similar for both sexes and for both methods, with differences of more than $10 \mathrm{~cm}$ between certain bones with no regular pattern.

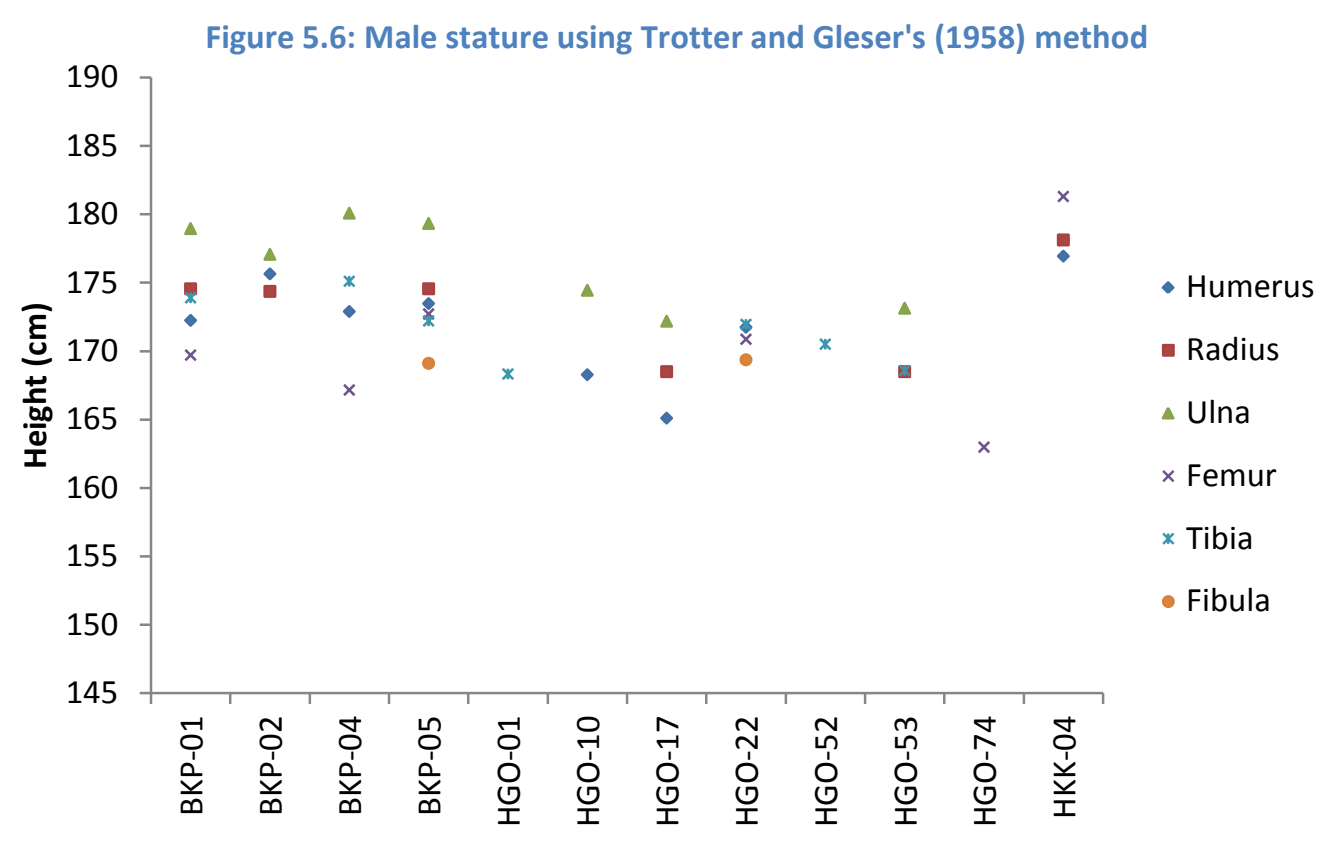

Figure 5.7: Male stature using Sjøvold's (1990) method

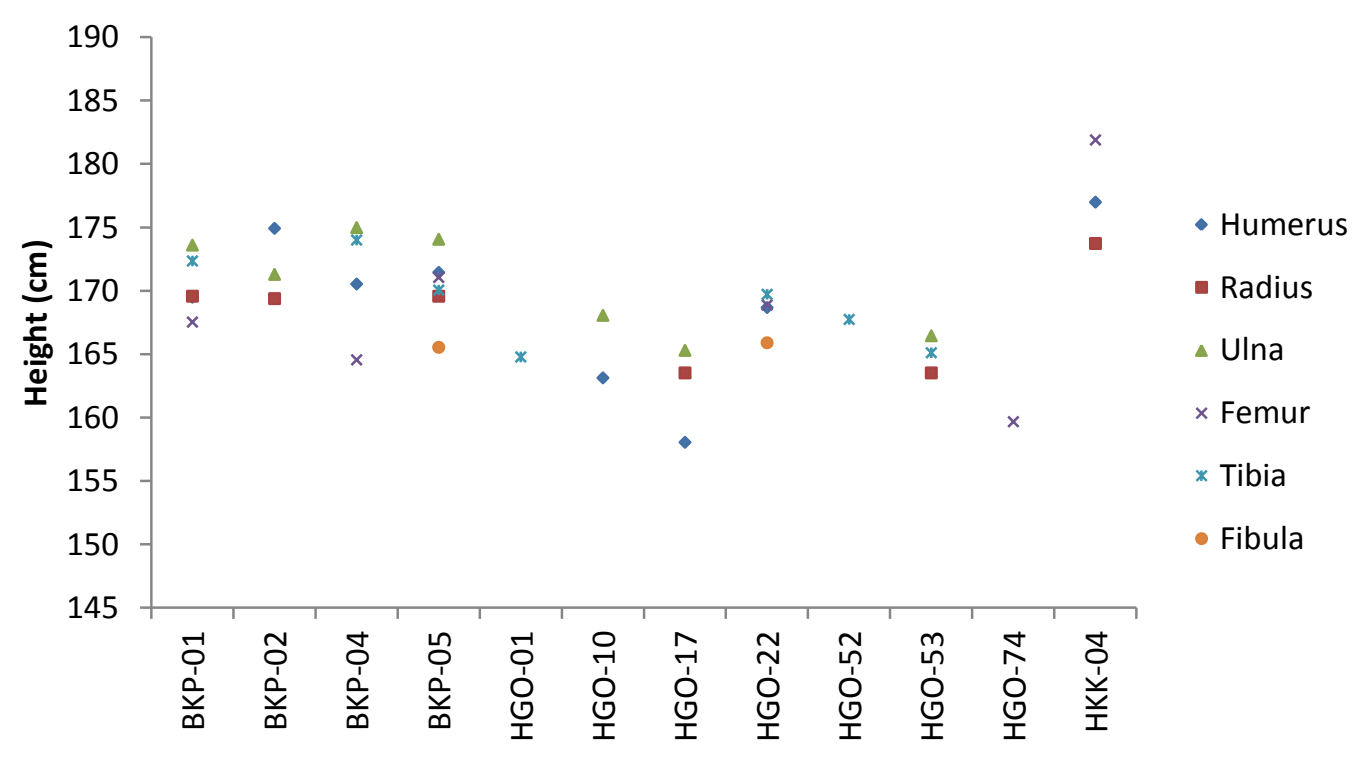


Figure 5.8: Female stature using Trotter and Gleser (1952) method, all bones
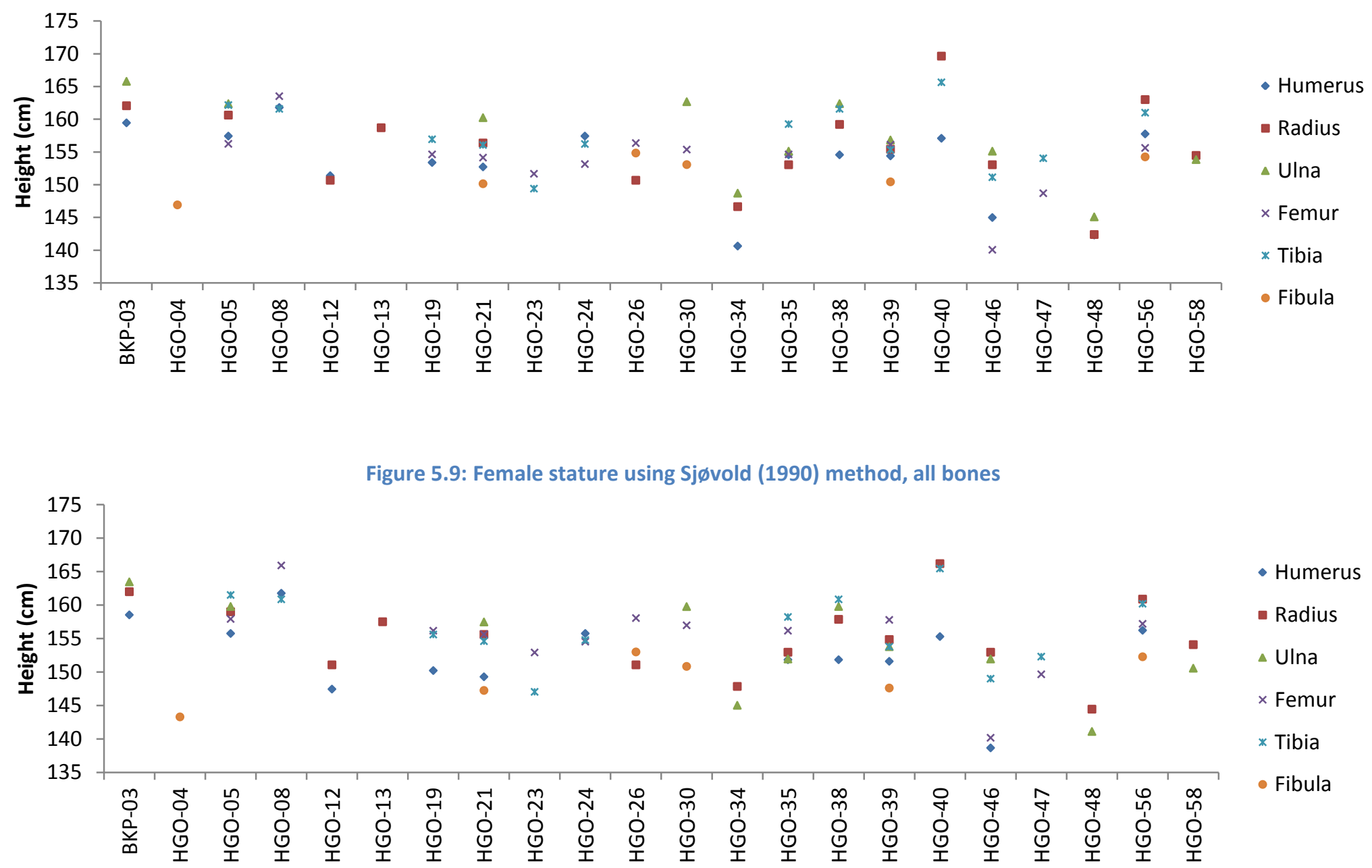
The two bones of the lower arm and of the lower leg were then averaged to compare upper/lower arm and upper/lower leg for individuals who presented at least one measurable bone for each category. The majority (87.5\%) of females had upper arm estimates lower than from the lower arms using Trotter and Gleser's method, and 100\% using Sjøvold's, while the upper leg estimate was lower than from the lower leg in $87.5 \%$ with the first method, but only in $50 \%$ of the cases using Sjøvold's method. Males had similar results with both methods: all provided a higher estimate from the lower arm than from the upper arm, and two out of three produced higher estimates from the lower leg compared to the upper leg. The upper limbs/lower limbs difference in estimates was on average $2.4 \mathrm{~cm}$ for Trotter and Gleser and $2 \mathrm{~cm}$ for Sjøvold in females with no regular pattern, and a difference of $3.8 \mathrm{~cm}$ and $2.1 \mathrm{~cm}$ respectively for males. A comparison of these averages and those of Éry's study (1998, see section 4.3.4 for further details) is given in table 5.9 below.

\begin{tabular}{ccccccc}
\hline & \multicolumn{2}{c}{ Trotter and Gleser } & \multicolumn{2}{c}{ Sjøvold } & \multicolumn{2}{c}{ From Éry } \\
\hline \multirow{3}{*}{$\begin{array}{c}\text { Upper } \\
\text { Limb }\end{array}$} & $\begin{array}{c}\text { Lower } \\
\text { Limb }\end{array}$ & $\begin{array}{c}\text { Upper } \\
\text { Limb }\end{array}$ & $\begin{array}{c}\text { Lower } \\
\text { Limb }\end{array}$ & $\begin{array}{c}\text { Upper } \\
\text { Limb }\end{array}$ & $\begin{array}{c}\text { Lower } \\
\text { Limb }\end{array}$ \\
\hline Males & 173.84 & 169.23 & 169.36 & 166.9 & 165.18 & 164.64 \\
\hline Females & 155.44 & 155 & 153.07 & 154.65 & 153.29 & 153.98 \\
\hline
\end{tabular}

Table 5.9: Stature estimates from the upper and lower limbs

Average stature estimated from all six long bones can be seen in table 5.10 overleaf. All estimates apart from one (the female femur) presented higher stature with Trotter and Gleser's method. Box plots of the height estimates by the two methods for all long bones were also created. They revealed more graphically the difference in estimates on the same bones. The same overaging pattern can be seen clearly in males (fig. 5.10) and in females (fig. 5.11), albeit on a smaller scale in the latter. 
Table 5.10: Summary table for all stature estimations on this sample based on two methods

\begin{tabular}{|c|c|c|c|c|c|c|c|c|c|c|c|c|}
\hline & Long bone & Sex & $\mathbf{N}$ & $\begin{array}{c}\text { Mean } \\
\text { stature }\end{array}$ & SD & Min - Max & & Sex & $\mathbf{N}$ & $\begin{array}{c}\text { Mean } \\
\text { stature }\end{array}$ & SD & Min - Max \\
\hline \multirow{12}{*}{ 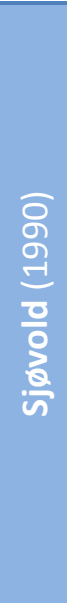 } & \multirow{2}{*}{ Humerus } & $M$ & 8 & 169.17 & \pm 4.89 & $158.06-177$ & \multirow{3}{*}{$\begin{array}{l}\infty \\
\stackrel{2}{2} \\
\text { ? }\end{array}$} & $M$ & 8 & 172.04 & \pm 4.57 & $165.09-176.94$ \\
\hline & & $\mathrm{F}$ & 15 & 150.23 & \pm 4.89 & $132.65-161.76$ & & $\mathrm{~F}$ & 15 & 153.74 & \pm 4.45 & $140.63-163.64$ \\
\hline & \multirow{2}{*}{ Radius } & $M$ & 6 & 168.23 & \pm 5.01 & $163.53-173.74$ & & $\mathrm{M}$ & 6 & 173.19 & \pm 4.66 & $168.49-178.72$ \\
\hline & & $\mathrm{F}$ & 14 & 155.16 & \pm 5.01 & $144.44-166.18$ & \multirow[b]{2}{*}{$=$} & $\mathrm{F}$ & 14 & 155.85 & \pm 4.24 & $142.39-169.64$ \\
\hline & \multirow{2}{*}{ Ulna } & $M$ & 7 & 170.54 & \pm 4.97 & 165.31 - 174.99 & & $M$ & 7 & 176.45 & \pm 4.72 & $172.18-180.08$ \\
\hline & & $\mathrm{F}$ & 10 & 154.41 & \pm 4.97 & $141.11-163.46$ & \multirow[t]{2}{*}{ 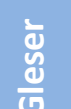 } & $\mathrm{F}$ & 10 & 157.4 & \pm 4.30 & $145.09-165.79$ \\
\hline & \multirow{2}{*}{ Femur } & $M$ & 6 & 168.8 & \pm 4.49 & $159.68-181.09$ & & $M$ & 6 & 170.78 & \pm 3.94 & $162.97-181.30$ \\
\hline & & $\mathrm{F}$ & 13 & 155.43 & \pm 4.49 & $140.17-165.91$ & \multirow[b]{2}{*}{$\frac{5}{10}$} & $\mathrm{~F}$ & 13 & 153.86 & \pm 3.72 & $140.06-163.52$ \\
\hline & \multirow{2}{*}{ Tibia } & $\mathrm{M}$ & 7 & 169.12 & \pm 4.15 & $164.79-174.01$ & & $M$ & 7 & 171.5 & \pm 4.00 & $168.32-175.10$ \\
\hline & & $\mathrm{F}$ & 13 & 156.47 & \pm 4.15 & $147.03-165.45$ & \multirow[t]{3}{*}{ 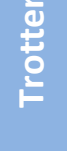 } & $\mathrm{F}$ & 13 & 157.72 & \pm 3.66 & $149.40-165.64$ \\
\hline & \multirow{2}{*}{ Fibula } & $\mathrm{M}$ & 2 & 165.73 & \pm 4.10 & $165.55-165.91$ & & $M$ & 2 & 169.23 & \pm 3.86 & $169.10-169.36$ \\
\hline & & $\mathrm{F}$ & 6 & 149.04 & \pm 4.10 & $143.29-152.99$ & & $\mathrm{~F}$ & 6 & 151.61 & \pm 3.57 & $146.92-154.84$ \\
\hline
\end{tabular}


Figure 5.10: Height estimates for males from the Tisza Culture using two methods: Trotter and Gleser (1952) in green, Sjøvold (1990) in lilac.

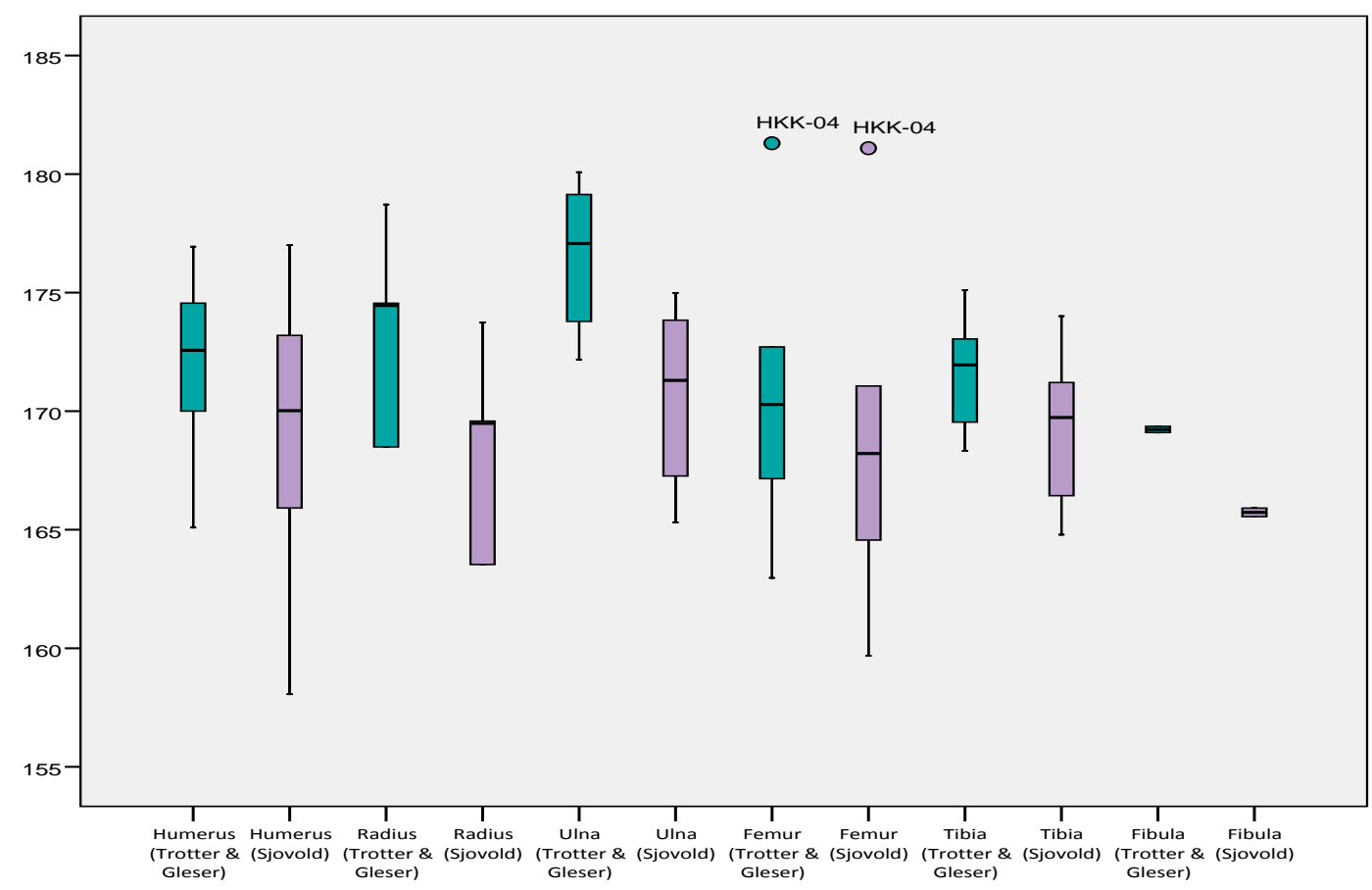

Figure 5.11: Height estimates for females from the Tisza Culture using two methods: Trotter and Gleser (1952) in green, Sjøvold (1990) in lilac.

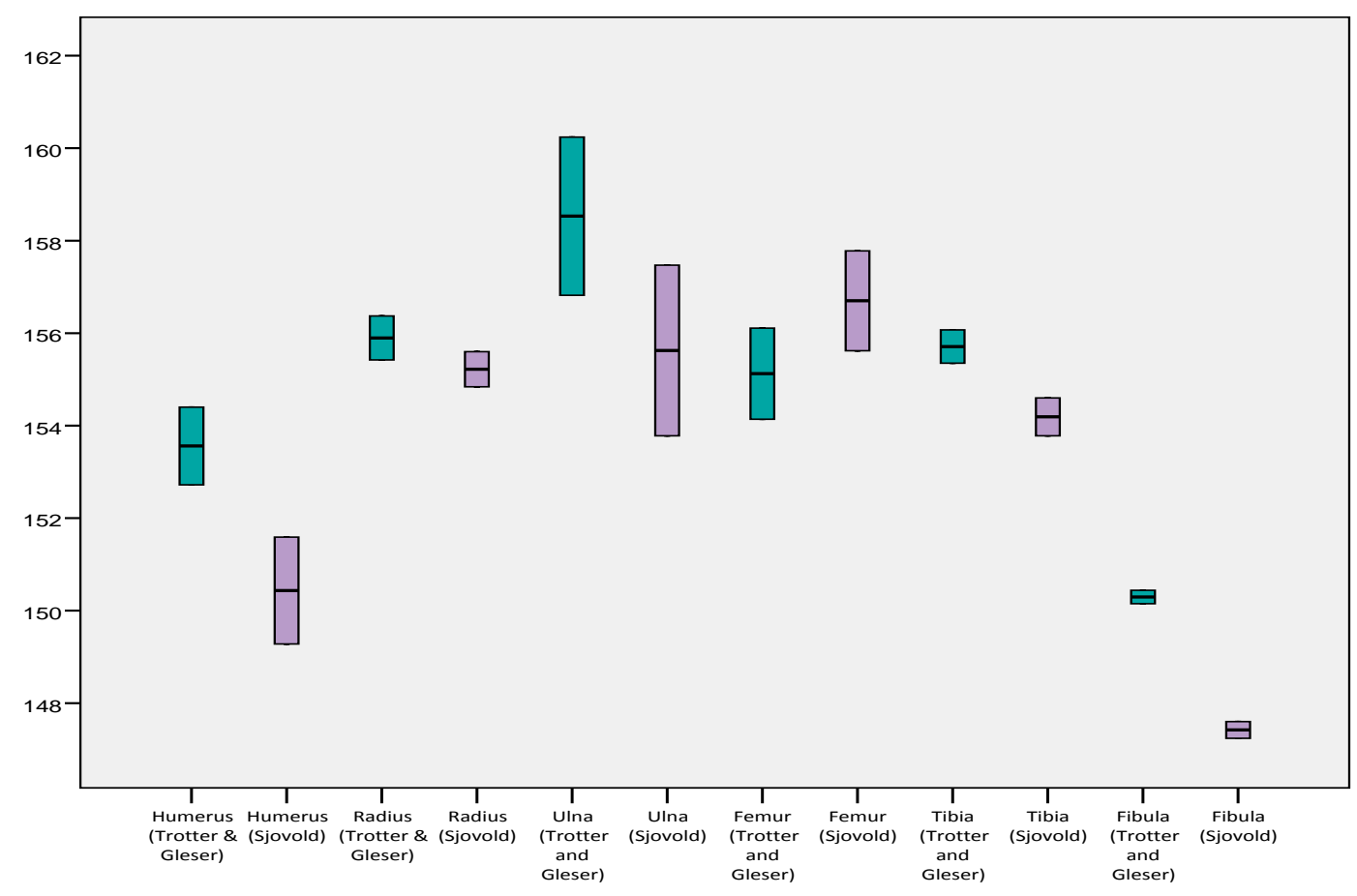


The average male stature of this Late Neolithic population estimated from the humerus and the femur was also compared using earlier methods described previously (see 4.3.1 and 4.3.2). Figures 5.12 and 5.13 below show wide range of results obtained.

Figure 5.12: Male stature estimates from average humerus length of the Tisza Culture

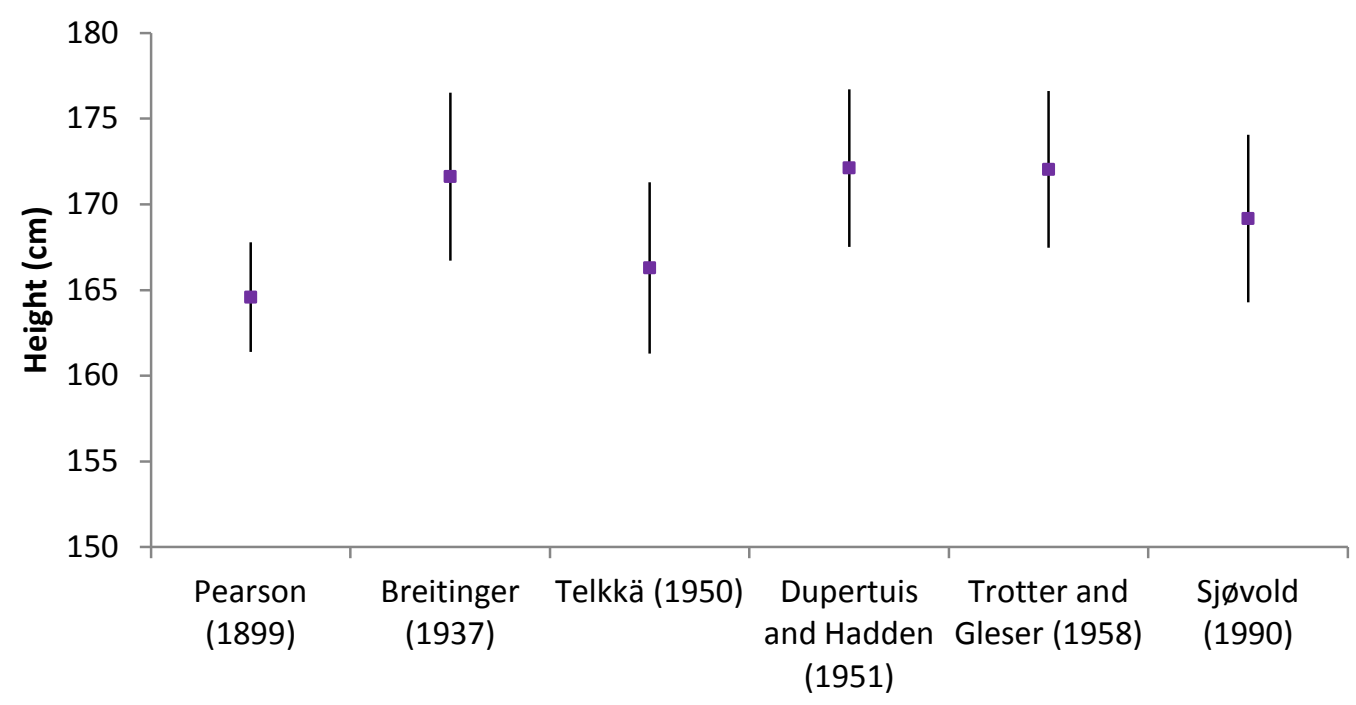

Figure 5.13: Male stature estimates from average femur length of the Tisza Culture

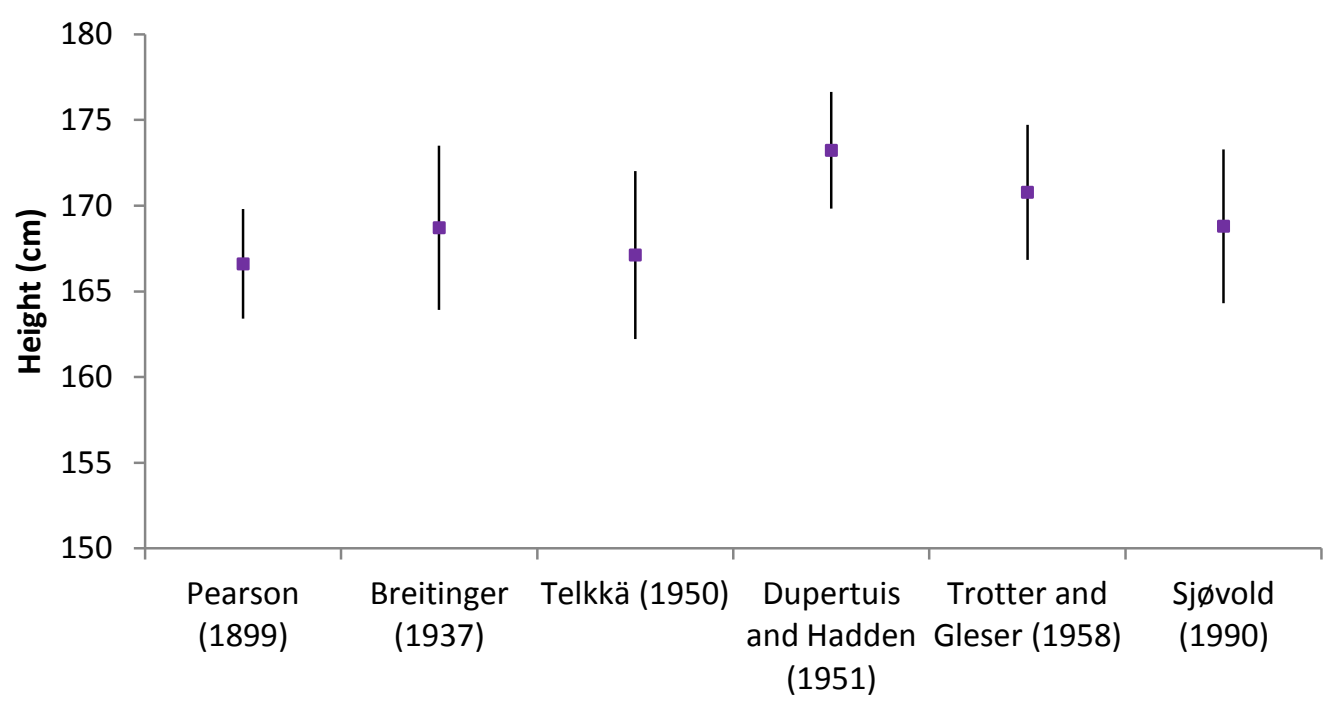


Results were also compared using Martin's categories (from very small to very tall) as reproduced in Éry (1998). The males were significantly taller than in Éry's results. This could be due to the low number of males available for this doctoral research, although Éry's minimum male stature of $146.85 \mathrm{~cm}$ appears abnormally low. There was also a vast difference between the results from Sjøvold's method compared to Trotter and Gleser's for the male population, though hardly noticeable in females (Figs. 5.14 and 5.15). It should be noted that this Neolithic sample from the Great Plain of Hungary was similar on average to modern Hungarians, with a difference of only less than a centimetre for males and and below $3 \mathrm{~cm}$ for females between Sjøvold's mean stature in this study sample and the stature obtained from over 10,000 Hungarians between 1956 and 1989 (as published in Éry 1998).

Figure 5.14: Male stature per categories

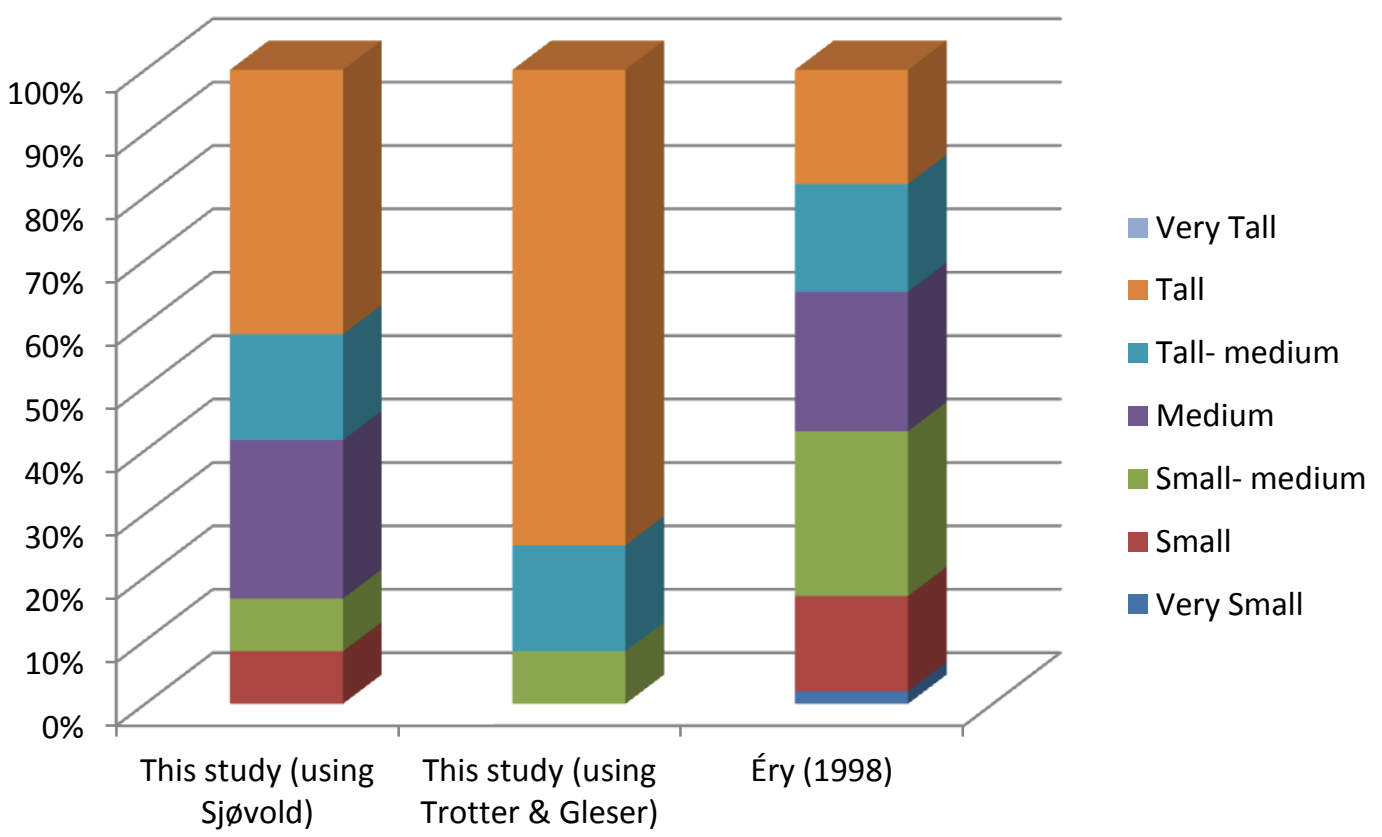


Figure 5.15: Females stature per categories

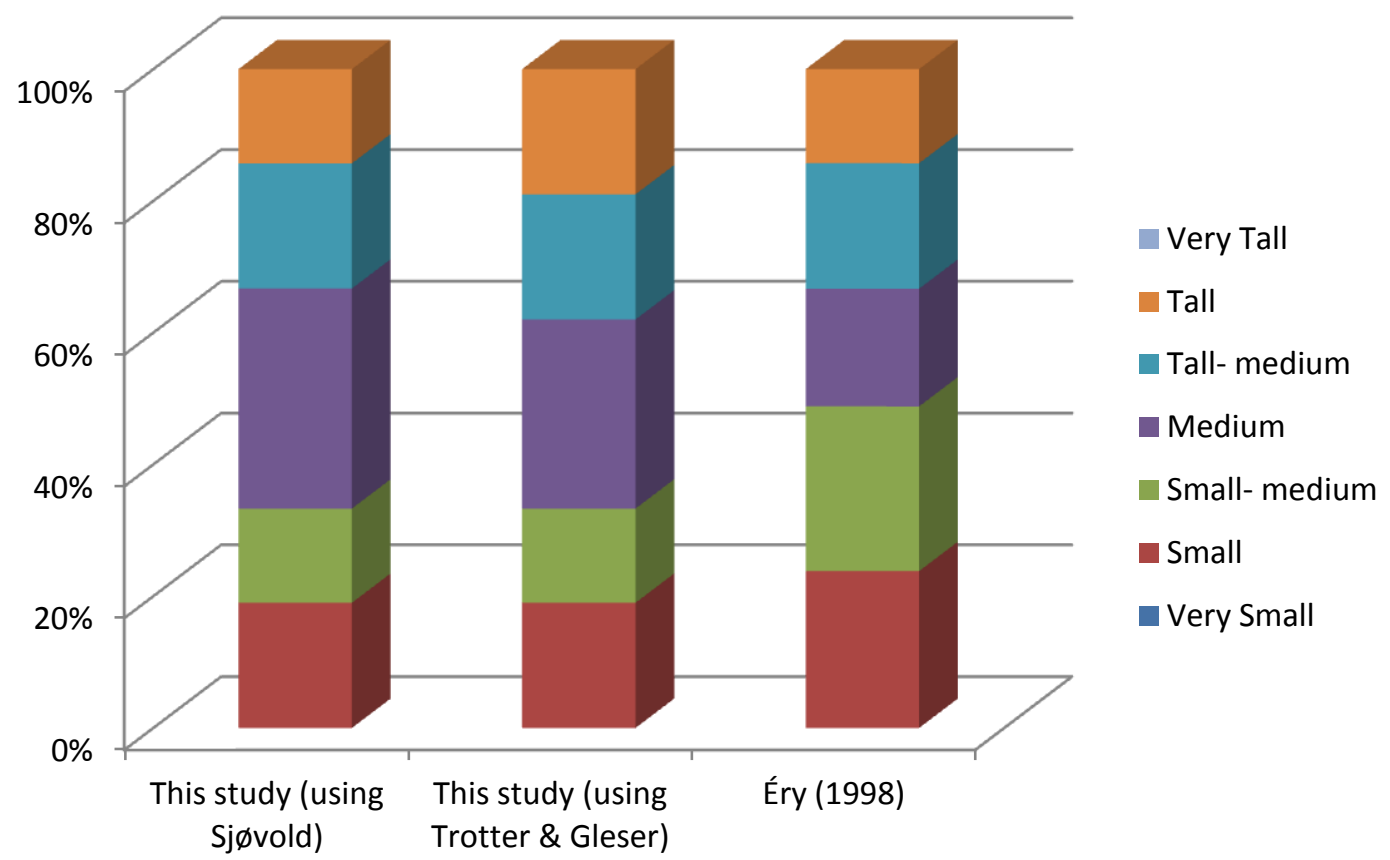

The mean stature obtained from the average of all estimations in this Late Neolithic sample was $167.9 \mathrm{~cm}$ for males and $153.3 \mathrm{~cm}$ for females from Sjøvold's formulae, or $171.5 \mathrm{~cm}$ for males and $154.7 \mathrm{~cm}$ for females when employing Trotter \& Gleser's method. The means of the results from the calculations with the least standard error, instead of the overall average, were $167.7 \mathrm{~cm}$ for males and $151.9 \mathrm{~cm}$ for females (Sjøvold), or $169.7 \mathrm{~cm}$ and 154.7 cm (Trotter \& Gleser).

Results for all analysed individuals are presented in Appendix 30. Height estimations of both females from the Early Neolithic Körös fitted within the range of their Late Neolithic counterparts. Interestingly, marked differences were observed between different sites within the Tisza Culture (table 5.11).

\begin{tabular}{|ccccc|}
\hline \multirow{2}{*}{ Site } & \multicolumn{2}{c}{ Males } & \multicolumn{2}{c|}{ Females } \\
& $n$ & Stature & $n$ & Stature \\
\hline Hódmezővásárhely-Kökénydomb & 1 & 177.3 & 0 & - \\
\hline Békés-Povádzug & 4 & 171 & 1 & 160.7 \\
\hline Hódmezővásárhely-Gorzsa & 7 & 164.8 & 20 & 152.9 \\
\hline
\end{tabular}


A single result was available from the Middle Neolithic AVK Culture, with a female stature estimate of $160.1 \mathrm{~cm}$, which fitted with both Early and Late Neolithic results (Guba et al. 2007). Comparable stature estimates were also available from Northeastern Hungary for the Neolithic period (Ubelaker et al. 2006, 15 males and 12 females), Copper Age (Ubelaker \& Pap 2009, 22 males and 13 females) and Iron Age (Ubelaker \& Pap 1998, 23 males and 27 females). These are presented in figure 5.16 below. Male stature means were very similar in the four different samples, as were their ranges. According to these samples, female stature seems to have increased from the Neolithic to the Copper Age, before decreasing again to the lowest level in the Iron Age. Although this was particularly visible in the ranges, it only translated to a difference of $2 \mathrm{~cm}$ between the means.

Fig.5.16: Comparison of stature estimates

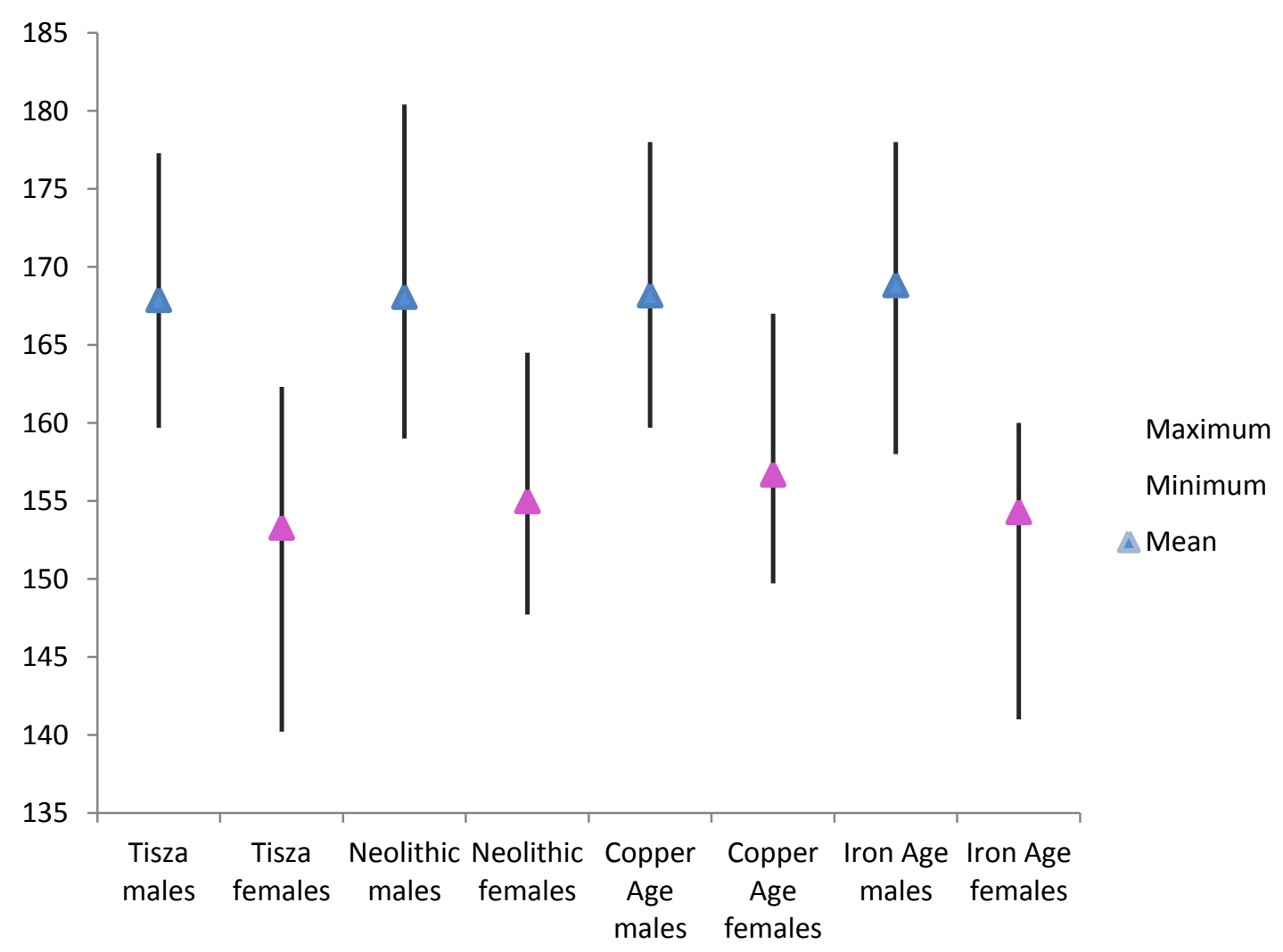


Finally, the male stature mean from this Late Neolithic population can also be compared with means of other archaeological and historic populations on which juvenile ageing and growth methods were based. These can be found in table 5.12 below and show that estimated mean stature for Tisza males was very close to the stature of male prehistoric and protohistoric Amerindians as well as the historic English sample. The two historic populations from Central Europe had slightly higher stature means for males than the other samples.

\begin{tabular}{|c|c|c|c|}
\hline Population & Period & Male stature & Reference \\
\hline Tisza Culture, Hungary & $5^{\text {th }}$ millennium BC & $167.9 \mathrm{~cm}$ & This thesis \\
\hline Indian Knoll, Kentucky & $3000 \mathrm{BC}$ & $167 \mathrm{~cm}$ & (Sundick 1972) \\
\hline Altenerding, Germany & AD $6-7^{\text {th }} c$. & $173 \mathrm{~cm}$ & (Sundick 1978) \\
\hline Mikulčice, Czech Republic & $A D 9^{\text {th }} c$. & $171 \mathrm{~cm}$ & $\begin{array}{c}\text { (Stloukal \& } \\
\text { Hanáková 1978) }\end{array}$ \\
\hline Wharram Percy, England & AD $10-16^{\text {th }} c$. & $168.9 \mathrm{~cm}$ & (Mays 1998) \\
\hline Arikara, Dakota & AD $18^{\text {th }} \mathrm{c}$. & $169 \mathrm{~cm}$ & $\begin{array}{c}\text { (Merchant \& } \\
\text { Ubelaker 1977) }\end{array}$ \\
\hline
\end{tabular}

Table 5.12: Comparison of mean male stature between prehistoric and historic samples

\subsubsection{Conclusion}

Dental and skeletal development enabled nearly all the juveniles in this sample to be aged. Dental age estimation produced concurring results between the different methods employed, including Ubelaker and Schour \& Massler charts, Smith stages, and Gustafson and Koch diagram. The latter was particularly helpful for such ancient and fragmentary remains with a mixture of more or less damaged dentitions with loose teeth as well as complete mandibles when radiographic facilities are unavailable and root development therefore invisible. Black, Scheuer and Schaefer's work was the main source of skeletal developmental age for juveniles, with the added research of Fazekas and Kósa for fetuses and neonates. However, Rissech and Black's scapular functions were 
unsuccessful in this population. Age estimates based on diaphyseal lengths were also confirmed to suffer the effect of population variability so that populationspecific data should be preferred. For ancient archaeological remains with no other suitable baseline sample, the ranges of measurements provided by Stloukal and Hanáková (1978) from their historic sample could be used as a guideline. Although juvenile sexing estimation was not successful in this instance, morphological traits were easy to record. It is therefore hoped that further research on documented samples will help refine these methods to eventually afford sexing of juvenile remains. Visser's calculations were useful for juvenile stature estimates. Growth in this Late Neolithic population demonstrated the same prolonged growth compared to modern populations as historic samples, although the Late Neolithic growth rate was closer to contemporary populations than historic populations.

Adult sex estimation based on commonly used cranial and pelvic morphological traits was shown to benefit from the addition of the mandibular ramus flexure trait, while Bruzek's pelvic traits and those from the distal humerus did not prove useful. Although morphological traits remain the preferred sex indicators, osteometric analyses were also shown to yield valuable additional information. Foot and hand bones for example provided an alternative solution for sex estimation. These bones often survive when ancient remains are excavated, and would therefore enable more information to be detected on otherwise fragmented or damaged material. However, population-specific baselines are essential due to population variability. A baseline dataset was created for this Tisza population based on the author's analyses and successfully provided sex estimates for thirteen out of fourteen individuals who had remained unsexed or inderterminate after morphological observations. Interestingly, while osteometric sexual dimorphism in this geographical area seemed to remain the same throughout the Neolithic in addition to little change in robusticity compared to the following two period of prehistory, cranial morphometrics appeared to show some differences between the Early and Late Neolithic. 
Dental wear based on Smith and Brothwell methods was shown to have significant potential in ageing adults in such ancient populations. Although these age estimates were still constrained to a placement in young adult, old adult or adult categories, they could still provide additional details and help ageing as "young" or "old" some adults whose age could not be estimated by other methods. Four individuals who had been aged as "adults" from their skeletal maturation were further classified as young or old based on their dental wear.

Finally, stature estimates showed the same trends as previous research with higher stature obtained from Trotter and Gleser's equations than from Sjøvold's. Unfortunately, no anatomical statures (either length in situ in the grave or anatomical method such as Fully's technique) were available in this population, which would have helped providing a more accurate picture of the living stature of these individuals. Sjøvold's weighted lines of correlation were already demonstrated in Chapter 4 to be methodologically the best system currently available for stature estimation of archaeological populations. Following the irregular pattern of estimates differences with each limb element demonstrated in this research and the limited number of bones sufficiently complete for analyses, the average of all estimations available should also be preferred to restricting estimation to the femurs, tibiae or humeri. The calculated average height of nearly $168 \mathrm{~cm}$ for males and slightly above $153 \mathrm{~cm}$ for females living at the heart of the Carpathian Basin during the Neolithic was only slightly shorter than that of their modern Hungarian counterparts. Comparison with other prehistoric and historic samples worldwide also showed little difference between those geographically and chronologically distant populations. However, differences between sites of the same period were also demonstrated. 
PART TWO

PALAEOPATHOLOGY 


\section{Chapter 6: Palaeopathology - Methodology and Pathologies}

\subsection{Introduction}

Palaeopathology is the study of ancient disease, which does not merely cover infections but "myriads of other conditions that affect health - for example arthropathies (diseases of joints); congenital anomalies; circulatory, endocrine, growth (dysplasias), haematological and metabolic disorders; oral pathologies; neoplastic conditions; and trauma" (Buikstra 2010:395). While undertaking palaeopathological analyses, osteoarchaeologists look for "variations from normal anatomy [which] provide the initial evidence of disease" (Ortner 2003:45). These bony changes must be established to have occurred before or close to the time of death, to distinguish them from pseudopathologies caused by post-mortem processes. This is then followed by "the exploration of singular or multifactoral causes of these alterations and the ramifications of the conditions on our understanding of human life" (Grauer 2012:2).

\subsection{Methodology}

All pathological examinations of the human remains presented in this study (see inventory in appendix 6) were carried out by the author at the Biological Anthropology Department of Szeged University. These were performed macroscopically, also known as gross study or visual observation, and the palaeopathological analysis based on these macromorphological observations were undertaken by the author at the same laboratory. Any anatomical variation visible was noted unless linked to the taphonomic process, and its aetiology established when possible based on palaeopathological references such as the work of Roberts and Manchester (1995), Aufderheide and 
Rodríguez-Martín (1998), Salter (1999), Ortner (2003), Mann and Hunt (2005), Lewis (2007), Pinhasi and Mays (2008), Brickley and Ives (2008), and Waldron (2009). Literature relevant to the specific conditions observed was also systematically consulted.

\subsection{Skeletal pathologies}

The Cambridge Encyclopedia of Human Paleopathology presented all skeletal pathologies under twelve sections: traumatic conditions, congenital anomalies, circulatory disorders, joint diseases, infectious diseases, diseases of the viscera, metabolic diseases, endocrine disorders, haematological disorders, skeletal dysplasias, neoplastic conditions and miscellaneous (Aufderheide \& RodríguezMartín 1998). Roberts and Manchester (1995) grouped pathologies under six categories: congenital disease, trauma, joint disease, infectious disease, metabolic and endocrine disease, and neoplastic disease. Mays (1998) proposed five categories of bone disease in addition to trauma and to metric/non-metric variations: congenital abnormalities, infections, neoplasms, diseases due to metabolic or dietary deficiency or imbalance, and arthropathies (joint diseases). Waldron (2009) presented skeletal pathologies under seven yet again different groups: joint diseases, bone forming and DISH, infectious diseases, metabolic diseases, trauma, tumours, growth and developmental disorders. Finally, Ragsdale (Ortner \& Ragsdale 2007; Ragsdale \& Lehmer 2012) asserted that there are seven basic categories of bone disease, easily remembered from the acronym VITAMIN: Vascular, Innervation/Mechanical, Trauma/Repair, Anomaly, Metabolic, Inflammatory/Immune, and Neoplastic.

Based on this variety of categories and the range of pathologies observed in the populations analysed in this study, six skeletal pathology groups were used for recording purposes as can be seen in appendix 31. These include trauma, metabolic diseases, inflammatory/immune/infectious diseases, mechanical changes and joint diseases (including osteoarthritis and entheseal changes), 
anomalies or anatomic variations, and non-specific stress indicators. A glossary of the types of disorders encountered in the populations under study can be found divided into these groups below. However, it should be kept in mind that all "classificatory categories are artificial mental constructs that, although often convenient and critical, can interfere with our understanding of the disease process" (Ortner 2012:250).

\subsubsection{Trauma}

Trauma may be caused by an accident or can be the result of a deliberate act of violence.

\section{( Fractures}

Fractures are the most common sign of trauma on skeletal remains in such assemblages. Specific types of fractures observed in this collection include: avulsion fracture, also known as tension fracture, associated with injury to the muscle, tendons or ligaments; compression or crushing fracture, resulting from the compression of the joint surface; and depressed skull fracture, where the external surface of the skull is forced inwards, with the possible associated risk of brain damage (for more detailed information, see C. Roberts \& Manchester 1995:65-98; Ortner 2003:120-59; Waldron 2009:138-67).

\section{Spondylolysis}

Spondylolysis is the separation of the neural arch from the vertebral body. It is mostly agreed to be the result of stress fracture from repetitive strain in the lower back (C. Roberts \& Manchester 1995; Merbs 2002a; Ortner 2003; Bennike 2008), and could also be caused by acute trauma such as a fall (Waldron 2009). A congenital or hereditary factor has also been highlighted causing some debate about its exact aetiology (C. Roberts \& Cox 2003; Mays 
2007). Although congenital spondylolysis does occur, it is mainly found on the cervical and thoracic vertebrae, whereas the "classic" spondylolysis in the fourth and fifth lumbar, and to a lesser extent the first three lumbar and the first sacral vertebrae, have been linked to activity patterns and hard physical work (Waldron 1991; Merbs 1996; Weiss 2009). Spondylolysis also exists in unilateral and asymmetric forms (Waldron 1992; Merbs 2002a) and its symptoms tend to be restricted to mild back pain (Merbs 1996).

\subsubsection{Metabolic diseases}

Metabolic (bone) diseases are "conditions in which the processes of bone modelling and remodelling are specifically disrupted" (Brickley \& Ives 2008:2).

\section{- Osteoporosis}

Osteoporosis is the loss of bone density, resulting in thinner and more breakable bones. It can affect both males and females, and is particularly associated with post-menopausal women. Osteoporosis can also result from other pathological conditions, such as trauma, diseases and dietary imbalance (Brickley \& Ives 2008:151-216).

\section{- Scurvy}

Scurvy is a metabolic disease caused by a deficiency in vitamin C mostly found in fruits and vegetables, resulting in defective bone formation and haemorrhages. It has a high mortality rate when untreated. Symptoms include tiredness, lethargy, musculoskeletal pain and weakness, reddening, swelling and bleeding of the gums, sub-periosteal bleeding in juveniles, swelling in the joints due to bleeding in adults, and weakening of the bone structure (see Aufderheide \& Rodríguez-Martín 1998:310-3; Ortner 2003:383-93; and Brickley \& Ives 2008:41-74 for more information). 


\subsubsection{Inflammatory / Immune / Infectious diseases}

\section{( Chronic rhinitis}

Chronic rhinitis is an ongoing (chronic) inflammation of the nose, more specifically the mucous membrane.

\section{(- Hypertrophic Osteoarthropathy (HOA)}

Hypertrophic Osteoarthropathy, also known as Marie-Bamberger disease, is a periosteal phenomenon characterised by the symmetrical (diffuse or distal) appearance of new bone mainly on the shaft of the long bones. The reaction can result in "appliqué" (new bone with sharply defined edges distinguishable from the underlying bone) or surface form that covers the entire bone with no visible edge. It is extremely rare as a primary pathology and is usually encountered in its secondary form, also known as Hypertrophic Pulmonary Osteopathy (HPO). Today, its most common causes are intrathoracic cancer and chronic intrathoracic infection (Rothschild \& Rothschild 1998, 1999). However, in the past tuberculosis would have been a more likely cause.

\section{(๑) Leprosy}

Leprosy is an infectious disease caused by Mycobacterium leprae. The infection has two forms: tuberculoid and lepromatous. Lepromatous leprosy involves the facial features, with atrophy and disappearance or recession of the anterior nasal spine and the maxillar alveolar process (rhinomaxillary syndrome), and also produces nerve, skin and bone lesions. It often starts as chronic rhinitis. Tuberculoid leprosy does not involve facial bones (no rhinomaxillary syndrome) and produces fewer skin lesions than lepromatous leprosy. This form of the infection is mostly a peripheral neuropathic disease, leading to loss of sensation particularly in the extremities, resulting in damage to the bones. 
Bone lesions appear earlier in tuberculoid leprosy than in its lepromatous form, and are much more extensive. Pathological diaphyseal remodelling of the proximal phalanges, metacarpals and metatarsals are typical of leprosy, and periostitis is often observed on the long bones, especially on the tibia and the fibula (see Aufderheide \& Rodríguez-Martín 1998:141-54 for further details).

\section{(๑) Mastoiditis}

Mastoiditis is an inflammatory process of the mastoid. In pre-antibiotic times, mastoiditis was a common complication of middle ear infection (acute or chronic otitis media) and could have serious consequences from hearing loss to facial paralysis and even death (Titche et al. 1981; Hamman \& Wang 2006; Flohr \& Schultz 2009a, 2009b). Mastoiditis has also been linked to tuberculosis. Although rare nowadays (Mustafa et al. 2004), tuberculous otitis media and tuberculous mastoiditis were much more common in the past, mainly in children under 15 years of age. At the beginning of the $20^{\text {th }}$ century, tuberculosis was found to be the cause of suppurative otitis media in $50 \%$ of the cases for under-one year olds and 27\% for those under two (Jeanes \& Friedmann 1960; Meher et al. 2006). Tuberculous mastoiditis and tuberculous otitis media are often attributed to a secondary reaction to active pulmonary disease (Joshi et al. 2002) and have also been linked to congenital tuberculosis (Park et al. 2010), although numerous cases described in the literature did not present any other sign of tuberculosis (Vital et al. 2002; Vaamonde et al. 2004). Without treatment, symptoms can last for years.

\section{๑) Meningitis}

Meningitis is an inflammation of the meninges surrounding the brain and can be hemorrhagic, inflammatory, or a mixture of both. Skeletal evidence may include abnormal blood vessel impressions on the endocranial (internal) surface of the skull, which are linked to the haemorrhagic process or extensive meningeal 
inflammation. Meningitis can be non-specific (of unknown aetiology) or a specific bacterial or viral infection. In the case of tuberculous meningitis (Leptomeningitis tuberculosa), blood vessel impressions are accompanied by granular impressions. Meningitis is a very severe condition and can frequently lead to death if untreated, particularly in infancy (see Ortner 2003:93-5 for further details on specific and non-specific meningitis).

\section{Osteitis}

Osteitis is an inflammation of the bone.

\section{- Tuberculosis (TB)}

Tuberculosis is an infection with Mycobacterium tuberculosis as its main causative agent. It is a disease of infancy, young adults and the elderly. It is important not to restrict the diagnosis of tuberculosis in palaeopathological cases to the modern clinical diagnostic criteria for TB, as skeletal changes may have differed in the past (Santos \& Roberts 2001). Classical tuberculosis pathology includes vertebral fusion and collapse leading to Pott's disease, knee joint ankylosis, hip joint destruction, cold abscess on the sacrum or vertebrae and endocranial TB. Other osseous change probably related to tuberculosis include rib periostitis, hypervascularization, diffuse symmetrical periostitis (HOA), endocranial changes such as serpens endocrania symmetrica (SES) and abnormal blood vessel impressions (Maczel 2003). Rib changes may include sharply demarcated lytic lesions or diffuse periostitis on the ventral side of the ribs, possibly caused by adjacent soft tissue infection. Most rib changes are associated with individuals suffering from pulmonary TB, particularly in the left chest. Although those lesions cannot be considered specifically characteristic of pulmonary tuberculosis, they can indicate a non-specific chronic pulmonary disease, with tuberculosis as the most likely cause (C. Roberts 1999; Matos \& Santos 2006). Porotic hyperostoses, such as cribra orbitalia and cribra cranii, 
are generally attributed to iron-deficiency anaemia, which can develop from the interaction of several factors, such as weaning practices, diet, hygiene, parasites and infectious diseases, so may also be associated with tuberculosis.

\section{(๑) Cancer}

Malignant neoplasms / cancer have been added to this category as only one potential case was found which had a possible alternative diagnosis of (pubic) osteitis. A neoplasm, or tumour, can be benign in which case it does not spread, or malignant where the primary tumour spreads with the formation of secondary tumours, also known as metastasis (see C. Roberts \& Manchester 1995:186-95; and Waldron 2009:168-90 for more information). Osteologically, although some primary malignant tumours can be visible on the skeleton, cancer is often identified through metastasis affecting the bones secondary to primary soft-tissue tumours (Brothwell 2008). Without treatment, cancer can spread rapidly and has a high mortality rate.

\subsubsection{Mechanical changes}

This group includes enthesopathic lesions and other Musculoskeletal Stress Markers (MSM, also known as Entheseal Changes or EC), Osteoarthritis (OA), Degenerative bony changes (DBC), and Degenerative Joint Disease (DJD).

\section{Enthesopathies / MSM}

An enthesis is the insertion or attachment site on the bone of a ligament or tendon. Enthesopathies, or enthesopathic lesions, correspond to entheses which differ from the aspect that would normally be expected, for example with an unexpected (and usually larger) than normal size as well as possible signs of inflammation or destruction. Such pathology was described several decades ago as "activity-induced pathology" (Merbs 1983), later refined to "musculoskeletal 
stress markers" or MSM (Hawkey \& Merbs 1995). These enthesopathies have been continuously utilised since then as a basis to reconstruct past activities, which has been described itself as a "kind of "Holy Grail" by an entire subfield of human osteology" (Villotte \& Knüsel 2013).

However, in the last few years, dedicated research to the subject based on historical skeletal populations with associated records of occupation has demonstrated the multi-factorial aetiology of such changes (Henderson 2008; Alves Cardoso \& Henderson 2010). In order to avoid implying that all enthesopathies are the results of activities, Jurmain and colleagues (2012) proposed the new terminology of Entheseal Change, or EC. Most recently, EC have been demonstrated to be influenced by age (Alves Cardoso \& Henderson 2013) and to potentially reflect numerous activities throughout a lifetime rather than a single activity around the time of death (Henderson et al. 2013).

\section{( ) Arthropathies}

Osteoarthritis (OA), degenerative bone changes (DBC), and degenerative joint disease (DJD) with a mechanical aetiology are also included in this category. These can be brought on by age from repeated use over time, or occur secondary to previous damage to the joint through high level of activity or trauma, particularly over an extended amount of time. Lipping, created by osteophytes, appears at the margins of the degenerating joint, and pitting or erosion can also often be seen on the joint surface.

Other arthropathies with non-mechanical aetiologies would have fitted under other categories. For example, septic or rheumatoid arthritis and spondyloarthropathies would have been recorded in the inflammatory / immune / infectious category, whereas gout would have come under the metabolic category. However, none of these arthropathies were observed on these populations, and therefore they do not appear in this glossary. 


\subsubsection{Anomalies / anatomic variations}

\section{- Anomaly / anatomic variation}

This category includes any developmental anomaly or anatomic variation, for example the presence of supernumerary vertebrae. Anatomic variations in particular may be useful in funerary archaeology to try linking geneticallyrelated individuals in the same burial ground.

\section{- Benign neoplasms}

Benign neoplasms / tumours have also been added to this category as only one case was found, which was a button osteoma. This common benign tumour is usually found on the ectocranial (external) surface of the skull, with a variety of shapes and sizes. Its aetiology is still uncertain, with some mentioning a possible link to trauma as an ossification of haematomas in some cases (Brothwell 2008) while others maintain osteomas can only be "the overgrowth of normal bone formed in the periosteum" (Waldron 2009:171).

\subsubsection{Non-Specific Stress Indicators (NSSI)}

The following skeletal signs indicate stress on the body. They can result from disease, malnutrition, trauma or environmental stress. However, they cannot provide a more specific aetiology on their own.

\section{- Cribra cranii}

Cribra cranii appears as porosity or pitting on the ectocranial (external) surface of the skull. 


\section{- Cribra orbitalia}

Cribra orbitalia is indicated by porosity or pitting in the orbits, with or without subperiosteal bone formation.

\section{Periostitis}

Periostitis is the inflammation of the periosteum, with porosity and/or subperiosteal bone formation. It is mostly associated with infectious processes, but can also follow trauma and other diseases. Woven bone formation indicates a rapid response active at the time of death, while compact bone shows that remodelling had already taken place and is associated with more chronic conditions (Ortner 2008).

\section{- Increased vascularisation}

Increased vascularisation of the bones also indicate a response to a pathological condition, infectious or otherwise.

\subsection{Oral pathologies}

Oral pathologies include caries, gum or alveolar resorption, antemortem tooth loss (AMTL), calculus, periodontal disease (also referred to as periodontitis), abscesses or cysts, anomalies or anatomic variations, and non-specific stress indicators such as enamel hypoplasia and in particular linear enamel hypoplasis (LEH). Tooth wear or dental attrition was also considered under ageing, see sections 3.3.5 for the theory and 5.3.2 for the results (for more information on dental anthropology and oral pathology, see Hillson 1996; Hillson 2000; Hadjouis \& Mafart 2001; Hillson 2005; and Ogden 2008). 


\subsection{Conclusion}

A catalogue of all the individuals analysed has been provided in appendix 31, with estimates of age, sex and stature (see Part One, chapters 2-4 for the methods and Chapter 5 for the results) as well as the type of pathologies observed on the skeleton and the dentition, recorded according to the categories of pathologies presented in this chapter.

The next chapter presents the results of the palaeopathological analysis of the Neolithic populations under study for this doctoral research, following the methodology and criteria presented in this chapter. 


\section{Chapter 7: Osteological analysis - Palaeopathological results}

\subsection{Introduction}

The pathological analyses of the populations from this study were hindered by the condition of the skeletal remains. A combination of taphonomy, seven millennia in the ground and storage in boxes for several decades resulted in very fragmentary material. These time-consuming osteological analyses were also restricted by lack of funding to macromorphology only. However, as will be seen in this chapter, the painstaking careful observations of these tens of thousands of bone fragments yielded very rewarding results. Microbiological and DNA specialists were contacted for the most outstanding cases and were able to confirm the author's diagnosis. Although this thesis is concerned specifically with macroscopic methods, basic results from these other techniques will accompany the relevant cases in this section, if only to confirm that extensive macroscopic analyses of what are too often disregarded as boxes of worthless fragments may indeed reveal hidden treasures.

Results of the palaeopathological analyses are presented below. These do not follow the disease categories used to record the pathological data, but are divided instead into time periods and age categories more appropriate for such a report. For the largest group, the adult population from the Tisza culture, further subdivisions separate cases of trauma, non-specific pathologies, specific infections, a case of multiple pathologies, and other skeletal pathologies. A glossary of the pathological conditions encountered in these Neolithic populations is available in chapter 6 . 


\subsection{Palaeopathology in the Early Neolithic}

\subsubsection{Skeletal Pathologies}

Of the twelve individuals available for study, all adults, only the two females from Deszk-Olajkút were complete while the other seven males and three females lacked any postcranial remains. For full details on the demographic estimates obtained for these individuals, see Appendices 11 (sex), 26 (age) and 30 (stature).

Both of the mostly complete individuals showed degenerative bone changes, with DOL-01, estimated to have died around 60 years of age, presenting signs of osteoarthritis on most of the spine as well as more signs of degenerative joint

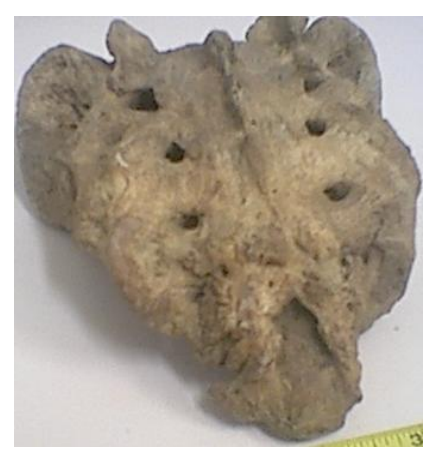

Figure 7.1: DOL-01 Sacrum with evidence of trauma disease at the shoulders, elbows, and hips, knees and feet, and widespread musculoskeletal stress markers. Evidence of trauma was also observed on the sacrum with a subsequent periosteal reaction leading to reactive bone formation (fig.7.1). Evidence of osteoarthritis on the other woman [DOL-02] was found on her thoracic vertebrae and her shoulders, and squatting facets could also be observed on her tibiae.

Non specific stress indicators were also present in this population with two cases of cribra orbitalia, both males, one [HKO-06] from Hódmezővásárhely Kotac and the other [HBP-01] from Hódmezővásárhely - Bodzás Part with added frontal hyperostosis.

\subsubsection{Oral Pathologies}

Eight dentitions were available, every single one of them showing signs of oral pathology.

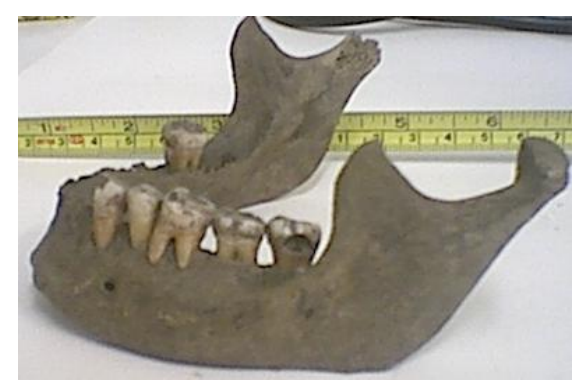

Figure 7.2: HKO-07 - mandible with caries, alveolar resorption, abscesses, periodontal disease and antemortem tooth loss 
Calculus, gum resorption and periodontal disease were the most frequent (see table 7.1 below).

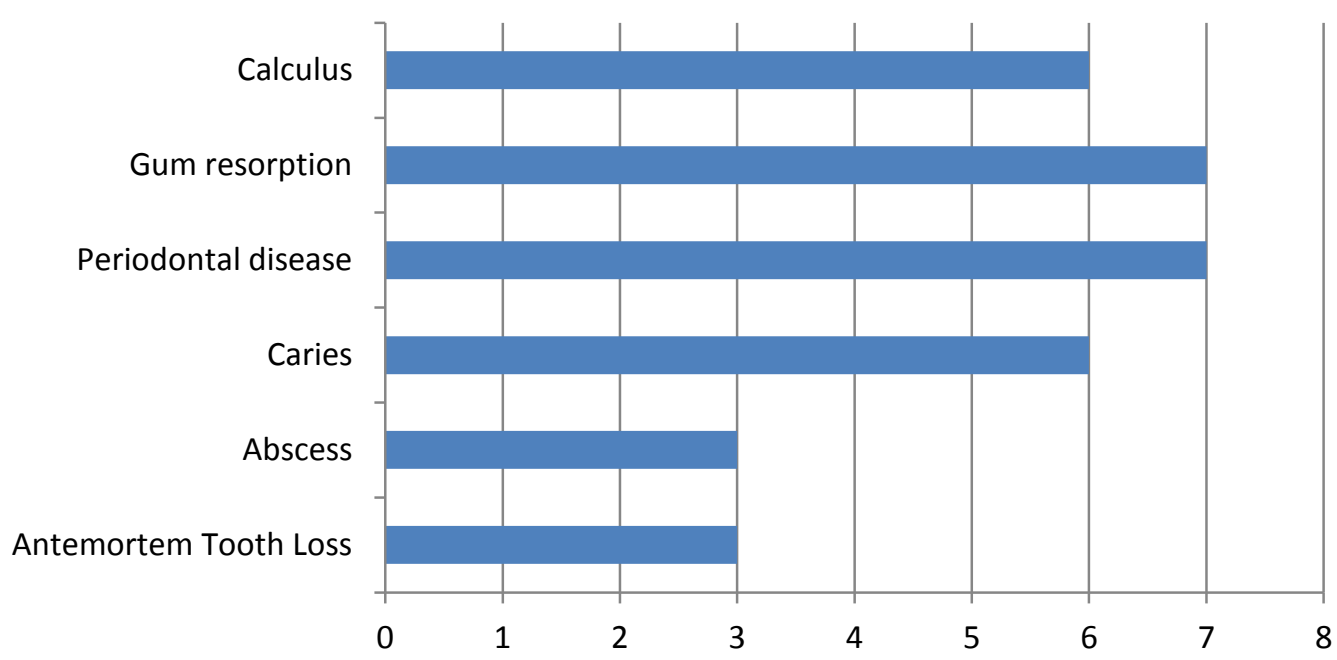

Table 7.1: Oral pathology rates in the Early Neolithic

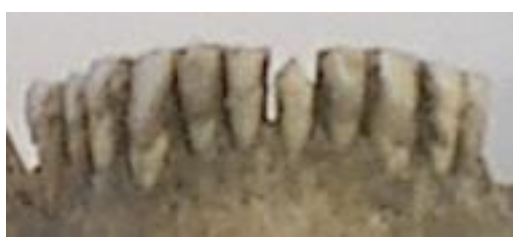

Figure 7.3: HKJ-01 - Unique tooth wear, probably activity-related
A unique example of irregular tooth wear with HKJ-01 lower left permanent central incisor giving it a sharp triangular appearance might indicate an activity-related wear pattern (fig.6.3).

\subsection{Palaeopathology among individuals from Neolithic sites with unspecified dates}

The adult from Hódmezővásárhely Z bérház [HZB-01], and the adult from Ószentiván [OSZ-01] could only be dated to the Neolithic as a whole, and were represented by very partial skeletons, the former by her skull without mandible, and the latter by her skull and upper vertebrae only. For more details on the demographic estimates obtained for these two individuals, see Appendices 11 (sex) and 26 (age). 
HZB-01, possibly female, presented with age-related non-pathological endocranial pitting and evidence of oral pathology, with signs of calculus, antemortem tooth loss, and periodontal disease.

OSZ-01, a young adult possibly aged between 18 and 24 years, with anatomical variations in the form of extra-sutural bones and rotation with misalignment of the upper right first premolar, also presented early signs of oral pathology indicated by calculus and a slight gum resorption.

\subsection{Palaeopathology in the Late Neolithic - Juveniles}

A third of this Late Neolithic sample was juvenile, with half of the cases pathological (table 7.2).

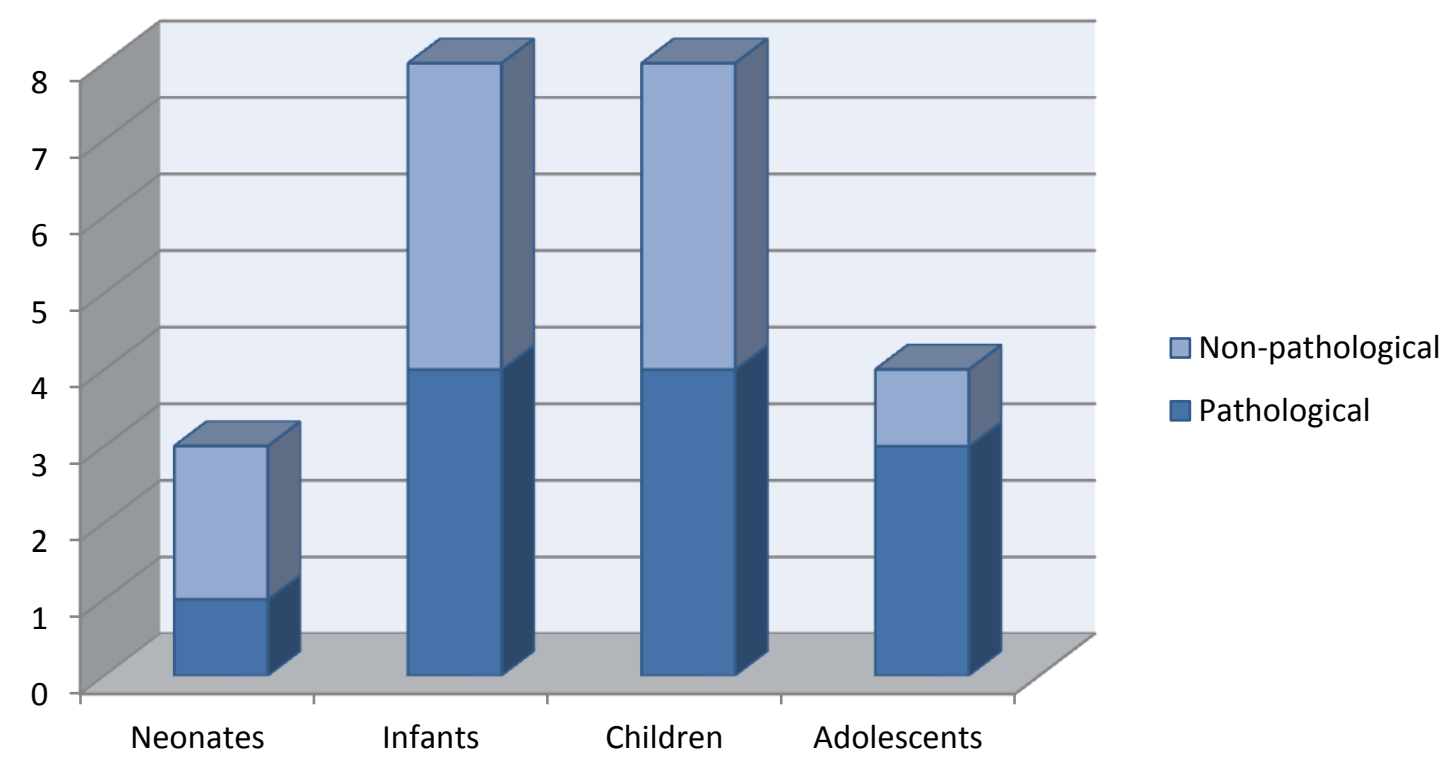

Table 7.2: Pathology frequency in Late Neolithic juveniles 


\subsubsection{Neonates}

Three juvenile skeletons were aged as neonates based on their bone formation and their measurements (see appendix 7 for more details).

Out of the three, one neonate was pathological (fig.7.4). The skull of HGO-55 was found to be very porotic. Severe periostitis was observed on the endocranial and ectocranial surface of nearly all skull bones and fragments including the sphenoid and particularly the mandible, as well as the shaft of the clavicles, the spine of the scapulae, and in some areas of the humeri, ulnae, ilia, ischia, femurs, tibiae and fibulae. Pronounced cribra orbitalia was also present.

The character of these lesions and their location on the skeleton affecting the cranium, pelvis, scapulae and long bones fit all the criteria typical of scurvy (Ortner \& Ericksen 1997; Lewis 2004; Brickley \& Ives

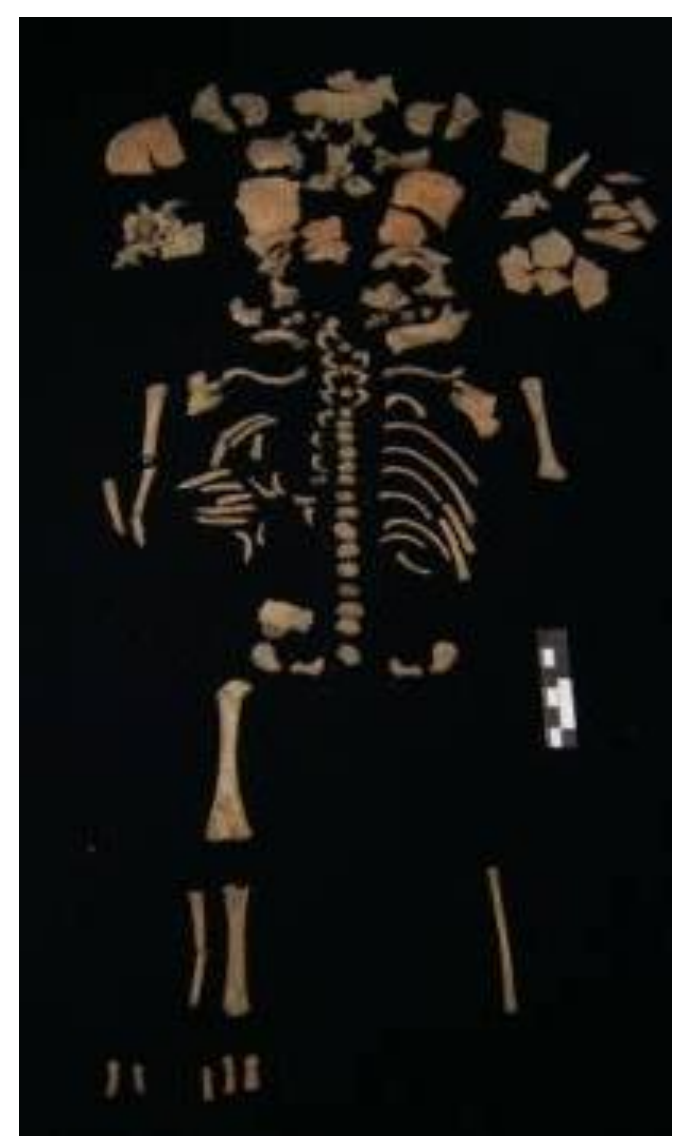

Figure 7.4: Skeletal remains of HGO-55 2008).

Photographs of the probable scorbutic signs on the skull, mandible, scapula and femur of HGO-55 are presented overleaf. 

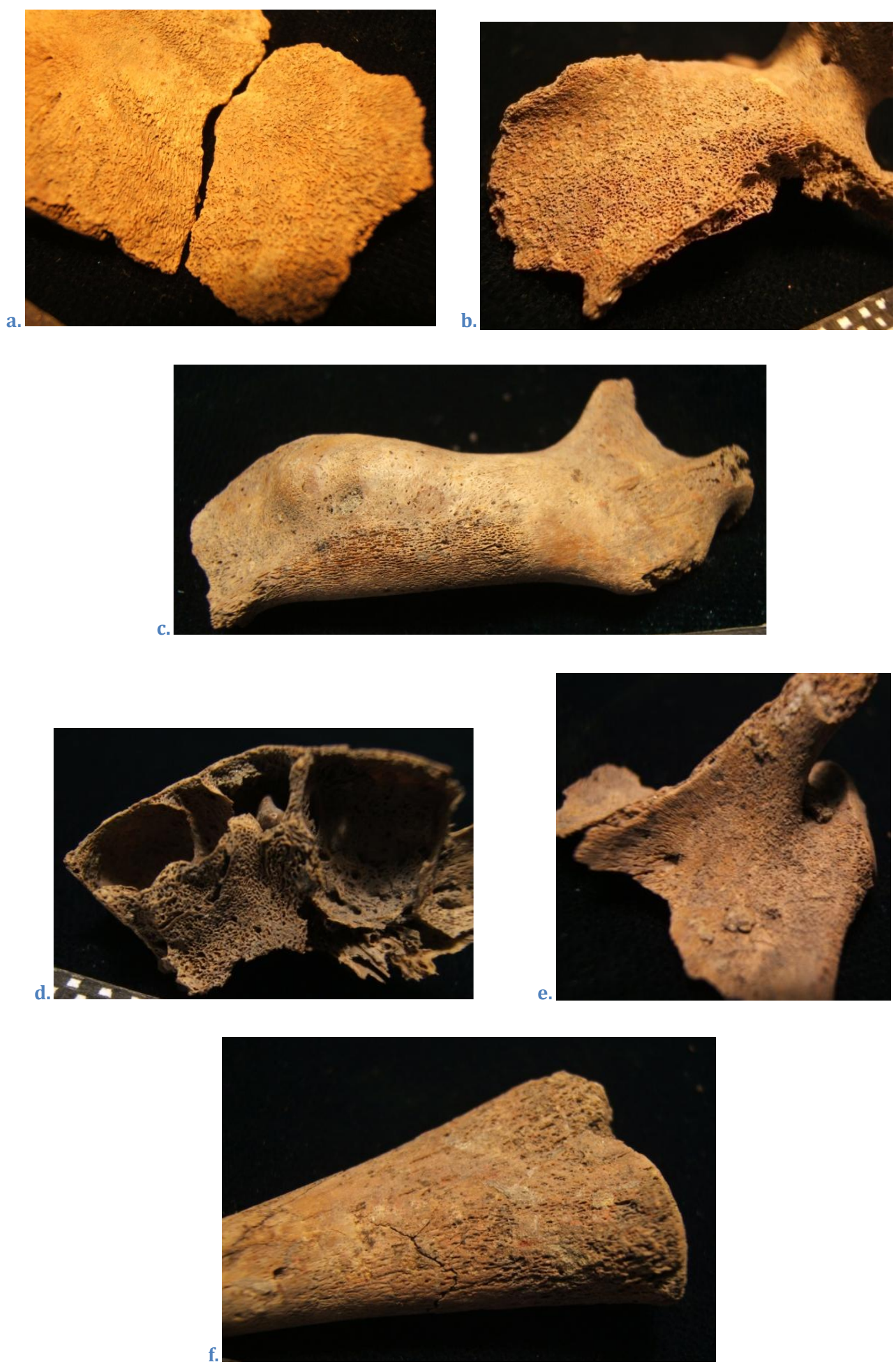

Figure 7.5: Signs of probable scurvy on HGO-55

a. cranium, b. sphenoid, c. mandible, d. palate, e. scapula, f. femur. 


\subsubsection{Infants ( $\leq 1$ year $)$}

Half of the infants provided evidence of pathology. Details of the age estimates of all infants can be found in table 5.1 on page 175 and table 5.2 on page 176 .

HGO-43, aged c. six months, presented another probable case of scurvy with widespread porosity as well as irregular and spiculated new bone with proliferative bone growth on the endo- and ectocranial surfaces of all cranial bones, a fragment of long bone shaft towards its distal end, and along the spine of the scapula (fig.7.6).

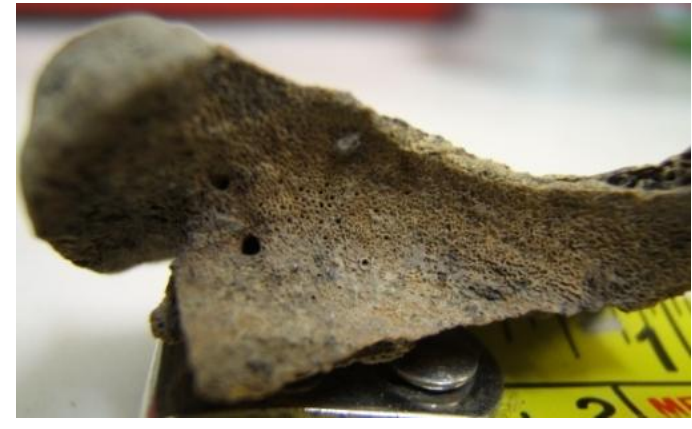

Figure 7.6: Signs of probable scurvy on HGO43. Above: scapula. Right: pars interalis.

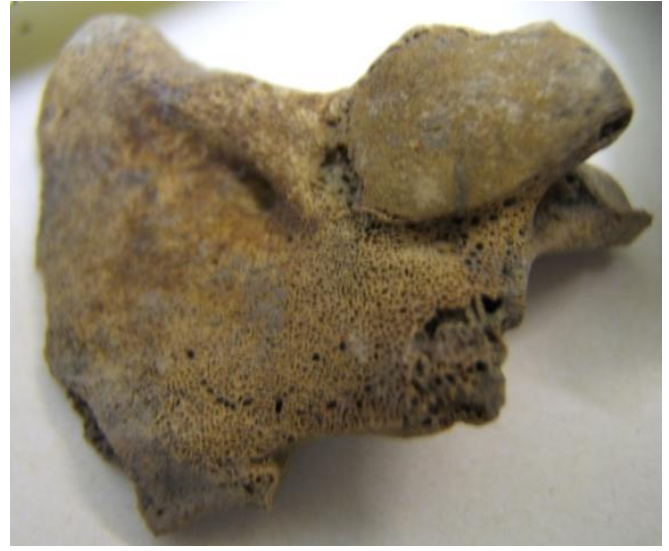

HGO-57, also c. six months, showed signs of a severe non-specific infection, with bony changes from meningeal reaction, widespread periostosis with new bone formation on the endocranial surface of the frontal, temporals, parietals and squamous occipital, in addition to periostosis with woven bone formation on the ventral surface of eight ribs, the proximal half of both humeri and shafts of both fibulae, and with very pronounced periostitis on the left ilium and both tibiae (figs.7.7-7.9). Two cases of periostitis were also observed. Traces of light periostitis, a non-specific stress indicator, were observed along the shaft of both

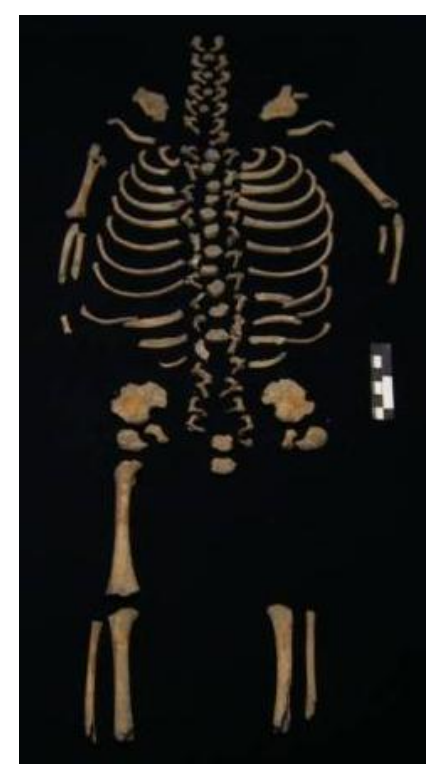

Figure 7.7: Remains of HGO-57 
femurs of HGO-42, aged c. nine months. Periosteal new bone formation of probable inflammatory or infectious origins was found along the shafts of both femurs, both tibiae and the fibula of HKS-04, aged c. one year.

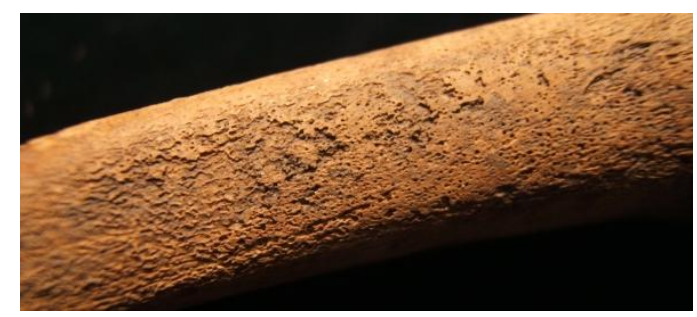

Figure 7.8: Periostitis with woven bone formation on humeral shaft of HGO-57
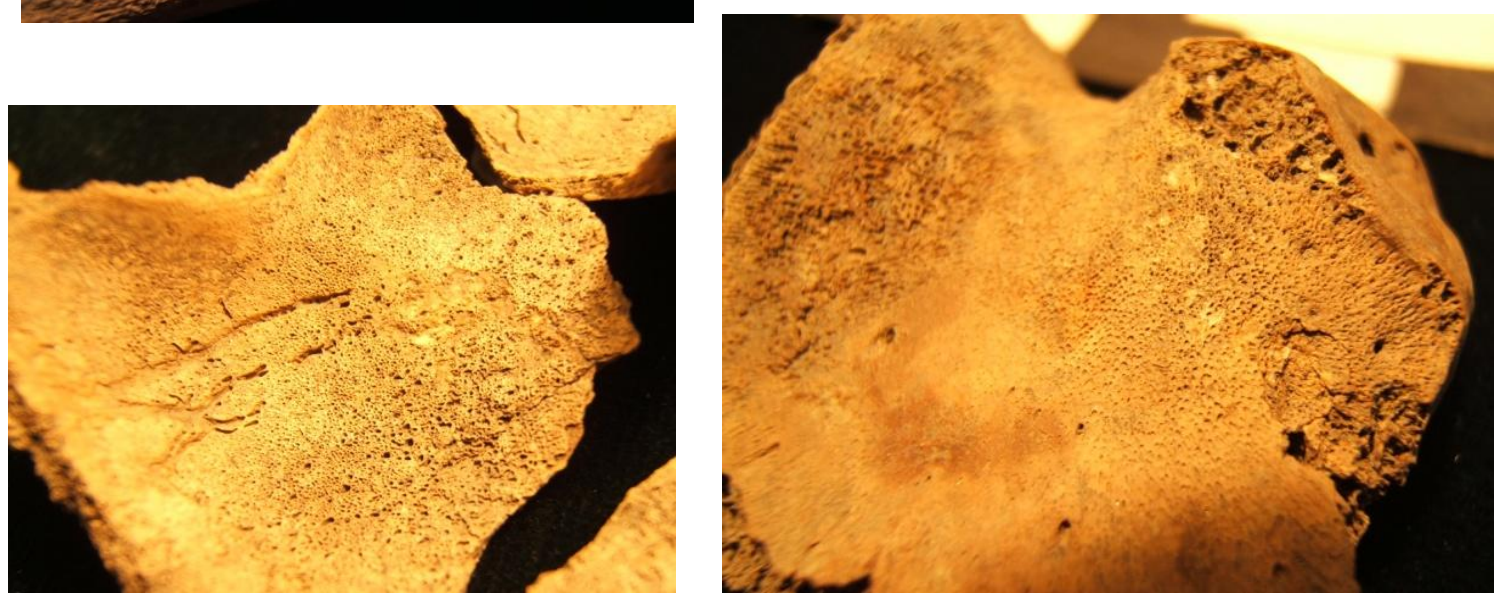

Figure 7.9: Signs of severe infection on HGO-57 skeleton. Left: endocranial surface. Right: ilium.

\subsubsection{Children (1-12 years)}

Half of the children's remains were also pathological or anomalous. More details on the children's age estimates can be found in table 5.2 on page 176, and their stature estimates are available in Appendix 10.

A case of non-specific infection was observed on HGO-06, a child around five year of age. Osteological evidence included cribra orbitalia in the left orbit, meningeal reaction visible on the right parietal with abnormal vessel impressions on the endocranial surface and pitting on the external surface towards the occipital, mastoiditis indicated by a large foramen through the mastoid process and an anomalous cavity in the endocranial surface below the petrous bone endocranially (fig.7.10 overleaf). 

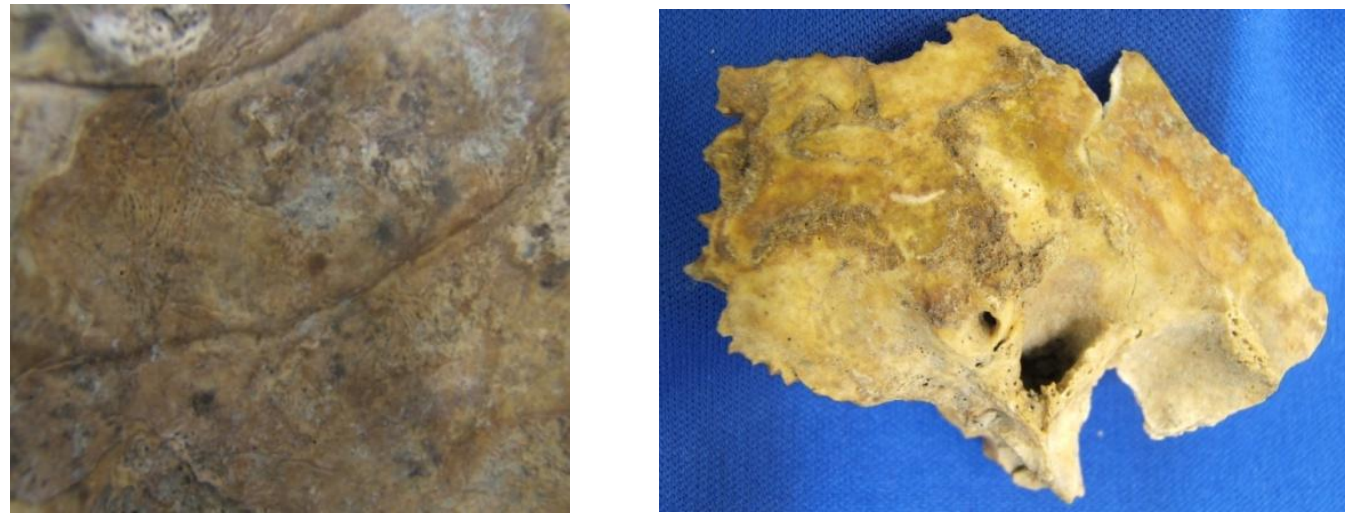

Figure 7.10: Signs of infection on the skull of HGO-06. Left: Abnormal blood vessel impressions. Right: Mastoiditis

Finally, this child also had severe oral pathology with periodontitis and gum resorption visible in addition to abnormal calcification of all permanent teeth (fig.7.11), possibly attesting a period of infection.
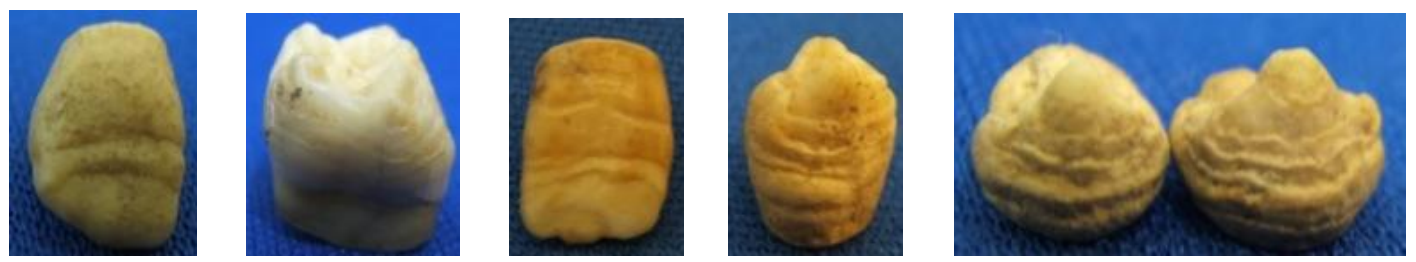

Figure 7.11: Developmental anomaly of the enamel of HGO-06 permanent teeth.

Cribra orbitalia, a non-specific stress indicator (NSSI) were observed on the remains of HGO-54, c. eleven years, together with a developmental anomaly in the form of a fifth lumbar bifid vertebra with right arch missing (fig.7.12).
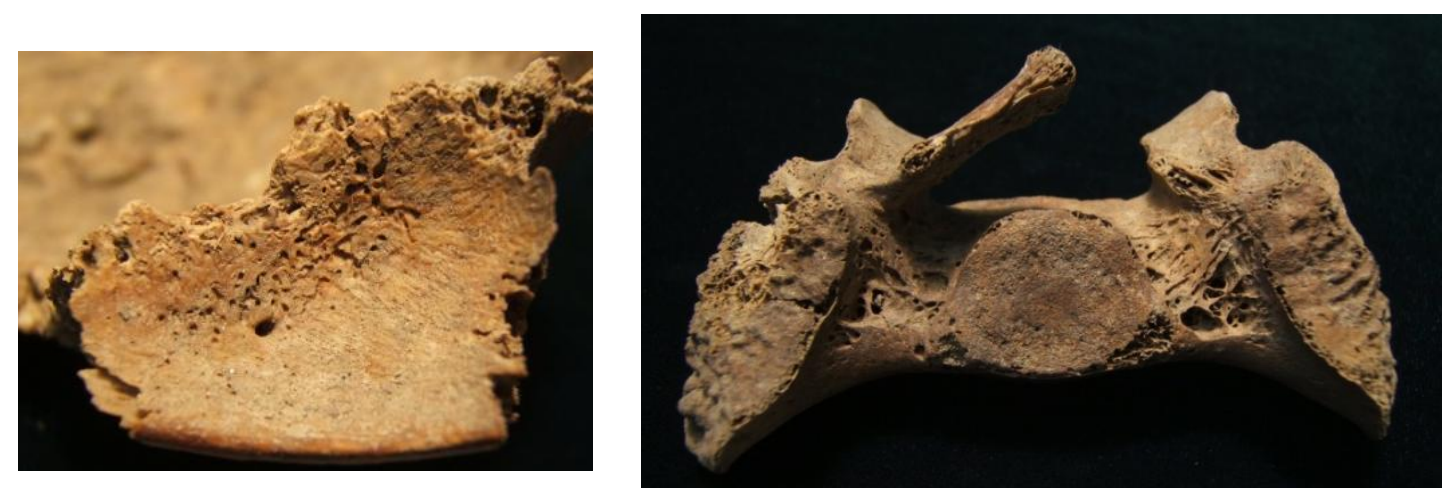

Figure 7.12: HGO-54. Left: cribra orbitalia. Right: bifid L5. 
NSSIs were also visible on HKK-01, c. six to seven years, with light cribra orbitalia and linear enamel hypoplasia (as well as caries) on the dentition.

Finally, oral developmental anomalies were detected on HGO-09, c. 8 years, with a foramen caecum on the upper first permanent molars on both side, the right lower first permanent molar, both lower second permanent molars, and a crown root groove on the upper lateral incisors on both sides.

\subsubsection{Adolescents (13-17 years)}

Three out of the four adolescents displayed pathologies (see page 169 for a description of their age estimates).

Pronounced cribra orbitalia (fig.7.13) were observed in both orbits of HGO-50. Although this young adolescent was aged at around 13 years based on his skeletal development, stature was only estimated at 4'1 (see appendix 10). This could be an indication of restricted growth due to disease or metabolic disorders, especially associated with cribra orbitalia. Oral pathology included caries and calculus as well as gum resorption.
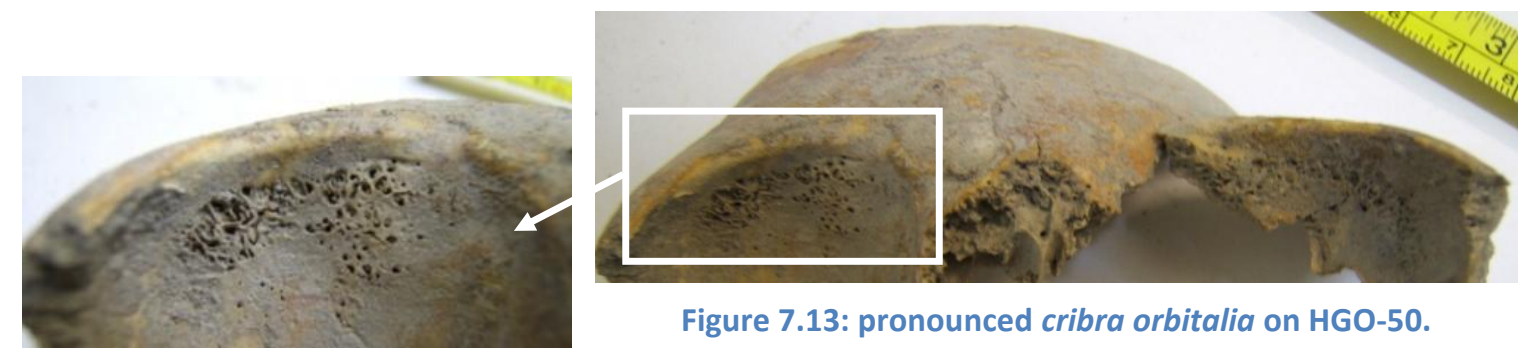

Figure 7.13: pronounced cribra orbitalia on HGO-50.

HKK-04, a male in his late teens, showed mechanical changes extensive for such a young age and consistent with an active life undertaking very strenuous activities. These changes comprised numerous enthesopathic lesions and other musculoskeletal stress markers on the shoulders, upper and lower limbs, schmorl's nodes on three thoracic vertebrae and aseptic osteonecrosis of the 
right femoral lateral condyle with corresponding discolouration of the patella (fig.7.14). Oral pathology included light periodontal disease, gum resorption, and inflammation with a cyst at the level of the lower right permanent third molar.
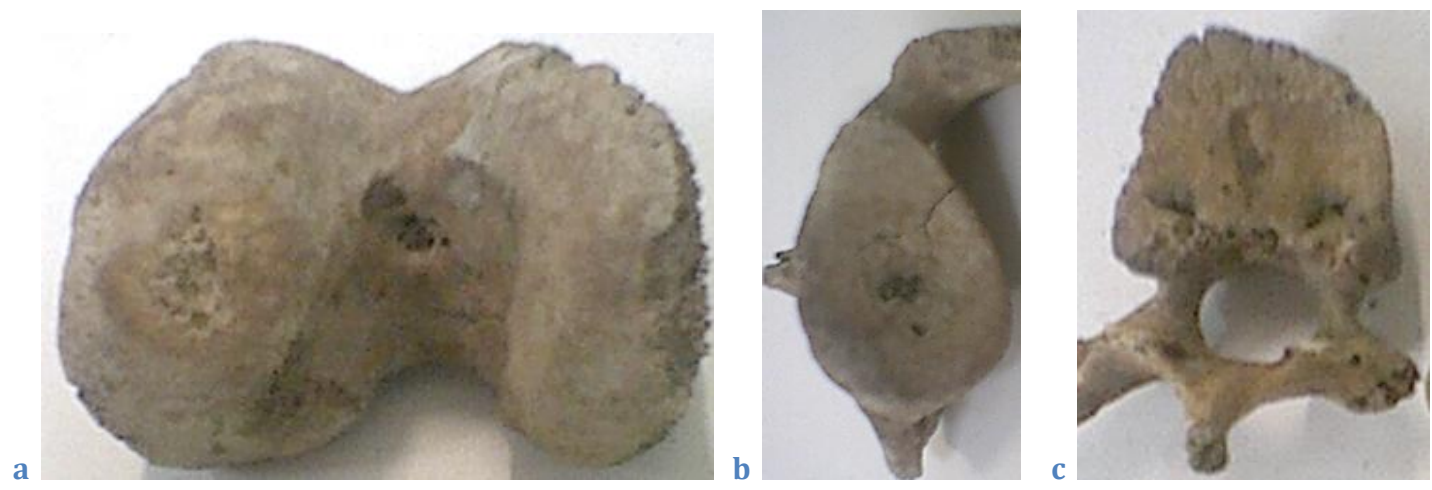

Figure 7.14: Signs of strenuous physical activities on HKK-04:

a. femur, b. scapula, c. 8th thoracic vertebra.

HGO-26 was also in her late teens. Her remains indicated that she may have suffered from an infection, with cribra orbitalia, cribra cranii, a slightly porotic palate (fig.7.15), granular impression with new bone formation on the endocranial surface of the occipital (fig.7.16), and finally light periostitis on both tibiae. Both her femoral fovea capites were abnormally large (fig.7.15), and musculoskeletal stress markers were visible on her clavicle and her

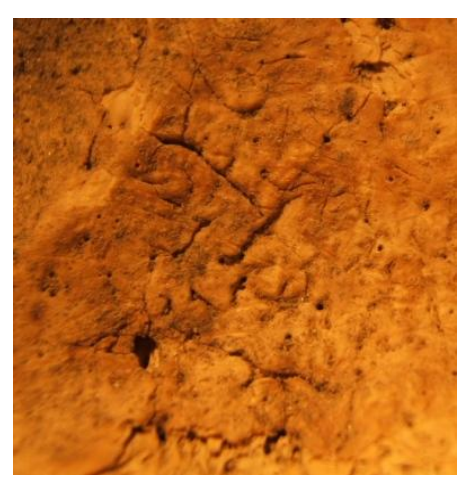

Figure 7.16: Endocranial new bone formation on HGO-26. left tibia.
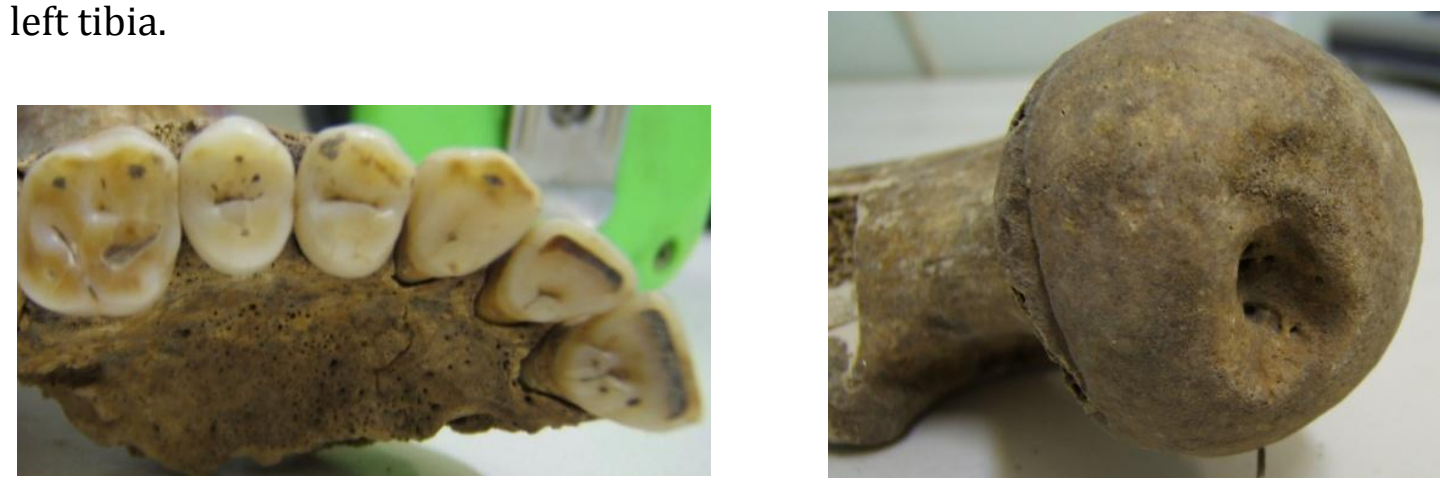

Figure 7.15: HGO-26. Left: Porotic palate from a possible inflammatory reaction.

Right: Anomalous fovea capitis. 


\subsection{Palaeopathology in the Late Neolithic - Adults}

For more details on the demographic data of these individuals, refer to appendices 11 (based on morphology) and 25 (based on measurement for otherwise unidentified individuals) for sex estimates, appendix 26 for age estimates, and appendix 30 for estimation of stature.

\subsubsection{Trauma}

Evidence of what appears to be mostly accidental trauma was observed on eleven individuals: two young males, two young females, one mature to old male, one mature to old female, three elderly males, and two elderly females. These eleven well-healed injuries comprised two cases of superficial skull trauma including one with an additional well-healed depressed skull fracture, one rib fracture, one forearm fracture, one hand fracture, one case of spondylolysis, one fracture at the knee, two post-traumatic ankle injuries, and two foot fractures. One potential case of interpersonal violence was also observed, although multiple aetiologies are possible.

The case of spondylolysis will be discussed in section 7.5.5 together with the potential case of interpersonal violence, both found on a young adult female [HGO-05, see 7.5.4].

\section{(.) Skull and thorax}

Two males, a mature adult [HGO-41] and an old adult [HGO-22], showed evidence of superficial trauma, with porosity and new bone formation on top of their skulls (figs.7.17 \& 7.18).

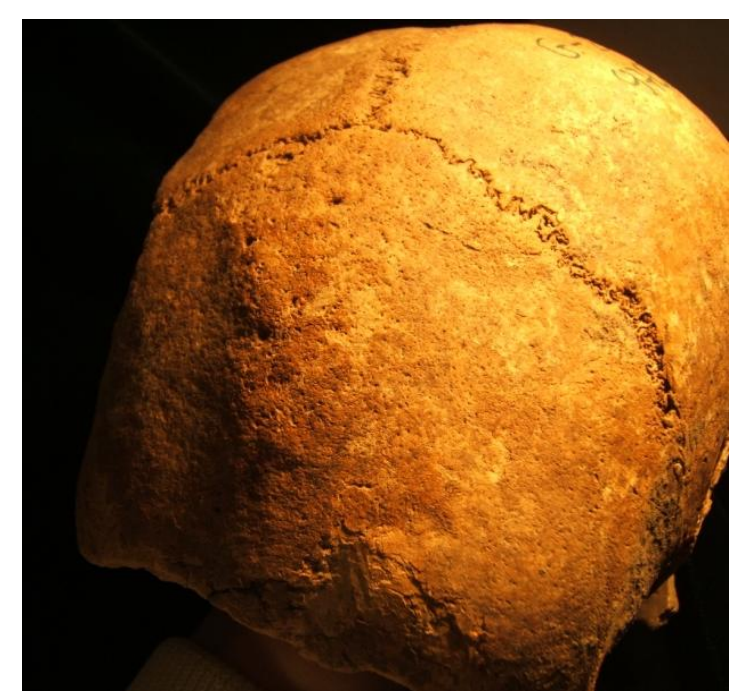

Figure 7.17: Sign of superficial skull trauma on HGO-22. 
HGO-41 also presented a healed depression fracture about $20 \mathrm{~mm}$ in diameter on his right parietal (fig.7.18, right).
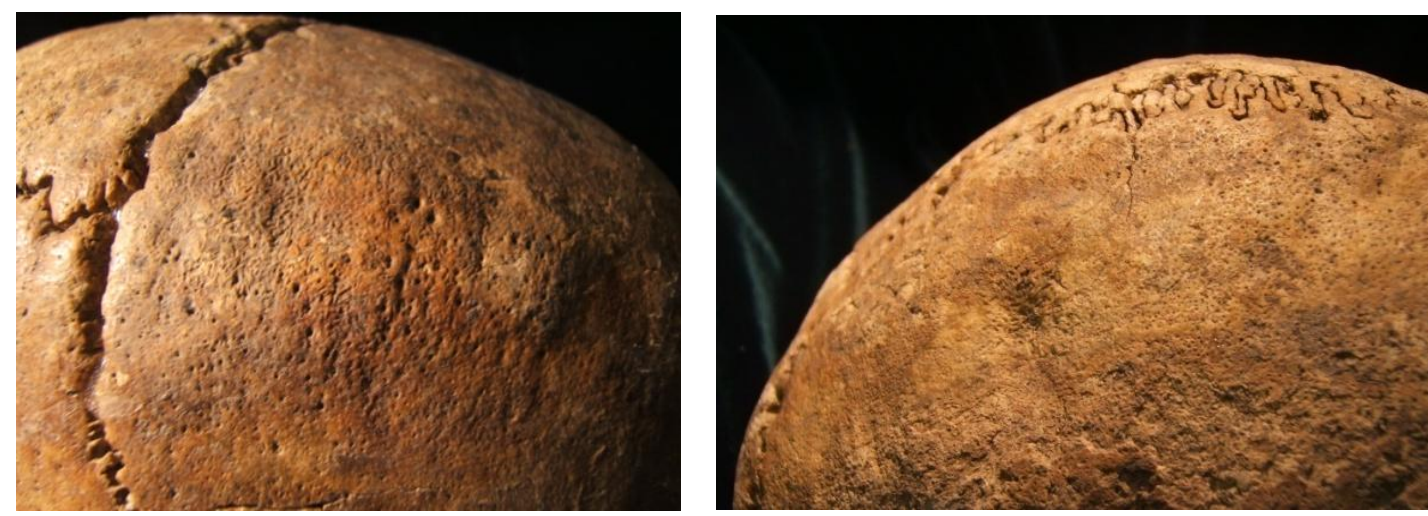

Figure 7.18: skull of HGO-41.

Left: Superficial trauma with bone formation.

Right: Depressed healed fracture.

Evidence of a completely healed rib fracture was observed in a male in his late teens / early twenties [HGO-01] with bone formation on the superior side.

\section{(๑) Upper Limbs}

BKP-02, an old adult male, had suffered from a fracture of the left distal radius a long time before death. Although it is probable that this indicates an old juvenile greenstick fracture, pointing to accidental trauma, it is also possible that this was a parry fracture maybe caused by interpersonal violence.

HGO-10, a young adult male, presented an avulsion fracture of the lateral articular surface of the first right metacarpal with corresponding osteophytes towards the base of the right first proximal phalanx dorsal side, especially on the left side (fig.7.19).

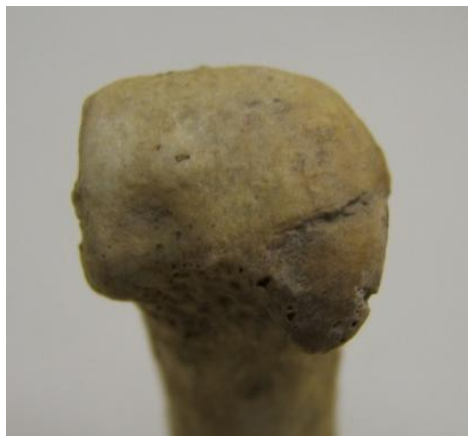

Figure 7.19: HGO-10 - right MC1 with avulsion fracture 


\section{(๑) Lower Limbs}

A small compression fracture of the left tibial plateau towards the fibula articular facet was found on HGO-28, an old adult female.

Two elderly individuals presented evidence of post-traumatic pathology, following a trauma of the soft tissues around the ankle. BKP-04, a male, showed signs of a possible subluxation of the left ankle, with marked attachments at the tibiofibular syndesmosis and a slight depression on the lateral side towards the distal end, a bony protuberance on the medial side of the left fibula towards the distal end, and an enthesopathic lesion on the calcaneus at the insertion of achilles tendon. The other case was noted on a

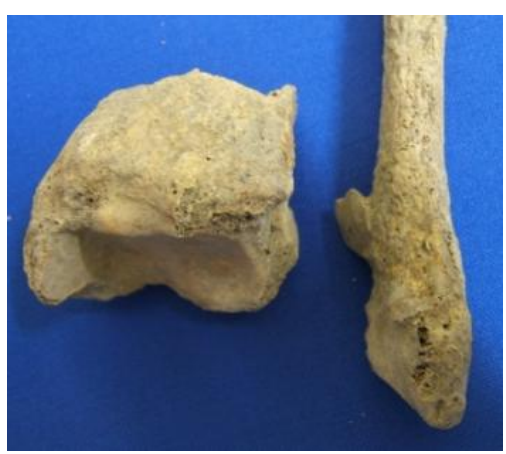

Figure 7.20: Pathological left ankle of HGO-12. female [HGO-12], with periostitis on the medial side of the left fibula towards the distal end, lipping of the articular surface and protrusion of an anomalous articular facet which articulated with a small corresponding facet on the tibia at the tibiofibular syndesmosis (fig.7.20) and enthesophytes of the Achilles tendon attachment on the left calcaneus.

An avulsion fracture of the lateral tubercle of the right talus posterior process (fig.7.21) was observed on a young female adult [HGO-56].

Figure 7.21: Avulsion fracture on the right talus of HGO-56

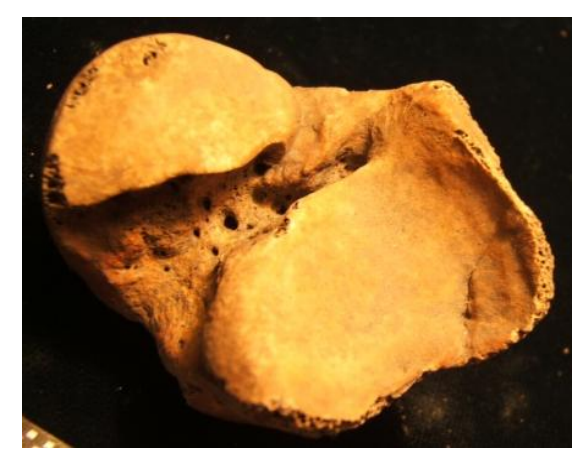




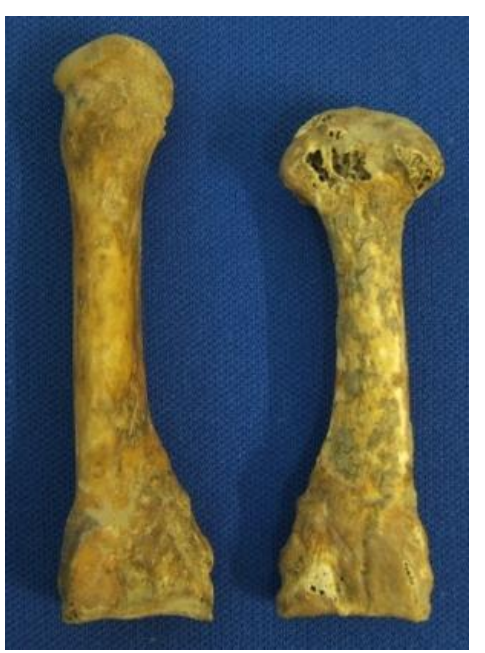

Finally, a mature or elderly female [HGO-19] had experienced a crushing fracture of the third right metatarsal's head, completely healed at the time of death. This had resulted in a much smaller metatarsal with an enlarged flat distal end and an extension of the proximal phalanx at the proximal articular surface (fig.7.22).

Figure 7.22: HGO-19 -

Crushing fracture of head of MT3

\subsubsection{Non-Specific Pathologies}

\section{Non-Specific Stress Indicators (NSSI)}

Eleven occurrences of NSSI were observed, including cribra orbitalia, cribra cranii, periostitis and increased vascularisation.

\section{( ) Non-Specific Infections}

Deep vessel grooves on the maxilla at the nose were noted on a mature adult [HGO-41], signalling chronic rhinitis.

The remains of HGO-44, an adult female, provided evidence of mastoiditis (fig.7.23) with large perforations on the mastoid process and behind the edge of the temporal, and smaller apertures along the ear canal on the petrous bone.
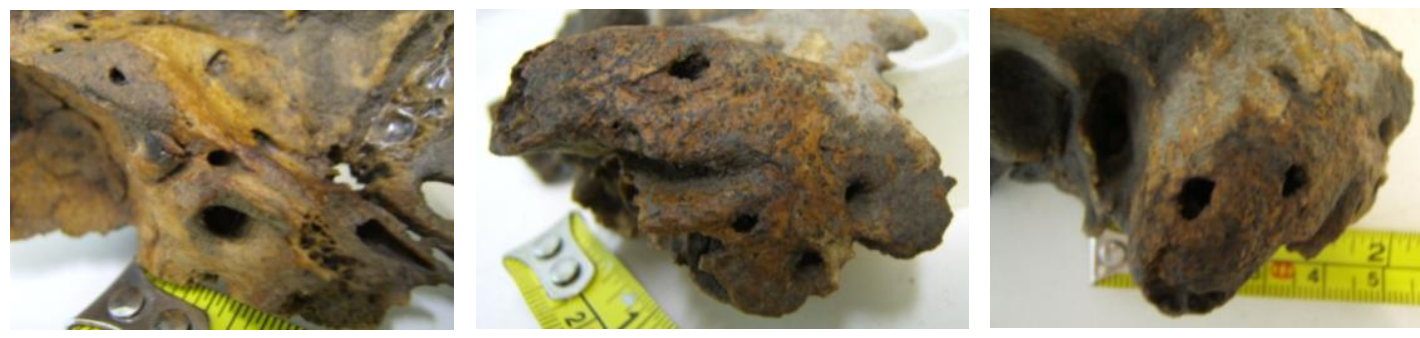

Figure 7.23: Traces of probable mastoiditis observed on HGO-44 
Meningitis of unknown aetiology was discovered in the following six cases.

HGO-22, an old adult male mentioned in section 7.5.1 for his superficial skull trauma, showed abnormal endocranial vessel impressions, with abnormally marked meningeal groove and pitting (fig.7.24).

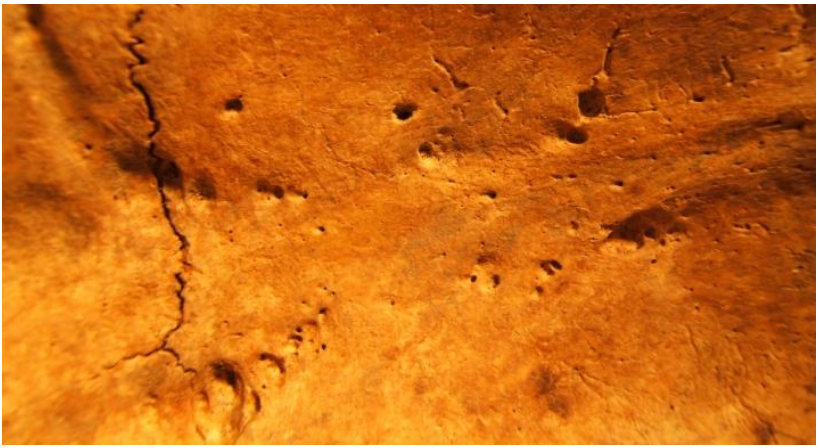

Figure 7.24: abnormal blood vessel impressions (abvi) on HGO-22

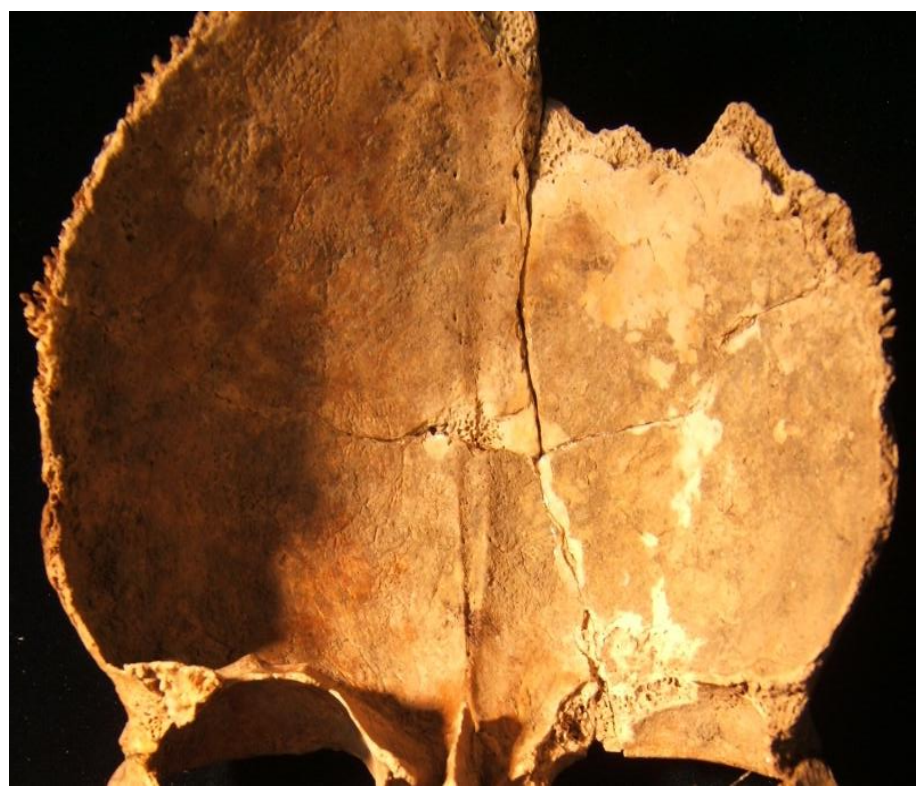

Abnormal blood vessel impressions on the endocranial surface of all skull bones (fig.7.25) as well as overall endocranial irregularity, were observed on HGO-23, a young adult female with cribra orbitalia in the right orbit and severe oral pathology.

Figure 7.25: HGO-23 - abvi

Abnormal blood vessel impressions were also visible on two old adult females, HGO-24 (fig.7.26a) and HGO-34 (fig.7.26b), the latter with granular impressions on both temporal.

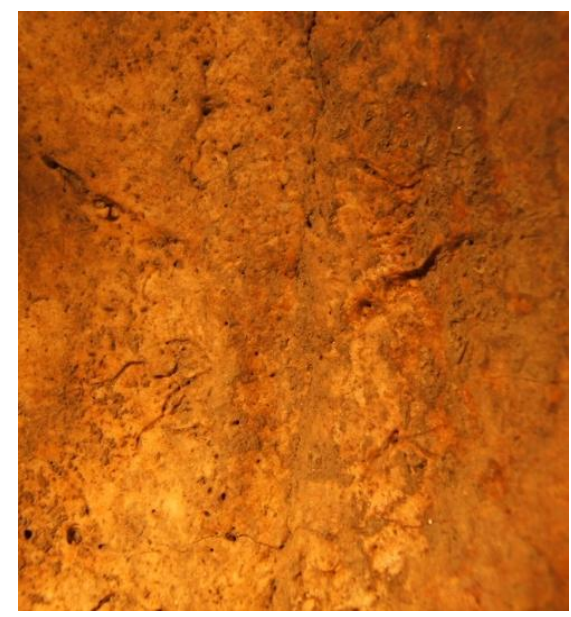

Figure 7.26a: HGO-24 - abvi.

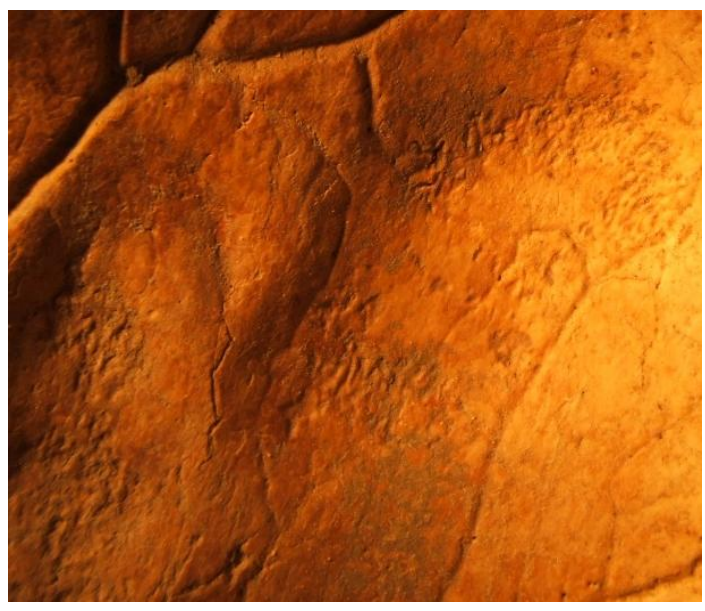

Figure 7.26b: HGO-34 - granular impressions 
Abnormal blood vessel impressions were detected on the frontal of HGO-56, a young adult female, in addition to endocranial pitting on the occipital, suggesting meningitis. Further signs of infections were found on the postcranial remains, including sacroiliitis, remodelling on the anterior surface of vertebrae, light periostitis along the shafts of both femurs, tibiae and fibulae.

Finally, cribra orbitalia and strong vessel impressions on the endocranial surface were also observed on an adult male, HGO-84.

\subsubsection{Specific infections}

\section{(- Hypertrophic pulmonary osteopathy secondary to tuberculosis}

HGO-53 was a young adult male, probably between 19 and 20 years old, with an estimated height of around 5'5 (165 cm). His remains were very fragmentary with over one thousand fragments though his skeleton was mostly complete. Pathology was observed on his skull, thorax, shoulder, upper limbs, spine, lower limbs and feet (fig.7.27).
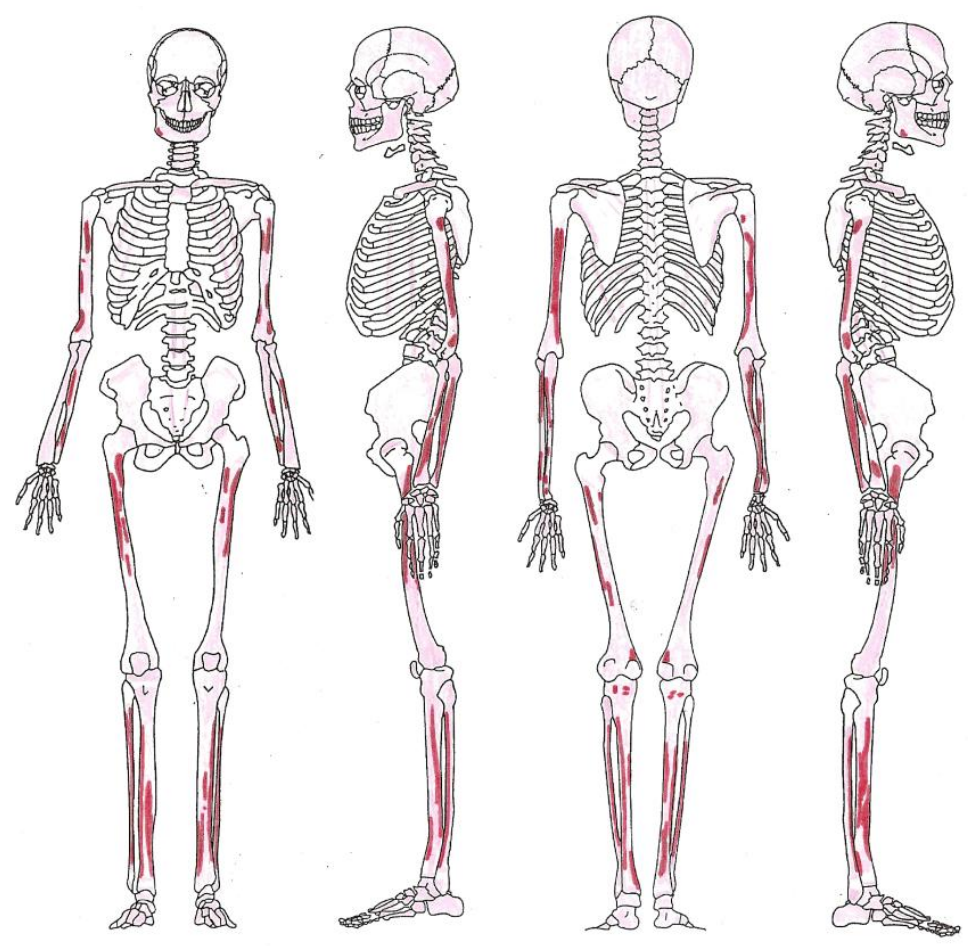

Figure 7.27: HGO-53 - Location of periostitis. 


\section{Pathology}

Light cribra orbitalia and cribra cranii were visible on the skull, and a small area of periostitis was visible on the right ramus of the mandible, on the buccal side towards the gonial angle. The only dental pathology was evidence of calculus.

Active diffuse periostitis with severe bone formation on the ventral surface of the heads of nine left ribs was observed, although none on the heads of right ribs. Sixty-three unsided fragments of ribs also showed active diffuse periostitis on all fragments with a focal lytic lesion accompanied by reactive surface new bone formation in one case (fig.7.28).
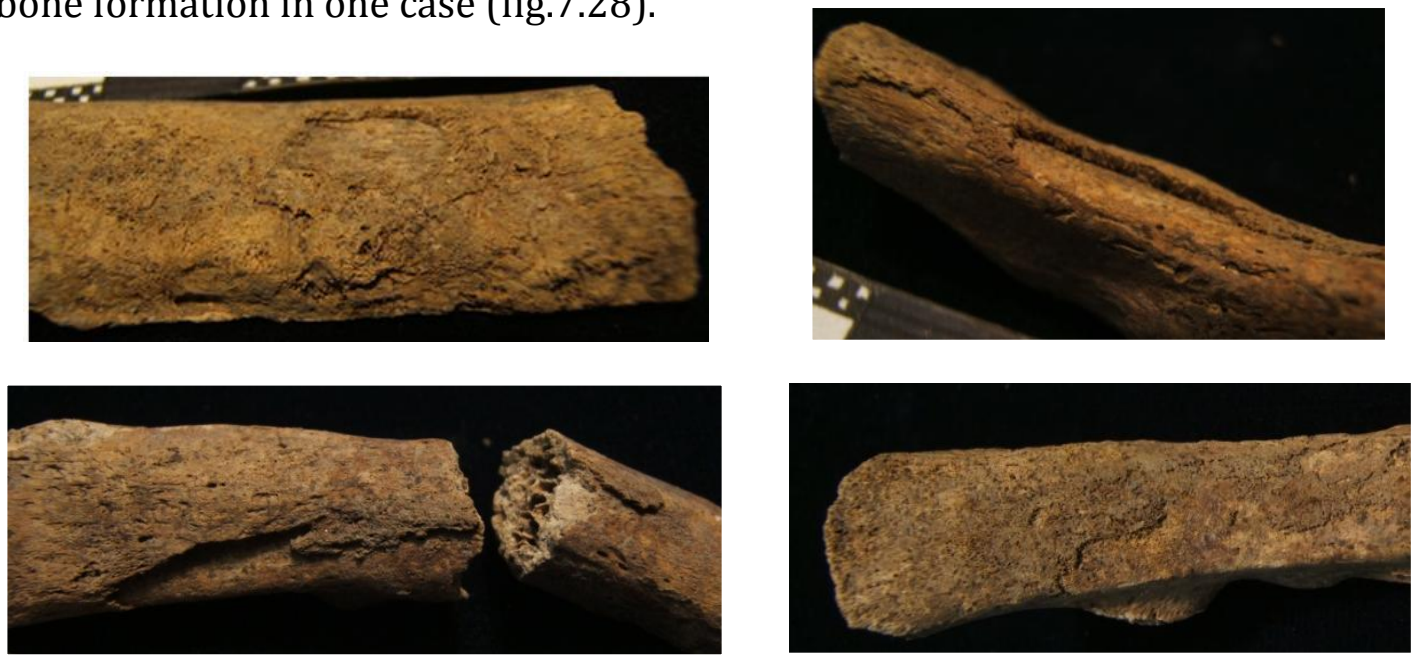

Figure 7.28: HGO-53 - Lytic lesion and active diffuse periostitis on ribs

An enthesopathic lesion at the site of the costo-clavicular ligaments was observed on the medial end of the right clavicle. Cavitations (Schmorl's nodes or lytic lesions) were observed on six fragments of vertebral bodies (fig.7.29).
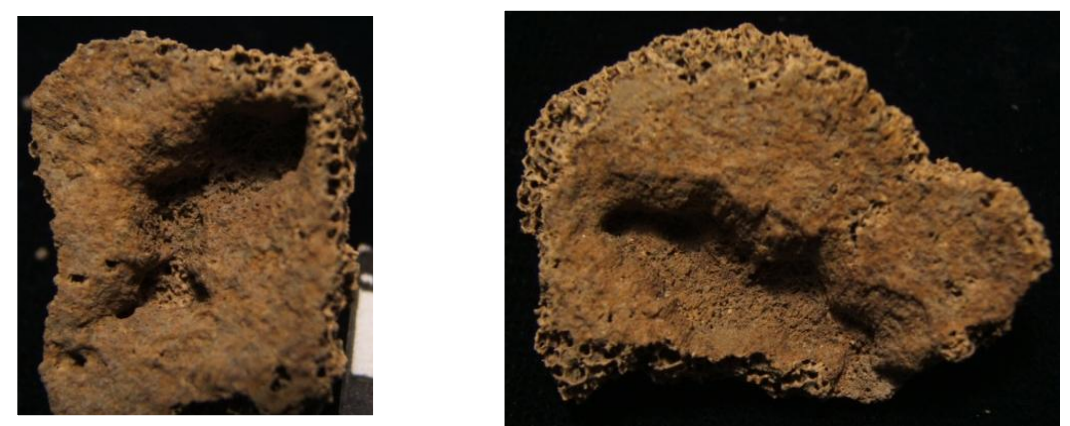

Figure 7.29: HGO-53 - Cavitations on two fragments of vertebral bodies 
The left and right humeri also presented with periostitis along the shaft, mainly on the posterior side, with bone destruction and periostitis on the crest of the lesser tubercle (fig.7.30a). Periostitis was observed along the entire shaft of the left radius, though scarce along the anterior side. The active woven bone formation was mainly located towards the middle of the shaft on the posterior side. The right radius also showed periostitis along the shaft, mainly on the posterior side, with an active woven bone formation. Widespread active periostitis was visible along the shaft of both left and right ulnae, mainly on the posterior side and specifically the lateral half (fig.7.30b).

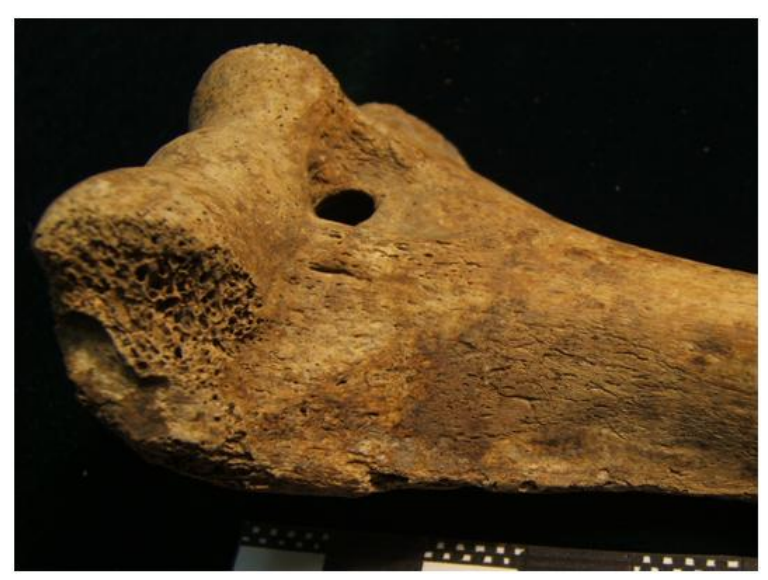

Figure 7.30a: HGO-53 - Periostitis on distal end of humerus

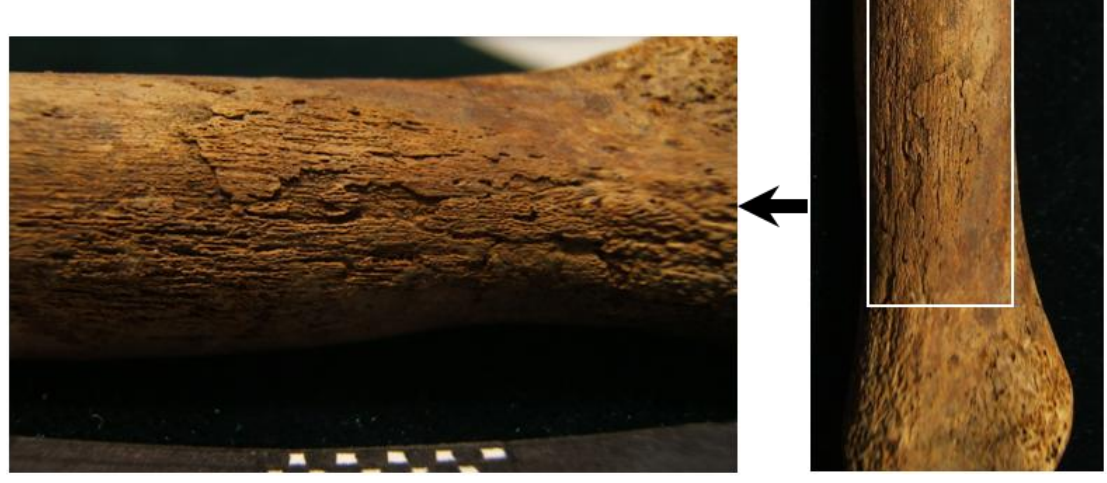

Figure 7.30b: HGO-53 - Traces of widespread woven bone formation on ulna, indicating active periostitis shortly before death

Both femurs had widespread active periostitis along the shaft, mainly on the lateral side, with woven bone formation (see fig.7.31a below). Both tibiae showed widespread active periostitis along the shaft with woven bone 
formation (fig.7.31b). Widespread active periostitis with woven bone formation was also observed on both fibulae, mainly along the medial side (fig.7.32).

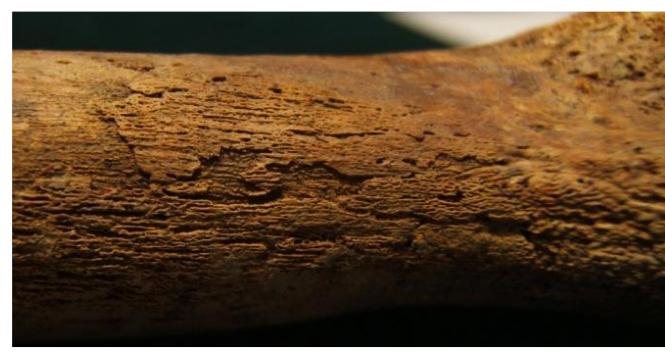

Figure 7.31a: HGO-53 - Periostis on femur

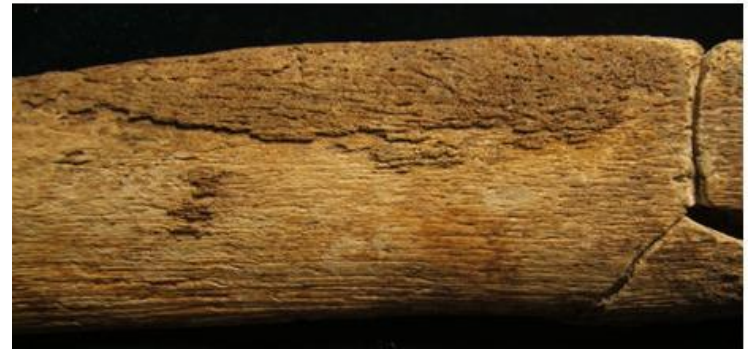

Figure 7.31b: HGO-53 - Periostitis on tibia

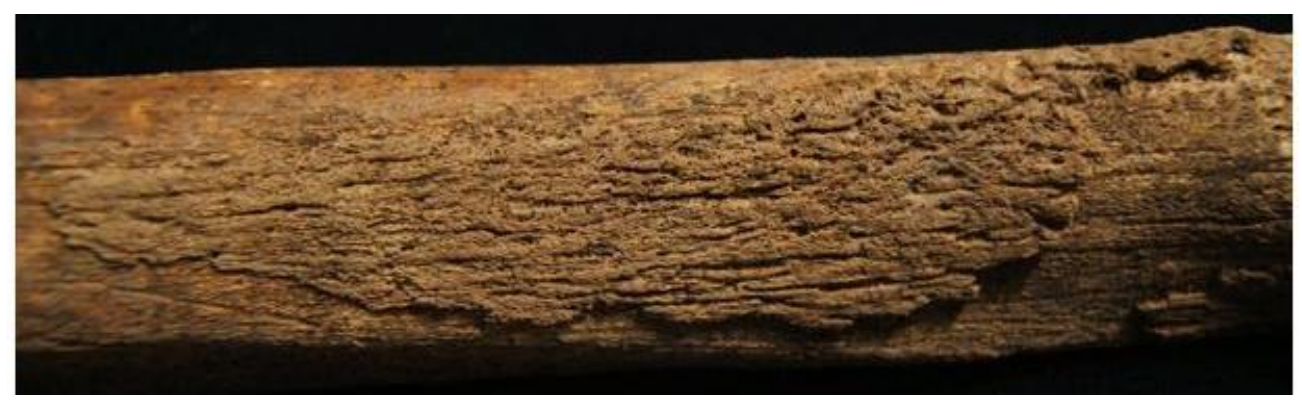

Figure 7.32: HGO-53 - active diffuse woven bone formation on fibula

Slight periostitis below the sustentacular sulcus was visible on the left calcaneus, with a cavitation between the posterior and middle talar articular surface next to the medial edge (fig.7.33). The right calcaneus also presented evidence of periostitis below the sustentacular sulcus. Periostitis was observed on the first, fourth and fifth metatarsals on both sides, and on the right second metatarsal. The first left proximal phalanx had periostitis on the shaft and a slight cavitation in the metatarsal articular surface, and the first right proximal phalanx had a stronger cavitation on the

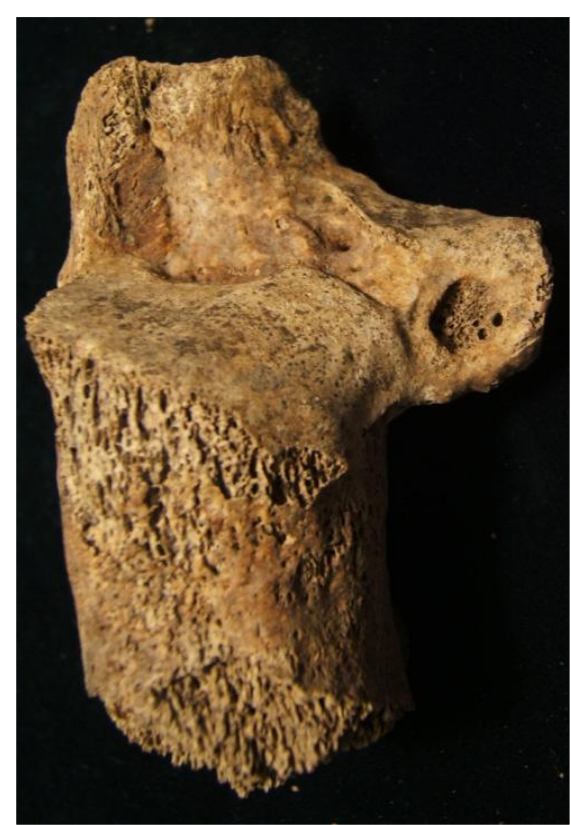

Figure 7.33: HGO-53 cavitation on calcaneus metatarsal articular surface. 


\section{Macroscopic Diagnosis}

The macromorphological analysis revealed a strikingly symmetrical diffuse "appliqué" periostitis on the bones of this young adult male. This characteristic sign of Secondary Hypertrophic Osteoarthropathy (HOA), also known as Hypertrophic Pulmonary Osteopathy (HPO) strongly advocated that this individual had been suffering from a chronic pulmonary disease. In addition, the analysis also brought to light revealing changes on the ribs of the left chest, cavitations in the vertebral bodies and signs of porotic hyperostosis. The strong association with tuberculosis, (see section 6.4), the antiquity of this population and the importance this case would have for palaeopathology made further studies of this 7000 year-old skeleton imperative to ascertain the presence of tuberculosis at the Tisza Culture site. Without any complimentary microbiological studies however, all that could be stated with high certainty was that HGO-53 was one of the earliest evidence of chronic pulmonary disease in the archaeological record. Detection of aDNA and lipid biomarkers can offer decisive support for the presence of tuberculosis in archaeological material, so there was good expectation of finding such biomarkers in HGO-53 (Hershkovitz et al. 2008; Minnikin et al. 2012). It was therefore decided that in this instance, further biomolecular analyses would be organised.

$\underline{\text { aDNA }}$

The DNA analysis was undertaken in the former Department for Medical Microbiology, University College London, by Dr. Helen D. Donoghue, Centre for Clinical Microbiology and Centre for the History of Medicine, UCL. She had previously published an article on the potential of aDNA for the study of tuberculosis although warning that DNA was not a stable molecule and would degrade with age (Donoghue et al. 2004). 
Due to the condition of the fragments, DNA was very hard to recover from this sample. DNA was nevertheless successfully extracted from the bones, and analyses provided a partial sequence for Mycobacterium tuberculosis, albeit fragmented and of poor quality. A PCR using primers of specific for Mycobacterium tuberculosis gave an amplicon of 113bp (fig.7.34).

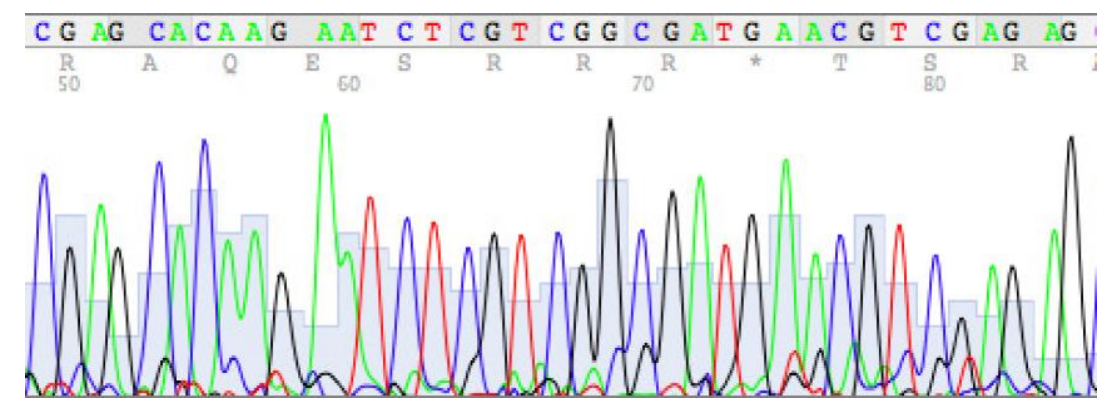

Figure 7.34: Results from DNA analyses on HGO-53

\section{Lipid biomarkers}

As an alternative to DNA, Gernaey and colleagues $(1999 ; 2001)$ recommended the use of mycolic acids, these lipid biomarkers being complementary to and possibly more reliable than DNA especially in an archaeological context. They also do not suffer as much from contamination problems, as the sensitive methods used involve no amplification. This new technique has already been refined several times to ensure maximum potential (Hershkovitz et al. 2008; Donoghue et al. 2010). Redman and colleagues (2009) demonstrated that mycocerosic acid biomarkers were also robust indicators of tuberculosis in ancient remains. Both these classes of lipid biomarkers are totally distinct from anything found in mammalian tissue. In addition, the mycocerosis and mycolipenic acid cell wall lipid biomarkers appear to be more stable than aDNA, and can thus offer conclusive support as demonstrated in a very ancient, 17,000 year-old bison metacarpal (Lee et al. 2012). 
Prof. David E. Minnikin and Dr. Oona Y.-C. Lee from the School of Biosciences, University of Birmingham, analysed this sample for lipid biomarkers and obtained a mycocerosate profile typical of M. tuberculosis (fig.7.35), in addition to a weak mycolic acid trace with severe degradation (fig.7.48). This case has now been published in full (Masson et al. 2013).

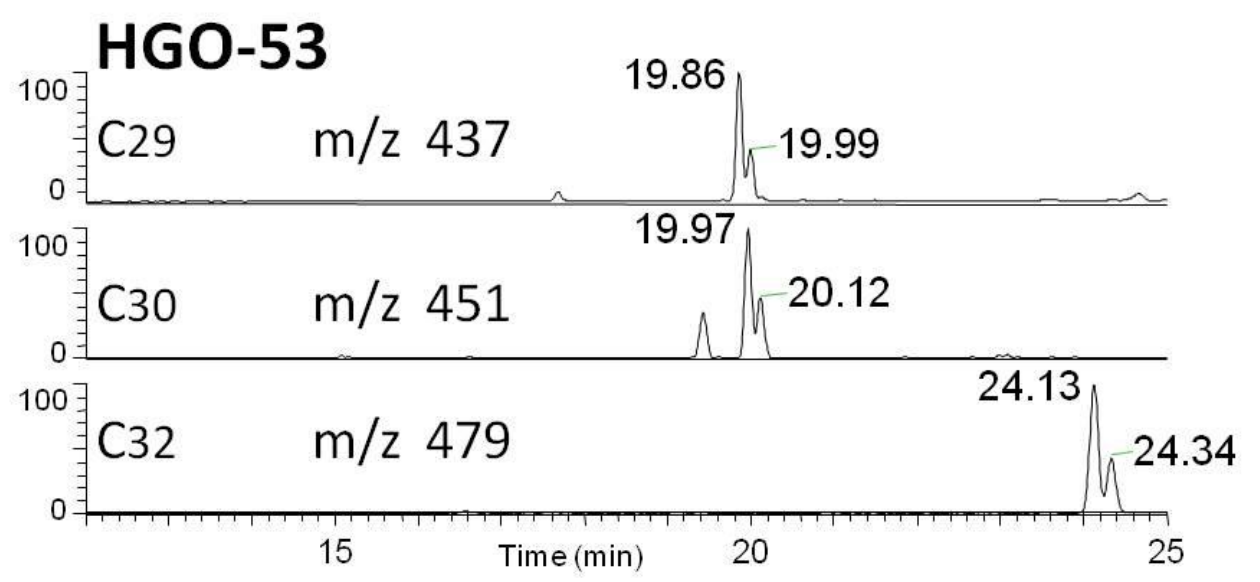

Figure 7.35: HGO-53 - Mycocerosate profile (kindly provided by Prof. David Minnikin and Dr. Oona Lee)

\section{๑) Tuberculosis}

Four further probable cases of tuberculosis were discovered in this population, all young adults. All have now been confirmed by positive results from lipid biomarkers analyses, and two have also produced positive DNA results.

HG0-08 was a young female aged 17-22 years. Light cribra orbitalia was visible in both her orbits. Resorptive lesions were observed on the anterior aspect of all thoracic vertebrae from T5 (fig.7.36 overleaf) and on two lumbar vertebrae. Schmorl's nodes were found on each thoracic vertebra from the T7 to T12. An extensive lesion on both the inferior side of the first lumbar vertebra and the superior side of the second lumbar vertebra was found with adjacent osteophytes (fig.7.36). This individual also showed anatomical variations including a thirteenth thoracic vertebra and a lumbarised first sacral vertebra, 


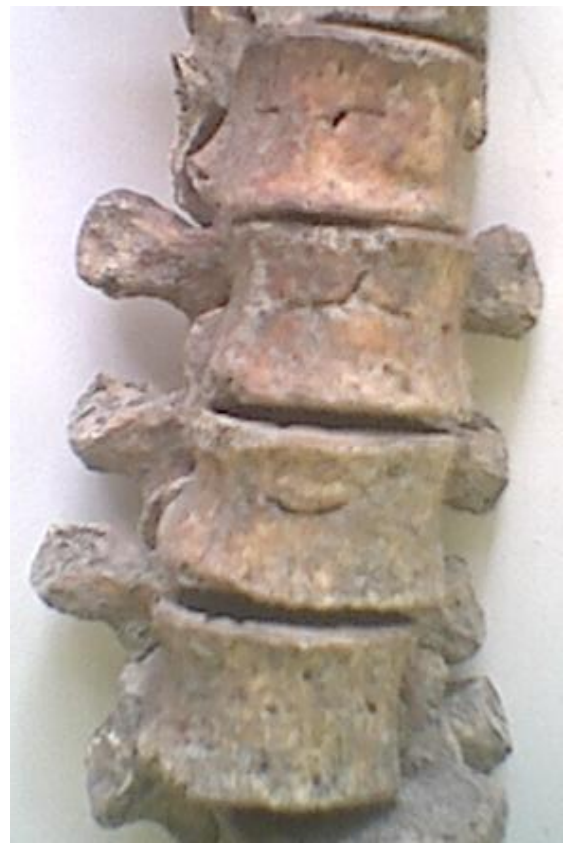

Figure 7.36: HGO-08 - Resorptive lesions on thoracic (above) and lumbar (right) vertebrae. humeral facets on the acromions and marked asymmetry in the clavicle and humerus. Musculoskeletal stress markers were present on the left clavicle, pelvis and naviculars, the latter anomalous in shape. The case was confirmed by lipid biomarkers analyses (figs.7.37 \& 7.48).
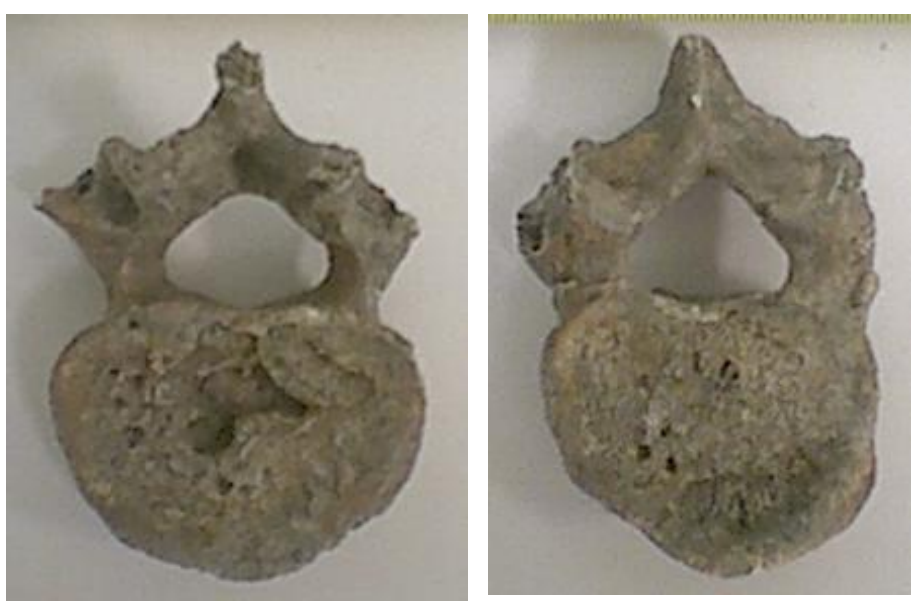

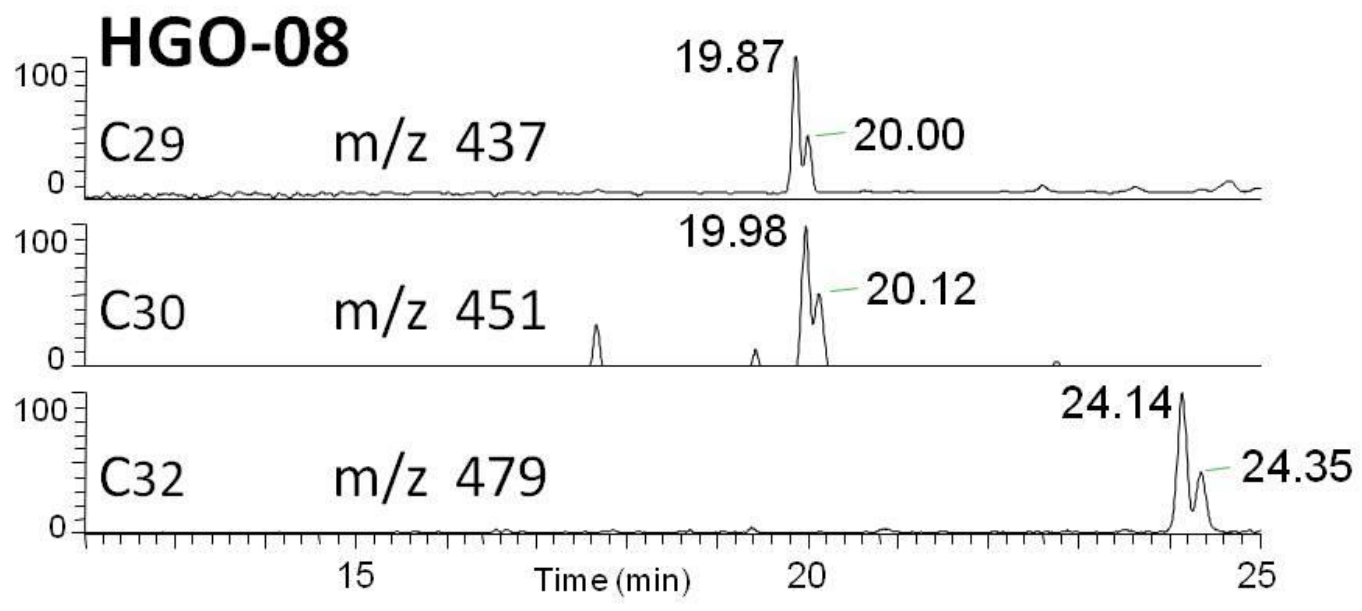

Figure 7.37: HGO-08 - Mycocerosate profile typical of M.tuberculosis (kindly provided by Prof. David Minnikin and Dr. Oona Lee) 
HGO-10, a male in his early twenties, was also diagnosed as a possible case of tuberculosis based on the evidence of hypervascularisation (pits and grooves) on the anterior aspect of five consecutive thoracic vertebrae (fig.7.38) and two lumbars (pits only), in addition to some level of periosteal remodeling and a slight hypervascularisation on the visceral surface of his ribs. Linear enamel hypoplasia was visible on his dentition. The case was confirmed by lipid biomarkers analyses (figs.7.39 \& 7.48).

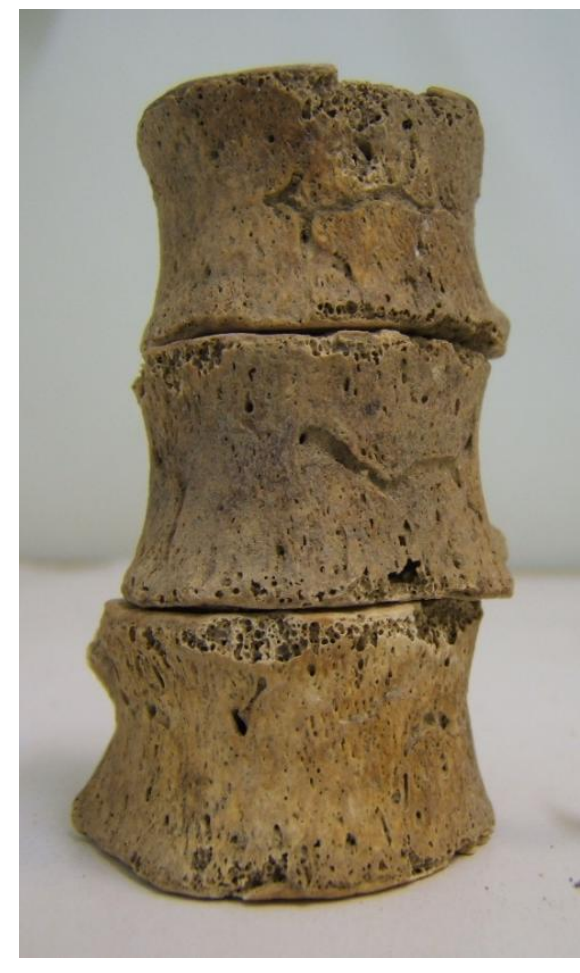

Figure 7.38: HGO-10 hypervascularisation of thoracic

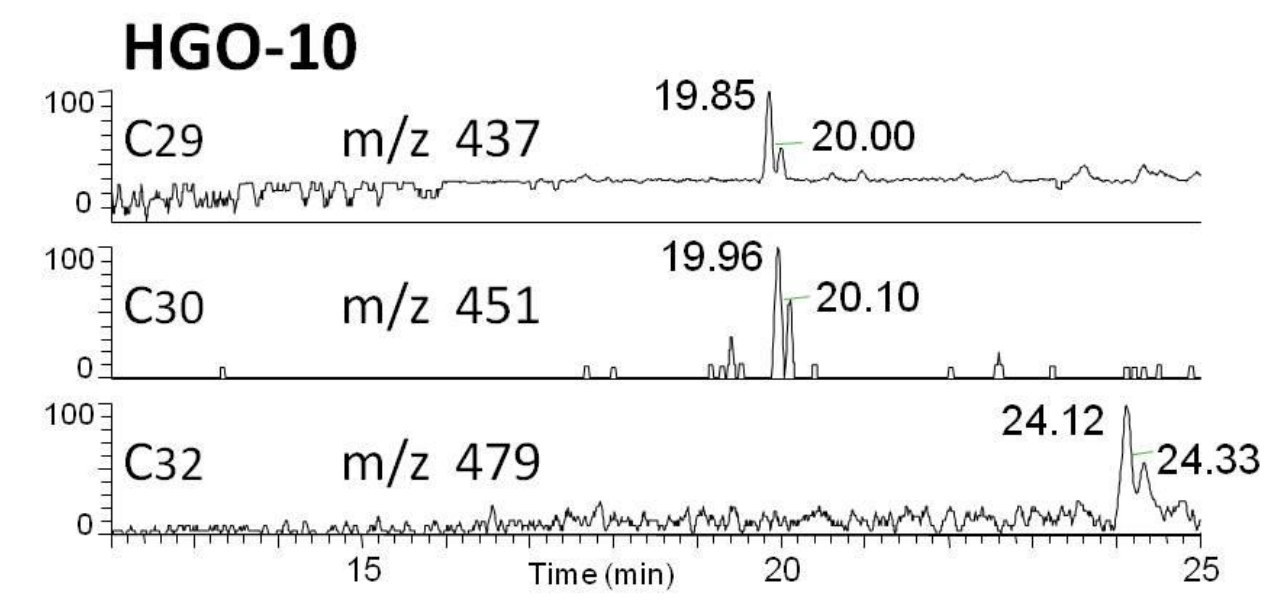

Figure 7.39: HGO-10 - Mycocerosate profile typical of M.tuberculosis (kindly provided by Prof. David Minnikin and Dr. Oona Lee)

HGO-21 was a female in her early twenties. Small endocranial pits, resorptive lesion on the ninth thoracic vertebra (fig.7.40 overleaf), increased vascularisation on the ventral side of one rib, cribra orbitalia, and light periostitis on the external surface of two fragments of ribs towards their sternal 
end (fig.7.41), all pointed to a possible case of tuberculosis. This was confirmed by the mycocerosate (fig.7.42) and to a lesser extent the mycolic acid (fig.7.48) profiles, and by the results of DNA analyses (fig.7.43).

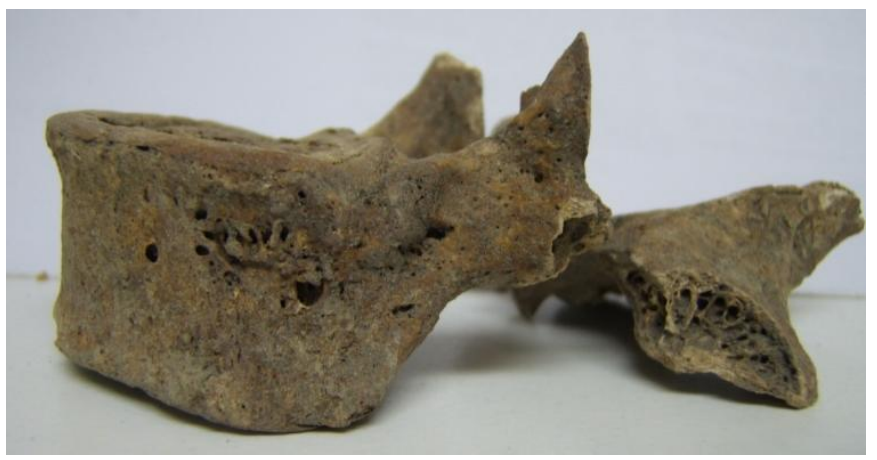

Figure 7.40: HGO-21 - Resorptive lesions on $9^{\text {th }}$ thoracic vertebra
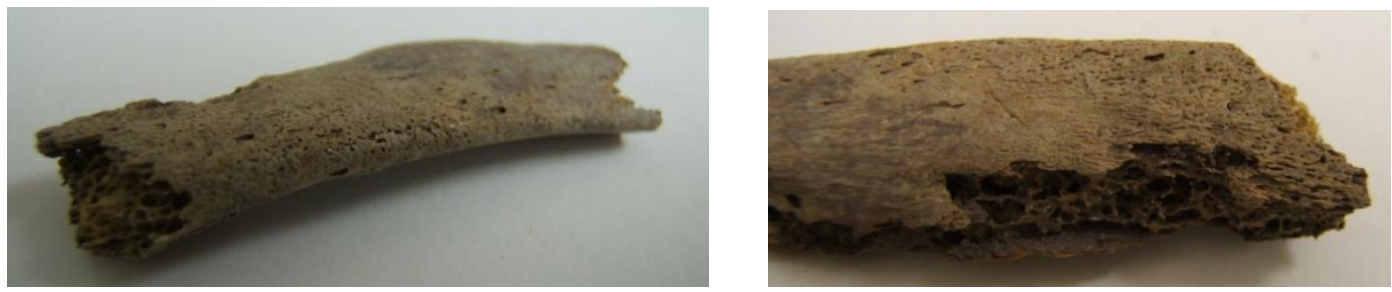

Figure 7.41: HGO-21 - Periostitis on two rib fragments

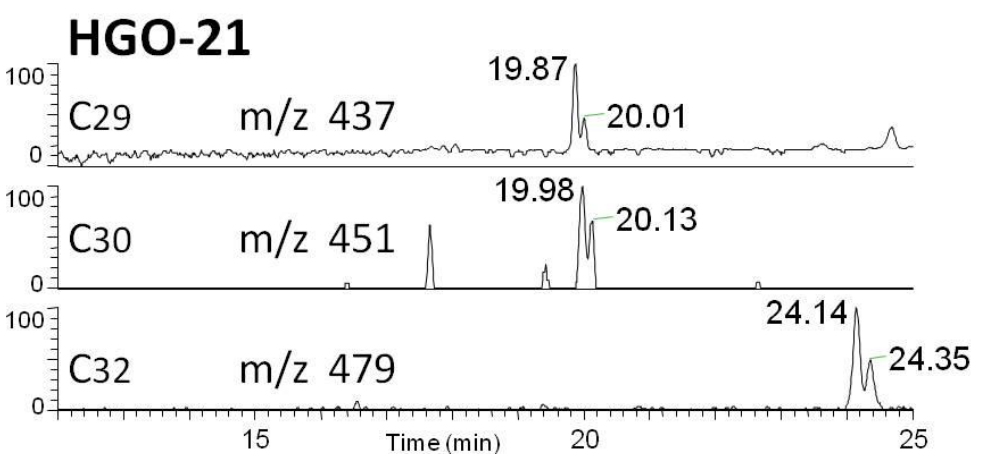

Figure 7.42: HGO-21

Mycocerosate profile, typical of $M$. tuberculosis (kindly provided by Prof. David Minnikin and Dr. Oona Lee)

Figure 7.43: HGO-21 Initial results of aDNA analysis. These PCRs used primers and a specific fluorescent probe for the

Mycobacterium tuberculosis complex region IS1081 (6 copies/cell), as confirmatory as the more traditional sequencing used for HGO-53 but better suited to fragmentary DNA as this probe target a shorter $72 \mathrm{bp}$ sequence.

The + indicates that the DNA extraction included treatment with PTB to enhance strand separation of the DNA. Kindly provided by Dr. Helen Donoghue

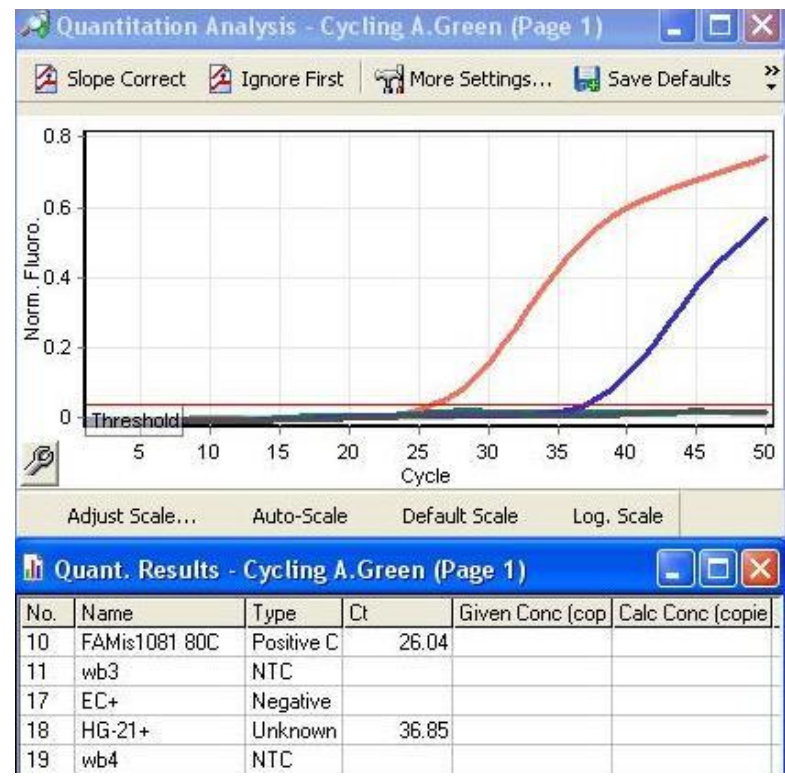


The last confirmed case of tuberculosis so far is HG0-48, a young adult female showing abnormal blood vessel impressions (abvi) over most of the frontal endocranial surface with SESlike pattern (Serpens endocrania symmetrica), indicator of meningitis caused by infection, metabolic disease and particularly tuberculosis

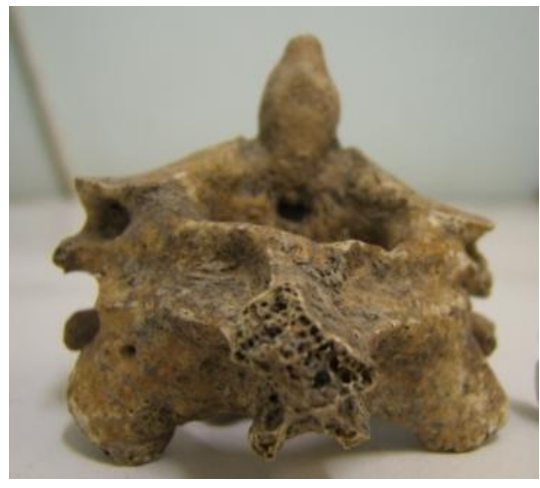
(fig.7.45). A large round depression around $1 \mathrm{~cm}$ in diameter on the endocranial surface deep in the right parietal towards the coronal and sagittal sutures could be a sign of tuberculous lesion. Very slight cribra orbitalia was found in her left orbit, and this young female's axis and third vertebra were fused (fig.7.44). Results from the mycocerosate profile (fig.7.46) and DNA analysis (fig.7.47) were positive for M. tuberculosis.

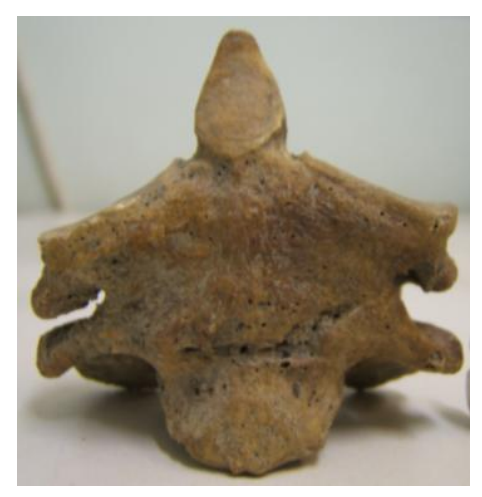

Figure 7.44: HGO-48 Axis / C3 fusion

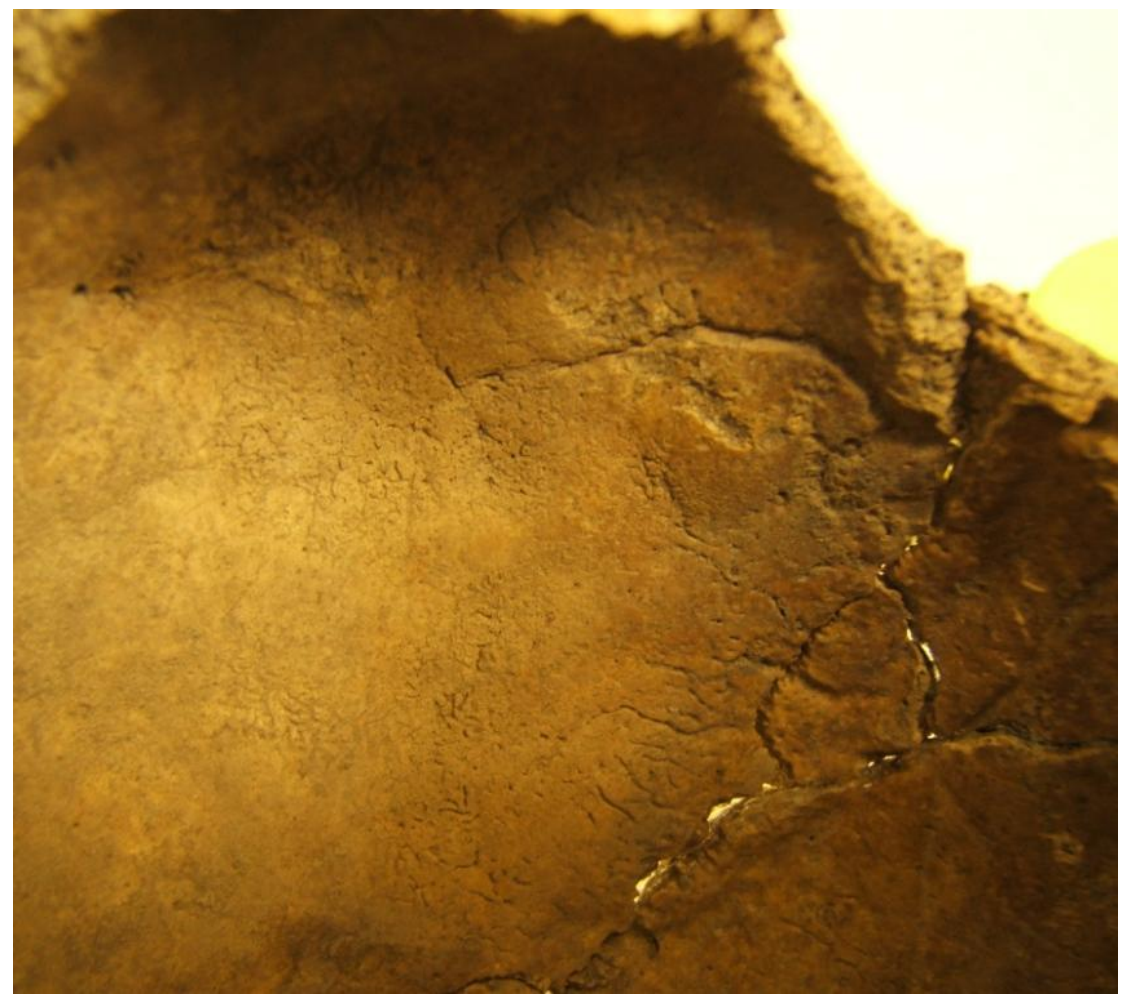

Figure 7.45: HGO-48 - Abnormal blood vessel impressions in SES-like pattern 


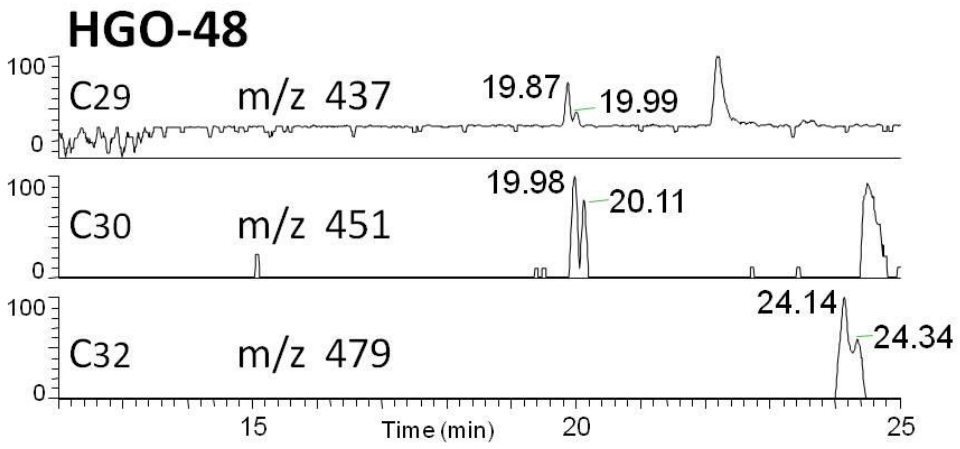

Figure 7.46: HGO-48

Mycocerosate profile, typical of $M$. tuberculosis (kindly provided by Prof. David Minnikin and Dr. Oona Lee)

Figure 7.47: HGO-48

Initial results of aDNA analysis. These PCRs used primers and a fluorescent specific probe for the MTb

complex region IS1081

(6 copies/cell).

The + indicates that

the DNA extraction included treatment with PTB to enhance strand separation of the DNA.

Kindly provided by

Dr. Helen Donoghue
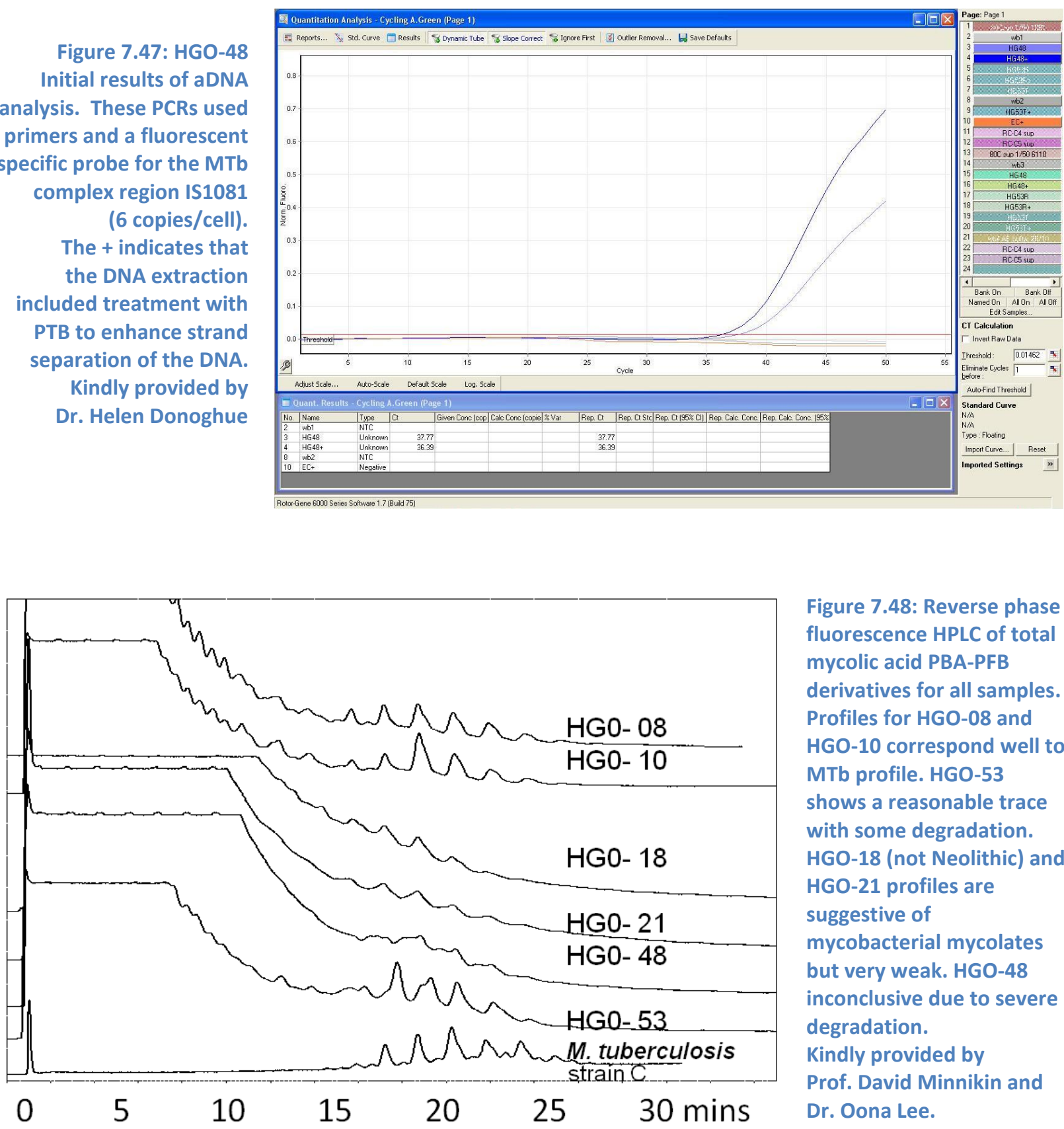

Figure 7.48: Reverse phase fluorescence HPLC of total mycolic acid PBA-PFB derivatives for all samples. Profiles for HGO-08 and HGO-10 correspond well to MTb profile. HGO-53 shows a reasonable trace with some degradation. HGO-18 (not Neolithic) and HGO-21 profiles are suggestive of mycobacterial mycolates but very weak. HGO-48 inconclusive due to severe degradation.

Kindly provided by Prof. David Minnikin and Dr. Oona Lee. 


\section{- Another possible HOA case}

HG0-11 was an adult female only represented by fragments of her lower body. All long bones and foot bones showed osteoporosis. A possible case of $\mathrm{HOA}$ was indicated by periostitis with severe widespread active appliqué woven bone formation all around the shafts (fig.7.50), especially on the anterior and lateral side of the left femur (right femur missing), left and right tibiae, left and right fibulae, left and right fourth metatarsals and left fifth metatarsal, as well as periostitis on the medial side of the right calcaneus and right talus and light periostitis on the shafts of the right second metatarsal and both sides of the third metatarsal (fig.7.49).

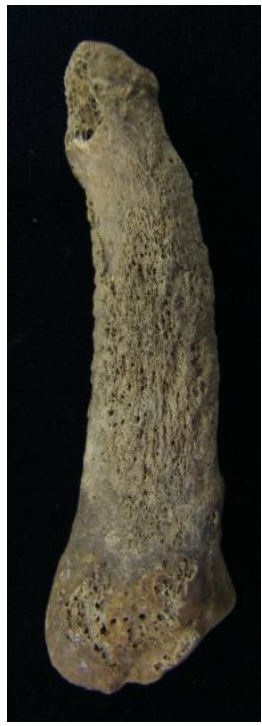

Figure 7.49: HGO-11

Periostitis on metatarsal
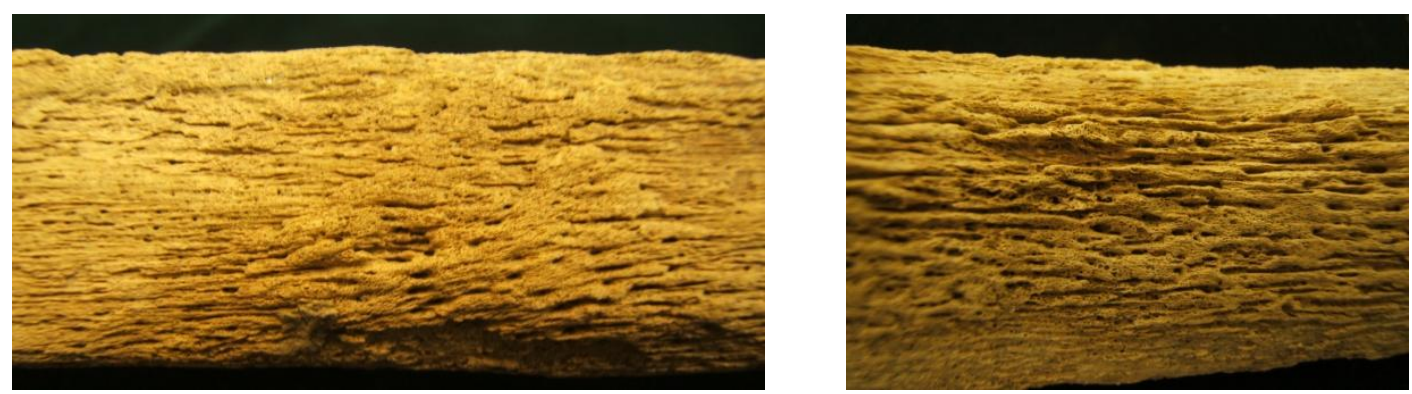

Figure 7.50: HGO-11 - Severe widespread active woven bone formation. Left: tibia

Right: fibula

\subsubsection{Multiple pathology case study}

HGO-05 was a young woman in her early twenties and around 5'2 in height. Multiple pathologies were observed on her mostly complete though fragmentary skeleton.

\section{(๑) Mastoiditis}

Signs of mastoiditis, an inflammatory process of the mastoid, were clearly visible on the left temporal (fig.7.51). The mastoid process on that

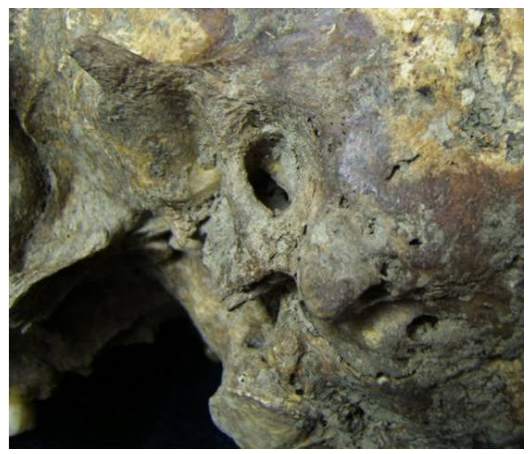

Figure 7.51: HGO-05 - Mastoiditis and abnormal zygomatic process 
side showed severe bone destruction, making it much smaller than its right counterpart, and its tip had been perforated (fig.7.52 below), which may have led to a Bezold's abscess (Parker 1994; Castillo et al. 1998; Spiegel et al. 1998). Signs of superficial alterations were also present above the external auditory meatus (see fig.7.51 on previous page).
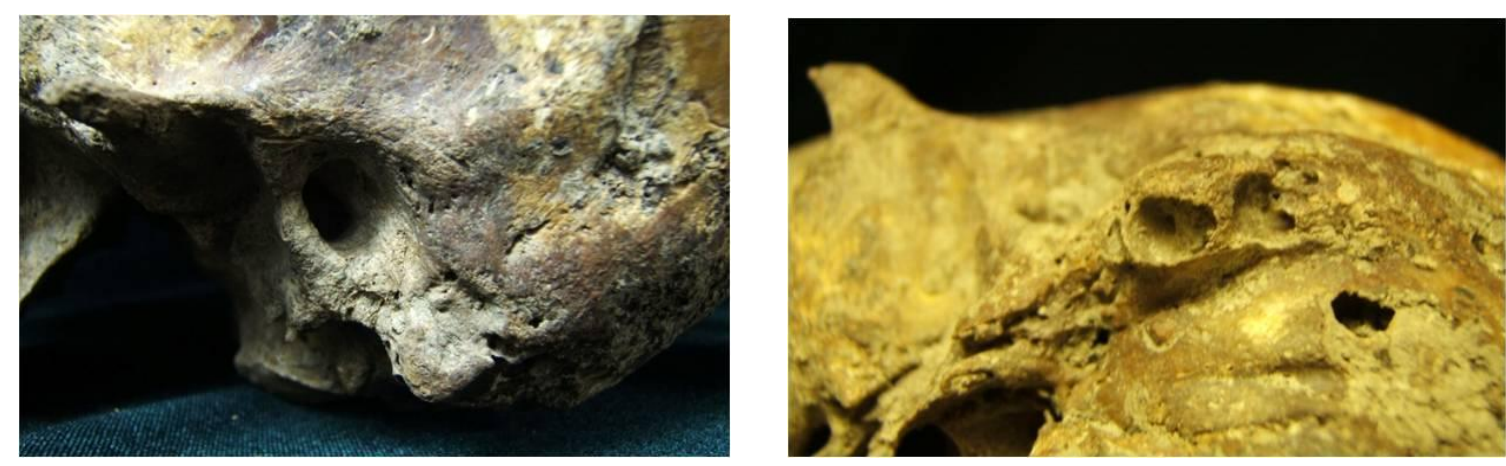

Figure 7.52: HGO-05 - Left temporal with mastoid process much reduced in size and its tip perforated

\section{Absent left zygomatic process}

This young woman's left zygomatic process had completely disappeared (fig.7.53 and 7.54), creating a noticeable facial asymmetry (fig.7.54). This could be linked to the mastoiditis, which can spread to surrounding bones including the zygomatic arch, causing further

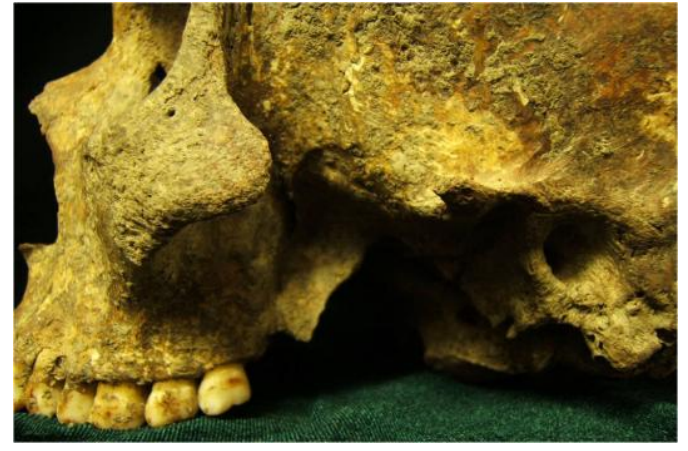

Figure 7.53: HGO-05 - Missing zygomatic process bone destruction (Warnaar et al. 1989; Kuczkowski et al. 2005; Araújo et al. 2008). An unusual developmental anomaly cannot be ruled out either. However, it seems more probable that this striking pathology originated from trauma, more precisely an isolated depressed zygomatic fracture, which combines three fracture points, the first where impact was made and the other two at both ends caused by the arch being forced inward (Galloway 1999). Extensive remodelling at both ends (figs.7.51 \& 7.52) would indicate that the trauma occurred a long time before death, when she might still have been a juvenile. 

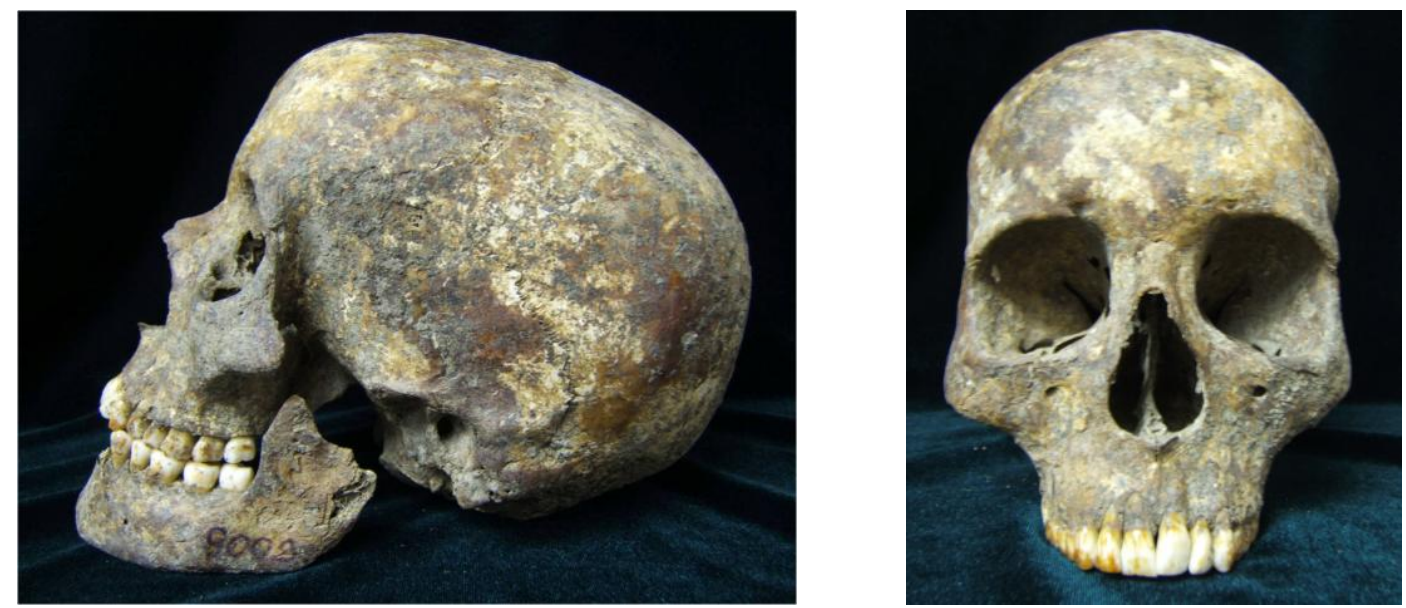

Figure 7.54: HGO-05 - Left side of skull with missing zygomatic (left) and facial asymmetry (right)

\section{๑) Spondylolysis}

This young woman also displayed evidence of spondylolysis, the separation of the neural arch from the vertebral body, on the fourth and fifth lumbar vertebrae. Remodelling could be observed in both cases. Spondylolysis was visible on the left pars interarticularis of fourth lumbar vertebra,
Fig.7.55: HGO-05 $-4^{\text {th }}$ lumbar vertebra with spondylolysis

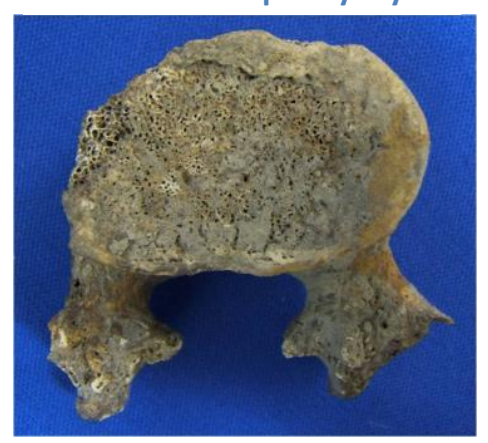
under the superior articular facet (fig.7.55). Unfortunately, the right side was unobservable due to post-mortem damage although irregularities on the right superior articular facet were antemortem, which could indicate bilateral spondylolysis. The fifth lumbar vertebra presented with a slightly asymmetrical bilateral spondylolysis on the partes interarticularis under the left articular facet and above the right inferior articular facet (fig.7.56). Part of the vertebral

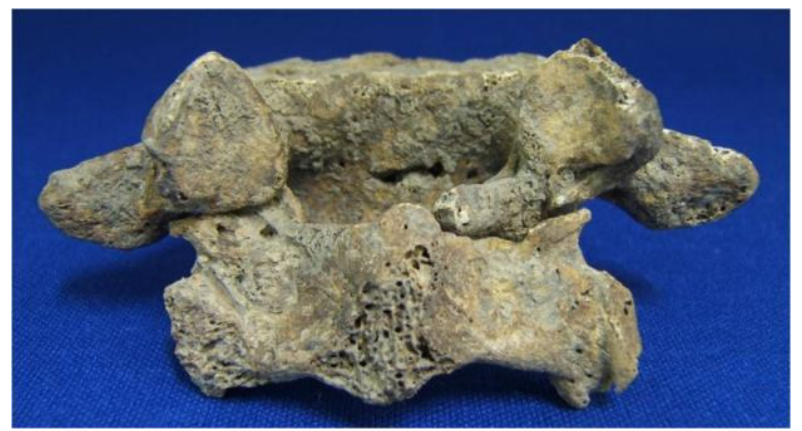

Figure 7.55: HGO-05

$5^{\text {th }}$ lumbar vertebra with spondylolysis arch was still on the main element and remodelling of the connecting surface without any fusion provided evidence for a separation quite a long time before death (fig.7.57 overleaf). 
This young woman's strenuous physical activities had also already led to the start of osteoarthritis, with lipping on the thoracic vertebrae from T4 to T11 and corresponding ribs $(5,6 \& 7$ right ribs with osteophytes on rim of vertebral articular facets). Some periosteal reaction was also visible on both coxals on the iliac tuberosity together with bone irregularity at the right greater sciatic notch.
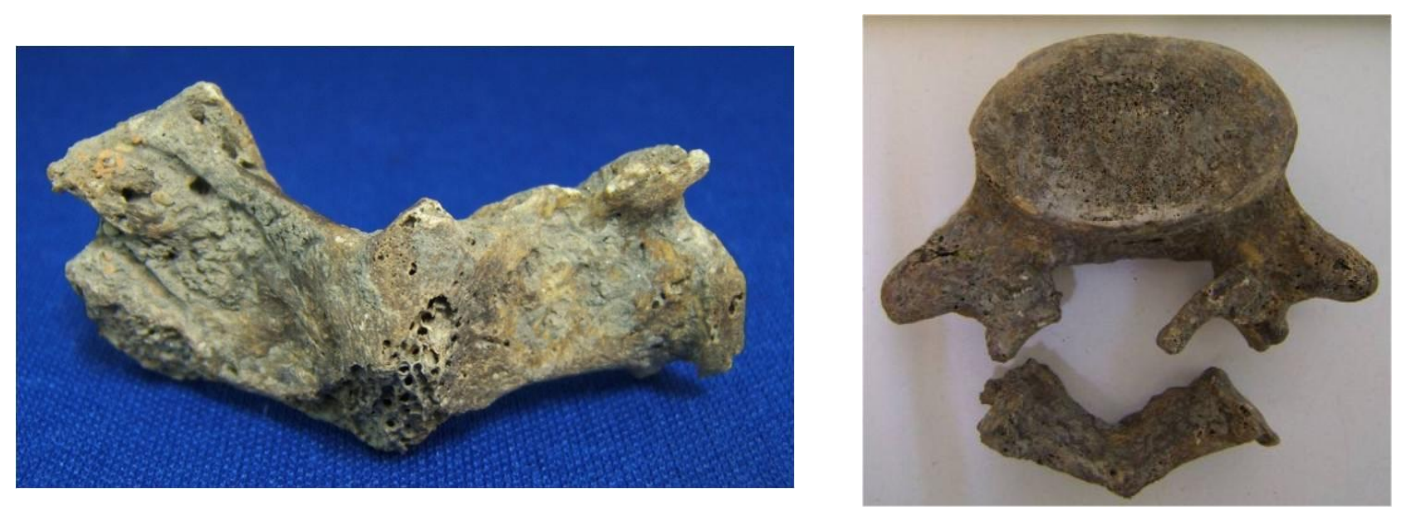

Figure 7.56: HGO-05 - separate neural arch of $5^{\text {th }}$ lumbar vertebra

\section{- Aetiology}

From the macroscopic analysis of the skeletal remains of this young Neolithic woman, it was possible to establish that during her short life, she had suffered from trauma and infection. It is unfortunately impossible at present to identify the exact aetiology of the mastoiditis, which could have been caused by a nonspecific or specific infection (including tuberculosis). It is interesting to note that acute otitis media, which could be at the origin of this case of mastoiditis, is a complication of upper respiratory tract infections, and could therefore indicate another ailment undergone by this individual.

One of the most common causes of such facial fractures, especially in children, is a fall (Fortunato et al. 1982; Iida \& Matsuya 2002; Brasileiro \& Passeri 2006; Zimmermann et al. 2006; Yamamoto et al. 2007). Nevertheless, as this type of fracture requires blunt trauma directly over the zygomatic arch, it is mostly associated with interpersonal violence and a blow from a fist (Martin et al. 1956; Kaewlai 2007). The fact that this fracture occurred on the left side and that this individual was a female could point more specifically to an act of domestic violence (Le et al. 2001; O.Dutour pers. comm.). 
Ear infection and mastoiditis have both been linked to vertigo and consequent accidental trauma from falls (Kasson-Jones \& Smith 2008). The mastoiditis could therefore potentially have caused a fall which could have led to the zygomatic fracture, and also to the spondylolysis. It seems more probable however that this unlucky individual suffered the effects of infection, domestic violence and long-term strenuous physical labour as evidenced by her classic spondylolysis and early onset of osteoarthritis, all possibly when she was still a child or a teenager. She might also have had to endure some hearing loss and her asymmetric face would have impaired her physical appearance.

It is unlikely that the precise aetiology of her pathological conditions, or their chronological order, will ever be known. Further analyses using histology, microscopy and radiography would help retrieve more information from her remains. Isotope analysis could also shed some light as to her geographical origins as well as her diet, and her place within the group or as an outsider.

\subsubsection{Other skeletal pathologies}

\section{- Degenerative changes and musculoskeletal} stress markers were very common, affecting $62.5 \%$ of young adults and all the old adults (figs.7.58 \& 7.59). They were grouped in this study under the term "mechanical changes" (see section 6.2.2). These included musculoskeletal stress markers (MSM),

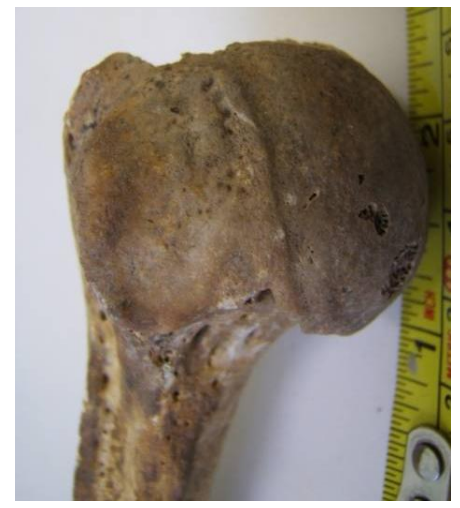
occupational stress markers,

Figure 7.57: Advance OA with lipping on a vertebra

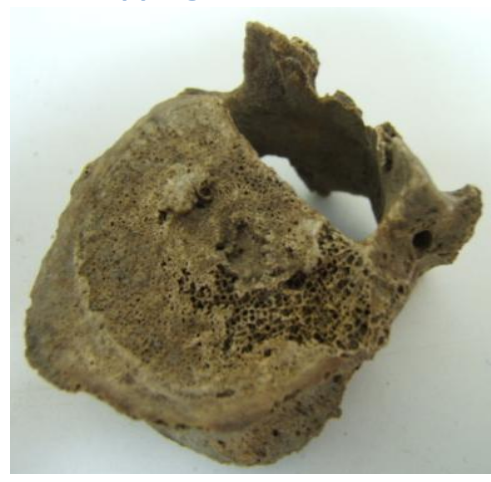
enthesopathies, degenerative joint disease (DJD), degenerative bony changes (DBC) and osteoarthritis (OA). In addition, further specific cases included aseptic osteonecroses, two cases of central osteophytes and one case of temporomandibular joint disease.

Figure 7.58: OA and enthesopathy on head of humerus 
Seventy percent of the young adults presenting mechanical changes are females. Sixty percent of young adult females and $70 \%$ of all adult females suffered some degree of mechanical changes.

(- Evidence of osteoporosis was observed in four females, all from the site of Hódmezővásárhely - Gorzsa.

( A button osteoma (or "button hamartoma" according to Eshed et al. 2002 new terminology) was noted on one skull (fig.7.60).

Figure 7.59: HGO-28 button osteoma

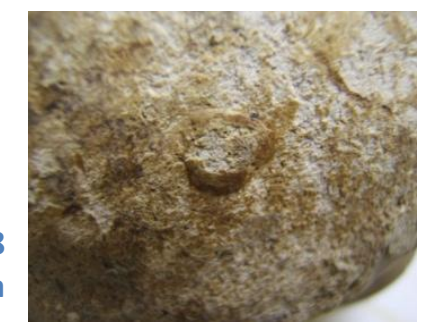

- Numerous anomalous or anatomical variations were observed, including fossae of Allen, secondary articular facet on the first rib, bifid vertebrae,

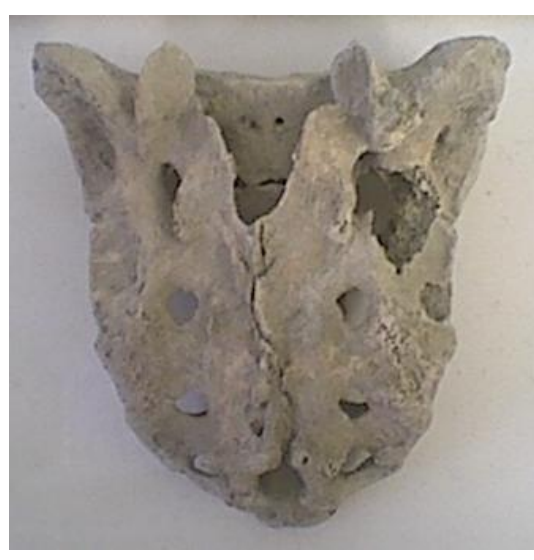

Figure 7.60: BKP-01 Spina Bifida Occulta sacralised fifth lumbar vertebrae, lumbarised first sacral vertebrae, supernumerary cervical or thoracic vertebrae, irregular vertebral body shape, spinous process deviation, bone formation on medullar surface of vertebral body, developmental anomaly of the sternum, mandibular condyle hypoplasia, spina bifida occulta (fig.7.61), and crouching facets on tibiae and metatarsals.

Two further promising pathological cases were discovered from the site of Hódmezővásárhely-Gorzsa. However, they will both require supplementary investigations to confirm the aetiology of their lesions, which would not be achievable based on macromorphological information alone. 


\section{- Potential case of cancer (metastasis)}

HGO-13 was a young adult female with very fragmentary remains. It is likely that this individual was a female, as a neonate ilium was found in the pelvic area during excavation. Severe coarse porosity was observed on the pubis and a sharp edged irregular osteolytic lesion $5 \mathrm{~mm}$ in diameter was found on the left ventral side, $15 \mathrm{~mm}$ from the pubic symphysis (fig.7.62). This could indicate an infection (pubic osteitis) or a potential metastasis (secondary to breast cancer for example, see Marks \& Hamilton 2007).

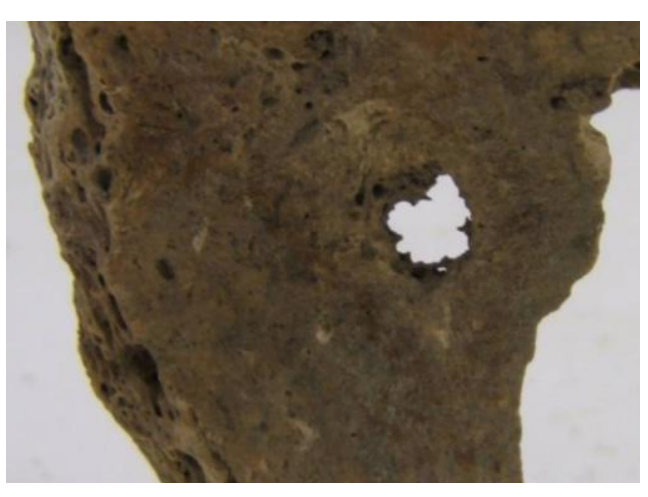

Figure 7.61: HGO-13 Signs of metastasis or pubic osteitis

\section{- Potential case of leprosy}

HGO-30 was only discovered after the main investigation was concluded, having been mislabeled as belonging to a later period in the original collection. After confirmation that this individual belonged to the Tisza culture occupation of the site Hódmezővásárhely - Gorzsa, osteological analyses revealed that this mature male showed signs of osteolytic activity on the left temporal (fig.7.63), deep vessel impressions on the endocranial surface and mechanical changes in the spine and all limbs. Moreover, evidence was observed of possible osteomyelitis or secondary infection on the distal end of the radius, tibia and fibula, diaphyseal remodelling of the proximal phalange of the feet, alterations with bony protuberance and bone loss on the first metatarsal, and osteolysis /resorption/neuropathic destruction of the second metatarsal head with typical "pencilling” (fig.7.64), all possibly consistent with leprosy (Ortner 2003). 


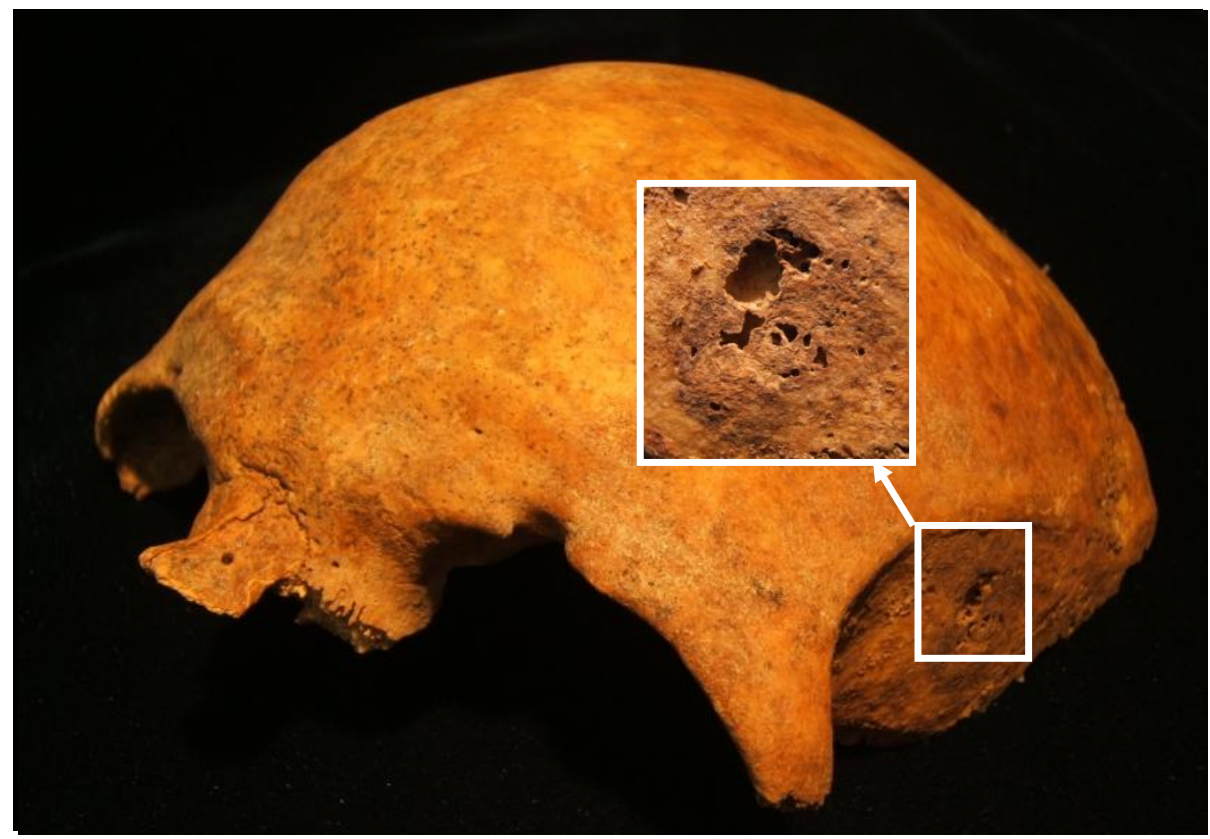

Figure 7.62: HGO-30 - Osteolytic activity on left temporal
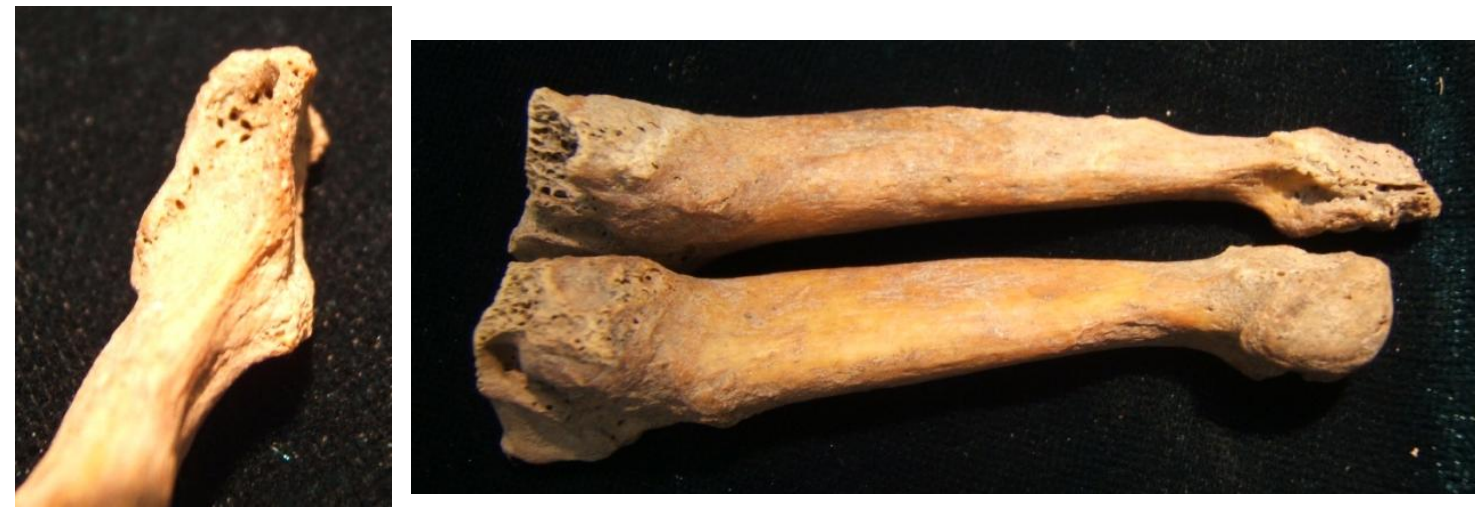

Figure 7.63: HGO-30 - "pencilling" of the left second metatarsal with head destruction

\subsubsection{Oral pathologies}

Nearly one hundred percent of the adult population showed signs of oral pathology, with fifteen out of sixteen young adults, all eleven old adults, and 39 out of the 40 dentitions available to study overall.

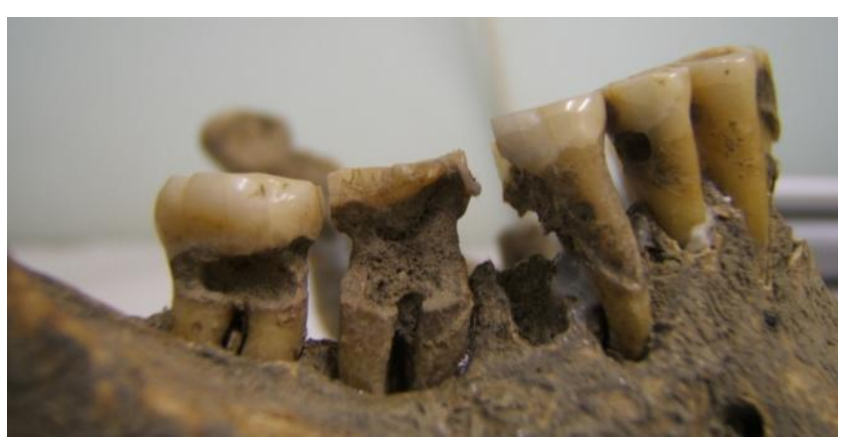

Figure 7.64: Severe carious lesions and alveolar resorption 
The most common ailment was alveolar resorption of all severity noted in three quarters of the dentitions, followed by periodontal disease and calculus in nearly sixty percent of the dentition, as well as caries in just under half of the cases (fig. 7.65). Antemortem tooth loss (fig.7.66) and anomalies or anatomical

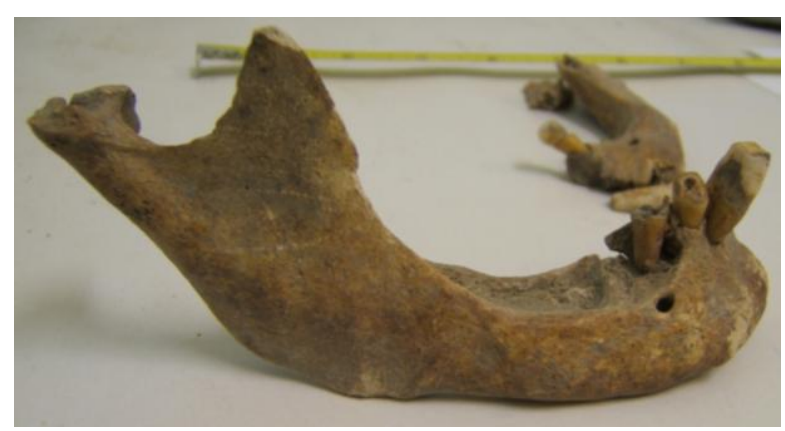

Figure 7.65: Antemortem tooth loss and extreme dental attrition variations were both seen in a third of dentitions. There were also thirteen percent of abscesses or alveolitis. Traces of linear enamel hypoplasia were only observed on three adults.

The severe dental attrition observed corresponds to other early agricultural populations, as already reported in section 3.3.5. One case of activity-related wear pattern (fig.7.67) was recognised on HGO-34, an old adult female who had lost all her mandibular teeth by the time of her death and showed severe mechanical changes, from osteoarthritis and enthesopathies to clear crouching facets on her metatarsals. Similar non-dietary occlusal grooves on the anterior maxillary dentition have been reported on two adult females from the AVK (Middle Neolithic) sites of Ecsegfalva 23 and Tiszavasvári-Deákhalmi dűlő and an adult from the Late Neolithic site of Kisköre-Gát, suggesting the manufacture of thread from plant fibres or animal sinew (Schulting et al. 2007).

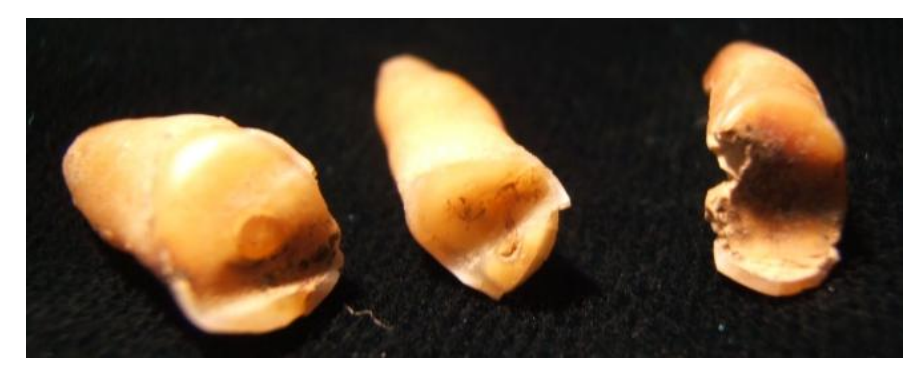

Figure 7.66: HGO-34 Activity-related pattern of tooth 


\subsection{Significance for the palaeopathological record}

Numerous discoveries made during this analysis are, or in some cases have the potential to be, of great importance for Palaeopathology worldwide. The Late Neolithic Tisza population of Hódmezővásárhely-Gorzsa in particular has provided a very significant contribution to this specialist field of research.

\subsubsection{Hypertrophic Osteoarthropathy (HOA) / Hypertrophic Pulmonary Osteopathy (HPO)}

HOA /HPO is a rare find in the archaeological record. The oldest documented cases in Europe include a Merovingian skeleton from the site of Les Rues des Vignes (Nord, France) dated AD500 to 700 (Blondiaux et al. 1992), and a medieval 40-50 year-old male from Czarna Wielka (Grozish, Poland) (Gladykowska-Rzeczycka \& Prejzner 1993). In a collection of one thousand individuals from Pre-Hispanic Mexico, two presented with HOA (Martínez-Lavín 1997): a young female from a Maya site from the Classic period (AD 300 to 900) and a young adult male from the Ticoman site from the Formative period (2000 $\mathrm{BC}$ to $\mathrm{AD} 100)$. Most recently in the Middle East, the skeletal remains of a twelve-month old infant recovered from the underwater Neolithic site of AtlitYam, Israel, dated to 9250 - $8160 \mathrm{BP}$, were described as showing evidence of HOA, in addition to Mycobacterium tuberculosis aDNA and mycolic cell wall biomarkers (Hershkovitz et al. 2008).

In their study, Webb and Thomas (1986) associated HOA particularly with severe and untreated pulmonary tuberculosis, and TB was successfully identified as the possible primary cause of HOA in a medieval case from England (Mays \& Taylor 2002). In their recent study of a Portuguese population from a pre-antibiotic era, Assis and colleagues (2011) also found a strong statistical correlation between HOA and tuberculosis in the skeletal remains. 
HGO-53, and probably HGO-11, are the earliest occurrences of fully-developed HPO on adult human skeletons to date, confirming the presence of this pathology already in Neolithic Europe and enriching the sparse archaeological record of this disease. Thanks to the additional successful microbiological analyses, the case of HGO-53 also provides another example of tuberculosis as probable primary cause of HOA /HPO in the archaeological record.

\subsubsection{Tuberculosis (TB)}

The Atlit-Yam study (Hershkovitz et al. 2008) provides the earliest biomolecular evidence of tuberculosis in humans. Both DNA and lipid biomarkers analyses confirmed that the 25-year old female and the 12-month old infant were infected with a human lineage of the Mycobacterium tuberculosis complex. The osteological pathological evidence however was very scarce on the adult female. In the infant, it consisted of endocranial changes (SES) and periostitis on tubular bones, consistent with tuberculosis. Although the periostitis was described as HOA, there is no evidence of symmetry of lesions.

Prior to this study, the oldest recognised cases of tuberculosis came from Neolithic Europe. A 15-year old juvenile and a 30-year old female from Liguria, Italy, dating from the Middle Neolithic in the first half of the $4^{\text {th }}$ millennium BC, were both diagnosed on the basis of spinal osteolytic lesions (Formicola et al. 1987; Canci et al. 1996). Another probable case originated from Zlota, Poland, based on the spine of a Neolithic male (Gladykowska-Rzeczycka 1999). Tuberculosis had also been previously confirmed by DNA analyses in a predynastic Egyptian skeleton aged 12-14 years with Pott's disease and new bone formation on one rib dated to 3400 BC (Zink et al. 2001; Zink et al. 2007).

In Hungary, Pott's disease in an adult male, dating from the Late Neolithic / Early Copper Age (5 $5^{\text {th }}$ millennium BC) was discovered recently at the site of Alsónyék-Bátaszék (Köhler et al. 2012). This has not yet been confirmed by molecular biomarkers, but the morphological observations unequivocally 
indicate an advanced stage of vertebral tuberculosis. Several other possible tuberculosis cases have also been discovered recently from the 5000 year-old site of Vésztô-Mágor, Hungary, associated with archaeological material from the Tisza Culture (Spekker et al. 2012). Palaeomicrobial analysis of the dental pulp region in the teeth of one of the cases confirmed the presence of M. tuberculosis aDNA (Pósa et al. 2012).

With the successful combination of different scientific methods, including morphological observations and palaeomicrobiological analyses, the presence of the Mycobacterium tuberculosis complex in Neolithic Europe was verified conclusively as early as 7000 years ago. Hódmezővásárhely-Gorzsa presents the oldest confirmed cases of TB in Hungary and Europe to date, second only to Atlit-Yam worldwide. It is also unprecedented in the archaeological record by providing so many ancient cases of tuberculosis, with five confirmed already and several relevant cases of non-specific infections as well as one possible HOA case as yet not biochemically analysed but also potential TB cases. It is hoped that this will encourage more osteoarchaeologists to look for less typical or obvious osteological signs of tuberculosis than for example the classic Pott's disease. By discovering more palaeopathological cases of tuberculosis, it will help us gain a unique insight into the evolution of this still ever present disease, "second only to HIV/AIDS as the greatest killer worldwide due to a single infectious agent" (WHO 2013).

\subsubsection{Scurvy}

Scurvy has historically been connected with sailors and long sea crossing journeys without adequate diet, mostly from AD 16 th century onwards (Maat 2004). Brickley and Ives (2006) found six infantile cases of scurvy among the 164 juveniles with documented history examined from St Martin's Churchyard, Birmingham, five of them aged birth to six months. The two researchers associated the lack of scurvy cases in the palaeopathological record to a 
misunderstanding and under-recognition of the skeletal manifestation of this disease. Most recently in Hungary, osteological examination of the remains of the late medieval population of Bácsalmás-Óalmás, dated 16-17th century AD, provided four new cases for publication of juveniles aged between 6 months and 3 years old, as well as highlighting 43 further possible cases, with a potential frequency of scurvy in this juvenile population of over 20\% (Lovász et al. 2013).

The origins of agriculture having led to a marked decline in food diversity with concentration on fewer plant foods such as cereal grains which lack vitamin C, would have dramatically increased the risks of scurvy (Cohen \& Armelagos 1984; Cordain 1999).

The oldest published scorbutic case comes from Early Neolithic Germany dated 3500 - 2900 BC (Carli-Thiele 1996). A probable case of scurvy was also identified in a child skeleton from England, dating to 2200-1970 BC (Mays 2008). A possible scurvy case was published based on the skeletal remains of an eight-year old child from Middle Bronze Age Israel (c. 2200 BC), although no postcranial pathology was observed (Mogle \& Zias 1995). Finally, a case of scurvy in a 14-16 year old adolescent from Early Bronze Age Franzhausen, Austria, dated 2200-1800 BC, has been confirmed by histological analysis (Schmidt-Schultz \& Schultz 2004).

Both probable cases of scurvy (one neonatal and one infantile) brought to light in this thesis originated from the Tisza site of Hódmezővásárhely-Gorzsa. They do not only confirm the presence of scurvy in Neolithic populations, but also push backwards the earliest evidence of this metabolic disease by a considerable millennium and a half. It is hoped that additional analyses including histological techniques will help to explore further these oldest scurvy cases in the world so far, enriching the palaeopathological record by providing further details for comparative case studies to osteoarchaeologists worldwide, and helping to address the under-recognition of scurvy in skeletal remains. 


\subsubsection{Leprosy}

The earliest evidence for this chronic infectious disease based on iconographic and literary material goes as far back as the second millennium BC (Aufderheide \& Rodríguez-Martín 1998). Until very recently, the earliest skeletal evidence of this infection was on two skulls from Dakhleh Oasis in Egypt, dated to 200 BC (Dzierzykray-Rogalski 1980) . However, earlier osteological evidence of leprosy surfaced very recently from the site of Abony 36 in Pest County, Hungary, where an 18-22 year-old male and a 40-45 year-old female presented macroscopic manifestions of the infection in the form of leprous rhinomaxillary changes (Köhler et al. 2009). This site is as yet undated but pottery typology places it in the Late Copper Age, and it is mostly similar with Abony site 49, which itself was dated to 3800-3500 BC. Other early osteological evidence of leprosy from Hungary include the $7^{\text {th }}$ century AD Avar age series of Kiskundorozsma (Szeged) with eight out of 94 individuals presenting osteological signs of leprosy (Pálfi \& Molnár 2009).

Mycobacterium leprae has been successfully investigated on archaeological remains with aDNA analysis (Donoghue et al. 2002) and mycolic acids analysis (Taylor et al. 2009) in the same manner as M.tuberculosis. The presence of M.leprae was confirmed by DNA analysis in all the Hungarian cases mentioned directly above. It is hoped that undertaking similar biomolecular analyses will help to shed light on the possible leprosy case of likely tuberculoid form discovered through this analysis of the late Neolithic population of Hódmezővásárhely-Gorzsa. If presence of M.leprae was confirmed following these further investigations, this case would vastly extend the palaeopathological record all the way to the origins of agriculture. It would also potentially increase the osteological evidence for tuberculoid leprosy, which is currently missing from the prehistoric record. 


\subsubsection{Cancer}

Prehistoric osteological evidence of cancer currently extends to the Neolithic. A 40-50 year old female buried in a Neolithic (Lengyel culture) stone quarry at Mauer (Vienna XXIII), Austria, dated to the beginning of the fourth millennium $\mathrm{BC}$, showed signs of multiple osteolytic lesions and was diagnosed as a case of multiple myeloma (Strouhal \& Kritscher 1990). Meningiomas were observed on a Neolithic adult skull from a necropolis at Sant Quirze del Vallès, Catalonia, Spain (Campillo 1991) and skeletal remains from the Middle/Late Neolithic in Denmark (Bennike 1985). Finally, a British Neolithic skeleton presented evidence of osteochondroma (Chamberlain et al. 1992) and a young male from Late Neolithic Catalonia, Spain, was diagnosed with Ewing's sarcoma (Campillo \& Marí-Barcells 1984).

In Hungary thirteen cases of malignant bone tumours were discovered in twelve anthropological series totalling close to 4000 individuals and dating from the $3^{\text {rd }}$ to the $16^{\text {th }}$ centuries AD (E. Molnár et al. 2009). These were mostly cases of metastatic carcinoma, with some possible multiple myelomas. Although only one possible case of cancer, more precisely metastatic carcinoma, was revealed during this doctoral research, it has the potential to push back the osteological evidence for cancer in Hungary by five millennia. It would also confirm other skeletal evidence of cancer in Europe during the Neolithic, and would predate chronologically the oldest cases currently on record. A future microscopic investigation will hopefully establish more clearly a diagnosis for this important case.

\subsubsection{Mastoiditis}

The frequencies of mastoiditis reported from archaeological material vary widely, ranging from under 20\% (Titche et al. 1981) to over 80\% (Flohr \& Schultz 2009). It is likely that methodology would affect greatly these results as the highest prevalences are found when using microscopic methods. One of the 
most famous cases of mastoiditis was that of the seventeen-year-old King Francois II of France who died in 1560 from this infection (Liston 1994; Spiegel et al. 1998). Osteological evidence of this infection goes back to the Neolithic, with two cases of mastoiditis observed in the Late PPNB population of Basta, Jordan (Schultz et al. 2004).

In total, three cases of mastoiditis were found in this population, one on a female adult with multiple abscesses in her mastoid process and the corresponding petrous bone, one on a child with a non-specific infection and one on the young adult female with multiple pathologies. These cases significantly reinforce the Neolithic osteological evidence for this infection, and increase the palaeopathological record. The link between mastoiditis and tuberculosis (see 6.2.2) is of particular interest as tuberculosis was present in the population under study (see 6.6.2). Further biomolecular studies of these three cases of mastoiditis could therefore also potentially reveal additional cases of tuberculosis.

\subsubsection{Spondylolysis}

The frequency of spondylolysis seems to vary greatly between population from about 50\% in Eskimos to less than 1\% in some European historical populations and reaching 20\% in living athletes (Lester \& Shapiro 1968; Bridges 1989; Merbs 2002). These wide variations could be due to distinct activity patterns, but also to a difference in the methodology used to assess prevalence (Fibiger \& Knüsel 2005). No other cases of spondylolysis were observed in this Hungarian prehistoric population, although it must be kept in mind that all the remains were very fragmentary and often incomplete. No other Neolithic case of spondylolysis from Europe could be found in the literature, and only one in the Old World from further afield at Çatalhöyük, Turkey, dated 7400-6000 BC (Agarwal et al. 2011). This Neolithic case from Hungary will therefore be a very useful contribution to the palaeopathological record. 


\subsection{Conclusion}

Skeletal remains available for this study were damaged and very fragmentary, and had consequently never been fully analysed previously. In addition, only macromorphological methods were available for the palaeopathological investigation. However, the painstaking and careful osteological analysis of these tens of thousands of bone fragments resulted in remarkable discoveries, contributing very significantly to the palaeopathological record.

Only two individuals from the Early Neolithic in the collection had postcranial remains. Both showed evidence for mechanical changes, but no other skeletal pathology was observed. No juvenile remains were available from the Körös Culture either. This unfortunately limits the comparisons that could have been made between individuals from the Körös and the Tisza Cultures, only appropriate for adult oral pathology.

The proportion of juveniles recovered from Late Neolithic sites is in itself indicative of a low health status: the fact that a third of individuals died so young is a clear sign of poor health, regardless of the presence or absence of visible pathologies on their skeletal remains. Osteological evidence increased with age: a third of neonates, half of infants and children, and three quarters of adolescents were pathological.

Despite the limits of the osteological material, this truly exceptional sample has provided a wealth of new information about the health status of the Neolithic populations of the Southern Great Plain of Hungary between 8000 and 7000 years ago. 


\section{- High rate of dental disease}

The Early Neolithic Körös Culture was represented by eight adult dentitions, while the Late Neolithic Tisza Culture sample provided 39 adult dentitions for observation. Oral pathology touched nearly one hundred percent of Tisza adults and all Körös individuals. The two adults originating from Neolithic sites of unspecified Culture also both presented signs of dental disease. Very high rates of calculus, gum disease and caries were observed, as well as frequent tooth loss, but very few cases of linear enamel hypoplasia (LEH) or abnormal calcification. Rates of specific pathologies for Late Neolithic individuals (see table 7.3 below) were slightly lower than their Körös counterparts, particularly for caries and abscesses, although they remained high.

\begin{tabular}{|c|c|c|c|c|c|c|}
\hline & $\begin{array}{c}\text { Körös } \\
\text { (this study) } \\
n=8\end{array}$ & $\begin{array}{c}\text { Tisza } \\
\text { (this study) } \\
n=39\end{array}$ & $\begin{array}{c}\text { Kisköre- } \\
\text { Gát }^{1} \\
n=6\end{array}$ & $\begin{array}{c}\text { Vésztő- } \\
\text { Mágor }^{1} \\
n=10\end{array}$ & $\begin{array}{c}\text { Early/Middle } \\
\text { Neolithic }^{2} \\
n=24\end{array}$ & $\begin{array}{c}\text { Late } \\
\text { Neolithic }^{2} \\
n=44\end{array}$ \\
\hline Caries & $75 \%$ & $48.7 \%$ & $60 \%$ & $60 \%$ & $54.2 \%$ & $44.7 \%$ \\
\hline $\begin{array}{l}\text { Periodontal } \\
\text { disease }\end{array}$ & $87.5 \%$ & $59 \%$ & $47 \%$ & $80 \%$ & ? & ? \\
\hline Abscess & $37.5 \%$ & $12.8 \%$ & $3.2 \%$ & $1.3 \%$ & $25 \%$ & $31.9 \%$ \\
\hline $\begin{array}{l}\text { Alveolar } \\
\text { resorption }\end{array}$ & $87.5 \%$ & $74.4 \%$ & $79.6 \%$ & $82.5 \%$ & $?$ & $?$ \\
\hline LEH & $0 \%$ & $7.7 \%$ & $0 \%$ & $0 \%$ & ? & ? \\
\hline AMTL & $37.5 \%$ & $33.3 \%$ & ? & ? & $12.5 \%$ & $38.3 \%$ \\
\hline Calculus & $75 \%$ & $59 \%$ & ? & ? & $?$ & $?$ \\
\hline
\end{tabular}

Table 7.3: Oral pathology comparison between Early and Late Neolithic adults from Hungary $\left({ }^{1}\right.$ described as Tisza (Late Neolithic) Culture, although currently dated to the $3^{\text {rd }}$ millennium BC, data from Molnár \& Molnár $1985 ;^{2}$ data from Schulting et al. 2007, see text below for details)

Table 7.3 also presents the results from two sites attributed to the same Tisza culture, although currently dated the first half of the $3^{\text {rd }}$ millennium BC (S. Molnár \& Molnár 1985). Rates of caries, periodontal disease and alveolar resorption were similarly very high, with no instance of enamel hypoplasia recorded. These two samples provided lower rates of abscess than in the Körös 
and Tisza populations investigated in this doctoral research. The AVK Middle Neolithic female discovered at Ecsegfalva 23A was also reported to have "poor" dental health similar to the samples presented in this thesis, with nine caries and three abscesses observed on this single individual (Guba et al. 2007). Another study based on 24 Körös and AVK adults and 44 Late Neolithic adults from Hungary was also published recently, although numerous individuals from the later period might be attributed to the Copper Age (Schulting et al. 2007). This study utilised five of the Körös individuals and three of the Tisza individuals from the sample analysed in this thesis, and reported that such rates were higher than in Neolithic North-western Europe but lower than in Southern Europe.

Three children and two adolescents from the Tisza culture also presented evidence of extensive dental disease despite their young age with periodontitis, calculus, gum resorption, and caries. The three children all had some type of tooth malformation, with one case of LEH, one of developmental anomalies, and one child with abnormal calcification of all permanent teeth. One of the adolescents also had an abscess.

A recent study of a North-eastern Neolithic sample from Hungary (Ubelaker et al. 2006) also mentioned a much higher rate of caries compared to the Copper Age, Bronze Age and Iron Age, as well as higher frequencies of abscesses and antemortem tooth loss, and a low rate of enamel hypoplasia. Unfortunately the rates of these pathologies as a percentage of individuals affected were not reported, with frequencies within tooth count reported instead and given separately for deciduous and permanent teeth.

\section{- Stressful life physically}

Mechanical changes were very common in the Neolithic populations studied, affecting over sixty percent of young adults and all the old adults. Although musculoskeletal stress markers (MSM) and entheseal changes (EC) are still 
generally accepted to represent stress from an occupation or activity, there has been considerable debate on the subject over the last few years (Villotte 2006; Alves Cardoso \& Henderson 2010; Villotte et al. 2010, and see section 6.2.2. for more details). The high prevalence of females in the mechanical changes category would seem to favour a link to certain types of activity.

These results might be influenced partly by the lower proportion of males in this sample, only accounting for $38 \%$ of the adults available for analyses, and more critically representing only a quarter of the young adults. In the view of the ongoing debate on the validity of such bony changes as occupational markers, and the underrepresentation of males in this sample, care must be taken not to over-interpret these results, especially for gender-related studies.

Over sixty percent of the adult population showing signs of mechanical changes and the majority of young adults as well as juveniles in their late teens would seem to indicate nevertheless long term strenuous physical labour in these Neolithic populations.

In addition, non-specific stress indicators were observed on a fifth of these populations. Two probable cases of infantile scurvy also provided evidence for insufficient or inadequate diet. These oldest cases in the archaeological record so far confirmed the incidence of scurvy in the Neolithic and pushed the earliest osteological evidence of this metabolic disease back by a millennium and a half.

\section{(-) Mostly non-violent populations}

In a recent study of 71 individuals from several Neolithic sites of North-eastern Hungary, evidence of trauma was observed in $7 \%$ of the sample, all on males and mostly in the form of depressed skull fractures (Ubelaker et al. 2006). This was attributed to higher rates of interpersonal violence in the Neolithic, shifting to more accidental traumas in the following Copper and Iron Ages. 
Contrary to these findings, the analysis presented in this thesis of one hundred individuals from Neolithic sites of South-eastern Hungary revealed a high rate of accidental trauma against a low rate of apparent interpersonal violence, particularly in Late Neolithic Tisza populations. In this sample, trauma was present in about twelve percent of the population, exclusively on adults, and may have affected males disproportionately with over half of the cases discovered on males, even though males accounted for only 38\% of recovered individuals. These trauma cases were mostly accidental fractures, with potential signs of violence affecting only three heads and one arm, all of which as likely to have resulted from accidents.

This apparent lack of violence might not be indicative of these cultures overall. It is possible that males (as well as potentially females and juveniles) who had experienced violence had died and/or were buried elsewhere. However, it is likely to reflect at least the life of the individuals recovered from these Neolithic settlements.

A single example of possible interpersonal or domestic violence was observed on a young adult female, although this case could potentially have been accidental. The same individual also provided a useful contribution to the palaeopathological record by presenting the only Neolithic case of spondylolysis in Europe reported so far, with only one more in the Old World originating from Çatalhöyük.

\section{( Prone to infections, including tuberculosis}

A fifth of the populations under study showed osteological signs of infection, including both juveniles and adults. This is much higher than the study of Northeastern Neolithic individuals mentioned further above (Ubelaker et al. 2006), which did not report any specific infections but noted signs of periostitis in $9 \%$ of bones observed (percentage of population unknown). 
One of the most important discoveries in this sample was the young adult male diagnosed with HPO secondary to tuberculosis. This and the other probable case of HPO on a female adult are the earliest occurrences of fully-developed HPO on adult human skeletons to date. Tuberculosis (TB) was confirmed by biomolecular analyses in this first HPO case, providing additional archaeological evidence of TB as probable primary cause of HPO. Following this initial case, four additional individuals yielded positive biomolecular results. These five confirmed cases, all young adults in their late teens - early twenties, successfully established the presence of tuberculosis in Neolithic Europe as early as 7000 years ago. They are the oldest confirmed TB cases in Hungary and Europe to date, second only to Atlit-Yam worldwide, and are unprecedented in such numbers in the prehistoric record.

Six adult cases of meningitis of unconfirmed aetiology were also discovered, including one potentially linked to a head trauma on an elderly man, and one with sacroiliitis, periostitis on the lower limbs and remodelling of the anterior surface of the vertebrae representing another case of possible TB on a young female. One case of meningitis on an infant with periostitis on its skeleton including some of its ribs represents an additional possible TB case.

Three cases of mastoiditis were observed: one on a child with abnormal vessel impressions on the endocranial surface of its skull, one on a female adult with multiple abscesses, and one on a young female adult with multiple pathologies. These significantly reinforce the Neolithic osteological evidence for this infection, and all three are also potential tuberculosis cases.

Another case of infection, this time on an adolescent female in her late teens, showed potential for tuberculosis. Altogether, these amount to seven possible TB cases on juveniles and young adults in addition to the five already confirmed. 
Two other cases of periostis were observed on infants, one of non-specific origin and one caused by a non-specific infection or inflammation. A case of chronic rhinitis was also observed on a mature adult.

Two more cases could prove of crucial importance to the palaeopathological record. A mature male was found to have possibly suffered from leprosy, which would vastly extend the palaeopathological record of this infection all the way to the Neolithic if confirmed by biomolecular analysis. Finally, a young female adult presented a potential case of metastatic carcinoma. If confirmed by further investigation, it would provide osteological evidence of cancer five millennia before its current earliest occurrence in Hungary, and would chronologically predate all other cases currently on record worldwide.

\section{- Potential for further studies}

Further microscopic, histological and biomolecular analyses are of the utmost importance to confirm the additional possible cases of tuberculosis, the probable scurvy cases, the unique case of possible leprosy and that of potential cancer.

A plan of the burials would greatly enhance the value of these pathological findings, allowing for more advanced palaeoepidemiologic studies to try establishing the chronological occurrences of these contagious infections, as well as their effect on burial practices. Numerous anomalies and anatomical variations already recorded during this doctoral research could also be used to assess potential connections between individuals.

Radiocarbon dating of the pathological skeletal remains would also be invaluable, and isotopic analyses could provide key information to accompany these pathological results. 


\section{DISCUSSION AND CONCLUSION}

The remains of one hundred Neolithic individuals from the Southern Great Plain of Hungary were analysed for this doctoral research. A catalogue summarising the information gathered from this rare Neolithic sample is provided as the final appendix to this thesis, presenting data on age, sex, stature and pathology for all individuals (appendix 31, explanation of pathology categories in Chapter 6).

\subsection{Palaeodemographic and palaeopathological analyses based on macroscopic observations}

Analyses of the remains of undocumented past populations have numerous obstacles, including variability of bone preservation, sampling and representativeness issues, inter- and intra-population variations, and lack of any similar documented sample for reference. It is therefore important to always keep in mind that any results produced are only ever mere estimates, particularly as results from different methods may vary markedly. State of preservation, which can fluctuate between individuals of the same site, will also influence which methods can be applied.

\subsubsection{Juvenile demographic characteristics}

\section{- Age}

Nearly all the juveniles in this sample could be given age estimates based on their dental and skeletal development. 
Gustafson and Koch's diagram was particularly useful for macroscopic dental age estimation of ancient and fragmentary remains. Ubelaker and Schour \& Massler charts, MFH/Smith stages, and Gustafson and Koch diagram all produced concurring dental age estimates.

Black, Scheuer and Schaefer's work was demonstrated to be the most up-todate source of skeletal developmental age estimates for juveniles at present, with the added research of Fazekas and Kósa for fetuses and neonates. Rissech and Black's scapular functions were unsuccessful in this population. Age estimates based on diaphyseal lengths suffered from the effect of population variability so that population-specific data should be preferred. However, for ancient archaeological remains with no other suitable baseline sample, the ranges of measurements provided by Stloukal and Hanáková (1978) from their historic sample were found to be the most appropriate to use as a guideline for age estimates.

\section{- Sex}

Juvenile sexing estimates were deemed unsuccessful in this study based on the wide variation of their results. However, morphological sexing traits were easy to record, including chin protrusion, shape of the anterior dental arcade, gonial eversion and ramus angle for the skull, as well as angle and depth of the greater sciatic notch, arch criterion, iliac crest curvature, and auricular elevation for the pelvis. It would therefore remain useful to note these traits in the hope that further research on documented samples will help refine these methods to eventually afford sexing of juvenile remains. The combined facial score method and Mays' plotting method were also found to offer great potential for archaeological remains methods, although they could not be applied to this particular sample due to the limited number of juveniles and the lack of undamaged orbits. 


\section{Stature}

The best method to estimate fetal stature available at present remains Fazekas and Kósa's formulae based on various bone dimensions, while Visser's calculations utilising diaphyseal lengths were found to be particularly useful for postnatal juvenile stature estimates of archaeological remains. Percentage of attained growth can also be utilised for comparison with other populations, as long as the same methodologies are used on all samples.

\subsubsection{Adult demographic characteristics}

\section{- Age}

Skeletal and dental development as described above can be utilised to estimate the age of adult remains before full maturation. Once the individuals are fully mature, broad age-ranges should be presented instead of merely giving the mean estimate, utilising as many age indicators as can possibly be observed. These age classes should also always be specified and no assumption made on the age ranges covered when those are not detailed. Although these age categories limit severely any analysis of the demography and health status of past populations, even the broad definition of infant, child, adolescent, young adult and mature or old adult can provide essential information on the life and death of those former communities. Dental wear based on Smith and Brothwell methods was shown to have significant potential in ageing adults in such ancient populations within broad age categories while cranial sutures and ribs were demonstrated not to be suitable age indicators for the macroscopic analyses of such ancient archaeological remains. See figure 8.1 overleaf for the age category distribution of the Late Neolithic Tisza population under study. 


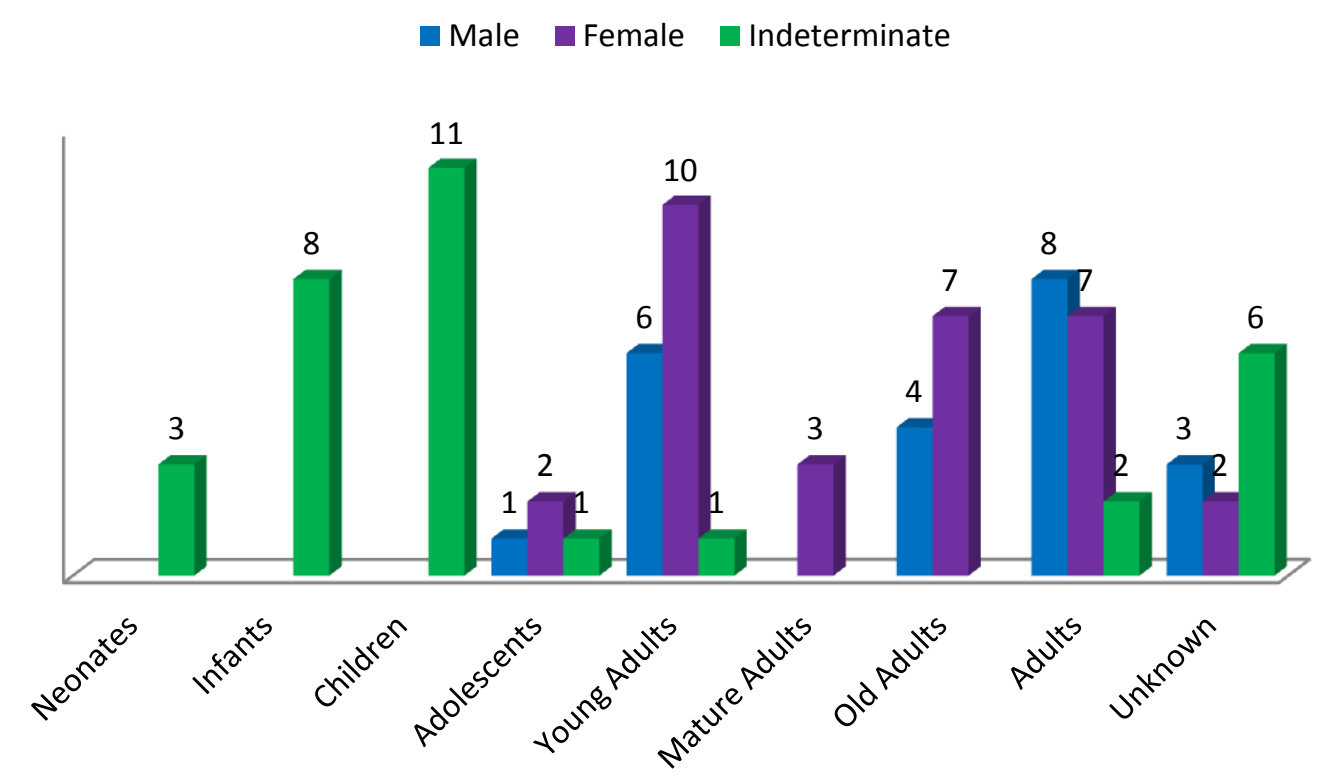

Figure 8.1: Distribution of the Tisza population under study based on macromophological observations

\section{( Sex}

Adult sex estimation based on commonly used morphological traits of the skull (including the nuchal crest, mastoid process, supra-orbital margin, glabella, and mental eminence) and the pelvis (greater sciatic notch, pre-auricular sulcus, overall pelvis shape, and shape of the pubis) was shown to benefit from the addition of the mandibular ramus flexure trait, while Bruzek's pelvic traits and those from the distal humerus did not prove useful. Osteometric analyses also yielded valuable additional information, including foot and hand bones dimensions which provided a useful alternative solution for sex estimation of ancient remains. As population-specific baselines were shown to be essential due to population variability, a baseline dataset was created for this Tisza population based on these analyses. 


\section{Stature}

Anatomical statures (ideally from length in situ in the grave or if not available utilising an anatomical method such as Fully's technique) remain the most appropriate source of stature estimates in archaeological populations. However, for damaged and incomplete "bones in a box" as in this Neolithic sample, Sjøvold's weighted lines of correlation offer the best system currently available to estimate maximum adult height of archaeological populations from their long bone lengths, utilising the average of all estimations available.

\subsubsection{Palaeopathology}

The skeletal remains of the populations under study were damaged and very fragmented, lacking contextual data, and their analyses restricted to macromorphological observations. Despite these severe limitations, remarkable information on the health status of these early farmers were revealed thanks to a very careful and painstakingly time-consuming macroscopic pathological analysis. Cases of particular interest could then be referred to specialists for further analyses in an attempt to confirm macromorphological diagnosis while limiting costs as well as sample destruction to the bare minimum. For example, the detailed macromorphological analysis of the thousands of bone fragments of HGO-53 led to the diagnosis of a case HPO secondary to tuberculosis, which in turn helped identifying four other probable cases in the same sample. The five tuberculosis cases were then confirmed by microbiological analyses, providing evidence of the presence of this infection in Europe seven millennia ago. Without the initial macroscopic study of these thousands of fragments, this major discovery would not have taken place. 


\subsection{Early farmers of Europe}

One year before the start of this doctoral research, the author's dissertation based on the published osteological evidence available at the time had to conclude that the health status of European populations was not radically changed by the transition to farming, with a steady evolution and progressive transformation from hunting-gathering to farming. Violence seemed to be present both in the Mesolithic and the Neolithic, and at that time there were no signs of diseases linked to agriculture and domestication such as tuberculosis with the first farmers of Europe (Masson 2002). However, the osteological evidence presented in this thesis has greatly improved our knowledge, both specifically for the Neolithic populations of the Southern Great Plain of Hungary, but also for early farmers of Europe in general.

\subsubsection{Origins}

Unfortunately, as no Mesolithic human remains were available in Hungary, a direct comparison with preceding hunting-gathering populations could not be undertaken. The gracility of the skeletal remains would appear compatible with a reported loss of robusticity between the Mesolithic and the Neolithic, attributed to the arrival of a new population (Menk \& Nemeskéri 1989). Osteometric sexual dimorphism in this small geographical study area seemed to remain the same throughout the Neolithic with little change in robusticity compared to the following two periods of prehistory, although cranial morphometrics appeared to show some differences between the Early and Late Neolithic. This would agree with the results of Zoffmann's distance analyses mostly concentrating on the taxonomy of Hungarian ancient populations, and which reported a continuity overall with a new type added during the Late Neolithic (Zoffmann 2000, 2005). It would also agree with the evidence based on Neolithic faunal remains (Bartosiewicz 2005), and the excavations of Mesolithic and Early Neolithic sites in the region (Kertész 1996; Makkay 1996; 
Kertész \& Sümegi 2001; Sümegi et al. 2003), with the neolithisation of former Mesolithic indigenous groups and the slow assimilation of both populations. Differences in mean male stature between different sites of the same period also demonstrated that intra-population variations were present within contemporary populations.

\subsubsection{Health status}

Growth in the Tisza population demonstrated the same prolonged growth compared to modern populations as historic samples, although the Late Neolithic growth rate was closer to contemporary populations than historic populations. The calculated average height of nearly $168 \mathrm{~cm}$ for males and slightly above $153 \mathrm{~cm}$ for females living at the heart of the Carpathian Basin during the Neolithic was only slightly shorter than that of their modern Hungarian counterparts. Comparison with other prehistoric and historic samples worldwide also showed little difference between those geographically and chronologically distant populations. There was also very little incidence of enamel hypoplasia among these individuals, particularly in adult remains. The adoption of farming did not seem to have had a negative impact on these indicators of health.

However, the rate of dental disease in these populations was very high and similar to other published Neolithic individuals from Hungary, illustrating the negative impact of their diet on their oral health. Metabolic diseases also seemed to be present, with several probable cases of scurvy noted on juveniles, denoting a severe lack of vitamin $\mathrm{C}$ in the diet of these farmers, on some occasions at least. 
While the rates of accidental trauma and of infection reported for Northeastern Hungary were low (Ubelaker et al. 2006), the analyses of this substantial sample from a small geographical area in Southeastern Hungary revealed a high rate of accidental trauma and mechanical changes, indicating that the way of life of these early farmers was physically very stressful. The incidence of infections in the Tisza population was also very high, with a fifth of the population affected, including adults and juveniles. Most importantly, five cases of tuberculosis were confirmed following the author's initial macromorphological diagnosis, and more possible cases have been highlighted. As detailed in Chapter 1, the Tisza Culture had a complex socio-economic system, centred on densely occupied tellsettlements with smaller farmsteads in the vicinity. In particular, the site of Gorzsa, from where all five cases of tuberculosis were recovered, also produced the largest Neolithic house in the country with six separate rooms. Such changes in settlement system may have precipitated the rise of infection, and would certainly have facilitated the spread of infections among these early farmers.

\subsubsection{Violence}

Interestingly, there were little signs of inter-personal violence in this relatively large sample of one hundred Neolithic individuals from South-eastern Hungary, in contradiction with findings from the same period reported in the Northeastern part of the country (Ubelaker et al. 2006), and more generally across Europe (Guilaine \& Zammit 2005). This could be linked to sample bias, with individuals having suffered (and particularly died from) violence not necessarily buried in the same place as the rest of the community (Smith \& Brickley 2009), as well as the fragmented and incomplete nature of the remains. Nevertheless, the osteological evidence of this population would agree with the theory that violence during the Neolithic may not have been common (Barker 2006). 


\subsection{Areas for future research}

\section{- Advanced pathological analyses}

Further microscopic, histological and biomolecular analyses will be undertaken to confirm the additional possible cases of tuberculosis, the probable scurvy cases, the unique case of possible leprosy and that of potential cancer.

\section{- Archaeological data}

Contextual data for the site of Gorzsa would be of crucial importance. A plan of the burials would allow for more advanced palaeoepidemiologic studies to try establishing the chronological occurrences of these contagious infections, as well as their effect on burial practices. Numerous anomalies and anatomical variations already recorded during this doctoral research could also be used to assess potential connections between individuals.

\section{- Radiocarbon Dating and Isotopic Analyses}

Radiocarbon dating of the pathological skeletal remains would also be invaluable, while isotopic analyses could also provide key information to accompany the pathological results.

\section{- Sample}

More skeletal remains from the Körös Culture would greatly improve the comparison of Early and Late Neolithic populations of the Southern Great Plain of Hungary, and it is still hoped that Mesolithic human remains will one day be recovered. The investigations of additional Tisza individuals from other sites yet to be uncovered would also provide a helpful comparison for the findings of this thesis and increase the data available for further statistical studies. 


\subsection{Conclusion}

This doctoral research provides a unique contribution to our knowledge of the early farmers of Europe in general and the Neolithic populations from the Southern Great Plain of Hungary in particular. The potential of macroscopic observations for the demographic and pathological analyses of past populations was demonstrated on this exceptional Neolithic sample, revealing despite the lack of contextual data, the damaged and fragmentary skeletal remains, and the restriction to morphological analyses, a mostly non-violent population prone to infections, with a physically stressful way of life and a diet leading to a high rate of dental disease and (at least occasionally) lack of vitamin C. In particular, the palaeopathological analysis uncovered the oldest cases tuberculosis in Europe so far, as well as the probable oldest cases of scurvy. In addition, a potential case of leprosy and one of cancer were also discovered, which would be of great significance for the palaeopathological record if these can be confirmed by further studies. 


\section{BIBLIOGRAPHY}

Acharya, A. B. \& S. Mainali, 2008. Sex Discrimination Potential of Buccolingual and Mesiodistal Tooth Dimensions. Journal of Forensic Sciences, 53(4), 790-2.

Acsádi, G. \& J. Nemeskéri, 1970. History of Human Life Span and Mortality, Budapest: Akadémiai Kiado.

Agarwal, S., B. Glencross \& P. Beauchesne, 2011. Bone Growth, Maintenance and Loss in the Neolithic Community of Çatalhöyük, Turkey: Preliminary Results, in Archaeological Research Facility Laboratory Reports University of Berkeley, California.

Albert, A. M. \& W. R. Maples, 1995. Stages of epiphyseal union for thoracic and lumbar vertebral centra as a method of age determination for teenage and young adult skeletons. Journal of Forensic Sciences, 40, 623-33.

Alves Cardoso, F. \& C. Y. Henderson, 2010. Enthesopathy formation in the humerus: Data from known age-at-death and known occupation skeletal collections. American Journal of Physical Anthropology, 141(4), 550-60.

Alves Cardoso, F. \& C. Y. Henderson, 2013. The Categorisation of Occupation in Identified Skeletal Collections: A Source of Bias? International Journal of Osteoarchaeology, 23(2), 186-96.

Ammerman, A. J. \& L. L. Cavalli-Sforza, 1971. Measuring the rate of spread or early farming in Europe. Man, 6(4), 674-88.

Andersen, B. C., 1987. Pelvic scarring analysis: parturition or excess motion. American Journal of Physical Anthropology, 75, 181.

Anderson, D. L., G. W. Thompson \& F. Popovic, 1976. Age of attainment of mineralization stages of the permanent dentition. Journal of Forensic Sciences, 21, 191-200.

Anderson, J. E., 1962. The Human Skeleton: A manual for archaeologists, Ottawa: National Museum of Canada.

Anderson, M., W. T. Green \& M. B. Messner, 1963. Growth and predictions of growth in the lower extremities. Journal of Bone and Joint Surgery, 45A, 1-4.

Anderson, M., M. B. Messner \& W. T. Green, 1964. Distribution of lengths of the normal femur and tibia in children from one to eighteen years of age. Journal of Bone and Joint Surgery, 46A, 1197-202.

Angel, J. L., 1969. The bases of paleodemography. American Journal of Physical Anthropology, 30(3), 427-38.

Arany, S., M. Iino \& N. Yoshioka, 2004. Radiographic Survey of Third Molar Development in Relation to Chronological Age Among Japanese Juveniles. Journal of Forensic Sciences, 49(3), 534-48.

Aranzadi de, T., 1913. Algunas observaciones acera del diagnostico de la edad en el craneo. Boletin de la Real Sociedad Española de Historia Natural, (March), 161-79. 
Araújo, P. d. P., J. O. Matos, F. B. Madeira, S. Araujo Ade, A. M. Arruda \& S. Tomita, 2008. Squamozygomatic mastoiditis. Brazilian Journal of Otorhinolaryngology, 74(4), 617-20.

Arsuaga, J. L. \& J. M. Carretero, 1994. Multivariate analysis of the sexual dimorphism of the hip bone in a modern human population and in early hominids. American Journal of Physical Anthropology, 93(2), 241-57.

Asala, S. A., 2001. Sex determination from the head of the femur of South African whites and blacks. Forensic Science International, 117, 15-21.

Assis, S., A. L. Santos \& C. Roberts, 2011. Evidence of hypertrophic osteoarthropathy in individuals from the Coimbra Skeletal Identified Collection (Portugal). International Journal of Paleopathology, 1, 155-63.

Aufderheide, A. C. \& C. Rodríguez-Martín (eds.), 1998. The Cambridge Encyclopedia of Human Paleopathology, Cambridge: Cambridge University Press.

Baccino, E., D. H. Ubelaker, L.-A. C. Hayek \& A. Zerilli, 1999. Evaluation of Seven Methods of Estimating Age at Death from Mature Human Skeletal Remains. Journal of Forensic Sciences, 44, 931-6.

Bailit, H. \& E. E. Hunt, 1964. The sexing of children's skeletons from teeth alone and its genetic implications. American Journal of Physical Anthropology, 22(2), 171-4.

Bainbridge, D. \& S. Genovés, 1956. A study of sex differences in the scapula. Journal of the Royal Anthropological Institute, 86(2), 109-34.

Balthazard, V. \& Dervieux, 1921. Etudes anthropologiques sur le foetus humain. Annales de médecine légale, 1, 37-42.

Bang, G., 1989. Age changes in teeth: Developmental and regressive, in Age Markers in the Human Skeleton, ed. M. Y. İşcan, Springfield: Charles C Thomas, 211-36.

Banner, J., 1930a. A kökénydombi neolithkori telep. Dolgozatok, 6 (1930), 49158.

Banner, J., 1930b. A tiszai-kultúra két ismeretlen lelőhelyéről. Archaeológiai Értesítő, 44, 220-5.

Banner, J., 1931. A Kökénydombi Neolithkori Telep, Szeged: Szeged Városi Nyomda és Könyvkiadó.

Banner, J., 1935. Ásatás a hódmezővásárhelyi Kotacparton. Dolgozatok, 11, $97-$ 125.

Banner, J., 1939. Bádeni sírok Hódmezővásárhelyen, a Bodzásparton. Folia Archaeologica, 1-2, 13-23.

Banner, J., 1940a. Hódmezővásárhely története a honfoglalás koráig - Első rész: A legrégibb időktől a bronzkor kialakulásáig, Hódmezővásárhely: A Városi Múzeum Kiadása.

Banner, J., 1940b. A Régiségtudományi Intézet ásatásai 1940-ben. Újabb ásatás a Hódmezővásárhelyi Kökénydombon. Dolgozatok, 16, 181-2, 8.

Banner, J., 1940c. Újabb adatok a bodrogkeresztúri kultúra elterjedéséhez. Archaeológiai Értesítő, 1, 13-8.

Banner, J., 1942. A Régiségtudományi Intézet ásatásai 1942-ben. Újabb ásatás a 
Hódmezővásárhelyi Kökénydombon. Dolgozatok, 18, 138-43.

Banner, J., 1951. A harmadik ásatás a Hódmezővásárhelyi kökénydombon

(1941. augusztus 4-30.). Archaeológiai Értesítő, 78 (1951), 27-37.

Banner, J., 1954. Funde der Körös-Kultur von Hódmezővásárhely - Bodzáspart.

Acta Archaeologica Academiae Scientiarum Hungaricae, 4, 1-8.

Banner, J., 1957. Mit adott Hódmezővásárhely a magyar régészetnek? A Morá Ferenc Múzeum Évkönyve, 133-50.

Banner, J. \& I. Foltiny, 1945. Újabb ásatás a Hódmezővásárhelyi Kökénydombon. Folia Archaeologica, 5, 8-34.

Banner, J. \& J. Korek, 1949. Negyedik és ötödik ásatás a Hódmezővásárhelyi Kökénydombon. (1942. aug. 3-28. és aug. 2-szept. 8.). Archaeológiai Értesítő, 76, 9-25.

Barker, G., 2006. The Agricultural Revolution in Prehistory: Why did Foragers become Farmers?, Oxford: Oxford University Press.

Barrio, P. A., G. J. Trancho \& J. A. Sánchez, 2006. Metacarpal Sexual Determination in a Spanish Population. Journal of Forensic Sciences, 51(5), 990-5.

Bartosiewicz, L., 2005. Plain talk: animals, environment and culture in the Neolithic of the Carpathian Basin and adjacent areas, in (un)settling the Neolithic, eds. D. Bailey, A. Whittle \& V. Cummings, Oxford: Oxbow books, 51-63.

Bass, W. M., 1995. Human Osteology : A Laboratory and Field Manual, Columbia: Missouri Archaeological Society, Inc.

Bedford, M. E., K. F. Russell, C. O. Lovejoy, R. S. Meindl, S. W. Simpson \& P. StuartMacadam, 1993. Test of the Multifactorial Aging Method Using Skeletons With Known Ages-at-Death From the Grant Collection. American Journal of Physical Anthropology, 91, 287-97.

Belcastro, M. G., E. Rastelli \& V. Mariotti, 2008. Variation of the Degree of Sacral Vertebral Body Fusion in Adulthood in Two European Modern Skeletal Collections. American Journal of Physical Anthropology, 135, 149-60.

Bennike, P., 1985. Paleopathology of Danish Skeletons, Copenhagen: Akademisk Forlag.

Bennike, P., 2008. Trauma, in Advances in Human Palaeopathology, eds. R. Pinhasi \& S. Mays, Chichester: John Wiley and Sons, 309-28.

Berg, G. E., 2008. Pubic Bone Age Estimation in Adult Women. Journal of Forensic Sciences, 53(3), 569-77.

Bethard, J. D. \& N. L. Shirley, 2007. A test of the Igarashi et al. method for estimating age-at-death from the auricular surface of the ilium, in 76th Annual Meeting of the AAPA Philadelphia.

Bidmos, M. A., 2005. On the Non-equivalence of Documented Cadaver Lengths to Living Stature Estimates Based on Fully's Method on Bones in the Raymond A. Dart Collection. Journal of Forensic Sciences, 50(3), 1-6.

Bidmos, M. A. \& S. A. Asala, 2003. Discriminant function sexing of the calcaneus of the South African Whites. Journal of Forensic Sciences, 48, 1213-8.

Bidmos, M. A. \& S. A. Asala, 2004. Sexual Dimorphism of the Calcaneus of South 
African Blacks. Journal of Forensic Sciences, 49(3), 1-5.

Bidmos, M. A. \& M. R. Dayal, 2003. Sex determination from the talus of South African whites by discriminant function analysis. American Journal of Forensic Medicine and Pathology, 24(4), 322-8.

Bidmos, M. A. \& M. R. Dayal, 2004. Further evidence to show population specificity of Discriminant function equations for sex determination using the talus of South African Blacks. Journal of Forensic Sciences, 49, 1165-70.

Black, S. \& L. Scheuer, 1996. Age Changes in the Clavicle: from the Early Neonatal Period to Skeletal Maturity. International Journal of Osteoarchaeology, 6, 425-34.

Black, T. K., 1978a. Sexual dimorphism in the tooth-crown diameters of the deciduous teeth. American Journal of Physical Anthropology, 48, 77-82.

Black, T. K., III, 1978b. A new method for assessing the sex of fragmentary skeletal remains: Femoral shaft circumference. American Journal of Physical Anthropology, 48, 227-31.

Blankenship, J. A., H. H. Mincer, K. M. Anderson, M. A. Woods \& E. L. Burton, 2007. Third Molar Development in the Estimation of Chronologic Age in American Blacks as Compared with Whites. Journal of Forensic Sciences, 52(2), 428-33.

Blondiaux, J., C.-A. Baud, N. Boscher-Barré, C. Dardenne, N. Deschamps, P. Trocellier \& L. Buchet, 1992. Trace elements in palaeopathology: quantitative analysis of a case of hypertrophic osteoarthropathy by instrumental neutron activation analysis. International Journal of Osteoarchaeology, 2(3), 241-4.

Bogin, B., 1999. Evolutionary Perspective on Human Growth. Annual Review of Anthropology, 28, 109-53.

Bonsall, C., M. Horvat, K. McSweeney, M. Masson, T. F. G. Higham, C. Pickard \& G. T. Cook, 2007. Chronological and Dietary Aspects of the Human Burials from Ajdovska Cave, Slovenia. Radiocarbon, 49(2), 727-40.

Boucher, B. J., 1955. Sex differences in the foetal sciatic notch. Journal of Forensic Medicine, 2, 51-4.

Boucher, B. J., 1957. Sex differences in the foetal pelvis. American Journal of Physical Anthropology, 15(4), 581-600.

Boulinier, G., 1968. La détermination du sexe des crânes humains à l'aide des fonctions discriminantes. Bulletins et Mémoires de la Société d'anthropologie de Paris, XII ${ }^{\circ}$ Série, 3(4), 301-16.

Boulinier, G., 1969. Variations avec l'âge du dimorphisme sexuel des crânes humains adultes : influence sur les possibilités de discrimination statistique des sujets. Bulletins et Mémoires de la Société d'anthropologie de Paris, XII ${ }^{\circ}$ Série, 4(2), 127-38.

Bowman, J. E., S. M. MacLaughlin \& L. Scheuer, 1992. The relationship between biological and chronological age in the juveniles from St Bride's Church, Fleet Street. Annals of Human Biology, 19, 216.

Boyd, J. D. \& J. C. Trevor, 1953. Problems in reconstruction: race, sex, age and 
stature from skeletal material, in Modern Trends in Forensic Medicine, ed. K. Simpson, London: Butterworth and Co., 133-52.

Brasileiro, B. F. \& L. A. Passeri, 2006. Epidemiological analysis of maxillofacial fractures in Brazil: A 5-year prospective study. Oral Surgery, Oral Medicine, Oral Pathology, Oral Radiology \& Endodontics, 102, 28-34.

Breitinger, E., 1937. Zur Berechnung der Körperhöhe aus den langen Gliedmassenknochen. Anthropologischer Anzeiger, 14, 249-74.

Brickley, M., 2004. Guidance on recording age at death in juvenile skeletons, in Guidelines to the Standards for Recording Human Remains, eds. M. Brickley \& J. I. McKinley, Southampton and Reading: BABAO and the Institute of Field Archaeologists, 21-2.

Brickley, M. \& R. Ives, 2006. Skeletal Manifestations of Infantile Scurvy. American Journal of Physical Anthropology, 129, 163-72.

Brickley, M. \& R. Ives, 2008. The Bioarchaeology of Metabolic Bone Disease, Oxford: Elsevier.

Bridges, P. S., 1989. Spondylolysis and Its Relationship to Degenerative Joint Disease in the Prehistoric Southeastern United States. American Journal of Physical Anthropology, 79, 321-9.

Broca, $\mathrm{P} ., 1875$. Instructions craniologiques et craniométriques de la Société d'anthropologie de Paris, Paris: G, Masson.

Bronk Ramsey, C., 2009. Bayesian analysis of radiocarbon dates. Radiocarbon, 51(1), 337-60.

Brooks, S. T., 1955. Skeletal age at death: the reliability of cranial and pubic age indicators. American Journal of Physical Anthropology, 13, 567-97.

Brooks, S. T. \& J. M. Suchey, 1990. Skeletal age determination based on the os pubis: A comparison of the Acsádi-Nemeskéri and Suchey-Brooks methods. Human Evolution, 5, 227-38.

Brothwell, D. R., 1963. Digging up Bones: The Excavation, Treatment and Study of Human Skeletal Remains, London: British Museum (Natural History).

Brothwell, D. R., 1965. Digging up Bones: The Excavation, Treatment and Study of Human Skeletal Remains, London: British Museum (Natural History).

Brothwell, D. R. (ed.) 1968. The Skeletal Biology of Earlier Human Populations, London: Pergamon Press.

Brothwell, D. R., 1972. Digging Up Bones, London.

Brothwell, D. R., 1989. The relationship of tooth wear to aging, in Age Markers in the Human Skeleton, ed. M. Y. İșcan, Springfield: Charles C Thomas, 30318.

Brothwell, D. R., 2008. Tumours and Tumour-like processes, in Advances in Human Palaeopathology, eds. R. Pinhasi \& S. Mays, Chichester: John Wiley and Son, 253-82.

Brown, R. P., D. H. Ubelaker \& M. S. Schanfield, 2007. Evaluation of Purkait's Triangle Method for Determining Sexual Dimorphism. Journal of Forensic Sciences, 52(3), 553-6.

Brown, W. A. B., 1985. Identification of Human Teeth. Bulletin of the Institute of Archaeology, University of London, 21-22, 1-30. 
Bruzek, J., 2002. A Method for Visual Determination of Sex Using the Human Hip Bone. American Journal of Physical Anthropology, 117, 157-68.

Buckberry, J. L. \& A. T. Chamberlain, 2002. Age estimation from the auricular surface of the ilium: A revised method American Journal of Physical Anthropology, 119(3), 231-9.

Buikstra, J. E., 2010. Paleopathology: A Contemporary Perspective, in $A$ Companion to Biological Anthropology, ed. C. S. Larsen, Wiley-Blackwell, 395-411.

Buikstra, J. E. \& D. H. Ubelaker (eds.), 1994. Standards for Data Collection from Human Skeletal Remains, Fayetteville, Arkansas: Arkansas Archaeological Survey Report Number 44.

Burrows, A. M., V. P. Zanella \& T. M. Brown, 2003. Testing the validity of metacarpal use in sex assessment of human skeletal remains. Journal of Forensic Sciences, 48, 17-20.

Byers, S. N., 2008. Introduction to Forensic Anthropology, Boston: Pearson, Allyn and Bacon.

Byers, S. N., K. Akoshima \& B. Curran, 1989. Determination of Adult Stature From Metatarsal Length. American Journal of Physical Anthropology, 79, 275-9.

Camacho, F. J., L. G. Pellico \& R. F. Rodriguez, 1993. Osteometry of the Human Iliac Crest: Patterns of Normality and Its Utility in Sexing Human Remains. Journal of Forensic Sciences, 38(4), 779-87.

Campillo, D., 1991. The possibility of diagnosing meningiomas in paleopathology. International Journal of Osteoarchaeology, 1(3), 225-30.

Campillo, D. \& V. J. Marí-Barcells, 1984. Microscopy of osteal tumours in paleopathology., in Proceedings of the Vth European meeting of the Paleopathology Association Siena, 35-43.

Canci, A., S. Minozzi \& S. M. Borgognini Tarli, 1996. New Evidence of Tuberculous Spondylitis from Neolithic Liguria (Italy). International Journal of Osteoarchaeology, 6, 497-501.

Cardoso, H. F. V., 2007a. Environmental Effects on Skeletal Versus Dental Development: Using a Documented Subadult Skeletal Sample to Test a Basic Assumption in Human Osteological Research. American Journal of Physical Anthropology, 132, 223-33.

Cardoso, H. F. V., 2007b. A Test of the Differential Accuracy of the Maxillary Versus the Mandibular Dentition in Age Estimations of Immature Skeletal Remains Based on Developing Tooth Length. Journal of Forensic Sciences, 52(2), 434-7.

Cardoso, H. F. V., 2008. Epiphyseal Union at the Innominate and Lower Limb in a Modern Portuguese Skeletal Sample, and Age Estimation in Adolescent and Young Adult Male and Female Skeletons. American Journal of Physical Anthropology, 135, 161-70.

Carli-Thiele, P., 1996. Spuren von Mangelerkrankungen an steinzeitlichen Kinderskeleten, Göttingen: Verlag Erich Goltze.

Case, D. T. \& A. H. Ross, 2007. Sex Determination form Hand and Foot Bone 
Lengths. Journal of Forensic Sciences, 52(2), 264-70.

Castellana, C. \& F. Kósa, 2001. Estimation of fetal age from dimensions of atlas and axis ossification centers. Forensic Science International, 117, 31-43.

Castillo, M., V. S. Albernaz, S. K. Mukherji, M. M. Smith \& J. L. Weissman, 1998. Imaging of Bezold's Abscess. American Journal of Roentgenology, 171(6), 1491-5.

Chagula, W. K., 1960. The age of eruption of third permanent molars in male East Africans. American Journal of Physical Anthropology, 18, 77-82.

Chamberlain, A., S. Rogers \& A. J. Romanowski, 1992. Osteochondroma in a British Neolithic Skeleton. British Journal of Hospital Medicine, 47(1), 513.

Cohen, M. N. \& G. J. Armelagos (eds.), 1984. Paleopathology at the Origins of Agriculture, New York: Academic Press Inc.

Connell, B., 2004. Compiling a dental inventory, in Guidelines to the Standards for Recording Human Remains, eds. M. Brickley \& J. I. McKinley, Southampton and Reading: BABAO and the Institute of Field Archaeologists, 8.

Coqueugniot, H. \& T. D. Weaver, 2007. Brief Communication: Infracranial Maturation in the Skeletal Collection from Coimbra, Portugal: New Aging Standards for Epiphyseal Union. American Journal of Physical Anthropology, 134, 424-37.

Cordain, L., 1999. Cereal grains: Humanity's double-edged sword. World Review of Nutrition and Dietetics, 84, 19-73.

Cox, M., 2000a. Ageing adults from the skeleton, in Human Osteology In Archaeology and Forensic Science, eds. M. Cox \& S. Mays, London: Greenwich Medical Media, 61-81.

Cox, M., 2000b. Assessment of parturition, in Human Osteology In Archaeology and Forensic Science, eds. M. Cox \& S. Mays, London: Greenwich Medical Media, 131-42.

Cox, M. \& A. Scott, 1992. Evaluation of the obstetric significance of some pelvic characters in an 18th century British sample of known parity. American Journal of Physical Anthropology, 89, 431-40.

Crossner, C. G. \& L. Mansfield, 1983. Determination of dental age in adopted non-European children. Swedish Dental Journal, 7, 1-10.

Crowder, C. \& D. Austin, 2005. Age Ranges of Epiphyseal Fusion in the Distal Tibia and Fibula of Contemporary Males and Females. Journal of Forensic Sciences, 50(5), ??

Dayal, M. R. \& M. A. Bidmos, 2005. Discriminating Sex in the South African Blacks Using Patella Dimensions. Journal of Forensic Sciences, 50(6), 1-4.

De Vito, C. \& S. R. Saunders, 1990. A discriminant function analysis of deciduous teeth to determine sex. Journal of Forensic Sciences, 35, 845-58.

Dedouit, F., S. Bindel, D. Gainza, A. Blanc, F. Joffre, D. Rougé \& N. Telmon, 2008. Application of the Iscan Method of Two- and Three-Dimensional Imaging of the Sternal End of the Right Fourth Rib. Journal of Forensic Sciences, 53(2), 288-95.

Demirjian, A. \& H. Goldstein, 1976. New systems for dental maturity based on 
seven and four teeth. Annals of Human Biology, 3, 411-21.

Demirjian, A., H. Goldstein \& J. M. Tanner, 1973. A new system of dental age assessment. Human Biology, 45, 211-27.

Demirjian, A. \& G.-Y. Levesque, 1980. Sexual differences in dental development and prediction of emergence. Journal of Dental Research, 59, 1110-22.

Demoulin, F., 1972. Importance de certaines mesures crâniennes (en particulier de la longueur sagittale de la mastoïde) dans la dénomination sexuelle des crânes Bulletins et Mémoires de la Société d'anthropologie de Paris, $X I{ }^{\circ}$ Série, 9(3), 259-64.

Deter, C. A., 2009. Gradients of Occlusal Wear in Hunter-Gatherers and Agriculturalists. American Journal of Physical Anthropology, 138, 247-54.

Di-Loreto, A., 2006. The Correlation Between Aging Results Obtained from Four Juvenile Dental and Long Bone Aging Methods, in BABAO 8th Annual Conference Birmingham, UK: (poster presentation).

DiBennardo, R. \& J. V. Taylor, 1979. Sex assessment of the femur: A test of a new method. American Journal of Physical Anthropology, 50, 635-8.

DiGangi, E. A., J. D. Bethard, E. H. Kimmerle \& L. Konigsberg, 2009. A New Method for Estimating Age-At-Death From the First Rib. American Journal of Physical Anthropology, 138, 164-76.

Dittrick, J. \& J. M. Suchey, 1986. Sex determination of prehistoric central California skeletal remains using discriminant analysis of the femur and humerus. American Journal of Physical Anthropology, 70(1), 3-9.

Djurić, M., D. Djonić, S. Nikolić, D. Popović \& J. Marinković, 2007. Evaluation of the Suchey-Brooks Method for Aging Skeletons in Balkans. Journal of Forensic Sciences, 52(1), 21-3.

Donnelly, S. M., S. M. Hens, N. L. Rogers \& K. L. Schneider, 1998. Technical note: A blind test of mandibular ramus flexure as a morphological indicator of sexual dimorphism in the human skeleton. American Journal of Physical Anthropology, 107, 363-6.

Donoghue, H. D., J. J. Gladykowska-Rzeczycka, A. Marcsik, J. Holton \& M. Spigelman, 2002. Mycobacterium leprae in archaeological samples, in The Past and Present of Leprosy, eds. C. Roberts, M. E. Lewis \& K. Manchester, Oxford: Archaeopress, 271-86.

Donoghue, H. D., O. Y.-C. Lee, D. E. Minnikin, G. S. Besra, J. H. Taylor \& M. Spigelman, 2010. Tuberculosis in Dr Granville's mummy: a molecular reexamination of the earliest known Egyptian mummy to be scientifically examined and given a medical diagnosis. Proceedings of the Royal Society of Biological Sciences, 277, 51-6.

Donoghue, H. D., M. Spigelman, C. L. Greenblatt, G. Lev-Maor, G. K. Bar-Gal, C. Matheson, K. Vernon, A. G. Nerlich \& A. R. Zink, 2004. Tuberculosis: from prehistory to Robert Koch, as revealed by ancient DNA. Lancet Infectious Diseases, 4, 584-92.

Drew, R., 2013. A Review of the Ischium-Pubis Index: Accuracy, Reliability, and Common Errors. Human Biology, 85(4), 579-96.

Dupertuis, C. W. \& J. A. Hadden Jr., 1951. On the reconstruction of stature from 
long bones. American Journal of Physical Anthropology, 9, 15-54.

Dwight, T., 1881. The sternum as an index of sex and age. J Anat, 15, 327-30.

Dwight, T., 1894. The range and significance of variation in the human skeleton. Boston Medical Surgery Journal, 13, 73-6.

Dwight, T., 1904-1905. The size of auricular surfaces of the long bones as characteristics of sex as an anthropological study. American Journal of Anatomy, 4, 19-32.

Dwight, T., 1905. The size of articular surfaces of the long bones as characteristics of sex as an anthropological study. American Journal of Anatomy, (1904-1905) 4, 19-32.

Dzierzykray-Rogalski, T., 1980. Paleopathology of the Ptolemaic inhabitants of Dakhleh Oasis (Egypt). Journal of Human Evolution, 9, 71-4.

El-Nofely, A. \& M. Y. İșcan, 1989. Assessment of age from the dentition in children, in Age Markers in the Human Skeleton, ed. M. Y. İscan, Springfield: Charles C Thomas, 237-54.

Éry, K., 1998. Length of limb bones and stature in ancient populations in the Carpathians basin, Budapest.

Eshed, V., B. Latimer, C. M. Greenwald, L. M. Jellema, B. M. Rothschild, S. WishBaratz \& I. Hershkovitz, 2002. Button Osteoma: Its Etiology and Pathophysiology. American Journal of Physical Anthropology, 118, 21730.

Falsetti, A. B., 1995. Sex assessment from metacarpals of the human hand. Journal of Forensic Sciences, 40(5), 774-6.

Falys, C. G., H. Schutkowski \& D. A. Weston, 2005. The Distal Humerus - A Blind Test of Rogers' Sexing Technique Using a Documented Skeletal Collection. Journal of Forensic Sciences, 50(6).

Falys, C. G., H. Schutkowski \& D. A. Weston, 2006. Auricular Surface Aging: Worse Than Expected? A Test of the Revised Method on a Documented Historic Skeletal Assemblage. American Journal of Physical Anthropology, 130, 508-13.

Farkas, G., 1975. A Délalföld őskorának paleoantropológiája. Kandidátusi Értekezés, Embertani Intézete, József Attila Tudományegyetem, Szeged.

Farkas, G., 1977. Anthropological Outlines of the Prehistory of the Southern Part of the Great Hungarian Plain and of Northern Jugoslavia. Acta Biologica Szegediensis, 23(1-4), 139-67.

Farkas, G., 2005. Szakvélemény a Hódmezővásárhely-Gorzsa Kovács tanya lelőhelyen Gazdapusztai Gyula régész által 1955-ben feltárt hamvasztott csontokról, in Szakvélemény a Hódmezővásárhely-Gorzsa Kovács tanya lelőhelyen Gazdapusztai Gyula régész által 1955-ben feltárt hamvasztott csontokról, ed. O. Trogmayer, Zalai Múzeum.

Farkas, G. \& A. Marcsik, 1988. Dél-magyarországi késő neolitikus emberi csontvázak (Gorzsa, Deszk). A Morá Ferenc Múzeum Évkönyve, 1987-1, 51-67.

Farkas, G. \& F. Szalai, 1992. Újabb neolitikus (Tiszai kultúrás) antropológiai leletek Hódmezővásárhely-Kökénydombról. A Morá Ferenc Múzeum 
Évkönyve, 1989-1990/1, 231-5.

Fazekas, I. G. \& F. Kósa, 1978. Forensic Fetal Osteology, Budapest: Akadémiai Kiadó.

Feldesman, M. R., 1992. Femur/ Stature Ratio and Estimates of Stature in Children. American Journal of Physical Anthropology, 87, 447-59.

Feldesman, M. R., J. G. Kleckner \& J. K. Lundy, 1990. The femur/stature ratio and estimates of stature in mid- and late-Pleistocene fossil hominids. American Journal of Physical Anthropology, 83, 359-71.

Ferembach, D., I. Schwidetzky \& M. Stloukal, 1980. Recommendations for age and sex diagnoses of skeletons. Journal of Human Evolution, 9, 517-49.

Ferraz de Macedo, F., 1892. Crime et criminel, Lisboa: National Press.

Fibiger, L. \& C. J. Knüsel, 2005. Prevalence Rates of Spondylolysis in British Skeletal Populations. International Journal of Osteoarchaeology, 15, 16474.

Flander, L. B., 1978. Univariate and multivariate methods for sexing the sacrum. American Journal of Physical Anthropology, 49, 103-10.

Flecker, H., 1942. Time of Appearance and Fusion of Ossification Centers as observed by roentgenographic methods. American Journal of Roentgenology, 47(1), 97-159.

Flohr, S. \& M. Schultz, 2009a. Mastoiditis-Paleopathological Evidence of a Rarely Reported Disease. American Journal of Physical Anthropology, 138, 266-73.

Flohr, S. \& M. Schultz, 2009b. Osseous Changes due to Mastoiditis in Human Skeletal Remains. International Journal of Osteoarchaeology, 19, 99-106.

Folayan, M., F. Owotade, E. Adejuyigbe, S. Sen, B. Lawal \& K. Ndukwe, 2007. The Timing of Eruption of the Primary Dentition in Nigerian Children. American Journal of Physical Anthropology, 134(4), 443-8.

Formicola, V., 1993. Stature Reconstruction From Long Bones in Ancient Population Samples: An Approach to the Problem of its Reliability. American Journal of Physical Anthropology, 90(3), 351-8.

Formicola, V. \& M. Franceschi, 1996. Regression Equations for Estimating Stature From Long Bones or Early Holocene European Samples. American Journal of Physical Anthropology, 100, 83-8.

Formicola, V., Q. Milanesi \& C. Scarsini, 1987. Evidence of Spinal Tuberculosis at the Beginning of the Fourth Millennium BC From Arene Candide Cave (Liguria, Italy). American Journal of Physical Anthropology, 72, 1-6.

Fortunato, M. A., A. F. Fielding \& L. H. Guernsey, 1982. Facial bone fractures in children. Oral Surgery, Oral Medicine, Oral Pathology, Oral Radiology \& Endodontics, 53(3), 225-30.

France, D. L., 1983. Sexual dimorphism in the human humerus. Department of Anthropology, University of Colorado.

France, D. L., 1985. Occupational effects on the determination of sex in the humerus, in 37th Annual Meeting of the Amercian Academy of Forensic Sciences Las Vegas.

France, D. L., 1998. Observational and metric analysis of sex in the skeleton, in 
Forensic Osteology, Advances in the Identification of Human Remains, ed.

K. J. Reichs, Springfield: Charles C. Thomas, 163-86.

Franklin, D., C. E. Oxnard, P. O'Higgins \& I. Dadour, 2007. Sexual Dimorphism in the Subadult Mandible: Quantification Using Geometric Morphometrics. Journal of Forensic Sciences, 52(1), 6-10.

Frédéric, J., 1906. Untersuchungen über die normale Obliteration der Schädelnähte. Zeitschrift für Morphologie und Anthropologie, 9, 373-456.

Fully, G., 1956. Une nouvelle méthode de détermination de la taille. Annales de médecine légale, 36, 266-73.

Galera, V., D. H. Ubelaker \& L.-A. C. Hayek, 1998. Comparison of Macrosopic Cranial Methods of Age Estimation Applied to Skeletons from the Terry Collection. Journal of Forensic Sciences, (September 1998), 933-9.

Galloway, A. (ed.) 1999. Broken Bones, Anthropological Analysis of Blunt Force Trauma, Springfield, Illinois: Charles C. Thomas.

Gapert, R., S. Black \& J. Last, 2009. Sex Determination from the Occipital Condyle: Discriminant Function Analysis in an Eighteenth and Nineteenth Century British Sample. American Journal of Physical Anthropology, Early View.

Garn, S. M., A. B. Lewis \& B. Bonne, 1962. Third Molar Formation and Its Developmental Course. The Angle Orthodontist, 32, 270-9.

Garn, S. M., C. G. Rohmann \& T. Blumenthal, 1966. Ossification sequence polymorphism in skeletal development. American Journal of Physical Anthropology, 24, 101-16.

Gat, H., H. Sarnat, K. Bjorvatn \& D. Dayan, 1984. Dental age evaluation. A new six-developmental-stage method. Clin Prevent Dent, 6, 18-22.

Gazdapusztai, G., 1957. A Körös kultúra lakótelepe Hódmezővásárhely-Gorzsán - La colonie d'habitation de la civilisation de Körös à HódmezővásárhelyGorzsa. Archaeológiai Értesítő, 84, 3-13.

Gazdapusztai, G., 1963. Későneolitkori telep és temető HódmezővásárhelyGorzsán. A Morá Ferenc Múzeum Évkönyve, 21-48.

Gernaey, A. M., D. E. Minnikin, M. S. Copley, A. M. S. Ahmed, D. J. Robertson, J. Nolan \& A. Chamberlain, 1999. Correlation of the occurrence of mycolic acids with tuberculosis in an archaeological population, in Tuberculosis, Past and Present, eds. G. Pálfi, O. Dutour, J. Deák \& I. Hutás, Golden Book Publisher Ltd., Tuberculosis Foundation, 275-82.

Gernaey, A. M., D. E. Minnikin, M. S. Copley, R. A. Dixon, J. C. Middleton \& C. Roberts, 2001. Mycolic acids and ancient DNA confirm an osteological diagnosis of tuberculosis. Tuberculosis, 81(4), 259-65.

Gilbert, B. M. \& T. W. McKern, 1973. A Method of Aging the Female Os pubis. American Journal of Physical Anthropology, 38, 31-8.

Giles, E., 1964. Sex determination by discriminant function analysis of the mandible. American Journal of Physical Anthropology, 22(2), 129-35.

Giles, E., 1970. Discriminant function sexing of the human skeleton, in Personal Identification in Mass Disasters, ed. T. D. Stewart, Washington: National Museum of Natural History, 99-107. 
Giles, E., 1991. Corrections for age in estimating older adults' stature from long bones. Journal of Forensic Sciences, 36, 898-901.

Giles, E. \& O. Elliot, 1962. Race identification from cranial measurements. Journal of Forensic Sciences, 7(2), 147-57.

Giles, E. \& O. Elliot, 1963. Sex determination by discriminant function analysis of crania. American Journal of Physical Anthropology, 21, 53-68.

Gindhart, P. S., 1973. Growth Standards for the Tibia and Radius in Children Aged One Month through Eighteen Years. American Journal of Physical Anthropology, 39, 41-8.

Gladykowska-Rzeczycka, J. J., 1999. Tuberculosis in the past and present in Poland, in Tuberculosis: Past and Present, eds. G. Pálfi, O. Dutour, J. Deák \& I. Hutás, Budapest / Szeged: Golden Book Publishers and Tuberculosis Foundation, 561-73.

Gladykowska-Rzeczycka, J. J. \& W. Prejzner, 1993. A case of probable pulmonary osteoarthropathy fom the Polish Mediaeval Cemetery of Czarna Wielka, District of Grodzisk. Journal of Paleopathology, 5, 159-65.

Grauer, A., 2012. Introduction: The Scope of Paleopathology, in A Companion to Paleopathology, ed. A. Grauer, Wiley-Blackwell, 1-14.

Graw, M., A. Czarnetzki \& H.-T. Haffner, 1999. The form of supraorbital margin as a criterion in identification of sex from the skull: Investigations based on modern human skulls. American Journal of Physical Anthropology, 108, 91-6.

Gray, H., 1967. Gray's Anatomy, London: Churchill Livingston.

Greulich, W. W. \& S. I. Pyle, 1950. Radiographic Atlas of Skeletal Development of the Hand and Wrist, Stanford: Stanford University Press.

Guba, Z., I. Szikossy \& I. Pap, 2007. Anthropological Analysis of the Human Skeletal Remains, in The Early Neolithic on the Great Hungarian Plain: Investigations of the Körös Culture site of Ecsegfalva 23, Country Békés., ed. A. Whittle, Budapest: Institute of Archaeology, Hungarian Academy of Sciences, 461-8.

Gülekon, I. N. \& H. B. Turgut, 2003. The external occipital protuberance: can it be used as a criterion in the determination of sex? Journal of Forensic Sciences, 48, 513-6.

Guilaine, J. \& J. Zammit, 2005. The Origins of War, Oxford: Blackwell Publishing.

Gustafson, G., 1966. Forensic Odontology, London: Staples Press.

Gustafson, G. \& G. Koch, 1974. Age estimation up to 16 years of age based on dental development. Odontologisk Revy, 25, 297-306.

Haavikko, K., 1970. The formation and the alveolar and clinical eruption of the permanent teeth. Proceedings of the Finnish Dental Society, 66, 101-70.

Hadjouis, D. \& B. Mafart, 2001. La Paléo-odontologie, analyses et méthodes d'étude, Paris: Artcom'.

Hägg, U. \& L. Matsson, 1985. Dental maturity as an indicator of chronological age: the accuracy and precision of three methods. European Journal of Orthodontics, 7, 25-34.

Hamman, A. K. \& N. E. Wang, 2006. Mastoiditis, in eMedicine. 
Hanihara, K., 1958. Sexual diagnosis of Japanese long bones by means of discriminant functions. Journal of Anthropological Society of Nippon, 66(4), 187-96.

Hanihara, K., 1959. Sex diagnosis of Japanese skulls and scapulae by means of discriminant functions. Journal of Anthropological Society of Nippon, 67(4), 191-7.

Hanna, R. E. \& S. L. Washburn, 1953. The determination of the sex of skeletons, as illustrated by a study of the Eskimo pelvis. Human Biology, 25, 21-7.

Haret, G., A. Dariaux \& J. Quenu, 1927. Atlas de radiographie du système osseux normal, Paris.

Harris, E. F. \& A. L. Buck, 2002. Tooth mineralization: A Technical Note on the Moorrees-Fanning-Hunt Standards. Dental Anthropology, 16(1), 15-21.

Harris, E. F. \& J. H. McKee, 1990. Tooth mineralization standards for Blacks and Whites from the middle southern United States. Journal of Forensic Sciences, 34, 859-72.

Haun, S. J., 2000. Brief communication: A study of the predictive accuracy of mandibular ramus flexure as a single morphologic indicator of sex in an archaeological sample. American Journal of Physical Anthropology, 111, 429-32.

Hawkey, D. E. \& C. F. Merbs, 1995. Activity-induced Musculoskeletal Stress Markers (MSM) and Subsistence Strategy Changes among Ancient Hudson Bay Eskimos. International Journal of Osteoarchaeology, 5(4), 324-38.

Henderson, C. Y., 2008. When hard work is disease: the interpretation of enthesopathies., in Proceedings of the Eighth Annual Conference of the British Association for Biological Anthropology and Osteoarchaeology, eds. M. Brickley \& M. Smith British Archaeological Reports: International Series, 17-25.

Henderson, C. Y., D. D. Craps, A. C. Caffell, A. R. Millard \& R. Gowland, 2013. Occupational Mobility in the 19th Century Rural England: The Interpretation of Entheseal Changes. International Journal of Osteoarchaeology, 23(2), 197-210.

Hens, S. M., E. Rastelli \& M. G. Belcastro, 2008. Age Estimation from the Human os Coxa: A Test on a Documented Italian Collection. Journal of Forensic Sciences, 53(5), 1040-3.

Hershkovitz, I., H. D. Donoghue, D. E. Minnikin, G. S. Besra, O. Y.-C. Lee, A. M. Gernaey, E. Galili, V. Eshed, C. L. Greenblatt, E. Lemma, G. K. Bar-Gal \& M. Spigelman, 2008. Detection and Molecular Characterization of 9000Year-Old Mycobacterium tuberculosis from a Neolithic Settlement in the Eastern Mediterranean. PLoS One, 3(10), e3426.

Hershkovitz, I., B. Latimer, O. Dutour, L. M. Jellema, S. Wish-Baratz, B. M. Rothschild \& C. Rothschild, 1997a. The Elusive Petroexoccipital Articulation. American Journal of Physical Anthropology, 103, 365-73.

Hershkovitz, I., B. Latimer, O. Dutour, L. M. Jellema, S. Wish-Baratz, C. Rothschild \& B. M. Rothschild, 1997b. Why Do We Fail in Aging the Skull From the 
Sagittal Suture? American Journal of Physical Anthropology, 103, 393-9. Hertelendi, E. \& F. Horváth, 1992. Radiocarbon Chronology of Late Neolithic Settlements in the Tisza-Maros Region, Hungary. Radiocarbon, 34(3), 859-66.

Hertelendi, E., É. Svingor, P. Raczky, F. Horváth, I. Futó \& L. Bartosiewicz, 1998a. Duration of Tell Settlements at four Prehistoric Sites in Hungary. Radiocarbon, 40(1-2).

Hertelendi, E., É. Svingor, P. Raczky, F. Horváth, I. Futó, L. Bartosiewicz \& M. Molnár, 1998b. Radiocarbon Chronology of the Neolithic and Time Span of Tell Settlements in Eastern Hungary Based on Calibrated Radiocarbon Dates, in Archaeometrical Research in Hungary II., eds. L. Költő \& L. Bartosiewicz, Budapest - Kaposvár - Veszprém, 61-9.

Hill, C. A., 2000. Technical note: Evaluating mandibular ramus flexure as a morphological indicator of sex. American Journal of Physical Anthropology, 111, 573-7.

Hillson, S., 1996. Dental Anthropology, Cambridge: Cambridge University Press.

Hillson, S., 2000. Dental Pathology, in Biological Anthropology of the Human Skeleton, eds. M. A. Katzenberg \& S. R. Saunders, Wiley-Liss, 249-86.

Hillson, S., 2005. Teeth, Cambridge: Cambridge University Press.

Himes, J. H., D. C. Yarbrough \& R. Martorell, 1977. Estimation of stature in children from radiographically determined metacarpal length. Journal of Forensic Sciences, 22, 452-6.

Hoffman, B. E., 1979. Age estimations from diaphyseal lengths: Two months to twelve years. Journal of Forensic Sciences, 24, 461-9.

Holcomb, S. M. C. \& L. Konigsberg, 1995. Statistical study of sexual dimorphism in the human fetal sciatic notch. American Journal of Physical Anthropology, 97, 113-25.

Holland, T. D., 1986. Sex determination of fragmentary crania by analysis of the cranial base. American Journal of Physical Anthropology, 70(2), 203-8.

Holt, C. A., 1978. A Re-examination of Parturition Scars on the Human Female Pelvis. American Journal of Physical Anthropology, 49(1), 91-4.

Holtby, J. R. D., 1918. Some indices and measurements of the modern femur. Journal of Anatomy, 52(4), 363-82.

Hoppa, R. D. \& S. Saunders, 1998. Two quantitative methods for rib seriation in human skeletal remains. Journal of Forensic Sciences, 43, 174-7.

Horváth, F., 1976. Deszk - Ordos. Régészeti Füzetek, 1, 5.

Horváth, F., 1982. A Gorzsai halom későneolit rétege. Archaeológiai Értesítő, 109, 201-22.

Horváth, F., 1987a. Hódmezővásárhely-Gorzsa - A tiszai kultúra települése, in $A$ Tisza-vidék késői neolitikuma, eds. L. Tálas \& L. Madaras, Szolnok: Szolnok Megyei Múzeumok Igazgatósága, 12-6.

Horváth, F., 1987b. Hódmezővásárhely-Gorzsa: A settlement of the Tisza culture, in The Late Neolithic of the Tisza Region, ed. P. Raczky, Budapest and Szolnok: Szolnok County Museums, 31-46.

Horváth, F., 2003a. Hódmezővásárhely-Gorzsa: A Late Neolithic Settlement in 
the Tisza Region, in Hungarian Archaeologz at the Turn of the Millennium, eds. Z. Visy, M. Nagy \& Z. B. Kiss, Budapest: Ministry of National Culture Heritage, Teleki László Foundation, 106-7.

Horváth, F., 2003b. The Neolithic in the Southern Part of the Great Hungarian Plain, in Hungarian Archaeology at the turn of the Millennium, eds. Z. Visy, M. Nagy \& Z. B. Kiss, Budapest: Ministry of National Cultural Heritage, Teleki László Foundation, 100-1.

Horváth, F., 2005a. Gorzsa. Előzetes eredmények az újkőkori tell 1978 és 1996 közötti feltárásából, in Hétköznapok Vénuszai, eds. L. Bende \& L. G., Hódmezővásárhely: Tornyai János Múzeum, Móra Ferenc Múzeum, 5183.

Horváth, F., 2005b. Gorzsa. Preliminary results of the Excavation of the Neolithic Tell between 1978-1996, in Hétköznapok Vénuszai, eds. L. Bende \& L. G., Hódmezővásárhely: Tornyai János Múzeum, Móra Ferenc Múzeum, 67-8.

Horváth, F., 2005c. Neolithic settlement under the Gorzsa mound (5th millennium BC), in Everyday Venuses, Late 7th millennium mid - 5th millennium BC, Guide to the Permanent Archaeological Exhibition of the Tornyai János Museum, eds. L. Bende \& G. Lőrinczy, Hódmezővásárhely: Móra Ferenc Museum, 27-43.

Horváth, F. \& E. Hertelendi, 1994. Contributions to the 14C based absolute chronology of the Early and Middle Neolithic Tisza region. A Nyíregyházi Jósa András Múzeum Évkönyve, 36, 111-33.

Houghton, P., 1974. The relationship of pre-auricular groove of the ilium to pregnancy. American Journal of Physical Anthropology, 41(3), 381-9.

Houghton, P., 1975. The bony imprint of pregnancy. Bulleting of the New York Academy of Medicine, 51(5), 655-61.

Howells, W. W., 1965. Détermination du sexe du bassin par fonction discriminante: Étude du matériel du Doctor Gaillard. Bulletins et Mémoires de la Société d'anthropologie de Paris, XI ${ }^{\circ}$ Série, 7(1), 95-105.

Humphrey, L., 1998. Growth Patterns in the Modern Human Skeleton. American Journal of Physical Anthropology, 105, 57-72.

Humphrey, L., 2003. Linear growth in the archaeological record, in Patterns of Growth and Development in the Genus Homo, eds. J. L. Thompson, G. E. Krovitz \& A. J. Nelson, Cambridge: Cambridge University Press, 144-69.

Humphrey, L. \& L. Scheuer, 2006. Age of Closure of the Foramen of Huschke: An Osteological Study. International Journal of Osteoarchaeology, 16, 47-60.

Hunt, D. R., 1990. Sex determination in the subadult ilia: an indirect test of Weaver's non-metric sexing method. Journal of Forensic Sciences, 35, 881-5.

Hunt, E. E. \& I. Gleiser, 1955. The estimation of age and sex of preadolescent children from bones and teeth. American Journal of Physical Anthropology, 13, 479-87.

Hurme, V. 0., 1948. Standards of variation in the eruption of the first six permanent teeth. Child Development, 19, 213-31.

Igarashi, Y., K. Uesu, T. Wakebe \& E. Kanazawa, 2005. New Method for 
Estimation of Adult Skeletal Age at Death From the Morphology of the Auricular Surface of the Ilium. American Journal of Physical Anthropology, 128, 324-39.

Iida, S. \& T. Matsuya, 2002. Paediatric maxillofacial fractures: their aetiological characters and fracture patterns. Journal of Cranio-Maxillofacial Surgery, 30, 237-41.

Introna Jr, F., G. Di Vella \& C. P. Campobasso, 1998. Sex determination by discriminant function analysis of patella measurements. Forensic Science International, 95, 39-45.

Introna Jr, F., G. Di Vella, C. P. Campobasso \& M. Dragone, 1997. Sex determination by discriminant analysis of calcanei measurements. Journal of Forensic Sciences, 42(4), 725-8.

Iordanidis, P., 1961. Détermination du sexe par les os du squelette. Annales de médecine légale, 41, 280-91.

İșcan, M. Y., 1989. Research strategies in age estimation: The multiregional approach, in Age Markers in the Human Skeleton, ed. M. Y. İșcan, Springfield: Charles C Thomas, 325-40.

İşcan, M. Y. \& K. Derrick, 1984. Determination of sex from the sacroiliac: a visual assessment technique. Florida Sci, 47, 94-8.

İșcan, M. Y. \& S. R. Loth, 1986a. Determination of age from the sternal rib in White females: A test of the phase method. Journal of Forensic Sciences, 31, 990-9.

İşcan, M. Y. \& S. R. Loth, 1986b. Determination of age from the sternal rib in White males: A test of the phase method. Journal of Forensic Sciences, 31, 122-32.

İşcan, M. Y. \& S. R. Loth, 1998. Estimation of age and determination of sex from the sternal rib, in Forensic osteology. Advances in the identification of human remains, ed. K. J. Reichs, Springfield, IL.: Charles C. Thomas, 68-89.

İşcan, M. Y., S. R. Loth, C. A. King, D. Shihai \& M. Yoshino, 1998. Sexual dimorphism in the humerus: a comparative analysis of Chinese, Japanese and Thais. Forensic Science International, 98, 17-29.

İşcan, M. Y., S. R. Loth \& R. K. Wright, 1984. Age estimation from the rib by phase analysis: White males. Journal of Forensic Sciences, 29, 1094-104.

İşcan, M. Y., S. R. Loth \& R. K. Wright, 1985. Age estimation from the rib by phase analysis: White females. Journal of Forensic Sciences, 30, 853-63.

İșcan, M. Y. \& P. Miller-Shaivitz, 1984a. Determination of sex from the femur in Blacks and Whites. Collegium Anthropologicum, 8(2), 169-77.

İscan, M. Y. \& P. Miller-Shaivitz, 1984b. Determination of sex from the Tibia. American Journal of Physical Anthropology, 64(1), 53-7.

İscan, M. Y. \& P. Miller-Shaivitz, 1984c. Discriminant Function Sexing of the Tibia. Journal of Forensic Sciences, 29(4), 1087-93.

İşcan, M. Y., M. Yoshino \& S. Kato, 1994. Sex determination from the Tibia: Standards for Contemporary Japan. Journal of Forensic Sciences, 39(3), 785-92.

Jackes, M., 2000. Building the Bases for Paleodemographic Analysis: Adult Age 
Determination, in Biological Anthropology of the Human Skeleton, eds. M. A. Katzenberg \& S. R. Saunders, Wiley-Liss, 417-66.

Jantz, R. L., D. R. Hunt \& L. Meadows, 1994. Maximum Length of the Tibia: How Did Trotter Measure It? American Journal of Physical Anthropology, 93, 525-8.

Jantz, R. L., D. R. Hunt \& L. Meadows, 1995. The measure and mismeasure of the tibia: Implications for stature estimation. Journal of Forensic Sciences, 40, 758-61.

Jantz, R. L., E. H. Kimmerle \& J. P. Baraybar, 2008. Sexing and Stature Estimation Criteria for Balkan Populations. Journal of Forensic Sciences, 53(3), 601-5.

Jantz, R. L. \& D. W. Owsley, 1984. Long bone growth variation among Arikara skeletal populations. American Journal of Physical Anthropology, 63, 1320.

Jeanes, A. L. \& I. Friedmann, 1960. Tuberculosis of the Middle Ear. Tubercle, 41, 109-16.

Jit, I., V. Jhingan \& M. Kulkarni, 1980. Sexing the Human Sternum. American Journal of Physical Anthropology, 53(2), 217-24.

Jit, I. \& S. Singh, 1966. The sexing of the adult clavicles. Indian Journal of Medical Research, 54, 551-71.

Johnson, D. R., P. O'Higgins, W. J. Moore \& T. J. McAndrew, 1989. Determination of race and sex of the human skull by discriminant function analysis of linear and angular dimensions. Forensic Science International, 41, 41-53.

Johnston, F. E., 1961. Sequence of Epiphyseal Union in a Prehistoric Kentucky Population from Indian Knoll. Human Biology, 33, 66-81.

Johnston, F. E., 1962. Growth of long bones of infants and young children at Indian Knoll. American Journal of Physical Anthropology, 20, 249-54.

Johnston, F. E., 1968. Growth of the skeleton in Earlier Peoples, in The Skeletal Biology of Earlier Human Populations, ed. D. R. Brothwell, London: Pergamon Press, 57-66.

Joshi, A. R., M. A. Lawande, S. I. Shaikh \& S. S. Nalavde, 2002. Tuberculous mastoiditis. Indian Journal of Otolaryngology and Head and Neck Surgery, 54(4), 299-300.

Jurmain, R., F. Alves Cardoso, C. Y. Henderson \& S. Villotte, 2012. Bioarchaeology's Holy Grail: The Reconstruction of Activity., in $A$ Companion to Paleopathology, ed. A. Grauer, New York: Wiley-Blackwell, 531-52.

Kaewlai, R., 2007. Imaging of Facial Trauma. Part 3: Pathology (Zygomatic, Maxillary and Mandibular Fractures), in www.RadiologyInThai.com.

Kalicz, N. \& P. Raczky, 1987. The Late Neolithic of the Tisza Region: A survey of recent archaeological research, in The Late Neolithic of the Tisza Region, eds. L. Tálas \& P. Raczky, Budapest and Szolnok: Szolnok County Museums, 11-29.

Kasson-Jones, D. A. \& S. K. Smith, 2008. Mastoiditis in Cretan Cranial Remains from the 1st - 2nd century A.D., in 35th Annual Meeting (North America) of the Paleopathology Association Columbus, Ohio. 
Katz, D. \& J. M. Suchey, 1986. Age determination of the Male os pubis. American Journal of Physical Anthropology, 69, 427-35.

Keen, E. N., 1953. Estimation of stature from the long bones. A discussion of its reliability. Journal of Forensic Medicine, 1, 46-51.

Keen, E. N., 1955. Estimation of stature from the long bones. Journal of Forensic Medicine, 2, 190-1.

Keen, J. A., 1950. Sex differences in skulls. American Journal of Physical Anthropology, 8(1), 65-79.

Kelley, M. A., 1979a. Parturition and pelvic changes. American Journal of Physical Anthropology, 51, 541-6.

Kelley, M. A., 1979b. Sex determination with fragmented remains. Journal of Forensic Sciences, 24, 154-8.

Kemkes, A. \& T. Göbel, 2006. Metric Assessment of the "Mastoid Triangle" for Sex Determination: A Validation Study. Journal of Forensic Sciences, 51(5), 985-9.

Kertész, R., 1996. The Mesolithic in the Great Hungarian Plain: A Survey of the Evidence, in At The Fringes of the Three Worlds: Hunter-gatherers and Farmers in the Middle Tisza Valley, ed. L. Tálas, Szolnok: Damjanich Museum Press, 5-34.

Kertész, R. \& P. Sümegi, 2001. Theories, critiques and a model: Why did the expansion of the Körös-Starčevo culture stop in the centre of the Carpathian Basin?, in From the Mesolithic to the Neolithic, eds. R. Kertész \& J. Makkay, Budapest, 225-46.

Kimmerle, E. H., L. Konigsberg, R. L. Jantz \& J. P. Baraybar, 2008. Analysis of Ageat-Death Estimation Through the Use of Pubic Symphyseal Data. Journal of Forensic Sciences, 53(3), 558-68.

Kimura, K., 1982a. A base-wing index for sexing the sacrum. Journal of Anthropological Society of Nippon, 90((Suppl)), 153-62.

Kimura, K., 1982b. Sex differences of the hip bone among several populations. Okajima's Folia Anatomica Japonica, 58, 266-73.

King, C. A., S. R. Loth \& M. Y. Iscan, 1998. Metric and comparative analysis of sexual dimorphism in the Thai femur. Journal of Forensic Sciences, 43, 954-8.

Klepinger, L. L., D. Katz, M. S. Micozzi \& L. Carroll, 1992. Evaluation of cast methods for estimating age from the os pubis. Journal of Forensic Sciences, 37(3), 763-70.

Knußmann, R. \& R. Martin (eds.), 1988. Anthropologie, Handbuch der vergleichenden Biologie des Menschen, Stuttgart: Gustav Fischer Verlag.

Kocsis, G. S., 1988. Hódmezővásárhely Gorzsa-Czukor major újkőkori temető embertani anyagának fogantropológiai és patológiai leírása. A Morá Ferenc Múzeum Évkönyve, 1987(1), 69-79.

Köhler, K., A. Marcsik, H. D. Donoghue, L. Mark \& T. Hajdu, 2009. Előzetes eredmények az Abony 36. lelőhely késő rézkori áldozati gödreiből feltárt embertani leletek vizsgálata alapján. Folia Anthropologica, 8, 41-6.

Köhler, K., G. Pálfi, E. Molnár, I. Zalai-Gaál, A. Osztás, E. Bánffy, K. Kirinó, K. K. 
Kiss \& B. G. Mende, 2012. A Late Neolithic Case of Pott's Disease from Hungary. International Journal of Osteoarchaeology.

Konigsberg, L. W., S. M. Hens, L. M. Jantz \& W. L. Jungers, 1998. Stature estimation and calibration: bayesian likelihood perspectives in physical anthropology. Yearbook of Physical Anthropology, 41, 65-92.

Korek, J., 1943. Az 1943. évi ószentiváni ásatások. Dolgozatok, 19, 208-11.

Kósa, F., 1989. Age estimation from the fetal skeleton, in Age Markers in the Human Skeleton, ed. M. Y. İşcan, Springfield: Charles C Thomas, 21-54.

Kósa, F. \& C. Castellana, 2005. New forensic anthropological approachment for the age determination of human fetal skeletons on the base morphometry of vertebral column. Forensic Science International, 147S, S69-S74.

Krogman, W. M., 1939. A guide to the identification of human skeletal material. FBI Law Enforcement Bull, 8(8), 1-29.

Krogman, W. M., 1955. The human skeleton in forensic medicine. Postgrad Med, 17(2), A48-A62.

Krogman, W. M., 1962. The human skeleton in forensic medicine, Springfield, IL: C.C. Thomas.

Krogman, W. M. \& M. Y. İșcan, 1986. The Human Skeleton in Forensic Medicine, Springfield: Thomas.

Kuczkowski, J., W. Narozny, C. Stankiewicz, B. Mikaszewski \& E. IzyckaSwieszewska, 2005. Zygomatic abscess with temporal myositis - a rare extracranial complication of acute otitis media. International Journal of Pediatric Otorhinolaryngology, 69(4), 555-9.

Kunos, C. A., S. W. Simpson, K. F. Russell \& I. Hershkovitz, 1999. First rib metamorphosis: Its possible utility for human age-at-death estimation. American Journal of Physical Anthropology, 110, 303-23.

Kurihara, Y., Y. Kurihara, K. Ohashi, A. Kitagawa, M. Miyasaka, E. Okamoto \& T. Ishikawa, 1996. Radiologic Evidence of Sex Differences: Is the Patient a Woman or a Man? American Journal of Radiology, 167, 1037-40.

Kurki, H., 2005. Use of the First Rib for Adult Age Estimation: A Test of One Method. International Journal of Osteoarchaeology, 15, 342-50.

Kurth, G., 1950. Über die Verwendbarkeit der Grablänge vor- und frühgeschichtlicher Reihengräberserien zur Bestimmung einer genauen Körperhöhe. Zeitschrift für Morphologie und Anthropologie, 42, 293-306.

Kustár, A. \& K. Árpás, 2005. Facial reconstruction of the Neolithic Woman from the Körös site at Deszk, Oil Well 1, in Hétköznapok vénuszai, eds. L. Bende \& G. Lőrinczy, Hódmezővásárhely: Móra Ferenc Múzeum (Szeged), 166.

Lazenby, R., 1994. Identification of Sex from Metacarpals: Effect of Side Asymmetry. Journal of Forensic Sciences, 39(5), 1188-94.

Le, B. T., E. J. Dierks, B. Ueeck, L. D. Homer \& B. F. Potter, 2001. Maxillofacial injuries associated with domestic violence. Journal of Oral and Maxillofacial Surgery, 59(11), 1277-83.

Lee, O. Y.-C., H. H. T. Wu, H. D. Donoghue, M. Spigelman, C. L. Greenblatt, I. D. Bull, B. M. Rothschild, L. D. Martin, D. E. Minnikin \& G. S. Besra, 2012. 
Mycobacterium tuberculosis Complex Lipid Virulence Factors Preserved in the 17,000-Year-Old Skeleton of an Extinct Bison, Bison antiquus. PLoS One, 7(7), e41923.

Lester, C. W. \& H. L. Shapiro, 1968. Vertebral Arch Defects in the Lumbar Vertebrae of Pre-historic American Eskimos. American Journal of Physical Anthropology, 28(1), 43-8.

Levesque, G.-Y., A. Demirjian \& R. Tanguay, 1981. Sexual Dimorphism in the Development, Emergence and Agenesis of the Mandibular Third Molar. Journal of Dental Research, 60, 1735-41.

Lewis, M. E., 2004. Endocranial Lesions in Non-adult Skeletons: Understanding their Aetiology. International Journal of Osteoarchaeology, 14, 82-97.

Lewis, M. E., 2007. The Bioarchaeology of Children, Perspectives from Biological and Forensic Anthropology, Cambridge: Cambridge University Press.

Lipták, P., 1975. Neolitikus Csontvázmaradványok Deszk mellett. A Morá Ferenc Múzeum Évkönyve, 1974-1975(1), 311-5.

Lipták, P. \& G. Farkas, 1967. A Békés-Povádzugi őskori és 10-12. Századi temető csontvázanyagának embertani vizsgálata. Anthropológiai Közlemények, XI(3-4), 127-63.

Listi, G. A. \& H. E. Bassett, 2006. Test of an Alternative Method for Determining Sex from the Os Coxae: Applications for Modern Americans. Journal of Forensic Sciences, 51(2), 248-52.

Liston, S. L., 1994. Ambroise Paré and the King's Mastoiditis. American Journal of Surgery, 167, 440-2.

Liu, W., 1989. Sex determination of Chinese femur by discriminate function. Journal of Forensic Sciences, 34, 1222-7.

Liversidge, H. M., 1994. Accuracy of age estimation from developing teeth of a population of known age ( 0 - 5.4 years). International Journal of Osteoarchaeology, 4, 37-45.

Liversidge, H. M., M. C. Dean \& T. Molleson, 1993. Increasing Human Tooth Length Between Birth and 5.4 years. American Journal of Physical Anthropology, 90, 307-13.

Liversidge, H. M., B. Herdeg \& F. W. Rösing, 1998. Dental age estimation of nonadults. A review of methods and principles, in Dental Anthropology, Fundamentals, Limits and Prospects, eds. K. W. Alt, F. W. Rösing \& M. Teschler-Nicola, Vienna: Springer, 419-42.

Liversidge, H. M., F. Lyons \& M. P. Hector, 2003. The accuracy of three methods of age estimation using radiographic measurements of developing teeth. Forensic Science International, 131, 22-9.

Liversidge, H. M. \& T. Molleson, 1999a. Deciduous tooth size and morphogenetic fields in children from Christ Church, Spitalfields. Archives of Oral Biology, 44, 7-13.

Liversidge, H. M. \& T. Molleson, 1999b. Developing permanent tooth length as an estimate of age. Journal of Forensic Sciences, 44(5), 917-20.

Liversidge, H. M. \& T. Molleson, 2004. Variation in crown and root formation and eruption of human deciduous teeth. American Journal of Physical 
Anthropology, 123, 172-80.

Logan, W. H. G. \& R. Kronfeld, 1933. Development of the human jaws and surrounding structures from birth to the age of fifteen years. Journal of the American Dental Association, 20, 379-427.

Loth, S. R. \& M. Henneberg, 1996. Mandibular ramus flexure: a new morphologic indicator of sexual dimorphism in the human skeleton. American Journal of Physical Anthropology, 99, 473-85.

Loth, S. R. \& M. Henneberg, 2001. Sexually dimorphic mandibular morphology in the first few years of life. American Journal of Physical Anthropology, 115, 179-86.

Loth, S. R. \& M. Y. İşcan, 1989. Morphological assessment of age in the adult: The thoracic region, in Age Markers in the Human Skeleton, ed. M. Y. İscan, Springfield: Charles C Thomas, 105-36.

Lovász, G., M. Schultz, J. Gödde, Z. Bereczki, G. Pálfi, A. Marcsik \& E. Molnár, 2013. Skeletal manifestations of infantile scurvy in a late medieval anthropological series from Hungary. Anthropological Science, Submitted manuscript.

Lovejoy, C. O., 1985. Dental Wear in the Libben Population: Its Functional Pattern and Role in the Determination of Adult Skeletal Age at Death. American Journal of Physical Anthropology, 68, 47-56.

Lovejoy, C. O., R. S. Meindl, R. P. Mensforth \& T. J. Barton, 1985a. Multifactorial Determination of Skeletal Age at Death: A Method and Blind Tests of Its Accuracy. American Journal of Physical Anthropology, 68, 1-14.

Lovejoy, C. O., R. S. Meindl, T. R. Pryzbeck \& R. P. Mensforth, 1985b.

Chronological Metamorphosis of the Auricular Surface of the Ilium: A New Method for the Determination of Adult Skeletal Age at Death. American Journal of Physical Anthropology, 68, 15-28.

Lovejoy, C. O., K. F. Russell \& M. L. Harrison, 1990. Long bone growth velocity in the Libben population. American Journal of Human Biology, 2, 533-42.

Lovell, N. C., 1989. Test of Phenice's technique for determining sex from the os pubis. American Journal of Physical Anthropology, 79, 117-20.

Maat, G. J. R., 2004. Scurvy in Adults and Youngsters: the Dutch Experience. A Review of the History and Pathology of a Disregarded Disease. International Journal of Osteoarchaeology, 14, 77-81.

Maat, G. J. R. \& R. W. Mastwijk, 1995. Fusion Status of the Jugular Growth Plate: an Aid for Age at Death Determination. International Journal of Osteoarchaeology, 5, 163-7.

MacCord, K., 2009. Human Skeletal Growth: Observations from Analyses of Three Skeletal Population. Faculty of Arts and Sciences, University of Pittsburgh.

MacLaughlin, S. M. \& M. F. Bruce, 1986. The sciatic notch / acetabulum index as a discriminator of sex in European skeletal remains. Journal of Forensic Sciences, 31, 1380-90.

MacLaughlin, S. M. \& M. F. Bruce, 1990. The accuracy of sex identification in European skeletal remains using the Phenice characters. Journal of Forensic Sciences, 35, 1384-92. 
Maczel, M., 2003. On the Traces of Tuberculosis: Diagnostic Criteria of Tuberculous Affection of the Human Skeleton and their Application in Hungarian and French Anthropological Series. University of La Méditerranée - Aix-Marseilles II, University of Szeged.

Makkay, J., 1996. Theories about the Origin, the Distribution and the End of the Körös Culture, in At the Fringes of Three Worlds: Hunter-gatherers and Farmers in the Middle Tisza Valley, ed. L. Tálas, Szolnok: Damjanich Museum Press, 35-49.

Mall, G., M. Graw, K. D. Gehring \& M. Hubig, 2000. Determination of sex from femora. Forensic Science International, 113, 315-21.

Mann, R. W. \& D. R. Hunt, 2005. Photographic Regional Atlas of Bone Disease, Springfield: Charles C. Thomas.

Manouvrier, L., 1892. Determination de la taille d'après les grands os des membres. Revue Mensuelle de l'École d'Anthropologie, 2, 227-33.

Manouvrier, L., 1893. La determination de la taille d'après les grands os des membres. Mémoires de la Société d'anthropologie, 2e série(4), 347-402.

Maples, W. R., 1989. The practical application of age estimation techniques, in Age Markers in the Human Skeleton, ed. M. Y. İșcan, Springfield: Charles C Thomas, 319-24.

Maresh, M., 1943. Growth of major long bones in healthy children. American Journal of Diseases of Children, 66, 227-57.

Maresh, M., 1955. Linear Growth of Long Bones of Extremities from Infancy Through Adolescence. American Journal of Diseases of Children, 89(3), 725-42.

Maresh, M., 1961. Bone, muscle and fat measurements. Longitudinal measurements of the bone, muscle and fat widths from roentgenograms of the extremities during the first six years of life. Paediatrics, 28, 971-84.

Maresh, M., 1970. Measurements from roentgenograms, heart size, long bone lengths, bone, muscle, and fat widths, skeletal maturation, in Human Growth and Development, ed. R. W. McCammon, Springfield: Charles C Thomas, 155-200.

Marks, M. K. \& M. D. Hamilton, 2007. Metastatic Carcinoma: Palaeopathology and Differential Diagnosis. International Journal of Osteoarchaeology, 17, 217-34.

Martin, B. C., J. C. Trabue \& T. R. Leech, 1956. An Analysis of the Etiology, Treatment and Complications of Fractures of the Malar Compound and Zygomatic Arch. American Journal of Surgery, 92, 920-4.

Martin, R., 1928. Lehrbuch der Anthropologie in systematischer Darstellung: Gustav Fisher.

Martínez-Lavín, M., 1997. Hypertrophic osteoarthropathy. Current Opinion in Rheumatology, 9, 83-6.

Martrille, L., D. H. Ubelaker, C. Cattaneo, F. Seguret, M. Tremblay \& E. Baccino, 2007. Comparison of Four Skeletal Methods for the Estimation of Age at Death on White and Black Adults. Journal of Forensic Sciences, 52(2), 3027. 
Masset, C., 1989. Age estimation on the basis of cranial sutures, in Age Markers in the Human Skeleton, ed. M. Y. İșcan, Springfield: Charles C Thomas, 71104.

Massler, M., I. Schour \& H. G. Poncher, 1941. Developmental pattern of the child as reflected in the calcification pattern of the teeth. American Journal of Diseases of Children, 62, 33-67.

Masson, M., 2002. Human Osteological Evidence for the Transition from HuntingGathering to Farming in Europe (unpublished). Undergraduate dissertation, Department of Archaeology, University of Edinburgh, Edinburgh.

Masson, M., E. Molnár, H. D. Donoghue, G. S. Besra, D. E. Minnikin, H. H. T. Wu, O. Y.-C. Lee, I. D. Bull \& G. Pálfi, 2013. Osteological and Biomolecular Evidence of a 7000-year-old case of Hypertrophic Pulmonary Osteopathy secondary to Tuberculosis from Neolithic Hungary. PLoS One. doi: 10.1371/journal.pone.0078252

Matos, V. \& A. L. Santos, 2006. On the Trail of Pulmonary Tuberculosis Based on Rib Lesions: Results From the Human Identified Skeletal Collection From the Museu Bocage (Lisbon, Portugal). American Journal of Physical Anthropology, 130, 190-200.

Mays, S., 1998. The Archaeology of Human Bones, London: Routledge.

Mays, S., 1999. Linear and appositional long bone growth in earlier human populations: a case study from Mediaeval England, in Humand Growth in the Past: Studies from bones and teeth, eds. R. D. Hoppa \& C. M. Fitzgerald, Cambridge: Cambridge University Press, 290-312.

Mays, S., 2002. The relationship between molar wear and age in an early 19th century AD archaeological human skeletal series of documented age at death. Journal of Archaeological Science, 29, 861-71.

Mays, S., 2007. Spondylolysis in the Lower Thoracic-upper Lumbar Spine in a British Medieval Population. International Journal of Osteoarchaeology, 17, 608-18.

Mays, S., 2008. A Likely Case of Scurvy from Early Bronze Age Britain. International Journal of Osteoarchaeology, 18, 178-87.

Mays, S., M. Brickley \& R. Ives, 2008. Growth in an English Population From the Industrial Revolution. American Journal of Physical Anthropology, 136, 85-92.

Mays, S. \& M. Cox, 2000. Sex determination in skeletal remains, in Human Osteology In Archaeology and Forensic Science, eds. M. Cox \& S. Mays, London: Greenwich Medical Media, 117-30.

Mays, S., C. de la Rua \& T. Molleson, 1995. Molar Crown Height as a Means of Evaluating Existing Dental Wear Scales for Estimating Age at Death in Human Skeletal Remains. Journal of Archaeological Science, 22, 659-70.

Mays, S. \& G. M. Taylor, 2002. Osteological and Biomolecular Study of Two Possible Cases of Hypertrophic Osteoarthropathy from Medieval England. Journal of Archaeological Science, 29, 1267-76.

McCormick, W. F., J. H. Stewart \& L. A. Langford, 1985. Sex Determination From 
Chest Plate Roentgenograms. American Journal of Physical Anthropology, 68, 173-95.

McKern, T. W. \& T. D. Stewart, 1957. Skeletal Age Changes in Young American Males Analyzed from the Standpoint of Identification, Natick, Massachusetts.

Meadows, L. \& L. M. Jantz, 1995. Allometric secular change in the long bones from the 1800 s to the present. Journal of Forensic Sciences, 40, 762-7.

Meadows, L. \& R. L. Jantz, 1992. Estimation of stature from metacarpal lengths. Journal of Forensic Sciences, 37, 147-54.

Meher, R., I. Singh, S. P. S. Yadav \& G. Gathwala, 2006. Tubercular otitis media in children. Otolaryngology - Head and Neck Surgery, 135, 650-2.

Mehta, L. \& H. M. Singh, 1972. Determination of crown-rump length from fetal long bones. American Journal of Physical Anthropology, 36, 165-8.

Meindl, R. S. \& C. O. Lovejoy, 1985. Ectocranial suture closure: a revised method for the determination of skeletal age at death based on the lateralanterior sutures. American Journal of Physical Anthropology, 68, 57-66.

Meindl, R. S. \& C. O. Lovejoy, 1989. Age changes in the pelvis: Implications for paleodemography, in Age Markers in the Human Skeleton, ed. M. Y. İșcan, Springfield: Charles C Thomas, 137-68.

Meindl, R. S., C. O. Lovejoy \& R. P. Mensforth, 1983. Skeletal Age at Death: Accuracy of Determination and Implications for Human Demography. Human Biology, 55(1), 73-87.

Meindl, R. S., C. O. Lovejoy, R. P. Mensforth \& L. D. Carlos, 1985a. Accuracy and Direction of Error in the Sexing of the Skeleton: Implications for Paleodemography. American Journal of Physical Anthropology, 68, 79-85.

Meindl, R. S., C. O. Lovejoy, R. P. Mensforth \& P. L. Walker, 1985b. A Revised Method of Age Determination Using the Os Pubis, With a Review and Tests of Accuracy of Other Current Methods of Pubic Symphyseal Aging. American Journal of Physical Anthropology, 68, 29-45.

Menk, R. \& J. Nemeskéri, 1989. The Transition from Mesolithic to Early Neolithic in Southeastern and Eastern Europe: An Anthropological Outline, in People and Culture in Change, Proceedings of the Second Symposium on Upper Palaeolithic, Mesolithic and Neolithic Populations of Europe and the Mediterranean Basin, ed. I. Hershkovitz, Oxford, 531-40.

Mensforth, R. P., 1985. Relative tibia long bone growth in the Libben and Bt-5 prehistoric skeletal populations. American Journal of Physical Anthropology, 68, 247-62.

Merbs, C. F., 1983. Patterns of Activity-induced Pathology in a Canadian Inuit Population, Ottawa: National Museums of Canada.

Merbs, C. F., 1996. Spondylolysis and spondylolisthesis: A Cost of Being an Erect Biped or a Clever Adaptation? American Journal of Physical Anthropology, 101(S23), 201-28.

Merbs, C. F., 2002a. Asymmetrical Spondylolysis. American Journal of Physical Anthropology, 119, 156-74.

Merbs, C. F., 2002b. Spondylolysis in Inuit Skeletons from Arctic Canada. 
International Journal of Osteoarchaeology, 12, 279-90.

Merchant, V. L. \& D. H. Ubelaker, 1977. Skeletal growth of the protohistoric Arikara. American Journal of Physical Anthropology, 46, 61-72.

Miles, A. E. W., 1962. Assessment of the Ages of a Population of Anglo-Saxons from Their Dentitions. Proceedings of the Royal Society of Medicine, 55, 881-6.

Mincer, H. H., E. F. Harris \& H. E. Berryman, 1993. The A.B.F.O. study of third molar development and its use as an estimator of chronological age. Journal of Forensic Sciences, 38, 379-90.

Minnikin, D. E., O. Y.-C. Lee, H. H. T. Wu, G. S. Besra \& H. D. Donoghue, 2012. Molecular biomarkers for ancient tuberculosis., in Understanding Tuberculosis - Deciphering the Secret Life of the Bacilli., ed. P.-J. Cardona, Rijeka, Croatia.: InTech - Open Access Publisher, 1-36.

Mittler, D. M. \& S. G. Sheridan, 1992. Sex determination in subadults using auricular surface morphology: a forensic science perspective. Journal of Forensic Sciences, 37, 1068-75.

Mogle, P. \& J. Zias, 1995. Trephination as a Possible Treatment for Scurvy in a Middle Bronze Age (ca. 2200 BC) Skeleton. International Journal of Osteoarchaeology, 5, 77-81.

Molleson, T. \& M. Cox, 1993. The Spitalfields Project, in CBA Research Report, ed. C. f. B. Archaeology.

Molleson, T., K. Cruse \& S. Mays, 1998. Some sexually dimorphic features of the human juvenile skull and their value in sex determination in immature skeletal remains. Journal of Archaeological Science, 25, 719-28.

Molnár, E., A. Marcsik, Z. Bereczki, T. H. Schmidt-Schultz, M. Schultz \& G. Pálfi, 2009. Malignant tumors in ostearchaeological samples from Hungary. Acta Biologica Szegediensis, 53(2), 117-24.

Molnar, S., 1971. Human tooth wear, tooth function, and cultural variability. American Journal of Physical Anthropology, 34, 175-90.

Molnár, S. \& I. Molnár, 1985. Observations of Dental Diseases Among Prehistoric Populations of Hungary. American Journal of Physical Anthropology, 67, 51-63.

Montagu, J. D., 1994. Length of life in the ancient world: a controlled study. Journal of the Royal Society of Medicine, 87(January), 25-6.

Moorrees, C. F. A., E. A. Fanning \& E. E. J. Hunt, 1963. Formation and Resorption of Three Deciduous Teeth in Children. American Journal of Physical Anthropology, 21, 205-13.

Müller, G., 1935. Zur Bestimmung der Länge beschadigter Extremitätenknochen. Anthropologischer Anzeiger, 12, 70-2.

Mulhern, D. M. \& E. B. Jones, 2005. Test of Revised Method of Age Estimation From the Auricular Surface of the Ilium. American Journal of Physical Anthropology, 126, 61-5.

Muller-Bolla, M., L. Lupi-Pegurier, G. Quatrehomme, A. M. Velly \& M. Bolla, 2003. Age estimation from teeth in children and adolescents. Journal of Forensic Sciences, 48, 140-8. 
Murphy, T., 1959. The changing pattern of dentine exposure in human tooth attrition. American Journal of Physical Anthropology, 17, 167-78.

Murray, K. A. \& T. A. Murray, 1991. A test of auricular surface ageing techniques. Journal of Forensic Sciences, 36, 1162-9.

Musgrave, J. H. \& N. K. Harneja, 1978. The Estimation of Adult Stature from Metacarpal Bone Length. American Journal of Physical Anthropology, 48, 113-20.

Mustafa, A., M. Wiorowski, P. Schultz \& C. Debry, 2004. La mastoïdite aiguë tuberculeuse : Un diagnostic rare. A propos d'un cas. Revue de laryngologie, d'otologie et de rhinologie, 125(3), 171-3.

Nemeskéri, J., 1972. Die archaeologischen und antrhopologischen Voraussetzungen palaeodemographischer forschungen. Praehistorische Zeitschrift, 47, 5-46.

Nemeskéri, J., L. Harsanyi \& G. Acsádi, 1960. Methoden zur Diagnose der Lebensalters von Skelettfunden. Anthropologischer Anzieger, 24, 70-95.

Norris, S. P., 2002. Mandibular ramus height as an indicator of human infant age. Journal of Forensic Sciences, 47, 8-11.

Nyström, M. \& H. Ranta, 2003. Tooth formation and the Mandibular symphisis during the first five postnatal months. Journal of Forensic Sciences, 48(373-378).

O'Connell, L., 2004. Guidance on recording age at death in adults, in Guidelines to the Standards for Recording Human Remains, eds. M. Brickley \& J. I. McKinley, Southampton and Reading: BABAO and the Institute of Field Archaeologists, 18-20.

Oettlé, A. C. \& M. Styn, 2000. Age estimation from sternal ends of ribs by phase analysis in South African Blacks. Journal of Forensic Sciences, 45, 1071-9.

Ogden, A., 2008. Advances in the Palaeopathology of Teeth and Jaws, in Advances in Human Palaeopathology, eds. R. Pinhasi \& S. Mays, Chichester: John Wiley and Sons, 283-308.

Okazaki, K., 2004. A morphological study on the growth patterns of ancient people in the northern Kyushu-Yamaguchi region, Japan. Anthropological Science, 112(3), 219-34.

Oliveira, R. N., S. F. S. M. Silva, A. Kawano \& J. L. F. Antunes, 2006. Estimating Age by Tooth Wear of Prehistoric Human Remains in Brazilian Archaeological Sites. International Journal of Osteoarchaeology, 16, 40714.

Olivier, G., 1969. Practical Anthropology, Springfield: Thomas.

Olivier, G. \& H. Pineau, 1958. Détermination de l'âge du foetus et de l'embryon. Archives d'anatomie (La Semaine des hôpitaux), 6, 21-8.

Olivier, G. \& H. Pineau, 1960. Nouvelle détermination de la taille foetale d'après les longueurs diaphysaires des os longs. Annales de médecine légale, 40, 141-4.

Olze, A., P. v.Niekerk, R. Schulz \& A. Schmeling, 2007. Studies of the Chronological Course of Wisdom Tooth Eruption in a Black African Population. Journal of Forensic Sciences, 52(5), 1161-3. 
Ortner, D. J., 2003. Identifications of Pathological Conditions in Human Skeletal Remains, San Diego: Academic Press, Elsevier Science.

Ortner, D. J., 2008. Differential Diagnosis of Skeletal Lesions in Infectious Disease, in Advances in Human Palaeopathology, eds. R. Pinhasi \& S. Mays, Chichester: John Wiley and Sons, 191-214.

Ortner, D. J., 2012. Differential Diagnosis and Issues in Disease Classification, in A Companion to Paleopathology, ed. A. Grauer, Wiley-Blackwell, 250-67.

Ortner, D. J. \& M. F. Ericksen, 1997. Bone changes in the human skull probably resulting from scurvy in infancy and childhood. International Journal of Osteoarchaeology, 7 212-20.

Ortner, D. J. \& B. D. Ragsdale, 2007. Paleopathology Association Workshop \#18: Bone Lesions: Basic Pathogenesis and Anatomy, in 34th Annual Meeting (North America) of the Paleopathology Association Philadelphia, PA.

Osborne, D. L., T. L. Simmons \& S. P. Nawrocki, 2004. Reconsidering the Auricular Surface as an Indicator of Age at Death. Journal of Forensic Sciences, 49(5).

Otuyemi, O. D., V. I. Ugboko, K. C. Ndukwe \& C. A. Adekoya-Sofowora, 1997. Eruption times of third molars in young rural Nigerians. International Dental Journal, 47, 266-70.

Owers, S. K. \& R. F. Pastor, 2005. Analysis of quantitative methods for rib seriation using the Spitalfields documented skeletal collection. American Journal of Physical Anthropology, 127, 210-8.

Owsley, D. W. \& R. S. Webb, 1983. Misclassification probability in dental discrimination functions for sex determination. Journal of Forensic Sciences, 28, 181-5.

Pálfi, G. \& E. Molnár, 2009. The Paleopathology of specific infectious diseases from Southeastern Hungary: a brief overview. Acta Biologica Szegediensis, 53(2), 111-6.

Palkama, A., P. Virtama \& A. Telkkä, 1962. Estimation of Stature from Radiographs of Long Bones in Children. II, Children under one year of age. Annales Medicinae Experimentalis et Biologiae Fenniae, 40, 219-22.

Paluch, T. \& K. Tóth, 2005. Everyday Venuses, in Everyday Venuses, Late 7 th millennium mid - 5th millennium $B C$, Guide to the Permanent Archaeological Exhibition of the Tornyai János Museum, eds. L. Bende \& G. Lőrinczy, Hódmezővásárhely: Móra Ferenc Museum, 7-26.

Park, S.-H., E.-K. Goh, S.-K. Kong \& J.-K. Lee, 2010. Tuberculous otitis media with facial nerve paralysis in an infant: A case of maternal transmission. Otolaryngology - Head and Neck Surgery, 142, 774-5.

Parker, J. L., 1994. Acute Mastoiditis, Bobby R.Alford Department of Otolaryngology - Head and Neck Surgery.

Parsons, F. G., 1914. The characters of the English thigh-bone: I. Journal of Anatomy and Physiology, 48(3rd Series Vol.9), 238-67.

Parsons, F. G., 1915. The characters of the English thigh-bone: II. The Difficulty of Sexing. Journal of Anatomy and Physiology, 49(4)(Series 10), 345-61.

Patel, M. M., B. D. Gupta \& T. C. Singel, 2005. Sexing of sacrum by sacral index 
and Kimura's base-wing's index. Journal of Indian Academy of Forensic Medicine, 27(1), 5-9.

Pearson, K., 1899. IV. Mathematical contributions to the theory of evolution. V. On the reconstruction of the stature of prehistoric races. Philosophical Transactions of the Royal Society, Series A(192), 169-244.

Pearson, K. \& J. Bell, 1917/1919. A study of the long bones of the English skeleton. I. The femur., in Company Research, Memoirs, ed. D. o. A. Statistics, University of London: University College.

Petersen, H. C., 2005. On the Accuracy of Estimating Living Stature from Skeletal Length in the Grave and by Linear Regression. International Journal of Osteoarchaeology, 15, 106-14.

Pfitzner, W., 1901. Der Einfluss des Geschleschts auf die anthropologischen charaktere. Zeitschrift für Morphol Anthropol, 3, 385-575.

Phenice, T. W., 1969. A Newly Developed Visual Method of Sexing the Os Pubis. American Journal of Physical Anthropology, 30, 297-302.

Piercecchi-Marti, M. D., P. Adalian, B. Bourliere-Najean, J. Gouvernet, M. Maczel, O. Dutour \& G. Leonetti, 2002. Validation of a radiographic method to establish new fetal growth standrads: radio-anatomical correlation. Journal of Forensic Sciences, 47, 328-31.

Pinhasi, R. \& S. Mays (eds.), 2008. Advances in Human Palaeopathology, Chichester: John Wiley and Sons.

Pinhasi, R. \& M. Pluciennik, 2004. A Regional Biological Approach to the Spread of Farming in Europe. Current Anthropology, 45(Supplement), S59-S82.

Pinhasi, R., P. Shaw, B. White \& A. Ogden, 2006. Morbidity, rickets and long-bone growth in post-medieval Britain-a cross-population analysis. Annals of Human Biology, 33, 372-89.

Piontek, J., B. Jerszyńska \& S. Segeda, 2001. Long bones growth variation among prehistoric agricultural and pastoral populations from Ukraine (Bronze Era to Iron Age). Variability and Evolution, 9, 61-73.

Pons, J., 1955. The sexual diagnosis of isolated bones of the skeleton. Human Biology, 27(1), 12-21.

Pósa, A., F. Maixner, A. R. Zink, G. Lovász, E. Molnár, Z. Bereczki, P. Perrin, O. Dutour, C. Sola \& G. Pálfi, 2012. Ancient human tooth samples used for TB paleomicrobial research. Acta Biologica Szegediensis, 56(2), 125-31.

Powell, T. V. \& A. G. Brodie, 1963. Closure of the Spheno-occipital Synchondrosis. The Anatomical Record, 147(1), 15-23.

Prince, D. A., E. H. Kimmerle \& L. Konigsberg, 2008. A Bayesian Approiach to Estimate Skeletal Age-at-Death Utilizing Dental Wear. Journal of Forensic Sciences, 53(3), 588-93.

Purkait, R., 2001. Measurements of ulna - a new method for determination of sex. Balkans. Journal of Forensic Sciences, 46, 924-7.

Putschar, W. G. J., 1976. The structure of the human symphisis pubis with special consideration of parturition and its sequelae. American Journal of Physical Anthropology, 45, 589-94.

Ragsdale, B. D. \& L. M. Lehmer, 2012. A Knowledge of Bone at the Cellular 
(Histological) Level is Essential to Paleopathology, in A Companion to Paleopathology, ed. A. Grauer, Wiley-Blackwell, 227-49.

Rauber, A. \& F. Kopsch, 1952. Lehrbuch und Atlas der Anatomie des Menschen, Leipzig.

Raxter, M. H., B. M. Auerbach \& C. B. Ruff, 2006. Revision of the Fully Technique for Estimating Statures. American Journal of Physical Anthropology, 130(3), 374-84.

Raxter, M. H., C. B. Ruff \& B. M. Auerbach, 2007. Technical Note: Revised Fully Stature Estimation Technique. American Journal of Physical Anthropology, 133, 817-8.

Raxter, M. H., C. B. Ruff, A. Azab, M. Erfan, M. Soliman \& A. El-Sawaf, 2008. Stature Estimation in Ancient Egyptians: A New Technique Based on Anatomical Reconstruction of Stature. American Journal of Physical Anthropology, 136(2), 147-55.

Redfield, A., 1970. A New Aid to Aging Immature Skeletons: Development of the Occipital Bone. American Journal of Physical Anthropology, 33, 207-20.

Redman, J. E., M. J. Shaw, A. I. Mallet, A. L. Santos, C. Roberts, A. M. Gernaey \& D. E. Minnikin, 2009. Mycocerosic acid biomarkers for the diagnosis of tuberculosis in the Coimbra Skeletal Collection. Tuberculosis, 89, 267-77.

Reimer, P. J., M. G. L. Baillie, E. Bard, A. Bayliss, J. W. Beck, C. J. Bertrand, P. G. Blackwell, C. E. Buck, G. S. Burr, K. B. Cutler, P. E. Damon, R. L. Edwards, R. G. Fairbanks, M. Friedrich, T. P. Guilderson, A. G. Hogg, K. A. Hughen, B. Kromer, G. McCormac, S. Manning, C. Bronk Ramsey, R. W. Reimer, S. Remmele, J. R. Southon, M. Stuiver, S. Talamo, F. W. Taylor, J. van der Plicht \& C. E. Weyhenmeyer, 2004. IntCal04 terrestrial radiocarbon age calibration, 0-26 cal kyr BP. Radiocarbon, 46(3), 1029-58.

Reynolds, E., 1945. The Bony Pelvic Girdle in Early Infancy. A roentgenometric study. American Journal of Physical Anthropology, 3, 321-54.

Reynolds, E., 1947. The bony pelvis in prepubertal children. American Journal of Physical Anthropology, 5, 165-200.

Ribot, I. \& C. Roberts, 1996. A Study of Non-specific Stress Indicators and Skeletal Growth in Two Medieval Subadult Populations. Journal of Archaeological Science, 23, 67-79.

Riepert, T., T. Drechsler, H. Schild, B. Nafe \& R. Mattern, 1996. Estimation of sex on the basis of radiographs of the calcaneus. Forensic Science International, 77, 133-40.

Ríos, L. \& H. F. V. Cardoso, 2009. Age Estimation From Stages of Union of the Vertebral Epiphyses of the Ribs. American Journal of Physical Anthropology, early view.

Rissech, C. \& S. Black, 2007. Scapular Development from the Neonatal Period to Skeletal Maturity: A Preliminary Study. International Journal of Osteoarchaeology, 17, 451-64.

Rissech, C., G. F. Estabrook, E. Cunha \& A. Malgosa, 2006. Using the Acetabulum to Estimate Age at Death. Journal of Forensic Sciences, 51(2), 213-29.

Rissech, C., G. F. Estabrook, E. Cunha \& A. Malgosa, 2007. Estimation of Age-at- 
Death for Adult Males Using the Acetabulum, Applied to Four Western European Populations. Journal of Forensic Sciences, 52(4), 774-8.

Rissech, C., M. Garcia \& A. Malgosa, 2003. Sex and age diagnosis by ischium morphometric analysis. Forensic Science International, 135, 188-96.

Roberts, C., 1999. Rib lesions and tuberculosis: the current state of play, in Tuberculosis, Past and Present, eds. G. Pálfi, O. Dutour, J. Deák \& I. Hutás, Golden Book Publisher Ltd., Tuberculosis Foundation, 311-6.

Roberts, C. \& M. Cox, 2003. Health and Disease in Britain, From Prehistory to the Present Day, Stroud: Sutton Publishing.

Roberts, C. \& K. Manchester, 1995. The Archaeology of Disease: Sutton Publishing.

Robling, A. G. \& D. H. Ubelaker, 1997. Sex Estimation from the Metatarsals. Journal of Forensic Sciences, (November 1997), 1062-9.

Rogers, T. L., 1999. A visual method of determining the sex of skeletal remains using the distal humerus. Journal of Forensic Sciences, 44, 57-60.

Rogers, T. L., 2005. Determining the sex of Human Remains Through Cranial Morphology. Journal of Forensic Sciences, 50(3).

Rogers, T. L., 2009. Sex determination of adolescent skeletons using the distal humerus. American Journal of Physical Anthropology, 140(1), 143-8.

Rogers, T. L. \& S. Saunders, 1994. Accuracy of sex determination using morphological traits of the human pelvis. Journal of Forensic Sciences, 39, 1047-56.

Rollet, E., 1888. De la mensuration des os longs des membres. . Thesis pour le doctorat en médecine, 1st series(43), 1-128.

Rösing, F. W., 1988. Körperhöhenrekonstruktion aus Skelettmaßen, in Anthropologie. Handbuch der vergleichenden Anthropologie des Menschen, ed. R. Knußmann, Stuttgart: Fischer, 586-600.

Ross, A. H. \& L. Konigsberg, 2002. New formulae for estimating stature in the Balkans. Journal of Forensic Sciences, 47, 165-7.

Rothschild, B. M. \& C. Rothschild, 1998. Recognition of Hypertrophic Osteoarthropathy in Skeletal Remains. Journal of Rheumatology, 25, 2221-7.

Rothschild, B. M. \& C. Rothschild, 1999. Evolution of osseous / radiologic signs of tuberculosis, in Tuberculosis, Past and Present, eds. G. Pálfi, O. Dutour, J. Deák \& I. Hutás, Golden Book Publisher Ltd., Tuberculosis Foundation, 293-8.

Rougé-Maillart, C., N. Telmon, C. Rissech, A. Malgosa \& D. Rougé, 2004. The Determination of Male Adult Age at Death by Central and Posterior Coxal Analysis - A Preliminary Study. Journal of Forensic Sciences, 49(2), ??

Ruff, C. B., 2007. Body Size Prediction From Juvenile Skeletal Remains. American Journal of Physical Anthropology, 133, 698-716.

Russell, K. F., S. W. Simpson, J. Genovese, M. D. Kinkel, R. S. Meindl \& C. 0. Lovejoy, 1993. Independent test of the fourth rib aging technique. American Journal of Physical Anthropology, 92, 53-62.

Sahni, D., I. Jit, Neelam \& S. Suri, 1998. Time of fusion of the basisphenoid with 
the basilar part of the occipital bone in northwest Indian subjects. Forensic Science International, 98, 41-5.

Salter, R. B., 1999. Textbook of Disorders and Injuries of the Musculoskeletal System, Baltimore: Lippincott Williams \& Wilkins.

Santos, A. L. \& C. Roberts, 2001. A Picture of Tuberculosis in Young Portuguese People in the Early 20th Century: A Multidisciplinary Study of the Skeletal and Historical Evidence. American Journal of Physical Anthropology, 115, 38-49.

Saunders, E., 1837. Teeth as a Test of Age, Considered with Reference to the Factory Children: Addressed to the Members of bouth Houses of Parliament, London: Renshaw.

Saunders, S. R., 2000. Subadult Skeletons and Growth-Related Studies, in Biological Anthropology of the Human Skeleton, eds. M. A. Katzenberg \& S. R. Saunders, Wiley-Liss, 135-61.

Saunders, S. R., 2008. Juvenile Skeletons and Growth-related Studies, in Biological Anthropology of the Human Skeleton, eds. M. A. Katzenberg \& S. R. Saunders, Hoboken, New Jersey: John Wiley \& Sons, 117-47.

Saunders, S. R., C. M. Fitzgerald, T. L. Rogers, J. C. Dudar \& H. McKillop, 1992. A test of several methods of skeletal age estimation using a documented archaeological sample. Canadian Society of Forensic Science Journal, 25, 97-117.

Saunders, S. R., R. D. Hoppa \& R. Southern, 1993. Diaphyseal growth in a nineteenth century skeletal sample of subadults from St. Thomas' Church, Belleville, Ontario. International Journal of Osteoarchaeology, 3, 265-81.

Scammon, R. E. \& L. A. Calkins, 1929. The Development and Growth of the External Dimensions of the Human Body in the Fetal Period, Minneapolis: University of Minnesota Press.

Schaefer, M. C., 2008. A Summary of Epiphyseal Union Timings in Bosnian Males. International Journal of Osteoarchaeology, Early View, ?

Schaefer, M. C. \& S. Black, 2007. Epiphyseal Union Sequencing: Aiding in the Recognition and Sorting of Commingled Remains. Journal of Forensic Sciences, 52(2), 277-85.

Schaefer, M. C., S. Black \& L. Scheuer, 2009. Juvenile Osteology: A Laboratory and Field Manual: Academic Press.

Scheuer, L., 2002. Brief communication: a blind test of mandibular morphology for sexing mandibles in the first few years of life. American Journal of Physical Anthropology, 119, 189-91.

Scheuer, L. \& S. Black, 1980. The estimation of late fetal and perinatal age from limb bone length by linear and logarithimic regression. Annals of Human Biology, 7(257-265).

Scheuer, L. \& S. Black, 2000. Developmental Juvenile Osteology, London: Academic Press.

Scheuer, L. \& S. Black, 2004. The Juvenile Skeleton, London: Elsevier Academic Press. 
Scheuer, L. \& N. M. Elkington, 1993. Sex determination from metacarpals and the first proximal phalanx. Journal of Forensic Sciences, 38(4), 769-78.

Scheuer, L. \& S. M. MacLaughlin-Black, 1994. Age estimation from the pars basilaris of the fetal and juvenile occipital bone. International Journal of Osteoarchaeology, 4, 377-80.

Schmidt-Schultz, T. H. \& M. Schultz, 2004. Bone Protects Proteins Over Thousands of Years: Extraction, Analysis, and Interpretation of Extracellular Matrix Proteins in Archeological Skeletal Remains. American Journal of Physical Anthropology, 123, 30-9.

Schour, I. \& M. Massler, 1940. Studies in tooth development: the growth pattern of human teeth. Journal of the American Dental Association, 27, 1778-92.

Schour, I. \& M. Massler, 1941. The Development of the Human Dentition. Journal of the American Dental Association, 28, 1153-60.

Schulter-Ellis, F. P., L. C. Hayek \& D. J. Schmidt, 1983. Determination of sex with a discriminate analysis of new pelvic bone measurements : part I. Journal of Forensic Sciences, 28(1), 169-80.

Schulter-Ellis, F. P., L. C. Hayek \& D. J. Schmidt, 1985. Determination of sex with a discriminate analysis of new pelvic bone measurements : part II. Journal of Forensic Sciences, 30(1), 178-85.

Schulting, R., A. Marcsik \& I. Pap, 2007. The Evidence of Teeth, in The Early Neolithic of the Great Hungarian Plain: Investigations of the Körös culture site of Ecsegfalva 23, County Békés, ed. A. Whittle, Budapest: Archaeological Institute of the Hungarian Academy of Sciences, 469-89.

Schultz, M., M. Berner \& T. H. Schmidt-Schultz, 2004. Preliminary Results on Morbidity and Mortality in the Late PPNB Population from Basta, Jordan., in Central Settlements in Neolithic Jordan, eds. H. D. Bienert, H. G. K. Gebel \& R. Neef, Berlin: Ex oriente, 259-69.

Schutkowski, H., 1993. Sex determination of Infant and Juvenile Skeletons: I. Morphognostic Features. American Journal of Physical Anthropology, 90, 199-205.

Sciulli, P. W. \& S. H. Blatt, 2008. Evaluation of Juvenile Stature and Body Mass Prediction. American Journal of Physical Anthropology, 136(4), 387-93.

Scoles, P. V., R. Salverno, K. Villalba \& D. Riew, 1988. Relationship of the iliac crest to skeletal and chronological age. Journal of Pediatric Orthopaedics, 8, 639-44.

Scott, E. C., 1979. Dental wear scoring technique. American Journal of Physical Anthropology, 51, 213-8.

Sherwood, R. J., 2000. Fetal Age: Methods of Estimation and Effects of Pathology. American Journal of Physical Anthropology, 113, 305-15.

Shirley, N. L. \& J. D. Bethard, 2007. A Test of the Buckberry and Chamberlain Method for Auricular Surface Aging, in 76th Annual Meeting of the AAPA Philadelphia.

Shourie, K. L., 1946. Eruption age of teeth in India. Indian Journal of Medical Research, 34, 105-18.

Singh, G. \& S. P. Singh, 1976. Identification of sex from the fibula. Journal of the 
Indian Academy of Forensic Sciences, 15, 29-34.

Sjøvold, T., 1978. Inference concerning the age distribution of skeletal populations and some consequences for paleodemography. Anthropológiai Közlemények, 22, 99-114.

Sjøvold, T., 1990. Estimation of stature from long bones utilizing the line of organic correlation. Human Evolution, 5(5), 431-47.

Šlaus, M., D. Strinović, J. Škavić \& V. Petrovečki, 2003. Discriminant function sexing of fragmentary and complete femora: standards for contemporary Croatia. Journal of Forensic Sciences, 48, 509-12.

Smith, B. H., 1984. Patterns of Molar Wear in Hunter-Gatherers and Agriculturalists. American Journal of Physical Anthropology, 63(1), 39-56.

Smith, B. H., 1991. Standards of human tooth formation and dental age assessment, in Advances in dental anthropology, eds. M. A. Kelly \& C. S. Larsen, New York: Wiley-Liss, 143-68.

Smith, M. \& M. Brickley, 2009. People of the Long Barrows: Life, Death and Burial in the Earlier Neolithic, Stroud: The History Press.

Smith, S., 1939. Forensic Medicine, Boston: Little, Brown Co.

Smith, S. L., 1996. Attribution of hand bones to sex and population groups. Journal of Forensic Sciences, 41(3), 469-77.

Smith, S. L., 1997. Attribution of foot bones to sex and population groups. Journal of Forensic Sciences, 42(2), 186-95.

Smith, S. L., 2007. Stature Estimation of 3-10 Year-Old Children from Long Bone Lengths. Journal of Forensic Sciences, 52(3), 538-46.

Snodgrass, J. J. \& A. Galloway, 2003. Utility of pits and pubic tubercle height in parity assessment. Journal of Forensic Sciences, 48, 1226-30.

Spekker, O., G. Pálfi, G. Kozocsay, A. Pósa, Z. Bereczki \& E. Molnár, 2012. New cases of probable skeletal tuberculosis from the Neolithic period in Hungary - A morphological study. Acta Biologica Szegediensis, 56(2), 11523.

Spiegel, J. H., L. R. Lustig, K. C. Lee, A. H. Murr \& R. A. Schindler, 1998. Contemporary presentation and management of a spectrum of mastoid abscesses. Laryngoscope, 108(6), 822-8.

Steckel, R. H., C. S. Larsen, P. W. Sciulli \& P. L. Walker, 2005. Data Collection Codebook, The Global History of Health Project.

Steel, F. L. D., 1972. The sexing of the long bones, with reference to the St. Bride series of identified skeletons. Journal of the Royal Anthropological Institute, 92, 212-22.

Steele, D. G., 1970. Estimation of stature from fragments of long limb bones, in Personal Identification in Mass Disasters, ed. T. D. Stewart, Washington: Smithsonian Institution, 85-97.

Steele, D. G. \& T. W. McKern, 1969. A method for assessment of maximum long bone length and living stature from fragmentary long bones. American Journal of Physical Anthropology, 31, 215-28.

Steele, G., 1976. The Estimation of Sex on the Basis of the Talus and Calcaneus. American Journal of Physical Anthropology, 45, 581-8. 
Stermer Beyer-Olsen, E. M. \& S. Risnes, 1994. Radiographic Analysis of Dental Development Used in Age Determination of Infant and Juvenile Skulls from a Medieval Archaeological Site in Norway. International Journal of Osteoarchaeology, 4, 299-303.

Stevenson, P. H., 1924. Age order of epiphyseal union in man. American Journal of Physical Anthropology, 7, 53-93.

Stevenson, P. H., 1929. On racial differences in stature long bone regression formulae, with special reference to stature reconstruction formulae for the Chinese. Biometrika, 21, 303-21.

Stewart, J. H. \& W. F. McCormick, 1983. The gender predictive value of sternal length. American Journal of Forensic Medicine and Pathology, 4(3), 21720.

Stewart, T. D., 1934. Sequence of epiphyseal union, third molar eruption and sutrue closure in Eskimos and American Indians. American Journal of Physical Anthropology, 19, 433-52.

Stewart, T. D., 1948. Medico-legal aspects of the skeleton. I. Age, sex, race and stature. American Journal of Physical Anthropology, 6(3), 315-21.

Stewart, T. D., 1951. What the bones tell. FBI Law Enforcement Bull, 20(2), 2-5, 19.

Stewart, T. D., 1954. Evaluation of evidence from the skeleton, in Legal Medicine, ed. R. B. H. Gradwohl, St. Louis: Mosby.

Stewart, T. D., 1958. The rate of development of vertebral osteoarthritis in American Whites and its significance in skeletal age identification. The Leech, 28(3-5), 144-51.

Stewart, T. D., 1970. Identification of the scars of parturition in the skeletal remains of females, in Personal Identification in Mass Disasters, ed. T. D. Stewart, Washington: National Museum of Natural History, 127-35.

Stewart, T. D., 1976. Identification by the Skeletal Structures, in Gradwohl's Legal Medicine, ed. F. E. Camps, Chicago: Year Book Medical Publishers, 109-35.

Stewart, T. D., 1979. Essentials of Forensic Anthropology, Springfield, IL: Charles C Thomas.

Steyn, M. \& M. Henneberg, 1996. Skeletal growth of children from the Iron Age site at K2 (South Africa). American Journal of Physical Anthropology, 100, 389-96.

Steyn, M. \& M. Y. Iscan, 1997. Sex determination from the femur and tibia in South African whites. Forensic Science International, 90, 111-9.

Steyn, M. \& M. Y. Iscan, 1999. Osteometric variation in the humerus: sexual dimorphism in South Africans. Forensic Science International, 106, 7785.

Steyn, M. \& M. Y. İşcan, 1998. Sexual dimorphism in the crania and mandibles of South African whites. Forensic Science International, 98, 9-16.

Stloukal, M. \& H. Hanáková, 1978. Die Lange der Langsknochen altslawischer Bevölkerungen - Unter besondered Berücksichtigung von Wachstumsfragen. Homo, 29, 53-69. 
Stojanowski, C. M., 1999. Sexing potential of fragmentary and pathological metacarpals. American Journal of Physical Anthropology, 109, 245-52.

Strádalová, V., 1974. Determination from metrical characteristics of the sacrum. Folia Morphologica, 22, 408-12.

Strádalová, V., 1975. Sex differences and sex determination on the sacrum. Anthropologie, 13, 237-44.

Straus, W. L., 1927. The human ilium: Sex and stock. American Journal of Physical Anthropology, 11, 1-28.

Strouhal, E. \& H. Kritscher, 1990. Neolithic Case of a Multiple Myeloma from Mauer (Vienna, Austria). . Anthropologie, 28, 78-87.

Suchey, J. M., 1979. Problems in the aging of females using the os pubis. American Journal of Physical Anthropology, 51(3), 467-70.

Suchey, J. M. \& D. Katz, 1998. Applications of pubic age determination in a forensic setting, in Forensic Osteology, ed. K. J. Reichs, 204-36.

Suchey, J. M., P. A. Owings Webb, D. V. Wiseley \& T. T. Noguchi, 1984. Skeletal Aging of Unidentified Persons, in Human Identification: Case Studies in Forensic Anthropology, eds. T. A. Rathbun \& J. E. Buikstra, Springfield, Illinois: Charles C. Thomas, 278-97.

Suchey, J. M., D. V. Wiseley, R. F. Green \& T. T. Noguchi, 1979. Analysis of dorsal pitting in the os pubis in an extensive sample of modern American females. American Journal of Physical Anthropology, 51, 517-40.

Sümegi, P., R. Kertész, I. Juhász, G. Tímár \& S. Gulyás, 2003. Mesolithic/Neolithic transition in the Carpathian Basin - was there an ecological trap during the Neolithic?, in The Fifth World Archaeological Congress, Past Human Environments in Modern Contexts Washington D.C.

Sulzmann, C. E., J. L. Buckberry \& R. F. Pastor, 2008. The Utility of Carpals for Sex Assessment: A Preliminary Study. American Journal of Physical Anthropology, 135, 252-62.

Sundick, R. I., 1972. Human skeletal growth and dental development as observed in the Indian Knoll population. PhD dissertation, University of Toronto, Toronto.

Sundick, R. I., 1977. Age and Sex determination of subadult skeletons. Journal of Forensic Sciences, 22(1), 141-4.

Sundick, R. I., 1978. Human skeletal growth and age determination. Homo, 29, 228-49.

Sutherland, L. D. \& J. M. Suchey, 1991. Use of the ventral arc in pubic sex determination. Journal of Forensic Sciences, 36(2), 501-11.

Sutter, R. C., 2003. Nonmetric subadult skeletal sexing traits: I. A blind test of the accurfacy of eight previously proposed methods using prehistoric known-sex mummies from Northern Chile. Journal of Forensic Sciences, 48, 927-35.

Symes, S. A. \& R. L. Jantz, 1983. Discriminant fuction sexing of the tibia, in 35th Annual Meeting of the American Academy of Forensic Sciences Cincinnati.

Szathmáry, L., 1987. An Attempt at the Allowance for Human Genetics Aspects at the Quantitative Comparison of Craniological Samples. A Debreceni 
Déri Múzeum Évkönyvé, 1986, 73-85.

Tálas, L. (ed.) 1996. At the Fringes of Three Worlds: Hunter-gatherers and Farmers in the Middle Tisza Valley, Szolnok: Damjanich Museum Press.

Tanner, J. M., P. C. R. Hughs \& R. H. Waterhouse, 1981. Radiographically determined widths of bone muscle and fat in the upper arm and calf from age 3-18 years. Annals of Human Biology, 8, 495-517.

Tanner, J. M., R. H. Whitehouse \& M. Takaishi, 1966. Standards from birth to maturity for height, weight, height velocityand weight velocity. Archives of Disease in Childhood, 41, 454-71 and 613-35.

Taylor, G. M., S. Blau, S. Mays, M. Monot, O. Y.-C. Lee, D. E. Minnikin, G. S. Besra, S. T. Cole \& P. Rutland, 2009. Mycobacterium leprae genotype amplified from an archaeological case of lepromatous leprosy in Central Asia. Journal of Archaeological Science, 36, 2408-14.

Telkkä, A., 1950. On the prediction of human stature from the long bones. Acta Anatomica, 9, 103-17.

Telkkä, A., A. Palkama \& P. Virtama, 1962. Estimation of Stature from Radiographs of Long Bones in Children. I, Children Aged from One to Nine. Annales Medicinae Experimentalis et Biologiae Fenniae, 40, 91-6.

Temple, D. H., 2007. Human Biological Variation During the Agricultural Transition in Prehistoric Japan. Department of Anthropology, The Ohio State University.

Temple, D. H., 2008. What Can Variation in Stature Reveal About Environmental Differences Between Prehistoric Jomon Foragers? Understanding the Impact of Systemic Stress on Developmental Stability. American Journal of Human Biology, 20, 431-9.

Thieme, F. P., 1957. Sex in Negro skeletons. Journal of Forensic Medicine, 4, 7281.

Thomson, A., 1899. Sexual differences of the foetal pelvis. Journal of Anatomy, 33(3), 359-80.

Titche, L. L., S. W. Coulthard, R. D. Wachter, A. C. Thies \& L. L. Harries, 1981. Prevalence of Mastoid Infection in Prehistoric Arizona Indians. American Journal of Physical Anthropology, 56, 269-73.

Todd, T. W., 1920. Age changes in the pubic bone. American Journal of Physical Anthropology, 3(3), 285-339.

Todd, T. W., 1930a. Age Changes in the Pubic Bone American Journal of Physical Anthropology, 14, 225-72.

Todd, T. W., 1930b. The anatomical features of epiphyseal union. Child Dev, 1(3), 186-94.

Todd, T. W. \& A. Lindala, 1928. Dimensions of the body; Whites and American Negroes of both sexes. American Journal of Physical Anthropology, 12(1), 35-119.

Todd, T. W. \& J. Lyon, D.W., 1924. Endocranial suture closure, its progress and age relationship. Part I. Adult males of White stock. American Journal of Physical Anthropology, 7, 325-84.

Todd, T. W. \& J. Lyon, D.W., 1925a. Cranial suture closure, its progress and age 
relationship. Part II. Ectocranial closure in adult males of White stock. American Journal of Physical Anthropology, 8, 23-45.

Todd, T. W. \& J. Lyon, D.W., 1925b. Cranial suture closure, its progress and age relationship. Part III. Endocranial closure in adult males of Negro stock. American Journal of Physical Anthropology, 8, 47-71.

Todd, T. W. \& J. Lyon, D.W., 1925c. Cranial suture closure, its progress and age relationship. Part IV. Ectocranial closure in adult males of Negro stock. American Journal of Physical Anthropology, 8, 149-68.

Tomenchuk, J. \& J. T. Mayhall, 1979. A Correlation of Tooth Wear and Age Among Modern Iglooik Eskimos. American Journal of Physical Anthropology, 51, 67-78.

Tóth, T., 1987. Men and nutrition in the Carpathian postglacial millennia. Annales Historico-Naturales Musei Nationalis Hungarici, 79, 281-92.

Trancho, G. J., B. Robledo, I. Lopez-Bueis \& J. A. Sanchez, 1997. Sexual determination of the femur using discrimate functions. Analysis of a Spanish population of known sex and age. Journal of Forensic Sciences, 42, 181-5.

Trogmayer, 0., 1959. Archaeologische Forschungen im Jahre 1958. BékésPovádzug. Archaeológiai Értesítő, 86, 213.

Trogmayer, 0., 1962. X-XII. századi Magyar temető Békésen. A Morá Ferenc Múzeum Évkönyve, 1960-1962, 9-38.

Trogmayer, 0., 1969. Die Bestattung der Körös - Gruppe. A Morá Ferenc Múzeum Évkönyve, 2, 5-15.

Trotter, M. \& G. C. Gleser, 1951. Trends in stature of American Whites and Negroes born between 1840 and 1924. American Journal of Physical Anthropology, 9, 427-40.

Trotter, M. \& G. C. Gleser, 1952. Estimation of stature from long bones of American Whites and Negroes. American Journal of Physical Anthropology, 10(4), 463-514.

Trotter, M. \& G. C. Gleser, 1958. A re-evaluation of stature based on measurements taken during life and long bones after death. American Journal of Physical Anthropology, 16(1), 79-124.

Trotter, M. \& G. C. Gleser, 1977. Corrigenda to "Estimation of Stature from Long Limb Bones of American Whites and Negroes," American Journal of Physical Anthropology (1952). American Journal of Physical Anthropology, 47(2), 355-6.

Tupman, G. S., 1962. A study of bone growth in normal children and its relationship to skeletal maturation. Journal of Bone and Joint Surgery, 44B, 42-67.

Ubelaker, D. H., 1978. Human Skeletal Remains: Excavation, Analysis, Interpretation, Chicago: Aldine.

Ubelaker, D. H., 1982. The Development of American Paleopathology, in $A$ History of American Physical Anthropology, 1930-1980Academic Press, 337-55.

Ubelaker, D. H., 1984. Human Skeletal Remains: Excavation, Analysis, 
Interpretation, Washington: Taraxacum.

Ubelaker, D. H., 1989a. The estimation of age at death from immature human bone, in Age Markers in the Human Skeleton, ed. M. Y. İşcan, Springfield: Charles C Thomas, 55-70.

Ubelaker, D. H., 1989b. Human Skeletal Remains: Excavation, Analysis, Interpretation, Washington D.C.: Taraxacum.

Ubelaker, D. H. \& I. Pap, 1998. Skeletal Evidence for Health and Disease in the Iron Age of Northeastern Hungary. International Journal of Osteoarchaeology, 8, 231-51.

Ubelaker, D. H. \& I. Pap, 2009. Skeletal Evidence for Morbidity and Mortality in Copper Age Samples from Northeastern Hungary. International Journal of Osteoarchaeology, 19, 23-35.

Ubelaker, D. H., I. Pap \& S. Graver, 2006. Morbidity and Mortality in the Neolithic of Northeastern Hungary. Anthropologie, 44(3), 241-57.

Ubelaker, D. H. \& C. G. Volk, 2002. A Test of the Phenice Method for the Estimation of Sex. Journal of Forensic Sciences, 47, 19-24.

Ullrich, H., 1975. Estimation of fertility by means of pregnancy and childbirth alterations at the pubis, ilium and the sacrum. Ossa, 2, 23-39.

Vaamonde, P., C. Castro, N. Garcia-Soto, T. Labella \& A. Lozano, 2004. Tuberculous otitis media: A significant diagnostic challenge. Otolaryngology - Head and Neck Surgery, 130(6), 759-66.

Vallois, H. V., 1960. Vital Statistics in Prehistoric Populations as Determined from Archaeological Data, in The Application of Quantitative Methods in Archaeology, eds. R. F. Heizer \& S. F. Cook, Chicago: Quadrangle Books, 186-204.

Vercellotti, G., A. M. Agnew, H. M. Justus \& P. W. Sciulli, 2009. Stature Estimation in an Early Medieval (XI-XII c.) Polish Population: Testing the Accuracy of Regression Equations in a Bioarcheological Sample. American Journal of Physical Anthropology, Early view.

Verneau, R., 1875. Le bassin dans les sexes et dans les races, Paris: J.B. Bailliere.

Villemin, F., 1937. La différentiation sexuelle précoce de la grande échancrure sciatique, et son retentissement sur l'évolution du bassin chez les hommes. Strasbourg médical, 33, 593-625.

Villotte, S., 2006. Connaissances médicales actuelles, cotation des enthésopathies : nouvelle méthode. Bulletins et Mémoires de la Société d'anthropologie de Paris, Nouvelle Série, 18(1-2), 65-85.

Villotte, S., D. Castex, V. Couallier, O. Dutour, C. J. Knüsel \& D. Henry-Gambier, 2010. Enthesopathies as Occupational Stress Markers: Evidence From the Upper Limb. American Journal of Physical Anthropology, 142, 224-34.

Villotte, S. \& C. J. Knüsel, 2013. Understanding Entheseal Changes: Definition and Life Course Changes. International Journal of Osteoarchaeology, 23(2), 135-46.

Virtama, P., R. Kiviluoto, A. Palkama \& A. Telkkä, 1962. Estimation of Stature from Radiographs of Long Bones in Children. III, Children aged ten to fifteen. Annales Medicinae Experimentalis et Biologiae Fenniae, 40, 283-5. 
Visser, E. P., 1998. Little Waifs: Estimating Child Body Size from Historic Skeletal Material. International Journal of Osteoarchaeology, 8, 413-23.

Vital, V., A. Printza \& T. Zaraboukas, 2002. Tuberculous Otitis Media: A Difficult Diagnosis and Report of Four Cases. Pathology Research and Practice, 198, 31-5.

Vlak, D., M. Roksandic \& M. A. Schillaci, 2008. Greater Sciatic Notch as a Sex Indicator in Juveniles. American Journal of Physical Anthropology, 137(3), 309-15.

Waldron, T., 1991. Variations in the rate of Spondylolysis in Early Populations. International Journal of Osteoarchaeology, 1, 63-5.

Waldron, T., 1992. Unilateral Spondylolysis. International Journal of Osteoarchaeology, 2, 177-81.

Waldron, T., 2009. Palaeopathology, Cambridge: Cambridge University Press.

Walker, P. L., 1969. The linear growth of long bones in Late Woodland Indian children. Proceedings of the Indiana Academy of Science, 78, 83-7.

Walker, P. L., 2005. Greater sciatic notch morphology: sex, age and population differences. American Journal of Physical Anthropology, 127, 385-91.

Walker, P. L., 2008. Sexing Skulls Using Discrimant Function Analysis of Visually Assessed Traits. American Journal of Physical Anthropology, 136, 39-50.

Walker, R. A. \& C. O. Lovejoy, 1985. Radiographic Changes in the Clavicle and Proximal Femur and Their Use in the Determination of Skeletal Age at Death. American Journal of Physical Anthropology, 68, 67-78.

Walrath, D., P. Turner \& J. Bruzek, 2004. Reliability test of the visual assessment of cranial traits for sex determination. American Journal of Physical Anthropology, 125, 132-7.

Warnaar, A., G. Snoep \& F. S. Stals, 1989. A swollen cheek, an unusual course of acute mastoiditis. International Journal of Pediatric Otorhinolaryngology, 17(2), 179-83.

Warren, M. W., 1999. Radiographic determination of developmental age in fetuses and stillborns. Journal of Forensic Sciences, 44, 708-12.

Washburn, S. L., 1948. Sex differences in the pubic bone. American Journal of Physical Anthropology, 6, 199-207.

Washburn, S. L., 1949. Sex differences in the pubic bone of Bantu and Bushman. American Journal of Physical Anthropology, 7(3), 425-32.

Watanabe, S. \& K. Terazawa, 2006. Age estimation from the degree of osteophyte formation of vertebral columns in Japanese. Legal Medicine, 8(3), 156-60.

Weaver, D. S., 1980. Sex differences in the ilia of a known age and sex sample of fetal and infant skeletons. American Journal of Physical Anthropology, 52, 191-5.

Webb, J. G. \& P. Thomas, 1986. Hypertrophic Osteoarthropathy and Pulmonary Tuberculosis. Tubercle, 67, 225-8.

Webb, P. A. O. \& J. M. Suchey, 1985. Epiphyseal Union of the Anterior Iliac Crest and Medial Clavcle in a Modern Multiracial Sample of American Males and Females. American Journal of Physical Anthropology, 68, 457-66. 
Weiss, E., 2009. Spondylolysis in a Pre-Contact San Francisco Bay Population: Behavioural and Anatomical Sex Differences. International Journal of Osteoarchaeology, 19, 375-85.

White, T. D. \& P. A. Folkens, 2000. Human Osteology, San Diego: Academic Press.

White, T. D. \& P. A. Folkens, 2005. The Human Bone Manual, San Diego: Elsevier Academic Press.

Whittle, A., 1996. Europe in the Neolithic, The creation of new worlds, Cambridge: Cambridge University Press.

Whittle, A., 2003. The archaeology of people: dimensions of Neolithic life, London: Routledge.

WHO, 2013. Tuberculosis. World Health Organization Fact sheet $N^{\circ} 104$, Reviewed February 2013.

Williams, B. A. \& T. L. Rogers, 2006. Evaluating the Accuracy and Precision of Cranial Morphological Traits for Sex Determination. Journal of Forensic Sciences, 51(4), 729-35.

Wilson, L. A., N. MacLeod \& L. Humphrey, 2008. Morphometric Criteria for Sexing Juvenile Human Skeletons Using the Ilium. Journal of Forensic Sciences, 53(2), 269-78.

Wolff-Heidegger, G., 1954. Atlas der systematischen Anatomie des Menschen, Basel.

Xinzhi, W., S. Xinzhou \& W. Heng, 1982. Sex differences and sex determination of the innominate bone of modern Han Nationality. Acta Anthropologica Sinica, 1, 118-31.

Y'Edynak, G., 1976. Long bone growth in western Eskimo and Aleut skeletons. American Journal of Physical Anthropology, 45, 569-74.

Yamamoto, K., K. Murakami, T. Sugiura, M. Fujimoto, M. Noue, M. Kawakami, K. Ohgi \& T. Kirita, 2007. Clinical analysis of isolated zygomatic arch fractures. Journal of Oral and Maxillofacial Surgery, 65(3), 457-61.

Yerkes, R. W., A. Gyucha \& W. Parkinson, 2009. A Multiscalar approach to modeling the end of the Neolithic on the Great Hungarian Plain using Calibrated Radiocarbon dates. Radiocarbon, 51(3), 1071-109.

Yoder, C., D. H. Ubelaker \& J. F. Powell, 2001. Examination of variation in sternal rib end morphology relevant to age assessment. Journal of Forensic Sciences, 46, 223-7.

Zakrzewski, S. R. \& A. Di-Loreto, 2008. Growth and development in medieval Écija (Spain). American Journal of Physical Anthropology, 135(S46), 2278.

Zimmermann, C. E., M. J. Troulis \& L. B. Kaban, 2006. Pediatric facial fractures: recent advances in prevention, diagnosis and management. International Journal of Oral and Maxillofacial Surgery, 35, 2-13.

Zink, A. R., C. J. Haas, U. Reischl, U. Szeimies \& A. G. Nerlich, 2001. Molecular analysis of skeletal tuberculosis in an ancient Egyptian population. Journal of Medical Microbiology, 50, 355-66.

Zink, A. R., E. Molnár, N. Motamedi, G. Pálfi, A. Marcsik \& A. G. Nerlich, 2007. Molecular History of Tuberculosis from Ancient Mummies and Skeletons. 
International Journal of Osteoarchaeology, 17, 380-91.

Zoffmann, Z. K., 1980. Eine Übersicht über das Anthropologische Material der Neolithischen und Kupferzeitlichen Kulturen im Karpatenbecken. Alba Regia, 18, 9-29.

Zoffmann, Z. K., 1984. A Kárpát-medence neolitikus és rézkori embertani leleteinek főbb metrikus és taxonómiai jellemzői. Anthropológiai Közlemények, 28, 79-90.

Zoffmann, Z. K., 1992. Kelet Kárpát-medence Neolitikus és rézkori népességeinek embertani vázlata. Kandidátusi értekezés, Budapest.

Zoffmann, Z. K., 1994. A kelet kárpát-medence neolitikus és rézkori népességeinek embertani vázlata. Anthropológiai Közlemények, 36, 7984.

Zoffmann, Z. K., 2000. Anthropological sketch of the prehistoric population of the Carpathian Basin. Acta Biologica Szegediensis, 44(1-4), 75-9.

Zoffmann, Z. K., 2005. Anthropological Data to the Biological and Historical Reconstruction of the Neolithic of the Southern Part of the Great Hungarian Plain, in Hétköznapok Vénuszai, eds. L. Bende \& G. Lőrinczy, Hódmezővásárhely: Tornyai János Múzeum, Móra Ferenc Múzeum, 1515.

Zuhrt, R., 1955. Stomatologische Untersuchungen an Spätmittelalterlichen Funden von Reckkahn (12-14 Jh.) I. Die Zahnkaries und ihre Folgen. Deutsche Zahn-, Mund-, und Kieferheilkunde, 25, 1-15. 